\title{
Highly Chemoselective, Transition-Metal-Free Transamidation of Unactivated Amides and Direct Amidation of Alkyl Esters by $\mathrm{N}-\mathrm{C} / \mathrm{O}-\mathrm{C}$ Cleavage
}

\author{
Guangchen $\mathrm{Li}^{\dagger}{ }^{\dagger}$ Chong-Lei Ji, ${ }^{\ddagger}$ Xin Hong ${ }^{\ddagger} * *$ and Michal Szostak ${ }^{\S, \dagger, *}$ \\ ${ }^{\S}$ College of Chemistry and Chemical Engineering and Key Laboratory of Auxiliary Chemistry and \\ Technology for Chemical Industry, Ministry of Education, Shaanxi University of Science and Technology, \\ Xi'an 710021, China \\ ${ }^{\dagger}$ Department of Chemistry, Rutgers University, 73 Warren Street, Newark, NJ 07102, USA \\ ${ }^{\ddagger}$ Department of Chemistry, Zhejiang University, Hangzhou 310027, China \\ hxchem@zju.edu.cn; michal.szostak@rutgers.edu \\ Supporting Information
}

Table of Contents 1

Computational Details $\quad 2$

Computed Relative Stabilities of (LiHMDS) 2 and (LiHMDS) 3

Thermodynamics of Ketene Formation 3

DFT-Calculated Resonance Energies of Amides 3

DFT-Calculated Free Energies of the Rate-Determining Nucleophilic Addition Step 4

DFT-Calculated Reactivity Scale for the Selected Amides using the Resting State 4

Energies of the Structures Calculated at the M06-2X/6-311+G(d,p)-SMD(toluene)// 5

B3LYP-D3(BJ)/6-31G(d) Level of Theory

Cartesian Coordinates of the Structures 9

List of Known Compounds/General Methods 171

Experimental Procedures and Characterization Data 172

Mechanistic Studies Referred to from the Main Manuscript 174

Optimization Studies Referred to from the Main Manuscript 177

Characterization Data of Starting Materials 186

Characterization Data of Products 196

$\begin{array}{ll}\text { References } & 238\end{array}$

HPLC Traces $\quad 241$

${ }^{1} \mathrm{H}$ and ${ }^{13} \mathrm{C}$ NMR Spectra $\quad 244$

\section{Corresponding Author:}

Department of Chemistry, Zhejiang University

Hangzhou 310027, China

E-mail: $\underline{\text { hxchem@zju.edu.cn }}$

Department of Chemistry, Rutgers University

73 Warren Street, Newark, NJ 07102, United States

E-mail: michal.szostak@ rutgers.edu 


\section{Computational Details}

All density functional theory (DFT) calculations were performed with Gaussian 09 software package. ${ }^{1}$ Geometry optimizations were carried out at the B3LYP ${ }^{2,3}$ level of theory with Grimme's D3(BJ) empirical dispersion correction, ${ }^{4}$ with the 6-31G(d) basis set for all atoms. Vibrational frequencies were computed at the same level of theory to evaluate its zero-point vibrational energy (ZPVE) and thermal corrections at $298 \mathrm{~K}$, and to check whether each optimized structure is an energy minimum or a transition state. On the basis of the gas-phase optimized structures, the single point energies were calculated with the M06-2X level of theory ${ }^{5}$ and $6-311+G(d, p)$ for all atoms. Solvation energy corrections were calculated using the SMD model $^{6}$ with toluene as the solvent. The $3 \mathrm{D}$ diagrams of computed species were generated using CYLView. ${ }^{7}$ 


\section{Computed Relative Stabilities of (LiHMDS) 2 and (LiHMDS) 3}

The experimental determination of LiHMDS oligomer-dimer equilibrium indicated a 1:11 ratio in favor of dimer form at $-80{ }^{\circ} \mathrm{C}$ on $0.1 \mathrm{M}$ toluene solution of LiHMDS. ${ }^{8}$ This refers to a 0.32 $\mathrm{kcal} / \mathrm{mol}$ free energy preference for LiHMDS dimer.

[dimer] : [trimer] $=11: 1$ (the oligomers are all treated as trimer for simplification purpose)

[dimer $]=0.044 \mathrm{M},[$ trimer $]=0.004 \mathrm{M}$

trimer $\leftrightarrow 3 / 2$ dimer

$$
\Delta G=-\mathrm{RT} \operatorname{lnK}=-0.00198588 * 193.15 * \ln \left[(0.044)^{1.5} / 0.004\right]=-0.32 \mathrm{kcal} / \mathrm{mol}
$$

Comparing the experimental and computational results, our computational approach provides a satisfying consistency.

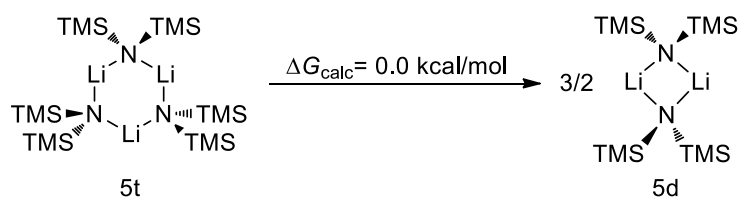

Scheme S1. DFT-computed relative stabilities of (LiHMDS)2 and (LiHMDS)3.
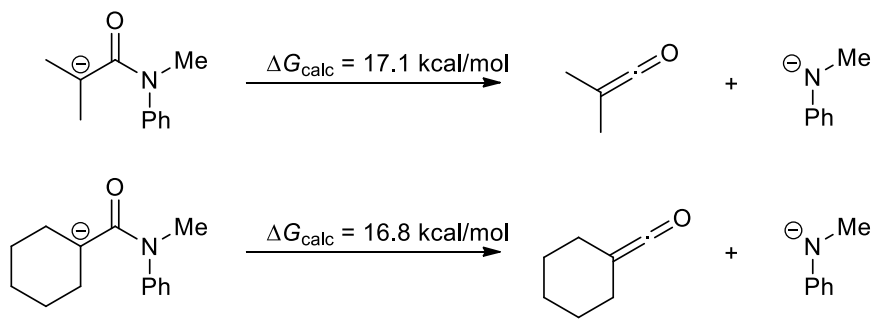

Scheme S2. DFT-computed thermodynamics of ketene formation from the deprotonated amide.

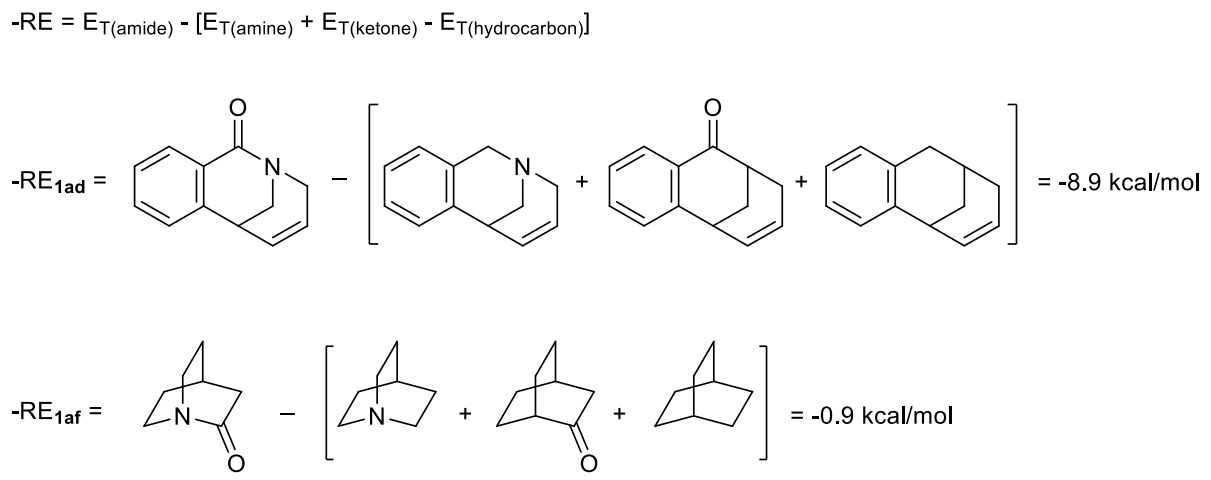

Scheme S3. DFT-calculated resonance energies of amides 1ad and 1af using M06-2X/6$311+\mathbf{G}(\mathbf{d}, \mathbf{p})$ in gas phase. ${ }^{9-11}$ 


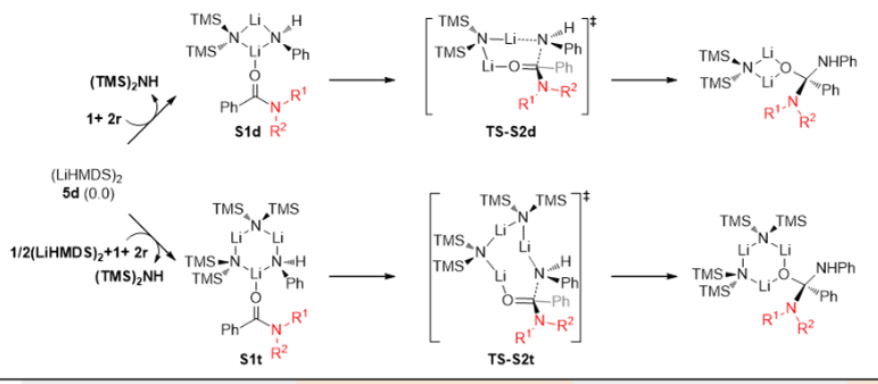

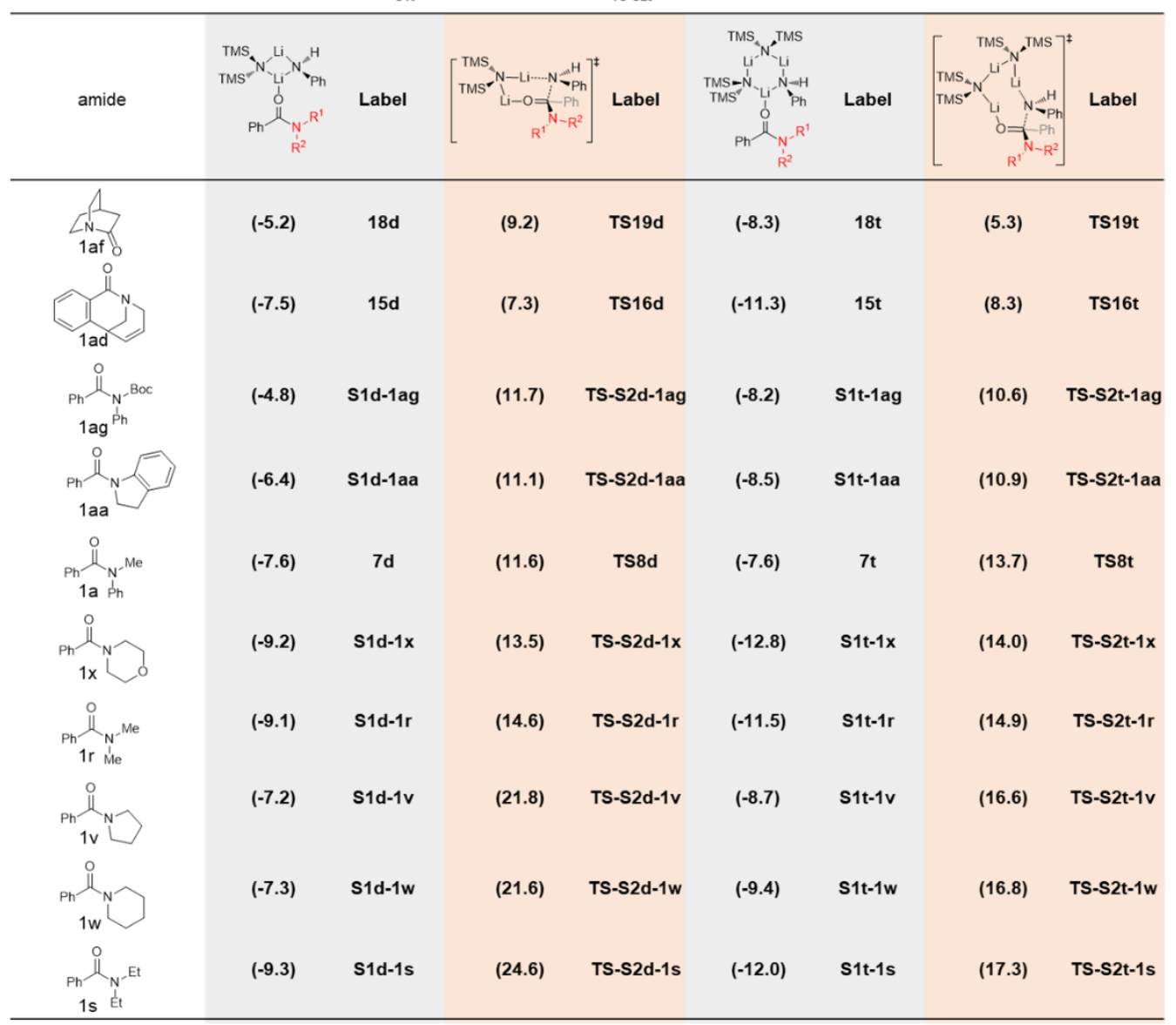

Table S1. DFT-calculated free energies of the rate-determining nucleophilic addition step in the transamidation reaction.

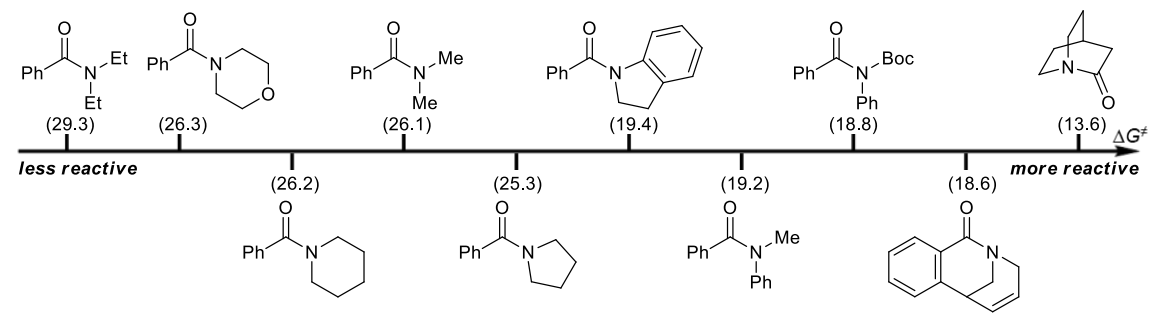

Scheme S4. DFT-calculated reactivity scale for the selected amides using the resting state reference. All barrier details can be found in Table S1. 
Zero-point correction ( $\mathrm{ZPE})$, thermal correction to enthalpy $(T C H)$, thermal correction to Gibbs free energy (TCG), energies $(E)$, enthalpies $(H)$, and Gibbs free energies $(G)$ (in Hartree) of the structures calculated at the M06-2X/6311+G(d,p)-SMD(toluene)// B3LYP-D3(BJ)/6-31G(d) level of theory.

Table S2. Energies in Figure 13, Figure 14, Figure 15, Scheme S1, Scheme S2, Scheme S3, Table S1 and Scheme S4.

\begin{tabular}{|c|c|c|c|c|c|c|c|}
\hline Structures & $Z P E$ & $t c H$ & $t c G$ & $\boldsymbol{E}$ & $\boldsymbol{H}$ & $G$ & $\begin{array}{l}\text { Imaginary } \\
\text { Frequency }\end{array}$ \\
\hline $1 \mathrm{a}$ & 0.237330 & 0.251848 & 0.195743 & -671.214069 & -670.962221 & -671.018326 & \\
\hline $1 \mathrm{aa}$ & 0.245286 & 0.259395 & 0.204433 & -709.324562 & -709.065167 & -709.120129 & \\
\hline $1 \mathrm{ad}$ & 0.206237 & 0.216980 & 0.171105 & -593.792180 & -593.575200 & -593.621075 & \\
\hline $1 \mathrm{af}$ & 0.176607 & 0.184806 & 0.144513 & -403.278867 & -403.094061 & -403.134354 & \\
\hline $1 \mathrm{ag}$ & 0.336251 & 0.357534 & 0.285929 & -977.699396 & -977.341862 & -977.413467 & \\
\hline $1 \mathrm{r}$ & 0.184761 & 0.196210 & 0.148259 & -479.498056 & -479.301846 & -479.349797 & \\
\hline $1 \mathrm{~s}$ & 0.242273 & 0.256302 & 0.200954 & -558.110217 & -557.853915 & -557.909263 & \\
\hline $1 \mathrm{v}$ & 0.222262 & 0.234157 & 0.184715 & -556.913601 & -556.679444 & -556.728886 & \\
\hline $1 \mathrm{w}$ & 0.251628 & 0.264431 & 0.212810 & -596.222376 & -595.957945 & -596.009566 & \\
\hline $1 \mathrm{x}$ & 0.227440 & 0.240014 & 0.188703 & -632.121918 & -631.881904 & -631.933215 & \\
\hline $2 \mathrm{r}$ & 0.117524 & 0.124250 & 0.088373 & -287.563199 & -287.438949 & -287.474826 & \\
\hline $4 a$ & 0.144299 & 0.154018 & 0.109952 & -460.072220 & -459.918202 & -459.962268 & \\
\hline $5 \mathrm{~d}$ & 0.463633 & 0.500337 & 0.398686 & -1761.694103 & -1761.193766 & -1761.295417 & \\
\hline $5 \mathrm{t}$ & 0.699147 & 0.753610 & 0.616736 & -2642.559850 & -2641.806240 & -2641.943114 & \\
\hline $6 \mathrm{~d}$ & 0.340080 & 0.365973 & 0.285528 & -1175.392885 & -1175.026912 & -1175.107357 & \\
\hline $6 \mathrm{t}$ & 0.573086 & 0.618084 & 0.495576 & -2056.259112 & -2055.641028 & -2055.763536 & \\
\hline $7 d$ & 0.579575 & 0.620715 & 0.504493 & -1846.641739 & -1846.021024 & -1846.137246 & \\
\hline $7 \mathrm{t}$ & 0.812593 & 0.872534 & 0.716244 & -2727.501296 & -2726.628762 & -2726.785052 & \\
\hline TS8d & 0.579221 & 0.619207 & 0.505968 & -1846.612758 & -1845.993551 & -1846.106790 & $196.3 i$ \\
\hline $\mathrm{TS} 8 \mathrm{t}$ & 0.814064 & 0.871957 & 0.723642 & -2727.474736 & -2726.602779 & -2726.751094 & $84.4 i$ \\
\hline $9 \mathrm{~d}$ & 0.581237 & 0.621002 & 0.509019 & -1846.633332 & -1846.012330 & -1846.124313 & \\
\hline $9 t$ & 0.816055 & 0.874365 & 0.723537 & -2727.497864 & -2726.623499 & -2726.774327 & \\
\hline TS10d & 0.581658 & 0.620243 & 0.512834 & -1846.628764 & -1846.008521 & -1846.115930 & $17.8 i$ \\
\hline TS10t & 0.815600 & 0.872836 & 0.726305 & -2727.493651 & -2726.620815 & -2726.767346 & $33.0 i$ \\
\hline $11 d$ & 0.581793 & 0.621276 & 0.510764 & -1846.628766 & -1846.007490 & -1846.118002 & \\
\hline $11 \mathrm{t}$ & 0.816618 & 0.874081 & 0.727311 & -2727.501134 & -2726.627053 & -2726.773823 & \\
\hline
\end{tabular}




\begin{tabular}{|c|c|c|c|c|c|c|c|}
\hline $\mathrm{TS} 12 \mathrm{~d}$ & 0.579705 & 0.619143 & 0.509182 & -1846.619406 & -1846.000263 & -1846.110224 & $179.0 i$ \\
\hline TS12t & 0.813951 & 0.871587 & 0.725086 & -2727.485055 & -2726.613468 & -2726.759969 & $112.8 i$ \\
\hline $13 \mathrm{~d}$ & 0.579434 & 0.620148 & 0.507167 & -1846.626704 & -1846.006556 & -1846.119537 & \\
\hline $13 \mathrm{t}$ & 0.812799 & 0.871871 & 0.721278 & -2727.489495 & -2726.617624 & -2726.768217 & \\
\hline $14 d$ & 0.335990 & 0.359317 & 0.280823 & -972.769152 & -972.409835 & -972.488329 & \\
\hline $14 \mathrm{t}$ & 0.568662 & 0.610808 & 0.492457 & -1853.631251 & -1853.020443 & -1853.138794 & \\
\hline $15 \mathrm{~d}$ & 0.548100 & 0.585596 & 0.478590 & -1769.218559 & -1768.632963 & -1768.739969 & \\
\hline $15 \mathrm{t}$ & 0.781598 & 0.837708 & 0.690624 & -2650.084361 & -2649.246653 & -2649.393737 & \\
\hline TS16d & 0.547032 & 0.583859 & 0.478962 & -1769.195311 & -1768.611452 & -1768.716349 & $83.8 i$ \\
\hline TS16t & 0.783000 & 0.837301 & 0.698563 & -2650.060946 & -2649.223645 & -2649.362383 & $46.0 i$ \\
\hline $17 d$ & 0.549711 & 0.586132 & 0.482591 & -1769.212520 & -1768.626388 & -1768.729929 & \\
\hline $17 \mathrm{t}$ & 0.782863 & 0.838305 & 0.692803 & -2650.084817 & -2649.246512 & -2649.392014 & \\
\hline $18 \mathrm{~d}$ & 0.518351 & 0.553387 & 0.450847 & -1578.700301 & -1578.146914 & -1578.249454 & \\
\hline $18 \mathrm{t}$ & 0.751932 & 0.805484 & 0.663421 & -2459.565559 & -2458.760075 & -2458.902138 & \\
\hline TS19d & 0.518979 & 0.552537 & 0.454812 & -1578.681456 & -1578.128919 & -1578.226644 & $147.2 i$ \\
\hline TS19t & 0.754470 & 0.805606 & 0.673368 & -2459.553907 & -2458.748301 & -2458.880539 & $111.2 i$ \\
\hline $20 \mathrm{~d}$ & 0.521651 & 0.555144 & 0.456570 & -1578.717193 & -1578.162049 & -1578.260623 & \\
\hline $20 \mathrm{t}$ & 0.756196 & 0.807799 & 0.674046 & -2459.590753 & -2458.782954 & -2458.916707 & \\
\hline $21 d$ & 0.485695 & 0.522147 & 0.417077 & -1635.495778 & -1634.973631 & -1635.078701 & \\
\hline $21 \mathrm{t}$ & 0.719398 & 0.774335 & 0.630579 & -2516.362224 & -2515.587889 & -2515.731645 & \\
\hline $\mathrm{TS} 22 \mathrm{~d}$ & 0.485606 & 0.521003 & 0.418331 & -1635.473199 & -1634.952196 & -1635.054868 & $207.8 i$ \\
\hline $\mathrm{TS} 22 \mathrm{t}$ & 0.720865 & 0.774096 & 0.635625 & -2516.339426 & -2515.565330 & -2515.703801 & $115.6 i$ \\
\hline $23 \mathrm{~d}$ & 0.487851 & 0.523139 & 0.420824 & -1635.493660 & -1634.970521 & -1635.072836 & \\
\hline $23 \mathrm{t}$ & 0.721664 & 0.775582 & 0.633516 & -2516.363038 & -2515.587456 & -2515.729522 & \\
\hline $\mathrm{TS} 24 \mathrm{~d}$ & 0.487808 & 0.522265 & 0.422322 & -1635.487535 & -1634.965270 & -1635.065213 & $75.1 i$ \\
\hline $\mathrm{TS} 24 \mathrm{t}$ & 0.721333 & 0.774522 & 0.635712 & -2516.356403 & -2515.581881 & -2515.720691 & $93.0 i$ \\
\hline $25 \mathrm{~d}$ & 0.488633 & 0.523422 & 0.423405 & -1635.487394 & -1634.963972 & -1635.063989 & \\
\hline $25 \mathrm{t}$ & 0.721857 & 0.775572 & 0.635181 & -2516.360100 & -2515.584528 & -2515.724919 & \\
\hline TS26d & 0.486273 & 0.521215 & 0.420585 & -1635.477272 & -1634.956057 & -1635.056687 & $199.8 i$ \\
\hline $\mathrm{TS} 26 \mathrm{t}$ & 0.721078 & 0.774084 & 0.636805 & -2516.346072 & -2515.571988 & -2515.709267 & $144.3 i$ \\
\hline $27 \mathrm{~d}$ & 0.484713 & 0.521909 & 0.413881 & -1635.481448 & -1634.959539 & -1635.067567 & \\
\hline
\end{tabular}




\begin{tabular}{|c|c|c|c|c|c|c|}
\hline $27 \mathrm{t}$ & 0.719139 & 0.774193 & 0.630656 & -2516.348016 & -2515.573823 & -2515.717360 \\
\hline $28 \mathrm{~d}$ & 0.242649 & 0.261780 & 0.193116 & -761.641249 & -761.379469 & -761.448133 \\
\hline $28 \mathrm{t}$ & 0.475293 & 0.513188 & 0.402896 & -1642.500440 & -1641.987252 & -1642.097544 \\
\hline$(\mathrm{TMS})_{2} \mathrm{NH}$ & 0.240166 & 0.257869 & 0.197446 & -873.860800 & -873.602931 & -873.663354 \\
\hline 1aa-anion & 0.292498 & 0.307987 & 0.25069 & -674.2740988 & -673.9661118 & -674.0234088 \\
\hline 1aa-ketene & 0.15742 & 0.165736 & 0.125543 & -347.9135168 & -347.7477808 & -347.7879738 \\
\hline 1z-anion & 0.225471 & 0.23945 & 0.186344 & -557.5541134 & -557.3146634 & -557.3677694 \\
\hline 1z-ketene & 0.090062 & 0.097106 & 0.060966 & -231.1927733 & -231.0956673 & -231.1318073 \\
\hline amine-anion & 0.130334 & 0.138125 & 0.099586 & -326.3082467 & -326.1701217 & -326.2086607 \\
\hline 1ad-amide & 0.206237 & 0.216980 & 0.171105 & -593.775261 & -593.558281 & -593.604156 \\
\hline 1ad-amine & 0.224369 & 0.234706 & 0.189725 & -519.737011 & -519.502305 & -519.547286 \\
\hline $\begin{array}{c}1 \text { ad- } \\
\text { hydrocarbon }\end{array}$ & 0.236207 & 0.246739 & 0.201137 & -503.712352 & -503.465613 & -503.511215 \\
\hline 1ad-ketone & 0.217652 & 0.228686 & 0.182021 & -577.736396 & -577.507710 & -577.554375 \\
\hline 1af-amide & 0.176607 & 0.184806 & 0.144513 & -403.265776 & -403.080970 & -403.121263 \\
\hline 1af-amine & 0.196487 & 0.203958 & 0.165753 & -329.243353 & -329.039395 & -329.077600 \\
\hline 1af-hydrocarbon & 0.207959 & 0.215705 & 0.176740 & -313.217278 & -313.001573 & -313.040538 \\
\hline 1af-ketone & 0.188429 & 0.196849 & 0.156201 & -387.238327 & -387.041478 & -387.082126 \\
\hline S1d-1aa & 0.587181 & 0.628090 & 0.511317 & -1884.748513 & -1884.120423 & -1884.237196 \\
\hline S1d-1ag & 0.678710 & 0.726457 & 0.597648 & -2153.125604 & -2152.399147 & -2152.527956 \\
\hline S1d-1r & 0.527148 & 0.565164 & 0.456831 & -1654.927996 & -1654.362832 & -1654.471165 \\
\hline S1d-1s & 0.584799 & 0.625202 & 0.510921 & -1733.541958 & -1732.916756 & -1733.031037 \\
\hline S1d-1v & 0.564095 & 0.602867 & 0.492041 & -1732.339252 & -1731.736385 & -1731.847211 \\
\hline S1d-1w & 0.593454 & 0.633091 & 0.520727 & -1771.648803 & -1771.015712 & -1771.128076 \\
\hline S1d-1x & 0.569619 & 0.608937 & 0.496740 & -1807.551507 & -1806.942570 & -1807.054767 \\
\hline S1t-1aa & 0.820608 & 0.880163 & 0.724895 & -2765.613219 & -2764.733056 & -2764.888324 \\
\hline S1t-1ag & 0.912042 & 0.978589 & 0.809559 & -3033.990655 & -3033.012066 & -3033.181096 \\
\hline S1t-1r & 0.761187 & 0.817496 & 0.670610 & -2535.793323 & -2534.975827 & -2535.122713 \\
\hline S1t-1s & 0.818603 & 0.877477 & 0.725250 & -2614.408239 & -2613.530762 & -2613.682989 \\
\hline S1t-1v & 0.797524 & 0.854920 & 0.705421 & -2613.202762 & -2612.347842 & -2612.497341 \\
\hline S1t-1w & 0.827320 & 0.885427 & 0.734285 & -2652.513383 & -2651.627956 & -2651.779098 \\
\hline
\end{tabular}




\begin{tabular}{|c|c|c|c|c|c|c|c|}
\hline S1t-1x & 0.803177 & 0.861052 & 0.708367 & -2688.416581 & -2687.555529 & -2687.708214 & \\
\hline TS-S2d-1aa & 0.587735 & 0.626924 & 0.517592 & -1884.726839 & -1884.099915 & -1884.209247 & $172.6 i$ \\
\hline TS-S2d-1ag & 0.678408 & 0.725198 & 0.597502 & -2153.099171 & -2152.373973 & -2152.501669 & $145.0 i$ \\
\hline TS-S2d-1r & 0.525974 & 0.562824 & 0.457387 & -1654.890735 & -1654.327911 & -1654.433348 & $175.2 i$ \\
\hline TS-S2d-1s & 0.584971 & 0.623854 & 0.515928 & -1733.492914 & -1732.869060 & -1732.976986 & $153.3 i$ \\
\hline TS-S2d-1v & 0.564465 & 0.601621 & 0.497384 & -1732.298460 & -1731.696839 & -1731.801076 & $122.7 i$ \\
\hline TS-S2d-1w & 0.593459 & 0.631661 & 0.523797 & -1771.605757 & -1770.974096 & -1771.081960 & $188.3 i$ \\
\hline TS-S2d-1x & 0.569317 & 0.607443 & 0.498447 & -1807.517061 & -1806.909618 & -1807.018614 & $171.7 i$ \\
\hline TS-S2t-1aa & 0.822193 & 0.879503 & 0.732529 & -2765.589831 & -2764.710328 & -2764.857302 & $75.3 i$ \\
\hline TS-S2t-1ag & 0.913642 & 0.978243 & 0.815984 & -3033.967215 & -3032.988972 & -3033.151231 & $179.8 i$ \\
\hline TS-S2t-1r & 0.762736 & 0.816712 & 0.679756 & -2535.760469 & -2534.943757 & -2535.080713 & $79.5 i$ \\
\hline TS-S2t-1s & 0.820322 & 0.876913 & 0.733454 & -2614.369732 & -2613.492819 & -2613.636278 & $56.3 i$ \\
\hline TS-S2t-1v & 0.798936 & 0.854456 & 0.712032 & -2613.168957 & -2612.314501 & -2612.456925 & $170.4 i$ \\
\hline TS-S2t-1w & 0.829390 & 0.885078 & 0.743644 & -2652.480904 & -2651.595826 & -2651.737260 & $60.6 i$ \\
\hline TS-S2t-1x & 0.804419 & 0.860454 & 0.717280 & -2688.382786 & -2687.522332 & -2687.665506 & $101.1 i$ \\
\hline
\end{tabular}




\section{Cartesian coordinates of the structures}

\begin{tabular}{|c|c|c|c|}
\hline $\mathrm{C}$ & 1.470382 & 0.318125 & 0.023669 \\
\hline $\mathrm{C}$ & 1.265235 & -0.437931 & 1.182961 \\
\hline $\mathrm{C}$ & 2.430662 & -0.093554 & -0.908243 \\
\hline $\mathrm{C}$ & 1.992192 & -1.609123 & 1.390793 \\
\hline $\mathrm{H}$ & 0.528788 & -0.110456 & 1.909135 \\
\hline $\mathrm{N}$ & 0.729060 & 1.515586 & -0.209207 \\
\hline $\mathrm{C}$ & 3.168509 & -1.254984 & -0.686695 \\
\hline $\mathrm{H}$ & 2.580135 & 0.490971 & -1.811248 \\
\hline $\mathrm{C}$ & 2.947341 & -2.020713 & 0.460141 \\
\hline $\mathrm{H}$ & 1.818113 & -2.194516 & 2.288976 \\
\hline $\mathrm{C}$ & 1.476272 & 2.776596 & -0.211264 \\
\hline $\mathrm{C}$ & -0.636152 & 1.619285 & 0.007691 \\
\hline $\mathrm{H}$ & 3.908426 & -1.568332 & -1.417650 \\
\hline $\mathrm{H}$ & 3.517024 & -2.929944 & 0.627443 \\
\hline $\mathrm{H}$ & 1.638764 & 3.142534 & 0.810372 \\
\hline $\mathrm{H}$ & 2.443682 & 2.615362 & -0.689421 \\
\hline $\mathrm{H}$ & 0.907638 & 3.528655 & -0.758161 \\
\hline $\mathrm{C}$ & -1.477284 & 0.382205 & -0.109034 \\
\hline $\mathrm{O}$ & -1.153844 & 2.708092 & 0.241055 \\
\hline $\mathrm{C}$ & -2.642786 & 0.330556 & 0.665875 \\
\hline $\mathrm{C}$ & -1.199475 & -0.660035 & -1.003243 \\
\hline $\mathrm{C}$ & -3.499866 & -0.763878 & 0.577552 \\
\hline $\mathrm{H}$ & -2.862981 & 1.162835 & 1.325880 \\
\hline $\mathrm{C}$ & -2.067715 & -1.745413 & -1.104330 \\
\hline $\mathrm{H}$ & -0.310999 & -0.621546 & -1.622776 \\
\hline $\mathrm{C}$ & -3.213003 & -1.805324 & -0.307670 \\
\hline $\mathrm{H}$ & -4.394775 & -0.801514 & 1.192172 \\
\hline $\mathrm{H}$ & -1.849216 & -2.545502 & -1.805802 \\
\hline $\mathrm{H}$ & -3.883149 & -2.657259 & -0.382129 \\
\hline \multicolumn{4}{|c|}{$1 \mathbf{a a}$} \\
\hline $\mathrm{N}$ & 0.403672 & 0.128286 & 0.085541 \\
\hline $\mathrm{C}$ & 0.232567 & 1.591385 & -0.078929 \\
\hline $\mathrm{C}$ & -0.590123 & -0.823597 & 0.005334 \\
\hline $\mathrm{C}$ & 1.584838 & 2.079278 & -0.642712 \\
\hline $\mathrm{H}$ & 0.041839 & 2.054259 & 0.896138 \\
\hline $\mathrm{H}$ & -0.615098 & 1.804727 & -0.730075 \\
\hline $\mathrm{C}$ & 1.789490 & -0.171942 & 0.103539 \\
\hline $\mathrm{C}$ & -2.013570 & -0.346988 & 0.028685 \\
\hline
\end{tabular}




$\begin{array}{llll}\mathrm{O} & -0.341439 & -2.023014 & -0.086971 \\ \mathrm{C} & 2.527990 & 0.962046 & -0.261949 \\ \mathrm{H} & 1.866487 & 3.050809 & -0.225596 \\ \mathrm{H} & 1.540012 & 2.191569 & -1.734297 \\ \mathrm{C} & 2.418679 & -1.370480 & 0.440160 \\ \mathrm{C} & -2.487401 & 0.603974 & 0.940860 \\ \mathrm{C} & -2.916209 & -0.972361 & -0.841488 \\ \mathrm{C} & 3.915088 & 0.918523 & -0.282359 \\ \mathrm{C} & 3.816894 & -1.398712 & 0.418162 \\ \mathrm{H} & 1.836630 & -2.243985 & 0.695489 \\ \mathrm{C} & -3.840713 & 0.941439 & 0.966079 \\ \mathrm{H} & -1.806674 & 1.061246 & 1.651530 \\ \mathrm{C} & -4.263117 & -0.619151 & -0.830581 \\ \mathrm{H} & -2.544795 & -1.738172 & -1.514288 \\ \mathrm{C} & 4.563683 & -0.271874 & 0.066777 \\ \mathrm{H} & 4.488426 & 1.796921 & -0.567201 \\ \mathrm{H} & 4.327228 & -2.320731 & 0.681958 \\ \mathrm{C} & -4.728375 & 0.339762 & 0.073122 \\ \mathrm{H} & -4.201410 & 1.670036 & 1.686546 \\ \mathrm{H} & -4.953096 & -1.098104 & -1.519362 \\ \mathrm{H} & 5.648621 & -0.319346 & 0.060214 \\ \mathrm{H} & -5.780686 & 0.609064 & 0.087741 \\ & & & \\ \mathrm{H} & & & \end{array}$

1ad

$\begin{array}{lllll}\text { C } & 0.791328 & 0.622374 & 0.148743\end{array}$

$\begin{array}{llll}\text { C } & 1.981023 & 1.326250 & -0.048420\end{array}$

$\begin{array}{llll}\text { C } & 0.789513 & -0.786913 & 0.157424\end{array}$

$\begin{array}{llll}\text { C } & 3.179741 & 0.635738 & -0.216273\end{array}$

$\begin{array}{llll}\mathrm{H} & 1.942972 & 2.410301 & -0.081711\end{array}$

C $\quad-0.518350 \quad 1.348663 \quad 0.160125$

$\begin{array}{llll}\text { C } & -0.540871 & -1.481575 & 0.415811\end{array}$

$\begin{array}{llll}\text { C } & 1.990349 & -1.468973 & -0.029183\end{array}$

$\begin{array}{lllll}\text { C } & 3.181577 & -0.761384 & -0.211201\end{array}$

$\mathrm{H} \quad 4.107460 \quad 1.181436 \quad-0.360963$

$\begin{array}{llll}\mathrm{N} & -1.607324 & 0.662427 & 0.728814\end{array}$

$\begin{array}{llll}\text { O } & -0.657560 & 2.431563 & -0.389179\end{array}$

$\begin{array}{lllll}\text { C } & -1.276502 & -0.592039 & 1.418108\end{array}$

$\begin{array}{lllll}\text { C } & -1.411042 & -1.597810 & -0.829830\end{array}$

$\begin{array}{llll}\mathrm{H} & -0.353877 & -2.481676 & 0.826026\end{array}$

$\mathrm{H} \quad \begin{array}{llll}\mathrm{H} & 1.997351 & -2.556167 & -0.023587\end{array}$

$\mathrm{H} \quad 4.113484 \quad-1.303237 \quad-0.347116$

$\begin{array}{lllll}\text { C } & -2.709965 & 0.455376 & -0.219895\end{array}$ 


\begin{tabular}{|c|c|c|c|}
\hline $\mathrm{H}$ & -2.211069 & -1.045335 & 1.761587 \\
\hline $\mathrm{H}$ & -0.657137 & -0.377550 & 2.293669 \\
\hline $\mathrm{C}$ & -2.413854 & -0.750126 & -1.091628 \\
\hline $\mathrm{H}$ & -1.189517 & -2.412318 & -1.515735 \\
\hline $\mathrm{H}$ & -2.807033 & 1.361026 & -0.823550 \\
\hline $\mathrm{H}$ & -3.650940 & 0.329159 & 0.332545 \\
\hline $\mathrm{H}$ & -3.037631 & -0.892618 & -1.9721 \\
\hline \multicolumn{4}{|l|}{$1 \mathrm{af}$} \\
\hline $\mathrm{N}$ & 0.477251 & -1.054227 & -0.250374 \\
\hline $\mathrm{C}$ & -0.539980 & -0.862724 & -1.309287 \\
\hline $\mathrm{C}$ & -0.210530 & -1.207738 & 1.056556 \\
\hline $\mathrm{C}$ & -1.535856 & 0.291416 & -0.988779 \\
\hline $\mathrm{H}$ & -0.006494 & -0.677135 & -2.246605 \\
\hline $\mathrm{H}$ & -1.060452 & -1.818914 & -1.424217 \\
\hline $\mathrm{C}$ & 1.297317 & 0.138703 & -0.141422 \\
\hline $\mathrm{C}$ & -1.006144 & 0.079492 & 1.444252 \\
\hline $\mathrm{H}$ & 0.542605 & -1.456974 & 1.808916 \\
\hline $\mathrm{H}$ & -0.875145 & -2.072638 & 0.960886 \\
\hline $\mathrm{C}$ & -0.989670 & 1.040533 & 0.238090 \\
\hline $\mathrm{H}$ & -2.537528 & -0.098957 & -0.7742 \\
\hline $\mathrm{H}$ & -1.630939 & 0.970919 & -1.843651 \\
\hline $\mathrm{C}$ & 0.474491 & 1.424228 & -0.052984 \\
\hline $\mathrm{O}$ & 2.499678 & 0.092319 & -0.052632 \\
\hline $\mathrm{H}$ & -2.038686 & -0.168016 & 1.715331 \\
\hline $\mathrm{H}$ & -0.553004 & 0.568475 & 2.315070 \\
\hline $\mathrm{H}$ & -1.586671 & 1.933753 & 0.448637 \\
\hline $\mathrm{H}$ & 0.560723 & 1.982295 & -0.995334 \\
\hline H & 0.909650 & 2.054769 & 0.73029 \\
\hline
\end{tabular}

1ag

N $\quad 0.923638-0.109420-0.105826$

$\begin{array}{llll}\text { C } & 0.276919 & 1.100953 & 0.206707\end{array}$

C $\quad 0.260749-1.205103-0.736006$

$\begin{array}{llll}\text { O } & -0.899611 & 1.164413 & -0.429665\end{array}$

$\begin{array}{llll}\text { O } & 0.767026 & 1.953100 & 0.918011\end{array}$

$\begin{array}{llll}\text { C } & 2.353920 & -0.164909 & 0.007403\end{array}$

C $\quad-1.098589-1.555220 \quad-0.230857$

O $\quad 0.827003-1.870528-1.581164$

$\begin{array}{lllll}\text { C } & -1.882645 & 2.219458 & -0.124548\end{array}$

$\begin{array}{llll}\text { C } & 2.936165 & -1.247771 & 0.667584\end{array}$

$\begin{array}{lllll}\text { C } & 3.153690 & 0.839266 & -0.538977\end{array}$ 


\begin{tabular}{|c|c|c|c|}
\hline $\mathrm{C}$ & -1.457472 & -1.368802 & 1.109658 \\
\hline $\mathrm{C}$ & -2.000804 & -2.155641 & -1.115259 \\
\hline $\mathrm{C}$ & -3.094639 & 1.777398 & -0.943799 \\
\hline $\mathrm{C}$ & -1.338110 & 3.563148 & -0.609230 \\
\hline $\mathrm{C}$ & -2.210719 & 2.227834 & 1.369414 \\
\hline $\mathrm{C}$ & 4.323040 & -1.329673 & 0.774634 \\
\hline $\mathrm{H}$ & 2.301998 & -2.021477 & 1.088906 \\
\hline $\mathrm{C}$ & 4.539342 & 0.760858 & -0.414231 \\
\hline $\mathrm{H}$ & 2.692589 & 1.678561 & -1.047490 \\
\hline $\mathrm{C}$ & -2.719106 & -1.757965 & 1.554544 \\
\hline $\mathrm{H}$ & -0.748134 & -0.924205 & 1.800869 \\
\hline $\mathrm{C}$ & -3.269830 & -2.524006 & -0.674269 \\
\hline $\mathrm{H}$ & -1.695165 & -2.316507 & -2.143634 \\
\hline $\mathrm{H}$ & -2.838548 & 1.718148 & -2.006197 \\
\hline $\mathrm{H}$ & -3.909555 & 2.498137 & -0.821233 \\
\hline $\mathrm{H}$ & -3.442508 & 0.793037 & -0.616183 \\
\hline $\mathrm{H}$ & -1.072329 & 3.503656 & -1.669931 \\
\hline $\mathrm{H}$ & -0.454878 & 3.852274 & -0.037424 \\
\hline $\mathrm{H}$ & -2.106329 & 4.335146 & -0.491413 \\
\hline $\mathrm{H}$ & -3.012440 & 2.950413 & 1.555881 \\
\hline $\mathrm{H}$ & -1.341251 & 2.510284 & 1.964814 \\
\hline $\mathrm{H}$ & -2.560240 & 1.240488 & 1.685728 \\
\hline $\mathrm{C}$ & 5.128352 & -0.323758 & 0.238086 \\
\hline $\mathrm{H}$ & 4.772561 & -2.176433 & 1.284885 \\
\hline $\mathrm{H}$ & 5.159482 & 1.546664 & -0.835468 \\
\hline $\mathrm{C}$ & -3.631920 & -2.323584 & 0.660537 \\
\hline $\mathrm{H}$ & -2.990668 & -1.620259 & 2.597194 \\
\hline $\mathrm{H}$ & -3.975032 & -2.972052 & -1.368169 \\
\hline $\mathrm{H}$ & 6.208958 & -0.384298 & 0.328050 \\
\hline $\mathrm{H}$ & -4.619373 & -2.616414 & 1.005777 \\
\hline \multicolumn{4}{|c|}{$1 \mathbf{r}$} \\
\hline $\mathrm{C}$ & -0.445140 & 0.240795 & -0.078992 \\
\hline $\mathrm{C}$ & -1.338426 & 1.162697 & 0.480385 \\
\hline $\mathrm{C}$ & -0.939559 & -0.954431 & -0.617133 \\
\hline $\mathrm{C}$ & -2.700031 & 0.875482 & 0.540118 \\
\hline $\mathrm{H}$ & -0.948666 & 2.103414 & 0.854999 \\
\hline $\mathrm{C}$ & 0.999486 & 0.641222 & -0.184392 \\
\hline $\mathrm{C}$ & -2.305686 & -1.233013 & -0.574803 \\
\hline $\mathrm{H}$ & -0.257046 & -1.655336 & -1.088484 \\
\hline $\mathrm{C}$ & -3.186443 & -0.323729 & 0.013370 \\
\hline $\mathrm{H}$ & -3.384423 & 1.590250 & 0.988145 \\
\hline
\end{tabular}




$\begin{array}{llll}\mathrm{N} & 1.958629 & -0.322419 & 0.040176 \\ \mathrm{O} & 1.295174 & 1.789275 & -0.509269 \\ \mathrm{H} & -2.682114 & -2.156745 & -1.005108 \\ \mathrm{H} & -4.249531 & -0.544004 & 0.052338 \\ \mathrm{C} & 3.348358 & 0.040993 & -0.192350 \\ \mathrm{C} & 1.763593 & -1.506806 & 0.862624 \\ \mathrm{H} & 3.887989 & -0.825946 & -0.590146 \\ \mathrm{H} & 3.839784 & 0.360608 & 0.737886 \\ \mathrm{H} & 3.384122 & 0.863782 & -0.904667 \\ \mathrm{H} & 2.043087 & -2.416734 & 0.315720 \\ \mathrm{H} & 2.392822 & -1.444727 & 1.761698 \\ \mathrm{H} & 0.725267 & -1.591092 & 1.177576\end{array}$

$1 \mathrm{~s}$

$\begin{array}{llll}\text { C } & 0.535900 & -0.678753 & -0.377022\end{array}$

$\begin{array}{llll}\mathrm{N} & 1.492969 & 0.174546 & 0.117659\end{array}$

$\begin{array}{llll}\text { C } & -0.908516 & -0.371399 & -0.093273\end{array}$

C $\quad 2.890514-0.191558-0.153480$

$\begin{array}{llll}\text { C } & 1.223796 & 1.570432 & 0.475422\end{array}$

$\begin{array}{lllll}\text { O } & 0.832909 & -1.687248 & -1.016322\end{array}$

C $\quad-1.812314-0.396927-1.161338$

$\begin{array}{llll}\text { C } & -1.386725 & -0.171229 & 1.207772\end{array}$

C $\quad 3.411200-1.223888 \quad 0.845802$

$\mathrm{H} \quad 3.487000 \quad 0.724365-0.105205$

$\mathrm{H} \quad 2.959271 \quad-0.589034 \quad-1.170146$

C $\quad 1.226421 \quad 2.503193 \quad-0.738676$

$\begin{array}{llll}\mathrm{H} & 1.986099 & 1.876840 & 1.200159\end{array}$

$\begin{array}{llll}\mathrm{H} & 0.261604 & 1.634355 & 0.982113\end{array}$

$\begin{array}{llll}\text { C } & -3.171031 & -0.181293 & -0.937565\end{array}$

$\mathrm{H} \quad \begin{array}{llll}-1.438293 & -0.596217 & -2.160606\end{array}$

$\begin{array}{llll}\text { C } & -2.749072 & 0.024494 & 1.433553\end{array}$

$\begin{array}{llll}\mathrm{H} & -0.692204 & -0.189432 & 2.043064\end{array}$

$\mathrm{H} \quad 4.458662 \quad-1.467761 \quad 0.634469$

$\begin{array}{llll}\mathrm{H} & 2.821972 & -2.141674 & 0.772157\end{array}$

$\begin{array}{llll}\mathrm{H} & 3.344586 & -0.844642 & 1.871697\end{array}$

$\begin{array}{lllll}\mathrm{H} & & 1.066145 & 3.541783 & -0.427483\end{array}$

$\begin{array}{llll}\mathrm{H} & 0.424398 & 2.223212 & -1.430076\end{array}$

$\begin{array}{llll}\mathrm{H} & 2.177272 & 2.451869 & -1.280105\end{array}$

$\begin{array}{llll}\text { C } & -3.641920 & 0.030852 & 0.360173\end{array}$

$\begin{array}{lllll}\mathrm{H} & & -3.864431 & -0.189165 & -1.773747\end{array}$

$\begin{array}{llll}\mathrm{H} & -3.113602 & 0.167655 & 2.446872\end{array}$

$\mathrm{H} \quad-4.702048 \quad 0.190450 \quad 0.535583$ 


$\begin{array}{llll}\text { 1v } & & & \\ \mathrm{C} & -1.091529 & 0.329731 & -0.116434 \\ \mathrm{C} & -1.431109 & -0.879439 & -0.735928 \\ \mathrm{C} & -2.071597 & 1.033920 & 0.595126 \\ \mathrm{C} & -2.725724 & -1.387567 & -0.625985 \\ \mathrm{H} & -0.693217 & -1.411554 & -1.327284 \\ \mathrm{C} & 0.259957 & 0.970320 & -0.246327 \\ \mathrm{C} & -3.358678 & 0.517520 & 0.720339 \\ \mathrm{H} & -1.804475 & 1.989200 & 1.034350 \\ \mathrm{C} & -3.688258 & -0.696065 & 0.110892 \\ \mathrm{H} & -2.983274 & -2.320194 & -1.120049 \\ \mathrm{~N} & 1.365286 & 0.161813 & -0.264190 \\ \mathrm{O} & 0.368049 & 2.193218 & -0.342956 \\ \mathrm{H} & -4.108486 & 1.064075 & 1.285285 \\ \mathrm{H} & -4.694347 & -1.095826 & 0.202041 \\ \mathrm{C} & 2.685491 & 0.811132 & -0.341478 \\ \mathrm{C} & 1.467702 & -1.211654 & 0.255433 \\ \mathrm{C} & 3.672561 & -0.323882 & -0.041186 \\ \mathrm{H} & 2.738397 & 1.615720 & 0.401467 \\ \mathrm{H} & 2.830470 & 1.269312 & -1.324238 \\ \mathrm{C} & 2.867070 & -1.249377 & 0.882964 \\ \mathrm{H} & 0.665452 & -1.421632 & 0.965845 \\ \mathrm{H} & 1.396749 & -1.946057 & -0.558968 \\ \mathrm{H} & 4.598605 & 0.041419 & 0.411374 \\ \mathrm{H} & 3.936764 & -0.854399 & -0.964041 \\ \mathrm{H} & 3.269889 & -2.264433 & 0.946436 \\ \mathrm{H} & 2.830760 & -0.831898 & 1.896268\end{array}$

1w

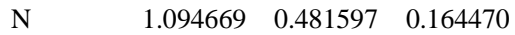

$\begin{array}{llll}\text { C } & 2.361040 & 1.189189 & -0.023260\end{array}$

C $\quad 1.209932 \quad-0.729747 \quad 0.976744$

$\begin{array}{llll}\text { C } & 3.381832 & 0.262571 & -0.691861\end{array}$

$\mathrm{H} \quad 2.741103 \quad 1.524460 \quad 0.955339$

$\mathrm{H} \quad 2.157310 \quad 2.077505 \quad-0.620361$

$\begin{array}{lllll}\text { C } & & -0.078486 & 1.154557 & -0.092338\end{array}$

$\begin{array}{llll}\text { C } & 2.192026 & -1.715028 & 0.336578\end{array}$

$\begin{array}{llll}\mathrm{H} & 0.226259 & -1.175947 & 1.113157\end{array}$

$\mathrm{H} \quad 1.582080 \quad-0.446206 \quad 1.975396$

C $\quad 3.551056-1.042000 \quad 0.099416$

$\begin{array}{llll}\mathrm{H} & 4.341600 & 0.785160 & -0.783532\end{array}$ 


$\begin{array}{llll}\mathrm{H} & 3.036023 & 0.035865 & -1.708887 \\ \mathrm{C} & -1.361194 & 0.371882 & -0.057652 \\ \mathrm{O} & -0.098254 & 2.346352 & -0.394111 \\ \mathrm{H} & 1.776643 & -2.061176 & -0.619411 \\ \mathrm{H} & 2.301196 & -2.593697 & 0.983694 \\ \mathrm{H} & 4.011641 & -0.815971 & 1.072223 \\ \mathrm{H} & 4.232604 & -1.723977 & -0.422278 \\ \mathrm{C} & -2.490715 & 0.986493 & 0.495443 \\ \mathrm{C} & -1.489179 & -0.888202 & -0.656407 \\ \mathrm{C} & -3.721009 & 0.332979 & 0.489022 \\ \mathrm{H} & -2.388335 & 1.980536 & 0.918215 \\ \mathrm{C} & -2.724989 & -1.534375 & -0.679326 \\ \mathrm{H} & -0.624700 & -1.350670 & -1.123638 \\ \mathrm{C} & -3.840459 & -0.929534 & -0.097296 \\ \mathrm{H} & -4.589805 & 0.811358 & 0.932197 \\ \mathrm{H} & -2.817682 & -2.506379 & -1.155579 \\ \mathrm{H} & -4.801713 & -1.435566 & -0.109325\end{array}$

$\mathbf{1 x}$

$\begin{array}{llll}\mathrm{N} & 1.095587 & 0.441230 & 0.097962\end{array}$

C $\quad 2.364866 \quad 1.148943 \quad-0.059546$

$\begin{array}{llll}\text { C } & 1.220987 & -0.760333 & 0.923185\end{array}$

C $\quad 3.403909 \quad 0.176356-0.612152$

$\mathrm{H} \quad 2.206226 \quad 1.993140 \quad-0.731456$

$\begin{array}{llll}\mathrm{H} & 2.700944 & 1.541087 & 0.911967\end{array}$

C $\quad-0.073865 \quad 1.139390 \quad-0.117736$

$\begin{array}{llll}\text { C } & 2.300146 & -1.664416 & 0.331547\end{array}$

$\begin{array}{lllll}\mathrm{H} & 0.266130 & -1.280834 & 0.985087\end{array}$

$\mathrm{H} \quad \begin{array}{llll}\mathrm{H} & 1.520424 & -0.470356 & 1.942338\end{array}$

$\begin{array}{llll}\text { O } & 3.534675 & -0.976146 & 0.211476\end{array}$

$\begin{array}{lllll}\mathrm{H} & 4.391531 & 0.644579 & -0.645327\end{array}$

$\mathrm{H} \quad 3.117809 \quad-0.121837 \quad-1.634182$

$\begin{array}{lllll}\text { C } & -1.363621 & 0.372555 & -0.070395\end{array}$

$\begin{array}{lllll}\text { O } & -0.073465 & 2.336684 & -0.391871\end{array}$

$\begin{array}{lllll}\mathrm{H} & 2.483997 & -2.522506 & 0.984426\end{array}$

$\begin{array}{llll}\mathrm{H} & 1.970998 & -2.032224 & -0.655237\end{array}$

$\begin{array}{llll}\text { C } & -2.471850 & 0.985945 & 0.525359\end{array}$

$\begin{array}{llll}\text { C } & -1.517888 & -0.874550 & -0.690119\end{array}$

$\begin{array}{llll}\text { C } & -3.707443 & 0.342604 & 0.539315\end{array}$

$\begin{array}{lllll}\mathrm{H} & -2.349443 & 1.970090 & 0.965647\end{array}$

$\begin{array}{llll}\text { C } & -2.759429 & -1.509930 & -0.691852\end{array}$

$\mathrm{H} \quad-\quad-0.670613 \quad-1.332662 \quad-1.192191$ 


$\begin{array}{llll}\mathrm{C} & -3.853216 & -0.907260 & -0.067712 \\ \mathrm{H} & -4.559786 & 0.819069 & 1.015105 \\ \mathrm{H} & -2.873860 & -2.471168 & -1.184755 \\ \mathrm{H} & -4.818723 & -1.405118 & -0.063374 \\ & & & \\ \mathrm{2r} & & & \\ \mathrm{C} & -0.938119 & 0.000106 & -0.010717 \\ \mathrm{~N} & -2.335887 & 0.000020 & -0.078334 \\ \mathrm{C} & -0.221637 & -1.208152 & -0.005418 \\ \mathrm{H} & -2.774555 & -0.835583 & 0.288432 \\ \mathrm{H} & -2.774972 & 0.835358 & 0.288407 \\ \mathrm{C} & -0.221351 & 1.208261 & -0.005316 \\ \mathrm{C} & 1.171204 & -1.202639 & 0.003461 \\ \mathrm{H} & -0.764564 & -2.150767 & -0.013166 \\ \mathrm{C} & 1.171267 & 1.202574 & 0.003434 \\ \mathrm{H} & -0.764055 & 2.150992 & -0.013682 \\ \mathrm{C} & 1.880768 & -0.000115 & 0.008467 \\ \mathrm{H} & 1.705057 & -2.149373 & 0.008890 \\ \mathrm{H} & 1.705101 & 2.149341 & 0.009211 \\ \mathrm{H} & 2.966403 & -0.000316 & 0.016775\end{array}$

$4 a$

$\begin{array}{lllll}\text { C } & & -1.233003 & 0.390896 & -0.000077\end{array}$

O $\quad-1.729832 \quad 1.500656 \quad-0.000379$

$\begin{array}{llll}\mathrm{O} & -1.968793 & -0.744793 & 0.000321\end{array}$

$\begin{array}{llll}\text { C } & 0.229857 & 0.120142 & 0.000000\end{array}$

$\begin{array}{llll}\text { C } & 1.097168 & 1.220156 & 0.000152\end{array}$

$\begin{array}{llll}\text { C } & 0.750300 & -1.180697 & -0.000173\end{array}$

$\begin{array}{llll}\text { C } & 2.474986 & 1.021246 & 0.000167\end{array}$

$\mathrm{H} \quad 0.669862 \quad 2.217071 \quad 0.000241$

C $\quad 2.130092-1.375370-0.000140$

$\mathrm{H} \quad 0.072820 \quad-2.026560-0.000394$

$\begin{array}{llll}\text { C } & 2.992759 & -0.276685 & 0.000045\end{array}$

$\begin{array}{llll}\mathrm{H} & 3.145900 & 1.875342 & 0.000286\end{array}$

$\begin{array}{lllll}\mathrm{H} & 2.533267 & -2.383907 & -0.000291\end{array}$

$\begin{array}{llll}\mathrm{H} & 4.068094 & -0.431813 & 0.000070\end{array}$

$\begin{array}{lllll}\text { C } & -3.388773 & -0.547365 & 0.000094\end{array}$

$\begin{array}{lllll}\mathrm{H} & -3.823332 & -1.547325 & -0.002798\end{array}$

$\begin{array}{llll}\mathrm{H} & -3.699506 & 0.005719 & 0.890512\end{array}$

$\begin{array}{llll}\mathrm{H} & -3.698415 & 0.010630 & -0.887570\end{array}$ 


\begin{tabular}{|c|c|c|c|}
\hline $\mathrm{Li}$ & 0.006099 & -0.028732 & -1.152322 \\
\hline $\mathrm{N}$ & -1.571283 & -0.003378 & -0.008910 \\
\hline $\mathrm{Si}$ & -2.320388 & 1.312652 & 0.792574 \\
\hline $\mathrm{Si}$ & -2.358977 & -1.310156 & -0.787463 \\
\hline $\mathrm{C}$ & -1.099791 & 1.878200 & 2.161660 \\
\hline $\mathrm{H}$ & -0.110682 & 2.163387 & 1.773663 \\
\hline $\mathrm{H}$ & -1.488331 & 2.764011 & 2.677370 \\
\hline $\mathrm{H}$ & -0.960484 & 1.115425 & 2.946016 \\
\hline $\mathrm{C}$ & -3.938397 & 0.878398 & 1.677071 \\
\hline $\mathrm{H}$ & -4.716343 & 0.567196 & 0.969844 \\
\hline $\mathrm{H}$ & -3.786474 & 0.052359 & 2.382782 \\
\hline $\mathrm{H}$ & -4.330329 & 1.733779 & 2.241329 \\
\hline $\mathrm{C}$ & -2.626845 & 2.805977 & -0.328835 \\
\hline $\mathrm{H}$ & -3.126976 & 3.627829 & 0.198370 \\
\hline $\mathrm{H}$ & -1.685169 & 3.195743 & -0.735037 \\
\hline $\mathrm{H}$ & -3.257536 & 2.518651 & -1.178980 \\
\hline $\mathrm{C}$ & -1.073535 & -2.072820 & -1.988417 \\
\hline $\mathrm{H}$ & -1.487083 & -2.964376 & -2.473934 \\
\hline $\mathrm{H}$ & -0.797211 & -1.393157 & -2.812496 \\
\hline $\mathrm{H}$ & -0.151943 & -2.393384 & -1.482383 \\
\hline $\mathrm{C}$ & -2.911703 & -2.680863 & 0.395388 \\
\hline $\mathrm{H}$ & -2.066468 & -3.070393 & 0.976403 \\
\hline $\mathrm{H}$ & -3.646778 & -2.289451 & 1.109223 \\
\hline $\mathrm{H}$ & -3.373713 & -3.524801 & -0.131866 \\
\hline $\mathrm{C}$ & -3.845247 & -0.802518 & -1.847944 \\
\hline $\mathrm{H}$ & -4.652395 & -0.384870 & -1.234374 \\
\hline $\mathrm{H}$ & -3.559987 & -0.035405 & -2.579000 \\
\hline $\mathrm{H}$ & -4.259866 & -1.654390 & -2.401312 \\
\hline $\mathrm{Li}$ & -0.006228 & -0.028996 & 1.152046 \\
\hline$N$ & 1.571162 & -0.003562 & 0.008720 \\
\hline $\mathrm{Si}$ & 2.320078 & 1.312705 & -0.792595 \\
\hline $\mathrm{Si}$ & 2.359288 & -1.309970 & 0.787465 \\
\hline $\mathrm{C}$ & 1.099425 & 1.878409 & -2.161593 \\
\hline $\mathrm{H}$ & 0.110333 & 2.163637 & -1.773592 \\
\hline $\mathrm{H}$ & 1.488093 & 2.764275 & -2.677117 \\
\hline $\mathrm{H}$ & 0.960065 & 1.115828 & -2.946128 \\
\hline $\mathrm{C}$ & 3.938114 & 0.878787 & -1.677210 \\
\hline $\mathrm{H}$ & 4.716115 & 0.567585 & -0.970042 \\
\hline $\mathrm{H}$ & 3.786285 & 0.052824 & -2.383030 \\
\hline $\mathrm{H}$ & 4.329925 & 1.734300 & -2.241353 \\
\hline $\mathrm{C}$ & 2.626381 & 2.805986 & 0.328922 \\
\hline $\mathrm{H}$ & 3.126319 & 3.627955 & -0.198285 \\
\hline
\end{tabular}




$\begin{array}{llll}\mathrm{H} & 1.684664 & 3.195566 & 0.735210 \\ \mathrm{H} & 3.257187 & 2.518745 & 1.179006 \\ \mathrm{C} & 1.073982 & -2.072849 & 1.988417 \\ \mathrm{H} & 1.487553 & -2.964292 & 2.474114 \\ \mathrm{H} & 0.797456 & -1.393096 & 2.812355 \\ \mathrm{H} & 0.152507 & -2.393597 & 1.482279 \\ \mathrm{C} & 2.912472 & -2.680627 & -0.395219 \\ \mathrm{H} & 2.067297 & -3.070499 & -0.976096 \\ \mathrm{H} & 3.647357 & -2.289096 & -1.109183 \\ \mathrm{H} & 3.374785 & -3.524344 & 0.132122 \\ \mathrm{C} & 3.845290 & -0.801681 & 1.848014 \\ \mathrm{H} & 4.652312 & -0.383632 & 1.234555 \\ \mathrm{H} & 3.559621 & -0.034756 & 2.579108 \\ \mathrm{H} & 4.260233 & -1.653410 & 2.401362\end{array}$

$5 t$

$\mathrm{N} \quad-2.213463 \quad-0.334651 \quad 0.066077$

$\mathrm{Li} \quad-0.603706-1.447750 \quad 0.020326$

$\mathrm{Li} \quad-1.049331 \quad 1.209921 \quad 0.279351$

$\begin{array}{llll}\mathrm{N} & 0.686112 & 1.987430 & 0.025805\end{array}$

$\mathrm{Li} \quad 1.517495 \quad 0.289175 \quad-0.410260$

$\mathrm{N} \quad 1.334124-1.610079-0.080784$

Si $\quad \begin{array}{llll}\text { Si } & 1.845832 & 2.359558 & 1.254780\end{array}$

$\begin{array}{lllll}\mathrm{Si} & 0.399759 & 3.061008 & -1.301293\end{array}$

$\begin{array}{llll}\mathrm{Si} & -3.232316 & 0.128799 & 1.388101\end{array}$

Si $\quad \begin{array}{llll}-2.949570 & -1.093336 & -1.306332\end{array}$

$\begin{array}{lllll}\mathrm{Si} & 1.549424 & -2.429770 & 1.427878\end{array}$

$\mathrm{Si} \quad 2.323878 \quad-2.017738 \quad-1.439466$

$\begin{array}{llll}\text { C } & 1.333741 & 1.673026 & 2.942010\end{array}$

$\begin{array}{llll}\mathrm{H} & 0.523879 & 2.267472 & 3.380139\end{array}$

$\begin{array}{llll}\mathrm{H} & 0.990364 & 0.635158 & 2.875070\end{array}$

$\begin{array}{llll}\mathrm{H} & 2.175547 & 1.693401 & 3.645141\end{array}$

$\begin{array}{llll}\text { C } & 2.242244 & 4.193986 & 1.505520\end{array}$

$\begin{array}{llll}\mathrm{H} & 3.014473 & 4.305513 & 2.277104\end{array}$

$\begin{array}{llll}\mathrm{H} & 2.616075 & 4.672136 & 0.593287\end{array}$

$\begin{array}{llll}\mathrm{H} & 1.359259 & 4.751865 & 1.837639\end{array}$

$\begin{array}{llll}\text { C } & 3.498631 & 1.521807 & 0.776734\end{array}$

$\begin{array}{llll}\mathrm{H} & 3.876027 & 1.937556 & -0.167006\end{array}$

$\mathrm{H} \quad 4.262766 \quad 1.698459 \quad 1.543806$

$\begin{array}{lllll}\mathrm{H} & 3.440723 & 0.429681 & 0.665108\end{array}$

$\begin{array}{llll}\text { C } & 1.947401 & 3.450639 & -2.326447\end{array}$

$\mathrm{H} \quad 1.723892 \quad 4.176737 \quad-3.117963$ 


\begin{tabular}{|c|c|c|c|}
\hline H & 2.745568 & 3.874205 & -1.705424 \\
\hline $\mathrm{H}$ & 2.344111 & 2.548695 & -2.807576 \\
\hline $\mathrm{C}$ & -0.390776 & 4.703237 & -0.777871 \\
\hline $\mathrm{H}$ & -0.730667 & 5.272791 & -1.652093 \\
\hline $\mathrm{H}$ & -1.265939 & 4.523245 & -0.138853 \\
\hline $\mathrm{H}$ & 0.300730 & 5.338031 & -0.215243 \\
\hline $\mathrm{C}$ & -0.863097 & 2.229675 & -2.448423 \\
\hline $\mathrm{H}$ & -1.038926 & 2.840547 & -3.342309 \\
\hline $\mathrm{H}$ & -0.536660 & 1.238278 & -2.785177 \\
\hline $\mathrm{H}$ & -1.836265 & 2.098948 & -1.958593 \\
\hline $\mathrm{C}$ & 2.336701 & -0.484213 & -2.584828 \\
\hline $\mathrm{H}$ & 2.888131 & 0.371020 & -2.164697 \\
\hline $\mathrm{H}$ & 2.851466 & -0.729498 & -3.521229 \\
\hline $\mathrm{H}$ & 1.332386 & -0.137674 & -2.861961 \\
\hline $\mathrm{C}$ & 4.144628 & -2.311105 & -0.992884 \\
\hline $\mathrm{H}$ & 4.573760 & -1.434806 & -0.491718 \\
\hline $\mathrm{H}$ & 4.277651 & -3.169684 & -0.325078 \\
\hline $\mathrm{H}$ & 4.739145 & -2.501667 & -1.895182 \\
\hline $\mathrm{C}$ & 1.745247 & -3.499410 & -2.461639 \\
\hline $\mathrm{H}$ & 2.476425 & -3.740796 & -3.243408 \\
\hline $\mathrm{H}$ & 1.632024 & -4.387049 & -1.828302 \\
\hline $\mathrm{H}$ & 0.783663 & -3.311334 & -2.948930 \\
\hline $\mathrm{C}$ & 2.984215 & -1.793741 & 2.485050 \\
\hline $\mathrm{H}$ & 3.052539 & -2.356687 & 3.424535 \\
\hline $\mathrm{H}$ & 3.940095 & -1.905600 & 1.961031 \\
\hline $\mathrm{H}$ & 2.866658 & -0.735740 & 2.739274 \\
\hline $\mathrm{C}$ & -0.051392 & -2.198231 & 2.434820 \\
\hline $\mathrm{H}$ & -0.321745 & -1.145620 & 2.581587 \\
\hline $\mathrm{H}$ & -0.920009 & -2.702264 & 1.986522 \\
\hline $\mathrm{H}$ & 0.070021 & -2.636709 & 3.432712 \\
\hline $\mathrm{C}$ & 1.762767 & -4.305035 & 1.234608 \\
\hline $\mathrm{H}$ & 1.828525 & -4.796995 & 2.213100 \\
\hline $\mathrm{H}$ & 0.910872 & -4.743272 & 0.698865 \\
\hline $\mathrm{H}$ & 2.669090 & -4.564769 & 0.675882 \\
\hline $\mathrm{C}$ & -2.332945 & 1.514462 & 2.348165 \\
\hline $\mathrm{H}$ & -2.955638 & 1.817136 & 3.198586 \\
\hline $\mathrm{H}$ & -1.369773 & 1.197717 & 2.763650 \\
\hline $\mathrm{H}$ & -2.162512 & 2.435369 & 1.767315 \\
\hline $\mathrm{C}$ & -4.886777 & 0.889716 & 0.855558 \\
\hline $\mathrm{H}$ & -5.446881 & 1.237313 & 1.732752 \\
\hline $\mathrm{H}$ & -4.733110 & 1.752472 & 0.195533 \\
\hline $\mathrm{H}$ & -5.525493 & 0.177107 & 0.321631 \\
\hline
\end{tabular}




$\begin{array}{llll}\mathrm{C} & -3.616854 & -1.245881 & 2.629015 \\ \mathrm{H} & -4.292780 & -0.887004 & 3.415388 \\ \mathrm{H} & -4.105101 & -2.092423 & 2.132731 \\ \mathrm{H} & -2.709787 & -1.621938 & 3.112448 \\ \mathrm{C} & -3.869116 & 0.042356 & -2.508481 \\ \mathrm{H} & -4.360593 & -0.554059 & -3.287945 \\ \mathrm{H} & -4.645219 & 0.623227 & -1.999158 \\ \mathrm{H} & -3.195753 & 0.747018 & -3.006075 \\ \mathrm{C} & -1.568679 & -1.951046 & -2.298661 \\ \mathrm{H} & -0.772387 & -1.261187 & -2.602974 \\ \mathrm{H} & -1.097972 & -2.789475 & -1.766021 \\ \mathrm{H} & -1.990587 & -2.378792 & -3.216063 \\ \mathrm{C} & -4.150911 & -2.481731 & -0.819145 \\ \mathrm{H} & -5.031475 & -2.108946 & -0.283279 \\ \mathrm{H} & -4.511308 & -3.015725 & -1.707386 \\ \mathrm{H} & -3.658326 & -3.214678 & -0.167096\end{array}$

6d

$1.213197-0.118042-0.156991$

$\begin{array}{llll}\mathrm{Si} & 1.279502 & 1.598819 & -0.202231\end{array}$

Si $\quad 2.532677 \quad-1.151972 \quad 0.205283$

$\begin{array}{llll}\text { C } & 0.905350 & 2.402822 & 1.468605\end{array}$

$\begin{array}{llll}\text { C } & 2.905556 & 2.308417 & -0.865790\end{array}$

$\begin{array}{llll}\text { C } & -0.082964 & 2.166225 & -1.431827\end{array}$

$\mathrm{Li} \quad \begin{array}{llll}-0.228418 & -0.211648 & -1.474462\end{array}$

$\mathrm{Li} \quad-0.127540 \quad-1.373227 \quad 0.539051$

$\begin{array}{lllll}\text { C } & & 3.632378 & -1.535061 & -1.285349\end{array}$

$\begin{array}{llll}\text { C } & 3.624597 & -0.554149 & 1.632568\end{array}$

$\begin{array}{llll}\text { C } & 1.769383 & -2.817593 & 0.786952\end{array}$

$\begin{array}{llll}\mathrm{H} & 0.896104 & 3.498457 & 1.413234\end{array}$

$\begin{array}{llll}\mathrm{H} & -0.070057 & 2.079284 & 1.853029\end{array}$

$\begin{array}{llll}\mathrm{H} & 1.662630 & 2.111738 & 2.207127\end{array}$

$\begin{array}{lllll}\mathrm{H} & 2.854518 & 3.400267 & -0.960063\end{array}$

$\begin{array}{lllll}\mathrm{H} & & 3.138963 & 1.897632 & -1.855807\end{array}$

$\mathrm{H} \quad 3.749735 \quad 2.075753 \quad-0.206202$

$\begin{array}{llll}\mathrm{H} & -1.101670 & 1.859320 & -1.151100\end{array}$

$\begin{array}{llll}\mathrm{H} & 0.114649 & 1.839239 & -2.467275\end{array}$

$\begin{array}{llll}\mathrm{H} & -0.106550 & 3.261172 & -1.475035\end{array}$

$\mathrm{N} \quad \quad-1.572792 \quad-1.429013 \quad-0.762546$

$\mathrm{H} \quad \begin{array}{llll}4.435161 & -2.241908 & -1.041541\end{array}$

$\mathrm{H} \quad 3.043741 \quad-1.964358 \quad-2.106101$

$\mathrm{H} \quad 4.098723 \quad-0.616269-1.661156$ 


$\begin{array}{llll}\mathrm{H} & 3.023893 & -0.373188 & 2.532575 \\ \mathrm{H} & 4.397742 & -1.289535 & 1.887598 \\ \mathrm{H} & 4.133274 & 0.384707 & 1.384715 \\ \mathrm{H} & 1.099749 & -3.279059 & 0.044799 \\ \mathrm{H} & 1.226225 & -2.735952 & 1.744491 \\ \mathrm{H} & 2.564670 & -3.548949 & 0.970736 \\ \mathrm{C} & -2.755347 & -0.837625 & -0.318518 \\ \mathrm{H} & -1.798608 & -2.267091 & -1.297914 \\ \mathrm{C} & -2.693948 & 0.290171 & 0.540420 \\ \mathrm{C} & -4.045783 & -1.294950 & -0.673455 \\ \mathrm{C} & -3.847910 & 0.919593 & 1.002669 \\ \mathrm{H} & -1.719238 & 0.672128 & 0.843541 \\ \mathrm{C} & -5.193430 & -0.661169 & -0.204988 \\ \mathrm{H} & -4.132144 & -2.160421 & -1.328098 \\ \mathrm{C} & -5.111983 & 0.453769 & 0.634983 \\ \mathrm{H} & -3.754331 & 1.782312 & 1.658186 \\ \mathrm{H} & -6.167082 & -1.044016 & -0.502308 \\ \mathrm{H} & -6.010948 & 0.944192 & 0.995217\end{array}$

$6 \mathbf{t}$

$\mathrm{N} \quad 1.292198-1.498272-1.567400$

$\begin{array}{lllll}\mathrm{Li} & 1.673873 & 0.038468 & -0.437705\end{array}$

$\mathrm{Li} \quad-0.535654-1.628363-1.062426$

$\mathrm{N} \quad-1.952565-0.752468-0.094006$

Li $\quad-0.946849 \quad 0.867259 \quad 0.299223$

$\begin{array}{lllll}\mathrm{N} & 0.828223 & 1.648096 & 0.184968\end{array}$

$\begin{array}{lllll}\mathrm{Si} & & -2.495638 & -1.619696 & 1.300305\end{array}$

Si $\quad-2.978210-0.206360-1.371550$

$\begin{array}{llll}\mathrm{Si} & 1.110996 & 1.951659 & 1.862947\end{array}$

$\mathrm{Si} \quad 1.079061 \quad 2.802987-1.078844$

$\begin{array}{llll}\text { C } & -1.003052 & -2.035731 & 2.395625\end{array}$

$\begin{array}{llll}\mathrm{H} & -0.366665 & -2.794092 & 1.922015\end{array}$

$\mathrm{H} \quad \begin{array}{llll}0.374767 & -1.164852 & 2.612757\end{array}$

$\begin{array}{llll}\mathrm{H} & -1.330699 & -2.448872 & 3.357517\end{array}$

$\begin{array}{lllll}\text { C } & -3.301070 & -3.282566 & 0.871632\end{array}$

$\mathrm{H} \quad \begin{array}{llll}-3.513620 & -3.864493 & 1.777299\end{array}$

$\begin{array}{llll}\mathrm{H} & -4.244890 & -3.156511 & 0.331081\end{array}$

$\begin{array}{llll}\mathrm{H} & -2.636462 & -3.889975 & 0.241914\end{array}$

$\begin{array}{llll}\text { C } & -3.736030 & -0.624422 & 2.335759\end{array}$

$\mathrm{H} \quad \begin{array}{llll}\mathrm{H} & -4.625072 & -0.369953 & 1.746159\end{array}$

$\mathrm{H} \quad \begin{array}{llll}-4.073107 & -1.187779 & 3.214802\end{array}$

$\begin{array}{llll}\mathrm{H} & -3.302047 & 0.317094 & 2.694345\end{array}$ 


\begin{tabular}{|c|c|c|c|}
\hline $\mathrm{C}$ & -3.093393 & 1.692821 & -1.310339 \\
\hline $\mathrm{H}$ & -3.678969 & 2.091632 & -2.147774 \\
\hline $\mathrm{H}$ & -3.588630 & 2.011428 & -0.382743 \\
\hline $\mathrm{H}$ & -2.116050 & 2.190768 & -1.356492 \\
\hline $\mathrm{C}$ & -4.750355 & -0.868556 & -1.40123 \\
\hline $\mathrm{H}$ & -5.319391 & -0.386197 & -2.20584 \\
\hline $\mathrm{H}$ & -4.770015 & -1.948688 & -1.58408 \\
\hline $\mathrm{H}$ & -5.281251 & -0.679308 & -0.461809 \\
\hline $\mathrm{C}$ & -2.183589 & -0.708189 & -3.0289 \\
\hline $\mathrm{H}$ & -2.726401 & -0.278700 & -3.87973 \\
\hline $\mathrm{H}$ & -1.141673 & -0.375013 & -3.12405 \\
\hline $\mathrm{H}$ & -2.205574 & -1.800250 & -3.16041 \\
\hline $\mathrm{C}$ & 0.620386 & 1.999509 & -2.737584 \\
\hline $\mathrm{H}$ & -0.446307 & 1.760967 & -2.804231 \\
\hline $\mathrm{H}$ & 0.858164 & 2.671986 & -3.570872 \\
\hline $\mathrm{H}$ & 1.174547 & 1.066102 & -2.902119 \\
\hline $\mathrm{C}$ & 0.031504 & 4.373730 & -0.894650 \\
\hline $\mathrm{H}$ & -1.035644 & 4.137986 & -0.798193 \\
\hline $\mathrm{H}$ & 0.322505 & 4.947522 & -0.007600 \\
\hline $\mathrm{H}$ & 0.147091 & 5.031086 & -1.765427 \\
\hline $\mathrm{C}$ & 2.896561 & 3.325266 & -1.237248 \\
\hline $\mathrm{H}$ & 3.051455 & 3.970553 & -2.111075 \\
\hline $\mathrm{H}$ & 3.244637 & 3.873097 & -0.354988 \\
\hline $\mathrm{H}$ & 3.548508 & 2.448773 & -1.358202 \\
\hline $\mathrm{C}$ & -0.571384 & 1.917882 & 2.762687 \\
\hline $\mathrm{H}$ & -0.447788 & 2.056268 & 3.843856 \\
\hline $\mathrm{H}$ & -1.219715 & 2.729692 & 2.403631 \\
\hline $\mathrm{H}$ & -1.117368 & 0.972237 & 2.644090 \\
\hline $\mathrm{C}$ & 2.196845 & 0.585382 & 2.605312 \\
\hline $\mathrm{H}$ & 1.773413 & -0.416017 & 2.464167 \\
\hline $\mathrm{H}$ & 3.194733 & 0.585585 & 2.145966 \\
\hline $\mathrm{H}$ & 2.336213 & 0.727103 & 3.684283 \\
\hline $\mathrm{C}$ & 1.926196 & 3.600697 & 2.307782 \\
\hline $\mathrm{H}$ & 1.996519 & 3.698646 & 3.398379 \\
\hline $\mathrm{H}$ & 2.943009 & 3.669114 & 1.905500 \\
\hline $\mathrm{H}$ & 1.360315 & 4.461160 & 1.935011 \\
\hline $\mathrm{H}$ & 1.425593 & -1.368720 & -2.570899 \\
\hline $\mathrm{C}$ & 2.339518 & -2.259933 & -1.054028 \\
\hline $\mathrm{C}$ & 2.363070 & -2.560296 & 0.331672 \\
\hline $\mathrm{C}$ & 3.434557 & -2.716994 & -1.827263 \\
\hline $\mathrm{C}$ & 3.423175 & -3.261136 & 0.906595 \\
\hline $\mathrm{H}$ & 1.522030 & -2.250254 & 0.949477 \\
\hline
\end{tabular}




\begin{tabular}{|c|c|c|c|}
\hline $\mathrm{C}$ & 4.482710 & -3.422013 & -1.244532 \\
\hline $\mathrm{H}$ & 3.443143 & -2.511265 & -2.896040 \\
\hline $\mathrm{C}$ & 4.494476 & -3.699031 & 0.127431 \\
\hline $\mathrm{H}$ & 3.401280 & -3.471993 & 1.972918 \\
\hline $\mathrm{H}$ & 5.304718 & -3.760396 & -1.870862 \\
\hline & 5.3174 & 4.247846 & 0.57 \\
\hline
\end{tabular}

7d

Li $\quad-2.513721 \quad-0.810273 \quad 1.770328$

$\mathrm{N} \quad-3.292070 \quad-0.048189 \quad 0.194356$

O $\quad-0.029782 \quad 0.350343-1.369103$

$\mathrm{Si} \quad-3.902062 \quad 1.548513 \quad 0.081672$

$\mathrm{Si} \quad-3.866605-1.465291 \quad-0.576612$

$\begin{array}{llll}\mathrm{Li} & -1.320800 & 0.238448 & 0.000601\end{array}$

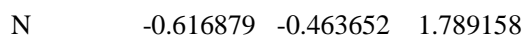

$\begin{array}{llll}\text { C } & 1.168126 & 0.494252 & -1.031974\end{array}$

$\begin{array}{llll}\text { C } & -5.657488 & 1.763377 & 0.765924\end{array}$

$\begin{array}{llll}\text { C } & -3.879865 & 2.203010 & -1.700478\end{array}$

$\begin{array}{llll}\text { C } & -2.755941 & 2.669718 & 1.110334\end{array}$

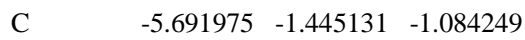

$\begin{array}{lllll}\text { C } & -2.838967 & -1.907744 & -2.108665\end{array}$

$\begin{array}{llll}\text { C } & -3.662064 & -2.897725 & 0.680276\end{array}$

$\begin{array}{llll}\text { C } & 0.366917 & -1.428197 & 1.718115\end{array}$

$\mathrm{H} \quad-0.323169 \quad 0.265716 \quad 2.440816$

$\begin{array}{lllll}\mathrm{N} & 2.126214 & -0.351354 & -1.477340\end{array}$

$\begin{array}{llll}\text { C } & 1.522247 & 1.619029 & -0.111966\end{array}$

$\begin{array}{llll}\mathrm{H} & -6.383286 & 1.181635 & 0.186412\end{array}$

$\mathrm{H} \quad \begin{array}{llll}-5.716312 & 1.420476 & 1.806980\end{array}$

$\begin{array}{llll}\mathrm{H} & -5.975696 & 2.813445 & 0.741173\end{array}$

$\begin{array}{lllll}\mathrm{H} & -4.539724 & 1.610007 & -2.346382\end{array}$

$\mathrm{H} \quad \begin{array}{llll}\mathrm{H} & -2.867750 & 2.133043 & -2.120582\end{array}$

$\mathrm{H} \quad \begin{array}{llll}\mathrm{H} & -4.203053 & 3.249607 & -1.767192\end{array}$

$\mathrm{H} \quad-2.753456 \quad 2.368611 \quad 2.166732$

$\begin{array}{llll}\mathrm{H} & -1.713157 & 2.644009 & 0.765643\end{array}$

$\mathrm{H} \quad \begin{array}{llll}3.077864 & 3.717869 & 1.069245\end{array}$

$\begin{array}{lllll}\mathrm{H} & & -5.985278 & -2.394134 & -1.550454\end{array}$

$\mathrm{H} \quad \begin{array}{llll}-6.343193 & -1.280898 & -0.217720\end{array}$

$\begin{array}{lllll}\mathrm{H} & & -5.895853 & -0.645453 & -1.806861\end{array}$

$\mathrm{H} \quad-3.148422 \quad-2.857178 \quad-2.563568$

$\mathrm{H} \quad\left[\begin{array}{llll}-1.772171 & -1.982958 & -1.863419\end{array}\right.$

$\begin{array}{llll}\mathrm{H} & -2.936619 & -1.120450 & -2.866501\end{array}$

$\mathrm{H} \quad \begin{array}{llll}-2.626034 & -3.039752 & 1.024184\end{array}$ 


\begin{tabular}{|c|c|c|c|}
\hline $\mathrm{H}$ & -4.302655 & -2.742453 & 1.561951 \\
\hline $\mathrm{H}$ & -3.962081 & -3.857945 & 0.243018 \\
\hline $\mathrm{C}$ & 0.253432 & -2.489389 & 0.779443 \\
\hline $\mathrm{C}$ & 1.532669 & -1.432953 & 2.529728 \\
\hline $\mathrm{C}$ & 1.751312 & $-1.519682-$ & -2.280317 \\
\hline $\mathrm{C}$ & 3.529566 & $-0.144483-$ & -1.266652 \\
\hline $\mathrm{C}$ & 0.870976 & 2.843617 & -0.317631 \\
\hline $\mathrm{C}$ & 2.370336 & 1.462244 & 0.991998 \\
\hline $\mathrm{C}$ & 1.219085 & -3.485676 & 0.680374 \\
\hline $\mathrm{H}$ & -0.612403 & -2.512494 & 0.122212 \\
\hline $\mathrm{C}$ & 2.497207 & -2.430502 & 2.418874 \\
\hline $\mathrm{H}$ & 1.656018 & -0.638873 & 3.263714 \\
\hline $\mathrm{H}$ & 2.031523 & -2.434400 & -1.753279 \\
\hline $\mathrm{H}$ & 2.266914 & -1.475594 & -3.244593 \\
\hline $\mathrm{H}$ & 0.674289 & -1.502053 & -2.430551 \\
\hline $\mathrm{C}$ & 4.260626 & -1.091948 & -0.545712 \\
\hline $\mathrm{C}$ & 4.157450 & 0.981843 & -1.801892 \\
\hline $\mathrm{C}$ & 1.086702 & 3.909407 & 0.553723 \\
\hline $\mathrm{H}$ & 0.194057 & 2.947465 & -1.159727 \\
\hline $\mathrm{C}$ & 2.563457 & 2.523243 & 1.874161 \\
\hline $\mathrm{H}$ & 2.844773 & 0.508385 & 1.180807 \\
\hline $\mathrm{C}$ & 2.357415 & -3.472712 & 1.495303 \\
\hline $\mathrm{H}$ & 1.079309 & -4.286441 & -0.043025 \\
\hline $\mathrm{H}$ & 3.369612 & -2.396554 & 3.068150 \\
\hline $\mathrm{C}$ & 5.626684 & -0.894965 & -0.348421 \\
\hline $\mathrm{H}$ & 3.752022 & -1.947681 & -0.112875 \\
\hline $\mathrm{C}$ & 5.521846 & 1.176259 & -1.590305 \\
\hline $\mathrm{H}$ & 3.572677 & 1.702147 & -2.364958 \\
\hline $\mathrm{C}$ & 1.931908 & 3.749435 & 1.653709 \\
\hline $\mathrm{H}$ & 0.586162 & 4.857388 & 0.381048 \\
\hline $\mathrm{H}$ & 3.208789 & 2.389781 & 2.737530 \\
\hline $\mathrm{H}$ & 3.102570 & -4.259531 & 1.425348 \\
\hline $\mathrm{C}$ & 6.258932 & 0.238302 & -0.865213 \\
\hline $\mathrm{H}$ & 6.194602 & -1.624004 & 0.222210 \\
\hline $\mathrm{H}$ & 6.008457 & 2.057739 & -1.997141 \\
\hline $\mathrm{H}$ & 2.093044 & 4.575747 & 2.340169 \\
\hline $\mathrm{H}$ & 7.322105 & 0.389978 & -0.703439 \\
\hline \multicolumn{4}{|l|}{$7 t$} \\
\hline $\mathrm{N}$ & -0.340419 & 1.458844 & -1.159633 \\
\hline $\mathrm{Li}$ & -2.191093 & 1.135994 & -0.834964 \\
\hline $\mathrm{Li}$ & 0.397287 & -0.014084 & -0.040923 \\
\hline
\end{tabular}




\begin{tabular}{|c|c|c|c|}
\hline $\mathrm{N}$ & -0.575830 & -1.420815 & 1.009201 \\
\hline $\mathrm{Li}$ & -2.430527 & -1.216718 & 0.506799 \\
\hline $\mathrm{N}$ & -3.635819 & -0.091885 & -0.505307 \\
\hline $\mathrm{Si}$ & -0.197504 & -1.113454 & 2.658931 \\
\hline $\mathrm{Si}$ & -0.335486 & -2.931853 & 0.215042 \\
\hline $\mathrm{Si}$ & -4.725059 & 0.444587 & 0.719954 \\
\hline $\mathrm{Si}$ & -4.101775 & -0.541099 & -2.107107 \\
\hline $\mathrm{C}$ & -0.601910 & 0.702561 & 3.048294 \\
\hline $\mathrm{H}$ & 0.012923 & 1.391939 & 2.457618 \\
\hline $\mathrm{H}$ & -1.649515 & 0.946615 & 2.832955 \\
\hline $\mathrm{H}$ & -0.423066 & 0.930117 & 4.106501 \\
\hline $\mathrm{C}$ & 1.640738 & -1.353834 & 3.086530 \\
\hline $\mathrm{H}$ & 1.878335 & -0.944994 & 4.076944 \\
\hline $\mathrm{H}$ & 1.908208 & -2.416373 & 3.094227 \\
\hline $\mathrm{H}$ & 2.276279 & -0.858400 & 2.344940 \\
\hline $\mathrm{C}$ & -1.172321 & -2.193088 & 3.882877 \\
\hline $\mathrm{H}$ & -0.929890 & -3.253715 & 3.744805 \\
\hline $\mathrm{H}$ & -0.934794 & -1.933928 & 4.922666 \\
\hline $\mathrm{H}$ & -2.255780 & -2.085780 & 3.754454 \\
\hline $\mathrm{C}$ & -2.059669 & -3.689750 & -0.137423 \\
\hline $\mathrm{H}$ & -1.968522 & -4.638236 & -0.681232 \\
\hline $\mathrm{H}$ & -2.587073 & -3.905630 & 0.803173 \\
\hline $\mathrm{H}$ & -2.714476 & -3.056321 & -0.752443 \\
\hline $\mathrm{C}$ & 0.619561 & -4.258506 & 1.174912 \\
\hline $\mathrm{H}$ & 0.680021 & -5.182455 & 0.585778 \\
\hline $\mathrm{H}$ & 1.642634 & -3.928103 & 1.377048 \\
\hline $\mathrm{H}$ & 0.145787 & -4.502602 & 2.132164 \\
\hline $\mathrm{C}$ & 0.545576 & -2.703726 & -1.445428 \\
\hline $\mathrm{H}$ & 0.531506 & -3.626769 & -2.038205 \\
\hline $\mathrm{H}$ & 0.076912 & -1.918870 & -2.051870 \\
\hline $\mathrm{H}$ & 1.589532 & -2.420864 & -1.283651 \\
\hline $\mathrm{C}$ & -2.544671 & -1.025423 & -3.081366 \\
\hline $\mathrm{H}$ & -2.097339 & -1.954062 & -2.711323 \\
\hline $\mathrm{H}$ & -2.785873 & -1.176308 & -4.141069 \\
\hline $\mathrm{H}$ & -1.769952 & -0.248073 & -3.032579 \\
\hline $\mathrm{C}$ & -5.297960 & -2.009299 & -2.194238 \\
\hline $\mathrm{H}$ & -4.930114 & -2.854882 & -1.599795 \\
\hline $\mathrm{H}$ & -6.292422 & -1.747057 & -1.817498 \\
\hline $\mathrm{H}$ & -5.415760 & -2.356607 & -3.228577 \\
\hline $\mathrm{C}$ & -4.877225 & 0.914276 & -3.050400 \\
\hline $\mathrm{H}$ & -5.148933 & 0.636941 & -4.076674 \\
\hline $\mathrm{H}$ & -5.781955 & 1.284663 & -2.554736 \\
\hline
\end{tabular}




\begin{tabular}{|c|c|c|c|}
\hline $\mathrm{H}$ & -4.172520 & 1.755445 & -3.114074 \\
\hline $\mathrm{C}$ & -4.391313 & -0.570263 & 2.297188 \\
\hline $\mathrm{H}$ & -5.042882 & -0.251218 & 3.119996 \\
\hline $\mathrm{H}$ & -4.583051 & -1.640191 & 2.131142 \\
\hline $\mathrm{H}$ & -3.362344 & -0.463321 & 2.6621 \\
\hline $\mathrm{C}$ & -4.413085 & 2.266220 & 1.151222 \\
\hline $\mathrm{H}$ & -3.389232 & 2.432943 & 1.509886 \\
\hline $\mathrm{H}$ & -4.561071 & 2.908266 & 0.272668 \\
\hline $\mathrm{H}$ & -5.091149 & 2.621212 & 1.93734 \\
\hline $\mathrm{C}$ & -6.571809 & 0.250720 & 0.345876 \\
\hline $\mathrm{H}$ & -7.167229 & 0.638787 & 1.181876 \\
\hline $\mathrm{H}$ & -6.874916 & 0.795815 & -0.55507 \\
\hline $\mathrm{H}$ & -6.843355 & -0.801550 & 0.20623 \\
\hline $\mathrm{H}$ & 0.068824 & 1.247088 & -2.071490 \\
\hline $\mathrm{C}$ & -0.121027 & 2.796906 & -0.868659 \\
\hline $\mathrm{C}$ & -0.645630 & 3.348332 & 0.330157 \\
\hline $\mathrm{C}$ & 0.576258 & 3.689339 & -1.720952 \\
\hline $\mathrm{C}$ & -0.520675 & 4.702995 & 0.628859 \\
\hline $\mathrm{H}$ & -1.151182 & 2.687196 & 1.030374 \\
\hline $\mathrm{C}$ & 0.705309 & 5.038149 & -1.408009 \\
\hline $\mathrm{H}$ & 1.010392 & 3.300531 & -2.640273 \\
\hline $\mathrm{C}$ & 0.153864 & 5.567741 & -0.235879 \\
\hline $\mathrm{H}$ & -0.951157 & 5.081968 & 1.553275 \\
\hline $\mathrm{H}$ & 1.247306 & 5.688319 & -2.091335 \\
\hline $\mathrm{H}$ & 0.250541 & 6.624079 & -0.003963 \\
\hline $\mathrm{C}$ & 3.369159 & 0.378705 & -0.116871 \\
\hline $\mathrm{O}$ & 2.266324 & -0.193331 & -0.100834 \\
\hline $\mathrm{C}$ & 3.457784 & 1.844058 & 0.132692 \\
\hline $\mathrm{C}$ & 2.683173 & 2.383292 & 1.167242 \\
\hline $\mathrm{C}$ & 4.195468 & 2.696209 & -0.699594 \\
\hline $\mathrm{C}$ & 2.670328 & 3.756402 & 1.386942 \\
\hline $\mathrm{H}$ & 2.094721 & 1.719336 & 1.790915 \\
\hline $\mathrm{C}$ & 4.162972 & 4.073012 & -0.489700 \\
\hline $\mathrm{H}$ & 4.767234 & 2.285328 & -1.526415 \\
\hline $\mathrm{C}$ & 3.405590 & 4.602693 & 0.556057 \\
\hline $\mathrm{H}$ & 2.056854 & 4.169457 & 2.179457 \\
\hline $\mathrm{H}$ & 4.716731 & 4.732855 & -1.150992 \\
\hline $\mathrm{H}$ & 3.368103 & 5.676235 & 0.710219 \\
\hline $\mathrm{N}$ & 4.498893 & -0.336073 & -0.377240 \\
\hline $\mathrm{C}$ & 4.349193 & -1.719833 & -0.748574 \\
\hline $\mathrm{C}$ & 4.392887 & -2.071348 & -2.096970 \\
\hline $\mathrm{C}$ & 4.180775 & -2.693882 & 0.236274 \\
\hline
\end{tabular}




$\begin{array}{llll}\text { C } & 4.263414 & -3.411317 & -2.464877 \\ \text { H } & 4.512324 & -1.294984 & -2.846724 \\ \text { C } & 4.052891 & -4.031174 & -0.136648 \\ \text { H } & 4.124461 & -2.396517 & 1.278529 \\ \text { C } & 4.093823 & -4.390598 & -1.485555 \\ \text { H } & 4.287601 & -3.686889 & -3.514854 \\ \text { H } & 3.907981 & -4.790612 & 0.625134 \\ \text { H } & 3.986689 & -5.432417 & -1.772378 \\ \text { C } & 5.868775 & 0.121994 & -0.143703 \\ \text { H } & 6.412505 & 0.238679 & -1.088141 \\ \text { H } & 5.861563 & 1.073595 & 0.385252 \\ \text { H } & 6.390299 & -0.622991 & 0.465261\end{array}$

\section{TS8d}

$\begin{array}{lllll}\text { C } & 0.758889 & 0.391659 & -0.628557\end{array}$

$\begin{array}{lllll}\text { O } & & -0.410984 & 0.476196 & -1.223199\end{array}$

$\begin{array}{lllll}\mathrm{N} & & 1.717725 & -0.377474 & -1.299031\end{array}$

Li $\quad-1.428011 \quad-0.660170 \quad-0.007718$

$\mathrm{Li} \quad-2.021152 \quad 1.229885-1.263310$

$\begin{array}{llll}\text { C } & 1.282655 & 1.667504 & 0.010395\end{array}$

C $\quad 1.296856-1.243114-2.394009$

$\begin{array}{llll}\text { C } & 3.123490 & -0.110358 & -1.229369\end{array}$

$\mathrm{N} \quad-3.239050 \quad 0.093440-0.304732$

$\begin{array}{lllll}\mathrm{N} & 0.259945 & -0.577165 & 0.953278\end{array}$

$\begin{array}{llll}\text { C } & 0.783463 & 2.889847 & -0.448671\end{array}$

$\begin{array}{llll}\text { C } & 2.255423 & 1.674665 & 1.019904\end{array}$

$\begin{array}{lllll}\mathrm{H} & 0.289658 & -1.616453 & -2.214987\end{array}$

$\mathrm{H} \quad 1.296137 \quad-0.711475-3.356852$

$\begin{array}{llll}\mathrm{H} & 1.984215 & -2.090416 & -2.460763\end{array}$

C $\quad 3.973250 \quad-0.985302-0.546485$

$\begin{array}{llll}\text { C } & 3.656818 & 1.007234 & -1.881802\end{array}$

$\mathrm{Si} \quad \quad \quad-4.021995 \quad-1.287084 \quad-0.948918$

$\begin{array}{llll}\mathrm{Si} & -3.840719 & 1.239001 & 0.816411\end{array}$

$\begin{array}{llll}\text { C } & 1.091719 & -1.627937 & 1.329798\end{array}$

$\begin{array}{llll}\mathrm{H} & 0.268977 & 0.142092 & 1.675056\end{array}$

$\begin{array}{llll}\text { C } & 1.233220 & 4.096654 & 0.091152\end{array}$

$\mathrm{H} \quad \begin{array}{llll}\mathrm{H} & 0.051193 & 2.892668 & -1.249311\end{array}$

$\begin{array}{llll}\text { C } & 2.704989 & 2.876404 & 1.560385\end{array}$

$\begin{array}{llll}\mathrm{H} & 2.670455 & 0.738229 & 1.371561\end{array}$

$\begin{array}{llll}\text { C } & 5.343528 & -0.726124 & -0.496938\end{array}$

$\begin{array}{llll}\mathrm{H} & 3.554395 & -1.851129 & -0.048346\end{array}$

$\begin{array}{llll}\text { C } & 5.024812 & 1.267185 & -1.823608\end{array}$ 


\begin{tabular}{|c|c|c|c|}
\hline H & 2.987512 & 1.679679 & -2.409137 \\
\hline $\mathrm{C}$ & -5.182818 & -2.164072 & 0.266162 \\
\hline $\mathrm{C}$ & -2.653264 & -2.539052 & -1.409762 \\
\hline $\mathrm{C}$ & -4.989029 & -0.903705 & -2.532924 \\
\hline $\mathrm{C}$ & -5.675505 & 1.667831 & 0.614639 \\
\hline $\mathrm{C}$ & -3.524372 & 0.736125 & 2.614286 \\
\hline $\mathrm{C}$ & -2.860717 & 2.856035 & 0.505514 \\
\hline $\mathrm{C}$ & 1.144033 & -2.790813 & 0.533691 \\
\hline $\mathrm{C}$ & 1.922795 & -1.578802 & 2.469580 \\
\hline $\mathrm{C}$ & 2.194266 & 4.093405 & 1.100768 \\
\hline $\mathrm{H}$ & 0.832402 & 5.035774 & -0.280173 \\
\hline $\mathrm{H}$ & 3.459253 & 2.862624 & 2.342075 \\
\hline $\mathrm{C}$ & 5.871742 & 0.400943 & -1.128777 \\
\hline $\mathrm{H}$ & 5.996402 & -1.404478 & 0.045236 \\
\hline $\mathrm{H}$ & 5.428893 & 2.145085 & -2.319859 \\
\hline $\mathrm{H}$ & -4.655080 & -2.414162 & 1.195148 \\
\hline $\mathrm{H}$ & -5.576887 & -3.096988 & -0.155931 \\
\hline $\mathrm{H}$ & -6.040410 & -1.535432 & 0.533518 \\
\hline $\mathrm{H}$ & -2.086743 & -2.890228 & -0.532739 \\
\hline $\mathrm{H}$ & -1.941714 & -2.109476 & -2.127998 \\
\hline $\mathrm{H}$ & -3.076013 & -3.437297 & -1.875461 \\
\hline $\mathrm{H}$ & -5.769363 & -0.159561 & -2.330778 \\
\hline $\mathrm{H}$ & -4.326299 & -0.485296 & -3.302321 \\
\hline $\mathrm{H}$ & -5.473254 & -1.791910 & -2.957720 \\
\hline $\mathrm{H}$ & -5.887208 & 2.017359 & -0.403714 \\
\hline $\mathrm{H}$ & -5.984594 & 2.457461 & 1.310927 \\
\hline $\mathrm{H}$ & -6.312736 & 0.795534 & 0.801425 \\
\hline $\mathrm{H}$ & -4.079700 & -0.178042 & 2.857924 \\
\hline $\mathrm{H}$ & -2.458973 & 0.521904 & 2.771300 \\
\hline $\mathrm{H}$ & -3.822321 & 1.511236 & 3.331325 \\
\hline $\mathrm{H}$ & -1.777915 & 2.736487 & 0.651850 \\
\hline $\mathrm{H}$ & -3.031929 & 3.262818 & -0.506329 \\
\hline $\mathrm{H}$ & -3.178921 & 3.645582 & 1.196647 \\
\hline $\mathrm{C}$ & 2.006786 & -3.839070 & 0.846669 \\
\hline $\mathrm{H}$ & 0.494760 & -2.864280 & -0.332497 \\
\hline $\mathrm{C}$ & 2.778055 & -2.631955 & 2.781857 \\
\hline $\mathrm{H}$ & 1.887766 & -0.700081 & 3.109763 \\
\hline $\mathrm{H}$ & 2.545859 & 5.029483 & 1.525104 \\
\hline $\mathrm{H}$ & 6.938033 & 0.603682 & -1.081348 \\
\hline $\mathrm{C}$ & 2.838082 & -3.766570 & 1.967239 \\
\hline $\mathrm{H}$ & 2.027068 & -4.720494 & 0.210785 \\
\hline $\mathrm{H}$ & 3.405745 & -2.564724 & 3.666749 \\
\hline
\end{tabular}


$\begin{array}{llll}\mathrm{H} & 3.509356 & -4.584647 & 2.210216\end{array}$

\section{TS8t}

Si $\quad \begin{array}{llll}\text { Si } & -1.963880 & 2.800982 & -1.405244\end{array}$

$\mathrm{N} \quad \begin{array}{llll}-1.978053 & 1.942814 & 0.110441\end{array}$

$\begin{array}{lllll}\text { C } & & -3.630297 & 3.553561 & -1.904834\end{array}$

$\mathrm{Si} \quad-2.464734 \quad 2.693651 \quad 1.601676$

$\mathrm{Li} \quad-3.435254 \quad 0.566466 \quad 0.355033$

Li $\quad-0.006830 \quad 1.846380 \quad 0.602991$

C $\quad-0.694339 \quad 4.209229-1.406001$

$\begin{array}{llll}\text { C } & -1.488443 & 1.567559 & -2.766248\end{array}$

$\mathrm{H} \quad \begin{array}{lllll}\mathrm{H} & -4.463482 & 2.855991 & -1.755667\end{array}$

$\begin{array}{llll}\mathrm{H} & -3.619775 & 3.824359 & -2.968082\end{array}$

$\mathrm{H} \quad \begin{array}{llll}\mathrm{H} & -3.851315 & 4.462830 & -1.337016\end{array}$

$\begin{array}{llll}\text { C } & -3.178381 & 4.439946 & 1.486929\end{array}$

$\begin{array}{llll}\text { C } & -1.028766 & 2.753689 & 2.837562\end{array}$

$\begin{array}{lllll}\text { C } & -3.848028 & 1.629709 & 2.387353\end{array}$

$\begin{array}{lllll}\mathrm{N} & & -2.771886 & -1.202281 & 0.107867\end{array}$

$\mathrm{Li} \quad \quad \quad-1.295856 \quad-0.018604 \quad-0.425135$

$\begin{array}{llll}\text { O } & 1.666537 & 1.658396 & 1.085417\end{array}$

$\begin{array}{lllll}\mathrm{H} & -0.999108 & 5.009879 & -0.721881\end{array}$

$\begin{array}{lllll}\mathrm{H} & -0.598600 & 4.651267 & -2.405559\end{array}$

$\mathrm{H} \quad 0.306814 \quad 3.883096-1.100743$

$\mathrm{H} \quad \begin{array}{llll}0.599763 & 0.972126 & -2.525577\end{array}$

$\mathrm{H} \quad \begin{array}{llll}\mathrm{H} & -1.257972 & 2.101443 & -3.696321\end{array}$

$\begin{array}{lllll}\mathrm{H} & -2.302630 & 0.871256 & -2.992917\end{array}$

$\begin{array}{llll}\mathrm{H} & -2.485443 & 5.146628 & 1.017684\end{array}$

$\begin{array}{llll}\mathrm{H} & -3.392289 & 4.812985 & 2.496287\end{array}$

$\begin{array}{llll}\mathrm{H} & -4.115560 & 4.463091 & 0.920886\end{array}$

$\begin{array}{llll}\mathrm{H} & -1.390043 & 3.128089 & 3.803375\end{array}$

$\begin{array}{llll}\mathrm{H} & -0.229640 & 3.434114 & 2.512770\end{array}$

$\begin{array}{llll}\mathrm{H} & -0.563424 & 1.778773 & 3.024783\end{array}$

$\begin{array}{llll}\mathrm{H} & -3.547411 & 0.598680 & 2.609064\end{array}$

$\begin{array}{llll}\mathrm{H} & -4.775232 & 1.603031 & 1.789486\end{array}$

$\mathrm{H} \quad-4.137171 \quad 2.073940 \quad 3.346509$

$\begin{array}{lllll}\mathrm{Si} & & -3.626863 & -1.761020 & -1.281935\end{array}$

$\begin{array}{llll}\mathrm{Si} & -2.530354 & -2.169717 & 1.516146\end{array}$

$\begin{array}{llll}\text { C } & 2.158527 & 0.624443 & 0.445835\end{array}$

$\begin{array}{lllll}\text { C } & -2.455176 & -2.175877 & -2.709198\end{array}$

$\begin{array}{lllll}\text { C } & & -4.757635 & -3.261169 & -1.036019\end{array}$

$\begin{array}{lllll}\text { C } & & -4.788451 & -0.336839 & -1.817442\end{array}$

$\begin{array}{llll}\text { C } & -1.981742 & -3.954793 & 1.166281\end{array}$ 


\begin{tabular}{|c|c|c|c|}
\hline $\mathrm{C}$ & -4.109165 & -2.311756 & 2.568089 \\
\hline $\mathrm{C}$ & -1.221999 & -1.367773 & 2.648580 \\
\hline $\mathrm{N}$ & 2.897958 & 0.891678 & -0.778655 \\
\hline $\mathrm{C}$ & 2.826455 & -0.436886 & 1.313481 \\
\hline $\mathrm{H}$ & -1.807094 & -3.012878 & -2.423523 \\
\hline $\mathrm{H}$ & -2.999616 & -2.462603 & -3.618027 \\
\hline $\mathrm{H}$ & -1.789881 & -1.345735 & -2.969917 \\
\hline $\mathrm{H}$ & -5.497849 & -3.092117 & -0.245526 \\
\hline $\mathrm{H}$ & -5.304994 & -3.472577 & -1.963331 \\
\hline $\mathrm{H}$ & -4.190597 & -4.161202 & -0.776412 \\
\hline $\mathrm{H}$ & -4.269994 & 0.616839 & -1.981762 \\
\hline $\mathrm{H}$ & -5.571976 & -0.173499 & -1.060956 \\
\hline $\mathrm{H}$ & -5.305792 & -0.567050 & -2.756759 \\
\hline $\mathrm{H}$ & -2.845292 & -4.612177 & 1.014816 \\
\hline $\mathrm{H}$ & -1.405761 & -4.361877 & 2.007242 \\
\hline $\mathrm{H}$ & -1.356333 & -4.013070 & 0.270295 \\
\hline $\mathrm{H}$ & -4.467278 & -1.339322 & 2.928371 \\
\hline $\mathrm{H}$ & -3.943208 & -2.945717 & 3.448860 \\
\hline $\mathrm{H}$ & -4.921247 & -2.763363 & 1.986134 \\
\hline $\mathrm{H}$ & -1.276999 & -0.272708 & 2.638667 \\
\hline $\mathrm{H}$ & -1.384278 & -1.683418 & 3.686928 \\
\hline $\mathrm{H}$ & -0.191057 & -1.648780 & 2.403348 \\
\hline $\mathrm{C}$ & 2.337391 & 1.989017 & -1.562063 \\
\hline $\mathrm{C}$ & 4.323137 & 1.004253 & -0.668398 \\
\hline $\mathrm{C}$ & 2.639185 & -0.365223 & 2.697471 \\
\hline $\mathrm{C}$ & 3.525845 & -1.526196 & 0.776060 \\
\hline $\mathrm{H}$ & 2.824994 & 2.014300 & -2.540563 \\
\hline $\mathrm{H}$ & 2.469751 & 2.965776 & -1.075185 \\
\hline $\mathrm{H}$ & 1.269080 & 1.823771 & -1.719866 \\
\hline $\mathrm{C}$ & 5.106325 & 0.510232 & -1.717399 \\
\hline $\mathrm{C}$ & 4.944408 & 1.616236 & 0.426934 \\
\hline $\mathrm{C}$ & 3.138222 & -1.363746 & 3.534240 \\
\hline $\mathrm{H}$ & 2.106248 & 0.490269 & 3.097648 \\
\hline $\mathrm{C}$ & 4.028108 & -2.521548 & 1.613286 \\
\hline $\mathrm{H}$ & 3.683557 & -1.591525 & -0.292911 \\
\hline $\mathrm{C}$ & 6.495149 & 0.619619 & -1.672485 \\
\hline $\mathrm{H}$ & 4.608561 & 0.022763 & -2.551101 \\
\hline $\mathrm{C}$ & 6.335722 & 1.705287 & 0.477817 \\
\hline $\mathrm{H}$ & 4.332123 & 2.005501 & 1.233130 \\
\hline $\mathrm{C}$ & 3.833768 & -2.446945 & 2.994011 \\
\hline $\mathrm{H}$ & 2.988341 & -1.292649 & 4.608360 \\
\hline $\mathrm{H}$ & 4.571843 & -3.358342 & 1.183475 \\
\hline
\end{tabular}




$\begin{array}{llll}\mathrm{C} & 7.115271 & 1.212974 & -0.570431 \\ \mathrm{H} & 7.094129 & 0.227030 & -2.489856 \\ \mathrm{H} & 6.811448 & 2.168880 & 1.337867 \\ \mathrm{H} & 4.225590 & -3.224893 & 3.643706 \\ \mathrm{H} & 8.198237 & 1.288043 & -0.528203 \\ \mathrm{~N} & 0.750311 & -0.250756 & -0.096351 \\ \mathrm{C} & 0.966802 & -1.341534 & -0.987848 \\ \mathrm{H} & 0.510246 & -0.667364 & 0.805431 \\ \mathrm{C} & 1.247734 & -1.118542 & -2.345515 \\ \mathrm{C} & 0.895737 & -2.666796 & -0.528526 \\ \mathrm{C} & 1.434314 & -2.193194 & -3.214060 \\ \mathrm{H} & 1.327210 & -0.106611 & -2.718375 \\ \mathrm{C} & 1.069591 & -3.738315 & -1.402208 \\ \mathrm{H} & 0.711492 & -2.852124 & 0.524207 \\ \mathrm{C} & 1.340678 & -3.507700 & -2.751986 \\ \mathrm{H} & 1.649355 & -1.999123 & -4.261318 \\ \mathrm{H} & 1.003464 & -4.753611 & -1.021137 \\ \mathrm{H} & 1.480783 & -4.340567 & -3.434568\end{array}$

9d

$\begin{array}{lllll}\text { C } & & 1.310205 & -0.263777 & 0.098994\end{array}$

O $\quad 0.052628-0.534396 \quad 0.524953$

$\begin{array}{llll}\mathrm{N} & 2.112176 & 0.285634 & 1.256985\end{array}$

Li $\quad-1.353297 \quad 0.641847 \quad 0.719979$

$\mathrm{Li} \quad-1.257733-1.563986-0.095158$

$\begin{array}{llll}\text { C } & 2.005134 & -1.516078 & -0.464364\end{array}$

$\begin{array}{llll}\text { C } & 1.938888 & -0.508521 & 2.471556\end{array}$

$\begin{array}{llll}\text { C } & 3.460969 & 0.662210 & 0.976191\end{array}$

$\mathrm{N} \quad-2.863532 \quad-0.538152 \quad 0.246624$

$\mathrm{N} \quad 1.320385 \quad 0.757055 \quad-0.983994$

$\begin{array}{llll}\text { C } & 1.577128 & -2.785568 & -0.055835\end{array}$

$\begin{array}{llll}\text { C } & 3.109082 & -1.427482 & -1.324920\end{array}$

$\mathrm{H} \quad 2.501378 \quad-0.033395 \quad 3.279313$

$\begin{array}{llll}\mathrm{H} & 2.280015 & -1.552833 & 2.378554\end{array}$

$\mathrm{H} \quad 0.880751 \quad-0.525517 \quad 2.731192$

$\begin{array}{llll}\text { C } & 4.551572 & -0.135340 & 1.355189\end{array}$

$\begin{array}{llll}\text { C } & 3.717788 & 1.875136 & 0.317065\end{array}$

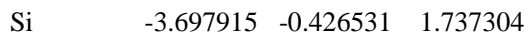

Si $\quad \begin{array}{llll}-3.526187 & -0.632076 & -1.329422\end{array}$

$\begin{array}{lllll}\text { C } & 0.430199 & 1.822989 & -0.931114\end{array}$

$\begin{array}{lllll}\mathrm{H} & & 1.335750 & 0.314057 & -1.894913\end{array}$

$\begin{array}{lllll}\text { C } & 2.211004 & -3.940945 & -0.517272\end{array}$ 


\begin{tabular}{|c|c|c|c|}
\hline $\mathrm{H}$ & 0.756834 & -2.861017 & 0.652389 \\
\hline $\mathrm{C}$ & 3.739034 & -2.580879 & -1.792973 \\
\hline $\mathrm{H}$ & 3.484533 & -0.453067 & -1.617721 \\
\hline $\mathrm{C}$ & 5.859362 & 0.262531 & 1.074974 \\
\hline $\mathrm{H}$ & 4.379249 & -1.081075 & 1.856801 \\
\hline $\mathrm{C}$ & 5.023560 & 2.260780 & 0.023838 \\
\hline $\mathrm{H}$ & 2.887541 & 2.515037 & 0.047121 \\
\hline $\mathrm{C}$ & -4.015978 & -2.117780 & 2.529856 \\
\hline $\mathrm{C}$ & -5.340160 & 0.514041 & 1.645873 \\
\hline $\mathrm{C}$ & -2.565313 & 0.553476 & 2.931895 \\
\hline $\mathrm{C}$ & -4.933512 & -1.892379 & -1.494922 \\
\hline $\mathrm{C}$ & -4.125114 & 1.023072 & -2.020230 \\
\hline $\mathrm{C}$ & -2.101159 & -1.241014 & -2.460007 \\
\hline $\mathrm{C}$ & 0.097824 & 2.456611 & 0.290147 \\
\hline $\mathrm{C}$ & -0.176690 & 2.299804 & -2.109582 \\
\hline $\mathrm{C}$ & 3.290677 & -3.841736 & -1.395689 \\
\hline $\mathrm{H}$ & 1.865062 & -4.916155 & -0.184905 \\
\hline $\mathrm{H}$ & 4.588574 & -2.491818 & -2.464381 \\
\hline $\mathrm{C}$ & 6.103526 & 1.457438 & 0.399939 \\
\hline $\mathrm{H}$ & 6.687442 & -0.373209 & 1.377340 \\
\hline $\mathrm{H}$ & 5.199020 & 3.204264 & -0.486405 \\
\hline $\mathrm{H}$ & -3.074019 & -2.664259 & 2.671612 \\
\hline $\mathrm{H}$ & -4.656620 & -2.728332 & 1.881283 \\
\hline $\mathrm{H}$ & -4.506572 & -2.039791 & 3.508128 \\
\hline $\mathrm{H}$ & -5.804291 & 0.615851 & 2.634748 \\
\hline $\mathrm{H}$ & -5.188377 & 1.521863 & 1.239951 \\
\hline $\mathrm{H}$ & -6.060011 & 0.002613 & 0.995278 \\
\hline $\mathrm{H}$ & -1.582078 & 0.078232 & 3.062465 \\
\hline $\mathrm{H}$ & -2.404901 & 1.592783 & 2.602062 \\
\hline $\mathrm{H}$ & -3.014581 & 0.621250 & 3.929832 \\
\hline $\mathrm{H}$ & -4.609920 & -2.887234 & -1.162897 \\
\hline $\mathrm{H}$ & -5.285852 & -1.981703 & -2.530211 \\
\hline $\mathrm{H}$ & -5.794180 & -1.606821 & -0.877670 \\
\hline $\mathrm{H}$ & -4.958789 & 1.408670 & -1.420907 \\
\hline $\mathrm{H}$ & -4.472667 & 0.935773 & -3.057528 \\
\hline $\mathrm{H}$ & -3.323435 & 1.769368 & -1.990557 \\
\hline $\mathrm{H}$ & -1.231533 & -0.571197 & -2.413070 \\
\hline $\mathrm{H}$ & -1.759510 & -2.264653 & -2.227359 \\
\hline $\mathrm{H}$ & -2.421842 & -1.274649 & -3.507819 \\
\hline $\mathrm{C}$ & -0.826041 & 3.507405 & 0.314028 \\
\hline $\mathrm{H}$ & 0.603014 & 2.130333 & 1.193659 \\
\hline $\mathrm{C}$ & -1.078854 & 3.359055 & -2.071920 \\
\hline
\end{tabular}




$\begin{array}{llll}\mathrm{H} & 0.061817 & 1.824590 & -3.058347 \\ \mathrm{H} & 3.784051 & -4.738241 & -1.760183 \\ \mathrm{H} & 7.121251 & 1.765897 & 0.178031 \\ \mathrm{C} & -1.424547 & 3.965945 & -0.860380 \\ \mathrm{H} & -1.060893 & 3.978083 & 1.265559 \\ \mathrm{H} & -1.531765 & 3.700969 & -2.998362 \\ \mathrm{H} & -2.139659 & 4.781704 & -0.835849\end{array}$

9t

$\mathrm{N} \quad-2.424391 \quad 0.758936-0.136295$

$\mathrm{Li} \quad-0.342779 \quad 1.338586 \quad-0.402763$

$\mathrm{Li} \quad 0.913908 \quad-1.399046 \quad 0.377286$

$\begin{array}{llll}\mathrm{N} & 2.760018 & -0.847501 & 0.264806\end{array}$

$\begin{array}{llll}\mathrm{Li} & 2.324902 & 1.041414 & 0.204801\end{array}$

$\begin{array}{llll}\mathrm{N} & 1.135670 & 2.540857 & -0.045887\end{array}$

Si $\quad 3.529780-1.362523-1.191829$

$\begin{array}{lllll}\mathrm{Si} & 3.382800 & -1.082409 & 1.853668\end{array}$

$\begin{array}{lllll}\mathrm{Si} & & 1.487000 & 3.349459 & -1.529289\end{array}$

$\begin{array}{lllll}\mathrm{Si} & 0.779553 & 3.331431 & 1.448710\end{array}$

$\begin{array}{llll}\text { C } & 2.400735 & -0.891288 & -2.643459\end{array}$

$\mathrm{H} \quad 1.481069-1.489100-2.617530$

$\begin{array}{llll}\mathrm{H} & 2.115105 & 0.166697 & -2.622617\end{array}$

$\mathrm{H} \quad 2.884683 \quad-1.079349 \quad-3.609648$

$\begin{array}{lllll}\text { C } & & 3.788193 & -3.235427 & -1.311040\end{array}$

$\mathrm{H} \quad 4.155306 \quad-3.515411-2.306917$

$\begin{array}{llll}\mathrm{H} & 4.514072 & -3.598409 & -0.575714\end{array}$

$\begin{array}{llll}\mathrm{H} & 2.843863 & -3.767493 & -1.145697\end{array}$

C $\quad 5.219794 \quad-0.532723 \quad-1.438692$

$\begin{array}{llll}\mathrm{H} & 5.918931 & -0.836952 & -0.649541\end{array}$

$\mathrm{H} \quad 5.672761 \quad-0.800668-2.401470$

$\mathrm{H} \quad 5.143695 \quad 0.561105 \quad-1.401155$

$\begin{array}{llll}\text { C } & 4.156895 & 0.538842 & 2.486855\end{array}$

$\mathrm{H} \quad 4.456782 \quad 0.473082 \quad 3.540172$

$\begin{array}{llll}\mathrm{H} & 5.055688 & 0.765859 & 1.898235\end{array}$

$\begin{array}{llll}\mathrm{H} & 3.486664 & 1.405299 & 2.405717\end{array}$

$\begin{array}{llll}\text { C } & 4.705370 & -2.423287 & 2.050790\end{array}$

$\mathrm{H} \quad 5.055836 \quad-2.464600 \quad 3.089815$

$\begin{array}{llll}\mathrm{H} & 4.311324 & -3.412899 & 1.792410\end{array}$

$\mathrm{H} \quad \begin{array}{llll}\mathrm{H} & 5.578131 & -2.236724 & 1.414555\end{array}$

$\begin{array}{llll}\text { C } & 1.936009 & -1.570290 & 2.990338\end{array}$

$\begin{array}{llll}\mathrm{H} & 2.244419 & -1.627035 & 4.041413\end{array}$

$\mathrm{H} \quad 1.102702-0.8597312 .935192$ 


\begin{tabular}{|c|c|c|c|}
\hline $\mathrm{H}$ & 1.549242 & -2.564263 & 2.719455 \\
\hline $\mathrm{C}$ & 0.502830 & 2.023435 & 2.795523 \\
\hline $\mathrm{H}$ & 1.431748 & 1.528371 & 3.098097 \\
\hline $\mathrm{H}$ & 0.070665 & 2.478860 & 3.695025 \\
\hline $\mathrm{H}$ & -0.178437 & 1.241220 & 2.444325 \\
\hline $\mathrm{C}$ & 2.167436 & 4.472093 & 2.053033 \\
\hline $\mathrm{H}$ & 3.112926 & 3.919004 & 2.128416 \\
\hline $\mathrm{H}$ & 2.334007 & 5.314480 & 1.372541 \\
\hline $\mathrm{H}$ & 1.945405 & 4.884588 & 3.045224 \\
\hline $\mathrm{C}$ & -0.836624 & 4.329241 & 1.364990 \\
\hline $\mathrm{H}$ & -1.125950 & 4.713970 & 2.350912 \\
\hline $\mathrm{H}$ & -0.758409 & 5.182841 & 0.683647 \\
\hline $\mathrm{H}$ & -1.661368 & 3.700795 & 1.001225 \\
\hline $\mathrm{C}$ & 3.278190 & 3.011555 & -2.060190 \\
\hline $\mathrm{H}$ & 3.511903 & 3.476925 & -3.025917 \\
\hline $\mathrm{H}$ & 3.978507 & 3.417948 & -1.318276 \\
\hline $\mathrm{H}$ & 3.490242 & 1.940363 & -2.164054 \\
\hline $\mathrm{C}$ & 0.340895 & 2.664130 & -2.888742 \\
\hline $\mathrm{H}$ & 0.461261 & 1.583493 & -3.035934 \\
\hline $\mathrm{H}$ & -0.715971 & 2.858977 & -2.654916 \\
\hline $\mathrm{H}$ & 0.545238 & 3.138113 & -3.856756 \\
\hline $\mathrm{C}$ & 1.247719 & 5.229317 & -1.546130 \\
\hline $\mathrm{H}$ & 1.528988 & 5.630821 & -2.527824 \\
\hline $\mathrm{H}$ & 0.203416 & 5.506930 & -1.364286 \\
\hline $\mathrm{H}$ & 1.863983 & 5.735540 & -0.795142 \\
\hline $\mathrm{H}$ & -2.261858 & 1.196564 & 0.770214 \\
\hline $\mathrm{C}$ & -3.786791 & 0.990463 & -0.483138 \\
\hline $\mathrm{C}$ & -4.523715 & 0.166042 & -1.342155 \\
\hline $\mathrm{C}$ & -4.394953 & 2.146497 & 0.032759 \\
\hline $\mathrm{C}$ & -5.836837 & 0.502484 & -1.676414 \\
\hline $\mathrm{H}$ & -4.082260 & -0.740459 & -1.731294 \\
\hline $\mathrm{C}$ & -5.702267 & 2.475861 & -0.309515 \\
\hline $\mathrm{H}$ & -3.830719 & 2.791742 & 0.702652 \\
\hline $\mathrm{C}$ & -6.434986 & 1.654267 & -1.169512 \\
\hline $\mathrm{H}$ & -6.394467 & -0.153204 & -2.339563 \\
\hline $\mathrm{H}$ & -6.150205 & 3.375897 & 0.102048 \\
\hline $\mathrm{H}$ & -7.456621 & 1.907885 & -1.435241 \\
\hline $\mathrm{C}$ & -1.786433 & -0.606562 & 0.044241 \\
\hline $\mathrm{O}$ & -0.510716 & -0.303686 & 0.423864 \\
\hline $\mathrm{C}$ & -2.590096 & -1.344067 & 1.125165 \\
\hline $\mathrm{C}$ & -2.266153 & -1.103502 & 2.466108 \\
\hline $\mathrm{C}$ & -3.670562 & -2.180308 & 0.827905 \\
\hline
\end{tabular}




\begin{tabular}{llll}
$\mathrm{C}$ & -3.010882 & -1.684669 & 3.491822 \\
$\mathrm{H}$ & -1.414794 & -0.473382 & 2.696917 \\
$\mathrm{C}$ & -4.413106 & -2.765601 & 1.854317 \\
$\mathrm{H}$ & -3.912938 & -2.394345 & -0.205386 \\
$\mathrm{C}$ & -4.089129 & -2.518670 & 3.188779 \\
$\mathrm{H}$ & -2.744179 & -1.490089 & 4.526956 \\
$\mathrm{H}$ & -5.244742 & -3.419751 & 1.607731 \\
$\mathrm{H}$ & -4.668288 & -2.975779 & 3.986034 \\
$\mathrm{C}$ & -1.631844 & -0.546736 & -2.463259 \\
$\mathrm{H}$ & -2.211059 & -0.965208 & -3.291277 \\
$\mathrm{H}$ & -1.996124 & 0.462952 & -2.296796 \\
$\mathrm{H}$ & -0.579252 & -0.500177 & -2.769920 \\
$\mathrm{~N}$ & -1.813310 & -1.352069 & -1.248011 \\
$\mathrm{C}$ & -1.112019 & -2.576321 & -1.263999 \\
$\mathrm{C}$ & -0.639139 & -3.222426 & -0.093354 \\
$\mathrm{C}$ & -0.818264 & -3.205832 & -2.493923 \\
$\mathrm{C}$ & 0.077637 & -4.419552 & -0.164403 \\
$\mathrm{H}$ & -0.873449 & -2.826683 & 0.885211 \\
$\mathrm{C}$ & -0.102839 & -4.399368 & -2.548878 \\
$\mathrm{H}$ & -1.155458 & -2.767012 & -3.423540 \\
$\mathrm{C}$ & 0.360722 & -5.020087 & -1.389000 \\
$\mathrm{H}$ & 0.413351 & -4.877543 & 0.762276 \\
$\mathrm{H}$ & 0.097435 & -4.842832 & -3.520297 \\
$\mathrm{H}$ & 0.926345 & -5.944649 & -1.439453 \\
& & & \\
\hline
\end{tabular}

\section{TS10d}

$\begin{array}{lllll}\mathrm{O} & 0.088360 & 0.963097 & -0.023830\end{array}$

$\begin{array}{llll}\text { C } & 1.380961 & 0.594422 & 0.123766\end{array}$

$\mathrm{Li} \quad-1.033562 \quad 0.786001 \quad-1.408050$

$\mathrm{N} \quad 1.620699-0.153822 \quad 1.384016$

$\mathrm{N} \quad 1.766449-0.292024-1.066839$

$\begin{array}{llll}\text { C } & 2.333410 & 1.804194 & 0.176069\end{array}$

$\mathrm{Li} \quad-1.423155 \quad 0.351457 \quad 0.820996$

$\begin{array}{lllll}\mathrm{N} & & -2.751686 & 0.329374 & -0.646655\end{array}$

$\begin{array}{llll}\text { C } & 0.602184 & -0.816351 & 2.051444\end{array}$

$\begin{array}{llll}\mathrm{H} & 2.212479 & 0.374291 & 2.011239\end{array}$

$\begin{array}{lllll}\text { C } & 1.770783 & 0.468837 & -2.313967\end{array}$

$\begin{array}{llll}\text { C } & 2.869988 & -1.188325 & -0.942391\end{array}$

$\begin{array}{llll}\text { C } & 3.704889 & 1.637446 & 0.420621\end{array}$

$\begin{array}{lllll}\text { C } & 1.837787 & 3.090637 & -0.048241\end{array}$

Si $\quad \begin{array}{llll}\text { Si } & -3.090699 & -0.875801 & -1.814570\end{array}$

$\begin{array}{llll}\mathrm{Si} & -3.900266 & 1.198605 & 0.280827\end{array}$ 


\begin{tabular}{|c|c|c|c|}
\hline $\mathrm{C}$ & 0.502674 & -0.720102 & 3.455297 \\
\hline $\mathrm{C}$ & -0.358145 & -1.600878 & 1.373138 \\
\hline $\mathrm{H}$ & 0.835981 & 1.027315 & -2.416004 \\
\hline $\mathrm{H}$ & 2.575766 & 1.214719 & -2.386449 \\
\hline $\mathrm{H}$ & 1.847210 & -0.221238 & -3.158059 \\
\hline $\mathrm{C}$ & 4.070124 & -1.003772 & -1.649834 \\
\hline $\mathrm{C}$ & 2.752583 & -2.331761 & -0.132974 \\
\hline $\mathrm{C}$ & 4.558841 & 2.740871 & 0.437520 \\
\hline $\mathrm{H}$ & 4.109016 & 0.644591 & 0.586893 \\
\hline $\mathrm{C}$ & 2.692544 & 4.194190 & -0.033768 \\
\hline $\mathrm{H}$ & 0.773866 & 3.209699 & -0.219911 \\
\hline $\mathrm{C}$ & -3.382362 & -2.610035 & -1.108274 \\
\hline $\mathrm{C}$ & -4.535636 & -0.455151 & -2.966713 \\
\hline $\mathrm{C}$ & -1.527228 & -1.011666 & -2.919472 \\
\hline $\mathrm{C}$ & -4.488273 & 2.790570 & -0.555953 \\
\hline $\mathrm{C}$ & -5.414041 & 0.180127 & 0.794437 \\
\hline $\mathrm{C}$ & -3.026673 & 1.701056 & 1.915595 \\
\hline $\mathrm{C}$ & -0.515202 & -1.366807 & 4.147770 \\
\hline $\mathrm{H}$ & 1.231208 & -0.120138 & 3.995517 \\
\hline $\mathrm{C}$ & -1.390780 & -2.229070 & 2.080211 \\
\hline $\mathrm{H}$ & -0.284950 & -1.715398 & 0.296975 \\
\hline $\mathrm{C}$ & 5.118385 & -1.919407 & -1.537520 \\
\hline $\mathrm{H}$ & 4.202108 & -0.133814 & -2.281729 \\
\hline $\mathrm{C}$ & 3.805107 & -3.233598 & -0.012345 \\
\hline $\mathrm{H}$ & 1.824534 & -2.520691 & 0.388823 \\
\hline $\mathrm{C}$ & 4.056416 & 4.023176 & 0.208304 \\
\hline $\mathrm{H}$ & 5.618937 & 2.596201 & 0.627547 \\
\hline $\mathrm{H}$ & 2.290873 & 5.189237 & -0.206178 \\
\hline $\mathrm{H}$ & -4.159798 & -2.593309 & -0.335064 \\
\hline $\mathrm{H}$ & -3.697712 & -3.317451 & -1.885347 \\
\hline $\mathrm{H}$ & -2.466592 & -3.007069 & -0.654190 \\
\hline $\mathrm{H}$ & -4.652195 & -1.199920 & -3.763872 \\
\hline $\mathrm{H}$ & -4.384370 & 0.523470 & -3.439278 \\
\hline $\mathrm{H}$ & -5.484268 & -0.409335 & -2.418207 \\
\hline $\mathrm{H}$ & -0.610275 & -1.245108 & -2.356783 \\
\hline $\mathrm{H}$ & -1.345116 & -0.105366 & -3.521199 \\
\hline $\mathrm{H}$ & -1.653166 & -1.823322 & -3.645214 \\
\hline $\mathrm{H}$ & -5.181220 & 3.366517 & 0.070015 \\
\hline $\mathrm{H}$ & -3.635310 & 3.437693 & -0.797377 \\
\hline $\mathrm{H}$ & -5.000493 & 2.557536 & -1.497506 \\
\hline $\mathrm{H}$ & -6.086760 & 0.753521 & 1.444255 \\
\hline $\mathrm{H}$ & -5.104740 & -0.718231 & 1.343043 \\
\hline
\end{tabular}




$\begin{array}{llll}\mathrm{H} & -5.996526 & -0.148766 & -0.074321 \\ \mathrm{H} & -2.125793 & 2.314106 & 1.760097 \\ \mathrm{H} & -2.755581 & 0.835100 & 2.542353 \\ \mathrm{H} & -3.704482 & 2.306316 & 2.528497 \\ \mathrm{C} & -1.481168 & -2.116929 & 3.467048 \\ \mathrm{H} & -0.565031 & -1.270014 & 5.228836 \\ \mathrm{H} & -2.124601 & -2.809057 & 1.530066 \\ \mathrm{C} & 4.998213 & -3.035348 & -0.712696 \\ \mathrm{H} & 6.034545 & -1.749053 & -2.096996 \\ \mathrm{H} & 3.684175 & -4.108065 & 0.621531 \\ \mathrm{H} & 4.722330 & 4.881545 & 0.220758 \\ \mathrm{H} & -2.281967 & -2.608740 & 4.009824 \\ \mathrm{H} & 5.814194 & -3.746812 & -0.624462\end{array}$

\section{TS10t}

$\begin{array}{lllll}\mathrm{N} & 2.457400 & -1.430891 & -0.412080\end{array}$

$\mathrm{Li} \quad-0.579977-1.381497-0.148675$

$\mathrm{Li} \quad-0.124648 \quad 1.413122-0.101908$

$\mathrm{N} \quad-1.905922 \quad 2.121190 \quad-0.199569$

$\mathrm{Li} \quad-2.771593 \quad 0.444520 \quad 0.264159$

$\mathrm{N} \quad-2.457668-1.462066 \quad 0.417388$

Si $\quad-1.930159 \quad 2.726430-1.815842$

Si $\quad \begin{array}{llll}2.351706 & 3.008719 & 1.210356\end{array}$

Si $\quad \begin{array}{llll}-3.634556 & -1.903379 & -0.760195\end{array}$

$\mathrm{Si} \quad \quad-2.102921 \quad-2.183070 \quad 1.944290$

$\begin{array}{lllll}\text { C } & -1.724242 & 1.276396 & -3.021584\end{array}$

$\mathrm{H} \quad \begin{array}{llll}0.922407 & 0.598207 & -2.709601\end{array}$

$\mathrm{H} \quad \begin{array}{llll}-2.636752 & 0.676875 & -3.107444\end{array}$

$\mathrm{H} \quad \begin{array}{llll}-1.472689 & 1.638027 & -4.026656\end{array}$

$\begin{array}{lllll}\text { C } & -0.436257 & 3.868543 & -2.104103\end{array}$

$\mathrm{H} \quad-0.407285 \quad 4.268596 \quad-3.125174$

$\mathrm{H} \quad \begin{array}{llll}-0.450330 & 4.718481 & -1.410510\end{array}$

$\mathrm{H} \quad 0.511223 \quad 3.331897 \quad-1.943526$

$\begin{array}{lllll}\text { C } & -3.508736 & 3.641079 & -2.322685\end{array}$

$\mathrm{H} \quad \begin{array}{llll}\mathrm{H} & -3.594967 & 4.622377 & -1.845885\end{array}$

$\mathrm{H} \quad \begin{array}{llll}\mathrm{H} & -3.529552 & 3.795894 & -3.408754\end{array}$

$\mathrm{H} \quad \begin{array}{llll}-4.399640 & 3.059171 & -2.054228\end{array}$

$\begin{array}{llll}\text { C } & -3.838986 & 2.126517 & 2.018478\end{array}$

$\begin{array}{llll}\mathrm{H} & -4.116553 & 2.607811 & 2.964374\end{array}$

$\begin{array}{llll}\mathrm{H} & -4.713904 & 2.176882 & 1.356002\end{array}$

$\begin{array}{llll}\mathrm{H} & -3.660854 & 1.070048 & 2.263089\end{array}$

$\begin{array}{llll}\text { C } & -2.878218 & 4.808454 & 0.948039\end{array}$ 


\begin{tabular}{|c|c|c|c|}
\hline $\mathrm{H}$ & -3.003251 & 5.308856 & 1.916478 \\
\hline $\mathrm{H}$ & -2.128499 & 5.372220 & 0.380145 \\
\hline $\mathrm{H}$ & -3.829687 & 4.882724 & 0.411099 \\
\hline $\mathrm{C}$ & -0.930519 & 3.044623 & 2.470252 \\
\hline $\mathrm{H}$ & -1.271953 & 3.412747 & 3.445722 \\
\hline $\mathrm{H}$ & -0.483681 & 2.057151 & 2.636878 \\
\hline $\mathrm{H}$ & -0.130634 & 3.716944 & 2.132342 \\
\hline $\mathrm{C}$ & -1.924839 & -0.861328 & 3.295029 \\
\hline $\mathrm{H}$ & -2.904423 & -0.570262 & 3.693720 \\
\hline $\mathrm{H}$ & -1.322746 & -1.228348 & 4.135401 \\
\hline $\mathrm{H}$ & -1.442639 & 0.045224 & 2.915356 \\
\hline $\mathrm{C}$ & -3.337352 & -3.451379 & 2.616092 \\
\hline $\mathrm{H}$ & -4.344810 & -3.032269 & 2.717286 \\
\hline $\mathrm{H}$ & -3.409115 & -4.344960 & 1.987763 \\
\hline $\mathrm{H}$ & -3.010988 & -3.771731 & 3.613993 \\
\hline $\mathrm{C}$ & -0.431305 & -3.102009 & 1.825736 \\
\hline $\mathrm{H}$ & -0.217591 & -3.625718 & 2.765823 \\
\hline $\mathrm{H}$ & -0.451673 & -3.859417 & 1.029055 \\
\hline $\mathrm{H}$ & 0.421598 & -2.434821 & 1.650555 \\
\hline $\mathrm{C}$ & -4.713541 & -0.358254 & -1.112845 \\
\hline $\mathrm{H}$ & -5.414628 & -0.567685 & -1.930003 \\
\hline $\mathrm{H}$ & -5.317752 & -0.094205 & -0.232995 \\
\hline $\mathrm{H}$ & -4.160361 & 0.539757 & -1.421194 \\
\hline $\mathrm{C}$ & -2.821284 & -2.420108 & -2.387393 \\
\hline $\mathrm{H}$ & -2.160719 & -1.640822 & -2.7799 \\
\hline $\mathrm{H}$ & -2.207127 & -3.318522 & -2.246906 \\
\hline $\mathrm{H}$ & -3.565009 & -2.644334 & -3.162601 \\
\hline $\mathrm{C}$ & -4.869872 & -3.264378 & -0.309151 \\
\hline $\mathrm{H}$ & -5.583340 & -3.390767 & -1.133407 \\
\hline $\mathrm{H}$ & -4.377051 & -4.229522 & -0.148725 \\
\hline $\mathrm{H}$ & -5.441460 & -3.028138 & 0.594350 \\
\hline $\mathrm{H}$ & 2.817753 & -2.150136 & 0.200372 \\
\hline $\mathrm{C}$ & 1.752150 & -1.947448 & -1.494194 \\
\hline $\mathrm{C}$ & 1.147896 & -1.119199 & -2.463771 \\
\hline $\mathrm{C}$ & 1.658504 & -3.344691 & -1.658864 \\
\hline $\mathrm{C}$ & 0.512974 & -1.682330 & -3.572258 \\
\hline $\mathrm{H}$ & 1.210895 & -0.044015 & -2.349190 \\
\hline $\mathrm{C}$ & 1.028739 & -3.891770 & -2.774720 \\
\hline $\mathrm{H}$ & 2.102013 & -3.996153 & -0.909224 \\
\hline $\mathrm{C}$ & 0.453078 & -3.065603 & -3.742994 \\
\hline $\mathrm{H}$ & 0.059398 & -1.023786 & -4.307117 \\
\hline $\mathrm{H}$ & 0.982997 & -4.972066 & -2.881368 \\
\hline
\end{tabular}




\begin{tabular}{|c|c|c|c|}
\hline 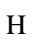 & -0.045469 & -3.492453 & -4.607102 \\
\hline $\mathrm{C}$ & 2.000811 & -0.252518 & 0.347374 \\
\hline $\mathrm{O}$ & 0.646797 & -0.118376 & 0.327615 \\
\hline $\mathrm{C}$ & 2.551253 & -0.428968 & 1.772627 \\
\hline $\mathrm{C}$ & 1.715473 & -0.211273 & 2.869931 \\
\hline $\mathrm{C}$ & 3.894168 & -0.766626 & 1.998318 \\
\hline $\mathrm{C}$ & 2.201603 & -0.331086 & 4.172021 \\
\hline $\mathrm{H}$ & 0.678806 & 0.034437 & 2.685973 \\
\hline $\mathrm{C}$ & 4.380732 & -0.888265 & 3.300800 \\
\hline $\mathrm{H}$ & 4.563389 & -0.924901 & 1.159536 \\
\hline $\mathrm{C}$ & 3.537465 & -0.670560 & 4.392005 \\
\hline $\mathrm{H}$ & 1.533093 & -0.163637 & 5.012143 \\
\hline $\mathrm{H}$ & 5.422605 & -1.151742 & 3.461102 \\
\hline $\mathrm{H}$ & 3.918895 & -0.766208 & 5.404725 \\
\hline $\mathrm{C}$ & 2.377129 & 2.191485 & 0.581546 \\
\hline $\mathrm{H}$ & 2.675428 & 3.089889 & 0.035511 \\
\hline $\mathrm{H}$ & 1.321792 & 2.293600 & 0.844527 \\
\hline $\mathrm{H}$ & 2.904805 & 2.166960 & 1.545520 \\
\hline $\mathrm{N}$ & 2.611553 & 1.018146 & -0.264445 \\
\hline $\mathrm{C}$ & 3.960247 & 0.948929 & -0.746221 \\
\hline $\mathrm{C}$ & 5.028840 & 1.565473 & -0.074649 \\
\hline $\mathrm{C}$ & 4.235818 & 0.280623 & -1.950248 \\
\hline $\mathrm{C}$ & 6.327892 & 1.502219 & -0.582486 \\
\hline $\mathrm{H}$ & 4.860474 & 2.087067 & 0.859442 \\
\hline $\mathrm{C}$ & 5.534056 & 0.208658 & -2.446573 \\
\hline $\mathrm{H}$ & 3.426949 & -0.171202 & -2.507263 \\
\hline $\mathrm{C}$ & 6.591642 & 0.817792 & -1.766636 \\
\hline $\mathrm{H}$ & 7.134417 & 1.988605 & -0.040205 \\
\hline $\mathrm{H}$ & 5.716215 & -0.316155 & -3.380514 \\
\hline $\mathrm{H}$ & 7.602381 & 0.767658 & -2.161207 \\
\hline
\end{tabular}

$\begin{array}{llll}\text { 11d } & & & \\ \mathrm{O} & -0.079694 & -0.766531 & -0.743294 \\ \mathrm{C} & -1.225121 & -0.237814 & -0.298594 \\ \mathrm{Li} & 1.486077 & -1.318085 & -0.048627 \\ \mathrm{~N} & -1.207792 & -0.140258 & 1.199180 \\ \mathrm{C} & -2.469961 & -0.995087 & -0.786335 \\ \mathrm{Li} & 0.820920 & 0.899596 & -0.610231 \\ \mathrm{~N} & 2.664998 & 0.256095 & -0.357700 \\ \mathrm{C} & -0.751780 & -1.344060 & 1.823030 \\ \mathrm{H} & -2.137396 & 0.092736 & 1.539275 \\ \mathrm{C} & -2.311677 & -2.051771 & -1.686252\end{array}$




\begin{tabular}{|c|c|c|c|}
\hline $\mathrm{C}$ & -3.762635 & -0.668100 & -0.351489 \\
\hline $\mathrm{Si}$ & 3.421476 & 1.187486 & 0.865466 \\
\hline $\mathrm{Si}$ & 3.363667 & -0.372050 & -1.792545 \\
\hline $\mathrm{C}$ & -1.587067 & -2.445204 & 2.047304 \\
\hline $\mathrm{C}$ & 0.597056 & -1.431930 & 2.199534 \\
\hline $\mathrm{C}$ & -3.416464 & -2.772976 & -2.141651 \\
\hline $\mathrm{H}$ & -1.307073 & -2.296791 & -2.010582 \\
\hline $\mathrm{C}$ & -4.869157 & -1.383565 & -0.809456 \\
\hline $\mathrm{H}$ & -3.919416 & 0.152923 & 0.340876 \\
\hline $\mathrm{C}$ & 4.734449 & 2.409631 & 0.248516 \\
\hline $\mathrm{C}$ & 4.235085 & 0.105460 & 2.198698 \\
\hline $\mathrm{C}$ & 2.085288 & 2.242715 & 1.722217 \\
\hline C & 5.253803 & -0.512540 & -1.808642 \\
\hline $\mathrm{C}$ & 2.827686 & 0.615031 & -3.321977 \\
\hline $\mathrm{C}$ & 2.690280 & -2.149237 & -2.041661 \\
\hline $\mathrm{C}$ & -1.071638 & -3.617795 & 2.603139 \\
\hline $\mathrm{H}$ & -2.632047 & -2.389319 & 1.759122 \\
\hline $\mathrm{C}$ & 1.118047 & -2.612625 & 2.735306 \\
\hline $\mathrm{H}$ & 1.221710 & -0.550188 & 2.098690 \\
\hline $\mathrm{C}$ & -4.699647 & -2.441106 & -1.705818 \\
\hline $\mathrm{H}$ & -3.273618 & -3.596679 & -2.836085 \\
\hline $\mathrm{H}$ & -5.863695 & -1.115507 & -0.463207 \\
\hline $\mathrm{H}$ & 5.038835 & 3.096586 & 1.048361 \\
\hline $\mathrm{H}$ & 4.346595 & 3.015568 & -0.580274 \\
\hline $\mathrm{H}$ & 5.635512 & 1.898837 & -0.107705 \\
\hline $\mathrm{H}$ & 5.000460 & -0.540241 & 1.749689 \\
\hline $\mathrm{H}$ & 3.502720 & -0.548200 & 2.689352 \\
\hline $\mathrm{H}$ & 4.719955 & 0.701600 & 2.981983 \\
\hline $\mathrm{H}$ & 1.259922 & 1.638551 & 2.119718 \\
\hline $\mathrm{H}$ & 1.653293 & 2.974722 & 1.024182 \\
\hline $\mathrm{H}$ & 2.502855 & 2.813954 & 2.560577 \\
\hline $\mathrm{H}$ & 5.625134 & -0.996454 & -0.896889 \\
\hline $\mathrm{H}$ & 5.592288 & -1.109728 & -2.664707 \\
\hline $\mathrm{H}$ & 5.732531 & 0.469963 & -1.881521 \\
\hline $\mathrm{H}$ & 3.154226 & 1.659214 & -3.235317 \\
\hline $\mathrm{H}$ & 1.733430 & 0.616114 & -3.419864 \\
\hline $\mathrm{H}$ & 3.239582 & 0.210717 & -4.255045 \\
\hline $\mathrm{H}$ & 1.597210 & -2.171172 & -2.154692 \\
\hline $\mathrm{H}$ & 2.976170 & -2.826142 & -1.219822 \\
\hline $\mathrm{H}$ & 3.105550 & -2.591203 & -2.955229 \\
\hline $\mathrm{C}$ & 0.282146 & -3.714409 & 2.933077 \\
\hline $\mathrm{H}$ & -1.730282 & -4.466500 & 2.765571 \\
\hline
\end{tabular}




$\begin{array}{llll}\mathrm{H} & 2.165541 & -2.657459 & 3.021904 \\ \mathrm{H} & -5.561029 & -3.001475 & -2.058513 \\ \mathrm{H} & 0.677588 & -4.632569 & 3.357168 \\ \mathrm{~N} & -1.244363 & 1.233805 & -0.822742 \\ \mathrm{C} & -1.384265 & 1.237064 & -2.289803 \\ \mathrm{C} & -2.094793 & 2.180407 & -0.156174 \\ \mathrm{H} & -1.218122 & 2.253424 & -2.658225 \\ \mathrm{H} & -2.362474 & 0.885045 & -2.641013 \\ \mathrm{H} & -0.629216 & 0.568929 & -2.709000 \\ \mathrm{C} & -3.320772 & 2.602010 & -0.693904 \\ \mathrm{C} & -1.677084 & 2.726169 & 1.068366 \\ \mathrm{C} & -4.114712 & 3.526777 & -0.013651 \\ \mathrm{H} & -3.666635 & 2.200818 & -1.638839 \\ \mathrm{C} & -2.479729 & 3.638345 & 1.750106 \\ \mathrm{H} & -0.723842 & 2.422457 & 1.478639 \\ \mathrm{C} & -3.704214 & 4.043684 & 1.214680 \\ \mathrm{H} & -5.060285 & 3.838747 & -0.448735 \\ \mathrm{H} & -2.136417 & 4.045368 & 2.697226 \\ \mathrm{H} & -4.323658 & 4.763022 & 1.742405\end{array}$

$11 t$

N $\quad \begin{array}{llll}2.377081 & -0.597301 & -1.219333\end{array}$

$\mathrm{Li} \quad-0.708378 \quad-1.475156-0.521952$

$\begin{array}{lllll}\mathrm{Li} & & 0.184177 & 1.248732 & 0.381668\end{array}$

$\begin{array}{llll}\mathrm{N} & -1.483285 & 2.236267 & 0.236224\end{array}$

$\mathrm{Li} \quad-2.545767 \quad 0.628113 \quad 0.212294$

$\begin{array}{lllll}\mathrm{N} & & -2.590945 & -1.288986 & 0.015441\end{array}$

Si $\quad-1.239073 \quad 3.045632-1.266611$

$\begin{array}{llll}\mathrm{Si} & -2.131364 & 2.937644 & 1.669280\end{array}$

$\mathrm{Si} \quad-3.795454-1.387739-1.209029$

$\begin{array}{llll}\mathrm{Si} & -2.508772 & -2.179597 & 1.493946\end{array}$

$\begin{array}{lllll}\text { C } & -0.733686 & 1.712907 & -2.527618\end{array}$

$\mathrm{H} \quad 0.138052 \quad 1.139013 \quad-2.188829$

$\begin{array}{llll}\mathrm{H} & -1.553317 & 1.000042 & -2.686817\end{array}$

$\mathrm{H} \quad-0.480674 \quad 2.146439 \quad-3.502751$

$\begin{array}{llll}\text { C } & 0.161932 & 4.321034 & -1.168695\end{array}$

$\begin{array}{llll}\mathrm{H} & 0.384602 & 4.766360 & -2.146729\end{array}$

$\begin{array}{llll}\mathrm{H} & -0.109854 & 5.136994 & -0.487637\end{array}$

$\begin{array}{llll}\mathrm{H} & 1.089670 & 3.873991 & -0.791682\end{array}$

$\begin{array}{llll}\text { C } & -2.757505 & 3.941416 & -1.967190\end{array}$

$\mathrm{H} \quad-2.987365 \quad 4.843038 \quad-1.389165$

$\begin{array}{lllll}\mathrm{H} & -2.578753 & 4.252574 & -3.004393\end{array}$ 


\begin{tabular}{|c|c|c|c|}
\hline $\mathrm{H}$ & -3.651198 & 3.306490 & -1.960005 \\
\hline $\mathrm{C}$ & -3.752944 & 2.021428 & 2.106532 \\
\hline $\mathrm{H}$ & -4.136013 & 2.350002 & 3.080726 \\
\hline $\mathrm{H}$ & -4.531950 & 2.235125 & 1.362379 \\
\hline $\mathrm{H}$ & -3.649301 & 0.928917 & 2.179115 \\
\hline $\mathrm{C}$ & -2.578999 & 4.775594 & 1.571822 \\
\hline $\mathrm{H}$ & -2.924997 & 5.136681 & 2.548398 \\
\hline $\mathrm{H}$ & -1.717220 & 5.387172 & 1.279900 \\
\hline $\mathrm{H}$ & -3.379482 & 4.960686 & 0.847232 \\
\hline $\mathrm{C}$ & -0.951458 & 2.722303 & 3.136896 \\
\hline $\mathrm{H}$ & -1.391535 & 3.100764 & 4.067834 \\
\hline $\mathrm{H}$ & -0.696990 & 1.668377 & 3.299555 \\
\hline $\mathrm{H}$ & -0.017454 & 3.272357 & 2.965201 \\
\hline $\mathrm{C}$ & -1.541945 & -1.179190 & 2.785746 \\
\hline $\mathrm{H}$ & -2.147300 & -0.364276 & 3.200169 \\
\hline $\mathrm{H}$ & -1.235420 & -1.813075 & 3.627460 \\
\hline $\mathrm{H}$ & -0.640246 & -0.744507 & 2.34391 \\
\hline $\mathrm{C}$ & -4.165401 & -2.610797 & 2.307132 \\
\hline $\mathrm{H}$ & -4.803642 & -1.722387 & 2.396311 \\
\hline $\mathrm{H}$ & -4.727194 & -3.370913 & 1.756080 \\
\hline $\mathrm{H}$ & -3.991160 & -2.993100 & 3.321071 \\
\hline $\mathrm{C}$ & -1.577727 & -3.815576 & 1.201286 \\
\hline $\mathrm{H}$ & -1.428557 & -4.380273 & 2.130342 \\
\hline $\mathrm{H}$ & -2.149045 & -4.451137 & 0.511972 \\
\hline $\mathrm{H}$ & -0.583685 & -3.670504 & 0.754281 \\
\hline $\mathrm{C}$ & -4.567415 & 0.345212 & -1.429223 \\
\hline $\mathrm{H}$ & -5.298156 & 0.343099 & -2.247360 \\
\hline $\mathrm{H}$ & -5.098782 & 0.671939 & -0.524755 \\
\hline $\mathrm{H}$ & -3.830607 & 1.119371 & -1.683789 \\
\hline $\mathrm{C}$ & -3.068630 & -1.855088 & -2.898145 \\
\hline $\mathrm{H}$ & -2.218148 & -1.220336 & -3.176268 \\
\hline $\mathrm{H}$ & -2.726814 & -2.896866 & -2.892679 \\
\hline $\mathrm{H}$ & -3.821158 & -1.757757 & -3.690502 \\
\hline $\mathrm{C}$ & -5.227992 & -2.586539 & -0.904203 \\
\hline $\mathrm{H}$ & -5.886740 & -2.616674 & -1.781270 \\
\hline $\mathrm{H}$ & -4.867746 & -3.606968 & -0.726059 \\
\hline $\mathrm{H}$ & -5.833267 & -2.291890 & -0.04090 \\
\hline $\mathrm{H}$ & 3.392505 & -0.543382 & -1.249847 \\
\hline $\mathrm{C}$ & 1.955992 & -1.844957 & -1.772575 \\
\hline $\mathrm{C}$ & 0.747351 & -1.906362 & -2.484221 \\
\hline $\mathrm{C}$ & 2.713183 & -3.015746 & -1.626817 \\
\hline $\mathrm{C}$ & 0.292453 & -3.119470 & -3.010120 \\
\hline
\end{tabular}




\begin{tabular}{|c|c|c|c|}
\hline $\mathrm{H}$ & 0.206921 & -0.982808 & -2.673071 \\
\hline $\mathrm{C}$ & 2.261507 & -4.222417 & -2.161501 \\
\hline $\mathrm{H}$ & 3.646247 & -2.980554 & -1.073813 \\
\hline $\mathrm{C}$ & 1.044673 & -4.283609 & -2.843051 \\
\hline $\mathrm{H}$ & -0.634734 & -3.143873 & -3.571511 \\
\hline $\mathrm{H}$ & 2.860014 & -5.120303 & -2.035015 \\
\hline $\mathrm{H}$ & 0.694313 & -5.224499 & -3.256639 \\
\hline $\mathrm{C}$ & 1.926397 & -0.405546 & 0.191690 \\
\hline $\mathrm{O}$ & 0.588984 & -0.548459 & 0.285947 \\
\hline $\mathrm{C}$ & 2.697193 & -1.367851 & 1.114055 \\
\hline $\mathrm{C}$ & 2.010850 & -2.302621 & 1.889286 \\
\hline $\mathrm{C}$ & 4.098678 & -1.344827 & 1.167954 \\
\hline $\mathrm{C}$ & 2.705871 & -3.211551 & 2.690870 \\
\hline $\mathrm{H}$ & 0.929744 & -2.310856 & 1.859145 \\
\hline $\mathrm{C}$ & 4.795008 & -2.244852 & 1.972693 \\
\hline $\mathrm{H}$ & 4.653262 & -0.612742 & 0.587054 \\
\hline $\mathrm{C}$ & 4.099226 & -3.187943 & 2.734541 \\
\hline $\mathrm{H}$ & 2.153506 & -3.937938 & 3.280825 \\
\hline $\mathrm{H}$ & 5.880681 & -2.211176 & 2.004904 \\
\hline $\mathrm{H}$ & 4.641092 & -3.893998 & 3.357382 \\
\hline $\mathrm{C}$ & 2.241585 & 1.229325 & 2.045011 \\
\hline $\mathrm{H}$ & 2.198092 & 2.297232 & 2.274778 \\
\hline $\mathrm{H}$ & 1.352337 & 0.743529 & 2.454508 \\
\hline $\mathrm{H}$ & 3.108331 & 0.787372 & 2.550907 \\
\hline $\mathrm{N}$ & 2.247236 & 1.057369 & 0.583714 \\
\hline $\mathrm{C}$ & 3.290948 & 1.765453 & -0.095410 \\
\hline $\mathrm{C}$ & 4.484988 & 2.132818 & 0.544396 \\
\hline $\mathrm{C}$ & 3.102508 & 2.150603 & -1.433962 \\
\hline $\mathrm{C}$ & 5.468005 & 2.850125 & -0.140836 \\
\hline $\mathrm{H}$ & 4.655877 & 1.859803 & 1.578997 \\
\hline $\mathrm{C}$ & 4.093310 & 2.851165 & -2.116302 \\
\hline $\mathrm{H}$ & 2.177031 & 1.892227 & -1.928897 \\
\hline $\mathrm{C}$ & 5.282745 & 3.206101 & -1.475330 \\
\hline $\mathrm{H}$ & 6.382504 & 3.126451 & 0.376765 \\
\hline $\mathrm{H}$ & 3.924087 & 3.137556 & -3.150490 \\
\hline $\mathrm{H}$ & 6.048128 & 3.763959 & -2.006709 \\
\hline
\end{tabular}

\section{2d}

$\mathrm{Li} \quad-0.562229 \quad 0.758379 \quad-0.913156$

$\mathrm{N} \quad-2.442273 \quad 0.573302-0.359576$

$\begin{array}{lllll}\mathrm{N} & & 1.280753 & 0.205205 & -1.305272\end{array}$

Si $\quad-2.939801 \quad 2.212094 \quad-0.314291$ 


\begin{tabular}{|c|c|c|c|}
\hline $\mathrm{Si}$ & -3.164404 & $4 \quad-0.837616$ & -1.01596 \\
\hline $\mathrm{Li}$ & -1.464449 & $9 \quad 0.427948$ & 1.29577 \\
\hline $\mathrm{O}$ & 0.202009 & 1.164574 & 0.837967 \\
\hline $\mathrm{C}$ & 1.823229 & 1.342075 & -2.049009 \\
\hline $\mathrm{C}$ & 1.838309 & -1.031688 & -1.627452 \\
\hline $\mathrm{C}$ & -4.665573 & 2.613036 & -0.977041 \\
\hline $\mathrm{C}$ & -1.700343 & 3.293074 & -1.280830 \\
\hline $\mathrm{C}$ & -2.851458 & 2.818058 & 1.494012 \\
\hline $\mathrm{C}$ & -5.050365 & -0.781445 & -1.202977 \\
\hline $\mathrm{C}$ & -2.783230 & -2.307837 & 0.122577 \\
\hline $\mathrm{C}$ & -2.438251 & -1.226027 & -2.730296 \\
\hline $\mathrm{C}$ & 1.348743 & 0.593945 & 0.658093 \\
\hline $\mathrm{H}$ & 2.893229 & 1.529741 & -1.887171 \\
\hline $\mathrm{H}$ & 1.659005 & 1.195778 & -3.125601 \\
\hline $\mathrm{H}$ & 1.297406 & 2.254328 & -1.747663 \\
\hline $\mathrm{C}$ & 3.120145 & -1.204911 & -2.196391 \\
\hline $\mathrm{C}$ & 1.099427 & -2.196818 & -1.321085 \\
\hline $\mathrm{H}$ & -4.759327 & 2.339583 & -2.034602 \\
\hline $\mathrm{H}$ & -4.865630 & 3.688703 & -0.893285 \\
\hline $\mathrm{H}$ & -5.449809 & 2.080931 & -0.427970 \\
\hline $\mathrm{H}$ & -0.694051 & 3.236308 & -0.843390 \\
\hline $\mathrm{H}$ & -1.636288 & 2.986155 & -2.334263 \\
\hline $\mathrm{H}$ & -1.989534 & 4.351330 & -1.268190 \\
\hline $\mathrm{H}$ & -3.530734 & 2.241230 & 2.137485 \\
\hline $\mathrm{H}$ & -1.831948 & 2.736812 & 1.900595 \\
\hline $\mathrm{H}$ & -3.134804 & 3.873894 & 1.586526 \\
\hline $\mathrm{H}$ & -5.536252 & -0.528647 & -0.252540 \\
\hline $\mathrm{H}$ & -5.430500 & -1.760931 & -1.520141 \\
\hline $\mathrm{H}$ & -5.372803 & -0.044092 & -1.945737 \\
\hline $\mathrm{H}$ & -3.144347 & -3.254541 & -0.298683 \\
\hline $\mathrm{H}$ & -3.265328 & -2.178853 & 1.100763 \\
\hline $\mathrm{H}$ & -1.710006 & $5 \quad-2.424694$ & 0.311694 \\
\hline $\mathrm{H}$ & -1.345754 & -1.333952 & -2.697932 \\
\hline $\mathrm{H}$ & -2.667380 & -0.414689 & -3.434115 \\
\hline $\mathrm{H}$ & -2.843837 & -2.154294 & -3.152567 \\
\hline $\mathrm{N}$ & 1.528190 & -0.692035 & 1.248180 \\
\hline $\mathrm{C}$ & 2.581593 & 1.446731 & 0.690791 \\
\hline $\mathrm{C}$ & 3.629030 & -2.481205 & -2.436034 \\
\hline $\mathrm{H}$ & 3.719414 & -0.338631 & -2.451246 \\
\hline $\mathrm{C}$ & 1.616648 & -3.467101 & -1.557841 \\
\hline $\mathrm{H}$ & 0.102987 & -2.082302 & -0.908171 \\
\hline $\mathrm{C}$ & 0.584327 & -1.348727 & 2.037437 \\
\hline
\end{tabular}




\begin{tabular}{|c|c|c|c|}
\hline $\mathrm{H}$ & 2.159516 & -1.307223 & 0.751584 \\
\hline $\mathrm{C}$ & 2.432477 & 2.835033 & 0.776509 \\
\hline $\mathrm{C}$ & 3.870813 & 0.902307 & 0.605864 \\
\hline $\mathrm{C}$ & 2.889230 & -3.620979 & -2.115528 \\
\hline $\mathrm{H}$ & 4.616948 & -2.583739 & -2.877866 \\
\hline $\mathrm{H}$ & 1.014127 & -4.340000 & -1.319418 \\
\hline $\mathrm{C}$ & 0.472521 & -2.749340 & 1.972835 \\
\hline $\mathrm{C}$ & -0.223634 & -0.657538 & 2.969652 \\
\hline $\mathrm{C}$ & 3.550487 & 3.668041 & 0.776820 \\
\hline $\mathrm{H}$ & 1.430527 & 3.243680 & 0.847128 \\
\hline $\mathrm{C}$ & 4.986989 & 1.736318 & 0.595671 \\
\hline $\mathrm{H}$ & 4.010731 & -0.170859 & 0.535456 \\
\hline $\mathrm{H}$ & 3.290663 & -4.611157 & -2.308917 \\
\hline $\mathrm{C}$ & -0.440310 & -3.427529 & 2.775410 \\
\hline $\mathrm{H}$ & 1.096943 & -3.296605 & 1.273878 \\
\hline $\mathrm{C}$ & -1.156084 & -1.350558 & 3.748433 \\
\hline $\mathrm{H}$ & -0.065539 & 0.404735 & 3.125809 \\
\hline $\mathrm{C}$ & 4.831539 & 3.122110 & 0.682265 \\
\hline $\mathrm{H}$ & 3.420751 & 4.744186 & 0.851066 \\
\hline $\mathrm{H}$ & 5.980681 & 1.302944 & 0.524899 \\
\hline $\mathrm{C}$ & -1.281069 & -2.736309 & 3.650896 \\
\hline $\mathrm{H}$ & -0.509348 & -4.508805 & 2.697336 \\
\hline $\mathrm{H}$ & -1.763347 & -0.796379 & 4.459726 \\
\hline $\mathrm{H}$ & 5.703573 & 3.769807 & 0.679081 \\
\hline $\mathrm{H}$ & -2.004902 & -3.269629 & 4.258369 \\
\hline
\end{tabular}

$12 \mathrm{t}$

O $\quad-0.469097 \quad-0.968866-0.349166$

C $\quad-1.736333-1.178354-0.214109$

Li $\quad-0.512892 \quad 0.948228 \quad-0.632289$

$\mathrm{N} \quad-2.238770 \quad-1.364730 \quad 1.087968$

C $\quad-2.487462-1.861934-1.296306$

$\mathrm{Li} \quad 1.028998 \quad-1.258994 \quad 0.685284$

$\begin{array}{lllll}\mathrm{N} & & 1.050263 & 2.139765 & -0.545680\end{array}$

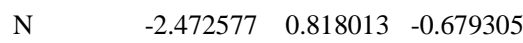

$\begin{array}{lllll}\text { C } & & -1.425180 & -1.645113 & 2.190511\end{array}$

$\mathrm{H} \quad \begin{array}{llll}-3.115918 & -0.897928 & 1.295796\end{array}$

$\begin{array}{lllll}\text { C } & -1.828300 & -2.170764 & -2.493968\end{array}$

$\begin{array}{llll}\text { C } & -3.845556 & -2.189408 & -1.158064\end{array}$

N $\quad 2.812489-1.030701 \quad-0.050445$

$\begin{array}{lllll}\mathrm{Si} & & 1.498878 & 2.931378 & -2.009709\end{array}$

$\begin{array}{lllll}\text { Si } & 0.746089 & 2.943385 & 0.951777\end{array}$ 


\begin{tabular}{|c|c|c|c|}
\hline $\mathrm{i}$ & 2.447120 & 0.811473 & -0.516326 \\
\hline $\mathrm{C}$ & -2.731967 & 0.891093 & -2.112977 \\
\hline $\mathrm{C}$ & -3.553952 & 1.102672 & 0.129973 \\
\hline $\mathrm{C}$ & -1.667329 & -1.046982 & 3.435559 \\
\hline $\mathrm{C}$ & -0.389644 & -2.597589 & 2.085444 \\
\hline $\mathrm{C}$ & -2.513031 & -2.794191 & $-3.53501 \epsilon$ \\
\hline $\mathrm{H}$ & -0.779203 & -1.922928 & -2.593778 \\
\hline $\mathrm{C}$ & -4.530283 & -2.797874 & -2.20706 \\
\hline $\mathrm{H}$ & -4.369224 & -1.963357 & -0.23661 \\
\hline $\mathrm{Si}$ & 4.059383 & -0.822429 & 1.119226 \\
\hline $\mathrm{Si}$ & 2.760302 & -2.062975 & -1.434139 \\
\hline $\mathrm{C}$ & 1.446207 & 4.824879 & -1.998777 \\
\hline $\mathrm{C}$ & 0.424497 & 2.368973 & -3.466387 \\
\hline $\mathrm{C}$ & 3.309965 & 2.460018 & -2.412318 \\
\hline $\mathrm{C}$ & 2.096616 & 4.143747 & 1.536355 \\
\hline $\mathrm{C}$ & -0.871528 & 3.930843 & 0.921301 \\
\hline $\mathrm{C}$ & 0.605010 & 1.580366 & 2.272487 \\
\hline $\mathrm{H}$ & -3.011742 & 1.915447 & -2.399094 \\
\hline $\mathrm{H}$ & -3.516745 & 0.211528 & -2.473101 \\
\hline $\mathrm{H}$ & -1.821276 & 0.632997 & -2.663592 \\
\hline $\mathrm{C}$ & -4.906330 & 1.090637 & -0.297732 \\
\hline $\mathrm{C}$ & -3.317706 & 1.371938 & 1.503416 \\
\hline $\mathrm{C}$ & -0.871982 & -1.366248 & 4.534723 \\
\hline $\mathrm{H}$ & -2.473766 & -0.327870 & 3.530199 \\
\hline $\mathrm{C}$ & 0.408880 & -2.896153 & 3.190321 \\
\hline $\mathrm{H}$ & -0.264657 & -3.151485 & 1.159242 \\
\hline $\mathrm{C}$ & -3.867252 & -3.104864 & -3.398283 \\
\hline $\mathrm{H}$ & -1.987231 & -3.037430 & -4.453952 \\
\hline $\mathrm{H}$ & -5.582692 & -3.040530 & -2.091171 \\
\hline $\mathrm{C}$ & 5.668823 & -1.783405 & 0.857193 \\
\hline $\mathrm{C}$ & 3.479297 & -1.265045 & 2.871412 \\
\hline $\mathrm{C}$ & 4.539189 & 1.022825 & 1.147813 \\
\hline $\mathrm{C}$ & 4.428157 & -2.644939 & -2.116431 \\
\hline $\mathrm{C}$ & 1.897684 & -1.160069 & -2.865569 \\
\hline $\mathrm{C}$ & 1.728889 & -3.622883 & -1.079281 \\
\hline $\mathrm{H}$ & 0.449171 & 5.198375 & -1.737858 \\
\hline $\mathrm{H}$ & 1.695021 & 5.214895 & -2.993789 \\
\hline $\mathrm{H}$ & 2.160816 & 5.252468 & -1.287605 \\
\hline $\mathrm{H}$ & 0.790014 & 2.787839 & -4.412293 \\
\hline $\mathrm{H}$ & -0.611205 & 2.705508 & 3. -3.335591 \\
\hline $\mathrm{H}$ & 0.413134 & 1.278438 & -3.575536 \\
\hline $\mathrm{H}$ & 3.991837 & 2.886941 & -1.664500 \\
\hline
\end{tabular}




\begin{tabular}{|c|c|c|c|}
\hline $\mathrm{H}$ & 3.500044 & 1.376977 & -2.457687 \\
\hline $\mathrm{H}$ & 3.610230 & 2.855075 & -3.390739 \\
\hline $\mathrm{H}$ & 3.103446 & 3.717390 & 1.464168 \\
\hline $\mathrm{H}$ & 1.929848 & 4.422977 & 2.584787 \\
\hline $\mathrm{H}$ & 2.086051 & 5.067606 & 0.948025 \\
\hline $\mathrm{H}$ & -0.752564 & 4.829942 & 0.304637 \\
\hline $\mathrm{H}$ & -1.177841 & 4.256146 & 1.923479 \\
\hline $\mathrm{H}$ & -1.691090 & 3.343643 & 0.493302 \\
\hline $\mathrm{H}$ & 0.283223 & 1.972636 & 3.244621 \\
\hline $\mathrm{H}$ & -0.118363 & 0.804078 & 1.993965 \\
\hline $\mathrm{H}$ & 1.572560 & 1.087519 & 2.429721 \\
\hline $\mathrm{C}$ & -5.944996 & 1.322901 & 0.600973 \\
\hline $\mathrm{H}$ & -5.141245 & 0.902270 & -1.338294 \\
\hline $\mathrm{C}$ & -4.364645 & 1.597916 & 2.392696 \\
\hline $\mathrm{H}$ & -2.290025 & 1.432672 & 1.843380 \\
\hline $\mathrm{C}$ & 0.181840 & -2.274493 & 4.419446 \\
\hline $\mathrm{H}$ & -1.072471 & -0.886737 & 5.488667 \\
\hline $\mathrm{H}$ & 1.198238 & -3.634659 & 3.088098 \\
\hline $\mathrm{H}$ & -4.402347 & -3.587185 & -4.211177 \\
\hline $\mathrm{H}$ & 5.507075 & -2.867564 & 0.868548 \\
\hline $\mathrm{H}$ & 6.369290 & -1.546465 & 1.668090 \\
\hline $\mathrm{H}$ & 6.155678 & -1.528985 & -0.089653 \\
\hline $\mathrm{H}$ & 4.239648 & -0.984234 & 3.611414 \\
\hline $\mathrm{H}$ & 2.543812 & -0.773729 & 3.162821 \\
\hline $\mathrm{H}$ & 3.319042 & -2.346521 & 2.960257 \\
\hline $\mathrm{H}$ & 3.688320 & 1.681412 & 1.362616 \\
\hline $\mathrm{H}$ & 4.979235 & 1.341899 & 0.192422 \\
\hline $\mathrm{H}$ & 5.285393 & 1.222574 & 1.926605 \\
\hline $\mathrm{H}$ & 4.267616 & -3.170222 & -3.066666 \\
\hline $\mathrm{H}$ & 5.099052 & -1.800484 & -2.315609 \\
\hline $\mathrm{H}$ & 4.947605 & -3.331370 & -1.440932 \\
\hline $\mathrm{H}$ & 2.544791 & -0.411730 & -3.338034 \\
\hline $\mathrm{H}$ & 1.595824 & -1.865910 & -3.649886 \\
\hline $\mathrm{H}$ & 0.994054 & -0.651507 & -2.514713 \\
\hline $\mathrm{H}$ & 2.090288 & -4.139421 & -0.179909 \\
\hline $\mathrm{H}$ & 0.668710 & -3.380018 & -0.935370 \\
\hline $\mathrm{H}$ & 1.781605 & -4.335522 & -1.911908 \\
\hline $\mathrm{C}$ & -5.690482 & 1.570293 & 1.952252 \\
\hline $\mathrm{H}$ & -6.970034 & 1.308747 & 0.238709 \\
\hline $\mathrm{H}$ & -4.140398 & 1.815816 & 3.434297 \\
\hline $\mathrm{H}$ & 0.807974 & -2.504890 & 5.275116 \\
\hline $\mathrm{H}$ & -6.507053 & 1.753458 & 2.643904 \\
\hline
\end{tabular}




\begin{tabular}{|c|c|c|c|}
\hline TS13d & & & \\
\hline $\mathrm{O}$ & 0.017559 & -0.319939 & -1.459701 \\
\hline $\mathrm{C}$ & 1.224694 & 0.025681 & -1.311101 \\
\hline $\mathrm{Li}$ & -1.687355 & 0.438057 & -1.323761 \\
\hline $\mathrm{N}$ & 1.550743 & 1.341580 & -1.244430 \\
\hline $\mathrm{C}$ & 2.321717 & -0.954754 & -1.326645 \\
\hline $\mathrm{Li}$ & -0.577339 & -0.879006 & 0.551110 \\
\hline $\mathrm{N}$ & -2.480148 & -0.473703 & 0.167776 \\
\hline $\mathrm{C}$ & 0.620824 & 2.406553 & -1.347579 \\
\hline $\mathrm{H}$ & 2.530788 & 1.583039 & -1.202548 \\
\hline $\mathrm{C}$ & 2.052354 & -2.233332 & -1.846320 \\
\hline $\mathrm{C}$ & 3.615040 & -0.655413 & -0.864858 \\
\hline $\mathrm{Si}$ & -3.190884 & 0.128357 & 1.620278 \\
\hline $\mathrm{Si}$ & -3.153041 & -1.727449 & -0.800234 \\
\hline $\mathrm{C}$ & 0.862334 & 3.438514 & -2.258786 \\
\hline $\mathrm{C}$ & -0.538377 & 2.435822 & -0.559834 \\
\hline $\mathrm{C}$ & 3.063692 & -3.182132 & -1.932574 \\
\hline $\mathrm{H}$ & 1.046783 & -2.457841 & -2.184868 \\
\hline $\mathrm{C}$ & 4.621540 & -1.616009 & -0.949210 \\
\hline $\mathrm{H}$ & 3.839636 & 0.293765 & -0.390451 \\
\hline $\mathrm{C}$ & -3.619392 & -1.261048 & 2.840341 \\
\hline $\mathrm{C}$ & -4.759596 & 1.148043 & 1.294510 \\
\hline $\mathrm{C}$ & -1.971814 & 1.258367 & 2.537417 \\
\hline $\mathrm{C}$ & -5.008995 & -2.048715 & -0.607937 \\
\hline $\mathrm{C}$ & -2.219399 & -3.362405 & -0.564244 \\
\hline $\mathrm{C}$ & -2.912941 & -1.233519 & -2.641007 \\
\hline $\mathrm{C}$ & -0.060307 & 4.476936 & -2.395571 \\
\hline $\mathrm{H}$ & 1.761315 & 3.416535 & -2.868101 \\
\hline $\mathrm{C}$ & -1.469428 & 3.463579 & -0.719046 \\
\hline $\mathrm{H}$ & -0.687059 & 1.691226 & 0.212047 \\
\hline $\mathrm{C}$ & 4.355814 & -2.872637 & -1.491680 \\
\hline $\mathrm{H}$ & 2.848084 & -4.163687 & -2.344213 \\
\hline $\mathrm{H}$ & 5.610118 & -1.380722 & -0.567529 \\
\hline $\mathrm{H}$ & -4.041283 & -0.864009 & 3.772139 \\
\hline $\mathrm{H}$ & -2.716216 & -1.827816 & 3.102434 \\
\hline $\mathrm{H}$ & -4.344366 & -1.971046 & 2.426309 \\
\hline $\mathrm{H}$ & -5.560334 & 0.532450 & 0.868997 \\
\hline $\mathrm{H}$ & -4.554922 & 1.958063 & 0.581121 \\
\hline $\mathrm{H}$ & -5.144688 & 1.605414 & 2.214702 \\
\hline $\mathrm{H}$ & -1.818116 & 2.224169 & 2.041039 \\
\hline $\mathrm{H}$ & -0.991141 & 0.782593 & 2.665629 \\
\hline
\end{tabular}




\begin{tabular}{|c|c|c|c|}
\hline $\mathrm{H}$ & -2.358704 & 1.478328 & 3.540448 \\
\hline $\mathrm{H}$ & -5.589142 & -1.150776 & -0.850532 \\
\hline $\mathrm{H}$ & -5.331414 & -2.848005 & -1.287005 \\
\hline $\mathrm{H}$ & -5.277501 & -2.350993 & 0.409813 \\
\hline $\mathrm{H}$ & -2.289438 & -3.698174 & 0.47818 \\
\hline $\mathrm{H}$ & -1.153485 & -3.245034 & -0.8037 \\
\hline $\mathrm{H}$ & -2.609908 & -4.165887 & -1.201104 \\
\hline $\mathrm{H}$ & -1.858248 & -1.161513 & -2.94184 \\
\hline $\mathrm{H}$ & -3.421359 & -0.286900 & -2.887712 \\
\hline $\mathrm{H}$ & -3.361783 & -1.993482 & -3.29167 \\
\hline $\mathrm{C}$ & -1.235623 & 4.485895 & -1.641826 \\
\hline $\mathrm{H}$ & 0.136348 & 5.274263 & -3.105950 \\
\hline $\mathrm{H}$ & -2.360225 & 3.470703 & -0.097584 \\
\hline $\mathrm{H}$ & 5.145214 & -3.615780 & -1.555984 \\
\hline $\mathrm{H}$ & -1.954328 & 5.290652 & -1.760244 \\
\hline $\mathrm{N}$ & 1.071247 & -0.902774 & 1.465331 \\
\hline $\mathrm{C}$ & 1.305424 & -2.333448 & 1.552846 \\
\hline $\mathrm{C}$ & 2.111900 & -0.111440 & 1.837481 \\
\hline $\mathrm{H}$ & 1.529716 & -2.674573 & 2.578333 \\
\hline $\mathrm{H}$ & 2.126813 & -2.688085 & 0.905502 \\
\hline $\mathrm{H}$ & 0.402002 & -2.879768 & 1.237272 \\
\hline $\mathrm{C}$ & 3.405020 & -0.585550 & 2.209090 \\
\hline $\mathrm{C}$ & 1.958518 & 1.307904 & 1.807496 \\
\hline $\mathrm{C}$ & 4.458598 & 0.293001 & 2.460308 \\
\hline $\mathrm{H}$ & 3.584858 & -1.651672 & 2.280943 \\
\hline $\mathrm{C}$ & 3.017885 & 2.168475 & 2.046902 \\
\hline $\mathrm{H}$ & 0.973299 & 1.710782 & 1.600077 \\
\hline $\mathrm{C}$ & 4.294633 & 1.674982 & 2.360851 \\
\hline $\mathrm{H}$ & 5.430345 & -0.117117 & 2.729427 \\
\hline $\mathrm{H}$ & 2.847480 & 3.242822 & 2.003696 \\
\hline $\mathrm{H}$ & 5.121810 & 2.351898 & 2.553078 \\
\hline
\end{tabular}

\section{TS13t}

$\begin{array}{llll}\mathrm{N} & 0.379155 & -3.333888 & -0.048991 \\ \mathrm{Li} & -1.815357 & -0.848274 & -0.187247 \\ \mathrm{Li} & 1.061293 & 0.439485 & -0.471402 \\ \mathrm{~N} & 0.174050 & 2.197609 & -0.345209 \\ \mathrm{Li} & -1.556588 & 1.804202 & 0.427790 \\ \mathrm{~N} & -2.943727 & 0.465560 & 0.671421 \\ \mathrm{Si} & 0.015250 & 2.517619 & -2.039556 \\ \mathrm{Si} & 0.757691 & 3.392459 & 0.756651 \\ \mathrm{Si} & -4.349144 & 0.769528 & -0.287194\end{array}$




\begin{tabular}{|c|c|c|c|}
\hline $\mathrm{Si}$ & -2.911526 & -0.084520 & 2.310698 \\
\hline $\mathrm{C}$ & -0.909182 & 1.014501 & -2.767533 \\
\hline $\mathrm{H}$ & -0.292796 & 0.111995 & -2.660671 \\
\hline $\mathrm{H}$ & -1.868195 & 0.844251 & -2.263801 \\
\hline $\mathrm{H}$ & -1.121476 & 1.132568 & -3.836871 \\
\hline $\mathrm{C}$ & 1.652077 & 2.638792 & -2.974008 \\
\hline $\mathrm{H}$ & 1.480217 & 2.737257 & -4.053997 \\
\hline $\mathrm{H}$ & 2.239688 & 3.504221 & -2.649783 \\
\hline $\mathrm{H}$ & 2.248625 & 1.735751 & -2.799560 \\
\hline $\mathrm{C}$ & -0.978432 & 4.089584 & -2.433948 \\
\hline $\mathrm{H}$ & -0.394928 & 4.983426 & -2.182461 \\
\hline $\mathrm{H}$ & -1.218358 & 4.148650 & -3.503214 \\
\hline $\mathrm{H}$ & -1.920417 & 4.149412 & -1.877991 \\
\hline $\mathrm{C}$ & -0.747558 & 4.323258 & 1.481011 \\
\hline $\mathrm{H}$ & -0.449958 & 5.071670 & 2.226206 \\
\hline $\mathrm{H}$ & -1.296802 & 4.843665 & 0.685598 \\
\hline $\mathrm{H}$ & -1.459139 & 3.653236 & 1.990153 \\
\hline $\mathrm{C}$ & 1.924858 & 4.683251 & 0.007573 \\
\hline $\mathrm{H}$ & 2.223047 & 5.418261 & 0.765839 \\
\hline $\mathrm{H}$ & 2.837474 & 4.206228 & -0.369301 \\
\hline $\mathrm{H}$ & 1.471003 & 5.232118 & -0.824676 \\
\hline $\mathrm{C}$ & 1.688528 & 2.651103 & 2.234498 \\
\hline $\mathrm{H}$ & 2.034279 & 3.457855 & 2.893499 \\
\hline $\mathrm{H}$ & 1.058744 & 1.988443 & 2.836745 \\
\hline $\mathrm{H}$ & 2.570245 & 2.083826 & 1.918313 \\
\hline $\mathrm{C}$ & -1.308996 & 0.533667 & 3.107751 \\
\hline $\mathrm{H}$ & -1.312678 & 1.622589 & 3.245313 \\
\hline $\mathrm{H}$ & -1.147124 & 0.084774 & 4.095247 \\
\hline $\mathrm{H}$ & -0.448715 & 0.274261 & 2.483677 \\
\hline $\mathrm{C}$ & -4.318245 & 0.484235 & 3.441716 \\
\hline $\mathrm{H}$ & -4.468424 & 1.568542 & 3.372771 \\
\hline $\mathrm{H}$ & -5.273467 & 0.000342 & 3.219547 \\
\hline $\mathrm{H}$ & -4.060511 & 0.252084 & 4.482925 \\
\hline $\mathrm{C}$ & -2.857233 & -1.988316 & 2.366534 \\
\hline $\mathrm{H}$ & -2.872627 & -2.366432 & 3.396426 \\
\hline $\mathrm{H}$ & -3.715801 & -2.423680 & 1.838558 \\
\hline $\mathrm{H}$ & -1.946005 & -2.382412 & 1.896426 \\
\hline $\mathrm{C}$ & -4.232249 & 2.492052 & -1.067703 \\
\hline $\mathrm{H}$ & -5.127839 & 2.729310 & -1.655105 \\
\hline $\mathrm{H}$ & -4.130296 & 3.267398 & -0.296117 \\
\hline $\mathrm{H}$ & -3.373588 & 2.576583 & -1.742168 \\
\hline $\mathrm{C}$ & -4.470965 & -0.487801 & -1.711309 \\
\hline
\end{tabular}




\begin{tabular}{|c|c|c|c|}
\hline $\mathrm{H}$ & -3.600321 & -0.463468 & -2.377652 \\
\hline $\mathrm{H}$ & -4.568109 & -1.510677 & -1.32555 \\
\hline $\mathrm{H}$ & -5.352341 & -0.286523 & -2.33343 \\
\hline $\mathrm{C}$ & -6.020857 & 0.671979 & 0.597986 \\
\hline $\mathrm{H}$ & -6.822976 & 0.854497 & -0.128500 \\
\hline $\mathrm{H}$ & -6.197671 & -0.315451 & 1.04052 \\
\hline $\mathrm{H}$ & -6.118348 & 1.417439 & 1.393668 \\
\hline $\mathrm{H}$ & 0.908245 & -4.117831 & 0.307823 \\
\hline $\mathrm{C}$ & -0.849617 & -3.623413 & -0.698986 \\
\hline $\mathrm{C}$ & -1.224225 & -2.934187 & -1.861780 \\
\hline $\mathrm{C}$ & -1.682478 & -4.616998 & -0.174221 \\
\hline $\mathrm{C}$ & -2.441347 & -3.231157 & -2.476790 \\
\hline $\mathrm{H}$ & -0.553204 & -2.196911 & -2.28632 \\
\hline $\mathrm{C}$ & -2.888619 & -4.917853 & -0.807461 \\
\hline $\mathrm{H}$ & -1.390534 & -5.138022 & 0.732995 \\
\hline $\mathrm{C}$ & -3.276436 & -4.221444 & -1.953459 \\
\hline $\mathrm{H}$ & -2.729474 & -2.692569 & -3.373504 \\
\hline $\mathrm{H}$ & -3.530213 & -5.690387 & -0.394749 \\
\hline $\mathrm{H}$ & -4.219910 & -4.450005 & -2.438755 \\
\hline $\mathrm{C}$ & 0.767099 & -2.098500 & 0.374382 \\
\hline $\mathrm{O}$ & -0.014222 & -1.107750 & 0.232493 \\
\hline $\mathrm{C}$ & 2.055708 & -2.002080 & 1.059886 \\
\hline $\mathrm{C}$ & 2.277908 & -0.933910 & 1.948532 \\
\hline $\mathrm{C}$ & 3.083379 & -2.945107 & 0.857942 \\
\hline $\mathrm{C}$ & 3.485740 & -0.818720 & 2.622036 \\
\hline $\mathrm{H}$ & 1.496632 & -0.201881 & 2.108256 \\
\hline $\mathrm{C}$ & 4.292448 & -2.814958 & 1.530586 \\
\hline $\mathrm{H}$ & 2.970524 & -3.734998 & 0.121074 \\
\hline $\mathrm{C}$ & 4.501213 & -1.757595 & 2.418506 \\
\hline $\mathrm{H}$ & 3.641673 & 0.019917 & 3.293021 \\
\hline $\mathrm{H}$ & 5.090783 & -3.523398 & 1.333603 \\
\hline $\mathrm{H}$ & 5.452385 & -1.653881 & 2.929224 \\
\hline $\mathrm{N}$ & 2.433612 & -0.491323 & -1.516158 \\
\hline $\mathrm{C}$ & 2.273994 & -1.545219 & -2.489809 \\
\hline $\mathrm{H}$ & 2.425670 & -2.567427 & -2.085279 \\
\hline $\mathrm{H}$ & 1.260224 & -1.511490 & -2.906910 \\
\hline $\mathrm{H}$ & 2.966936 & -1.450404 & -3.343502 \\
\hline $\mathrm{C}$ & 3.703818 & -0.284532 & -1.077535 \\
\hline $\mathrm{C}$ & 4.819355 & -1.139749 & -1.328375 \\
\hline $\mathrm{C}$ & 3.963712 & 0.860382 & -0.260684 \\
\hline $\mathrm{C}$ & 6.058541 & -0.894908 & -0.745672 \\
\hline $\mathrm{H}$ & 4.697479 & -2.008615 & -1.965779 \\
\hline
\end{tabular}




\begin{tabular}{|c|c|c|c|}
\hline $\mathrm{C}$ & 5.202588 & 1.089686 & 0.312054 \\
\hline $\mathrm{H}$ & 3.160357 & 1.578710 & -0.122262 \\
\hline $\mathrm{C}$ & 6.264589 & 0.198155 & 0.100781 \\
\hline $\mathrm{H}$ & 6.879818 & -1.580633 & -0.946430 \\
\hline $\mathrm{H}$ & 5.347985 & 1.972770 & 0.930980 \\
\hline & 7.237836 & 0.378701 & 0.547761 \\
\hline
\end{tabular}

14d

$\mathrm{N} \quad \begin{array}{llll}0.435873 & 0.411533 & 0.238384\end{array}$

$\begin{array}{llll}\text { C } & -0.811691 & -0.831380 & 0.029095\end{array}$

$\begin{array}{llll}\text { C } & -1.292241 & 1.522208 & 0.137839\end{array}$

C $\quad-2.227415-1.306954-0.065999$

$\begin{array}{llll}\text { O } & 0.106312 & -1.739983 & -0.069930\end{array}$

$\begin{array}{llll}\mathrm{Li} & 1.461322 & -0.034767 & 0.342485\end{array}$

$\begin{array}{llll}\text { C } & -1.282543 & 2.484176 & 1.159648\end{array}$

$\begin{array}{llll}\text { C } & -2.102494 & 1.742229 & -0.989151\end{array}$

$\begin{array}{llll}\text { C } & -2.516561 & -2.391113 & -0.905530\end{array}$

$\begin{array}{llll}\text { C } & -3.255342 & -0.740388 & 0.701122\end{array}$

$\begin{array}{llll}\mathrm{Li} & 1.524542 & -2.490863 & 0.671602\end{array}$

$\begin{array}{lllll}\mathrm{N} & & 2.871878 & -1.234464 & 1.184980\end{array}$

$\begin{array}{lllll}\text { C } & -2.087808 & 3.619094 & 1.073108\end{array}$

$\begin{array}{llll}\mathrm{H} & -0.645779 & 2.319299 & 2.024730\end{array}$

$\begin{array}{lllll}\text { C } & -2.903850 & 2.878574 & -1.070781\end{array}$

$\mathrm{H} \quad \begin{array}{llll}\mathrm{H} & -2.098066 & 1.012634 & -1.792788\end{array}$

$\begin{array}{lllll}\text { C } & -3.816907 & -2.883207 & -1.000869\end{array}$

$\mathrm{H} \quad-1.711602 \quad-2.834901 \quad-1.481616$

$\begin{array}{lllll}\text { C } & -4.550463 & -1.247830 & 0.620553\end{array}$

$\begin{array}{lllll}\mathrm{H} & -3.040843 & 0.092209 & 1.361019\end{array}$

$\begin{array}{llll}\text { C } & 3.316581 & -1.181480 & 2.569031\end{array}$

$\begin{array}{llll}\text { C } & 3.682090 & -0.596215 & 0.265619\end{array}$

$\begin{array}{lllll}\text { C } & -2.906368 & 3.821192 & -0.039912\end{array}$

$\begin{array}{llll}\mathrm{H} & -2.073883 & 4.348437 & 1.878648\end{array}$

$\mathrm{H} \quad-3.526800 \quad 3.030390 \quad-1.948251$

$\begin{array}{lllll}\text { C } & & -4.836731 & -2.312956 & -0.236307\end{array}$

$\mathrm{H} \quad-4.033550 \quad-3.714424 \quad-1.666025$

$\begin{array}{llll}\mathrm{H} & -5.338255 & -0.808228 & 1.225489\end{array}$

$\begin{array}{llll}\mathrm{H} & 3.421725 & -0.151997 & 2.954887\end{array}$

$\begin{array}{llll}\mathrm{H} & 2.579799 & -1.686984 & 3.206293\end{array}$

$\mathrm{H} \quad 4.287471 \quad-1.679549 \quad 2.730699$

$\begin{array}{llll}\text { C } & 4.860695 & 0.134333 & 0.577534\end{array}$

C $\quad 3.275356-0.585605-1.103683$

$\begin{array}{llll}\mathrm{H} & -3.530814 & 4.707112 & -0.108327\end{array}$ 


$\begin{array}{llll}\mathrm{H} & -5.849815 & -2.699517 & -0.304355 \\ \mathrm{C} & 5.558470 & 0.826787 & -0.407711 \\ \mathrm{H} & 5.229127 & 0.149648 & 1.596759 \\ \mathrm{C} & 3.981300 & 0.123406 & -2.073608 \\ \mathrm{H} & 2.413568 & -1.182233 & -1.407620 \\ \mathrm{C} & 5.129724 & 0.841385 & -1.738456 \\ \mathrm{H} & 6.458612 & 1.369567 & -0.127719 \\ \mathrm{H} & 3.633352 & 0.098209 & -3.103328 \\ \mathrm{H} & 5.682822 & 1.390061 & -2.494120\end{array}$

$14 t$

N $\quad 1.644893-0.156676-0.594289$

$\begin{array}{llll}\text { C } & 2.655275 & 0.749035 & -0.973125\end{array}$

$\begin{array}{llll}\text { C } & 1.810417 & -1.212033 & 0.196738\end{array}$

C $\quad 3.471691 \quad 1.387442-0.025081$

$\begin{array}{llll}\text { C } & 2.780510 & 1.101033 & -2.323934\end{array}$

$\mathrm{Li} \quad 0.069121 \quad-1.330716-1.253889$

$\begin{array}{lllll}\mathrm{Li} & 0.068867 & 0.084867 & 0.831119\end{array}$

$\begin{array}{lllll}\text { C } & 3.096028 & -1.822327 & 0.631307\end{array}$

$\begin{array}{llll}\text { O } & 0.701342 & -1.759167 & 0.592476\end{array}$

$\begin{array}{lllll}\text { C } & 4.409009 & 2.335450 & -0.428518\end{array}$

$\mathrm{H} \quad 3.366359 \quad 1.133683 \quad 1.025926$

$\begin{array}{llll}\text { C } & 3.722501 & 2.049039 & -2.722598\end{array}$

$\begin{array}{llll}\mathrm{H} & 2.131487 & 0.623390 & -3.052718\end{array}$

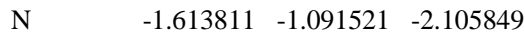

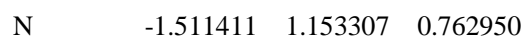

$\begin{array}{lllll}\text { C } & 4.265102 & -1.738637 & -0.139831\end{array}$

$\begin{array}{llll}\text { C } & 3.108402 & -2.548935 & 1.830843\end{array}$

$\begin{array}{llll}\text { C } & 4.542991 & 2.668618 & -1.778545\end{array}$

$\begin{array}{llll}\mathrm{H} & 5.032666 & 2.820721 & 0.316937\end{array}$

$\mathrm{H} \quad 3.809633 \quad 2.307452 \quad-3.774158$

$\begin{array}{lllll}\text { C } & -1.576829 & -0.632698 & -3.486366\end{array}$

C $\quad \begin{array}{llll}-2.747956 & -1.788249 & -1.728336\end{array}$

Li $\quad-2.278630 \quad 0.109552 \quad-0.661826$

$\begin{array}{llll}\mathrm{Si} & -1.203363 & 2.709159 & 0.072108\end{array}$

$\begin{array}{llll}\mathrm{Si} & -2.308844 & 0.889247 & 2.273730\end{array}$

$\begin{array}{lllll}\text { C } & & 5.431541 & -2.363820 & 0.294779\end{array}$

$\mathrm{H} \quad 4.261868 \quad-1.193969-1.076253$

$\begin{array}{llll}\text { C } & 4.283119 & -3.153695 & 2.272130\end{array}$

$\begin{array}{llll}\mathrm{H} & 2.188391 & -2.636107 & 2.398351\end{array}$

$\mathrm{H} \quad \begin{array}{llll}\mathrm{H} & \mathbf{2 7} 271723 & 3.411182 & -2.089099\end{array}$

$\mathrm{H} \quad \quad-1.609806 \quad-1.459981 \quad-4.214864$ 


\begin{tabular}{|c|c|c|c|}
\hline $\mathrm{H}$ & -2.407713 & 0.048747 & -3.746060 \\
\hline $\mathrm{H}$ & -0.648533 & -0.075069 & -3.655708 \\
\hline $\mathrm{C}$ & -3.894632 & -2.000870 & -2.539480 \\
\hline $\mathrm{C}$ & -2.827341 & -2.243049 & -0.379506 \\
\hline $\mathrm{C}$ & -2.687491 & 3.886314 & 0.187492 \\
\hline $\mathrm{C}$ & 0.306450 & 3.551043 & 0.850304 \\
\hline $\mathrm{C}$ & -0.831183 & 2.485992 & -1.775830 \\
\hline $\mathrm{C}$ & -4.170752 & 0.635611 & 2.033020 \\
\hline $\mathrm{C}$ & -1.588921 & -0.685819 & 3.064567 \\
\hline $\mathrm{C}$ & -2.052527 & 2.269445 & 3.550315 \\
\hline $\mathrm{C}$ & 5.446260 & -3.061749 & 1.504717 \\
\hline $\mathrm{H}$ & 6.330719 & -2.303728 & -0.311099 \\
\hline $\mathrm{H}$ & 4.288730 & -3.704640 & 3.207981 \\
\hline $\mathrm{C}$ & -5.035432 & -2.608593 & -2.018594 \\
\hline $\mathrm{H}$ & -3.882768 & -1.696199 & -3.579835 \\
\hline $\mathrm{C}$ & -3.977874 & -2.843092 & 0.124707 \\
\hline $\mathrm{H}$ & -1.945203 & -2.153927 & 0.253121 \\
\hline $\mathrm{H}$ & -3.577392 & 3.432370 & -0.268427 \\
\hline $\mathrm{H}$ & -2.489181 & 4.827336 & -0.340848 \\
\hline $\mathrm{H}$ & -2.943502 & 4.136639 & 1.222931 \\
\hline $\mathrm{H}$ & 1.213447 & 2.952383 & 0.693906 \\
\hline $\mathrm{H}$ & 0.170388 & 3.684668 & 1.930143 \\
\hline $\mathrm{H}$ & 0.493098 & 4.539511 & 0.412001 \\
\hline $\mathrm{H}$ & -0.597283 & 3.451560 & -2.241143 \\
\hline $\mathrm{H}$ & -1.678533 & 2.061483 & -2.331715 \\
\hline $\mathrm{H}$ & 0.030714 & 1.827687 & -1.929805 \\
\hline $\mathrm{H}$ & -4.691744 & 0.452311 & 2.981038 \\
\hline $\mathrm{H}$ & -4.373469 & -0.219109 & 1.374665 \\
\hline $\mathrm{H}$ & -4.617516 & 1.526918 & 1.574196 \\
\hline $\mathrm{H}$ & -1.684811 & -1.564307 & 2.416485 \\
\hline $\mathrm{H}$ & -2.100935 & -0.915387 & 4.007449 \\
\hline $\mathrm{H}$ & -0.521478 & -0.573198 & 3.307829 \\
\hline $\mathrm{H}$ & -2.543012 & 2.014470 & 4.498187 \\
\hline $\mathrm{H}$ & -0.985796 & 2.418687 & 3.760048 \\
\hline $\mathrm{H}$ & -2.459779 & 3.230286 & 3.217406 \\
\hline $\mathrm{H}$ & 6.360957 & -3.539025 & 1.844715 \\
\hline $\mathrm{C}$ & -5.099583 & -3.026951 & -0.686609 \\
\hline $\mathrm{H}$ & -5.893305 & -2.757437 & -2.670433 \\
\hline $\mathrm{H}$ & -3.989291 & -3.177082 & 1.159164 \\
\hline $\mathrm{H}$ & -5.997394 & -3.494583 & -0.295155 \\
\hline
\end{tabular}

15d 


\begin{tabular}{|c|c|c|c|}
\hline $\mathrm{O}$ & -0.243004 & 0.575493 & -0.777930 \\
\hline $\mathrm{C}$ & -1.380282 & 0.726605 & -0.301164 \\
\hline $\mathrm{Li}$ & 1.181364 & -0.565222 & -0.446946 \\
\hline $\mathrm{N}$ & -1.627459 & 1.711554 & 0.635645 \\
\hline $\mathrm{C}$ & -2.547637 & -0.021339 & -0.8279 \\
\hline $\mathrm{N}$ & 3.055202 & -0.209725 & 0.205126 \\
\hline $\mathrm{N}$ & 0.787649 & -2.363758 & 0.451678 \\
\hline $\mathrm{C}$ & -1.168047 & 3.055290 & 0.247547 \\
\hline $\mathrm{C}$ & -3.001587 & 1.757541 & 1.163202 \\
\hline $\mathrm{C}$ & -2.378363 & -1.248208 & -1.472842 \\
\hline $\mathrm{C}$ & -3.804886 & 0.619984 & -0.817921 \\
\hline $\mathrm{Si}$ & 4.001042 & -0.906556 & -1.042653 \\
\hline $\mathrm{Si}$ & 3.147750 & 1.395167 & 0.805904 \\
\hline $\mathrm{Li}$ & 2.458970 & -1.737687 & 1.187714 \\
\hline $\mathrm{C}$ & -0.434222 & -2.381899 & 1.103061 \\
\hline $\mathrm{H}$ & 0.850319 & -3.178174 & -0.161658 \\
\hline $\mathrm{C}$ & -2.211564 & 3.676542 & -0.661521 \\
\hline $\mathrm{H}$ & -0.206976 & 2.959793 & -0.260442 \\
\hline $\mathrm{H}$ & -0.998040 & 3.653474 & 1.151174 \\
\hline $\mathrm{C}$ & -3.916428 & 1.930499 & -0.046606 \\
\hline $\mathrm{H}$ & -3.207008 & 0.831314 & 1.703358 \\
\hline $\mathrm{H}$ & -3.063782 & 2.598466 & 1.858907 \\
\hline $\mathrm{C}$ & -3.464216 & -1.854213 & -2.100343 \\
\hline $\mathrm{H}$ & -1.400686 & -1.714802 & -1.465638 \\
\hline $\mathrm{C}$ & -4.877264 & 0.014168 & -1.465502 \\
\hline $\mathrm{C}$ & 5.654362 & -0.065565 & -1.422936 \\
\hline $\mathrm{C}$ & 2.996478 & -0.978455 & -2.660720 \\
\hline $\mathrm{C}$ & 4.375687 & -2.717193 & -0.566396 \\
\hline $\mathrm{C}$ & 1.825508 & 1.630636 & 2.149838 \\
\hline $\mathrm{C}$ & 2.806134 & 2.673698 & -0.559099 \\
\hline $\mathrm{C}$ & 4.816606 & 1.824248 & 1.600965 \\
\hline $\mathrm{C}$ & -1.447016 & -3.342762 & 0.858008 \\
\hline $\mathrm{C}$ & -0.742673 & -1.368772 & 2.047553 \\
\hline $\mathrm{C}$ & -3.429152 & 3.143923 & -0.830515 \\
\hline $\mathrm{H}$ & -1.920147 & 4.569319 & -1.210913 \\
\hline $\mathrm{H}$ & -4.958812 & 2.083589 & 0.256770 \\
\hline $\mathrm{C}$ & -4.707952 & -1.220059 & -2.10102 \\
\hline $\mathrm{H}$ & -3.341818 & -2.816630 & -2.58729 \\
\hline $\mathrm{H}$ & -5.850372 & 0.498293 & -1.464146 \\
\hline $\mathrm{H}$ & 6.314062 & -0.080554 & -0.547796 \\
\hline $\mathrm{H}$ & 6.174781 & -0.573408 & -2.244639 \\
\hline $\mathrm{H}$ & 5.515160 & 0.982088 & 15238 \\
\hline
\end{tabular}




\begin{tabular}{llll}
$\mathrm{H}$ & 2.071603 & -1.563329 & -2.539558 \\
$\mathrm{H}$ & 2.709487 & 0.030718 & -2.983301 \\
$\mathrm{H}$ & 3.557039 & -1.445228 & -3.480436 \\
$\mathrm{H}$ & 3.458904 & -3.299284 & -0.386261 \\
$\mathrm{H}$ & 4.996280 & -2.767686 & 0.339536 \\
$\mathrm{H}$ & 4.920903 & -3.242045 & -1.360498 \\
$\mathrm{H}$ & 0.814503 & 1.468112 & 1.759057 \\
$\mathrm{H}$ & 1.860834 & 2.644033 & 2.570054 \\
$\mathrm{H}$ & 1.976447 & 0.931294 & 2.984014 \\
$\mathrm{H}$ & 3.594523 & 2.657936 & -1.322191 \\
$\mathrm{H}$ & 2.741484 & 3.699066 & -0.172824 \\
$\mathrm{H}$ & 1.860998 & 2.435614 & -1.062889 \\
$\mathrm{H}$ & 4.804273 & 2.830051 & 2.040298 \\
$\mathrm{H}$ & 5.634438 & 1.789925 & 0.872646 \\
$\mathrm{H}$ & 5.058694 & 1.114236 & 2.402333 \\
$\mathrm{C}$ & -2.686290 & -3.274678 & 1.487962 \\
$\mathrm{H}$ & -1.246931 & -4.140164 & 0.143727 \\
$\mathrm{C}$ & -1.980886 & -1.316088 & 2.682044 \\
$\mathrm{H}$ & 0.007678 & -0.613954 & 2.270446 \\
$\mathrm{H}$ & -4.120222 & 3.582715 & -1.546375 \\
$\mathrm{H}$ & -5.554834 & -1.689774 & -2.593500 \\
$\mathrm{C}$ & -2.975558 & -2.258733 & 2.403659 \\
$\mathrm{H}$ & -3.438590 & -4.025566 & 1.257213 \\
$\mathrm{H}$ & -2.165471 & -0.529262 & 3.410683 \\
& -3.941019 & -2.213478 & 2.898267 \\
\hline
\end{tabular}

$15 \mathrm{t}$

$\mathrm{Si} \quad-4.113902-0.872616-1.844418$

$\begin{array}{lllll}\mathrm{N} & & -3.624802 & -0.847985 & -0.192186\end{array}$

C $\quad-5.683501 \quad-1.848800 \quad-2.250987$

$\mathrm{Si} \quad-4.509728-1.461505 \quad 1.157429$

$\mathrm{Li} \quad-1.880649-1.645984-0.251507$

$\mathrm{Li} \quad-2.849789 \quad 0.897940 \quad 0.134617$

C $\quad-4.381953 \quad 0.895350-2.481632$

$\begin{array}{lllll}\text { C } & -2.694229 & -1.652051 & -2.852941\end{array}$

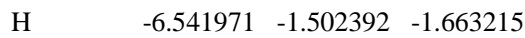

$\begin{array}{llll}\mathrm{H} & -5.939567 & -1.729668 & -3.311293\end{array}$

$\begin{array}{llll}\mathrm{H} & -5.556850 & -2.919324 & -2.056932\end{array}$

$\begin{array}{llll}\text { C } & -6.004365 & -0.376757 & 1.589207\end{array}$

$\begin{array}{llll}\text { C } & -5.138640 & -3.236860 & 0.925263\end{array}$

$\begin{array}{llll}\text { C } & -3.345840 & -1.530591 & 2.658479\end{array}$

$\begin{array}{lllll}\mathrm{N} & & -0.186457 & -1.603017 & 0.624326\end{array}$ 


\begin{tabular}{|c|c|c|c|}
\hline $\mathrm{N}$ & -1.201506 & 1.863188 & 0.351635 \\
\hline $\mathrm{H}$ & -4.630695 & 0.908340 & -3.550085 \\
\hline $\mathrm{H}$ & -5.211193 & 1.373386 & -1.943004 \\
\hline $\mathrm{H}$ & -3.495570 & 1.528302 & -2.357683 \\
\hline $\mathrm{H}$ & -1.732101 & -1.142385 & -2.701416 \\
\hline $\mathrm{H}$ & -2.557782 & -2.713026 & -2.595433 \\
\hline $\mathrm{H}$ & -2.893009 & -1.614078 & -3.930963 \\
\hline $\mathrm{H}$ & -6.706642 & -0.338979 & 0.746702 \\
\hline $\mathrm{H}$ & -5.705218 & 0.655243 & 1.810739 \\
\hline $\mathrm{H}$ & -6.551713 & -0.757481 & 2.460544 \\
\hline $\mathrm{H}$ & -5.500386 & -3.645674 & 1.877243 \\
\hline $\mathrm{H}$ & -4.335829 & -3.895994 & 0.568269 \\
\hline $\mathrm{H}$ & -5.960824 & -3.297287 & 0.205292 \\
\hline $\mathrm{H}$ & -3.891133 & -1.821731 & 3.564779 \\
\hline $\mathrm{H}$ & -2.555757 & -2.277406 & 2.501793 \\
\hline $\mathrm{H}$ & -2.853911 & -0.574316 & 2.864903 \\
\hline $\mathrm{C}$ & 0.793787 & -2.415694 & 0.069143 \\
\hline $\mathrm{Li}$ & 0.112405 & 0.377564 & 0.431439 \\
\hline $\mathrm{H}$ & -0.308603 & -1.848111 & 1.609032 \\
\hline $\mathrm{Si}$ & -1.321172 & 2.418804 & 1.976075 \\
\hline $\mathrm{Si}$ & -0.774626 & 2.844726 & -1.000679 \\
\hline $\mathrm{C}$ & 1.502298 & -3.406481 & 0.792970 \\
\hline $\mathrm{C}$ & 1.147918 & -2.249662 & -1.293041 \\
\hline $\mathrm{C}$ & -0.671884 & 4.161862 & 2.332912 \\
\hline $\mathrm{C}$ & -0.370547 & 1.233206 & 3.121888 \\
\hline $\mathrm{C}$ & -3.150320 & 2.413338 & 2.514947 \\
\hline $\mathrm{C}$ & -0.471168 & 1.711608 & -2.494204 \\
\hline $\mathrm{C}$ & 0.800724 & 3.882342 & -0.767993 \\
\hline $\mathrm{C}$ & -2.149352 & 4.081498 & -1.447121 \\
\hline $\mathrm{C}$ & 2.507513 & -4.162467 & 0.197059 \\
\hline $\mathrm{H}$ & 1.259332 & -3.560307 & 1.843016 \\
\hline $\mathrm{C}$ & 2.148904 & -3.016512 & -1.883953 \\
\hline $\mathrm{H}$ & 0.642048 & -1.480353 & -1.873055 \\
\hline $\mathrm{H}$ & -1.159584 & 4.917890 & 1.706310 \\
\hline $\mathrm{H}$ & -0.859583 & 4.428141 & 3.380791 \\
\hline $\mathrm{H}$ & 0.407385 & 4.230531 & 2.159134 \\
\hline $\mathrm{H}$ & -0.502214 & 1.498641 & 4.178132 \\
\hline $\mathrm{H}$ & 0.707106 & 1.267151 & 2.909355 \\
\hline $\mathrm{H}$ & -0.703039 & 0.194199 & 3.003682 \\
\hline $\mathrm{H}$ & -3.634859 & 1.434114 & 2.411201 \\
\hline $\mathrm{H}$ & -3.722262 & 3.134293 & 1.914501 \\
\hline $\mathrm{H}$ & -3.263320 & 2.700421 & 3.567903 \\
\hline
\end{tabular}




\begin{tabular}{|c|c|c|c|}
\hline I & 0.374817 & 1.041912 & -2.297082 \\
\hline $\mathrm{H}$ & -0.234122 & 2.290765 & -3.395516 \\
\hline $\mathrm{H}$ & -1.343042 & 1.090878 & -2.727902 \\
\hline $\mathrm{H}$ & 1.581995 & 3.294543 & -0.274707 \\
\hline $\mathrm{H}$ & 1.187264 & 4.221793 & -1.738451 \\
\hline $\mathrm{H}$ & 0.616822 & 4.775390 & -0.161398 \\
\hline $\mathrm{H}$ & -1.902378 & 4.663673 & -2.344174 \\
\hline $\mathrm{H}$ & -2.296735 & 4.792046 & -0.623383 \\
\hline $\mathrm{H}$ & -3.112837 & 3.589212 & -1.628504 \\
\hline $\mathrm{C}$ & 2.847857 & -3.977618 & -1.146905 \\
\hline $\mathrm{H}$ & 3.034212 & -4.904822 & 0.792173 \\
\hline $\mathrm{H}$ & 2.389819 & -2.851281 & -2.931546 \\
\hline $\mathrm{H}$ & 3.626873 & -4.575834 & -1.609953 \\
\hline $\mathrm{C}$ & 3.180523 & -0.827801 & 1.923317 \\
\hline $\mathrm{C}$ & 3.989895 & -1.219702 & 2.986574 \\
\hline $\mathrm{C}$ & 3.763012 & -0.423701 & 0.719575 \\
\hline $\mathrm{H}$ & 2.100175 & -0.832527 & 2.008299 \\
\hline $\mathrm{C}$ & 5.379738 & -1.185876 & 2.849921 \\
\hline $\mathrm{H}$ & 3.540847 & -1.546603 & 3.919405 \\
\hline $\mathrm{C}$ & 5.165655 & -0.394729 & 0.574289 \\
\hline $\mathrm{C}$ & 2.938160 & 0.179230 & -0.358595 \\
\hline $\mathrm{C}$ & 5.965257 & -0.772041 & 1.650010 \\
\hline $\mathrm{H}$ & 6.012869 & -1.486869 & 3.679899 \\
\hline $\mathrm{C}$ & 5.719364 & 0.020793 & -0.783767 \\
\hline $\mathrm{N}$ & 3.483558 & 0.223190 & -1.627461 \\
\hline $\mathrm{O}$ & 1.868609 & 0.751856 & -0.106001 \\
\hline $\mathrm{H}$ & 7.047279 & -0.752783 & 1.548937 \\
\hline $\mathrm{C}$ & 4.731858 & -0.535657 & -1.806802 \\
\hline $\mathrm{C}$ & 5.784695 & 1.533219 & -0.968574 \\
\hline $\mathrm{H}$ & 6.719569 & -0.409115 & -0.914285 \\
\hline $\mathrm{C}$ & 3.600744 & 1.574845 & -2.195926 \\
\hline $\mathrm{H}$ & 4.529137 & -1.597402 & -1.650765 \\
\hline $\mathrm{H}$ & 5.069135 & -0.385912 & -2.835843 \\
\hline $\mathrm{C}$ & 4.855248 & 2.223850 & -1.642425 \\
\hline $\mathrm{H}$ & 6.633831 & 2.050350 & -0.527896 \\
\hline $\mathrm{H}$ & 2.710926 & 2.145138 & -1.925369 \\
\hline $\mathrm{H}$ & 3.624167 & 1.506147 & -3.290803 \\
\hline $\mathrm{H}$ & 4.960683 & 3.297566 & -1.783056 \\
\hline
\end{tabular}

\section{TS16d}

$\begin{array}{llll}\mathrm{Si} & 3.398648 & 1.287997 & 0.876604\end{array}$

N $\quad 2.742688-0.142609 \quad 0.201101$ 


\begin{tabular}{|c|c|c|c|}
\hline $\mathrm{C}$ & 3.623818 & 2.703935 & -0.356301 \\
\hline $\mathrm{Si}$ & 3.603178 & -1.399023 & -0.592601 \\
\hline $\mathrm{Li}$ & 1.443470 & -0.510030 & 1.554866 \\
\hline $\mathrm{Li}$ & 1.042152 & -0.326720 & -0.844360 \\
\hline $\mathrm{C}$ & 5.045640 & 1.035158 & 1.783961 \\
\hline $\mathrm{C}$ & 2.148541 & 1.860883 & 2.217318 \\
\hline $\mathrm{H}$ & 4.365100 & 2.421740 & -1.114127 \\
\hline $\mathrm{H}$ & 3.977859 & 3.621776 & 0.129798 \\
\hline $\mathrm{H}$ & 2.692062 & 2.937305 & -0.883594 \\
\hline $\mathrm{C}$ & 5.067616 & -0.805862 & -1.638104 \\
\hline $\mathrm{C}$ & 4.225195 & -2.716826 & 0.622528 \\
\hline $\mathrm{C}$ & 2.386832 & -2.266661 & -1.782440 \\
\hline $\mathrm{O}$ & -0.040508 & -1.061703 & 0.774726 \\
\hline $\mathrm{N}$ & -0.603513 & 0.306782 & -1.602945 \\
\hline $\mathrm{H}$ & 5.374613 & 1.952527 & 2.288137 \\
\hline $\mathrm{H}$ & 4.958788 & 0.248440 & 2.544365 \\
\hline $\mathrm{H}$ & 5.843460 & 0.735509 & 1.094126 \\
\hline $\mathrm{H}$ & 1.153719 & 2.077289 & 1.805744 \\
\hline $\mathrm{H}$ & 2.030714 & 1.132800 & 3.040289 \\
\hline $\mathrm{H}$ & 2.495308 & 2.781525 & 2.701570 \\
\hline $\mathrm{H}$ & 4.741591 & -0.062186 & -2.375761 \\
\hline $\mathrm{H}$ & 5.533937 & -1.635533 & -2.184016 \\
\hline $\mathrm{H}$ & 5.844894 & -0.339433 & -1.021382 \\
\hline $\mathrm{H}$ & 4.740805 & -3.544234 & 0.119158 \\
\hline $\mathrm{H}$ & 3.388696 & -3.146156 & 1.191402 \\
\hline $\mathrm{H}$ & 4.924465 & -2.276673 & 1.344265 \\
\hline $\mathrm{H}$ & 1.488953 & -2.643047 & -1.272650 \\
\hline $\mathrm{H}$ & 2.059357 & -1.608159 & -2.600958 \\
\hline $\mathrm{H}$ & 2.867042 & -3.131594 & -2.255667 \\
\hline $\mathrm{C}$ & -1.159010 & -1.060342 & 0.156502 \\
\hline C & -1.109590 & 1.581683 & -1.483851 \\
\hline $\mathrm{H}$ & -1.130819 & -0.218202 & -2.296468 \\
\hline $\mathrm{N}$ & -1.440940 & -2.180423 & -0.653004 \\
\hline $\mathrm{C}$ & -2.286312 & -0.242092 & 0.650591 \\
\hline $\mathrm{C}$ & -0.360877 & 2.561769 & -0.787991 \\
\hline $\mathrm{C}$ & -2.393062 & 1.964297 & -1.943948 \\
\hline $\mathrm{C}$ & -2.757501 & -2.140966 & -1.302547 \\
\hline $\mathrm{C}$ & -1.241768 & -3.475966 & 0.019720 \\
\hline $\mathrm{C}$ & -2.047224 & 0.958525 & 1.329346 \\
\hline $\mathrm{C}$ & -3.611024 & -0.693919 & 0.476641 \\
\hline $\mathrm{C}$ & -0.870159 & 3.834437 & -0.549622 \\
\hline $\mathrm{H}$ & 0.636173 & 2.300030 & -0.446819 \\
\hline
\end{tabular}




$\begin{array}{llll}\mathrm{C} & -2.896638 & 3.237157 & -1.704009 \\ \mathrm{H} & -2.998600 & 1.232689 & -2.475501 \\ \mathrm{C} & -3.818321 & -2.036110 & -0.207595 \\ \mathrm{H} & -2.804932 & -1.283477 & -1.977554 \\ \mathrm{H} & -2.870600 & -3.054607 & -1.893482 \\ \mathrm{C} & -2.454492 & -3.854711 & 0.843428 \\ \mathrm{H} & -0.351386 & -3.397076 & 0.647019 \\ \mathrm{H} & -1.030183 & -4.238617 & -0.742303 \\ \mathrm{C} & -3.109210 & 1.730077 & 1.793698 \\ \mathrm{H} & -1.028158 & 1.289870 & 1.474645 \\ \mathrm{C} & -4.665799 & 0.077864 & 0.957467 \\ \mathrm{C} & -2.146057 & 4.184795 & -0.998902 \\ \mathrm{H} & -0.262420 & 4.560136 & -0.014030 \\ \mathrm{H} & -3.890615 & 3.493242 & -2.063065 \\ \mathrm{C} & -3.613431 & -3.192443 & 0.760133 \\ \mathrm{H} & -4.829374 & -2.081498 & -0.629732 \\ \mathrm{H} & -2.345478 & -4.694473 & 1.527276 \\ \mathrm{C} & -4.420064 & 1.293053 & 1.602900 \\ \mathrm{H} & -2.912246 & 2.674776 & 2.290298 \\ \mathrm{H} & -5.686535 & -0.272332 & 0.824347 \\ \mathrm{H} & -2.544885 & 5.177609 & -0.813318 \\ \mathrm{H} & -4.447507 & -3.458554 & 1.405369 \\ \mathrm{H} & -5.252420 & 1.894161 & 1.957650\end{array}$

\section{TS16t}

$\begin{array}{llll}\mathrm{Si} & -1.550242 & 2.764092 & -1.357749\end{array}$

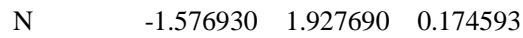

$\begin{array}{llll}\text { C } & -0.187175 & 4.069680 & -1.461277\end{array}$

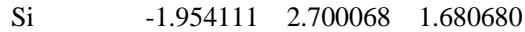

$\mathrm{Li} \quad-3.194324 \quad 0.740134 \quad 0.306754$

$\begin{array}{llll}\mathrm{Li} & 0.381234 & 1.768464 & 0.606667\end{array}$

$\begin{array}{llll}\text { C } & -3.202496 & 3.609923 & -1.755937\end{array}$

C $\quad-1.184040 \quad 1.496453 \quad-2.730693$

$\mathrm{H} \quad 0.789896 \quad 3.633662-1.218626$

$\mathrm{H} \quad-0.128511 \quad 4.485779-2.475279$

$\begin{array}{llll}\mathrm{H} & -0.363457 & 4.905101 & -0.773396\end{array}$

$\begin{array}{llll}\text { C } & -2.403857 & 4.533945 & 1.621319\end{array}$

$\begin{array}{llll}\text { C } & -0.490859 & 2.526888 & 2.882076\end{array}$

$\begin{array}{llll}\text { C } & -3.458579 & 1.812722 & 2.455248\end{array}$

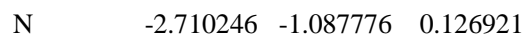

$\mathrm{Li} \quad-1.036704 \quad-0.106191 \quad-0.141912$

$\begin{array}{llll}\text { O } & 2.065158 & 2.000451 & 0.210868\end{array}$ 


\begin{tabular}{|c|c|c|c|}
\hline $\mathrm{H}$ & -4.067712 & 2.966541 & -1.555792 \\
\hline $\mathrm{H}$ & -3.246898 & 3.894027 & -2.814667 \\
\hline $\mathrm{H}$ & -3.326788 & 4.525186 & -1.167844 \\
\hline $\mathrm{H}$ & -1.592854 & 0.498167 & -2.534239 \\
\hline $\mathrm{H}$ & -1.618462 & 1.829355 & -3.681118 \\
\hline $\mathrm{H}$ & -0.104636 & 1.396735 & -2.896827 \\
\hline $\mathrm{H}$ & -1.622391 & 5.133830 & 1.142635 \\
\hline $\mathrm{H}$ & -2.534654 & 4.915703 & 2.641496 \\
\hline $\mathrm{H}$ & -3.339555 & 4.709503 & 1.079978 \\
\hline $\mathrm{H}$ & -0.752705 & 2.957905 & 3.855947 \\
\hline $\mathrm{H}$ & 0.406006 & 3.064125 & 2.540221 \\
\hline $\mathrm{H}$ & -0.209901 & 1.482628 & 3.065663 \\
\hline $\mathrm{H}$ & -3.315376 & 0.733972 & 2.596180 \\
\hline $\mathrm{H}$ & -4.380244 & 1.967703 & 1.869502 \\
\hline $\mathrm{H}$ & -3.669573 & 2.228550 & 3.447305 \\
\hline $\mathrm{Si}$ & -3.757609 & -1.550811 & -1.156431 \\
\hline $\mathrm{Si}$ & -2.347049 & -2.122498 & 1.458233 \\
\hline $\mathrm{C}$ & 2.505098 & 0.829926 & -0.180811 \\
\hline $\mathrm{C}$ & -2.854876 & -2.309868 & -2.636428 \\
\hline $\mathrm{C}$ & -5.159131 & -2.732277 & -0.670654 \\
\hline $\mathrm{C}$ & -4.627282 & 0.042911 & -1.764158 \\
\hline $\mathrm{C}$ & -1.832799 & -3.863305 & 0.927506 \\
\hline $\mathrm{C}$ & -3.782610 & -2.312257 & 2.687472 \\
\hline $\mathrm{C}$ & -0.929676 & -1.321522 & 2.447656 \\
\hline $\mathrm{N}$ & 3.177854 & 0.865626 & -1.492196 \\
\hline $\mathrm{C}$ & 3.248975 & 0.045873 & 0.901522 \\
\hline $\mathrm{H}$ & -2.142641 & -1.607782 & -3.084919 \\
\hline $\mathrm{H}$ & -3.552799 & -2.626475 & -3.421843 \\
\hline $\mathrm{H}$ & -2.280144 & -3.186042 & -2.314945 \\
\hline $\mathrm{H}$ & -5.779055 & -2.307324 & 0.128019 \\
\hline $\mathrm{H}$ & -5.811017 & -2.928422 & -1.531205 \\
\hline $\mathrm{H}$ & -4.782585 & -3.698802 & -0.317033 \\
\hline $\mathrm{H}$ & -5.342006 & -0.191313 & -2.562310 \\
\hline $\mathrm{H}$ & -5.219919 & 0.529225 & -0.970577 \\
\hline $\mathrm{H}$ & -3.933033 & 0.782906 & -2.178314 \\
\hline $\mathrm{H}$ & -2.681128 & -4.402101 & 0.487899 \\
\hline $\mathrm{H}$ & -1.472655 & -4.456743 & 1.777428 \\
\hline $\mathrm{H}$ & -1.034982 & -3.829914 & 0.179838 \\
\hline $\mathrm{H}$ & -3.472535 & -2.887885 & 3.569176 \\
\hline $\mathrm{H}$ & -4.149924 & -1.340206 & 3.040733 \\
\hline $\mathrm{H}$ & -4.629180 & -2.837045 & 2.231077 \\
\hline $\mathrm{H}$ & -0.080783 & -1.010058 & 1.827820 \\
\hline
\end{tabular}




\begin{tabular}{|c|c|c|c|}
\hline H & -0.524990 & -2.026117 & 3.184138 \\
\hline $\mathrm{H}$ & -1.286601 & -0.444614 & 3.000655 \\
\hline $\mathrm{C}$ & 4.001536 & -0.319790 & -1.763522 \\
\hline $\mathrm{C}$ & 3.956693 & 2.088712 & -1.728251 \\
\hline $\mathrm{C}$ & 2.701369 & -0.023699 & 2.185277 \\
\hline $\mathrm{C}$ & 4.478698 & -0.579639 & 0.647851 \\
\hline $\mathrm{C}$ & 5.117683 & -0.408156 & -0.718440 \\
\hline $\mathrm{H}$ & 3.382843 & -1.216540 & -1.745041 \\
\hline $\mathrm{H}$ & 4.419207 & -0.221127 & -2.771536 \\
\hline $\mathrm{C}$ & 5.379847 & 2.011263 & -1.226802 \\
\hline $\mathrm{H}$ & 3.425555 & 2.909961 & -1.244540 \\
\hline $\mathrm{H}$ & 3.958132 & 2.294340 & -2.810135 \\
\hline $\mathrm{C}$ & 3.329082 & -0.743182 & 3.199327 \\
\hline $\mathrm{H}$ & 1.768862 & 0.489988 & 2.392466 \\
\hline $\mathrm{C}$ & 5.107776 & -1.301077 & 1.668035 \\
\hline $\mathrm{C}$ & 5.917542 & 0.882591 & -0.758159 \\
\hline $\mathrm{H}$ & 5.776023 & -1.262059 & -0.923050 \\
\hline $\mathrm{H}$ & 5.965192 & 2.928746 & -1.265858 \\
\hline $\mathrm{C}$ & 4.536039 & -1.396326 & 2.935324 \\
\hline $\mathrm{H}$ & 2.882443 & -0.791978 & 4.188538 \\
\hline $\mathrm{H}$ & 6.055827 & -1.792143 & 1.460686 \\
\hline $\mathrm{H}$ & 6.931008 & 0.865278 & -0.362849 \\
\hline $\mathrm{H}$ & 5.032599 & -1.966859 & 3.715321 \\
\hline $\mathrm{N}$ & 1.090788 & -0.074190 & -0.537718 \\
\hline $\mathrm{C}$ & 1.164468 & -1.469314 & -0.847683 \\
\hline $\mathrm{H}$ & 0.918832 & 0.395584 & -1.429099 \\
\hline $\mathrm{C}$ & 1.554070 & -2.425915 & 0.105856 \\
\hline $\mathrm{C}$ & 0.799254 & -1.915353 & -2.131233 \\
\hline $\mathrm{C}$ & 1.613107 & -3.774943 & -0.237901 \\
\hline $\mathrm{H}$ & 1.827907 & -2.114710 & 1.103948 \\
\hline $\mathrm{C}$ & 0.836137 & -3.269553 & -2.458660 \\
\hline $\mathrm{H}$ & 0.495007 & -1.186918 & -2.878543 \\
\hline $\mathrm{C}$ & 1.254742 & -4.209612 & -1.515878 \\
\hline $\mathrm{H}$ & 1.929021 & -4.494831 & 0.511887 \\
\hline $\mathrm{H}$ & 0.543931 & -3.584691 & -3.456331 \\
\hline $\mathrm{H}$ & 1.293007 & -5.264607 & -1.769464 \\
\hline
\end{tabular}

\section{7d}

Si $\quad 3.457103 \quad 1.106686 \quad 0.925851$

$\begin{array}{lllll}\mathrm{N} & 2.699173 & -0.254477 & 0.215947\end{array}$

C $\quad 3.874058 \quad 2.493913 \quad-0.294951$

$\mathrm{Si} \quad 3.513483 \quad-1.576803 \quad-0.514339$ 


\begin{tabular}{|c|c|c|c|}
\hline $\mathrm{Li}$ & 1.233884 & -0.357355 & 1.497172 \\
\hline $\mathrm{Li}$ & 1.006995 & -0.518326 & -0.838910 \\
\hline $\mathrm{C}$ & 5.033244 & 0.708580 & 1.903919 \\
\hline $\mathrm{C}$ & 2.220273 & 1.812815 & 2.215493 \\
\hline $\mathrm{H}$ & 2.994634 & 2.825795 & -0.858741 \\
\hline $\mathrm{H}$ & 4.610744 & 2.136203 & -1.024991 \\
\hline $\mathrm{H}$ & 4.302936 & 3.369738 & 0.208218 \\
\hline $\mathrm{C}$ & 5.025195 & -1.088347 & -1.548930 \\
\hline $\mathrm{C}$ & 4.040073 & -2.895179 & 0.740986 \\
\hline $\mathrm{C}$ & 2.286263 & -2.410955 & -1.723344 \\
\hline $\mathrm{O}$ & -0.208939 & -0.743879 & 0.631934 \\
\hline $\mathrm{N}$ & -0.837853 & 0.176104 & -1.423059 \\
\hline $\mathrm{H}$ & 4.837455 & -0.056283 & 2.665931 \\
\hline $\mathrm{H}$ & 5.426045 & 1.597066 & 2.414191 \\
\hline $\mathrm{H}$ & 5.828007 & 0.325125 & 1.253227 \\
\hline $\mathrm{H}$ & 2.624144 & 2.726265 & 2.667985 \\
\hline $\mathrm{H}$ & 2.048034 & 1.125046 & 3.061941 \\
\hline $\mathrm{H}$ & 1.244894 & 2.082460 & 1.787401 \\
\hline $\mathrm{H}$ & 4.749520 & -0.351170 & -2.313421 \\
\hline $\mathrm{H}$ & 5.460324 & -1.955341 & -2.061595 \\
\hline $\mathrm{H}$ & 5.813318 & -0.641479 & -0.931352 \\
\hline $\mathrm{H}$ & 4.515182 & -3.763718 & 0.268042 \\
\hline $\mathrm{H}$ & 4.752086 & -2.474932 & 1.461816 \\
\hline $\mathrm{H}$ & 3.171877 & -3.257555 & 1.307520 \\
\hline $\mathrm{H}$ & 1.355731 & -2.749636 & -1.246303 \\
\hline $\mathrm{H}$ & 2.017976 & -1.759333 & -2.569967 \\
\hline $\mathrm{H}$ & 2.747819 & -3.302423 & -2.164364 \\
\hline $\mathrm{C}$ & -1.276608 & -0.678066 & -0.177345 \\
\hline $\mathrm{C}$ & -1.003516 & 1.596864 & -1.360500 \\
\hline $\mathrm{H}$ & -1.320975 & -0.195109 & -2.235621 \\
\hline $\mathrm{N}$ & -1.555717 & -1.990855 & -0.823948 \\
\hline $\mathrm{C}$ & -2.500889 & -0.058742 & 0.514477 \\
\hline $\mathrm{C}$ & -2.183577 & 2.224854 & -1.775663 \\
\hline $\mathrm{C}$ & 0.038124 & 2.377946 & -0.848073 \\
\hline $\mathrm{C}$ & -2.887583 & -2.045712 & -1.433506 \\
\hline $\mathrm{C}$ & -1.331227 & -3.165570 & 0.026305 \\
\hline $\mathrm{C}$ & -2.335672 & 1.091155 & 1.294519 \\
\hline $\mathrm{C}$ & -3.786071 & -0.610586 & 0.382134 \\
\hline $\mathrm{C}$ & -2.321139 & 3.607899 & -1.668397 \\
\hline $\mathrm{H}$ & -3.004795 & 1.620506 & -2.151610 \\
\hline $\mathrm{C}$ & -0.105573 & 3.759361 & -0.722327 \\
\hline $\mathrm{H}$ & 0.964422 & 1.896739 & -0.555215 \\
\hline
\end{tabular}




$\begin{array}{llll}\mathrm{C} & -3.955804 & -1.935036 & -0.342161 \\ \mathrm{H} & -3.003318 & -1.233768 & -2.160945 \\ \mathrm{H} & -2.981961 & -2.990654 & -1.979506 \\ \mathrm{C} & -2.543129 & -3.624812 & 0.800351 \\ \mathrm{H} & -0.513923 & -2.918352 & 0.707331 \\ \mathrm{H} & -0.974705 & -3.990303 & -0.611224 \\ \mathrm{C} & -3.423650 & 1.709058 & 1.906997 \\ \mathrm{H} & -1.342814 & 1.508300 & 1.413595 \\ \mathrm{C} & -4.875592 & 0.015230 & 0.996265 \\ \mathrm{C} & -1.287704 & 4.379274 & -1.131616 \\ \mathrm{H} & -3.244094 & 4.082271 & -1.989748 \\ \mathrm{H} & 0.712872 & 4.348744 & -0.318301 \\ \mathrm{C} & -3.748077 & -3.074321 & 0.639980 \\ \mathrm{H} & -4.962749 & -1.992708 & -0.774484 \\ \mathrm{H} & -2.395636 & -4.449194 & 1.496303 \\ \mathrm{C} & -4.703566 & 1.175264 & 1.748959 \\ \mathrm{H} & -3.272061 & 2.605759 & 2.501220 \\ \mathrm{H} & -5.865646 & -0.422494 & 0.888416 \\ \mathrm{H} & -1.400839 & 5.455559 & -1.040251 \\ \mathrm{H} & -4.600017 & -3.399463 & 1.232764 \\ \mathrm{H} & -5.560157 & 1.653301 & 2.216314\end{array}$

$\mathbf{1 7 t}$

$\begin{array}{llll}\mathrm{Li} & -0.198562 & 1.145908 & 0.206654\end{array}$

$\begin{array}{lllll}\mathrm{N} & 1.573218 & 1.794986 & 0.766604\end{array}$

$\mathrm{Li} \quad 0.747529-1.596391 \quad 0.152820$

$\begin{array}{lllll}\mathrm{Si} & 2.023663 & 3.101090 & -0.263788\end{array}$

$\begin{array}{llll}\mathrm{Si} & 1.525793 & 1.815877 & 2.488006\end{array}$

Li $\quad \begin{array}{llll}2.649846 & 0.412300 & -0.019401\end{array}$

$\mathrm{N} \quad 2.516796-1.407801-0.622888$

O $\quad-0.695681 \quad-0.631537 \quad 0.026530$

C $\quad 0.635151 \quad 3.483017 \quad-1.495524$

$\begin{array}{llll}\text { C } & 2.487786 & 4.729628 & 0.581760\end{array}$

$\begin{array}{lllll}\text { C } & & 3.556205 & 2.554525 & -1.269340\end{array}$

C $\quad 3.032325 \quad 2.605872 \quad 3.327230$

$\begin{array}{llll}\text { C } & -0.022018 & 2.722192 & 3.116751\end{array}$

$\begin{array}{llll}\text { C } & 1.470136 & 0.028717 & 3.139811\end{array}$

$\begin{array}{lllll}\mathrm{Si} & & 3.637440 & -2.261347 & 0.367413\end{array}$

Si $\quad 2.343108 \quad-1.552763 \quad-2.334597$

$\begin{array}{lllll}\text { C } & -2.042429 & -0.466858 & 0.088973\end{array}$

$\begin{array}{llll}\mathrm{H} & -0.271390 & 3.831313 & -0.983405\end{array}$

$\mathrm{H} \quad 0.931020 \quad 4.268884 \quad-2.201908$ 


\begin{tabular}{|c|c|c|c|}
\hline [ & 0.352018 & 2.608670 & -2.092670 \\
\hline $\mathrm{H}$ & 2.634547 & 5.517528 & -0.167489 \\
\hline $\mathrm{H}$ & 1.698777 & 5.066889 & 1.264697 \\
\hline $\mathrm{H}$ & 3.414087 & 4.641931 & 1.159254 \\
\hline $\mathrm{H}$ & 3.399569 & 1.640118 & -1.860010 \\
\hline $\mathrm{H}$ & 4.418823 & 2.386893 & -0.608171 \\
\hline $\mathrm{H}$ & 3.850615 & 3.327652 & -1.989818 \\
\hline $\mathrm{H}$ & 3.031073 & 2.388022 & 4.402896 \\
\hline $\mathrm{H}$ & 3.966545 & 2.205807 & 2.911570 \\
\hline $\mathrm{H}$ & 3.055394 & 3.693933 & 3.209803 \\
\hline $\mathrm{H}$ & -0.100815 & 2.707364 & 4.210922 \\
\hline $\mathrm{H}$ & 0.000866 & 3.771818 & 2.797167 \\
\hline $\mathrm{H}$ & -0.943664 & 2.284016 & 2.712327 \\
\hline $\mathrm{H}$ & 0.790209 & -0.607196 & 2.562795 \\
\hline $\mathrm{H}$ & 1.129854 & -0.002125 & 4.182634 \\
\hline $\mathrm{H}$ & 2.462069 & -0.434773 & 3.112793 \\
\hline $\mathrm{C}$ & 4.809240 & -3.456645 & -0.517093 \\
\hline $\mathrm{C}$ & 4.737484 & -0.993504 & 1.271474 \\
\hline $\mathrm{C}$ & 2.680182 & -3.281165 & 1.657183 \\
\hline $\mathrm{C}$ & 1.139237 & -0.214753 & -2.935759 \\
\hline $\mathrm{C}$ & 3.958436 & -1.316128 & -3.298453 \\
\hline $\mathrm{C}$ & 1.611723 & -3.237525 & -2.824263 \\
\hline $\mathrm{N}$ & -2.713479 & -0.947126 & -1.122621 \\
\hline $\mathrm{C}$ & -2.628760 & -1.057691 & 1.390259 \\
\hline $\mathrm{H}$ & 4.262495 & -4.183233 & -1.129391 \\
\hline $\mathrm{H}$ & 5.403416 & -4.020763 & 0.212727 \\
\hline $\mathrm{H}$ & 5.508010 & -2.926354 & -1.173440 \\
\hline $\mathrm{H}$ & 4.166265 & -0.223121 & 1.807530 \\
\hline $\mathrm{H}$ & 5.390491 & -0.482060 & 0.550376 \\
\hline $\mathrm{H}$ & 5.387345 & -1.469720 & 2.016151 \\
\hline $\mathrm{H}$ & 2.090975 & -4.062748 & 1.156399 \\
\hline $\mathrm{H}$ & 1.993675 & -2.669165 & 2.257062 \\
\hline $\mathrm{H}$ & 3.350773 & -3.786002 & 2.363542 \\
\hline $\mathrm{H}$ & 1.578700 & 0.787444 & -2.870274 \\
\hline $\mathrm{H}$ & 0.855121 & -0.376496 & -3.982841 \\
\hline $\mathrm{H}$ & 0.226228 & -0.217589 & -2.331847 \\
\hline $\mathrm{H}$ & 4.476047 & -0.403853 & -2.974735 \\
\hline $\mathrm{H}$ & 3.762948 & -1.222028 & -4.374040 \\
\hline $\mathrm{H}$ & 4.647640 & -2.155455 & -3.158706 \\
\hline $\mathrm{H}$ & 2.293155 & -4.055298 & -2.559355 \\
\hline $\mathrm{H}$ & 0.661787 & -3.425170 & -2.306131 \\
\hline $\mathrm{H}$ & 1.415695 & -3.304480 & -3.901730 \\
\hline
\end{tabular}




\begin{tabular}{|c|c|c|c|}
\hline $\mathrm{C}$ & -2.109036 & -2.071171 & -1.827049 \\
\hline $\mathrm{C}$ & -4.143856 & -1.158732 & -0.926095 \\
\hline $\mathrm{C}$ & -2.060895 & -0.694921 & 2.616736 \\
\hline $\mathrm{C}$ & -3.723539 & -1.937023 & 1.378518 \\
\hline $\mathrm{C}$ & -2.599415 & -3.422693 & -1.365968 \\
\hline $\mathrm{H}$ & -1.027315 & -1.995623 & -1.723186 \\
\hline $\mathrm{H}$ & -2.317110 & -1.955408 & -2.903485 \\
\hline $\mathrm{C}$ & -4.334461 & -2.331340 & 0.046243 \\
\hline $\mathrm{H}$ & -4.597748 & -0.241444 & -0.537231 \\
\hline $\mathrm{H}$ & -4.602888 & -1.371638 & -1.897475 \\
\hline $\mathrm{C}$ & -2.551773 & -1.204231 & 3.818156 \\
\hline $\mathrm{H}$ & -1.220544 & -0.012323 & 2.631870 \\
\hline $\mathrm{C}$ & -4.211514 & -2.445694 & 2.586328 \\
\hline $\mathrm{C}$ & -3.618302 & -3.550387 & -0.511988 \\
\hline $\mathrm{H}$ & -2.107778 & -4.299719 & -1.784651 \\
\hline $\mathrm{H}$ & -5.398322 & -2.560137 & 0.188055 \\
\hline $\mathrm{C}$ & -3.632357 & -2.087853 & 3.802931 \\
\hline $\mathrm{H}$ & -2.089661 & -0.914079 & 4.757494 \\
\hline $\mathrm{H}$ & -5.058956 & -3.127213 & 2.567358 \\
\hline $\mathrm{H}$ & -3.946505 & -4.530299 & -0.172376 \\
\hline $\mathrm{H}$ & -4.024736 & -2.491529 & 4.732046 \\
\hline $\mathrm{N}$ & -2.295826 & 1.060553 & 0.193616 \\
\hline $\mathrm{C}$ & -2.834771 & 1.824578 & -0.873137 \\
\hline $\mathrm{H}$ & -2.787987 & 1.268795 & 1.056164 \\
\hline $\mathrm{C}$ & -2.351613 & 1.705201 & -2.185861 \\
\hline $\mathrm{C}$ & -3.826484 & 2.777857 & -0.600802 \\
\hline $\mathrm{C}$ & -2.859572 & 2.522142 & -3.193095 \\
\hline $\mathrm{H}$ & -1.586447 & 0.975473 & -2.412357 \\
\hline $\mathrm{C}$ & -4.317419 & 3.603636 & -1.612084 \\
\hline $\mathrm{H}$ & -4.212244 & 2.871424 & 0.412591 \\
\hline $\mathrm{C}$ & -3.840837 & 3.477062 & -2.916919 \\
\hline $\mathrm{H}$ & -2.474425 & 2.414935 & -4.203479 \\
\hline $\mathrm{H}$ & -5.081124 & 4.339562 & -1.376204 \\
\hline $\mathrm{H}$ & -4.225701 & 4.114471 & -3.707272 \\
\hline
\end{tabular}

18d

Li $\quad-0.766052 \quad 0.141973 \quad-0.467459$

$\begin{array}{llll}\mathrm{N} & -0.242240 & 2.118022 & -0.544405\end{array}$

$\begin{array}{llll}\mathrm{N} & -2.658857 & 0.269587 & 0.165041\end{array}$

$\begin{array}{llll}\text { C } & 0.992464 & 2.367574 & 0.023072\end{array}$

Li $\quad-1.962896 \quad 2.040901 \quad 0.330542$

$\mathrm{H} \quad-0.268750 \quad 2.532527 \quad-1.477793$ 


\begin{tabular}{|c|c|c|c|}
\hline $\mathrm{O}$ & 0.572917 & -1.169560 & -0.214736 \\
\hline $\mathrm{Si}$ & -3.516494 & 0.289370 & -1.318488 \\
\hline $\mathrm{Si}$ & -2.923100 & -0.768721 & 1.509291 \\
\hline $\mathrm{C}$ & 2.046035 & 3.046341 & -0.643369 \\
\hline $\mathrm{C}$ & 1.276753 & 1.893144 & 1.331931 \\
\hline $\mathrm{C}$ & 1.788997 & -1.214090 & -0.329358 \\
\hline $\mathrm{C}$ & -5.194002 & -0.586728 & -1.337478 \\
\hline $\mathrm{C}$ & -2.438212 & -0.478736 & -2.692934 \\
\hline $\mathrm{C}$ & -3.809110 & 2.105140 & -1.828101 \\
\hline $\mathrm{C}$ & -2.758923 & -2.601245 & 1.044856 \\
\hline $\mathrm{C}$ & -1.586856 & -0.407696 & 2.810886 \\
\hline $\mathrm{C}$ & -4.611233 & -0.506582 & 2.334829 \\
\hline $\mathrm{C}$ & 3.292184 & 3.226751 & -0.046054 \\
\hline $\mathrm{H}$ & 1.865506 & 3.431497 & -1.645729 \\
\hline $\mathrm{C}$ & 2.523438 & 2.080761 & 1.920090 \\
\hline $\mathrm{H}$ & 0.496470 & 1.368878 & 1.878907 \\
\hline $\mathrm{C}$ & 2.574677 & -0.437147 & -1.362041 \\
\hline $\mathrm{N}$ & 2.577736 & -2.078518 & 0.495973 \\
\hline $\mathrm{H}$ & -5.894357 & -0.113476 & -0.639665 \\
\hline $\mathrm{H}$ & -5.645154 & -0.556909 & -2.337159 \\
\hline $\mathrm{H}$ & -5.098763 & -1.639350 & -1.046737 \\
\hline $\mathrm{H}$ & -1.491796 & 0.069375 & -2.821813 \\
\hline $\mathrm{H}$ & -2.189933 & -1.521207 & -2.453776 \\
\hline $\mathrm{H}$ & -2.938042 & -0.470462 & -3.669599 \\
\hline $\mathrm{H}$ & -2.869370 & 2.673557 & -1.902942 \\
\hline $\mathrm{H}$ & -4.459196 & 2.617128 & -1.104888 \\
\hline $\mathrm{H}$ & -4.293485 & 2.182170 & -2.809429 \\
\hline $\mathrm{H}$ & -1.765415 & -2.788811 & 0.618344 \\
\hline $\mathrm{H}$ & -2.881085 & -3.261610 & 1.912631 \\
\hline $\mathrm{H}$ & -3.504574 & -2.896260 & 0.295986 \\
\hline $\mathrm{H}$ & -1.733857 & -1.009028 & 3.716494 \\
\hline $\mathrm{H}$ & -0.588580 & -0.643764 & 2.421228 \\
\hline $\mathrm{H}$ & -1.588406 & 0.647445 & 3.118908 \\
\hline $\mathrm{H}$ & -4.715876 & -1.108445 & 3.246559 \\
\hline $\mathrm{H}$ & -5.433319 & -0.777149 & 1.662128 \\
\hline $\mathrm{H}$ & -4.747161 & 0.546693 & 2.613071 \\
\hline $\mathrm{C}$ & 3.553071 & 2.742538 & 1.239267 \\
\hline $\mathrm{H}$ & 4.069615 & 3.754383 & -0.594187 \\
\hline $\mathrm{H}$ & 2.691949 & 1.709998 & 2.928975 \\
\hline $\mathrm{C}$ & 4.022577 & -0.959451 & -1.359053 \\
\hline $\mathrm{H}$ & 2.081554 & -0.556543 & -2.335216 \\
\hline $\mathrm{H}$ & 2.526779 & 0.630881 & -1.110772 \\
\hline
\end{tabular}




$\begin{array}{llll}\mathrm{C} & 3.686828 & -1.281400 & 1.094598 \\ \mathrm{C} & 3.161883 & -3.116590 & -0.401591 \\ \mathrm{H} & 4.521276 & 2.894640 & 1.706155 \\ \mathrm{C} & 4.628099 & -0.661835 & 0.023222 \\ \mathrm{C} & 3.986614 & -2.486017 & -1.566680 \\ \mathrm{H} & 4.600774 & -0.473159 & -2.150340 \\ \mathrm{H} & 3.231404 & -0.503320 & 1.710275 \\ \mathrm{H} & 4.220284 & -1.964357 & 1.762242 \\ \mathrm{H} & 2.343152 & -3.739185 & -0.772854 \\ \mathrm{H} & 3.785724 & -3.748261 & 0.237452 \\ \mathrm{H} & 4.710110 & 0.418723 & 0.177579 \\ \mathrm{H} & 5.635645 & -1.088193 & 0.088692 \\ \mathrm{H} & 5.005303 & -2.888608 & -1.582498 \\ \mathrm{H} & 3.531233 & -2.723054 & -2.535644\end{array}$

$18 t$

Li $\quad \begin{array}{llll}0.542606 & 0.435391 & -0.177204\end{array}$

$\mathrm{N} \quad 0.757962 \quad 1.897607 \quad 0.075654$

$\mathrm{N} \quad-0.256166-1.482110-0.670739$

Si $\quad 0.747478 \quad 2.794778-1.391315$

$\mathrm{Si} \quad 0.485423 \quad 2.549052 \quad 1.652298$

$\begin{array}{lllll}\mathrm{Li} & 2.410275 & 0.918237 & -0.019636\end{array}$

$\begin{array}{llll}\mathrm{O} & -2.350737 & 0.752115 & 0.377430\end{array}$

C $\quad-1.243432-2.361782-0.255737$

$\begin{array}{llll}\mathrm{Li} & 1.481984 & -1.669772 & 0.095535\end{array}$

$\mathrm{H} \quad \begin{array}{llll}-0.154204 & -1.548416 & -1.685825\end{array}$

$\begin{array}{lllll}\text { C } & 0.075006 & 4.563054 & -1.302834\end{array}$

$\begin{array}{lllll}\text { C } & -0.312369 & 1.875432 & -2.679911\end{array}$

$\begin{array}{lllll}\text { C } & 2.523583 & 2.941263 & -2.070848\end{array}$

$\begin{array}{llll}\text { C } & 0.317025 & 1.086954 & 2.853349\end{array}$

$\begin{array}{llll}\text { C } & -1.098720 & 3.569501 & 1.844744\end{array}$

$\begin{array}{llll}\text { C } & 1.927184 & 3.653616 & 2.213587\end{array}$

N $\quad 3.208730 \quad-0.842009 \quad-0.059799$

$\begin{array}{llll}\text { C } & -3.466508 & 0.370469 & 0.065522\end{array}$

$\begin{array}{lllll}\text { C } & & -1.952421 & -3.221626 & -1.133189\end{array}$

$\begin{array}{llll}\text { C } & -1.604272 & -2.416247 & 1.115442\end{array}$

$\begin{array}{llll}\mathrm{H} & 0.605979 & 5.164647 & -0.555796\end{array}$

$\begin{array}{llll}\mathrm{H} & 0.187623 & 5.063290 & -2.273013\end{array}$

$\mathrm{H} \quad-0.988995 \quad 4.573999 \quad-1.041848$

$\mathrm{H} \quad \begin{array}{llll}-0.251737 & 2.343496 & -3.670059\end{array}$

$\begin{array}{llll}\mathrm{H} & -1.369182 & 1.886878 & -2.377933\end{array}$

$\mathrm{H} \quad \begin{array}{llll}0.011345 & 0.826596 & -2.797219\end{array}$ 


\begin{tabular}{|c|c|c|c|}
\hline $\mathrm{H}$ & 3.032477 & 1.976640 & -2.195856 \\
\hline $\mathrm{H}$ & 3.133673 & 3.552178 & -1.391197 \\
\hline $\mathrm{H}$ & 2.542782 & 3.427120 & -3.054533 \\
\hline $\mathrm{H}$ & -0.588093 & 0.509676 & 2.622555 \\
\hline $\mathrm{H}$ & 0.227290 & 1.425774 & 3.892637 \\
\hline $\mathrm{H}$ & 1.173449 & 0.405687 & 2.805808 \\
\hline $\mathrm{H}$ & -1.960704 & 2.994999 & 1.489211 \\
\hline $\mathrm{H}$ & -1.269871 & 3.809196 & 2.902184 \\
\hline $\mathrm{H}$ & -1.059264 & 4.514667 & 1.293735 \\
\hline $\mathrm{H}$ & 1.792213 & 4.018107 & 3.239707 \\
\hline $\mathrm{H}$ & 2.003582 & 4.531308 & 1.558693 \\
\hline $\mathrm{H}$ & 2.892592 & 3.133901 & 2.172579 \\
\hline $\mathrm{Si}$ & 3.861073 & -1.222254 & 1.489264 \\
\hline $\mathrm{Si}$ & 3.931699 & -1.181958 & -1.590706 \\
\hline $\mathrm{C}$ & -3.819655 & -0.198032 & -1.292609 \\
\hline $\mathrm{N}$ & -4.575122 & 0.498962 & 0.965274 \\
\hline $\mathrm{C}$ & -2.958643 & -4.067526 & -0.668696 \\
\hline $\mathrm{H}$ & -1.696734 & -3.213168 & -2.191719 \\
\hline $\mathrm{C}$ & -2.605786 & -3.269344 & 1.570512 \\
\hline $\mathrm{H}$ & -1.090823 & -1.759561 & 1.815045 \\
\hline $\mathrm{C}$ & 5.456241 & -2.240310 & 1.513643 \\
\hline $\mathrm{C}$ & 4.197070 & 0.356072 & 2.484547 \\
\hline $\mathrm{C}$ & 2.543110 & -2.249544 & 2.419783 \\
\hline $\mathrm{C}$ & 5.432168 & -0.073851 & -1.934521 \\
\hline $\mathrm{C}$ & 4.484554 & -2.985501 & -1.798163 \\
\hline $\mathrm{C}$ & 2.636873 & -0.891573 & -2.950482 \\
\hline $\mathrm{C}$ & -5.352599 & -0.285502 & -1.393191 \\
\hline $\mathrm{H}$ & -3.376706 & 0.442371 & -2.065517 \\
\hline $\mathrm{H}$ & -3.349772 & -1.186318 & -1.385566 \\
\hline $\mathrm{C}$ & -5.283330 & -0.808992 & 1.048378 \\
\hline $\mathrm{C}$ & -5.494035 & 1.507347 & 0.363534 \\
\hline $\mathrm{C}$ & -3.303446 & -4.100437 & 0.685699 \\
\hline $\mathrm{H}$ & -3.476468 & -4.711674 & -1.375788 \\
\hline $\mathrm{H}$ & -2.847263 & -3.281642 & 2.630926 \\
\hline $\mathrm{H}$ & 6.249441 & -1.767327 & 0.922519 \\
\hline $\mathrm{H}$ & 5.825998 & -2.340224 & 2.541787 \\
\hline $\mathrm{H}$ & 5.301164 & -3.249152 & 1.116389 \\
\hline $\mathrm{H}$ & 4.558362 & 0.130910 & 3.495616 \\
\hline $\mathrm{H}$ & 4.967641 & 0.958183 & 1.984347 \\
\hline $\mathrm{H}$ & 3.306485 & 0.984176 & 2.597733 \\
\hline $\mathrm{H}$ & 1.561025 & -1.757132 & 2.479911 \\
\hline $\mathrm{H}$ & 2.400891 & -3.234655 & 1.949869 \\
\hline
\end{tabular}




\begin{tabular}{llll}
$\mathrm{H}$ & 2.843637 & -2.440711 & 3.457087 \\
$\mathrm{H}$ & 6.204783 & -0.225334 & -1.170145 \\
$\mathrm{H}$ & 5.161088 & 0.989629 & -1.918415 \\
$\mathrm{H}$ & 5.884982 & -0.285419 & -2.911176 \\
$\mathrm{H}$ & 4.740380 & -3.193725 & -2.844811 \\
$\mathrm{H}$ & 3.681970 & -3.678936 & -1.512382 \\
$\mathrm{H}$ & 5.361994 & -3.225739 & -1.189518 \\
$\mathrm{H}$ & 3.088973 & -0.974487 & -3.946508 \\
$\mathrm{H}$ & 1.840282 & -1.645077 & -2.891582 \\
$\mathrm{H}$ & 2.161458 & 0.092657 & -2.888803 \\
$\mathrm{C}$ & -5.835969 & -1.282690 & -0.326459 \\
$\mathrm{C}$ & -5.937683 & 1.105210 & -1.077078 \\
$\mathrm{H}$ & -5.648689 & -0.611687 & -2.394805 \\
$\mathrm{H}$ & -4.579030 & -1.536046 & 1.456605 \\
$\mathrm{H}$ & -6.082836 & -0.671664 & 1.782491 \\
$\mathrm{H}$ & -4.986225 & 2.475893 & 0.377585 \\
$\mathrm{H}$ & -6.348239 & 1.579853 & 1.043082 \\
$\mathrm{H}$ & -4.081869 & -4.766000 & 1.045435 \\
$\mathrm{H}$ & -5.467350 & -2.289055 & -0.550299 \\
$\mathrm{H}$ & -6.931216 & -1.322238 & -0.320601 \\
$\mathrm{H}$ & -7.030229 & 1.080358 & -1.153914 \\
$\mathrm{H}$ & -5.579333 & 1.837155 & -1.810665 \\
& & & \\
\hline
\end{tabular}

\section{TS19d}

$\begin{array}{llll}\mathrm{Li} & -0.343207 & -0.932227 & 0.559623 \\ \mathrm{~N} & -2.179121 & -0.461815 & 0.068708 \\ \mathrm{O} & 1.251726 & -1.270645 & 1.333673 \\ \mathrm{Si} & -2.733343 & -1.905763 & -0.677391 \\ \mathrm{Si} & -3.098573 & 0.742416 & 0.880622 \\ \mathrm{Li} & -1.144268 & 0.684063 & -1.055285 \\ \mathrm{C} & 2.051176 & -0.933421 & 0.411946 \\ \mathrm{C} & -3.880869 & -2.969893 & 0.388269 \\ \mathrm{C} & -3.610897 & -1.560447 & -2.327528 \\ \mathrm{C} & -1.201083 & -2.968328 & -1.077773 \\ \mathrm{C} & -2.401855 & 1.074542 & 2.608210 \\ \mathrm{C} & -4.954009 & 0.396541 & 1.040001 \\ \mathrm{C} & -2.937924 & 2.362574 & -0.128552 \\ \mathrm{C} & 2.600527 & -2.030224 & -0.520518 \\ \mathrm{~N} & 3.058134 & 0.043209 & 0.748062 \\ \mathrm{H} & -3.442944 & -3.139509 & 1.379851 \\ \mathrm{H} & -4.052619 & -3.950255 & -0.073832 \\ \mathrm{H} & -4.857453 & -2.495973 & 0.533703\end{array}$




\begin{tabular}{|c|c|c|c|}
\hline $\mathrm{H}$ & -4.481075 & -0.909837 & -2.173507 \\
\hline $\mathrm{H}$ & -2.938930 & -1.047003 & -3.03047 \\
\hline $\mathrm{H}$ & -3.962061 & -2.475478 & -2.820775 \\
\hline $\mathrm{H}$ & -0.487213 & -2.435685 & -1.7202 \\
\hline $\mathrm{H}$ & -0.660300 & -3.285085 & -0.17436 \\
\hline $\mathrm{H}$ & -1.492750 & -3.883866 & -1.6065 \\
\hline $\mathrm{H}$ & -2.926829 & 1.889171 & 3.122389 \\
\hline $\mathrm{H}$ & -2.482843 & 0.175233 & 3.23168 \\
\hline $\mathrm{H}$ & -1.339813 & 1.343738 & 2.55615 \\
\hline $\mathrm{H}$ & -5.413909 & 0.185203 & 0.066863 \\
\hline $\mathrm{H}$ & -5.472077 & 1.262877 & 1.47015 \\
\hline $\mathrm{H}$ & -5.146352 & -0.462591 & 1.69228 \\
\hline $\mathrm{H}$ & -1.904935 & 2.732811 & -0.214567 \\
\hline $\mathrm{H}$ & -3.349798 & 2.236884 & -1.141436 \\
\hline $\mathrm{H}$ & -3.502501 & 3.177424 & 0.341588 \\
\hline $\mathrm{C}$ & 4.127954 & -2.073306 & -0.253971 \\
\hline $\mathrm{H}$ & 2.107571 & -2.968311 & -0.251257 \\
\hline $\mathrm{H}$ & 2.418325 & -1.844161 & -1.583564 \\
\hline $\mathrm{C}$ & 3.922089 & 0.395821 & -0.392504 \\
\hline $\mathrm{C}$ & 3.898212 & -0.610903 & 1.786111 \\
\hline $\mathrm{C}$ & 4.740146 & -0.824829 & -0.905714 \\
\hline $\mathrm{C}$ & 4.388680 & -2.000912 & 1.267395 \\
\hline $\mathrm{H}$ & 4.556632 & -2.988934 & -0.674653 \\
\hline $\mathrm{H}$ & 3.288760 & 0.805455 & -1.179387 \\
\hline $\mathrm{H}$ & 4.578784 & 1.205532 & -0.060365 \\
\hline $\mathrm{H}$ & 3.314366 & -0.705635 & 2.700860 \\
\hline $\mathrm{H}$ & 4.738063 & 0.062480 & 1.983844 \\
\hline $\mathrm{H}$ & 5.798436 & -0.736279 & -0.633191 \\
\hline $\mathrm{H}$ & 4.692337 & -0.894478 & -1.998997 \\
\hline $\mathrm{H}$ & 3.851597 & -2.809292 & 1.775966 \\
\hline $\mathrm{H}$ & 5.456890 & -2.138465 & 1.470965 \\
\hline $\mathrm{N}$ & 0.842748 & 0.151232 & -0.891615 \\
\hline $\mathrm{C}$ & 0.915127 & 1.544725 & -0.760966 \\
\hline $\mathrm{H}$ & 1.123665 & -0.121713 & -1.834013 \\
\hline $\mathrm{C}$ & 1.001240 & 2.417153 & -1.871746 \\
\hline $\mathrm{C}$ & 0.793049 & 2.122265 & 0.524901 \\
\hline $\mathrm{C}$ & 0.945905 & 3.801501 & -1.702256 \\
\hline $\mathrm{H}$ & 1.120628 & 1.994150 & -2.868678 \\
\hline $\mathrm{C}$ & 0.743626 & 3.504517 & 0.679076 \\
\hline $\mathrm{H}$ & 0.765392 & 1.466360 & 1.387791 \\
\hline $\mathrm{C}$ & 0.812097 & 4.356217 & -0.428203 \\
\hline $\mathrm{H}$ & 1.011568 & 4.447227 & -2.574016 \\
\hline
\end{tabular}


$\begin{array}{llll}\mathrm{H} & 0.649951 & 3.921626 & 1.678127\end{array}$

$\begin{array}{lllll}\mathrm{H} & 0.765394 & 5.432942 & -0.299074\end{array}$

\section{TS19t}

$\mathrm{Si} \quad 1.100239 \quad 2.750585-1.495181$

$\begin{array}{llll}\mathrm{N} & 0.977158 & 1.790674 & -0.053589\end{array}$

C $\quad 1.329790 \quad 4.613852 \quad-1.268545$

Si $\quad \begin{array}{llll}1.174367 & 2.466043 & 1.542359\end{array}$

$\begin{array}{llll}\mathrm{Li} & 2.580690 & 0.738330 & -0.684214\end{array}$

$\mathrm{Li} \quad-1.018642 \quad 1.343390 \quad-0.205405$

$\begin{array}{lllll}\text { C } & -0.399477 & 2.529812 & -2.629546\end{array}$

$\begin{array}{lllll}\text { C } & 2.643573 & 2.161305 & -2.479662\end{array}$

$\begin{array}{llll}\mathrm{H} & 0.573982 & 5.037962 & -0.597728\end{array}$

$\mathrm{H} \quad 1.227000 \quad 5.115748 \quad-2.238648$

$\begin{array}{lllll}\mathrm{H} & 2.314944 & 4.866787 & -0.865080\end{array}$

$\begin{array}{llll}\text { C } & 1.459057 & 1.073601 & 2.794791\end{array}$

$\begin{array}{llll}\text { C } & -0.368649 & 3.428774 & 2.074839\end{array}$

$\begin{array}{llll}\text { C } & 2.671535 & 3.621588 & 1.723320\end{array}$

N $\quad \begin{array}{llll}2.263553 & -1.142453 & -0.590689\end{array}$

$\mathrm{Li} \quad 0.706693 \quad-0.300071 \quad 0.277866$

$\begin{array}{lllll}\text { O } & -2.813595 & 1.551004 & -0.434826\end{array}$

$\begin{array}{llll}\mathrm{H} & -0.239237 & 3.090694 & -3.559010\end{array}$

$\begin{array}{llll}\mathrm{H} & -1.322025 & 2.910059 & -2.173720\end{array}$

$\begin{array}{llll}\mathrm{H} & -0.574077 & 1.485452 & -2.906819\end{array}$

$\mathrm{H} \quad 2.617126 \quad 1.107552-2.793632$

$\begin{array}{llll}\mathrm{H} & 3.584960 & 2.341706 & -1.934651\end{array}$

$\begin{array}{llll}\mathrm{H} & 2.721621 & 2.743409 & -3.404843\end{array}$

$\begin{array}{llll}\mathrm{H} & 2.461221 & 0.640955 & 2.715129\end{array}$

$\begin{array}{llll}\mathrm{H} & 1.367193 & 1.484393 & 3.808191\end{array}$

$\begin{array}{llll}\mathrm{H} & 0.726856 & 0.262506 & 2.733832\end{array}$

$\begin{array}{llll}\mathrm{H} & -0.245217 & 3.848549 & 3.081004\end{array}$

$\begin{array}{llll}\mathrm{H} & -0.574287 & 4.261322 & 1.391149\end{array}$

$\begin{array}{llll}\mathrm{H} & -1.262249 & 2.793657 & 2.097282\end{array}$

$\begin{array}{llll}\mathrm{H} & 2.936786 & 3.723087 & 2.783060\end{array}$

$\begin{array}{llll}\mathrm{H} & 3.556333 & 3.230519 & 1.203929\end{array}$

$\begin{array}{llll}\mathrm{H} & 2.476916 & 4.626072 & 1.335433\end{array}$

$\begin{array}{lllll}\mathrm{Si} & 1.759520 & -2.064381 & -1.953778\end{array}$

$\begin{array}{lllll}\mathrm{Si} & & 3.563701 & -1.579767 & 0.449541\end{array}$

$\begin{array}{lllll}\text { C } & -3.131473 & 0.332734 & -0.528692\end{array}$

$\begin{array}{lllll}\text { C } & 3.132259 & -2.426110 & -3.214342\end{array}$

$\begin{array}{lllll}\text { C } & 0.446350 & -1.098719 & -2.930836\end{array}$

$\begin{array}{llll}\text { C } & 0.983645 & -3.727752 & -1.463701\end{array}$ 


\begin{tabular}{|c|c|c|c|}
\hline $\mathrm{C}$ & 2.984492 & -2.301284 & 2.100623 \\
\hline $\mathrm{C}$ & 4.826587 & -2.807818 & -0.247213 \\
\hline $\mathrm{C}$ & 4.555841 & 0.030338 & 0.771535 \\
\hline $\mathrm{C}$ & -3.275520 & -0.303001 & -1.921096 \\
\hline $\mathrm{N}$ & -4.097132 & -0.175957 & 0.404799 \\
\hline $\mathrm{H}$ & 2.702968 & -2.809568 & -4.148931 \\
\hline $\mathrm{H}$ & 3.688810 & -1.511215 & -3.457393 \\
\hline $\mathrm{H}$ & 3.854665 & -3.164841 & -2.853852 \\
\hline $\mathrm{H}$ & 0.897714 & -0.275861 & -3.497303 \\
\hline $\mathrm{H}$ & -0.057553 & -1.750868 & -3.655334 \\
\hline $\mathrm{H}$ & -0.323458 & -0.665248 & -2.285844 \\
\hline $\mathrm{H}$ & 1.666839 & -4.312348 & -0.834725 \\
\hline $\mathrm{H}$ & 0.058303 & -3.586358 & -0.890203 \\
\hline $\mathrm{H}$ & 0.736256 & -4.337533 & -2.341840 \\
\hline $\mathrm{H}$ & 3.819925 & -2.443222 & 2.798013 \\
\hline $\mathrm{H}$ & 2.528091 & -3.283882 & 1.924677 \\
\hline $\mathrm{H}$ & 2.230512 & -1.683578 & 2.596666 \\
\hline $\mathrm{H}$ & 4.359825 & -3.766658 & -0.502210 \\
\hline $\mathrm{H}$ & 5.605101 & -3.011161 & 0.499123 \\
\hline $\mathrm{H}$ & 5.318359 & -2.426760 & -1.148770 \\
\hline $\mathrm{H}$ & 3.954712 & 0.868911 & 1.149574 \\
\hline $\mathrm{H}$ & 5.064819 & 0.359041 & -0.148004 \\
\hline $\mathrm{H}$ & 5.340436 & -0.130103 & 1.520583 \\
\hline $\mathrm{C}$ & -4.751400 & -0.770675 & -2.012602 \\
\hline $\mathrm{H}$ & -3.037806 & 0.465998 & -2.661773 \\
\hline $\mathrm{H}$ & -2.608778 & -1.152489 & -2.088812 \\
\hline $\mathrm{C}$ & -4.312343 & -1.628546 & 0.275445 \\
\hline $\mathrm{C}$ & -5.365719 & 0.521519 & 0.062267 \\
\hline $\mathrm{C}$ & -4.892153 & -2.010997 & -1.118056 \\
\hline $\mathrm{C}$ & -5.676922 & 0.334864 & -1.457626 \\
\hline $\mathrm{H}$ & -5.007098 & -1.001858 & -3.051851 \\
\hline $\mathrm{H}$ & -3.357852 & -2.122219 & 0.457321 \\
\hline $\mathrm{H}$ & -4.986317 & -1.925437 & 1.084303 \\
\hline $\mathrm{H}$ & -5.269783 & 1.573805 & 0.328811 \\
\hline $\mathrm{H}$ & -6.146723 & 0.080471 & 0.689245 \\
\hline $\mathrm{H}$ & -5.945594 & -2.305735 & -1.043151 \\
\hline $\mathrm{H}$ & -4.347479 & -2.860931 & -1.545808 \\
\hline $\mathrm{H}$ & -5.505767 & 1.271163 & -2.001142 \\
\hline $\mathrm{H}$ & -6.726231 & 0.056857 & -1.609155 \\
\hline $\mathrm{N}$ & -1.323637 & -0.625403 & 0.164566 \\
\hline $\mathrm{C}$ & -1.334339 & -1.001901 & 1.518439 \\
\hline $\mathrm{H}$ & -1.203383 & -1.456282 & -0.414738 \\
\hline
\end{tabular}




$\begin{array}{llll}\mathrm{C} & -0.764817 & -2.211876 & 1.970854 \\ \mathrm{C} & -1.858977 & -0.116609 & 2.485601 \\ \mathrm{C} & -0.698193 & -2.507478 & 3.332878 \\ \mathrm{H} & -0.349758 & -2.906679 & 1.244099 \\ \mathrm{C} & -1.792690 & -0.424992 & 3.840113 \\ \mathrm{H} & -2.339348 & 0.795022 & 2.151262 \\ \mathrm{C} & -1.206323 & -1.616494 & 4.278933 \\ \mathrm{H} & -0.237681 & -3.438894 & 3.651576 \\ \mathrm{H} & -2.204944 & 0.274398 & 4.562972 \\ \mathrm{H} & -1.151078 & -1.846900 & 5.338482\end{array}$

20d

$\mathrm{Li} \quad-0.427124 \quad-0.388260 \quad-0.666420$

$\begin{array}{llll}\mathrm{N} & -2.177950 & -0.566593 & 0.206102\end{array}$

$\begin{array}{lllll}\text { O } & 0.731347 & -0.963929 & 0.728836\end{array}$

$\begin{array}{lllll}\mathrm{Si} & & -2.879643 & -1.681699 & -0.886191\end{array}$

$\begin{array}{llll}\mathrm{Si} & -2.940146 & 0.716922 & 1.047635\end{array}$

$\begin{array}{llll}\mathrm{Li} & -0.807211 & -1.029208 & 1.522370\end{array}$

$\begin{array}{llll}\text { C } & 1.871656 & -0.725700 & 0.032527\end{array}$

$\begin{array}{llll}\text { C } & -4.225716 & -0.950298 & -2.002924\end{array}$

C $\quad-3.579228-3.218265-0.025155$

$\begin{array}{lllll}\text { C } & -1.478848 & -2.277445 & -2.047727\end{array}$

$\begin{array}{llll}\text { C } & -2.919953 & 2.349187 & 0.091246\end{array}$

$\begin{array}{llll}\text { C } & -4.721864 & 0.353996 & 1.587129\end{array}$

$\begin{array}{llll}\text { C } & -1.939681 & 0.978920 & 2.660725\end{array}$

$\begin{array}{llll}\text { C } & 2.427533 & -2.029814 & -0.641803\end{array}$

$\begin{array}{llll}\mathrm{N} & 2.920916 & -0.138158 & 0.907206\end{array}$

$\mathrm{N} \quad \begin{array}{llll}1.533571 & 0.243728 & -1.074227\end{array}$

$\begin{array}{lllll}\mathrm{H} & -3.854326 & -0.055407 & -2.518283\end{array}$

H $\quad \begin{array}{llll}-4.549749 & -1.667544 & -2.767412\end{array}$

$\begin{array}{llll}\mathrm{H} & -5.112789 & -0.655157 & -1.430467\end{array}$

$\mathrm{H} \quad-4.386271 \quad-2.936264 \quad 0.662472$

$\mathrm{H} \quad \begin{array}{llll}\mathrm{H} & -2.801905 & -3.719542 & 0.567033\end{array}$

$\begin{array}{llll}\mathrm{H} & -3.983798 & -3.952503 & -0.732996\end{array}$

$\mathrm{H} \quad-\quad-0.634430 \quad-2.710298 \quad-1.493305$

$\begin{array}{lllll}\mathrm{H} & & -1.089944 & -1.469721 & -2.688913\end{array}$

$\begin{array}{lllll}\mathrm{H} & & -1.842917 & -3.054964 & -2.729932\end{array}$

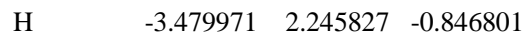

$\mathrm{H} \quad \begin{array}{llll}\mathrm{H} & -3.372498 & 3.171383 & 0.660262\end{array}$

$\mathrm{H} \quad \begin{array}{lllll}-1.893896 & 2.641984 & -0.161391\end{array}$

$\mathrm{H} \quad \begin{array}{llll}4.776181 & -0.580453 & 2.159827\end{array}$

$\begin{array}{llll}\mathrm{H} & -5.121965 & 1.157270 & 2.218615\end{array}$ 


\begin{tabular}{|c|c|c|c|}
\hline $\mathrm{H}$ & -5.390728 & 0.250276 & 0.724702 \\
\hline $\mathrm{H}$ & -0.883817 & 1.213132 & 2.467470 \\
\hline $\mathrm{H}$ & -1.989957 & 0.101062 & 3.326717 \\
\hline $\mathrm{H}$ & -2.341597 & 1.817866 & 3.241143 \\
\hline $\mathrm{C}$ & 3.883811 & -2.257061 & -0.206479 \\
\hline $\mathrm{H}$ & 1.792640 & -2.858374 & -0.314208 \\
\hline $\mathrm{H}$ & 2.357583 & -1.982002 & -1.737284 \\
\hline $\mathrm{C}$ & 4.131710 & 0.204583 & 0.145315 \\
\hline $\mathrm{C}$ & 3.276215 & -1.118881 & 1.945481 \\
\hline $\mathrm{C}$ & 1.356572 & 1.635075 & -0.836486 \\
\hline $\mathrm{H}$ & 2.059999 & 0.076430 & -1.924095 \\
\hline $\mathrm{C}$ & 4.712342 & -1.026388 & -0.613053 \\
\hline $\mathrm{C}$ & 3.920277 & -2.398027 & 1.327393 \\
\hline $\mathrm{H}$ & 4.282712 & -3.160557 & -0.681015 \\
\hline $\mathrm{H}$ & 3.899829 & 1.027092 & -0.535871 \\
\hline $\mathrm{H}$ & 4.857844 & 0.593129 & 0.866813 \\
\hline $\mathrm{H}$ & 2.364325 & -1.362125 & 2.492014 \\
\hline $\mathrm{H}$ & 3.963620 & -0.626820 & 2.641917 \\
\hline $\mathrm{C}$ & 1.413447 & 2.513619 & -1.930220 \\
\hline $\mathrm{C}$ & 1.038779 & 2.145679 & 0.432045 \\
\hline $\mathrm{H}$ & 5.769041 & -1.178843 & -0.363817 \\
\hline $\mathrm{H}$ & 4.660026 & -0.883980 & -1.700756 \\
\hline $\mathrm{H}$ & 4.956745 & -2.522700 & 1.664152 \\
\hline $\mathrm{H}$ & 3.371764 & -3.297121 & 1.632595 \\
\hline $\mathrm{C}$ & 1.150619 & 3.871943 & -1.762865 \\
\hline $\mathrm{H}$ & 1.660966 & 2.125020 & -2.916315 \\
\hline $\mathrm{C}$ & 0.777256 & 3.506963 & 0.585801 \\
\hline $\mathrm{H}$ & 1.030269 & 1.476251 & 1.279694 \\
\hline $\mathrm{C}$ & 0.825734 & 4.378640 & -0.503148 \\
\hline $\mathrm{H}$ & 1.200498 & 4.533728 & -2.623062 \\
\hline $\mathrm{H}$ & 0.532050 & 3.885506 & 1.574306 \\
\hline $\mathrm{H}$ & 0.616396 & 5.435916 & -0.372946 \\
\hline \multicolumn{4}{|c|}{$20 t$} \\
\hline $\mathrm{Li}$ & -0.975076 & 0.618154 & -0.527387 \\
\hline $\mathrm{N}$ & 0.032504 & 2.102009 & 0.229405 \\
\hline $\mathrm{N}$ & -2.266400 & -0.828813 & 0.413931 \\
\hline $\mathrm{Si}$ & -0.551321 & 2.403868 & 1.823383 \\
\hline $\mathrm{Si}$ & 0.057767 & 3.213334 & -1.086027 \\
\hline $\mathrm{Li}$ & 1.831449 & 1.424771 & 0.091776 \\
\hline $\mathrm{Li}$ & 0.925574 & -1.137853 & -0.078590 \\
\hline $\mathrm{C}$ & -3.487772 & -0.370426 & -0.125493 \\
\hline
\end{tabular}




\begin{tabular}{|c|c|c|c|}
\hline $\mathrm{H}$ & -2.341237 & -1.009682 & 1.405430 \\
\hline $\mathrm{C}$ & 0.338830 & 3.832477 & 2.702443 \\
\hline $\mathrm{C}$ & -2.406304 & 2.802684 & 1.860941 \\
\hline $\mathrm{C}$ & -0.275605 & 0.848590 & 2.885661 \\
\hline $\mathrm{C}$ & -0.691414 & 4.923687 & -0.779036 \\
\hline $\mathrm{C}$ & -0.875667 & 2.446125 & -2.554781 \\
\hline $\mathrm{C}$ & 1.871875 & 3.496139 & -1.605885 \\
\hline $\mathrm{N}$ & 2.664699 & -0.292847 & -0.151238 \\
\hline $\mathrm{O}$ & -0.604510 & -1.086042 & -1.182000 \\
\hline $\mathrm{C}$ & -4.561246 & -0.082498 & 0.732140 \\
\hline $\mathrm{C}$ & -3.624535 & -0.102229 & -1.500644 \\
\hline $\mathrm{H}$ & 1.426415 & 3.686576 & 2.711629 \\
\hline $\mathrm{H}$ & 0.009758 & 3.921522 & 3.745757 \\
\hline $\mathrm{H}$ & 0.138966 & 4.792087 & 2.211778 \\
\hline $\mathrm{H}$ & -2.779006 & 2.875233 & 2.890937 \\
\hline $\mathrm{H}$ & -2.999275 & 2.037819 & 1.345965 \\
\hline $\mathrm{H}$ & -2.613836 & 3.760770 & 1.369544 \\
\hline $\mathrm{H}$ & 0.758550 & 0.770417 & 3.237992 \\
\hline $\mathrm{H}$ & -0.920097 & 0.857953 & 3.773864 \\
\hline $\mathrm{H}$ & -0.491756 & -0.064582 & 2.320249 \\
\hline $\mathrm{H}$ & -0.624451 & 5.538571 & -1.685310 \\
\hline $\mathrm{H}$ & -0.172159 & 5.458137 & 0.024221 \\
\hline $\mathrm{H}$ & -1.749944 & 4.853479 & -0.504075 \\
\hline $\mathrm{H}$ & -1.947997 & 2.359082 & -2.328598 \\
\hline $\mathrm{H}$ & -0.787269 & 3.064313 & -3.456684 \\
\hline $\mathrm{H}$ & -0.501665 & 1.447234 & -2.815560 \\
\hline $\mathrm{H}$ & 2.412802 & 2.571689 & -1.850988 \\
\hline $\mathrm{H}$ & 2.423406 & 4.009922 & -0.805518 \\
\hline $\mathrm{H}$ & 1.936985 & 4.127857 & -2.500317 \\
\hline $\mathrm{Si}$ & 3.519970 & -0.455401 & 1.334453 \\
\hline $\mathrm{Si}$ & 3.305687 & -0.609692 & -1.725578 \\
\hline $\mathrm{C}$ & -1.394697 & -1.803763 & -0.342089 \\
\hline $\mathrm{C}$ & -5.735872 & 0.476155 & 0.233100 \\
\hline $\mathrm{H}$ & -4.464858 & -0.289259 & 1.795644 \\
\hline $\mathrm{C}$ & -4.810328 & 0.447462 & -1.988895 \\
\hline $\mathrm{H}$ & -2.804333 & -0.330751 & -2.173801 \\
\hline $\mathrm{C}$ & 5.326779 & -1.013733 & 1.225430 \\
\hline $\mathrm{C}$ & 2.635821 & -1.681203 & 2.484952 \\
\hline $\mathrm{C}$ & 3.556416 & 1.251261 & 2.190012 \\
\hline $\mathrm{C}$ & 3.766148 & -2.439045 & -1.958277 \\
\hline $\mathrm{C}$ & 1.992882 & -0.215845 & -3.034408 \\
\hline $\mathrm{C}$ & 4.838959 & 0.430218 & -2.140183 \\
\hline
\end{tabular}




\begin{tabular}{|c|c|c|c|}
\hline $\mathrm{C}$ & -2.224151 & -2.938285 & -1.016324 \\
\hline $\mathrm{N}$ & -0.483152 & -2.444963 & 0.703394 \\
\hline $\mathrm{C}$ & -5.869902 & 0.745852 & -1.130496 \\
\hline $\mathrm{H}$ & -6.551341 & 0.696697 & 0.916113 \\
\hline $\mathrm{H}$ & -4.899803 & 0.644345 & -3.053595 \\
\hline $\mathrm{H}$ & 5.750379 & -1.119199 & 2.232286 \\
\hline $\mathrm{H}$ & 5.426440 & -1.982429 & 0.723095 \\
\hline $\mathrm{H}$ & 5.944131 & -0.291033 & 0.680881 \\
\hline $\mathrm{H}$ & 3.059661 & -1.670430 & 3.496912 \\
\hline $\mathrm{H}$ & 2.740046 & -2.702407 & 2.094667 \\
\hline $\mathrm{H}$ & 1.564398 & -1.467128 & 2.569888 \\
\hline $\mathrm{H}$ & 2.563054 & 1.694931 & 2.338300 \\
\hline $\mathrm{H}$ & 4.156297 & 1.958369 & 1.599098 \\
\hline $\mathrm{H}$ & 4.013848 & 1.193505 & 3.185548 \\
\hline $\mathrm{H}$ & 2.888205 & -3.081294 & -1.807626 \\
\hline $\mathrm{H}$ & 4.535552 & -2.761847 & -1.247381 \\
\hline $\mathrm{H}$ & 4.144882 & -2.633338 & -2.969633 \\
\hline $\mathrm{H}$ & 1.040747 & -0.708733 & -2.807347 \\
\hline $\mathrm{H}$ & 2.326557 & -0.553670 & -4.023864 \\
\hline $\mathrm{H}$ & 1.797139 & 0.860016 & -3.111079 \\
\hline $\mathrm{H}$ & 5.128699 & 0.301362 & -3.190769 \\
\hline $\mathrm{H}$ & 4.644600 & 1.499061 & -1.981114 \\
\hline $\mathrm{H}$ & 5.701388 & 0.155735 & -1.523215 \\
\hline $\mathrm{C}$ & -2.120754 & -4.227420 & -0.178772 \\
\hline $\mathrm{H}$ & -1.818821 & -3.101038 & -2.020002 \\
\hline $\mathrm{H}$ & -3.269094 & -2.640478 & -1.132496 \\
\hline $\mathrm{C}$ & -1.223755 & -3.050026 & 1.831489 \\
\hline $\mathrm{C}$ & 0.255864 & -3.555783 & 0.033871 \\
\hline $\mathrm{H}$ & -6.786953 & 1.178619 & -1.517761 \\
\hline $\mathrm{C}$ & -2.406698 & -3.901941 & 1.301395 \\
\hline $\mathrm{C}$ & -0.679858 & -4.750599 & -0.285204 \\
\hline $\mathrm{H}$ & -2.832050 & -4.974033 & -0.546410 \\
\hline $\mathrm{H}$ & -1.541354 & -2.278257 & 2.539583 \\
\hline $\mathrm{H}$ & -0.507723 & -3.669475 & 2.380548 \\
\hline $\mathrm{H}$ & 0.688084 & -3.163183 & -0.890868 \\
\hline $\mathrm{H}$ & 1.080879 & -3.845323 & 0.691916 \\
\hline $\mathrm{H}$ & -3.354679 & -3.356864 & 1.385739 \\
\hline $\mathrm{H}$ & -2.507809 & -4.821084 & 1.889162 \\
\hline $\mathrm{H}$ & -0.517888 & -5.577731 & 0.416243 \\
\hline $\mathrm{H}$ & -0.479353 & -5.133265 & -1.291736 \\
\hline
\end{tabular}




\begin{tabular}{|c|c|c|c|}
\hline $\mathrm{Li}$ & 0.680557 & -0.361342 & -0.166849 \\
\hline $\mathrm{N}$ & 2.587281 & -0.164129 & 0.432576 \\
\hline $\mathrm{N}$ & -0.229334 & -0.846041 & 1.600277 \\
\hline $\mathrm{Si}$ & 3.139228 & -1.736674 & 0.036556 \\
\hline $\mathrm{Si}$ & 3.267354 & 1.354715 & 0.019844 \\
\hline $\mathrm{Li}$ & 1.549636 & -0.263445 & 2.040620 \\
\hline $\mathrm{O}$ & -0.352517 & 0.628172 & -1.380688 \\
\hline $\mathrm{C}$ & -1.432099 & -0.192910 & 1.799665 \\
\hline $\mathrm{H}$ & -0.378081 & -1.850616 & 1.713004 \\
\hline $\mathrm{C}$ & 4.797286 & -1.846398 & -0.870194 \\
\hline $\mathrm{C}$ & 1.832586 & -2.593430 & -1.067434 \\
\hline $\mathrm{C}$ & 3.252916 & -2.791145 & 1.615874 \\
\hline $\mathrm{C}$ & 3.078160 & 1.748848 & -1.827094 \\
\hline $\mathrm{C}$ & 2.311727 & 2.696696 & 0.974558 \\
\hline $\mathrm{C}$ & 5.096848 & 1.543386 & 0.482166 \\
\hline $\mathrm{C}$ & -1.566884 & 0.850375 & -1.424164 \\
\hline $\mathrm{C}$ & -2.653024 & -0.858169 & 2.073550 \\
\hline $\mathrm{C}$ & -1.493637 & 1.220966 & 1.692704 \\
\hline $\mathrm{H}$ & 5.620145 & -1.490898 & -0.239916 \\
\hline $\mathrm{H}$ & 5.019893 & -2.881526 & -1.157973 \\
\hline $\mathrm{H}$ & 4.793736 & -1.240424 & -1.784157 \\
\hline $\mathrm{H}$ & 0.866692 & -2.700132 & -0.550631 \\
\hline $\mathrm{H}$ & 1.664236 & -2.034030 & -1.998409 \\
\hline $\mathrm{H}$ & 2.144662 & -3.606553 & -1.349417 \\
\hline $\mathrm{H}$ & 2.289959 & -2.819160 & 2.148002 \\
\hline $\mathrm{H}$ & 4.003318 & -2.379715 & 2.303613 \\
\hline $\mathrm{H}$ & 3.528944 & -3.832140 & 1.406623 \\
\hline $\mathrm{H}$ & 2.023789 & 1.669784 & -2.119702 \\
\hline $\mathrm{H}$ & 3.430022 & 2.757154 & -2.080320 \\
\hline $\mathrm{H}$ & 3.644220 & 1.036900 & -2.441185 \\
\hline $\mathrm{H}$ & 1.249867 & 2.695043 & 0.698992 \\
\hline $\mathrm{H}$ & 2.702533 & 3.700709 & 0.768568 \\
\hline $\mathrm{H}$ & 2.372137 & 2.547922 & 2.062696 \\
\hline $\mathrm{H}$ & 5.450520 & 2.568268 & 0.311626 \\
\hline $\mathrm{H}$ & 5.731499 & 0.872256 & -0.107247 \\
\hline $\mathrm{H}$ & 5.259780 & 1.306136 & 1.541288 \\
\hline $\mathrm{C}$ & -2.602693 & -0.193759 & -1.327208 \\
\hline $\mathrm{O}$ & -2.047091 & 2.080269 & -1.598959 \\
\hline $\mathrm{C}$ & -3.845955 & -0.159112 & 2.210162 \\
\hline $\mathrm{H}$ & -2.647811 & -1.943085 & 2.157188 \\
\hline $\mathrm{C}$ & -2.694006 & 1.913478 & 1.829486 \\
\hline $\mathrm{H}$ & -0.571784 & 1.766243 & 1.500594 \\
\hline
\end{tabular}




$\begin{array}{llll}\mathrm{C} & -3.952693 & 0.133314 & -1.146012 \\ \mathrm{C} & -2.206413 & -1.538331 & -1.389144 \\ \mathrm{C} & -1.083405 & 3.147946 & -1.593953 \\ \mathrm{C} & -3.887848 & 1.234353 & 2.083119 \\ \mathrm{H} & -4.761946 & -0.711152 & 2.407327 \\ \mathrm{H} & -2.694279 & 2.997447 & 1.736633 \\ \mathrm{C} & -4.896386 & -0.881927 & -1.017175 \\ \mathrm{H} & -4.245996 & 1.172802 & -1.076217 \\ \mathrm{C} & -3.153596 & -2.547029 & -1.259923 \\ \mathrm{H} & -1.161404 & -1.780582 & -1.546002 \\ \mathrm{H} & -0.328057 & 2.987454 & -2.365920 \\ \mathrm{H} & -0.598943 & 3.204881 & -0.616638 \\ \mathrm{H} & -1.657104 & 4.052682 & -1.791676 \\ \mathrm{H} & -4.823663 & 1.774483 & 2.189955 \\ \mathrm{C} & -4.499572 & -2.218818 & -1.070637 \\ \mathrm{H} & -5.940601 & -0.629370 & -0.860714 \\ \mathrm{H} & -2.846731 & -3.587526 & -1.308570 \\ \mathrm{H} & -5.238894 & -3.007812 & -0.965214\end{array}$

21t

Li $\quad 0.573689 \quad 0.719941 \quad 0.203228$

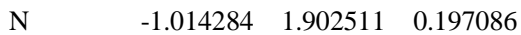

$\mathrm{N} \quad 0.674410-1.274665 \quad 0.373072$

$\begin{array}{llll}\mathrm{Si} & -1.177835 & 2.575783 & 1.773417\end{array}$

$\begin{array}{llll}\mathrm{Si} & -0.968732 & 2.834863 & -1.253707\end{array}$

$\begin{array}{lllll}\mathrm{Li} & & -2.443993 & 0.612263 & 0.217942\end{array}$

$\begin{array}{lllll}\text { O } & 2.251913 & 1.409625 & -0.391929\end{array}$

C $\quad 1.747751-1.877391-0.269685$

$\mathrm{Li} \quad-1.053720-1.688326-0.318433$

$\mathrm{H} \quad 0.698848 \quad-1.528809 \quad 1.362921$

$\begin{array}{llll}\text { C } & -0.859020 & 4.434749 & 1.937950\end{array}$

$\begin{array}{llll}\text { C } & 0.029372 & 1.709665 & 2.960103\end{array}$

$\begin{array}{llll}\text { C } & -2.957552 & 2.286834 & 2.400643\end{array}$

$\begin{array}{llll}\text { C } & 0.401040 & 4.146507 & -1.304319\end{array}$

$\begin{array}{llll}\text { C } & -0.638192 & 1.649093 & -2.700735\end{array}$

$\begin{array}{lllll}\text { C } & & -2.609226 & 3.738570 & -1.578503\end{array}$

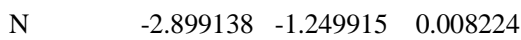

$\begin{array}{lllll}\text { C } & 3.393078 & 0.970649 & -0.547413\end{array}$

$\begin{array}{llll}\text { C } & 2.664565 & -2.744590 & 0.373212\end{array}$

C $\quad \begin{array}{llll}\text { C } & 1.974716 & -1.618658 & -1.645112\end{array}$

$\begin{array}{llll}\mathrm{H} & -1.518059 & 5.023957 & 1.289780\end{array}$

$\begin{array}{llll}\mathrm{H} & -1.030333 & 4.762794 & 2.970990\end{array}$ 


\begin{tabular}{|c|c|c|c|}
\hline $\mathrm{H}$ & 0.175139 & 4.683545 & 1.674971 \\
\hline $\mathrm{H}$ & -0.005283 & 0.617204 & 2.863341 \\
\hline $\mathrm{H}$ & 1.056852 & 2.036110 & 2.750466 \\
\hline $\mathrm{H}$ & -0.179524 & 1.951108 & 4.009492 \\
\hline $\mathrm{H}$ & -3.271639 & 1.234297 & 2.397392 \\
\hline $\mathrm{H}$ & -3.666364 & 2.846411 & 1.774663 \\
\hline $\mathrm{H}$ & -3.084062 & 2.636776 & 3.432823 \\
\hline $\mathrm{H}$ & 1.345298 & 3.717251 & -0.952731 \\
\hline $\mathrm{H}$ & 0.550702 & 4.516355 & -2.327279 \\
\hline $\mathrm{H}$ & 0.166472 & 5.010278 & -0.673587 \\
\hline $\mathrm{H}$ & 0.321817 & 1.138578 & -2.558229 \\
\hline $\mathrm{H}$ & -0.589449 & 2.182337 & -3.658065 \\
\hline $\mathrm{H}$ & -1.411394 & 0.879560 & -2.793650 \\
\hline $\mathrm{H}$ & -2.595289 & 4.289349 & -2.527502 \\
\hline $\mathrm{H}$ & -2.806879 & 4.464118 & -0.778961 \\
\hline $\mathrm{H}$ & -3.462115 & 3.049096 & -1.609761 \\
\hline $\mathrm{Si}$ & -3.558254 & -1.504969 & -1.562851 \\
\hline $\mathrm{Si}$ & -3.445462 & -1.970037 & 1.479652 \\
\hline $\mathrm{C}$ & 4.159525 & 0.264086 & 0.496292 \\
\hline $\mathrm{O}$ & 4.061840 & 1.127673 & -1.690363 \\
\hline $\mathrm{C}$ & 3.744437 & -3.291906 & -0.309654 \\
\hline $\mathrm{H}$ & 2.528090 & -2.956931 & 1.431403 \\
\hline $\mathrm{C}$ & 3.060832 & -2.171155 & -2.321719 \\
\hline $\mathrm{H}$ & 1.283120 & -0.961352 & -2.169531 \\
\hline $\mathrm{C}$ & -4.903466 & -2.827135 & -1.715380 \\
\hline $\mathrm{C}$ & -4.283219 & 0.104313 & -2.257955 \\
\hline $\mathrm{C}$ & -2.121443 & -2.055156 & -2.697105 \\
\hline $\mathrm{C}$ & -5.145907 & -1.323507 & 2.014828 \\
\hline $\mathrm{C}$ & -3.539207 & -3.864476 & 1.408287 \\
\hline $\mathrm{C}$ & -2.194769 & -1.570393 & 2.852112 \\
\hline $\mathrm{C}$ & 5.363289 & -0.388255 & 0.201003 \\
\hline $\mathrm{C}$ & 3.638347 & 0.223364 & 1.797147 \\
\hline $\mathrm{C}$ & 3.338489 & 1.719977 & -2.782089 \\
\hline $\mathrm{C}$ & 3.963551 & -3.008219 & -1.662717 \\
\hline $\mathrm{H}$ & 4.434307 & -3.939331 & 0.225997 \\
\hline $\mathrm{H}$ & 3.202338 & -1.940886 & -3.375380 \\
\hline $\mathrm{H}$ & -5.741553 & -2.635225 & -1.035141 \\
\hline $\mathrm{H}$ & -5.304362 & -2.844177 & -2.736590 \\
\hline $\mathrm{H}$ & -4.516082 & -3.827407 & -1.493583 \\
\hline $\mathrm{H}$ & -4.644407 & -0.021416 & -3.286219 \\
\hline $\mathrm{H}$ & -5.135029 & 0.428052 & -1.644934 \\
\hline $\mathrm{H}$ & -3.556557 & 0.924547 & -2.273673 \\
\hline
\end{tabular}




\begin{tabular}{llll}
$\mathrm{H}$ & -1.267533 & -1.362155 & -2.689838 \\
$\mathrm{H}$ & -1.749999 & -3.052005 & -2.414977 \\
$\mathrm{H}$ & -2.440839 & -2.127545 & -3.743813 \\
$\mathrm{H}$ & -5.904911 & -1.551548 & 1.256109 \\
$\mathrm{H}$ & -5.136124 & -0.234374 & 2.150062 \\
$\mathrm{H}$ & -5.475680 & -1.772176 & 2.960162 \\
$\mathrm{H}$ & -3.699963 & -4.285000 & 2.409021 \\
$\mathrm{H}$ & -2.603909 & -4.290263 & 1.019914 \\
$\mathrm{H}$ & -4.353893 & -4.216320 & 0.766963 \\
$\mathrm{H}$ & -2.526079 & -1.981977 & 3.813508 \\
$\mathrm{H}$ & -1.214975 & -2.012431 & 2.628280 \\
$\mathrm{H}$ & -2.043373 & -0.495185 & 2.992808 \\
$\mathrm{C}$ & 6.034301 & -1.083925 & 1.203088 \\
$\mathrm{H}$ & 5.745914 & -0.370561 & -0.811478 \\
$\mathrm{C}$ & 4.313661 & -0.470680 & 2.794220 \\
$\mathrm{H}$ & 2.705826 & 0.730863 & 2.012146 \\
$\mathrm{H}$ & 2.916645 & 2.683002 & -2.489062 \\
$\mathrm{H}$ & 2.535885 & 1.049997 & -3.097693 \\
$\mathrm{H}$ & 4.070847 & 1.840845 & -3.579651 \\
$\mathrm{H}$ & 4.810624 & -3.436341 & -2.189786 \\
$\mathrm{C}$ & 5.511610 & -1.127557 & 2.496742 \\
$\mathrm{H}$ & 6.960426 & -1.601446 & 0.972112 \\
$\mathrm{H}$ & 3.909270 & -0.500966 & 3.801602 \\
$\mathrm{H}$ & 6.036961 & -1.673927 & 3.275044 \\
& & & \\
\hline
\end{tabular}

\section{TS22d}

$\begin{array}{llll}\mathrm{N} & -2.589334 & -0.309792 & 0.064746\end{array}$

$\begin{array}{lllll}\text { C } & -4.586880 & -2.105484 & -1.393480\end{array}$

Si $\quad-3.554866 \quad 0.993510 \quad 0.614055$

Li $\quad-1.432974 \quad-1.247803 \quad 1.278918$

$\mathrm{Li} \quad-1.045450 \quad 0.756495 \quad-0.448510$

C $\quad-2.165458-0.873719-2.835090$

C $\quad-1.747420 \quad-3.001770-0.679750$

$\mathrm{H} \quad \begin{array}{llll}\mathrm{H} & -5.014961 & -2.445564 & -0.442544\end{array}$

$\begin{array}{lllll}\mathrm{H} & -4.619571 & -2.947975 & -2.095445\end{array}$

$\mathrm{H} \quad \begin{array}{llll}-5.239292 & -1.318908 & -1.787169\end{array}$

$\begin{array}{llll}\text { C } & -5.304554 & 1.090693 & -0.093438\end{array}$

$\begin{array}{llll}\text { C } & -3.651246 & 0.991043 & 2.506191\end{array}$

$\begin{array}{lllll}\text { C } & -2.674989 & 2.622521 & 0.105924\end{array}$

$\begin{array}{llll}\mathrm{O} & 0.240952 & -1.075412 & 1.768401\end{array}$

$\begin{array}{lllll}\mathrm{N} & 0.810004 & 0.265075 & -0.378684\end{array}$ 


\begin{tabular}{|c|c|c|c|}
\hline $\mathrm{H}$ & -2.732925 & 0.003926 & -3.172413 \\
\hline $\mathrm{H}$ & -1.107004 & -0.581535 & -2.774829 \\
\hline $\mathrm{H}$ & -2.243110 & -1.637135 & -3.619083 \\
\hline $\mathrm{H}$ & -0.684239 & -2.748929 & -0.564837 \\
\hline $\mathrm{H}$ & -2.087244 & -3.470891 & 0.256964 \\
\hline $\mathrm{H}$ & -1.803760 & -3.780525 & -1.449762 \\
\hline $\mathrm{H}$ & -5.287161 & 1.152796 & -1.188258 \\
\hline $\mathrm{H}$ & -5.832059 & 1.976279 & 0.281842 \\
\hline $\mathrm{H}$ & -5.894765 & 0.208538 & 0.178611 \\
\hline $\mathrm{H}$ & -4.182268 & 0.098837 & 2.862721 \\
\hline $\mathrm{H}$ & -2.648227 & 0.979989 & 2.953245 \\
\hline $\mathrm{H}$ & -4.176714 & 1.869271 & 2.900779 \\
\hline $\mathrm{H}$ & -2.604322 & 2.724939 & -0.987650 \\
\hline $\mathrm{H}$ & -1.661453 & 2.734155 & 0.523396 \\
\hline $\mathrm{H}$ & -3.232484 & 3.496259 & 0.464444 \\
\hline $\mathrm{C}$ & 1.273744 & -0.411465 & 1.427124 \\
\hline $\mathrm{C}$ & 1.738595 & 1.166434 & -0.909852 \\
\hline $\mathrm{H}$ & 0.922337 & -0.631860 & -0.854271 \\
\hline $\mathrm{C}$ & 2.531807 & -1.125550 & 1.039299 \\
\hline $\mathrm{O}$ & 1.561982 & 0.760916 & 2.082430 \\
\hline $\mathrm{C}$ & 2.731426 & 0.783200 & -1.834334 \\
\hline $\mathrm{C}$ & 1.721239 & 2.509853 & -0.481018 \\
\hline $\mathrm{C}$ & 2.464164 & -2.489125 & 0.728087 \\
\hline $\mathrm{C}$ & 3.753656 & -0.450835 & 0.924300 \\
\hline $\mathrm{C}$ & 0.458325 & 1.412462 & 2.684675 \\
\hline $\mathrm{C}$ & 3.651400 & 1.708136 & -2.322155 \\
\hline $\mathrm{H}$ & 2.778806 & -0.255028 & -2.154001 \\
\hline $\mathrm{C}$ & 2.650728 & 3.427675 & -0.963761 \\
\hline $\mathrm{H}$ & 0.977105 & 2.820711 & 0.247371 \\
\hline $\mathrm{C}$ & 3.602215 & -3.170389 & 0.302017 \\
\hline $\mathrm{H}$ & 1.515080 & -3.004060 & 0.836087 \\
\hline $\mathrm{C}$ & 4.891910 & -1.137531 & 0.501835 \\
\hline $\mathrm{H}$ & 3.803913 & 0.604764 & 1.160356 \\
\hline $\mathrm{H}$ & 0.007934 & 0.795608 & 3.468523 \\
\hline $\mathrm{H}$ & -0.313786 & 1.655127 & 1.943580 \\
\hline $\mathrm{H}$ & 0.849963 & 2.337859 & 3.110631 \\
\hline $\mathrm{C}$ & 3.621517 & 3.035989 & -1.889749 \\
\hline $\mathrm{H}$ & 4.404432 & 1.385210 & -3.036409 \\
\hline $\mathrm{H}$ & 2.616923 & 4.456471 & -0.614349 \\
\hline $\mathrm{C}$ & 4.820380 & -2.495034 & 0.186646 \\
\hline $\mathrm{H}$ & 3.541639 & -4.229482 & 0.066567 \\
\hline $\mathrm{H}$ & 5.835292 & -0.606310 & 0.412465 \\
\hline
\end{tabular}


$\mathrm{H} \quad 4.343143 \quad 3.754393 \quad-2.267109$

$\begin{array}{llll}\mathrm{H} & 5.708529 & -3.025827 & -0.145062\end{array}$

\section{TS22t}

N $\quad-1.386443-0.444996-0.350465$

$\begin{array}{lllll}\mathrm{Li} & 0.579796 & 0.205401 & -0.349268\end{array}$

$\mathrm{Li} \quad 0.220382 \quad-2.232012 \quad 0.253988$

N $\quad 2.029302-1.340679 \quad 0.044614$

$\begin{array}{llll}\mathrm{Li} & 2.723956 & 0.463724 & 0.604049\end{array}$

$\mathrm{N} \quad 1.380023 \quad 1.803478 \quad 0.470006$

Si $\quad \begin{array}{llll}2.495711 & -1.898077 & -1.537973\end{array}$

$\begin{array}{lllll}\mathrm{Si} & 2.647761 & -2.039495 & 1.510114\end{array}$

$\begin{array}{llll}\mathrm{Si} & 2.054396 & 2.862536 & -0.710660\end{array}$

$\begin{array}{llll}\mathrm{Si} & 0.601468 & 2.365317 & 1.903154\end{array}$

$\begin{array}{lllll}\text { C } & 1.665008 & -0.779351 & -2.821465\end{array}$

$\mathrm{H} \quad 0.590196 \quad-0.658835-2.644301$

$\begin{array}{llll}\mathrm{H} & 2.109872 & 0.220438 & -2.855493\end{array}$

$\mathrm{H} \quad \begin{array}{llll}1.767882 & -1.210951 & -3.824549\end{array}$

$\begin{array}{lllll}\text { C } & 1.904337 & -3.675264 & -1.841273\end{array}$

$\begin{array}{lllll}\mathrm{H} & 2.118001 & -3.991447 & -2.869904\end{array}$

$\mathrm{H} \quad 2.411256 \quad-4.379218-1.170355$

H $\quad \begin{array}{llll}0.824796 & -3.795994 & -1.687433\end{array}$

$\begin{array}{lllll}\text { C } & 4.359760 & -1.855709 & -1.873290\end{array}$

$\mathrm{H} \quad 4.883375 \quad-2.668117 \quad-1.359387$

$\mathrm{H} \quad 4.554328 \quad-1.966348 \quad-2.947411$

$\begin{array}{lllll}\mathrm{H} & 4.816016 & -0.909995 & -1.555763\end{array}$

$\begin{array}{llll}\text { C } & 3.340990 & -0.637704 & 2.608361\end{array}$

$\mathrm{H} \quad 3.688672 \quad-1.061949 \quad 3.557376$

$\begin{array}{llll}\mathrm{H} & 4.224969 & -0.142208 & 2.172017\end{array}$

$\begin{array}{llll}\mathrm{H} & 2.606287 & 0.133009 & 2.870099\end{array}$

$\begin{array}{llll}\text { C } & 4.053507 & -3.289739 & 1.333708\end{array}$

$\begin{array}{llll}\mathrm{H} & 4.303399 & -3.703678 & 2.318548\end{array}$

$\mathrm{H} \quad 3.790678 \quad-4.129859 \quad 0.681697$

$\mathrm{H} \quad 4.959376 \quad-2.824265 \quad 0.930704$

$\begin{array}{llll}\text { C } & 1.255200 & -2.889356 & 2.482239\end{array}$

$\begin{array}{llll}\mathrm{H} & 1.640287 & -3.234970 & 3.449256\end{array}$

$\mathrm{H} \quad 0.402113 \quad-2.233911 \quad 2.697523$

$\begin{array}{llll}\mathrm{H} & 0.863141 & -3.778873 & 1.968585\end{array}$

$\begin{array}{llll}\text { C } & -0.339974 & 0.930884 & 2.739566\end{array}$

$\begin{array}{llll}\mathrm{H} & 0.167079 & -0.033001 & 2.611314\end{array}$

$\begin{array}{llll}\mathrm{H} & -0.415600 & 1.108203 & 3.819637\end{array}$

$\begin{array}{llll}\mathrm{H} & -1.367690 & 0.817137 & 2.375537\end{array}$ 


\begin{tabular}{|c|c|c|c|}
\hline $\mathrm{C}$ & 1.839597 & 3.000611 & 3.199483 \\
\hline $\mathrm{H}$ & 2.551132 & 2.228499 & 3.518084 \\
\hline $\mathrm{H}$ & 2.423130 & 3.838583 & 2.800925 \\
\hline $\mathrm{H}$ & 1.320417 & 3.358585 & 4.097950 \\
\hline $\mathrm{C}$ & -0.636082 & 3.775551 & 1.622801 \\
\hline $\mathrm{H}$ & -1.351132 & 3.842752 & 2.452831 \\
\hline $\mathrm{H}$ & -0.128548 & 4.744030 & 1.551013 \\
\hline $\mathrm{H}$ & -1.204042 & 3.626070 & 0.699311 \\
\hline $\mathrm{C}$ & 3.738960 & 2.102622 & -1.217857 \\
\hline $\mathrm{H}$ & 4.196547 & 2.655546 & -2.047086 \\
\hline $\mathrm{H}$ & 4.452306 & 2.154693 & -0.379927 \\
\hline $\mathrm{H}$ & 3.676631 & 1.057916 & -1.549120 \\
\hline $\mathrm{C}$ & 0.948513 & 3.014202 & -2.237921 \\
\hline $\mathrm{H}$ & 0.726810 & 2.044417 & -2.696081 \\
\hline $\mathrm{H}$ & -0.016952 & 3.448526 & -1.951036 \\
\hline $\mathrm{H}$ & 1.393478 & 3.654614 & -3.010018 \\
\hline $\mathrm{C}$ & 2.471626 & 4.619628 & -0.137597 \\
\hline $\mathrm{H}$ & 2.967559 & 5.169161 & -0.947806 \\
\hline $\mathrm{H}$ & 1.577187 & 5.185714 & 0.142483 \\
\hline $\mathrm{H}$ & 3.149230 & 4.617438 & 0.724064 \\
\hline $\mathrm{H}$ & -1.473868 & -0.154873 & 0.625124 \\
\hline $\mathrm{C}$ & -1.990553 & 0.589098 & -1.127776 \\
\hline $\mathrm{C}$ & -1.937149 & 0.544743 & -2.528870 \\
\hline $\mathrm{C}$ & -2.638000 & 1.672860 & -0.514738 \\
\hline $\mathrm{C}$ & -2.493150 & 1.570519 & -3.291076 \\
\hline $\mathrm{H}$ & -1.466206 & -0.298099 & -3.018081 \\
\hline $\mathrm{C}$ & -3.180582 & 2.704494 & -1.279247 \\
\hline $\mathrm{H}$ & -2.717236 & 1.700872 & 0.567454 \\
\hline $\mathrm{C}$ & -3.109163 & 2.660586 & -2.672780 \\
\hline $\mathrm{H}$ & -2.438957 & 1.518609 & -4.374980 \\
\hline $\mathrm{H}$ & -3.668517 & 3.537850 & -0.781459 \\
\hline $\mathrm{H}$ & -3.533790 & 3.462138 & -3.269803 \\
\hline $\mathrm{C}$ & -2.252000 & -1.912062 & -0.262458 \\
\hline $\mathrm{O}$ & -1.432832 & -2.783014 & 0.253201 \\
\hline $\mathrm{O}$ & -2.705205 & -2.156286 & -1.570270 \\
\hline $\mathrm{C}$ & -3.455372 & -1.515974 & 0.580391 \\
\hline $\mathrm{C}$ & -3.368674 & -1.648246 & 1.970215 \\
\hline $\mathrm{C}$ & -4.596235 & -0.935779 & 0.015437 \\
\hline $\mathrm{C}$ & -4.403501 & -1.196281 & 2.788611 \\
\hline $\mathrm{H}$ & -2.488135 & -2.121398 & 2.392991 \\
\hline $\mathrm{C}$ & -5.633380 & -0.488259 & 0.835243 \\
\hline $\mathrm{H}$ & -4.664919 & -0.839481 & -1.061333 \\
\hline
\end{tabular}




$\begin{array}{llll}\mathrm{C} & -5.538603 & -0.611143 & 2.222479 \\ \mathrm{H} & -4.327552 & -1.306875 & 3.867156 \\ \mathrm{H} & -6.516252 & -0.039465 & 0.387879 \\ \mathrm{H} & -6.346075 & -0.258525 & 2.858507 \\ \mathrm{C} & -1.836506 & -2.920528 & -2.382500 \\ \mathrm{H} & -2.340862 & -3.025063 & -3.346227 \\ \mathrm{H} & -0.872778 & -2.417540 & -2.542326 \\ \mathrm{H} & -1.647472 & -3.907771 & -1.950011\end{array}$

23d

$\begin{array}{lllll}\text { C } & & 1.118254 & -0.874795 & -0.429469\end{array}$

N $\quad 0.912745 \quad 0.641277 \quad-0.405078$

$\begin{array}{lllll}\text { O } & 0.338315 & -1.386546 & -1.352234\end{array}$

$\begin{array}{llll}\text { C } & 1.838853 & 1.518627 & 0.232948\end{array}$

Li $\quad-0.787989 \quad 0.153921 \quad 0.599617$

$\mathrm{H} \quad 0.799363 \quad 0.892291 \quad-1.383170$

$\begin{array}{llll}\text { C } \quad 2.590817 & -1.252127 & -0.541822\end{array}$

$\begin{array}{llll}\text { O } & 0.619449 & -1.179235 & 0.959806\end{array}$

$\mathrm{Li} \quad \quad-1.420651 \quad-1.224064 \quad-1.441529$

$\begin{array}{llll}\text { C } & 1.963399 & 1.530297 & 1.630048\end{array}$

$\begin{array}{llll}\text { C } & 2.600605 & 2.415166 & -0.525140\end{array}$

$\begin{array}{llll}\text { C } & 3.471668 & -1.194940 & 0.544630\end{array}$

C $\quad 3.083443 \quad-1.612984-1.799251$

$\begin{array}{lllll}\text { C } & 0.599675 & -2.570335 & 1.266082\end{array}$

$\begin{array}{llll}\text { C } & 2.845329 & 2.414633 & 2.247952\end{array}$

$\mathrm{H} \quad 1.386552 \quad 0.826165 \quad 2.220850$

$\begin{array}{llll}\text { C } & 3.467677 & 3.311161 & 0.099372\end{array}$

$\mathrm{H} \quad 2.516213 \quad 2.401255 \quad-1.608959$

$\begin{array}{lllll}\text { C } & 4.824369 & -1.488749 & 0.372229\end{array}$

$\mathrm{H} \quad 3.098157 \quad-0.922734 \quad 1.524233$

C $\quad 4.436461 \quad-1.902550 \quad-1.973410$

$\mathrm{H} \quad 2.387708 \quad-1.669106-2.629206$

$\mathrm{H} \quad 0.230191 \quad-3.142855 \quad 0.409893$

$\mathrm{H} \quad \begin{array}{llll}\mathrm{H} & 1.602242 & -2.923976 & 1.528893\end{array}$

$\begin{array}{llll}\mathrm{H} & -0.072199 & -2.706247 & 2.116480\end{array}$

$\begin{array}{llll}\text { C } & 3.599228 & 3.311980 & 1.488302\end{array}$

$\begin{array}{llll}\mathrm{H} & 2.937564 & 2.406400 & 3.330525\end{array}$

$\begin{array}{lllll}\mathrm{H} & 4.049786 & 4.001407 & -0.504675\end{array}$

$\begin{array}{llll}\text { C } & 5.311755 & -1.840025 & -0.887471\end{array}$

$\mathrm{H} \quad \begin{array}{llll}\mathrm{H} & 5.497322 & -1.442705 & 1.224120\end{array}$

$\mathrm{H} \quad 4.806872-2.182354-2.956031$

$\mathrm{H} \quad 4.279959 \quad 4.004472 \quad 1.974201$ 


$\begin{array}{llll}\mathrm{H} & 6.365857 & -2.067465 & -1.020954 \\ \mathrm{~N} & -2.511360 & -0.128157 & -0.281957 \\ \mathrm{Si} & -3.377357 & -1.129733 & 0.817769 \\ \mathrm{Si} & -3.101866 & 1.312644 & -1.019538 \\ \mathrm{C} & -5.249062 & -0.856632 & 0.916979 \\ \mathrm{C} & -2.688999 & -0.883998 & 2.577849 \\ \mathrm{C} & -3.108698 & -2.949042 & 0.330012 \\ \mathrm{C} & -4.618722 & 1.026854 & -2.122574 \\ \mathrm{C} & -3.542076 & 2.645140 & 0.259069 \\ \mathrm{C} & -1.744030 & 2.042697 & -2.134346 \\ \mathrm{H} & -5.739697 & -1.092414 & -0.034192 \\ \mathrm{H} & -5.689976 & -1.502211 & 1.686987 \\ \mathrm{H} & -5.499755 & 0.179541 & 1.172033 \\ \mathrm{H} & -2.877461 & 0.143706 & 2.916954 \\ \mathrm{H} & -1.605468 & -1.059144 & 2.633049 \\ \mathrm{H} & -3.156046 & -1.560765 & 3.304125 \\ \mathrm{H} & -2.044392 & -3.209808 & 0.259988 \\ \mathrm{H} & -3.571714 & -3.156002 & -0.645322 \\ \mathrm{H} & -3.558965 & -3.640499 & 1.052860 \\ \mathrm{H} & -4.896084 & 1.939637 & -2.664708 \\ \mathrm{H} & -4.418776 & 0.245958 & -2.868453 \\ \mathrm{H} & -5.490404 & 0.708591 & -1.540622 \\ \mathrm{H} & -2.670588 & 2.889251 & 0.881911 \\ \mathrm{H} & -3.882447 & 3.576196 & -0.211047 \\ \mathrm{H} & -4.338108 & 2.302302 & 0.931312 \\ \mathrm{H} & -1.327253 & 1.294045 & -2.823208 \\ \mathrm{H} & -2.140687 & 2.860919 & -2.747541 \\ \mathrm{H} & -0.914189 & 2.447962 & -1.543069\end{array}$

$23 t$

$\mathrm{N} \quad-2.244483 \quad 0.301558 \quad 0.089096$

Li $\quad-0.300604 \quad 0.756493 \quad-0.281636$

Li $\quad \begin{array}{llll}1.162839 & -1.619827 & 0.302063\end{array}$

$\begin{array}{lllll}\mathrm{N} & 2.909139 & -0.953045 & -0.248037\end{array}$

$\begin{array}{llll}\mathrm{Li} & 2.532954 & 0.887949 & 0.099171\end{array}$

$\begin{array}{llll}\mathrm{N} & 1.021539 & 2.004222 & 0.434301\end{array}$

$\mathrm{Si} \quad 3.030071 \quad-1.388806-1.912026$

$\begin{array}{llll}\mathrm{Si} & 3.880070 & -1.513901 & 1.054036\end{array}$

$\mathrm{Si} \quad 1.190822 \quad 3.227095 \quad-0.764308$

$\begin{array}{llll}\mathrm{Si} & 0.584131 & 2.237934 & 2.084910\end{array}$

C $\quad 1.723164 \quad-0.435889-2.905230$

$\mathrm{H} \quad 0.723866 \quad-0.543000 \quad-2.467942$ 


\begin{tabular}{|c|c|c|c|}
\hline I & 1.950696 & 0.634018 & -2.965351 \\
\hline $\mathrm{H}$ & 1.669774 & -0.810466 & -3.935225 \\
\hline $\mathrm{C}$ & 2.668590 & -3.240236 & -2.154990 \\
\hline $\mathrm{H}$ & 2.702312 & -3.535193 & -3.211044 \\
\hline $\mathrm{H}$ & 3.394535 & -3.858891 & -1.613019 \\
\hline $\mathrm{H}$ & 1.669777 & -3.503769 & -1.777847 \\
\hline $\mathrm{C}$ & 4.715680 & -1.000243 & -2.682734 \\
\hline $\mathrm{H}$ & 5.516165 & -1.601415 & -2.237967 \\
\hline $\mathrm{H}$ & 4.715224 & -1.192307 & -3.762988 \\
\hline $\mathrm{H}$ & 4.973526 & 0.056549 & -2.534135 \\
\hline $\mathrm{C}$ & 4.428921 & -0.027143 & 2.103298 \\
\hline $\mathrm{H}$ & 4.964643 & -0.338393 & 3.008403 \\
\hline $\mathrm{H}$ & 5.109803 & 0.614136 & 1.526194 \\
\hline $\mathrm{H}$ & 3.584846 & 0.592171 & 2.434115 \\
\hline $\mathrm{C}$ & 5.434290 & -2.492565 & 0.599638 \\
\hline $\mathrm{H}$ & 5.938532 & -2.857308 & 1.503235 \\
\hline $\mathrm{H}$ & 5.201022 & -3.363142 & -0.024541 \\
\hline $\mathrm{H}$ & 6.149132 & -1.870883 & 0.048581 \\
\hline $\mathrm{C}$ & 2.805706 & -2.651056 & 2.152521 \\
\hline $\mathrm{H}$ & 3.347836 & -2.985612 & 3.045429 \\
\hline $\mathrm{H}$ & 1.892569 & -2.159047 & 2.520323 \\
\hline $\mathrm{H}$ & 2.509891 & -3.557230 & 1.602983 \\
\hline $\mathrm{C}$ & 0.479251 & 0.539531 & 2.934812 \\
\hline $\mathrm{H}$ & 1.464592 & 0.061460 & 2.992598 \\
\hline $\mathrm{H}$ & 0.106202 & 0.634332 & 3.961980 \\
\hline $\mathrm{H}$ & -0.184714 & -0.146078 & 2.395031 \\
\hline $\mathrm{C}$ & 1.848452 & 3.260118 & 3.063074 \\
\hline $\mathrm{H}$ & 2.857024 & 2.835960 & 2.973196 \\
\hline $\mathrm{H}$ & 1.893381 & 4.294206 & 2.702887 \\
\hline $\mathrm{H}$ & 1.597443 & 3.292883 & 4.130879 \\
\hline $\mathrm{C}$ & -1.111483 & 3.067449 & 2.295461 \\
\hline $\mathrm{H}$ & -1.439327 & 3.049505 & 3.342621 \\
\hline $\mathrm{H}$ & -1.083654 & 4.116431 & 1.978602 \\
\hline $\mathrm{H}$ & -1.881739 & 2.570709 & 1.692595 \\
\hline $\mathrm{C}$ & 2.938896 & 3.081710 & -1.519397 \\
\hline $\mathrm{H}$ & 3.068988 & 3.758030 & -2.373332 \\
\hline $\mathrm{H}$ & 3.698279 & 3.353867 & -0.772447 \\
\hline $\mathrm{H}$ & 3.177620 & 2.074597 & -1.889881 \\
\hline $\mathrm{C}$ & -0.090116 & 2.943447 & -2.140756 \\
\hline $\mathrm{H}$ & -0.026447 & 1.937029 & -2.576166 \\
\hline $\mathrm{H}$ & -1.106598 & 3.073695 & -1.745701 \\
\hline $\mathrm{H}$ & 0.033538 & 3.653593 & -2.967804 \\
\hline
\end{tabular}




\begin{tabular}{|c|c|c|c|}
\hline $\mathrm{C}$ & 1.003136 & 5.018701 & -0.184953 \\
\hline $\mathrm{H}$ & 1.143215 & 5.711076 & -1.024534 \\
\hline $\mathrm{H}$ & 0.007133 & 5.203488 & 0.232893 \\
\hline $\mathrm{H}$ & 1.740726 & 5.275720 & 0.583728 \\
\hline $\mathrm{H}$ & -2.228309 & 0.292186 & 1.108178 \\
\hline $\mathrm{C}$ & -3.515041 & 0.784699 & -0.347509 \\
\hline $\mathrm{C}$ & -3.684597 & 1.230286 & -1.664804 \\
\hline $\mathrm{C}$ & -4.589543 & 0.855964 & 0.546709 \\
\hline $\mathrm{C}$ & -4.919521 & 1.725720 & -2.077222 \\
\hline $\mathrm{H}$ & -2.848307 & 1.178365 & -2.350815 \\
\hline $\mathrm{C}$ & -5.818137 & 1.371925 & 0.133544 \\
\hline $\mathrm{H}$ & -4.461411 & 0.495059 & 1.564078 \\
\hline $\mathrm{C}$ & -5.990019 & 1.802360 & -1.181892 \\
\hline $\mathrm{H}$ & -5.042431 & 2.066329 & -3.101571 \\
\hline $\mathrm{H}$ & -6.642014 & 1.426277 & 0.839538 \\
\hline $\mathrm{H}$ & -6.947313 & 2.199200 & -1.507017 \\
\hline $\mathrm{C}$ & -1.765268 & -1.091847 & -0.306954 \\
\hline $\mathrm{O}$ & -0.465236 & -1.126986 & 0.032473 \\
\hline $\mathrm{O}$ & -1.963746 & -1.104516 & -1.721011 \\
\hline $\mathrm{C}$ & -2.627202 & -2.145385 & 0.384280 \\
\hline $\mathrm{C}$ & -2.172574 & -2.773356 & 1.547200 \\
\hline $\mathrm{C}$ & -3.917737 & -2.423741 & -0.084767 \\
\hline $\mathrm{C}$ & -2.991172 & -3.673264 & 2.231813 \\
\hline $\mathrm{H}$ & -1.175220 & -2.548114 & 1.910685 \\
\hline $\mathrm{C}$ & -4.734531 & -3.324442 & 0.597793 \\
\hline $\mathrm{H}$ & -4.271377 & -1.932755 & -0.984672 \\
\hline $\mathrm{C}$ & -4.274268 & -3.951371 & 1.757937 \\
\hline $\mathrm{H}$ & -2.626198 & -4.156747 & 3.133943 \\
\hline $\mathrm{H}$ & -5.732424 & -3.536150 & 0.223720 \\
\hline $\mathrm{H}$ & -4.912081 & -4.652559 & 2.288855 \\
\hline $\mathrm{C}$ & -1.526452 & -2.296828 & -2.356624 \\
\hline $\mathrm{H}$ & -1.673990 & -2.143420 & -3.427534 \\
\hline $\mathrm{H}$ & -0.465170 & -2.485723 & -2.163030 \\
\hline $\mathrm{H}$ & -2.112499 & -3.163420 & -2.026786 \\
\hline
\end{tabular}

\section{TS24d}

C $\quad 1.120778-0.701254 \quad 0.171768$

$\begin{array}{lllll}\text { O } & 0.080037 & -1.486790 & -0.114794\end{array}$

C $\quad 2.455408-1.420491 \quad 0.326309$

$\mathrm{Li} \quad-1.355090 \quad-1.114042 \quad 0.949864$

Li $\quad-0.826772 \quad 0.061243 \quad-0.990934$

N $\quad 1.199160 \quad 0.322322 \quad-0.973019$ 


\begin{tabular}{|c|c|c|c|}
\hline $\mathrm{O}$ & 0.840519 & 0.143301 & 1.326941 \\
\hline $\mathrm{C}$ & 3.592123 & -0.756630 & 0.809163 \\
\hline $\mathrm{C}$ & 2.557880 & -2.755635 & -0.072462 \\
\hline $\mathrm{N}$ & -2.562704 & -0.004366 & -0.106016 \\
\hline $\mathrm{C}$ & 2.123374 & 1.412858 & -0.911215 \\
\hline $\mathrm{H}$ & 1.444556 & -0.259822 & -1.774416 \\
\hline $\mathrm{C}$ & 0.907643 & -0.512741 & 2.583788 \\
\hline $\mathrm{C}$ & 4.813575 & -1.424599 & 0.887418 \\
\hline $\mathrm{H}$ & 3.516432 & 0.278298 & 1.124327 \\
\hline $\mathrm{C}$ & 3.781793 & -3.422317 & 0.004671 \\
\hline $\mathrm{H}$ & 1.665652 & -3.253033 & -0.436733 \\
\hline $\mathrm{Si}$ & -3.206854 & 1.389464 & 0.663818 \\
\hline $\mathrm{Si}$ & -3.426628 & -1.283597 & -0.861193 \\
\hline $\mathrm{C}$ & 3.262545 & 1.414867 & -1.725157 \\
\hline $\mathrm{C}$ & 1.889703 & 2.494428 & -0.052637 \\
\hline $\mathrm{H}$ & 1.937820 & -0.750181 & 2.869433 \\
\hline $\mathrm{H}$ & 0.338156 & -1.457719 & 2.590074 \\
\hline $\mathrm{H}$ & 0.474424 & 0.174598 & 3.314599 \\
\hline $\mathrm{C}$ & 4.912236 & -2.758237 & 0.483525 \\
\hline $\mathrm{H}$ & 5.689046 & -0.902810 & 1.263983 \\
\hline $\mathrm{H}$ & 3.851518 & -4.461176 & -0.306467 \\
\hline $\mathrm{C}$ & -4.433098 & 2.375768 & -0.394761 \\
\hline $\mathrm{C}$ & -1.778918 & 2.559555 & 1.100855 \\
\hline $\mathrm{C}$ & -4.082868 & 0.938596 & 2.291677 \\
\hline $\mathrm{C}$ & -5.292454 & -1.046111 & -1.065899 \\
\hline $\mathrm{C}$ & -2.673005 & -1.629039 & -2.572079 \\
\hline $\mathrm{C}$ & -3.165832 & -2.874369 & 0.169392 \\
\hline $\mathrm{C}$ & 4.157611 & 2.483740 & -1.685987 \\
\hline $\mathrm{H}$ & 3.454026 & 0.566541 & -2.378009 \\
\hline $\mathrm{C}$ & 2.798481 & 3.549695 & -0.003441 \\
\hline $\mathrm{H}$ & 1.007251 & 2.493627 & 0.572439 \\
\hline $\mathrm{H}$ & 5.865034 & -3.276887 & 0.545001 \\
\hline $\mathrm{H}$ & -4.691240 & 3.328791 & 0.084357 \\
\hline $\mathrm{H}$ & -4.000874 & 2.604308 & -1.377323 \\
\hline $\mathrm{H}$ & -5.365794 & 1.826518 & -0.563052 \\
\hline $\mathrm{H}$ & -2.122272 & 3.415107 & 1.695336 \\
\hline $\mathrm{H}$ & -1.305217 & 2.958873 & 0.194268 \\
\hline $\mathrm{H}$ & -1.001019 & 2.038219 & 1.671189 \\
\hline $\mathrm{H}$ & -4.911077 & 0.241285 & 2.112097 \\
\hline $\mathrm{H}$ & -3.388561 & 0.447512 & 2.988808 \\
\hline $\mathrm{H}$ & -4.492916 & 1.817525 & 2.804875 \\
\hline $\mathrm{H}$ & -5.780197 & -0.830582 & -0.107732 \\
\hline
\end{tabular}




$\begin{array}{llll}\mathrm{H} & -5.755324 & -1.950939 & -1.479121 \\ \mathrm{H} & -5.515261 & -0.216502 & -1.745893 \\ \mathrm{H} & -2.756241 & -0.746093 & -3.220374 \\ \mathrm{H} & -1.609762 & -1.895483 & -2.484920 \\ \mathrm{H} & -3.168053 & -2.462310 & -3.085924 \\ \mathrm{H} & -3.552337 & -2.751071 & 1.191950 \\ \mathrm{H} & -2.105688 & -3.166116 & 0.225068 \\ \mathrm{H} & -3.689937 & -3.732578 & -0.268924 \\ \mathrm{C} & 3.933522 & 3.552653 & -0.818332 \\ \mathrm{H} & 5.036037 & 2.471489 & -2.324995 \\ \mathrm{H} & 2.610588 & 4.382031 & 0.669174 \\ \mathrm{H} & 4.632946 & 4.382624 & -0.780145\end{array}$

\section{TS24t}

$\begin{array}{llll}\mathrm{N} & -2.214801 & 0.718101 & -0.021657 \\ \mathrm{Li} & -0.228920 & 1.034188 & 0.387041 \\ \mathrm{Li} & 0.498131 & -1.578696 & 0.330151 \\ \mathrm{~N} & 2.260764 & -1.505111 & -0.431180 \\ \mathrm{Li} & 2.521367 & 0.385672 & -0.066373 \\ \mathrm{~N} & 1.503939 & 1.906638 & 0.504475 \\ \mathrm{Si} & 2.230680 & -1.934011 & -2.103302 \\ \mathrm{Si} & 3.296770 & -2.174836 & 0.773621 \\ \mathrm{Si} & 1.690122 & 2.958769 & -0.848463 \\ \mathrm{Si} & 1.575187 & 2.381880 & 2.164776 \\ \mathrm{C} & 0.876042 & -0.919769 & -2.959491 \\ \mathrm{H} & -0.095927 & -1.100794 & -2.486684 \\ \mathrm{H} & 1.068431 & 0.158088 & -2.910436 \\ \mathrm{H} & 0.789740 & -1.188340 & -4.019607 \\ \mathrm{C} & 1.794804 & -3.762170 & -2.381248 \\ \mathrm{H} & 1.624021 & -3.977244 & -3.443625 \\ \mathrm{H} & 2.593907 & -4.426581 & -2.034063 \\ \mathrm{H} & 0.881315 & -4.037646 & -1.837863 \\ \mathrm{C} & 3.873400 & -1.604022 & -2.992863 \\ \mathrm{H} & 4.682708 & -2.209540 & -2.568160 \\ \mathrm{H} & 3.805575 & -1.846201 & -4.060939 \\ \mathrm{H} & 3.948805 & 0.090903 & 1.764404 \\ \mathrm{H} & 4.172976 & -0.552015 & -2.911026 \\ \mathrm{H} & 4.468118 & -0.807569 & 1.403417 \\ \mathrm{H} & 5.078536 & -1.160678 & 2.243670 \\ \mathrm{H} & 5.159621 & -0.499764 & 0.606480 \\ \mathrm{H} & 5.038130 & -3.936062 & 1.075683\end{array}$




\begin{tabular}{|c|c|c|c|}
\hline $\mathrm{H}$ & 3.782913 & -4.497160 & -0.037561 \\
\hline $\mathrm{H}$ & 5.031080 & -3.376790 & -0.602797 \\
\hline $\mathrm{C}$ & 2.232477 & -2.779823 & 2.229374 \\
\hline $\mathrm{H}$ & 2.849932 & -3.096486 & 3.079058 \\
\hline $\mathrm{H}$ & 1.547189 & -2.008966 & 2.604530 \\
\hline $\mathrm{H}$ & 1.631343 & -3.650646 & 1.929222 \\
\hline $\mathrm{C}$ & 1.390479 & 0.860218 & 3.279471 \\
\hline $\mathrm{H}$ & 2.248276 & 0.183531 & 3.199305 \\
\hline $\mathrm{H}$ & 1.314024 & 1.164175 & 4.331168 \\
\hline $\mathrm{H}$ & 0.490725 & 0.289200 & 3.023799 \\
\hline $\mathrm{C}$ & 3.195026 & 3.240204 & 2.648685 \\
\hline $\mathrm{H}$ & 4.059233 & 2.625754 & 2.363744 \\
\hline $\mathrm{H}$ & 3.307092 & 4.214921 & 2.162426 \\
\hline $\mathrm{H}$ & 3.247130 & 3.400352 & 3.732948 \\
\hline $\mathrm{C}$ & 0.124713 & 3.533182 & 2.601966 \\
\hline $\mathrm{H}$ & 0.149184 & 3.849642 & 3.652179 \\
\hline $\mathrm{H}$ & 0.121634 & 4.435969 & 1.979668 \\
\hline $\mathrm{H}$ & -0.835598 & 3.021431 & 2.440540 \\
\hline $\mathrm{C}$ & 3.081308 & 2.264565 & -1.954768 \\
\hline $\mathrm{H}$ & 3.209880 & 2.871436 & -2.859542 \\
\hline $\mathrm{H}$ & 4.042894 & 2.274189 & -1.421426 \\
\hline $\mathrm{H}$ & 2.896794 & 1.239400 & -2.301207 \\
\hline $\mathrm{C}$ & 0.094063 & 3.002321 & -1.874666 \\
\hline $\mathrm{H}$ & -0.240995 & 2.002917 & -2.177028 \\
\hline $\mathrm{H}$ & -0.725428 & 3.455038 & -1.300678 \\
\hline $\mathrm{H}$ & 0.216323 & 3.592161 & -2.791648 \\
\hline $\mathrm{C}$ & 2.163689 & 4.754701 & -0.474917 \\
\hline $\mathrm{H}$ & 2.161909 & 5.344003 & -1.400535 \\
\hline $\mathrm{H}$ & 1.461013 & 5.229336 & 0.219457 \\
\hline $\mathrm{H}$ & 3.165416 & 4.829023 & -0.037230 \\
\hline $\mathrm{H}$ & -2.550922 & 1.204253 & 0.810470 \\
\hline $\mathrm{C}$ & -3.148154 & 0.954882 & -1.076746 \\
\hline $\mathrm{C}$ & -2.831351 & 0.620139 & -2.399487 \\
\hline $\mathrm{C}$ & -4.374846 & 1.571161 & -0.800287 \\
\hline $\mathrm{C}$ & -3.740877 & 0.886389 & -3.420315 \\
\hline $\mathrm{H}$ & -1.878560 & 0.156868 & -2.617860 \\
\hline $\mathrm{C}$ & -5.273109 & 1.853217 & -1.829654 \\
\hline $\mathrm{H}$ & -4.629078 & 1.818348 & 0.227716 \\
\hline $\mathrm{C}$ & -4.963137 & 1.504613 & -3.144010 \\
\hline $\mathrm{H}$ & -3.484763 & 0.621252 & -4.442292 \\
\hline $\mathrm{H}$ & -6.218523 & 2.335935 & -1.598872 \\
\hline $\mathrm{H}$ & -5.662745 & 1.717794 & -3.946796 \\
\hline
\end{tabular}




$\begin{array}{llll}\mathrm{C} & -1.922254 & -0.694867 & 0.485733 \\ \mathrm{O} & -0.770048 & -0.606968 & 1.172295 \\ \mathrm{O} & -1.738055 & -1.469707 & -0.725993 \\ \mathrm{C} & -3.107656 & -1.200600 & 1.298447 \\ \mathrm{C} & -2.982293 & -1.337653 & 2.683256 \\ \mathrm{C} & -4.339843 & -1.472712 & 0.686235 \\ \mathrm{C} & -4.076554 & -1.739857 & 3.452122 \\ \mathrm{H} & -2.020696 & -1.125888 & 3.137780 \\ \mathrm{C} & -5.430106 & -1.875865 & 1.455620 \\ \mathrm{H} & -4.440092 & -1.370342 & -0.389143 \\ \mathrm{C} & -5.301733 & -2.009070 & 2.840725 \\ \mathrm{H} & -3.970029 & -1.844635 & 4.528318 \\ \mathrm{H} & -6.380593 & -2.086989 & 0.973515 \\ \mathrm{H} & -6.152747 & -2.323367 & 3.438497 \\ \mathrm{C} & -1.765518 & -2.883421 & -0.570154 \\ \mathrm{H} & -1.374482 & -3.298919 & -1.501111 \\ \mathrm{H} & -1.137231 & -3.223129 & 0.269528 \\ \mathrm{H} & -2.779107 & -3.257028 & -0.395810\end{array}$

25d

C $\quad 1.140344-0.760231 \quad 0.024622$

$\begin{array}{llll}\text { O } & 0.118338 & -1.523589 & -0.339563\end{array}$

O $\quad 0.695154-0.057585 \quad 1.262290$

$\mathrm{Li} \quad-1.072692 \quad-0.932789 \quad 0.991791$

Li $\quad-0.839482-0.054140-1.168779$

$\begin{array}{llll}\text { C } & 2.459437 & -1.505976 & 0.222630\end{array}$

$\begin{array}{lllll}\mathrm{N} & & 1.237602 & 0.329859 & -1.050761\end{array}$

$\begin{array}{llll}\text { C } & 1.556594 & 0.136968 & 2.380793\end{array}$

N $\quad \begin{array}{llll}-2.468888 & -0.036815 & -0.073425\end{array}$

$\begin{array}{llll}\text { C } & 3.695139 & -0.864539 & 0.391503\end{array}$

$\begin{array}{llll}\text { C } & 2.418908 & -2.904578 & 0.207460\end{array}$

$\begin{array}{llll}\text { C } & 2.030801 & 1.506403 & -0.903466\end{array}$

$\mathrm{H} \quad \begin{array}{llll}1.556160 & -0.179380 & -1.875127\end{array}$

$\begin{array}{llll}\mathrm{H} & 2.342250 & 0.866025 & 2.162071\end{array}$

$\begin{array}{llll}\mathrm{H} & 2.013498 & -0.802157 & 2.706969\end{array}$

$\mathrm{H} \quad 0.921525 \quad 0.532759 \quad 3.178314$

$\begin{array}{llll}\mathrm{Si} & -3.186244 & 1.329533 & 0.678563\end{array}$

$\begin{array}{lllll}\mathrm{Si} & & -3.320189 & -1.358136 & -0.776279\end{array}$

$\begin{array}{llll}\text { C } & 4.862342 & -1.612365 & 0.547774\end{array}$

$\begin{array}{lllll}\mathrm{H} & 3.757307 & 0.216377 & 0.399633\end{array}$

$\begin{array}{llll}\text { C } & 3.586156 & -3.651392 & 0.366897\end{array}$

$\begin{array}{llll}\mathrm{H} & 1.459718 & -3.384691 & 0.052472\end{array}$ 


\begin{tabular}{|c|c|c|c|}
\hline $\mathrm{C}$ & 1.707251 & 2.474949 & 0.055968 \\
\hline $\mathrm{C}$ & 3.119401 & 1.730538 & -1.758308 \\
\hline $\mathrm{C}$ & -4.345917 & 2.326280 & -0.445900 \\
\hline $\mathrm{C}$ & -4.168321 & 0.847948 & 2.235765 \\
\hline $\mathrm{C}$ & -1.825132 & 2.525572 & 1.244771 \\
\hline $\mathrm{C}$ & -5.177441 & -1.114602 & -1.061847 \\
\hline $\mathrm{C}$ & -3.103417 & -2.910687 & 0.305457 \\
\hline $\mathrm{C}$ & -2.538824 & -1.758120 & -2.46924 \\
\hline $\mathrm{C}$ & 4.812376 & -3.007317 & 0.538051 \\
\hline $\mathrm{H}$ & 5.812471 & -1.101234 & 0.676299 \\
\hline $\mathrm{H}$ & 3.537822 & -4.736780 & 0.351746 \\
\hline $\mathrm{C}$ & 2.483276 & 3.626959 & 0.173262 \\
\hline $\mathrm{H}$ & 0.847242 & 2.320885 & 0.691835 \\
\hline $\mathrm{C}$ & 3.878266 & 2.895073 & -1.649423 \\
\hline $\mathrm{H}$ & 3.380677 & 0.978159 & -2.498882 \\
\hline $\mathrm{H}$ & -5.249133 & 1.763274 & -0.704667 \\
\hline $\mathrm{H}$ & -4.663569 & 3.258685 & 0.038261 \\
\hline $\mathrm{H}$ & -3.842184 & 2.593957 & -1.383750 \\
\hline $\mathrm{H}$ & -4.622315 & 1.718333 & 2.726287 \\
\hline $\mathrm{H}$ & -3.515976 & 0.357982 & 2.972408 \\
\hline $\mathrm{H}$ & -4.974416 & 0.143056 & 1.997559 \\
\hline $\mathrm{H}$ & -1.087902 & 2.018640 & 1.879825 \\
\hline $\mathrm{H}$ & -2.240276 & 3.364879 & 1.816181 \\
\hline $\mathrm{H}$ & -1.287828 & 2.945397 & 0.385487 \\
\hline $\mathrm{H}$ & -5.705153 & -0.854416 & -0.136849 \\
\hline $\mathrm{H}$ & -5.628140 & -2.035616 & -1.45264 \\
\hline $\mathrm{H}$ & -5.367419 & -0.316604 & -1.788377 \\
\hline $\mathrm{H}$ & -3.606565 & -3.788618 & -0.118590 \\
\hline $\mathrm{H}$ & -3.520759 & -2.744731 & 1.308246 \\
\hline $\mathrm{H}$ & -2.041369 & -3.175517 & 0.410197 \\
\hline $\mathrm{H}$ & -1.486376 & -2.056299 & -2.365141 \\
\hline $\mathrm{H}$ & -2.598896 & -0.893778 & -3.147019 \\
\hline $\mathrm{H}$ & -3.054013 & -2.589699 & -2.965812 \\
\hline $\mathrm{H}$ & 5.723197 & -3.586980 & 0.659870 \\
\hline $\mathrm{C}$ & 3.570990 & 3.845621 & -0.675019 \\
\hline $\mathrm{H}$ & 2.221588 & 4.367260 & 0.924226 \\
\hline$\Pi$ & 4.717601 & 3.051480 & -2.321170 \\
\hline $\mathrm{H}$ & 492 & 4.749641 & 363 \\
\hline
\end{tabular}

$25 t$

$\begin{array}{llll}\mathrm{N} & 2.306515 & 0.897905 & -0.236075\end{array}$

$\mathrm{Li} \quad 0.248406 \quad 1.155823 \quad-0.700723$ 


\begin{tabular}{|c|c|c|c|}
\hline $\mathrm{Li}$ & -0.116583 & -1.610698 & 0.125867 \\
\hline $\mathrm{N}$ & -1.952374 & -1.590761 & 0.733220 \\
\hline $\mathrm{Li}$ & -2.307591 & 0.174396 & -0.006935 \\
\hline $\mathrm{N}$ & -1.599452 & 1.773082 & -0.805778 \\
\hline $\mathrm{Si}$ & -2.020806 & -1.708664 & 2.450659 \\
\hline $\mathrm{Si}$ & -2.862949 & -2.550970 & -0.372690 \\
\hline $\mathrm{Si}$ & -1.885311 & 2.994483 & 0.376484 \\
\hline $\mathrm{Si}$ & -1.843792 & 1.955763 & -2.508010 \\
\hline $\mathrm{C}$ & -0.777507 & -0.478938 & 3.193340 \\
\hline $\mathrm{H}$ & 0.245586 & -0.815525 & 2.989148 \\
\hline $\mathrm{H}$ & -0.879631 & 0.530067 & 2.777303 \\
\hline $\mathrm{H}$ & -0.889803 & -0.400213 & 4.281545 \\
\hline $\mathrm{C}$ & -1.497809 & -3.413838 & 3.102695 \\
\hline $\mathrm{H}$ & -1.403152 & -3.411982 & 4.196042 \\
\hline $\mathrm{H}$ & -2.217487 & -4.194213 & 2.833450 \\
\hline $\mathrm{H}$ & -0.523546 & -3.706176 & 2.686949 \\
\hline $\mathrm{C}$ & -3.741310 & -1.329105 & 3.155632 \\
\hline $\mathrm{H}$ & -4.481797 & -2.050647 & 2.789601 \\
\hline $\mathrm{H}$ & -3.750517 & -1.373626 & 4.251971 \\
\hline $\mathrm{H}$ & -4.085320 & -0.330158 & 2.861771 \\
\hline $\mathrm{C}$ & -4.201622 & -1.460929 & -1.183710 \\
\hline $\mathrm{H}$ & -4.737596 & -2.002885 & -1.972875 \\
\hline $\mathrm{H}$ & -4.943129 & -1.154168 & -0.433282 \\
\hline $\mathrm{H}$ & -3.809931 & -0.549537 & -1.655505 \\
\hline $\mathrm{C}$ & -3.762370 & -4.054569 & 0.347885 \\
\hline $\mathrm{H}$ & -4.362020 & -4.544553 & -0.429608 \\
\hline $\mathrm{H}$ & -3.054276 & -4.796075 & 0.735158 \\
\hline $\mathrm{H}$ & -4.439772 & -3.781734 & 1.165040 \\
\hline $\mathrm{C}$ & -1.699999 & -3.200865 & -1.728191 \\
\hline $\mathrm{H}$ & -2.250071 & -3.711392 & -2.528293 \\
\hline $\mathrm{H}$ & -1.114885 & -2.397692 & -2.191974 \\
\hline $\mathrm{H}$ & -0.995276 & -3.932127 & -1.305442 \\
\hline $\mathrm{C}$ & -1.485648 & 0.319103 & -3.394938 \\
\hline $\mathrm{H}$ & -2.243900 & -0.444214 & -3.187827 \\
\hline $\mathrm{H}$ & -1.472225 & 0.475615 & -4.481168 \\
\hline $\mathrm{H}$ & -0.515141 & -0.092809 & -3.096615 \\
\hline $\mathrm{C}$ & -3.604772 & 2.475423 & -2.982922 \\
\hline $\mathrm{H}$ & -4.343903 & 1.817475 & -2.507459 \\
\hline $\mathrm{H}$ & -3.830997 & 3.502360 & -2.677894 \\
\hline $\mathrm{H}$ & -3.753609 & 2.409921 & -4.068109 \\
\hline $\mathrm{C}$ & -0.622553 & 3.217802 & -3.240495 \\
\hline $\mathrm{H}$ & -0.760066 & 3.342620 & -4.321870 \\
\hline
\end{tabular}




\begin{tabular}{|c|c|c|c|}
\hline $\mathrm{H}$ & -0.725333 & 4.203947 & -2.7732 \\
\hline $\mathrm{H}$ & 0.414428 & 2.886237 & -3.082175 \\
\hline $\mathrm{C}$ & -3.145475 & 2.341517 & 1.646568 \\
\hline $\mathrm{H}$ & -3.332924 & 3.073804 & 2.441856 \\
\hline $\mathrm{H}$ & -4.109608 & 2.133158 & 1.161770 \\
\hline $\mathrm{H}$ & -2.815388 & 1.421905 & 2.145137 \\
\hline $\mathrm{C}$ & -0.276519 & 3.382216 & 1.308573 \\
\hline $\mathrm{H}$ & 0.161646 & 2.493332 & 1.778353 \\
\hline $\mathrm{H}$ & 0.481294 & 3.795130 & 0.628704 \\
\hline $\mathrm{H}$ & -0.435146 & 4.119118 & 2.105755 \\
\hline $\mathrm{C}$ & -2.575319 & 4.646927 & -0.245613 \\
\hline $\mathrm{H}$ & -2.643133 & 5.359024 & 0.586614 \\
\hline $\mathrm{H}$ & -1.940101 & 5.099652 & -1.015328 \\
\hline $\mathrm{H}$ & -3.580649 & 4.535361 & -0.66643 \\
\hline $\mathrm{H}$ & 2.756598 & 1.235894 & -1.085555 \\
\hline $\mathrm{C}$ & 3.052712 & 1.343970 & 0.893091 \\
\hline $\mathrm{C}$ & 2.563897 & 1.172663 & 2.195785 \\
\hline $\mathrm{C}$ & 4.255851 & 2.039956 & 0.706180 \\
\hline $\mathrm{C}$ & 3.286955 & 1.657090 & 3.283479 \\
\hline $\mathrm{H}$ & 1.622939 & 0.663397 & 2.347789 \\
\hline $\mathrm{C}$ & 4.962743 & 2.542903 & 1.798454 \\
\hline $\mathrm{H}$ & 4.638234 & 2.185767 & -0.301767 \\
\hline $\mathrm{C}$ & 4.488031 & 2.344470 & 3.094940 \\
\hline $\mathrm{H}$ & 2.894101 & 1.510873 & 4.285738 \\
\hline $\mathrm{H}$ & 5.889808 & 3.083970 & 1.631480 \\
\hline $\mathrm{H}$ & 5.040175 & 2.729747 & 3.946687 \\
\hline $\mathrm{C}$ & 1.960442 & -0.565847 & -0.513865 \\
\hline $\mathrm{O}$ & 0.772776 & -0.570213 & -1.122985 \\
\hline $\mathrm{O}$ & 1.723576 & -1.232230 & 0.777152 \\
\hline $\mathrm{C}$ & 3.066364 & -1.263335 & -1.306208 \\
\hline $\mathrm{C}$ & 2.692687 & -2.144526 & -2.326736 \\
\hline $\mathrm{C}$ & 4.427205 & -1.056696 & -1.046178 \\
\hline $\mathrm{C}$ & 3.663124 & -2.818414 & -3.068294 \\
\hline $\mathrm{H}$ & 1.637134 & -2.272890 & -2.537796 \\
\hline C & 5.397345 & -1.728218 & -1.791025 \\
\hline $\mathrm{H}$ & 4.735670 & -0.375551 & -0.260298 \\
\hline $\mathrm{C}$ & 5.018156 & -2.612864 & -2.801881 \\
\hline $\mathrm{H}$ & 3.360549 & -3.497086 & -3.860841 \\
\hline $\mathrm{H}$ & 6.449656 & -1.558416 & -1.580784 \\
\hline $\mathrm{H}$ & 5.774269 & -3.134190 & -3.382100 \\
\hline $\mathrm{C}$ & 2.754105 & -1.957502 & 1.449532 \\
\hline $\mathrm{H}$ & 2.289986 & -2.349472 & 2.357860 \\
\hline
\end{tabular}


$\begin{array}{llll}\mathrm{H} & 3.123338 & -2.784184 & 0.835639\end{array}$

$\begin{array}{llll}\mathrm{H} & 3.586308 & -1.306390 & 1.728517\end{array}$

\section{TS26d}

$\begin{array}{llll}\mathrm{Si} & -2.820928 & 1.493850 & -0.846065\end{array}$

$\begin{array}{lllll}\mathrm{N} & & -2.484895 & -0.006176 & -0.091774\end{array}$

$\begin{array}{llll}\text { C } & -4.647112 & 1.986861 & -0.973357\end{array}$

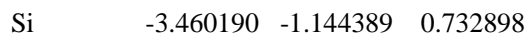

$\mathrm{Li} \quad-1.218722-1.275021-0.944321$

$\begin{array}{llll}\mathrm{Li} & -0.798747 & 0.166003 & 0.890826\end{array}$

$\begin{array}{llll}\text { C } & -1.970550 & 2.906229 & 0.116934\end{array}$

C $\quad-2.144811 \quad 1.517450 \quad-2.623409$

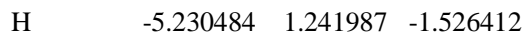

$\begin{array}{llll}\mathrm{H} & -4.742533 & 2.943504 & -1.502869\end{array}$

$\begin{array}{llll}\mathrm{H} & -5.112274 & 2.105751 & 0.011612\end{array}$

$\begin{array}{lllll}\text { C } & -5.330659 & -0.853778 & 0.742014\end{array}$

$\begin{array}{llll}\text { C } & -2.910031 & -1.296481 & 2.549717\end{array}$

$\begin{array}{lllll}\text { C } & & -3.197631 & -2.861287 & -0.070179\end{array}$

$\begin{array}{lllll}\text { O } & 0.403842 & -0.985934 & -1.821049\end{array}$

$\begin{array}{lllll}\text { O } & 0.109834 & -1.406245 & 0.647870\end{array}$

$\mathrm{H} \quad \begin{array}{llll}\mathrm{H} & -0.883056 & 2.778048 & 0.201321\end{array}$

$\begin{array}{llll}\mathrm{H} & -2.375050 & 2.961289 & 1.137267\end{array}$

$\mathrm{H} \quad \begin{array}{llll}\mathrm{H} & -2.140994 & 3.882451 & -0.354426\end{array}$

$\begin{array}{lllll}\mathrm{H} & -1.111034 & 1.157240 & -2.694504\end{array}$

$\mathrm{H} \quad \begin{array}{llll}\mathrm{H} & -2.747097 & 0.853011 & -3.257583\end{array}$

$\begin{array}{llll}\mathrm{H} & -2.186799 & 2.521018 & -3.065242\end{array}$

$\begin{array}{llll}\mathrm{H} & -5.741990 & -0.815278 & -0.272763\end{array}$

$\begin{array}{lllll}\mathrm{H} & & -5.836221 & -1.667184 & 1.277928\end{array}$

$\begin{array}{llll}\mathrm{H} & -5.592779 & 0.086352 & 1.239858\end{array}$

$\begin{array}{lllll}\mathrm{H} & & -1.838383 & -1.525825 & 2.624389\end{array}$

$\begin{array}{llll}\mathrm{H} & -3.092937 & -0.354928 & 3.085713\end{array}$

$\begin{array}{llll}\mathrm{H} & -3.449043 & -2.088428 & 3.084778\end{array}$

$\mathrm{H} \quad \begin{array}{llll}-2.149826 & -3.196263 & -0.069327\end{array}$

$\mathrm{H} \quad \begin{array}{llll}\mathrm{H} & -3.555876 & -2.860415 & -1.109330\end{array}$

$\mathrm{H} \quad \begin{array}{lllll} & -3.757546 & -3.639973 & 0.462563\end{array}$

$\begin{array}{llll}\text { C } & 1.080469 & -0.623313 & -0.817241\end{array}$

$\begin{array}{llll}\text { C } & 0.389346 & -2.741297 & 0.969995\end{array}$

$\begin{array}{lllll}\mathrm{N} & & 0.812476 & 0.750191 & -0.380477\end{array}$

$\begin{array}{llll}\text { C } & 2.452976 & -1.186415 & -0.592332\end{array}$

$\mathrm{H} \quad 1.322564 \quad-2.842400 \quad 1.541810$

$\begin{array}{llll}\mathrm{H} & 0.494534 & -3.361220 & 0.062903\end{array}$

$\begin{array}{llll}\mathrm{H} & -0.427377 & -3.161427 & 1.574131\end{array}$ 


$\begin{array}{llll}\mathrm{C} & 1.843074 & 1.575211 & 0.195551 \\ \mathrm{H} & 0.375940 & 1.204394 & -1.179711 \\ \mathrm{C} & 3.117365 & -1.141011 & 0.640067 \\ \mathrm{C} & 3.087123 & -1.771865 & -1.692725 \\ \mathrm{C} & 2.851868 & 2.127044 & -0.599393 \\ \mathrm{C} & 1.824633 & 1.839297 & 1.567235 \\ \mathrm{C} & 4.405191 & -1.655692 & 0.760874 \\ \mathrm{H} & 2.618325 & -0.720486 & 1.503649 \\ \mathrm{C} & 4.378773 & -2.285230 & -1.571045 \\ \mathrm{H} & 2.554192 & -1.814264 & -2.636129 \\ \mathrm{C} & 3.835662 & 2.929574 & -0.023850 \\ \mathrm{H} & 2.869386 & 1.909024 & -1.663314 \\ \mathrm{C} & 2.813552 & 2.635489 & 2.145349 \\ \mathrm{H} & 1.043058 & 1.405886 & 2.186901 \\ \mathrm{C} & 5.042037 & -2.224512 & -0.345416 \\ \mathrm{H} & 4.910926 & -1.618243 & 1.721485 \\ \mathrm{H} & 4.864307 & -2.733919 & -2.433063 \\ \mathrm{C} & 3.821186 & 3.182111 & 1.349534 \\ \mathrm{H} & 4.618075 & 3.352140 & -0.647306 \\ \mathrm{H} & 2.792678 & 2.831539 & 3.213285 \\ \mathrm{H} & 6.047618 & -2.624214 & -0.248392 \\ \mathrm{H} & 4.590503 & 3.804452 & 1.796848\end{array}$

\section{TS26t}

$\begin{array}{llll}\mathrm{Si} & -0.873689 & 2.885557 & -1.487999 \\ \mathrm{~N} & -1.104700 & 2.065835 & 0.018185 \\ \mathrm{C} & -0.773321 & 1.624570 & -2.900875 \\ \mathrm{Si} & -1.440683 & 2.825677 & 1.525970 \\ \mathrm{Li} & 0.445813 & 0.945050 & 0.231131 \\ \mathrm{Li} & -2.404268 & 0.625999 & 0.101362 \\ \mathrm{C} & -2.272010 & 4.090560 & -1.927819 \\ \mathrm{C} & 0.754170 & 3.868222 & -1.501359 \\ \mathrm{H} & -1.763892 & 1.255587 & -3.189525 \\ \mathrm{H} & -0.329506 & 2.085442 & -3.792926 \\ \mathrm{H} & -0.173891 & 0.747500 & -2.634264 \\ \mathrm{C} & -1.469702 & 4.718930 & 1.561384 \\ \mathrm{C} & -0.142725 & 2.290670 & 2.812490 \\ \mathrm{C} & -3.160302 & 2.250850 & 2.118252 \\ \mathrm{O} & 0.864642 & -0.811910 & 0.836895 \\ \mathrm{~N} & -2.548094 & -1.296725 & 0.058114 \\ \mathrm{H} & -2.168444 & 4.446344 & -2.960811 \\ \mathrm{H} & -3.252060 & 3.603841 & -1.843290\end{array}$




\begin{tabular}{|c|c|c|c|}
\hline $\mathrm{H}$ & -2.279965 & 4.969999 & -1.275203 \\
\hline $\mathrm{H}$ & 0.928662 & 4.351206 & -2.471223 \\
\hline $\mathrm{H}$ & 1.615786 & 3.220528 & -1.293442 \\
\hline $\mathrm{H}$ & 0.748887 & 4.654546 & -0.737093 \\
\hline $\mathrm{H}$ & -0.536739 & 5.147421 & 1.177496 \\
\hline $\mathrm{H}$ & -1.597301 & 5.073777 & 2.591953 \\
\hline $\mathrm{H}$ & -2.293664 & 5.126851 & 0.966419 \\
\hline $\mathrm{H}$ & -0.011245 & 1.201823 & 2.849459 \\
\hline $\mathrm{H}$ & 0.830650 & 2.741710 & 2.573690 \\
\hline $\mathrm{H}$ & -0.410706 & 2.616017 & 3.825145 \\
\hline $\mathrm{H}$ & -3.277098 & 1.158893 & 2.151258 \\
\hline $\mathrm{H}$ & -3.935630 & 2.652624 & 1.451189 \\
\hline $\mathrm{H}$ & -3.381936 & 2.609086 & 3.131167 \\
\hline $\mathrm{C}$ & 1.388869 & -1.343910 & 2.039835 \\
\hline $\mathrm{Si}$ & -2.631043 & -2.269306 & 1.473148 \\
\hline $\mathrm{Si}$ & -3.476612 & -1.400717 & -1.390703 \\
\hline $\mathrm{Li}$ & -0.631122 & -1.562147 & -0.302405 \\
\hline $\mathrm{H}$ & 1.715410 & -2.381211 & 1.907150 \\
\hline $\mathrm{H}$ & 2.244756 & -0.761121 & 2.404331 \\
\hline $\mathrm{H}$ & 0.601915 & -1.322959 & 2.799175 \\
\hline $\mathrm{C}$ & -4.297139 & -3.078350 & 1.868987 \\
\hline $\mathrm{C}$ & -2.180750 & -1.249518 & 3.011054 \\
\hline $\mathrm{C}$ & -1.366976 & -3.695970 & 1.328661 \\
\hline $\mathrm{C}$ & -4.951501 & -2.587767 & -1.389900 \\
\hline $\mathrm{C}$ & -2.392130 & -1.887580 & -2.866043 \\
\hline $\mathrm{C}$ & -4.215119 & 0.332528 & -1.722422 \\
\hline $\mathrm{O}$ & 0.723635 & -1.217349 & -1.497668 \\
\hline $\mathrm{H}$ & -5.103908 & -2.335447 & 1.887220 \\
\hline $\mathrm{H}$ & -4.254534 & -3.544426 & 2.861883 \\
\hline $\mathrm{H}$ & -4.574816 & -3.854012 & 1.148867 \\
\hline $\mathrm{H}$ & -3.054467 & -0.705920 & 3.390259 \\
\hline $\mathrm{H}$ & -1.818557 & -1.890302 & 3.824780 \\
\hline $\mathrm{H}$ & -1.404429 & -0.508179 & 2.797148 \\
\hline $\mathrm{H}$ & -1.637478 & -4.353980 & 0.491665 \\
\hline $\mathrm{H}$ & -0.333754 & -3.361045 & 1.159862 \\
\hline $\mathrm{H}$ & -1.345189 & -4.311529 & 2.236848 \\
\hline $\mathrm{H}$ & -4.633108 & -3.627341 & -1.249007 \\
\hline $\mathrm{H}$ & -5.469481 & -2.530480 & -2.355746 \\
\hline $\mathrm{H}$ & -5.677746 & -2.351994 & -0.605100 \\
\hline $\mathrm{H}$ & -2.944302 & -1.823770 & -3.812294 \\
\hline $\mathrm{H}$ & -2.049368 & -2.925827 & -2.756533 \\
\hline $\mathrm{H}$ & -1.500326 & -1.258729 & -2.954713 \\
\hline
\end{tabular}




\begin{tabular}{|c|c|c|c|}
\hline $\mathrm{H}$ & -3.478815 & 1.145861 & -1.776765 \\
\hline $\mathrm{H}$ & -4.945371 & 0.591200 & -0.94228 \\
\hline $\mathrm{H}$ & -4.746479 & 0.351654 & -2.68183 \\
\hline $\mathrm{C}$ & 1.698764 & -0.959307 & -0.711986 \\
\hline $\mathrm{N}$ & 2.123342 & 0.452148 & -0.877106 \\
\hline C & 2.743455 & -2.037299 & -0.506761 \\
\hline $\mathrm{C}$ & 3.332217 & 1.008537 & -0.354811 \\
\hline $\mathrm{H}$ & 2.015537 & 0.644686 & -1.869819 \\
\hline $\mathrm{C}$ & 2.501385 & -3.255266 & -1.157875 \\
\hline $\mathrm{C}$ & 3.899837 & -1.911439 & 0.277205 \\
\hline $\mathrm{C}$ & 4.457458 & 1.174050 & -1.168161 \\
\hline $\mathrm{C}$ & 3.378917 & 1.436694 & 0.978453 \\
\hline $\mathrm{C}$ & 3.389796 & -4.321575 & -1.033293 \\
\hline $\mathrm{H}$ & 1.612260 & -3.340628 & -1.771674 \\
\hline $\mathrm{C}$ & 4.788758 & -2.979409 & 0.399210 \\
\hline $\mathrm{H}$ & 4.113170 & -0.995646 & 0.809184 \\
\hline $\mathrm{C}$ & 5.619775 & 1.746547 & -0.651206 \\
\hline $\mathrm{H}$ & 4.422439 & 0.836472 & -2.200346 \\
\hline $\mathrm{C}$ & 4.549055 & 1.987472 & 1.498663 \\
\hline $\mathrm{H}$ & 2.503113 & 1.329528 & 1.609943 \\
\hline $\mathrm{C}$ & 4.539325 & -4.186861 & -0.253458 \\
\hline $\mathrm{H}$ & 3.185529 & -5.255332 & -1.549555 \\
\hline $\mathrm{H}$ & 5.677991 & -2.863844 & 1.012373 \\
\hline $\mathrm{C}$ & 5.674014 & 2.144588 & 0.685481 \\
\hline $\mathrm{H}$ & 6.487784 & 1.869388 & -1.292308 \\
\hline $\mathrm{H}$ & 4.575828 & 2.307198 & 2.536322 \\
\hline $\mathrm{H}$ & 5.234662 & -5.015666 & -0.154436 \\
\hline $\mathrm{H}$ & 6.582170 & 2.581990 & 1.088898 \\
\hline
\end{tabular}

27d

N $\quad-1.775445 \quad-0.709743 \quad 0.157510$

Si $\quad-2.689752-1.117093 \quad 1.544288$

$\mathrm{Si} \quad-2.261194-0.741249-1.491961$

C $\quad-4.547982 \quad-0.766241 \quad 1.422056$

$\begin{array}{llll}\text { C } & -2.448636 & -2.916875 & 2.077702\end{array}$

$\begin{array}{llll}\text { C } & -2.008994 & -0.011736 & 2.961883\end{array}$

$\begin{array}{llll}\mathrm{Li} & -0.762683 & 0.693406 & 0.856394\end{array}$

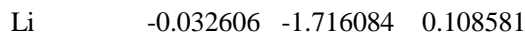

$\begin{array}{lllll}\text { C } & -3.034006 & -2.398851 & -1.986283\end{array}$

C $\quad-3.496832 \quad 0.641962 \quad-1.905052$

$\begin{array}{llll}\text { C } & -0.710936 & -0.470530 & -2.555579\end{array}$

$\begin{array}{llll}\mathrm{H} & -5.071884 & -1.024377 & 2.350838\end{array}$ 


\begin{tabular}{|c|c|c|c|}
\hline $\mathrm{H}$ & -4.737807 & 0.293327 & 1.210576 \\
\hline $\mathrm{H}$ & -5.003821 & -1.348460 & 0.612107 \\
\hline $\mathrm{H}$ & -1.386465 & -3.149796 & 2.230159 \\
\hline $\mathrm{H}$ & -2.982139 & -3.156696 & 3.006121 \\
\hline $\mathrm{H}$ & -2.813576 & -3.591321 & 1.29333 \\
\hline $\mathrm{H}$ & -0.930199 & -0.158109 & 3.12752 \\
\hline $\mathrm{H}$ & -2.191599 & 1.059655 & 2.776727 \\
\hline $\mathrm{H}$ & -2.498361 & -0.242069 & 3.915603 \\
\hline $\mathrm{H}$ & -2.323176 & -3.213785 & -1.80131 \\
\hline $\mathrm{H}$ & -3.312860 & -2.428800 & -3.04708 \\
\hline $\mathrm{H}$ & -3.937749 & -2.608025 & -1.399563 \\
\hline $\mathrm{H}$ & -3.738076 & 0.672345 & -2.975413 \\
\hline $\mathrm{H}$ & -3.084293 & 1.619715 & -1.625145 \\
\hline $\mathrm{H}$ & -4.436239 & 0.510899 & -1.354956 \\
\hline $\mathrm{H}$ & -0.243057 & 0.496766 & -2.327113 \\
\hline $\mathrm{H}$ & 0.033520 & -1.254759 & -2.364575 \\
\hline $\mathrm{H}$ & -0.945125 & -0.479469 & -3.62733 \\
\hline $\mathrm{C}$ & 2.073075 & 0.788882 & 0.402781 \\
\hline $\mathrm{N}$ & 1.964971 & 2.063082 & -0.098506 \\
\hline $\mathrm{C}$ & 3.414543 & 0.172752 & 0.344959 \\
\hline $\mathrm{O}$ & 1.073678 & 0.211150 & 0.875140 \\
\hline $\mathrm{C}$ & 0.727288 & 2.760340 & -0.147225 \\
\hline $\mathrm{H}$ & 2.711603 & 2.377621 & -0.703484 \\
\hline $\mathrm{C}$ & 3.511286 & -1.219640 & 0.188996 \\
\hline $\mathrm{C}$ & 4.571786 & 0.965928 & 0.447388 \\
\hline $\mathrm{C}$ & 0.183591 & 3.151314 & -1.373781 \\
\hline $\mathrm{C}$ & 0.023156 & 3.007873 & 1.042306 \\
\hline $\mathrm{C}$ & 4.779666 & -1.798204 & 0.121307 \\
\hline $\mathrm{H}$ & 2.602181 & -1.831320 & 0.073214 \\
\hline $\mathrm{C}$ & 5.827355 & 0.369639 & 0.392006 \\
\hline $\mathrm{H}$ & 4.491925 & 2.036636 & 0.617467 \\
\hline $\mathrm{C}$ & -1.063970 & 3.776464 & -1.412671 \\
\hline $\mathrm{H}$ & 0.722161 & 2.938862 & -2.292238 \\
\hline $\mathrm{C}$ & -1.237599 & 3.608945 & 0.991583 \\
\hline $\mathrm{H}$ & 0.470518 & 2.734781 & 1.993887 \\
\hline $\mathrm{C}$ & 5.930479 & -1.014703 & 0.222891 \\
\hline $\mathrm{H}$ & 4.862238 & -2.872087 & -0.014552 \\
\hline $\mathrm{H}$ & 6.721370 & 0.978267 & 0.489884 \\
\hline $\mathrm{C}$ & -1.783804 & 3.992386 & -0.236648 \\
\hline $\mathrm{H}$ & -1.485460 & 4.066671 & -2.369670 \\
\hline $\mathrm{H}$ & -1.782799 & 3.788073 & 1.913352 \\
\hline $\mathrm{H}$ & 6.911348 & -1.479858 & 0.175021 \\
\hline
\end{tabular}




\begin{tabular}{|c|c|c|c|}
\hline $\mathrm{H}$ & -2.764203 & 4.455742 & -0.275371 \\
\hline $\mathrm{C}$ & 1.600872 & -3.738540 & -1.349242 \\
\hline $\mathrm{H}$ & 2.689172 & -3.592283 & -1.541568 \\
\hline $\mathrm{H}$ & 1.497901 & -4.789307 & -1.010803 \\
\hline $\mathrm{H}$ & 1.115586 & -3.689138 & -2.348320 \\
\hline & .09504 & -2.8 & -0.4 \\
\hline
\end{tabular}

$27 t$

N $\quad 1.898955-2.863983-0.258680$

$\mathrm{Li} \quad-0.841627 \quad-1.236705 \quad 0.040460$

$\begin{array}{llll}\mathrm{Li} & 1.349335 & 0.787809 & -0.571159\end{array}$

$\mathrm{N} \quad 0.014363 \quad 2.251461 \quad-0.513845$

$\begin{array}{lllll}\mathrm{Li} & & -1.440871 & 1.419468 & 0.446025\end{array}$

$\mathrm{N} \quad-2.245217 \quad-0.261644 \quad 0.956052$

$\mathrm{Si} \quad-0.323964 \quad 2.533492 \quad-2.187095$

$\begin{array}{llll}\mathrm{Si} & 0.415661 & 3.515293 & 0.586426\end{array}$

$\begin{array}{llll}\text { Si } & -3.750912 & -0.377072 & 0.118979\end{array}$

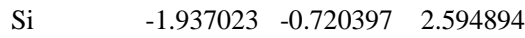

$\begin{array}{lllll}\text { C } & -0.915576 & 0.901313 & -2.953085\end{array}$

$\mathrm{H} \quad \begin{array}{llll}\mathrm{H} & -0.101148 & 0.170158 & -2.908420\end{array}$

$\mathrm{H} \quad \begin{array}{lllll}\mathrm{H} & -1.785065 & 0.485778 & -2.431738\end{array}$

$\begin{array}{lllll}\mathrm{H} & & -1.195431 & 1.028179 & -4.006133\end{array}$

$\begin{array}{llll}\text { C } & 1.212833 & 3.049034 & -3.166184\end{array}$

$\mathrm{H} \quad 1.008047 \quad 3.102460 \quad-4.243116$

$\mathrm{H} \quad \begin{array}{llll}1.592806 & 4.026738 & -2.849801\end{array}$

$\begin{array}{llll}\mathrm{H} & 2.007425 & 2.309257 & -3.011492\end{array}$

$\begin{array}{lllll}\text { C } & -1.674014 & 3.839488 & -2.484312\end{array}$

$\mathrm{H} \quad \begin{array}{lllll}-1.340966 & 4.837376 & -2.177479\end{array}$

$\begin{array}{lllll}\mathrm{H} & & -1.939685 & 3.895319 & -3.547735\end{array}$

$\mathrm{H} \quad \begin{array}{llll}\mathrm{H} & -2.591064 & 3.616287 & -1.926183\end{array}$

$\begin{array}{llll}\text { C } & -1.125193 & 3.904081 & 1.649484\end{array}$

$\begin{array}{llll}\mathrm{H} & -0.908591 & 4.645718 & 2.428581\end{array}$

$\begin{array}{llll}\mathrm{H} & -1.926370 & 4.310923 & 1.017819\end{array}$

$\begin{array}{llll}\mathrm{H} & -1.529928 & 3.023933 & 2.172961\end{array}$

$\begin{array}{llll}\text { C } & 0.987601 & 5.157809 & -0.168676\end{array}$

$\begin{array}{llll}\mathrm{H} & 1.165733 & 5.892751 & 0.626680\end{array}$

$\mathrm{H} \quad 1.928819 \quad 5.030260 \quad-0.716286$

$\begin{array}{llll}\mathrm{H} & 0.256788 & 5.589515 & -0.860205\end{array}$

$\begin{array}{llll}\text { C } & 1.804594 & 2.984517 & 1.766556\end{array}$

$\begin{array}{llll}\mathrm{H} & 2.123386 & 3.822999 & 2.398579\end{array}$

$\begin{array}{llll}\mathrm{H} & 1.489683 & 2.174862 & 2.432226\end{array}$

$\begin{array}{llll}\mathrm{H} & 2.687311 & 2.639454 & 1.211691\end{array}$ 


\begin{tabular}{|c|c|c|c|}
\hline $\mathrm{C}$ & -0.482659 & 0.287889 & 3.270790 \\
\hline $\mathrm{H}$ & -0.757858 & 1.330543 & 3.470007 \\
\hline $\mathrm{H}$ & -0.111637 & -0.137026 & 4.211800 \\
\hline $\mathrm{H}$ & 0.341622 & 0.283483 & 2.552829 \\
\hline $\mathrm{C}$ & -3.356717 & -0.478740 & 3.825532 \\
\hline $\mathrm{H}$ & -3.786699 & 0.527023 & 3.740750 \\
\hline $\mathrm{H}$ & -4.168134 & -1.200464 & 3.695355 \\
\hline $\mathrm{H}$ & -2.973037 & -0.588363 & 4.847953 \\
\hline $\mathrm{C}$ & -1.418861 & -2.552814 & 2.663588 \\
\hline $\mathrm{H}$ & -1.240406 & -2.890869 & 3.692119 \\
\hline $\mathrm{H}$ & -2.198126 & -3.194929 & 2.231839 \\
\hline $\mathrm{H}$ & -0.489832 & -2.730138 & 2.103526 \\
\hline $\mathrm{C}$ & -4.179432 & 1.321312 & -0.617057 \\
\hline $\mathrm{H}$ & -5.122688 & 1.292383 & -1.176459 \\
\hline $\mathrm{H}$ & -4.293016 & 2.072963 & 0.176498 \\
\hline $\mathrm{H}$ & -3.412392 & 1.681526 & -1.312467 \\
\hline $\mathrm{C}$ & -3.620333 & -1.616505 & -1.315432 \\
\hline $\mathrm{H}$ & -2.817775 & -1.373774 & -2.021006 \\
\hline $\mathrm{H}$ & -3.427676 & -2.629736 & -0.938485 \\
\hline $\mathrm{H}$ & -4.553060 & -1.654540 & -1.892559 \\
\hline $\mathrm{C}$ & -5.255163 & -0.931166 & 1.127677 \\
\hline $\mathrm{H}$ & -6.126711 & -1.008821 & 0.465257 \\
\hline $\mathrm{H}$ & -5.101255 & -1.915267 & 1.586029 \\
\hline $\mathrm{H}$ & -5.505449 & -0.225796 & 1.926444 \\
\hline $\mathrm{H}$ & 2.740121 & -3.423497 & -0.284759 \\
\hline $\mathrm{C}$ & 0.782633 & -3.290507 & -1.041573 \\
\hline $\mathrm{C}$ & 0.422970 & -2.540853 & -2.171357 \\
\hline $\mathrm{C}$ & 0.070032 & -4.438411 & -0.683827 \\
\hline $\mathrm{C}$ & -0.666983 & -2.952732 & -2.939098 \\
\hline $\mathrm{H}$ & 1.010072 & -1.646744 & -2.385553 \\
\hline $\mathrm{C}$ & -1.005092 & -4.851288 & -1.475649 \\
\hline $\mathrm{H}$ & 0.355595 & -4.994275 & 0.204192 \\
\hline $\mathrm{C}$ & -1.375157 & -4.109491 & -2.599222 \\
\hline $\mathrm{H}$ & -0.959109 & -2.367414 & -3.805646 \\
\hline $\mathrm{H}$ & -1.558395 & -5.745663 & -1.205398 \\
\hline $\mathrm{H}$ & -2.221208 & -4.424742 & -3.202089 \\
\hline $\mathrm{C}$ & 1.992432 & -1.610635 & 0.252881 \\
\hline $\mathrm{O}$ & 0.943631 & -0.930126 & 0.462406 \\
\hline $\mathrm{C}$ & 3.302824 & -1.211705 & 0.818867 \\
\hline$c$ & 3.320721 & -0.328982 & 1.906175 \\
\hline $\mathrm{C}$ & 4.515330 & -1.698482 & 0.307753 \\
\hline $\mathrm{C}$ & 4.530450 & 0.066829 & 2.470419 \\
\hline
\end{tabular}




\begin{tabular}{|c|c|c|c|}
\hline $\mathrm{H}$ & 2.383060 & 0.034435 & 2.305960 \\
\hline $\mathrm{C}$ & 5.723809 & -1.304257 & 0.877205 \\
\hline $\mathrm{H}$ & 4.522386 & -2.343082 & -0.565728 \\
\hline $\mathrm{C}$ & 5.735143 & -0.419896 & 1.958021 \\
\hline $\mathrm{H}$ & 4.530583 & 0.753560 & 3.311480 \\
\hline $\mathrm{H}$ & 6.657872 & -1.674658 & 0.465414 \\
\hline $\mathrm{H}$ & 6.679156 & -0.109581 & 2.396256 \\
\hline $\mathrm{O}$ & 2.343740 & -0.219111 & -1.634624 \\
\hline $\mathrm{C}$ & 3.428749 & -0.248454 & -2.481363 \\
\hline $\mathrm{H}$ & 4.392301 & -0.332406 & -1.935820 \\
\hline $\mathrm{H}$ & 3.397983 & -1.107892 & -3.185258 \\
\hline $\mathrm{H}$ & 3.513475 & 0.660607 & -3.108880 \\
\hline
\end{tabular}

28d

$\begin{array}{lllll}\text { C } & & 0.937501 & -1.348355 & -0.112629\end{array}$

C $\quad 1.393068-2.446709 \quad 0.628223$

$\begin{array}{llll}\text { C } & 1.830856 & -0.677949 & -0.959495\end{array}$

$\begin{array}{llll}\text { C } & 2.725039 & -2.848966 & 0.550838\end{array}$

$\mathrm{H} \quad 0.689220-2.976882 \quad 1.260962$

$\begin{array}{lllll}\text { C } & -0.514004 & -0.971588 & -0.019813\end{array}$

C $\quad 3.157373-1.094597-1.053216$

$\begin{array}{lllll}\mathrm{H} & & 1.487117 & 0.165936 & -1.546678\end{array}$

C $\quad 3.610604-2.173795-0.291654$

$\mathrm{H} \quad 3.070481-3.693465 \quad 1.140805$

$\begin{array}{llll}\mathrm{N} & -0.949518 & 0.281683 & -0.076080\end{array}$

$\begin{array}{lllll}\mathrm{O} & -1.336713 & -1.945785 & 0.078379\end{array}$

$\mathrm{H} \quad \begin{array}{llll}3.838724 & -0.573705 & -1.719955\end{array}$

$\mathrm{H} \quad 4.647837 \quad-2.490393 \quad-0.358858$

$\begin{array}{llll}\text { C } & -0.109112 & 1.403496 & 0.064310\end{array}$

$\begin{array}{lllll}\text { C } & -0.253289 & 2.475442 & -0.833407\end{array}$

$\begin{array}{llll}\text { C } & 0.803774 & 1.539996 & 1.126038\end{array}$

$\begin{array}{lllll}\text { C } & 0.507889 & 3.635662 & -0.690738\end{array}$

$\mathrm{H} \quad \begin{array}{llll}0.951315 & 2.371669 & -1.661482\end{array}$

$\begin{array}{llll}\text { C } & 1.564026 & 2.698872 & 1.261882\end{array}$

$\begin{array}{llll}\mathrm{H} & 0.911115 & 0.726279 & 1.836110\end{array}$

$\begin{array}{llll}\text { C } & 1.424477 & 3.753230 & 0.355359\end{array}$

$\mathrm{H} \quad 0.386902 \quad 4.447061 \quad-1.403662$

$\begin{array}{llll}\mathrm{H} & 2.267107 & 2.781328 & 2.086586\end{array}$

$\mathrm{H} \quad 2.017526 \quad 4.656056 \quad 0.467510$

$\begin{array}{lllll}\mathrm{O} & -4.219271 & -0.709481 & -0.011385\end{array}$

$\begin{array}{lllll}\text { C } & -5.607888 & -0.558497 & -0.028929\end{array}$

$\begin{array}{lllll}\mathrm{Li} & -3.120137 & -2.090514 & 0.077327\end{array}$ 


$\begin{array}{llll}\mathrm{H} & -6.059633 & -0.957308 & -0.954387 \\ \mathrm{H} & -5.913483 & 0.501528 & 0.038538 \\ \mathrm{H} & -6.095182 & -1.076890 & 0.815868 \\ \mathrm{Li} & -2.887800 & 0.447742 & -0.072443\end{array}$

$28 t$

$\begin{array}{llll}\mathrm{N} & 1.109895 & 0.349639 & 0.327432\end{array}$

$\begin{array}{llll}\text { C } & 1.994481 & 1.440498 & 0.200668\end{array}$

$\begin{array}{llll}\text { C } & 1.474495 & -0.927579 & 0.282603\end{array}$

$\begin{array}{llll}\text { C } & 1.680318 & 2.482962 & -0.682428\end{array}$

$\begin{array}{llll}\text { C } & 3.145048 & 1.550119 & 0.999101\end{array}$

$\mathrm{Li} \quad-0.668849-0.501418-0.310721$

$\begin{array}{llll}\mathrm{Li} & 0.100024 & -0.488140 & 2.078216\end{array}$

$\begin{array}{llll}\text { C } & 2.775623 & -1.477670 & -0.190319\end{array}$

$\begin{array}{lllll}\text { O } & 0.555543 & -1.762936 & 0.658749\end{array}$

$\begin{array}{llll}\text { C } & 2.516975 & 3.593906 & -0.788245\end{array}$

$\begin{array}{lllll}\mathrm{H} & 0.778829 & 2.411744 & -1.281368\end{array}$

$\begin{array}{llll}\text { C } & 3.975699 & 2.662699 & 0.890260\end{array}$

$\begin{array}{llll}\mathrm{H} & 3.385968 & 0.752338 & 1.696121\end{array}$

$\begin{array}{llll}\mathrm{N} & -2.544058 & -0.055161 & -0.048660\end{array}$

$\begin{array}{llll}\text { O } & -1.281214 & -0.117649 & 3.107852\end{array}$

$\begin{array}{llll}\text { C } & 3.569156 & -0.818801 & -1.141775\end{array}$

$\begin{array}{llll}\text { C } & 3.178113 & -2.729463 & 0.297257\end{array}$

$\begin{array}{lllll}\text { C } & 3.669813 & 3.688766 & -0.007210\end{array}$

$\mathrm{H} \quad 2.261906 \quad 4.389680-1.482594$

$\mathrm{H} \quad 4.863938 \quad 2.729565 \quad 1.512395$

$\begin{array}{lllll}\mathrm{Si} & & -3.277654 & -1.542545 & -0.516522\end{array}$

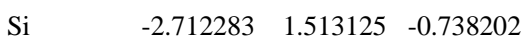

$\begin{array}{llll}\mathrm{Li} & -2.519740 & 0.036492 & 1.869944\end{array}$

$\begin{array}{llll}\text { C } & -1.375812 & 0.025725 & 4.494760\end{array}$

C $\quad 4.753876-1.402431 \quad-1.584772$

$\mathrm{H} \quad 3.261368 \quad 0.141551 \quad-1.537149$

$\begin{array}{lllll}\text { C } & 4.374791 & -3.298832 & -0.132043\end{array}$

$\begin{array}{llll}\mathrm{H} & 2.537045 & -3.243940 & 1.004558\end{array}$

$\mathrm{H} \quad 4.317577 \quad 4.556460 \quad-0.088576$

$\begin{array}{lllll}\text { C } & -4.873967 & -1.389548 & -1.521244\end{array}$

$\begin{array}{lllll}\text { C } & -3.645706 & -2.538820 & 1.058009\end{array}$

$\begin{array}{lllll}\text { C } & & -2.045671 & -2.557473 & -1.556751\end{array}$

$\begin{array}{lllll}\text { C } & & -1.853673 & 1.601467 & -2.436628\end{array}$

$\begin{array}{llll}\text { C } & -1.879839 & 2.752322 & 0.432447\end{array}$

$\begin{array}{lllll}\text { C } & -4.499805 & 2.089863 & -0.987672\end{array}$

$\begin{array}{llll}\mathrm{H} & -2.405515 & 0.264606 & 4.819802\end{array}$ 


\begin{tabular}{llll}
$\mathrm{H}$ & -0.732334 & 0.838753 & 4.875517 \\
$\mathrm{H}$ & -1.086256 & -0.895347 & 5.031321 \\
$\mathrm{C}$ & 5.164550 & -2.635847 & -1.073759 \\
$\mathrm{H}$ & 5.358346 & -0.891767 & -2.328500 \\
$\mathrm{H}$ & 4.685880 & -4.262885 & 0.259584 \\
$\mathrm{H}$ & -5.642638 & -0.834569 & -0.971354 \\
$\mathrm{H}$ & -5.280262 & -2.380538 & -1.758949 \\
$\mathrm{H}$ & -4.701883 & -0.865975 & -2.469464 \\
$\mathrm{H}$ & -2.739630 & -2.662828 & 1.666859 \\
$\mathrm{H}$ & -4.404386 & -2.041929 & 1.680442 \\
$\mathrm{H}$ & -4.025380 & -3.542764 & 0.832321 \\
$\mathrm{H}$ & -1.129511 & -2.797523 & -0.999095 \\
$\mathrm{H}$ & -1.762693 & -2.013005 & -2.469517 \\
$\mathrm{H}$ & -2.479704 & -3.513479 & -1.875204 \\
$\mathrm{H}$ & -1.852951 & 2.616078 & -2.854222 \\
$\mathrm{H}$ & -0.806375 & 1.270274 & -2.391417 \\
$\mathrm{H}$ & -2.366355 & 0.947753 & -3.154285 \\
$\mathrm{H}$ & -1.844464 & 3.760424 & 0.001218 \\
$\mathrm{H}$ & -2.428801 & 2.829398 & 1.382014 \\
$\mathrm{H}$ & -0.852927 & 2.455920 & 0.674083 \\
$\mathrm{H}$ & -4.530373 & 3.138031 & -1.311331 \\
$\mathrm{H}$ & -5.069555 & 2.016699 & -0.052179 \\
$\mathrm{H}$ & -5.024809 & 1.492831 & -1.740478 \\
$\mathrm{H}$ & 6.094475 & -3.082294 & -1.414490 \\
& & & \\
\hline
\end{tabular}

\section{$(\text { TMS })_{2} \mathbf{N H}$}

$\begin{array}{llll}\mathrm{N} & -0.000003 & -0.022736 & 0.825870 \\ \mathrm{Si} & -1.586292 & -0.010708 & 0.089797 \\ \mathrm{Si} & 1.586140 & 0.006296 & 0.089473 \\ \mathrm{C} & -2.818476 & -0.633586 & 1.377282 \\ \mathrm{H} & -2.811986 & -0.005680 & 2.277102 \\ \mathrm{H} & -3.840537 & -0.622937 & 0.980005 \\ \mathrm{H} & -2.588752 & -1.660981 & 1.682821 \\ \mathrm{C} & -1.588808 & -1.145176 & -1.419618 \\ \mathrm{H} & -0.862970 & -0.819319 & -2.174139 \\ \mathrm{H} & -1.336671 & -2.174087 & -1.139168 \\ \mathrm{H} & -2.574699 & -1.155901 & -1.900273 \\ \mathrm{C} & -2.089795 & 1.722599 & -0.469035 \\ \mathrm{H} & -3.081727 & 1.721227 & -0.938497 \\ \mathrm{H} & -2.120022 & 2.418384 & 0.377954 \\ \mathrm{H} & -1.375846 & 2.122151 & -1.198727 \\ \mathrm{C} & 2.820577 & 0.530001 & 1.418474\end{array}$




$\begin{array}{llll}\mathrm{H} & 3.842125 & 0.547404 & 1.020180 \\ \mathrm{H} & 2.593983 & 1.531946 & 1.801257 \\ \mathrm{H} & 2.813560 & -0.164397 & 2.267964 \\ \mathrm{C} & 2.077239 & -1.689164 & -0.584024 \\ \mathrm{H} & 2.103984 & -2.438848 & 0.215751 \\ \mathrm{H} & 1.360008 & -2.035952 & -1.336968 \\ \mathrm{H} & 3.068545 & -1.663099 & -1.053944 \\ \mathrm{C} & 1.599439 & 1.241457 & -1.338775 \\ \mathrm{H} & 0.870945 & 0.974288 & -2.113558 \\ \mathrm{H} & 1.355078 & 2.250229 & -0.987165 \\ \mathrm{H} & 2.585343 & 1.278311 & -1.818138 \\ \mathrm{H} & 0.000737 & -0.038601 & 1.840850\end{array}$

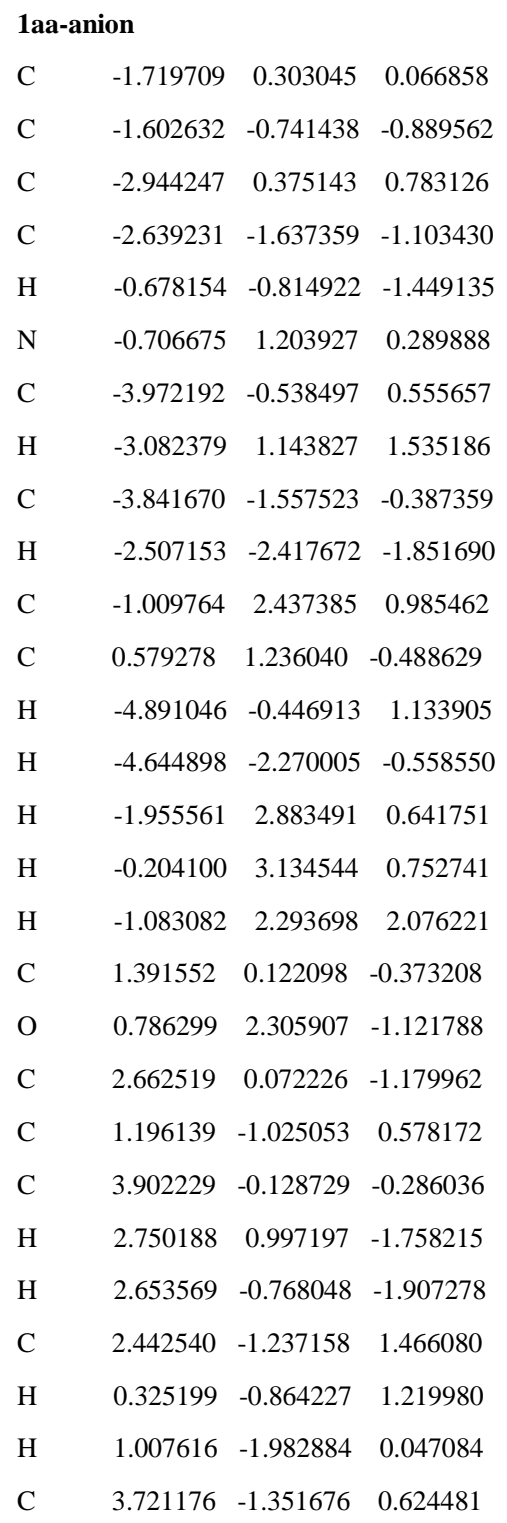




$\begin{array}{llll}\mathrm{H} & 4.816138 & -0.244404 & -0.891833 \\ \mathrm{H} & 4.037509 & 0.766302 & 0.338394 \\ \mathrm{H} & 2.325433 & -2.134964 & 2.094695 \\ \mathrm{H} & 2.539191 & -0.376152 & 2.143713 \\ \mathrm{H} & 4.600143 & -1.490190 & 1.272955 \\ \mathrm{H} & 3.651800 & -2.254448 & -0.004729\end{array}$

1aa-ketene

$\begin{array}{llll}\text { C } & -0.713979 & -0.000193 & 0.315128\end{array}$

C $\quad 0.062357 \quad-1.292994 \quad 0.500077$

$\begin{array}{llll}\text { C } & 0.062058 & 1.292816 & 0.500172\end{array}$

C $\quad 1.337663-1.271162-0.359906$

$\begin{array}{llll}\mathrm{H} & -0.553858 & -2.164482 & 0.255994\end{array}$

$\mathrm{H} \quad 0.346597 \quad-1.390047 \quad 1.559664$

$\begin{array}{lllll}\text { C } & -1.970608 & -0.000181 & -0.067182\end{array}$

$\begin{array}{llll}\text { C } & 1.337358 & 1.271356 & -0.359820\end{array}$

$\begin{array}{llll}\mathrm{H} & 0.346282 & 1.389865 & 1.559764\end{array}$

$\mathrm{H} \quad-0.554367 \quad 2.164174 \quad 0.256156$

C $\quad 2.160969 \quad 0.000187 \quad-0.106197$

H $\quad 1.053239-1.315694 \quad-1.419691$

$\mathrm{H} \quad \begin{array}{llll}1.941191 & -2.162933 & -0.148489\end{array}$

$\begin{array}{lllll}\text { O } & -3.099583 & 0.000062 & -0.403552\end{array}$

$\mathrm{H} \quad 1.052916 \quad 1.315886 \quad-1.419601$

$\mathrm{H} \quad 1.940679 \quad 2.163256 \quad-0.148354$

$\begin{array}{llll}\mathrm{H} & 2.511305 & 0.000193 & 0.936858\end{array}$

H $\quad 3.057786 \quad 0.000314 \quad-0.737516$

\begin{tabular}{llll}
\multicolumn{4}{l}{ 1z-anion } \\
C & -0.869376 & 0.359020 & 0.005789 \\
C & -0.941459 & -0.950857 & -0.541400 \\
C & -2.099347 & 0.952035 & 0.398743 \\
C & -2.156449 & -1.604286 & -0.680353 \\
H & -0.015916 & -1.419717 & -0.852860 \\
N & 0.323236 & 1.025325 & 0.142824 \\
C & -3.309479 & 0.275233 & 0.255004 \\
H & -2.101605 & 1.945054 & 0.834238 \\
C & -3.362973 & -1.009658 & -0.284720 \\
H & -2.163741 & -2.603979 & -1.112862 \\
C & 0.302273 & 2.458776 & 0.346382 \\
C & 1.645974 & 0.491265 & -0.340050 \\
H & -4.226354 & 0.768874 & 0.575997 \\
H & -4.309116 & -1.534710 & -0.391649
\end{tabular}




$\begin{array}{llll}\mathrm{H} & -0.434523 & 2.958133 & -0.301633 \\ \mathrm{H} & 1.292482 & 2.827723 & 0.076459 \\ \mathrm{H} & 0.068053 & 2.729043 & 1.389631 \\ \mathrm{C} & 2.102466 & -0.654304 & 0.285440 \\ \mathrm{O} & 2.201670 & 1.191411 & -1.230824 \\ \mathrm{C} & 3.376104 & -1.274388 & -0.218478 \\ \mathrm{C} & 1.468272 & -1.306105 & 1.479303 \\ \mathrm{H} & 3.749202 & -0.699113 & -1.071219 \\ \mathrm{H} & 3.249140 & -2.326737 & -0.541863 \\ \mathrm{H} & 0.697670 & -0.674757 & 1.932769 \\ \mathrm{H} & 0.987026 & -2.281901 & 1.263099 \\ \mathrm{H} & 2.221795 & -1.516967 & 2.262004 \\ \mathrm{H} & 4.173844 & -1.299895 & 0.550744\end{array}$

\begin{tabular}{lccc}
\multicolumn{4}{l}{ 1z-ketene } \\
C & 0.000019 & -0.337434 & -0.000000 \\
C & 0.000019 & -1.102355 & 1.305742 \\
C & 0.000019 & -1.102355 & -1.305742 \\
H & 0.000012 & -0.438682 & 2.175347 \\
H & -0.885595 & -1.748490 & 1.375556 \\
H & 0.885660 & -1.748464 & 1.375535 \\
C & -0.000054 & 0.978887 & 0.000000 \\
H & 0.000012 & -0.438682 & -2.175347 \\
H & -0.885595 & -1.748490 & -1.375556 \\
H & 0.885660 & -1.748464 & -1.375535 \\
O & -0.000023 & 2.156351 & 0.000000
\end{tabular}


$\begin{array}{llll}\mathrm{H} & -2.758352 & 0.982280 & 0.882706 \\ \mathrm{H} & -2.758298 & 0.982355 & -0.882701\end{array}$

$\begin{array}{llll}\mathrm{H} & -2.758298 & 0.982355 & -0.882701\end{array}$

\begin{tabular}{lccc}
\multicolumn{1}{l}{ lad-amine } & & & \\
$\mathrm{C}$ & 0.671976 & -0.570464 & 0.325626 \\
$\mathrm{C}$ & -0.679489 & -1.170283 & 0.678670 \\
$\mathrm{C}$ & 0.746427 & 0.801304 & 0.041691 \\
$\mathrm{C}$ & -1.536619 & -0.105349 & 1.387362 \\
$\mathrm{C}$ & -1.393001 & -1.585672 & -0.595766 \\
$\mathrm{H}$ & -0.532527 & -2.045319 & 1.324495 \\
$\mathrm{C}$ & 1.823548 & -1.357649 & 0.233449 \\
$\mathrm{C}$ & -0.475068 & 1.700016 & 0.211724 \\
$\mathrm{C}$ & 1.975684 & 1.358264 & -0.322617 \\
$\mathrm{~N}$ & -1.746430 & 1.031326 & 0.490444 \\
$\mathrm{H}$ & -1.041625 & 0.249853 & 2.298895 \\
$\mathrm{H}$ & -2.510106 & -0.522128 & 1.668899 \\
$\mathrm{C}$ & -2.222064 & -0.748267 & -1.221401 \\
$\mathrm{H}$ & -1.176081 & -2.570214 & -1.004142 \\
$\mathrm{C}$ & 3.043827 & -0.797949 & -0.142154 \\
$\mathrm{H}$ & 1.757969 & -2.419877 & 0.459348 \\
$\mathrm{H}$ & -0.269283 & 2.384240 & 1.049593 \\
$\mathrm{H}$ & -0.600758 & 2.334697 & -0.672358 \\
$\mathrm{C}$ & 3.119472 & 0.567464 & -0.424899 \\
$\mathrm{H}$ & 2.036223 & 2.425267 & -0.529832 \\
$\mathrm{C}$ & -2.526790 & 0.631845 & -0.684562 \\
$\mathrm{H}$ & -2.742340 & -1.050097 & -2.129054 \\
$\mathrm{H}$ & 3.930670 & -1.421770 & -0.211154 \\
$\mathrm{H}$ & 4.064774 & 1.013966 & -0.720806 \\
$\mathrm{H}$ & -2.382865 & 1.385049 & -1.468676 \\
& -3.596460 & 0.677496 & -0.421054
\end{tabular}

\section{1ad-hydrocarbon}

C $\quad 0.690795 \quad-0.556926 \quad 0.327173$

$\begin{array}{llll}\text { C } & -0.653798 & -1.178131 & 0.669377\end{array}$

$\begin{array}{lllll}\text { C } & & 0.754881 & 0.813086 & 0.029470\end{array}$

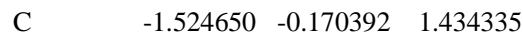

$\begin{array}{llll}\text { C } & -1.340987 & -1.581695 & -0.622125\end{array}$

$\begin{array}{llll}\mathrm{H} & -0.485054 & -2.071570 & 1.283078\end{array}$

$\begin{array}{llll}\text { C } & 1.848698 & -1.337568 & 0.252240\end{array}$

$\begin{array}{llll}\text { C } & -0.461066 & 1.717903 & 0.149457\end{array}$

C $\quad 1.987082 \quad 1.367153 \quad-0.337076$

$\begin{array}{llll}\text { C } & -1.796913 & 1.028788 & 0.514483\end{array}$ 


$\begin{array}{llll}\mathrm{H} & -1.009347 & 0.153702 & 2.346814 \\ \mathrm{H} & -2.467704 & -0.643425 & 1.732958 \\ \mathrm{C} & -2.210665 & -0.781948 & -1.242573 \\ \mathrm{H} & -1.054661 & -2.534610 & -1.062477 \\ \mathrm{C} & 3.069146 & -0.777684 & -0.120323 \\ \mathrm{H} & 1.786306 & -2.397670 & 0.488886 \\ \mathrm{H} & -0.238320 & 2.462522 & 0.926927 \\ \mathrm{H} & -0.582083 & 2.291776 & -0.778716 \\ \mathrm{C} & 3.137234 & 0.584417 & -0.419188 \\ \mathrm{H} & 2.042271 & 2.430943 & -0.560207 \\ \mathrm{C} & -2.619684 & 0.565969 & -0.707149 \\ \mathrm{H} & -2.402641 & 1.768032 & 1.052247 \\ \mathrm{H} & -2.670085 & -1.100456 & -2.177103 \\ \mathrm{H} & 3.960015 & -1.397307 & -0.174138 \\ \mathrm{H} & 4.081779 & 1.035409 & -0.710735 \\ \mathrm{H} & -2.555963 & 1.317905 & -1.506253 \\ \mathrm{H} & -3.684957 & 0.526921 & -0.429892\end{array}$

1ad-ketone

$1.960324-1.296063-0.235845$

$\begin{array}{llll}\text { C } & 0.758761 & -0.761469 & 0.266258\end{array}$

C $\quad 3.151368 \quad 0.585210 \quad-0.339986$

$\mathrm{H} \quad 1.924714 \quad 2.370353 \quad-0.384617$

$\begin{array}{llll}\text { C } & -0.493581 & 1.427212 & 0.175037\end{array}$

C $\quad-0.562383-1.457120 \quad 0.548019$

$\begin{array}{llll}\text { C } & 1.959629 & -1.464842 & 0.161173\end{array}$

$\begin{array}{llll}\text { C } & 3.148902 & -0.798481 & -0.138954\end{array}$

$\begin{array}{llll}\mathrm{H} & 4.077830 & 1.101161 & -0.574370\end{array}$

$\begin{array}{llll}\text { C } & -1.760543 & 0.715197 & 0.661156\end{array}$

$\begin{array}{lllll}\text { O } & -0.528995 & 2.614374 & -0.116170\end{array}$

$\begin{array}{llll}\text { C } & -1.422930 & -0.557346 & 1.448524\end{array}$

$\begin{array}{lllll}\text { C } & -1.280316 & -1.719631 & -0.764323\end{array}$

$\begin{array}{llll}\mathrm{H} & -0.359545 & -2.412138 & 1.047149\end{array}$

$\mathrm{H} \quad 1.963642 \quad-2.540846 \quad 0.317290$

$\begin{array}{llll}\mathrm{H} & 4.076304 & -1.359852 & -0.213566\end{array}$

$\begin{array}{lllll}\text { C } & -2.659987 & 0.358754 & -0.544883\end{array}$

$\begin{array}{llll}\mathrm{H} & -2.289201 & 1.444509 & 1.283389\end{array}$

$\begin{array}{llll}\mathrm{H} & -0.876703 & -0.314157 & 2.367200\end{array}$

$\mathrm{H} \quad \begin{array}{llll}-2.343643 & -1.078681 & 1.735124\end{array}$

$\begin{array}{lllll}\text { C } & & -2.213822 & -0.896796 & -1.249341\end{array}$

$\mathrm{H} \quad-0.969772 \quad-2.595034 \quad-1.330660$ 


\begin{tabular}{|c|c|c|}
\hline $\mathrm{H}$ & -2.683509 & 1.202792 \\
\hline $\mathrm{H}$ & -3.694307 & 0.231637 \\
\hline $\mathrm{H}$ & -2.691010 & -1.122760 \\
\hline
\end{tabular}

1af-amine

$\begin{array}{llll}\mathrm{N} & 1.292898 & -0.002552 & 0.002827\end{array}$

$\begin{array}{llll}\text { C } & 0.797260 & -1.099004 & -0.842100\end{array}$

C $\quad 0.794339-0.183641 \quad 1.373955$

C $\quad-0.762604-1.144694-0.881711$

$\mathrm{H} \quad 1.212692-0.960172-1.846576$

$\mathrm{H} \quad 1.208695 \quad-2.035469-0.448934$

C $\quad 0.801638 \quad 1.277653 \quad-0.526937$

$\begin{array}{lllll}\text { C } & & -0.765491 & -0.187673 & 1.431005\end{array}$

$\mathrm{H} \quad 1.204601-1.124963 \quad 1.756537$

$\begin{array}{llll}\mathrm{H} & 1.208777 & 0.623095 & 1.988955\end{array}$

$\begin{array}{lllll}\text { C } & & -1.294096 & 0.002857 & -0.002719\end{array}$

$\begin{array}{llll}\mathrm{H} & -1.133222 & -1.032260 & -1.907998\end{array}$

$\begin{array}{lllll}\mathrm{H} & & -1.137487 & -2.105843 & -0.508943\end{array}$

$\begin{array}{lllll}\text { C } & -0.757811 & 1.336824 & -0.553986\end{array}$

$\begin{array}{llll}\mathrm{H} & 1.217900 & 1.404602 & -1.532642\end{array}$

$\begin{array}{llll}\mathrm{H} & 1.215216 & 2.077910 & 0.097052\end{array}$

$\begin{array}{lllll}\mathrm{H} & & -1.141473 & -1.130843 & 1.846065\end{array}$

$\begin{array}{llll}\mathrm{H} & -1.137568 & 0.617609 & 2.076235\end{array}$

$\begin{array}{llll}\mathrm{H} & -2.389695 & 0.005234 & -0.004989\end{array}$

$\begin{array}{llll}\mathrm{H} & -1.130435 & 2.169751 & 0.054867\end{array}$

$\mathrm{H} \quad \begin{array}{llll}-1.127702 & 1.495286 & -1.574467\end{array}$

\section{1af-hydrocarbon}

$\begin{array}{llll}\text { C } & 1.298540 & 0.001324 & 0.000764\end{array}$

C $\quad 0.778658 \quad-0.826866-1.188570$

$\begin{array}{llll}\text { C } & 0.776927 & -0.615390 & 1.311646\end{array}$

$\begin{array}{lllll}\text { C } & -0.775459 & -0.899743 & -1.136630\end{array}$

H $\quad \begin{array}{llll}1.113519 & -0.366946 & -2.126471\end{array}$

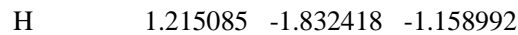

$\begin{array}{lllll}\text { C } & 0.775617 & 1.444484 & -0.121812\end{array}$

$\begin{array}{llll}\text { H } & 2.395367 & 0.002506 & 0.001370\end{array}$

$\begin{array}{lllll}\text { C } & & -0.777315 & -0.535119 & 1.346111\end{array}$

H $\quad \begin{array}{llll}\text { H } & 1.112346 & -1.657435 & 1.382019\end{array}$

$\mathrm{H} \quad \begin{array}{llll}\mathrm{H} & 1.211576 & -0.087424 & 2.168839\end{array}$

$\begin{array}{llll}\text { C } & -1.298538 & -0.001344 & -0.000728\end{array}$

$\begin{array}{lllll}\mathrm{H} & & -1.210682 & -0.581531 & -2.091504\end{array}$

H $\quad-1.109225 \quad-1.931006 \quad-0.967950$ 


$\begin{array}{llll}\mathrm{C} & -0.778421 & 1.432696 & -0.210787 \\ \mathrm{H} & 1.210948 & 1.922876 & -1.007292 \\ \mathrm{H} & 1.109374 & 2.027471 & 0.745452 \\ \mathrm{H} & -1.212051 & -1.521526 & 1.547351 \\ \mathrm{H} & -1.112917 & 0.126427 & 2.154225 \\ \mathrm{H} & -2.395367 & -0.002503 & -0.001365 \\ \mathrm{H} & -1.214864 & 2.099482 & 0.542441 \\ \mathrm{H} & -1.113158 & 1.801776 & -1.188085\end{array}$

1af-ketone

$\begin{array}{lllll}\text { C } & 0.459379 & -1.105651 & -0.000027\end{array}$

C $\quad-0.439541-1.054217-1.257869$

$\begin{array}{lllll}\text { C } & & -0.439248 & -1.054098 & 1.257987\end{array}$

C $\quad-1.298065 \quad 0.244778 \quad-1.255182$

H $\quad \begin{array}{llll}0.180952 & -1.111018 & -2.158605\end{array}$

$\mathrm{H} \quad-1.079531-1.944124-1.260342$

C $\quad 1.339491 \quad 0.133032-0.000185$

$\begin{array}{llll}\mathrm{H} & 1.093660 & -1.996141 & -0.000068\end{array}$

$\begin{array}{llll}\text { C } & -1.297158 & 0.245298 & 1.255819\end{array}$

$\begin{array}{llll}\mathrm{H} & 0.181335 & -1.111429 & 2.158624\end{array}$

$\mathrm{H} \quad-1.079708 \quad-1.943673 \quad 1.260366$

$\begin{array}{llll}\text { C } & -0.981230 & 1.079987 & 0.000029\end{array}$

$\begin{array}{llll}\mathrm{H} & -1.095999 & 0.839951 & -2.153312\end{array}$

$\mathrm{H} \quad-2.366621 \quad 0.001633 \quad-1.272976$

$\begin{array}{llll}\text { C } & 0.517637 & 1.423635 & -0.000566\end{array}$

$\begin{array}{llll}\text { O } & 2.554469 & 0.114432 & -0.000025\end{array}$

$\begin{array}{llll}\mathrm{H} & -2.365808 & 0.002644 & 1.274888\end{array}$

$\begin{array}{llll}\mathrm{H} & -1.093805 & 0.840628 & 2.153554\end{array}$

$\begin{array}{llll}\mathrm{H} & -1.579647 & 1.998179 & 0.000052\end{array}$

$\begin{array}{llll}\mathrm{H} & 0.801181 & 2.016171 & 0.878979\end{array}$

$\begin{array}{llll}\mathrm{H} & 0.800655 & 2.015149 & -0.880995\end{array}$

\section{S1d-1aa}

$\begin{array}{lllll}\mathrm{Si} & & -2.909523 & -1.088070 & -1.279324\end{array}$

$\begin{array}{lllll}\text { C } & -1.843504 & -0.061280 & -2.462119\end{array}$

$\begin{array}{lllll}\text { C } & -4.702442 & -0.499158 & -1.453593\end{array}$

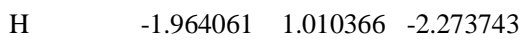

$\begin{array}{lllll}\mathrm{H} & -2.112930 & -0.253067 & -3.508563\end{array}$

$\mathrm{H} \quad \begin{array}{llll}-0.777827 & -0.292467 & -2.344277\end{array}$

$\begin{array}{llll}\text { C } & -2.866516 & -2.888285 & -1.964106\end{array}$

$\begin{array}{lllll}\mathrm{N} & & -2.296133 & -1.144589 & 0.313850\end{array}$

$\mathrm{H} \quad \begin{array}{lllll}4.796656 & 0.560674 & -1.188353\end{array}$ 


\begin{tabular}{|c|c|c|c|}
\hline I & -5.060342 & -0.614090 & -2.484360 \\
\hline $\mathrm{H}$ & -5.377264 & -1.064718 & -0.799700 \\
\hline $\mathrm{H}$ & -3.498298 & -3.564584 & -1.366013 \\
\hline $\mathrm{H}$ & -1.853760 & -3.319705 & -2.0259 \\
\hline $\mathrm{H}$ & -3.257191 & -2.927770 & -2.98795 \\
\hline 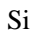 & -3.085427 & -0.910309 & 1.81368 \\
\hline $\mathrm{Li}$ & -0.347347 & -1.080471 & 0.7821 \\
\hline $\mathrm{C}$ & -4.094838 & 0.685840 & 1.980945 \\
\hline $\mathrm{C}$ & -1.746077 & -0.868366 & 3.166510 \\
\hline $\mathrm{C}$ & -4.252392 & -2.358808 & 2.224812 \\
\hline $\mathrm{N}$ & 0.176895 & -3.057871 & 0.701303 \\
\hline $\mathrm{O}$ & 0.658431 & 0.454017 & 0.792973 \\
\hline $\mathrm{H}$ & -4.887527 & 0.743368 & 1.226160 \\
\hline $\mathrm{H}$ & -4.571947 & 0.743755 & 2.967534 \\
\hline $\mathrm{H}$ & -3.458379 & 1.570898 & 1.869890 \\
\hline $\mathrm{H}$ & -1.139938 & -1.785371 & 3.173625 \\
\hline $\mathrm{H}$ & -2.188555 & -0.768577 & 4.165005 \\
\hline $\mathrm{H}$ & -1.063781 & -0.018407 & 3.028560 \\
\hline $\mathrm{H}$ & -3.710860 & -3.316705 & 2.226692 \\
\hline $\mathrm{H}$ & -5.052141 & -2.437819 & 1.476895 \\
\hline $\mathrm{H}$ & -4.726610 & -2.254914 & 3.208890 \\
\hline $\mathrm{C}$ & 1.242091 & -3.197885 & -0.168727 \\
\hline $\mathrm{Li}$ & -1.658436 & -2.993265 & 0.16727 \\
\hline $\mathrm{H}$ & 0.420773 & -3.477997 & 1.598859 \\
\hline $\mathrm{C}$ & 1.561107 & 1.256077 & 0.498542 \\
\hline $\mathrm{C}$ & 2.470506 & -3.817729 & 0.174139 \\
\hline $\mathrm{C}$ & 1.150939 & -2.665790 & -1.484019 \\
\hline $\mathrm{N}$ & 1.276605 & 2.481343 & -0.008121 \\
\hline $\mathrm{C}$ & 2.991431 & 0.885299 & 0.717195 \\
\hline $\mathrm{C}$ & 3.525377 & -3.892988 & -0.730407 \\
\hline $\mathrm{H}$ & 2.581762 & -4.237096 & 1.172305 \\
\hline $\mathrm{C}$ & 2.214473 & -2.739978 & -2.379669 \\
\hline $\mathrm{H}$ & 0.226625 & -2.179070 & -1.787057 \\
\hline $\mathrm{C}$ & 2.271354 & 3.370627 & -0.668913 \\
\hline $\mathrm{C}$ & -0.030268 & 3.002487 & -0.241325 \\
\hline $\mathrm{C}$ & 3.466621 & -0.306558 & 0.157458 \\
\hline $\mathrm{C}$ & 3.823595 & 1.652657 & 1.544605 \\
\hline $\mathrm{C}$ & 3.419109 & -3.350708 & -2.016296 \\
\hline $\mathrm{H}$ & 4.448156 & -4.382188 & -0.424787 \\
\hline $\mathrm{H}$ & 2.099206 & -2.313676 & -3.373801 \\
\hline $\mathrm{C}$ & 1.527272 & 4.707015 & -0.848709 \\
\hline $\mathrm{H}$ & 3.175968 & 3.456113 & -0.073287 \\
\hline
\end{tabular}




$\begin{array}{llll}\mathrm{H} & 2.536633 & 2.931227 & -1.637189 \\ \mathrm{C} & -1.273448 & 2.405005 & -0.027283 \\ \mathrm{C} & 0.076202 & 4.300484 & -0.761152 \\ \mathrm{C} & 4.781252 & -0.705916 & 0.396046 \\ \mathrm{H} & 2.816200 & -0.917292 & -0.458538 \\ \mathrm{C} & 5.129329 & 1.236827 & 1.796399 \\ \mathrm{H} & 3.438973 & 2.555064 & 2.012438 \\ \mathrm{H} & 4.245661 & -3.412938 & -2.717387 \\ \mathrm{H} & 1.786593 & 5.186171 & -1.797346 \\ \mathrm{H} & 1.783185 & 5.410577 & -0.045478 \\ \mathrm{C} & -2.413234 & 3.147923 & -0.355147 \\ \mathrm{H} & -1.365524 & 1.393963 & 0.339638 \\ \mathrm{C} & -1.064714 & 5.024212 & -1.079258 \\ \mathrm{C} & 5.612062 & 0.062212 & 1.213010 \\ \mathrm{H} & 5.137962 & -1.626471 & -0.052806 \\ \mathrm{H} & 5.766662 & 1.824522 & 2.450755 \\ \mathrm{C} & -2.319559 & 4.439567 & -0.875710 \\ \mathrm{H} & -3.387580 & 2.693422 & -0.206411 \\ \mathrm{H} & -0.981505 & 6.029224 & -1.484024 \\ \mathrm{H} & 6.632404 & -0.257466 & 1.405153 \\ \mathrm{H} & -3.220836 & 4.990524 & -1.127149\end{array}$

$\begin{array}{llll}\text { S1d-1ag } & & & \\ \mathrm{O} & 0.203211 & 0.878564 & -0.340726 \\ \mathrm{C} & -0.960614 & 0.688795 & -0.701543 \\ \mathrm{Li} & 1.650337 & -0.278048 & 0.164927 \\ \mathrm{~N} & -1.345829 & -0.563062 & -1.182618 \\ \mathrm{C} & -1.921764 & 1.817972 & -0.742370 \\ \mathrm{~N} & 1.217120 & -1.181745 & 1.938379 \\ \mathrm{~N} & 3.482679 & 0.451595 & 0.548037 \\ \mathrm{C} & -2.668532 & -1.096510 & -1.111435 \\ \mathrm{C} & -0.300995 & -1.481451 & -1.561448 \\ \mathrm{C} & -1.894731 & 2.756190 & 0.294571 \\ \mathrm{C} & -2.781701 & 2.001443 & -1.832617 \\ \mathrm{C} & -0.080333 & -1.138502 & 2.402220 \\ \mathrm{Li} & 2.706416 & -0.020274 & 2.236373 \\ \mathrm{H} & 1.507012 & -2.157453 & 1.848677 \\ \mathrm{Si} & 4.731360 & -0.635944 & 0.085893 \\ \mathrm{Si} & 3.495768 & 2.143295 & 0.223740 \\ \mathrm{O} & -3.404855 & -0.390874 & -0.267831 \\ \mathrm{O} & -2.983779 & -2.083770 & -1.737738 \\ \mathrm{C} & -0.128553 & -2.673545 & -0.856296\end{array}$




\begin{tabular}{|c|c|c|c|}
\hline $\mathrm{C}$ & 0.556320 & -1.141178 & -2.610140 \\
\hline $\mathrm{C}$ & -2.756185 & 3.850183 & 0.262003 \\
\hline $\mathrm{H}$ & -1.213904 & 2.606714 & 1.123729 \\
\hline $\mathrm{C}$ & -3.623007 & 3.109844 & -1.874262 \\
\hline $\mathrm{H}$ & -2.784305 & 1.282358 & -2.646075 \\
\hline $\mathrm{C}$ & -0.869520 & -2.293657 & 2.640627 \\
\hline $\mathrm{C}$ & -0.704660 & 0.112278 & 2.654243 \\
\hline $\mathrm{C}$ & 5.110324 & -0.582508 & -1.774079 \\
\hline $\mathrm{C}$ & 4.183524 & -2.408210 & 0.512337 \\
\hline $\mathrm{C}$ & 6.365248 & -0.364022 & 1.015890 \\
\hline $\mathrm{C}$ & 2.867835 & 2.547178 & -1.521246 \\
\hline $\mathrm{C}$ & 2.321298 & 2.977329 & 1.468424 \\
\hline $\mathrm{C}$ & 5.195497 & 2.968991 & 0.406738 \\
\hline $\mathrm{C}$ & -4.855768 & -0.638058 & -0.106473 \\
\hline $\mathrm{C}$ & 0.900126 & -3.540859 & -1.223270 \\
\hline $\mathrm{H}$ & -0.786111 & -2.902028 & -0.024882 \\
\hline $\mathrm{C}$ & 1.598148 & -2.003144 & -2.954980 \\
\hline $\mathrm{H}$ & 0.412618 & -0.205325 & -3.140811 \\
\hline $\mathrm{C}$ & -3.620541 & 4.028575 & -0.820614 \\
\hline $\mathrm{H}$ & -2.749086 & 4.566791 & 1.077656 \\
\hline $\mathrm{H}$ & -4.280329 & 3.257676 & -2.725911 \\
\hline $\mathrm{C}$ & -2.175804 & -2.200272 & 3.115158 \\
\hline $\mathrm{H}$ & -0.422000 & -3.272892 & 2.476499 \\
\hline $\mathrm{C}$ & -2.010886 & 0.196322 & 3.121461 \\
\hline $\mathrm{H}$ & -0.125873 & 1.017467 & 2.484795 \\
\hline $\mathrm{H}$ & 5.503020 & 0.398828 & -2.068027 \\
\hline $\mathrm{H}$ & 5.850422 & -1.337724 & -2.067370 \\
\hline $\mathrm{H}$ & 4.197634 & -0.758468 & -2.356184 \\
\hline $\mathrm{H}$ & 4.000446 & -2.527814 & 1.589357 \\
\hline $\mathrm{H}$ & 4.957620 & -3.134646 & 0.235020 \\
\hline $\mathrm{H}$ & 3.263939 & -2.690939 & -0.012081 \\
\hline $\mathrm{H}$ & 6.202978 & -0.392810 & 2.102037 \\
\hline $\mathrm{H}$ & 6.813944 & 0.606691 & 0.779673 \\
\hline $\mathrm{H}$ & 7.100375 & -1.141699 & 0.771799 \\
\hline $\mathrm{H}$ & 3.489901 & 2.052755 & -2.277419 \\
\hline $\mathrm{H}$ & 2.881153 & 3.625089 & -1.727239 \\
\hline $\mathrm{H}$ & 1.838457 & 2.192649 & -1.644943 \\
\hline $\mathrm{H}$ & 2.249625 & 4.059742 & 1.305410 \\
\hline $\mathrm{H}$ & 1.310148 & 2.563970 & 1.374058 \\
\hline $\mathrm{H}$ & 2.659699 & 2.832746 & 2.505603 \\
\hline $\mathrm{H}$ & 5.120422 & 4.055394 & 0.270444 \\
\hline $\mathrm{H}$ & 5.906364 & 2.592963 & -0.339254 \\
\hline
\end{tabular}




$\begin{array}{llll}\mathrm{H} & 5.628239 & 2.783277 & 1.397101 \\ \mathrm{C} & -5.258331 & 0.474916 & 0.858560 \\ \mathrm{C} & -5.055852 & -2.019829 & 0.512266 \\ \mathrm{C} & -5.560015 & -0.483382 & -1.454272 \\ \mathrm{C} & 1.766805 & -3.206518 & -2.266702 \\ \mathrm{H} & 1.035803 & -4.470284 & -0.678795 \\ \mathrm{H} & 2.271044 & -1.735893 & -3.763390 \\ \mathrm{H} & -4.285406 & 4.886970 & -0.848947 \\ \mathrm{C} & -2.765418 & -0.957591 & 3.364130 \\ \mathrm{H} & -2.736765 & -3.113363 & 3.303392 \\ \mathrm{H} & -2.445478 & 1.176337 & 3.306867 \\ \mathrm{H} & -5.053490 & 1.456089 & 0.419525 \\ \mathrm{H} & -6.328045 & 0.403399 & 1.080033 \\ \mathrm{H} & -4.694438 & 0.384877 & 1.790044 \\ \mathrm{H} & -4.451567 & -2.108086 & 1.419057 \\ \mathrm{H} & -6.110899 & -2.149036 & 0.777334 \\ \mathrm{H} & -4.769195 & -2.807519 & -0.186640 \\ \mathrm{H} & -5.362325 & 0.506951 & -1.876299 \\ \mathrm{H} & -6.640668 & -0.580063 & -1.305318 \\ \mathrm{H} & -5.237806 & -1.249153 & -2.162028 \\ \mathrm{H} & 2.578053 & -3.875812 & -2.535317 \\ \mathrm{H} & -3.777171 & -0.890230 & 3.752545\end{array}$

\section{S1d-1r}

$\begin{array}{lll}-0.694208 & -0.793580 & 0.594323\end{array}$

$\mathrm{N} \quad-2.410444-0.361501 \quad-0.392598$

$\begin{array}{lllll}\mathrm{N} & & -0.031798 & -2.392866 & -0.533204\end{array}$

$\mathrm{Si} \quad-3.546463 \quad-1.280986 \quad 0.501086$

$\begin{array}{llll}\mathrm{Si} & -2.497494 & 1.294301 & -0.831870\end{array}$

$\mathrm{Li} \quad-1.519293-1.639504-1.493156$

$\begin{array}{llll}\text { O } & 0.549618 & 0.067960 & 1.668749\end{array}$

C $\quad 1.293703-2.357899-0.917951$

$\mathrm{H} \quad-0.224604 \quad-3.291379-0.086504$

$\begin{array}{llll}\text { C } & -5.260834 & -0.511902 & 0.739834\end{array}$

$\begin{array}{lllll}\text { C } & -2.839477 & -1.672450 & 2.227942\end{array}$

$\begin{array}{lllll}\text { C } & -3.787264 & -2.963424 & -0.366699\end{array}$

$\begin{array}{lllll}\text { C } & & -0.928103 & 1.734162 & -1.806714\end{array}$

$\begin{array}{llll}\text { C } & -2.585042 & 2.436880 & 0.682845\end{array}$

$\begin{array}{lllll}\text { C } & -3.974153 & 1.709194 & -1.951344\end{array}$

$\begin{array}{lllll}\text { C } & 1.709174 & 0.476547 & 1.419424\end{array}$

$\begin{array}{llll}\text { C } & 2.215548 & -3.409466 & -0.674627\end{array}$

$\begin{array}{lllll}\text { C } & & 1.807705 & -1.213709 & -1.584805\end{array}$ 


\begin{tabular}{|c|c|c|c|}
\hline $\mathrm{H}$ & -5.766215 & -0.363957 & -0.221475 \\
\hline $\mathrm{H}$ & -5.897906 & -1.157615 & 1.357178 \\
\hline $\mathrm{H}$ & -5.198062 & 0.464267 & 1.235083 \\
\hline $\mathrm{H}$ & -3.528290 & -2.279289 & 2.828943 \\
\hline $\mathrm{H}$ & -2.635157 & -0.748407 & 2.784307 \\
\hline $\mathrm{H}$ & -1.896403 & -2.236961 & 2.16684 \\
\hline $\mathrm{H}$ & -2.835126 & -3.501978 & -0.48994 \\
\hline $\mathrm{H}$ & -4.234402 & -2.831470 & -1.3618 \\
\hline $\mathrm{H}$ & -4.448166 & -3.629522 & 0.20169 \\
\hline $\mathrm{H}$ & -0.910175 & 2.792658 & -2.092831 \\
\hline $\mathrm{H}$ & -0.023564 & 1.546345 & -1.218889 \\
\hline $\mathrm{H}$ & -0.854726 & 1.145942 & -2.73278 \\
\hline $\mathrm{H}$ & -1.801832 & 2.167506 & 1.400386 \\
\hline $\mathrm{H}$ & -2.452557 & 3.493413 & 0.417518 \\
\hline $\mathrm{H}$ & -3.550212 & 2.340352 & 1.195370 \\
\hline $\mathrm{H}$ & -3.948168 & 2.755139 & -2.283432 \\
\hline $\mathrm{H}$ & -4.927934 & 1.548545 & -1.435798 \\
\hline $\mathrm{H}$ & -3.973624 & 1.075537 & -2.847903 \\
\hline $\mathrm{N}$ & 2.792098 & -0.263276 & 1.722149 \\
\hline $\mathrm{C}$ & 1.853536 & 1.820313 & 0.776237 \\
\hline $\mathrm{C}$ & 3.552051 & -3.313418 & -1.051907 \\
\hline $\mathrm{H}$ & 1.857968 & -4.308933 & -0.175823 \\
\hline $\mathrm{C}$ & 3.143642 & -1.132007 & -1.967052 \\
\hline $\mathrm{H}$ & 1.133330 & -0.387440 & -1.800135 \\
\hline $\mathrm{C}$ & 4.176988 & 0.170645 & 1.581504 \\
\hline $\mathrm{C}$ & 2.625395 & -1.625059 & 2.223337 \\
\hline $\mathrm{C}$ & 2.660570 & 2.039088 & -0.347812 \\
\hline $\mathrm{C}$ & 1.025012 & 2.850242 & 1.241874 \\
\hline $\mathrm{C}$ & 4.040468 & -2.171351 & -1.695886 \\
\hline $\mathrm{H}$ & 4.221490 & -4.145625 & -0.844410 \\
\hline $\mathrm{H}$ & 3.487378 & -0.246999 & -2.499024 \\
\hline $\mathrm{H}$ & 4.731565 & -0.138661 & 2.474063 \\
\hline $\mathrm{H}$ & 4.634587 & -0.297463 & 0.702218 \\
\hline $\mathrm{H}$ & 4.235140 & 1.253948 & 1.493522 \\
\hline $\mathrm{H}$ & 3.238866 & -2.302187 & 1.624492 \\
\hline $\mathrm{H}$ & 2.932313 & -1.676319 & 3.275318 \\
\hline $\mathrm{H}$ & 1.582037 & -1.914767 & 2.131020 \\
\hline $\mathrm{C}$ & 2.640400 & 3.275531 & -0.992207 \\
\hline $\mathrm{H}$ & 3.264896 & 1.231471 & -0.741690 \\
\hline $\mathrm{C}$ & 1.020874 & 4.089120 & 0.607335 \\
\hline $\mathrm{H}$ & 0.378079 & 2.662796 & 2.091181 \\
\hline $\mathrm{H}$ & 5.079448 & -2.106452 & -2.004403 \\
\hline
\end{tabular}




\begin{tabular}{|c|c|c|c|}
\hline $\mathrm{C}$ & 1.826240 & 4.302885 & -0.513758 \\
\hline $\mathrm{H}$ & 3.252993 & 3.430848 & -1.875402 \\
\hline & 0.376939 & 4.881210 & 0.977200 \\
\hline
\end{tabular}

S1d-1s

Si $\quad-2.789864 \quad 1.470155 \quad-0.629793$

$\mathrm{N} \quad-2.705296-0.238607 \quad-0.481472$

$\begin{array}{llll}\text { C } & -1.289554 & 2.080457 & -1.620271\end{array}$

Si $\quad-3.782856-1.259812 \quad 0.373865$

$\mathrm{Li} \quad-1.903238-1.447164-1.722423$

$\mathrm{Li} \quad-0.940350 \quad-0.746250 \quad 0.343551$

$\begin{array}{llll}\text { C } & -2.741903 & 2.323822 & 1.067639\end{array}$

$\begin{array}{lllll}\text { C } & -4.338220 & 2.081294 & -1.542417\end{array}$

$\mathrm{H} \quad \begin{array}{lllll}\mathrm{H} & -1.300098 & 3.171879 & -1.730483\end{array}$

$\begin{array}{llll}\mathrm{H} & -1.277294 & 1.650864 & -2.631586\end{array}$

$\mathrm{H} \quad \begin{array}{llll}0.345290 & 1.817362 & -1.131660\end{array}$

$\begin{array}{llll}\text { C } & -5.490673 & -0.547498 & 0.773109\end{array}$

$\begin{array}{llll}\text { C } & -4.049038 & -2.846336 & -0.656312\end{array}$

$\begin{array}{llll}\text { C } & -2.988029 & -1.821785 & 2.013358\end{array}$

$\mathrm{N} \quad \begin{array}{llll}-0.313140 & -2.217440 & -0.934211\end{array}$

$\begin{array}{llll}\text { O } & 0.312790 & 0.054007 & 1.423087\end{array}$

$\begin{array}{llll}\mathrm{H} & -1.880418 & 1.959494 & 1.639450\end{array}$

$\begin{array}{llll}\mathrm{H} & -2.660361 & 3.415232 & 0.986187\end{array}$

$\begin{array}{llll}\mathrm{H} & -3.644100 & 2.100243 & 1.651088\end{array}$

$\mathrm{H} \quad \begin{array}{llll}-4.312635 & 3.168272 & -1.693374\end{array}$

$\mathrm{H} \quad \begin{array}{lllll}\mathrm{H} & -4.420183 & 1.609997 & -2.530404\end{array}$

$\mathrm{H} \quad \begin{array}{llll}-5.253232 & 1.844913 & -0.987476\end{array}$

$\begin{array}{llll}\mathrm{H} & -6.043105 & -0.306909 & -0.142381\end{array}$

$\begin{array}{llll}\mathrm{H} & -6.091315 & -1.261378 & 1.350459\end{array}$

$\begin{array}{llll}\mathrm{H} & -5.410968 & 0.373453 & 1.362720\end{array}$

$\mathrm{H} \quad\left[\begin{array}{llll}-4.547897 & -2.618607 & -1.608877\end{array}\right.$

$\mathrm{H} \quad \begin{array}{lllll} & -3.103978 & -3.364780 & -0.882303\end{array}$

$\mathrm{H} \quad-4.676854 \quad-3.574606 \quad-0.128153$

$\begin{array}{llll}\mathrm{H} & -2.768372 & -0.958793 & 2.655143\end{array}$

$\begin{array}{llll}\mathrm{H} & -2.041251 & -2.357187 & 1.844913\end{array}$

$\mathrm{H} \quad-3.637641 \quad-2.502077 \quad 2.578300$

$\begin{array}{lllll}\text { C } & & 0.948567 & -2.042864 & -1.471517\end{array}$

$\mathrm{H} \quad-0.386281 \quad-3.160036 \quad-0.548035$

$\begin{array}{llll}\text { C } & 1.510142 & 0.388713 & 1.260524\end{array}$

$\begin{array}{llll}\text { C } & 1.978810 & -3.017154 & -1.409607\end{array}$

$\begin{array}{lllll}\text { C } & 1.274075 & -0.823264 & -2.122465\end{array}$ 


\begin{tabular}{|c|c|c|c|}
\hline$\checkmark$ & 2.509506 & -0.468149 & 1.547467 \\
\hline $\mathrm{C}$ & 1.790220 & 1.757814 & 0.730837 \\
\hline $\mathrm{C}$ & 3.229265 & -2.793854 & -1.978137 \\
\hline $\mathrm{H}$ & 1.769792 & -3.965317 & -0.916521 \\
\hline $\mathrm{C}$ & 2.524835 & -0.612906 & -2.695341 \\
\hline $\mathrm{H}$ & 0.520246 & -0.041103 & -2.176989 \\
\hline $\mathrm{C}$ & 3.919273 & -0.086347 & 1.693699 \\
\hline $\mathrm{C}$ & 2.145683 & -1.834504 & 1.956656 \\
\hline $\mathrm{C}$ & 2.658743 & 1.978622 & -0.345608 \\
\hline $\mathrm{C}$ & 1.034764 & 2.822132 & 1.241264 \\
\hline $\mathrm{C}$ & 3.522225 & -1.591810 & -2.631776 \\
\hline $\mathrm{H}$ & 3.985868 & -3.573195 & -1.915220 \\
\hline $\mathrm{H}$ & 2.720085 & 0.330664 & -3.200734 \\
\hline $\mathrm{C}$ & 4.843659 & -0.738806 & 0.666601 \\
\hline $\mathrm{H}$ & 3.983628 & 1.000108 & 1.632134 \\
\hline $\mathrm{H}$ & 4.229570 & -0.367137 & 2.708794 \\
\hline $\mathrm{C}$ & 1.777241 & -1.925345 & 3.437315 \\
\hline $\mathrm{H}$ & 1.314587 & -2.155880 & 1.328114 \\
\hline $\mathrm{H}$ & 2.997842 & -2.476619 & 1.723263 \\
\hline $\mathrm{C}$ & 2.773928 & 3.253783 & -0.897535 \\
\hline $\mathrm{H}$ & 3.198159 & 1.146746 & -0.780717 \\
\hline $\mathrm{C}$ & 1.167716 & 4.098082 & 0.700703 \\
\hline $\mathrm{H}$ & 0.335086 & 2.634850 & 2.047925 \\
\hline $\mathrm{H}$ & 4.494168 & -1.428707 & -3.087625 \\
\hline $\mathrm{H}$ & 4.845825 & -1.829171 & 0.757006 \\
\hline $\mathrm{H}$ & 5.869103 & -0.386274 & 0.823641 \\
\hline $\mathrm{H}$ & 4.537908 & -0.505180 & -0.355759 \\
\hline $\mathrm{H}$ & 1.547470 & -2.963004 & 3.703398 \\
\hline $\mathrm{H}$ & 2.600210 & -1.588516 & 4.077992 \\
\hline $\mathrm{H}$ & 0.897117 & -1.311871 & 3.646579 \\
\hline $\mathrm{C}$ & 2.035250 & 4.315270 & -0.372433 \\
\hline $\mathrm{H}$ & 3.433037 & 3.414169 & -1.745741 \\
\hline $\mathrm{H}$ & 0.582080 & 4.918357 & 1.104717 \\
\hline $\mathrm{H}$ & 2.126661 & 5.307413 & -0.805080 \\
\hline
\end{tabular}

\section{S1d-1v}

\begin{tabular}{|c|c|c|c|}
\hline $\mathrm{Li}$ & -0.161947 & -1.014778 & -0.894180 \\
\hline $\mathrm{N}$ & -1.529491 & -2.428885 & -1.503534 \\
\hline $\mathrm{N}$ & 1.170983 & -2.143361 & 0.109932 \\
\hline $\mathrm{Li}$ & -0.248584 & -3.331017 & -0.405365 \\
\hline $\mathrm{C}$ & -2.769437 & -1.903380 & -1.185373 \\
\hline $\mathrm{H}$ & -1.410314 & -2.411988 & -2.518386 \\
\hline
\end{tabular}




\begin{tabular}{|c|c|c|c|}
\hline $\mathrm{O}$ & -0.206148 & 0.803940 & -1.080879 \\
\hline $\mathrm{Si}$ & 1.236890 & -1.981432 & 1.816340 \\
\hline $\mathrm{Si}$ & 2.539056 & -1.941073 & -0.916038 \\
\hline $\mathrm{C}$ & -3.574407 & -1.177345 & -2.102411 \\
\hline $\mathrm{C}$ & -3.276426 & -2.022054 & 0.134883 \\
\hline $\mathrm{C}$ & -0.169584 & 1.849147 & -0.393233 \\
\hline $\mathrm{C}$ & 2.787252 & -2.728688 & 2.617279 \\
\hline $\mathrm{C}$ & 1.121478 & -0.168970 & 2.380216 \\
\hline $\mathrm{C}$ & -0.263202 & -2.873737 & 2.578354 \\
\hline $\mathrm{C}$ & 3.646531 & -3.480207 & -0.977535 \\
\hline $\mathrm{C}$ & 3.620895 & -0.451519 & -0.453067 \\
\hline $\mathrm{C}$ & 1.907810 & -1.630634 & -2.687696 \\
\hline $\mathrm{C}$ & -4.774736 & -0.588905 & -1.713989 \\
\hline $\mathrm{H}$ & -3.228193 & -1.074101 & -3.129488 \\
\hline $\mathrm{C}$ & -4.474795 & -1.426058 & 0.516746 \\
\hline $\mathrm{H}$ & -2.715627 & -2.613050 & 0.854648 \\
\hline $\mathrm{N}$ & -1.213705 & 2.208648 & 0.367937 \\
\hline $\mathrm{C}$ & 1.066959 & 2.686634 & -0.427451 \\
\hline $\mathrm{H}$ & 2.869255 & -3.797725 & 2.385292 \\
\hline $\mathrm{H}$ & 2.765294 & -2.623596 & 3.709430 \\
\hline $\mathrm{H}$ & 3.702915 & -2.244746 & 2.257068 \\
\hline $\mathrm{H}$ & 0.195133 & 0.292770 & 2.014713 \\
\hline $\mathrm{H}$ & 1.134524 & -0.072799 & 3.473436 \\
\hline $\mathrm{H}$ & 1.959652 & 0.411041 & 1.977602 \\
\hline $\mathrm{H}$ & -1.206395 & -2.394536 & 2.289696 \\
\hline $\mathrm{H}$ & -0.313787 & -3.931320 & 2.277356 \\
\hline $\mathrm{H}$ & -0.217564 & -2.863044 & 3.674302 \\
\hline $\mathrm{H}$ & 4.489815 & -3.356958 & -1.669057 \\
\hline $\mathrm{H}$ & 3.074629 & -4.359075 & -1.304039 \\
\hline $\mathrm{H}$ & 4.056162 & -3.706469 & 0.013887 \\
\hline $\mathrm{H}$ & 4.063039 & -0.564763 & 0.544311 \\
\hline $\mathrm{H}$ & 4.445502 & -0.315971 & -1.164470 \\
\hline $\mathrm{H}$ & 3.028019 & 0.470290 & -0.450322 \\
\hline $\mathrm{H}$ & 1.242129 & -2.433074 & -3.034035 \\
\hline $\mathrm{H}$ & 1.363931 & -0.679577 & -2.769421 \\
\hline $\mathrm{H}$ & 2.745952 & -1.577723 & -3.393600 \\
\hline $\mathrm{C}$ & -5.237770 & -0.692230 & -0.397507 \\
\hline $\mathrm{H}$ & -5.356288 & -0.038554 & -2.450597 \\
\hline $\mathrm{H}$ & -4.823159 & -1.547032 & 1.540494 \\
\hline $\mathrm{C}$ & -2.427184 & 1.361053 & 0.411183 \\
\hline $\mathrm{C}$ & -1.436775 & 3.522621 & 1.007154 \\
\hline $\mathrm{C}$ & 1.648418 & 2.953194 & -1.673692 \\
\hline
\end{tabular}




$\begin{array}{llll}\mathrm{C} & 1.724358 & 3.085842 & 0.742872 \\ \mathrm{H} & -6.177117 & -0.235787 & -0.100250 \\ \mathrm{C} & -3.520598 & 2.316985 & 0.889279 \\ \mathrm{H} & -2.623299 & 0.922950 & -0.567459 \\ \mathrm{H} & -2.268068 & 0.536172 & 1.113491 \\ \mathrm{C} & -2.757774 & 3.324160 & 1.762064 \\ \mathrm{H} & -0.612645 & 3.796539 & 1.664639 \\ \mathrm{H} & -1.525783 & 4.297237 & 0.234972 \\ \mathrm{C} & 2.853483 & 3.647216 & -1.749004 \\ \mathrm{H} & 1.153397 & 2.602400 & -2.573331 \\ \mathrm{C} & 2.941739 & 3.761064 & 0.664562 \\ \mathrm{H} & 1.313675 & 2.822195 & 1.711425 \\ \mathrm{H} & -4.314606 & 1.787707 & 1.419810 \\ \mathrm{H} & -3.974085 & 2.823729 & 0.029473 \\ \mathrm{H} & -3.288686 & 4.268446 & 1.910975 \\ \mathrm{H} & -2.555631 & 2.890678 & 2.748693 \\ \mathrm{C} & 3.502273 & 4.051880 & -0.580046 \\ \mathrm{H} & 3.294069 & 3.859894 & -2.718476 \\ \mathrm{H} & 3.456946 & 4.049045 & 1.576067 \\ \mathrm{H} & 4.449188 & 4.580505 & -0.639281\end{array}$

\section{S1d-1w}

$\begin{array}{llll}\mathrm{Si} & 1.643274 & -1.850944 & 1.695514\end{array}$

C $\quad 0.053039 \quad-2.271793 \quad 2.652694$

C $\quad 3.036577 \quad-2.904862 \quad 2.437567$

$\mathrm{H} \quad 0.177894 \quad-2.093811 \quad 3.727951$

$\begin{array}{llll}\mathrm{H} & -0.804860 & -1.674972 & 2.319103\end{array}$

$\mathrm{H} \quad-0.220940 \quad-3.330336 \quad 2.534906$

$\begin{array}{llll}\text { C } & 2.056857 & -0.036875 & 2.105852\end{array}$

$\begin{array}{llll}\mathrm{N} & 1.377314 & -2.122008 & 0.023317\end{array}$

$\mathrm{H} \quad 4.004688-2.667983 \quad 1.979086$

$\begin{array}{llll}\mathrm{H} & 3.135471 & -2.745761 & 3.518829\end{array}$

$\begin{array}{llll}\mathrm{H} & 2.849442 & -3.972941 & 2.270894\end{array}$

$\begin{array}{llll}\mathrm{H} & 3.041962 & 0.231163 & 1.707390\end{array}$

$\begin{array}{llll}\mathrm{H} & 2.073076 & 0.141853 & 3.188511\end{array}$

$\begin{array}{llll}\mathrm{H} & 1.330147 & 0.652770 & 1.660678\end{array}$

Si $\quad \begin{array}{llll}2.629231 & -2.171928 & -1.156084\end{array}$

$\begin{array}{llll}\mathrm{Li} & 0.040050 & -0.855892 & -0.820726\end{array}$

$\begin{array}{llll}\text { C } & 3.919766 & -0.794432 & -0.955502\end{array}$

$\begin{array}{llll}\text { C } & 1.828589 & -1.931661 & -2.868418\end{array}$

C $\quad 3.543112 \quad-3.834674-1.194704$

$\mathrm{N} \quad \begin{array}{llll}-1.477215 & -2.112256 & -1.309757\end{array}$ 


\begin{tabular}{|c|c|c|c|}
\hline $\mathrm{O}$ & 0.538497 & 0.918815 & -1.117837 \\
\hline $\mathrm{H}$ & 4.491077 & -0.900891 & -0.025322 \\
\hline $\mathrm{H}$ & 4.638228 & -0.785824 & -1.784865 \\
\hline $\mathrm{H}$ & 3.417107 & 0.178950 & -0.929156 \\
\hline $\mathrm{H}$ & 1.044925 & -2.676193 & -3.065686 \\
\hline $\mathrm{H}$ & 2.573332 & -2.026522 & -3.668222 \\
\hline $\mathrm{H}$ & 1.376092 & -0.935488 & -2.967544 \\
\hline $\mathrm{H}$ & 4.028839 & -4.038483 & -0.233254 \\
\hline $\mathrm{H}$ & 2.845531 & -4.660519 & -1.389629 \\
\hline $\mathrm{H}$ & 4.314992 & -3.862893 & -1.974377 \\
\hline $\mathrm{C}$ & -2.759778 & -1.900987 & -0.827152 \\
\hline $\mathrm{Li}$ & -0.160531 & -3.175069 & -0.41453 \\
\hline $\mathrm{H}$ & -1.476811 & -1.960795 & -2.320574 \\
\hline $\mathrm{C}$ & 0.061067 & 1.993654 & -0.694185 \\
\hline $\mathrm{C}$ & -3.786100 & -1.272686 & -1.575148 \\
\hline $\mathrm{C}$ & -3.103112 & -2.312663 & 0.485826 \\
\hline $\mathrm{N}$ & 0.767855 & 3.144638 & -0.753828 \\
\hline $\mathrm{C}$ & -1.331664 & 2.007849 & -0.153555 \\
\hline $\mathrm{C}$ & -5.054805 & -1.066766 & -1.042985 \\
\hline $\mathrm{H}$ & -3.564191 & -0.942833 & -2.588736 \\
\hline $\mathrm{C}$ & -4.376444 & -2.108554 & 1.010324 \\
\hline $\mathrm{H}$ & -2.351658 & -2.816608 & 1.090741 \\
\hline $\mathrm{C}$ & 0.490483 & 4.315087 & 0.089394 \\
\hline $\mathrm{C}$ & 2.127162 & 3.124131 & -1.313759 \\
\hline $\mathrm{C}$ & -1.687173 & 1.077832 & 0.831168 \\
\hline $\mathrm{C}$ & -2.312127 & 2.840305 & -0.709783 \\
\hline $\mathrm{C}$ & -5.370017 & -1.477546 & 0.256568 \\
\hline $\mathrm{H}$ & -5.810732 & -0.577695 & -1.653785 \\
\hline $\mathrm{H}$ & -4.595027 & -2.452907 & 2.019198 \\
\hline $\mathrm{C}$ & 1.495739 & 4.376950 & 1.247607 \\
\hline $\mathrm{H}$ & -0.531509 & 4.257737 & 0.459684 \\
\hline $\mathrm{H}$ & 0.574602 & 5.211841 & -0.538403 \\
\hline $\mathrm{C}$ & 3.171130 & 3.150733 & -0.192804 \\
\hline $\mathrm{H}$ & 2.224142 & 2.234574 & -1.933857 \\
\hline $\mathrm{H}$ & 2.229346 & 4.012591 & -1.950712 \\
\hline $\mathrm{C}$ & -3.006607 & 0.989608 & 1.264982 \\
\hline $\mathrm{H}$ & -0.931176 & 0.425163 & 1.257584 \\
\hline $\mathrm{C}$ & -3.636745 & 2.731253 & -0.289364 \\
\hline $\mathrm{H}$ & -2.038411 & 3.547586 & -1.487691 \\
\hline $\mathrm{H}$ & -6.365133 & -1.323824 & 0.663411 \\
\hline $\mathrm{C}$ & 2.941400 & 4.352907 & 0.733224 \\
\hline $\mathrm{H}$ & 1.306200 & 5.281055 & 1.838399 \\
\hline
\end{tabular}




$\begin{array}{llll}\mathrm{H} & 1.321052 & 3.514457 & 1.904335 \\ \mathrm{H} & 3.095224 & 2.217991 & 0.377703 \\ \mathrm{H} & 4.174648 & 3.181893 & -0.632710 \\ \mathrm{C} & -3.984385 & 1.806843 & 0.697172 \\ \mathrm{H} & -3.279758 & 0.253392 & 2.010471 \\ \mathrm{H} & -4.398731 & 3.359078 & -0.741906 \\ \mathrm{H} & 3.642427 & 4.326628 & 1.574960 \\ \mathrm{H} & 3.143519 & 5.282127 & 0.180677 \\ \mathrm{H} & -5.018098 & 1.704474 & 1.010144\end{array}$

\section{S1d-1x}

$\begin{array}{llll}\text { O } & -0.495708 & -0.017893 & -1.284877\end{array}$

$\begin{array}{lllll}\text { C } & -1.488290 & 0.629583 & -0.877410\end{array}$

$\mathrm{Li} \quad 0.915724 \quad-0.863426-0.427462$

$\begin{array}{lllll}\mathrm{N} & -2.725310 & 0.099919 & -0.897023\end{array}$

$\begin{array}{llll}\mathrm{C} & -1.265110 & 2.016790 & -0.363651\end{array}$

N $\quad 2.824712-0.502849 \quad 0.137891$

$\begin{array}{llll}\mathrm{N} & 0.408180 & -2.299504 & 0.976381\end{array}$

$\begin{array}{llll}\text { C } & -3.975526 & 0.803336 & -0.606281\end{array}$

$\begin{array}{llll}\text { C } & -2.921945 & -1.317725 & -1.226066\end{array}$

C $\quad-0.390075 \quad 2.842025-1.081257$

$\begin{array}{llll}\text { C } & -1.777494 & 2.455533 & 0.864715\end{array}$

Si $\quad 3.661421 \quad-1.610156 \quad-0.866136$

$\begin{array}{llll}\mathrm{Si} & 3.224291 & 1.120409 & 0.514497\end{array}$

$\begin{array}{lllll}\mathrm{Li} & 2.086367 & -1.571431 & 1.543905\end{array}$

$\begin{array}{lllll}\text { C } & -0.839896 & -2.130373 & 1.540259\end{array}$

$\begin{array}{llll}\mathrm{H} & 0.481000 & -3.251680 & 0.612657\end{array}$

$\begin{array}{lllll}\text { C } & -4.963771 & 0.571290 & -1.752714\end{array}$

$\begin{array}{llll}\mathrm{H} & -3.784866 & 1.869759 & -0.493900\end{array}$

$\begin{array}{llll}\mathrm{H} & -4.385877 & 0.409812 & 0.331955\end{array}$

$\begin{array}{lllll}\text { C } & -3.947236 & -1.445385 & -2.347397\end{array}$

$\mathrm{H} \quad-1.960555 \quad-1.740253 \quad-1.512815$

$\mathrm{H} \quad-\quad-3.276463 \quad-1.825750 \quad-0.324213$

$\begin{array}{lllll}\text { C } & -0.051982 & 4.100665 & -0.591483\end{array}$

$\begin{array}{llll}\mathrm{H} & 0.030824 & 2.479833 & -2.012644\end{array}$

$\begin{array}{llll}\text { C } & -1.424279 & 3.709893 & 1.360278\end{array}$

$\begin{array}{llll}\mathrm{H} & -2.415201 & 1.800250 & 1.446131\end{array}$

$\begin{array}{lllll}\text { C } & 5.287639 & -1.004225 & -1.623730\end{array}$

$\begin{array}{lllll}\text { C } & 2.523497 & -2.149848 & -2.298218\end{array}$

$\begin{array}{llll}\text { C } & 4.040387 & -3.192162 & 0.130332\end{array}$

$\begin{array}{llll}\text { C } & 3.242044 & 2.258261 & -1.005357\end{array}$

$\begin{array}{llll}\text { C } & 1.923559 & 1.776787 & 1.734498\end{array}$ 


\begin{tabular}{|c|c|c|c|}
\hline $\mathrm{C}$ & 4.909958 & 1.303097 & 1.372356 \\
\hline $\mathrm{C}$ & -1.835416 & -3.141802 & 1.570953 \\
\hline $\mathrm{C}$ & -1.194638 & -0.881128 & 2.116679 \\
\hline $\mathrm{O}$ & -5.169618 & -0.806279 & -2.001167 \\
\hline $\mathrm{H}$ & -5.938644 & 0.995814 & -1.496556 \\
\hline $\mathrm{H}$ & -4.587627 & 1.071601 & -2.661218 \\
\hline $\mathrm{H}$ & -4.185395 & -2.497344 & -2.525157 \\
\hline $\mathrm{H}$ & -3.544643 & -1.010417 & -3.277160 \\
\hline $\mathrm{C}$ & -0.567291 & 4.535977 & 0.631330 \\
\hline $\mathrm{H}$ & 0.627918 & 4.732422 & -1.154595 \\
\hline $\mathrm{H}$ & -1.808526 & 4.035305 & 2.322417 \\
\hline $\mathrm{H}$ & 6.025296 & -0.767159 & -0.848533 \\
\hline $\mathrm{H}$ & 5.725392 & -1.768773 & -2.277647 \\
\hline $\mathrm{H}$ & 5.135443 & -0.099916 & -2.224473 \\
\hline $\mathrm{H}$ & 1.600207 & -2.626476 & -1.935138 \\
\hline $\mathrm{H}$ & 2.233670 & -1.289452 & -2.915841 \\
\hline $\mathrm{H}$ & 3.012913 & -2.879983 & -2.954835 \\
\hline $\mathrm{H}$ & 4.510141 & -3.969037 & -0.485729 \\
\hline $\mathrm{H}$ & 4.723866 & -2.974616 & 0.962460 \\
\hline $\mathrm{H}$ & 3.126098 & -3.638904 & 0.550835 \\
\hline $\mathrm{H}$ & 2.335810 & 2.112783 & -1.603532 \\
\hline $\mathrm{H}$ & 3.295430 & 3.318166 & -0.725861 \\
\hline $\mathrm{H}$ & 4.102628 & 2.040791 & -1.649745 \\
\hline $\mathrm{H}$ & 2.115980 & 2.819878 & 2.012982 \\
\hline $\mathrm{H}$ & 0.914913 & 1.737001 & 1.310624 \\
\hline $\mathrm{H}$ & 1.918064 & 1.191256 & 2.665797 \\
\hline $\mathrm{H}$ & 5.109659 & 2.343686 & 1.659169 \\
\hline $\mathrm{H}$ & 5.729530 & 0.976360 & 0.721612 \\
\hline $\mathrm{H}$ & 4.953180 & 0.694396 & 2.285465 \\
\hline $\mathrm{C}$ & -3.095303 & -2.912577 & 2.116737 \\
\hline $\mathrm{H}$ & -1.597896 & -4.117649 & 1.150412 \\
\hline $\mathrm{C}$ & -2.456799 & -0.663443 & 2.661444 \\
\hline $\mathrm{H}$ & -0.455272 & -0.082475 & 2.127379 \\
\hline $\mathrm{H}$ & -0.291798 & 5.511666 & 1.020843 \\
\hline $\mathrm{C}$ & -3.431199 & -1.667616 & 2.659989 \\
\hline $\mathrm{H}$ & -3.826780 & -3.717817 & 2.116800 \\
\hline $\mathrm{H}$ & -2.678914 & 0.301575 & 3.113228 \\
\hline $\mathrm{H}$ & -4.411211 & -1.494925 & 3.094015 \\
\hline
\end{tabular}

\section{S1t-1aa}

$\mathrm{Li} \quad 0.284116-0.044155-0.068356$

$\mathrm{N} \quad-0.647361 \quad 1.446782-0.956356$ 


\begin{tabular}{|c|c|c|c|}
\hline $\mathrm{N}$ & -0.494340 & -1.747436 & 0.650758 \\
\hline $\mathrm{C}$ & -0.813208 & 2.739696 & -0.495032 \\
\hline $\mathrm{Li}$ & -2.467580 & 0.979662 & -0.556663 \\
\hline $\mathrm{H}$ & -0.341039 & 1.472029 & -1.929806 \\
\hline $\mathrm{O}$ & 2.178756 & -0.014639 & -0.010347 \\
\hline $\mathrm{Si}$ & -0.075658 & -1.810989 & 2.319210 \\
\hline $\mathrm{Si}$ & -0.189128 & -2.978616 & -0.508818 \\
\hline $\mathrm{Li}$ & -2.395095 & -1.591549 & 0.283156 \\
\hline $\mathrm{C}$ & -0.661588 & 3.897893 & -1.302387 \\
\hline $\mathrm{C}$ & -1.223554 & 2.954890 & 0.849712 \\
\hline $\mathrm{N}$ & -3.770880 & -0.428440 & -0.425662 \\
\hline $\mathrm{C}$ & 3.116044 & 0.801166 & -0.082978 \\
\hline $\mathrm{C}$ & -0.557178 & -0.146716 & 3.106126 \\
\hline $\mathrm{C}$ & -0.952908 & -3.209858 & 3.261135 \\
\hline $\mathrm{C}$ & 1.775823 & -2.026103 & 2.682906 \\
\hline $\mathrm{C}$ & 0.534310 & -2.237997 & -2.100969 \\
\hline $\mathrm{C}$ & 0.944713 & -4.401220 & 0.030479 \\
\hline $\mathrm{C}$ & -1.850307 & -3.808460 & -0.970457 \\
\hline $\mathrm{C}$ & -0.919431 & 5.167793 & -0.801754 \\
\hline $\mathrm{H}$ & -0.329438 & 3.774527 & -2.331298 \\
\hline $\mathrm{C}$ & -1.491417 & 4.233530 & 1.335984 \\
\hline $\mathrm{H}$ & -1.299612 & 2.096099 & 1.513874 \\
\hline $\mathrm{Si}$ & -4.827281 & -0.264213 & 0.930434 \\
\hline $\mathrm{Si}$ & -4.315177 & -0.580722 & -2.059554 \\
\hline $\mathrm{N}$ & 4.393107 & 0.391225 & -0.288795 \\
\hline $\mathrm{C}$ & 2.858150 & 2.263172 & 0.069958 \\
\hline $\mathrm{H}$ & -1.608098 & 0.115092 & 2.931994 \\
\hline $\mathrm{H}$ & -0.395187 & -0.151083 & 4.191005 \\
\hline $\mathrm{H}$ & 0.052063 & 0.667224 & 2.690760 \\
\hline $\mathrm{H}$ & -0.619743 & -4.185543 & 2.885341 \\
\hline $\mathrm{H}$ & -0.724951 & -3.177258 & 4.334231 \\
\hline $\mathrm{H}$ & -2.042525 & -3.171280 & 3.151849 \\
\hline $\mathrm{H}$ & 2.146629 & -3.006776 & 2.364976 \\
\hline $\mathrm{H}$ & 1.966511 & -1.935428 & 3.760232 \\
\hline $\mathrm{H}$ & 2.368594 & -1.265711 & 2.164677 \\
\hline $\mathrm{H}$ & -0.058542 & -1.383638 & -2.449910 \\
\hline $\mathrm{H}$ & 0.550116 & -2.974500 & -2.914187 \\
\hline $\mathrm{H}$ & 1.561543 & -1.888476 & -1.949886 \\
\hline $\mathrm{H}$ & 0.526525 & -4.948426 & 0.883406 \\
\hline $\mathrm{H}$ & 1.073076 & -5.116421 & -0.791953 \\
\hline $\mathrm{H}$ & 1.937597 & -4.047000 & 0.324850 \\
\hline $\mathrm{H}$ & -2.611817 & -3.115074 & -1.352935 \\
\hline
\end{tabular}




\begin{tabular}{|c|c|c|c|}
\hline $\mathrm{H}$ & -2.275631 & -4.324768 & -0.09799 \\
\hline $\mathrm{H}$ & -1.710434 & -4.561186 & -1.75620 \\
\hline $\mathrm{C}$ & -1.341440 & 5.356421 & 0.520932 \\
\hline $\mathrm{H}$ & -0.788620 & 6.028737 & -1.4538 \\
\hline $\mathrm{H}$ & -1.808331 & 4.348837 & 2.37012 \\
\hline $\mathrm{C}$ & -6.660472 & -0.631831 & 0.62187 \\
\hline $\mathrm{C}$ & -4.709781 & 1.475483 & 1.677493 \\
\hline $\mathrm{C}$ & -4.267846 & -1.496215 & 2.27031 \\
\hline $\mathrm{C}$ & -5.379591 & -2.114155 & -2.39157 \\
\hline $\mathrm{C}$ & -2.807733 & -0.684796 & -3.2098 \\
\hline $\mathrm{C}$ & -5.289890 & 0.952058 & -2.61648 \\
\hline $\mathrm{C}$ & 5.595692 & 1.249607 & -0.107894 \\
\hline $\mathrm{C}$ & 4.814529 & -0.960337 & -0.445459 \\
\hline $\mathrm{C}$ & 2.208955 & 2.705183 & 1.227150 \\
\hline $\mathrm{C}$ & 3.170654 & 3.176424 & -0.945878 \\
\hline $\mathrm{H}$ & -1.549917 & 6.351948 & 0.900500 \\
\hline $\mathrm{H}$ & -7.093533 & 0.006032 & -0.156781 \\
\hline $\mathrm{H}$ & -7.233340 & -0.462677 & 1.542422 \\
\hline $\mathrm{H}$ & -6.814185 & -1.674674 & 0.32286 \\
\hline $\mathrm{H}$ & -3.682019 & 1.731651 & 1.964520 \\
\hline $\mathrm{H}$ & -5.040629 & 2.233039 & 0.954904 \\
\hline $\mathrm{H}$ & -5.333989 & 1.577816 & 2.574125 \\
\hline $\mathrm{H}$ & -3.234967 & -1.332640 & 2.60044 \\
\hline $\mathrm{H}$ & -4.350376 & -2.533921 & 1.915846 \\
\hline $\mathrm{H}$ & -4.893233 & -1.414455 & 3.167873 \\
\hline $\mathrm{H}$ & -4.891754 & -3.023637 & -2.0193 \\
\hline $\mathrm{H}$ & -5.550622 & -2.245588 & -3.46756 \\
\hline $\mathrm{H}$ & -6.358938 & -2.041467 & -1.907184 \\
\hline $\mathrm{H}$ & -3.124312 & -0.633212 & -4.259113 \\
\hline $\mathrm{H}$ & -2.104741 & 0.141060 & -3.038291 \\
\hline $\mathrm{H}$ & -2.250125 & -1.618368 & -3.08041 \\
\hline $\mathrm{H}$ & -5.619236 & 0.870874 & -3.659989 \\
\hline $\mathrm{H}$ & -4.669627 & 1.856109 & -2.537607 \\
\hline $\mathrm{H}$ & -6.179743 & 1.114581 & -1.997509 \\
\hline $\mathrm{C}$ & 6.755057 & 0.407797 & -0.676423 \\
\hline $\mathrm{H}$ & 5.471107 & 2.205445 & -0.609524 \\
\hline $\mathrm{H}$ & 5.729545 & 1.438518 & 0.963354 \\
\hline $\mathrm{C}$ & 4.054106 & -2.129263 & -0.408627 \\
\hline $\mathrm{C}$ & 6.200093 & -0.996021 & -0.650676 \\
\hline $\mathrm{C}$ & 1.909043 & 4.056394 & 1.385326 \\
\hline $\mathrm{H}$ & 1.936754 & 1.986751 & 1.994320 \\
\hline $\mathrm{C}$ & 2.847552 & 4.522403 & 3083 \\
\hline
\end{tabular}




$\begin{array}{llll}\mathrm{H} & 3.637895 & 2.825959 & -1.862320 \\ \mathrm{H} & 7.665188 & 0.524496 & -0.080703 \\ \mathrm{H} & 6.994904 & 0.712902 & -1.703455 \\ \mathrm{C} & 4.720906 & -3.344391 & -0.592169 \\ \mathrm{H} & 2.992806 & -2.092472 & -0.227218 \\ \mathrm{C} & 6.848907 & -2.210438 & -0.828255 \\ \mathrm{C} & 2.226310 & 4.964960 & 0.376913 \\ \mathrm{H} & 1.394347 & 4.393482 & 2.277880 \\ \mathrm{H} & 3.068713 & 5.224288 & -1.591710 \\ \mathrm{C} & 6.100909 & -3.392837 & -0.798642 \\ \mathrm{H} & 4.144844 & -4.264419 & -0.565359 \\ \mathrm{H} & 7.923530 & -2.240229 & -0.986643 \\ \mathrm{H} & 1.961481 & 6.010931 & 0.490007 \\ \mathrm{H} & 6.596376 & -4.349619 & -0.933069\end{array}$

\section{S1t-1ag}

$\begin{array}{llll}\mathrm{Li} & 0.626028 & 0.197701 & 0.045383 \\ \mathrm{~N} & 0.858764 & -0.784495 & -1.707969 \\ \mathrm{~N} & 1.893968 & 1.073902 & 1.261133 \\ \mathrm{C} & -0.150410 & -1.729249 & -1.814265 \\ \mathrm{Li} & 2.704572 & -1.157662 & -1.516316 \\ \mathrm{H} & 0.607227 & 0.026259 & -2.278188 \\ \mathrm{O} & -1.189109 & 0.523820 & 0.190871 \\ \mathrm{Si} & 1.421192 & 0.587736 & 2.846835 \\ \mathrm{Si} & 1.999941 & 2.700331 & 0.699872 \\ \mathrm{Li} & 3.574097 & 0.357621 & 0.647997 \\ \mathrm{C} & -1.402574 & -1.467254 & -2.425637 \\ \mathrm{C} & 0.022105 & -3.021785 & -1.254774 \\ \mathrm{~N} & 4.399897 & -0.806195 & -0.687235 \\ \mathrm{C} & -2.393854 & 0.325355 & 0.335048 \\ \mathrm{C} & 1.197938 & -1.298886 & 2.837988 \\ \mathrm{C} & 2.706699 & 1.055405 & 4.166415 \\ \mathrm{C} & -0.232139 & 1.302008 & 3.450352 \\ \mathrm{C} & 1.238151 & 2.824703 & -1.033683 \\ \mathrm{C} & 1.171848 & 4.019611 & 1.779045 \\ \mathrm{C} & 3.836862 & 3.197485 & 0.568155 \\ \mathrm{C} & -2.402308 & -2.433824 & -2.479894 \\ \mathrm{H} & -1.573675 & -0.482832 & -2.857774 \\ \mathrm{C} & -0.982680 & -3.984718 & -1.318605 \\ \mathrm{H} & 0.965039 & -3.258434 & -0.767661 \\ \mathrm{Si} & 4.936590 & -2.084629 & 0.341089 \\ \mathrm{Si} & 5.372588 & -0.036071 & -1.889837\end{array}$




\begin{tabular}{|c|c|c|c|}
\hline $\mathrm{N}$ & -3.302740 & 1.174387 & -0.298619 \\
\hline $\mathrm{C}$ & -2.889788 & -0.706977 & 1.276315 \\
\hline $\mathrm{H}$ & 2.110220 & -1.830066 & 2.545565 \\
\hline $\mathrm{H}$ & 0.904738 & -1.668728 & 3.828301 \\
\hline $\mathrm{H}$ & 0.402672 & -1.594667 & 2.140811 \\
\hline $\mathrm{H}$ & 2.812669 & 2.146898 & 4.217175 \\
\hline $\mathrm{H}$ & 2.413148 & 0.706545 & 5.164622 \\
\hline $\mathrm{H}$ & 3.699377 & 0.642363 & 3.950006 \\
\hline $\mathrm{H}$ & -0.164309 & 2.373325 & 3.665249 \\
\hline $\mathrm{H}$ & -0.550724 & 0.797255 & 4.371393 \\
\hline $\mathrm{H}$ & -1.016956 & 1.154051 & 2.702354 \\
\hline $\mathrm{H}$ & 1.714678 & 2.131324 & -1.736726 \\
\hline $\mathrm{H}$ & 1.346045 & 3.833710 & -1.449198 \\
\hline $\mathrm{H}$ & 0.166477 & 2.598452 & -1.009009 \\
\hline $\mathrm{H}$ & 1.579353 & 4.033113 & 2.796710 \\
\hline $\mathrm{H}$ & 1.326000 & 5.015677 & 1.344771 \\
\hline $\mathrm{H}$ & 0.093023 & 3.849806 & 1.850685 \\
\hline $\mathrm{H}$ & 4.423311 & 2.525422 & -0.072577 \\
\hline $\mathrm{H}$ & 4.302280 & 3.196418 & 1.563044 \\
\hline $\mathrm{H}$ & 3.957926 & 4.203059 & 0.145982 \\
\hline $\mathrm{C}$ & -2.209406 & -3.704344 & -1.928016 \\
\hline $\mathrm{H}$ & -3.346761 & -2.188711 & -2.959673 \\
\hline $\mathrm{H}$ & -0.803886 & -4.965304 & -0.883263 \\
\hline $\mathrm{C}$ & 6.580995 & -2.906522 & -0.112316 \\
\hline $\mathrm{C}$ & 3.621145 & -3.451529 & 0.400000 \\
\hline $\mathrm{C}$ & 5.139221 & -1.407134 & 2.110100 \\
\hline $\mathrm{C}$ & 6.835187 & 0.922190 & -1.153569 \\
\hline $\mathrm{C}$ & 4.278519 & 1.184877 & -2.849511 \\
\hline $\mathrm{C}$ & 6.067041 & -1.236144 & -3.185548 \\
\hline $\mathrm{C}$ & -4.612492 & 0.797405 & -0.703847 \\
\hline $\mathrm{C}$ & -2.806835 & 2.444607 & -0.766975 \\
\hline $\mathrm{C}$ & -2.291310 & -1.969484 & 1.267381 \\
\hline $\mathrm{C}$ & -3.875058 & -0.395734 & 2.221817 \\
\hline $\mathrm{H}$ & -2.989391 & -4.457765 & -1.979682 \\
\hline $\mathrm{H}$ & 6.515210 & -3.443164 & -1.065163 \\
\hline $\mathrm{H}$ & 6.860274 & -3.634155 & 0.660049 \\
\hline $\mathrm{H}$ & 7.398553 & -2.180879 & -0.193408 \\
\hline $\mathrm{H}$ & 2.645244 & -3.063731 & 0.717107 \\
\hline $\mathrm{H}$ & 3.492564 & -3.917975 & -0.586432 \\
\hline $\mathrm{H}$ & 3.893931 & -4.248402 & 1.102775 \\
\hline $\mathrm{H}$ & 4.216042 & -0.977882 & 2.520210 \\
\hline $\mathrm{H}$ & 5.912984 & -0.627670 & 2.138621 \\
\hline
\end{tabular}




\begin{tabular}{|c|c|c|c|}
\hline $\mathrm{H}$ & 5.441598 & -2.196360 & 2.809636 \\
\hline $\mathrm{H}$ & 6.501659 & 1.692632 & -0.447361 \\
\hline $\mathrm{H}$ & 7.428927 & 1.420161 & -1.930354 \\
\hline $\mathrm{H}$ & 7.505354 & 0.245891 & -0.608451 \\
\hline $\mathrm{H}$ & 4.842450 & 1.646144 & -3.669629 \\
\hline $\mathrm{H}$ & 3.407450 & 0.689114 & -3.302688 \\
\hline $\mathrm{H}$ & 3.900172 & 1.993275 & -2.216176 \\
\hline $\mathrm{H}$ & 6.520529 & -0.688421 & -4.021299 \\
\hline $\mathrm{H}$ & 5.272001 & -1.870639 & -3.600141 \\
\hline $\mathrm{H}$ & 6.833145 & -1.897938 & -2.769370 \\
\hline $\mathrm{O}$ & -4.747889 & -0.522702 & -0.642278 \\
\hline $\mathrm{O}$ & -5.422747 & 1.612263 & -1.085897 \\
\hline $\mathrm{C}$ & -2.686615 & 2.691354 & -2.133300 \\
\hline $\mathrm{C}$ & -2.424228 & 3.404140 & 0.168539 \\
\hline $\mathrm{C}$ & -2.699715 & -2.930423 & 2.189270 \\
\hline $\mathrm{H}$ & -1.540480 & -2.201794 & 0.522058 \\
\hline $\mathrm{C}$ & -4.260748 & -1.352133 & 3.157563 \\
\hline $\mathrm{H}$ & -4.325213 & 0.592588 & 2.229203 \\
\hline $\mathrm{C}$ & -6.060472 & -1.182851 & -0.800851 \\
\hline $\mathrm{C}$ & -2.185355 & 3.918579 & -2.565601 \\
\hline $\mathrm{H}$ & -2.987783 & 1.930267 & -2.845694 \\
\hline $\mathrm{C}$ & -1.914410 & 4.625463 & -0.269252 \\
\hline $\mathrm{H}$ & -2.516953 & 3.186469 & 1.227972 \\
\hline $\mathrm{C}$ & -3.679455 & -2.624011 & 3.136280 \\
\hline $\mathrm{H}$ & -2.250639 & -3.918593 & 2.164258 \\
\hline $\mathrm{H}$ & -5.011888 & -1.107281 & 3.902685 \\
\hline $\mathrm{C}$ & -5.739042 & -2.629984 & -0.432273 \\
\hline $\mathrm{C}$ & -6.500763 & -1.051627 & -2.257908 \\
\hline $\mathrm{C}$ & -7.073249 & -0.582065 & 0.174237 \\
\hline $\mathrm{C}$ & -1.797683 & 4.885095 & -1.635694 \\
\hline $\mathrm{H}$ & -2.091535 & 4.115454 & -3.629164 \\
\hline $\mathrm{H}$ & -1.605521 & 5.369585 & 0.457884 \\
\hline $\mathrm{H}$ & -3.988598 & -3.372223 & 3.860540 \\
\hline $\mathrm{H}$ & -4.933140 & -3.017027 & -1.060977 \\
\hline $\mathrm{H}$ & -6.629284 & -3.252259 & -0.569743 \\
\hline $\mathrm{H}$ & -5.417318 & -2.698237 & 0.610895 \\
\hline $\mathrm{H}$ & -5.736857 & -1.465102 & -2.924487 \\
\hline $\mathrm{H}$ & -7.428662 & -1.612591 & -2.412296 \\
\hline $\mathrm{H}$ & -6.674164 & -0.006041 & -2.519453 \\
\hline $\mathrm{H}$ & -6.690165 & -0.638740 & 1.197951 \\
\hline $\mathrm{H}$ & -8.001093 & -1.161652 & 0.125617 \\
\hline $\mathrm{H}$ & -7.297517 & 0.457343 & -0.069920 \\
\hline
\end{tabular}


$\mathrm{H} \quad-1.400171 \quad 5.836708 \quad-1.975434$

\section{S1t-1r}

$0.249679 \quad 2.804001 \quad 0.979590$

$\mathrm{N} \quad 0.928040 \quad 1.891564-0.316973$

$\begin{array}{llll}\text { C } & -0.440858 & 1.581721 & 2.257945\end{array}$

$\mathrm{Si} \quad 1.470832 \quad 2.551720-1.809278$

$\mathrm{Li} \quad-0.426274 \quad 0.510332 \quad-0.759461$

$\begin{array}{lllll}\mathrm{Li} & 2.497697 & 0.876664 & 0.152623\end{array}$

$\begin{array}{llll}\text { C } & -1.183247 & 3.942993 & 0.471526\end{array}$

$\begin{array}{llll}\text { C } & 1.536220 & 3.924069 & 1.820178\end{array}$

$\begin{array}{llll}\mathrm{H} & -0.856231 & 2.101570 & 3.130141\end{array}$

$\begin{array}{llll}\mathrm{H} & -1.253300 & 0.986632 & 1.821899\end{array}$

$\begin{array}{llll}\mathrm{H} & 0.325734 & 0.889153 & 2.623729\end{array}$

$\begin{array}{lllll}\text { C } & 0.990157 & 4.345789 & -2.183189\end{array}$

$\begin{array}{llll}\text { C } & 0.816598 & 1.500094 & -3.251477\end{array}$

$\begin{array}{llll}\text { C } & 3.380389 & 2.507702 & -1.850626\end{array}$

$\begin{array}{lllll}\mathrm{N} & & -0.110611 & -1.451163 & -1.103548\end{array}$

$\begin{array}{lllll}\mathrm{O} & -2.243680 & 0.920036 & -0.891176\end{array}$

$\begin{array}{llll}\mathrm{N} & 3.144138 & -0.925332 & 0.451304\end{array}$

$\begin{array}{lllll}\mathrm{H} & -1.827411 & 3.437191 & -0.255378\end{array}$

$\begin{array}{llll}\mathrm{H} & -1.792795 & 4.212382 & 1.343991\end{array}$

$\begin{array}{llll}\mathrm{H} & -0.826609 & 4.873706 & 0.018659\end{array}$

$\begin{array}{llll}\mathrm{H} & 1.099357 & 4.506173 & 2.641711\end{array}$

$\begin{array}{llll}\mathrm{H} & 1.937975 & 4.637364 & 1.088718\end{array}$

$\begin{array}{llll}\mathrm{H} & 2.385542 & 3.363597 & 2.229396\end{array}$

$\begin{array}{llll}\mathrm{H} & 1.331223 & 5.036028 & -1.402572\end{array}$

$\mathrm{H} \quad 1.441262 \quad 4.668422 \quad-3.130212$

$\begin{array}{lllll}\mathrm{H} & -0.095748 & 4.457653 & -2.275152\end{array}$

$\mathrm{H} \quad \begin{array}{llll}\mathrm{H} & 1.068120 & 0.439451 & -3.132389\end{array}$

$\mathrm{H} \quad-0.277188 \quad 1.581753 \quad-3.313941$

$\mathrm{H} \quad \begin{array}{llll}\mathrm{H} & 1.226062 & 1.828006 & -4.214851\end{array}$

$\mathrm{H} \quad 3.813477 \quad 1.516858 \quad-1.658852$

$\mathrm{H} \quad 3.784772 \quad 3.198593 \quad-1.097740$

$\mathrm{H} \quad 3.770475 \quad 2.821811 \quad-2.826817$

$\begin{array}{lllll}\text { C } & -1.160013 & -2.305714 & -0.808577\end{array}$

$\begin{array}{lllll}\mathrm{Li} & 1.407395 & -1.623503 & 0.036629\end{array}$

$\begin{array}{lllll}\mathrm{H} & & 0.144336 & -1.555687 & -2.087547\end{array}$

$\begin{array}{lllll}\text { C } & -3.357788 & 0.355008 & -0.826956\end{array}$

Si $\quad 3.274573 \quad-1.105757 \quad 2.161016$

$\mathrm{Si} \quad 4.262012 \quad-1.519618 \quad-0.723576$

$\begin{array}{lllll}\text { C } & & -1.768938 & -3.169347 & -1.754592\end{array}$ 


\begin{tabular}{|c|c|c|c|}
\hline $\mathrm{C}$ & -1.679944 & -2.343620 & 0.511028 \\
\hline $\mathrm{N}$ & -3.874556 & -0.281666 & -1.900242 \\
\hline $\mathrm{C}$ & -4.116836 & 0.416294 & 0.460218 \\
\hline $\mathrm{C}$ & 4.716664 & -2.154663 & 2.796669 \\
\hline $\mathrm{C}$ & 3.416142 & 0.597126 & 2.988367 \\
\hline $\mathrm{C}$ & 1.669952 & -1.918385 & 2.792196 \\
\hline $\mathrm{C}$ & 5.903736 & -0.569474 & -0.712326 \\
\hline $\mathrm{C}$ & 4.666103 & -3.363919 & -0.525804 \\
\hline $\mathrm{C}$ & 3.476930 & -1.361219 & -2.446508 \\
\hline $\mathrm{C}$ & -2.824930 & -4.005660 & -1.401708 \\
\hline $\mathrm{H}$ & -1.390191 & -3.174788 & -2.775167 \\
\hline $\mathrm{C}$ & -2.718943 & -3.201148 & 0.860028 \\
\hline $\mathrm{H}$ & -1.254824 & -1.675616 & 1.257885 \\
\hline $\mathrm{C}$ & -5.239530 & -0.780344 & -2.017456 \\
\hline $\mathrm{C}$ & -3.068805 & -0.418082 & -3.108623 \\
\hline $\mathrm{C}$ & -4.787393 & -0.686983 & 1.005015 \\
\hline $\mathrm{C}$ & -4.050275 & 1.611316 & 1.190077 \\
\hline $\mathrm{H}$ & 5.679223 & -1.810419 & 2.400464 \\
\hline $\mathrm{H}$ & 4.770460 & -2.099695 & 3.891149 \\
\hline $\mathrm{H}$ & 4.601033 & -3.208887 & 2.522792 \\
\hline $\mathrm{H}$ & 3.450689 & 0.513817 & 4.081720 \\
\hline $\mathrm{H}$ & 4.334021 & 1.107278 & 2.666360 \\
\hline $\mathrm{H}$ & 2.569084 & 1.251674 & 2.751438 \\
\hline $\mathrm{H}$ & 0.773280 & -1.342385 & 2.524443 \\
\hline $\mathrm{H}$ & 1.549811 & -2.934927 & 2.389466 \\
\hline $\mathrm{H}$ & 1.662684 & -2.008952 & 3.885351 \\
\hline $\mathrm{H}$ & 6.387057 & -0.648486 & 0.269719 \\
\hline $\mathrm{H}$ & 6.606666 & -0.956907 & -1.460359 \\
\hline $\mathrm{H}$ & 5.755235 & 0.498014 & -0.918082 \\
\hline $\mathrm{H}$ & 5.236595 & -3.730035 & -1.388803 \\
\hline $\mathrm{H}$ & 3.748710 & -3.964840 & -0.460837 \\
\hline $\mathrm{H}$ & 5.258111 & -3.566666 & 0.372184 \\
\hline $\mathrm{H}$ & 4.162480 & -1.729499 & -3.219829 \\
\hline $\mathrm{H}$ & 2.558876 & -1.960836 & -2.515594 \\
\hline $\mathrm{H}$ & 3.213238 & -0.330840 & -2.705497 \\
\hline $\mathrm{C}$ & -3.315200 & -4.035550 & -0.091213 \\
\hline $\mathrm{H}$ & -3.261331 & -4.654885 & -2.157917 \\
\hline $\mathrm{H}$ & -3.079177 & -3.201194 & 1.885820 \\
\hline $\mathrm{H}$ & -5.641259 & -0.474850 & -2.989989 \\
\hline $\mathrm{H}$ & -5.256040 & -1.874898 & -1.957763 \\
\hline $\mathrm{H}$ & -5.871736 & -0.364152 & -1.235666 \\
\hline $\mathrm{H}$ & -3.222240 & -1.418268 & -3.521008 \\
\hline
\end{tabular}




$\begin{array}{llll}\mathrm{H} & -3.362539 & 0.332755 & -3.853621 \\ \mathrm{H} & -2.017955 & -0.290509 & -2.862485 \\ \mathrm{C} & -5.392714 & -0.588313 & 2.256928 \\ \mathrm{H} & -4.792054 & -1.632878 & 0.477916 \\ \mathrm{C} & -4.672611 & 1.711885 & 2.431805 \\ \mathrm{H} & -3.502582 & 2.450775 & 0.779844 \\ \mathrm{C} & -5.344417 & 0.611560 & 2.968806 \\ \mathrm{H} & -5.894859 & -1.453969 & 2.679221 \\ \mathrm{H} & -4.622632 & 2.645215 & 2.984757 \\ \mathrm{H} & -5.820450 & 0.685903 & 3.942440 \\ \mathrm{H} & -4.124566 & -4.704963 & 0.184189\end{array}$

\section{S1t-1s}

\begin{tabular}{|c|c|c|c|}
\hline $\mathrm{Li}$ & -0.235300 & 0.549166 & -0.62808 \\
\hline $\mathrm{N}$ & 0.001762 & -1.386818 & -1.113940 \\
\hline $\mathrm{N}$ & 1.172108 & 1.885505 & -0.216219 \\
\hline $\mathrm{C}$ & -1.033554 & -2.253485 & -0.802024 \\
\hline $\mathrm{Li}$ & 1.554387 & -1.676004 & -0.040083 \\
\hline $\mathrm{H}$ & 0.214622 & -1.457967 & -2.110755 \\
\hline $\mathrm{O}$ & -2.027617 & 0.963801 & -0.411833 \\
\hline $\mathrm{Si}$ & 0.596043 & 2.787419 & 1.137333 \\
\hline $\mathrm{Si}$ & 1.677553 & 2.573316 & -1.708964 \\
\hline $\mathrm{Li}$ & 2.717575 & 0.790567 & 0.143285 \\
\hline $\mathrm{C}$ & -1.673889 & -3.094731 & -1.747394 \\
\hline $\mathrm{C}$ & -1.498790 & -2.331525 & 0.536319 \\
\hline $\mathrm{N}$ & 3.318759 & -1.041474 & 0.343556 \\
\hline $\mathrm{C}$ & -3.182775 & 0.491660 & -0.335949 \\
\hline $\mathrm{C}$ & -0.019853 & 1.564337 & 2.451981 \\
\hline $\mathrm{C}$ & 1.954033 & 3.876803 & 1.903595 \\
\hline $\mathrm{C}$ & -0.852338 & 3.950244 & 0.738739 \\
\hline $\mathrm{C}$ & 0.974357 & 1.559759 & -3.155277 \\
\hline $\mathrm{C}$ & 1.196242 & 4.377257 & -2.031781 \\
\hline $\mathrm{C}$ & 3.584460 & 2.512382 & -1.809030 \\
\hline $\mathrm{C}$ & -2.690559 & -3.967291 & -1.370356 \\
\hline $\mathrm{H}$ & -1.346357 & -3.058112 & -2.785099 \\
\hline $\mathrm{C}$ & -2.508489 & -3.215919 & 0.905263 \\
\hline $\mathrm{H}$ & -1.054633 & -1.674207 & 1.281537 \\
\hline $\mathrm{Si}$ & 3.522227 & -1.253487 & 2.042724 \\
\hline $\mathrm{S}$ & 4.395262 & -1.608613 & -0.882463 \\
\hline $\mathrm{N}$ & -3.813118 & 0.035966 & -1.440285 \\
\hline $\mathrm{C}$ & -3.851282 & 0.471487 & 1.001495 \\
\hline 1 & 0.774344 & 0.896083 & 2.802898 \\
\hline
\end{tabular}




\begin{tabular}{|c|c|c|c|}
\hline $\mathrm{H}$ & -0.418902 & 2.086778 & 3.330375 \\
\hline $\mathrm{H}$ & -0.830557 & 0.946542 & 2.047255 \\
\hline $\mathrm{H}$ & 2.318226 & 4.599154 & 1.161728 \\
\hline $\mathrm{H}$ & 1.584443 & 4.447454 & 2.765173 \\
\hline $\mathrm{H}$ & 2.818921 & 3.292968 & 2.241902 \\
\hline $\mathrm{H}$ & -0.519704 & 4.864366 & 0.236386 \\
\hline $\mathrm{H}$ & -1.373974 & 4.250297 & 1.657145 \\
\hline $\mathrm{H}$ & -1.571354 & 3.445090 & 0.085232 \\
\hline $\mathrm{H}$ & 1.220785 & 0.495434 & -3.068326 \\
\hline $\mathrm{H}$ & 1.360345 & 1.908638 & -4.121112 \\
\hline $\mathrm{H}$ & -0.119594 & 1.648432 & -3.189179 \\
\hline $\mathrm{H}$ & 1.573525 & 5.052159 & -1.254727 \\
\hline $\mathrm{H}$ & 1.609285 & 4.713091 & -2.991444 \\
\hline $\mathrm{H}$ & 0.107938 & 4.497117 & -2.075931 \\
\hline $\mathrm{H}$ & 4.011188 & 1.511848 & -1.654487 \\
\hline $\mathrm{H}$ & 4.017341 & 3.177980 & -1.049585 \\
\hline $\mathrm{H}$ & 3.950816 & 2.846887 & -2.787641 \\
\hline $\mathrm{C}$ & -3.121676 & -4.043477 & -0.040945 \\
\hline $\mathrm{H}$ & -3.151079 & -4.601024 & -2.125103 \\
\hline $\mathrm{H}$ & -2.826571 & -3.247486 & 1.944453 \\
\hline $\mathrm{C}$ & 4.930920 & -2.391356 & 2.593661 \\
\hline $\mathrm{C}$ & 1.907819 & -1.979370 & 2.746373 \\
\hline $\mathrm{C}$ & 3.813838 & 0.430526 & 2.871454 \\
\hline $\mathrm{C}$ & 6.065476 & -0.708646 & -0.854250 \\
\hline $\mathrm{C}$ & 3.587185 & -1.334030 & -2.578795 \\
\hline $\mathrm{C}$ & 4.746726 & -3.471668 & -0.784555 \\
\hline $\mathrm{C}$ & -5.247568 & -0.255936 & -1.534019 \\
\hline $\mathrm{C}$ & -3.064342 & 0.048263 & -2.706409 \\
\hline $\mathrm{C}$ & -3.668047 & 1.588894 & 1.827826 \\
\hline $\mathrm{C}$ & -4.546348 & -0.641572 & 1.491486 \\
\hline $\mathrm{H}$ & -3.903084 & -4.738945 & 0.250344 \\
\hline $\mathrm{H}$ & 4.749306 & -3.430682 & 2.299065 \\
\hline $\mathrm{H}$ & 5.029887 & -2.367537 & 3.686175 \\
\hline $\mathrm{H}$ & 5.893953 & -2.086850 & 2.167479 \\
\hline $\mathrm{H}$ & 1.031423 & -1.358942 & 2.514329 \\
\hline $\mathrm{H}$ & 1.718953 & -2.988783 & 2.352512 \\
\hline $\mathrm{H}$ & 1.944212 & -2.067582 & 3.839159 \\
\hline $\mathrm{H}$ & 3.003671 & 1.143264 & 2.676001 \\
\hline $\mathrm{H}$ & 4.748160 & 0.879097 & 2.507667 \\
\hline $\mathrm{H}$ & 3.892890 & 0.339875 & 3.961904 \\
\hline $\mathrm{H}$ & 5.944972 & 0.372559 & -0.998557 \\
\hline $\mathrm{H}$ & 6.741576 & -1.074313 & -1.637161 \\
\hline
\end{tabular}




\begin{tabular}{llll}
$\mathrm{H}$ & 6.565718 & -0.855565 & 0.111255 \\
$\mathrm{H}$ & 4.235669 & -1.704641 & -3.382252 \\
$\mathrm{H}$ & 2.634836 & -1.876614 & -2.653974 \\
$\mathrm{H}$ & 3.379062 & -0.279476 & -2.784942 \\
$\mathrm{H}$ & 5.310287 & -3.806359 & -1.664790 \\
$\mathrm{H}$ & 3.813017 & -4.049966 & -0.755138 \\
$\mathrm{H}$ & 5.329727 & -3.739574 & 0.102409 \\
$\mathrm{C}$ & -5.556863 & -1.731079 & -1.790665 \\
$\mathrm{H}$ & -5.727613 & 0.083841 & -0.616546 \\
$\mathrm{H}$ & -5.652296 & 0.356961 & -2.349891 \\
$\mathrm{C}$ & -3.073636 & 1.422471 & -3.376714 \\
$\mathrm{H}$ & -2.044842 & -0.273332 & -2.493358 \\
$\mathrm{H}$ & -3.515781 & -0.705848 & -3.355516 \\
$\mathrm{C}$ & -4.201376 & 1.605298 & 3.114233 \\
$\mathrm{H}$ & -3.100140 & 2.433242 & 1.455712 \\
$\mathrm{C}$ & -5.064107 & -0.628285 & 2.785798 \\
$\mathrm{H}$ & -4.638033 & -1.531726 & 0.882636 \\
$\mathrm{H}$ & -5.144641 & -2.069011 & -2.746368 \\
$\mathrm{H}$ & -6.642112 & -1.878319 & -1.821735 \\
$\mathrm{H}$ & -5.131047 & -2.370964 & -1.013857 \\
$\mathrm{H}$ & -4.095085 & 1.759207 & -3.587210 \\
$\mathrm{H}$ & -2.529566 & 1.382429 & -4.326642 \\
$\mathrm{H}$ & -2.589975 & 2.161660 & -2.732515 \\
$\mathrm{H}$ & -4.900133 & 0.496444 & 3.596439 \\
$\mathrm{H}$ & -4.061379 & 2.479055 & 3.743795 \\
\hline & -5.587635 & -1.501908 & 3.163262 \\
$\mathrm{H}$ & -5.306542 & 0.504746 & 4.603807
\end{tabular}

\section{S1t-1v}

$\begin{array}{llll}\mathrm{Li} & 0.381895 & -0.138420 & -0.503601 \\ \mathrm{~N} & -0.336235 & 1.629984 & -1.113316 \\ \mathrm{~N} & -0.586058 & -1.640625 & 0.424418 \\ \mathrm{C} & 0.301179 & 2.780882 & -0.666885 \\ \mathrm{Li} & -2.131584 & 1.478564 & -0.509560 \\ \mathrm{H} & -0.131525 & 1.512524 & -2.108202 \\ \mathrm{O} & 2.163195 & -0.511907 & -0.952484 \\ \mathrm{Si} & 0.028732 & -1.678345 & 2.030039 \\ \mathrm{Si} & -0.681144 & -2.994065 & -0.634748 \\ \mathrm{Li} & -2.440955 & -1.112872 & 0.287927 \\ \mathrm{C} & 1.162507 & 3.566450 & -1.472038 \\ \mathrm{C} & 0.084169 & 3.237083 & 0.658836 \\ \mathrm{~N} & -3.638356 & 0.311662 & -0.210499\end{array}$




\begin{tabular}{|c|c|c|c|}
\hline $\mathrm{C}$ & 3.371574 & -0.350567 & -0.691989 \\
\hline $\mathrm{C}$ & -0.116049 & 0.063873 & 2.772401 \\
\hline $\mathrm{C}$ & -0.896423 & -2.881865 & 3.173727 \\
\hline $\mathrm{C}$ & 1.866360 & -2.156996 & 2.158661 \\
\hline $\mathrm{C}$ & -0.058324 & -2.552128 & -2.369856 \\
\hline $\mathrm{C}$ & 0.226348 & -4.573285 & -0.096901 \\
\hline $\mathrm{C}$ & -2.520790 & -3.496320 & -0.800492 \\
\hline $\mathrm{C}$ & 1.735165 & 4.739514 & -0.991941 \\
\hline $\mathrm{H}$ & 1.368141 & 3.237761 & -2.489107 \\
\hline $\mathrm{C}$ & 0.653202 & 4.418963 & 1.127862 \\
\hline $\mathrm{H}$ & -0.551196 & 2.648705 & 1.317280 \\
\hline $\mathrm{Si}$ & -4.435334 & 0.726934 & 1.261829 \\
\hline $\mathrm{Si}$ & -4.392051 & 0.245467 & -1.763987 \\
\hline $\mathrm{N}$ & 4.235103 & -1.383910 & -0.759442 \\
\hline $\mathrm{C}$ & 3.892084 & 0.984727 & -0.276956 \\
\hline $\mathrm{H}$ & -1.153955 & 0.417669 & 2.788179 \\
\hline $\mathrm{H}$ & 0.253790 & 0.089271 & 3.804868 \\
\hline $\mathrm{H}$ & 0.461031 & 0.800675 & 2.200549 \\
\hline $\mathrm{H}$ & -0.745287 & -3.919486 & 2.852483 \\
\hline $\mathrm{H}$ & -0.542094 & -2.804059 & 4.209489 \\
\hline $\mathrm{H}$ & -1.976802 & -2.696044 & 3.179423 \\
\hline $\mathrm{H}$ & 2.036007 & -3.182113 & 1.810128 \\
\hline $\mathrm{H}$ & 2.212853 & -2.099042 & 3.198330 \\
\hline $\mathrm{H}$ & 2.495353 & -1.492361 & 1.558006 \\
\hline $\mathrm{H}$ & -0.555232 & -1.655314 & -2.758896 \\
\hline $\mathrm{H}$ & -0.246741 & -3.363973 & -3.083557 \\
\hline $\mathrm{H}$ & 1.016786 & -2.346366 & -2.358632 \\
\hline $\mathrm{H}$ & -0.128374 & -4.939014 & 0.873250 \\
\hline $\mathrm{H}$ & 0.058466 & -5.370209 & -0.832450 \\
\hline $\mathrm{H}$ & 1.308658 & -4.423269 & -0.016417 \\
\hline $\mathrm{H}$ & -3.176398 & -2.689104 & -1.157664 \\
\hline $\mathrm{H}$ & -2.915481 & -3.845288 & 0.164324 \\
\hline $\mathrm{H}$ & -2.643996 & -4.317192 & -1.517869 \\
\hline $\mathrm{C}$ & 1.483465 & 5.188060 & 0.309042 \\
\hline $\mathrm{H}$ & 2.390961 & 5.312142 & -1.643778 \\
\hline $\mathrm{H}$ & 0.444535 & 4.738385 & 2.146759 \\
\hline $\mathrm{C}$ & -6.315490 & 0.936206 & 1.176360 \\
\hline $\mathrm{C}$ & -3.709568 & 2.341161 & 1.954875 \\
\hline $\mathrm{C}$ & -4.104903 & -0.635374 & 2.548638 \\
\hline $\mathrm{C}$ & -5.798334 & -1.018824 & -1.890964 \\
\hline $\mathrm{C}$ & -3.085277 & -0.230769 & -3.055985 \\
\hline $\mathrm{C}$ & -5.061021 & 1.942311 & -2.296723 \\
\hline
\end{tabular}




\begin{tabular}{|c|c|c|c|}
\hline $\mathrm{C}$ & 5.604105 & -1.392380 & -0.197041 \\
\hline $\mathrm{C}$ & 3.744404 & -2.747079 & -1.029542 \\
\hline $\mathrm{C}$ & 3.271635 & 1.640429 & 0.792964 \\
\hline $\mathrm{C}$ & 4.935555 & 1.611437 & -0.967359 \\
\hline $\mathrm{H}$ & 1.927444 & 6.109053 & 0.674810 \\
\hline $\mathrm{H}$ & -6.618107 & 1.686736 & 0.438045 \\
\hline $\mathrm{H}$ & -6.701585 & 1.257914 & 2.151752 \\
\hline $\mathrm{H}$ & -6.813545 & -0.005095 & 0.918168 \\
\hline $\mathrm{H}$ & -2.630594 & 2.259147 & 2.142051 \\
\hline $\mathrm{H}$ & -3.862323 & 3.175494 & 1.256510 \\
\hline $\mathrm{H}$ & -4.175414 & 2.624060 & 2.907045 \\
\hline $\mathrm{H}$ & -3.035892 & -0.782348 & 2.745436 \\
\hline $\mathrm{H}$ & -4.523096 & -1.597354 & 2.220594 \\
\hline $\mathrm{H}$ & -4.566486 & -0.392992 & 3.513914 \\
\hline $\mathrm{H}$ & -5.465068 & -2.013060 & -1.567714 \\
\hline $\mathrm{H}$ & -6.156062 & -1.108456 & -2.924469 \\
\hline $\mathrm{H}$ & -6.653947 & -0.737532 & -1.267620 \\
\hline $\mathrm{H}$ & -3.491455 & -0.135721 & -4.070615 \\
\hline $\mathrm{H}$ & -2.199509 & 0.415706 & -2.997078 \\
\hline $\mathrm{H}$ & -2.745768 & -1.264953 & -2.935831 \\
\hline $\mathrm{H}$ & -5.522072 & 1.902822 & -3.291599 \\
\hline $\mathrm{H}$ & -4.250484 & 2.683693 & -2.342840 \\
\hline $\mathrm{H}$ & -5.812815 & 2.324392 & -1.596560 \\
\hline $\mathrm{C}$ & 6.022206 & -2.868569 & -0.285952 \\
\hline $\mathrm{H}$ & 6.270650 & -0.736493 & -0.759374 \\
\hline $\mathrm{H}$ & 5.576935 & -1.035806 & 0.838490 \\
\hline $\mathrm{C}$ & 4.690590 & -3.630555 & -0.213893 \\
\hline $\mathrm{H}$ & 2.699606 & -2.818724 & -0.730560 \\
\hline $\mathrm{H}$ & 3.808636 & -2.960268 & -2.104290 \\
\hline $\mathrm{C}$ & 3.717912 & 2.896100 & 1.193114 \\
\hline $\mathrm{H}$ & 2.446631 & 1.163387 & 1.309853 \\
\hline $\mathrm{C}$ & 5.359845 & 2.882679 & -0.582436 \\
\hline $\mathrm{H}$ & 5.392766 & 1.118729 & -1.820626 \\
\hline $\mathrm{H}$ & 6.723634 & -3.141191 & 0.507071 \\
\hline $\mathrm{H}$ & 6.512334 & -3.059704 & -1.247701 \\
\hline $\mathrm{H}$ & 4.754616 & -4.649389 & -0.604971 \\
\hline $\mathrm{H}$ & 4.337774 & -3.682218 & 0.822374 \\
\hline $\mathrm{C}$ & 4.758367 & 3.520008 & 0.503562 \\
\hline $\mathrm{H}$ & 3.226413 & 3.402005 & 2.015384 \\
\hline $\mathrm{H}$ & 6.155661 & 3.375646 & -1.133277 \\
\hline $\mathrm{H}$ & 5.087028 & 4.510969 & 0.802188 \\
\hline
\end{tabular}




\section{S1t-1w}

$\mathrm{Li} \quad \begin{array}{llll}0.436276 & -0.148890 & -0.473145\end{array}$

$\mathrm{N} \quad-0.381228 \quad 1.478489-1.251736$

$\mathrm{N} \quad-0.480580-1.682029 \quad 0.436215$

C $\quad-0.124561 \quad 2.744490 \quad-0.749516$

$\begin{array}{lllll}\mathrm{Li} & & -2.218659 & 1.303150 & -0.732849\end{array}$

$\mathrm{H} \quad-0.087518 \quad 1.439029 \quad-2.229043$

$\begin{array}{llll}\text { O } & 2.266490 & -0.503597 & -0.789397\end{array}$

$\begin{array}{lllll}\mathrm{Si} & 0.060619 & -1.791960 & 2.065693\end{array}$

$\mathrm{Si} \quad-0.385595 \quad-2.976968-0.697312$

$\begin{array}{llll}\mathrm{Li} & -2.339806 & -1.206777 & 0.268470\end{array}$

C $\quad 0.456432 \quad 3.798281 \quad-1.500111$

$\begin{array}{llll}\text { C } & -0.496211 & 3.050833 & 0.587159\end{array}$

$\begin{array}{lllll}\mathrm{N} & & -3.657094 & 0.126670 & -0.250127\end{array}$

$\begin{array}{lllll}\text { C } & & 3.386658 & 0.033895 & -0.688752\end{array}$

$\begin{array}{lllll}\text { C } & -0.305435 & -0.149177 & 2.945687\end{array}$

$\begin{array}{llll}\text { C } & -0.799154 & -3.174069 & 3.047218\end{array}$

$\begin{array}{llll}\text { C } & 1.928710 & -2.090352 & 2.256040\end{array}$

$\begin{array}{lllll}\text { C } & 0.082825 & -2.325943 & -2.419943\end{array}$

$\begin{array}{llll}\text { C } & 0.847240 & -4.364283 & -0.306136\end{array}$

$\begin{array}{lllll}\text { C } & -2.105125 & -3.793177 & -0.833225\end{array}$

$\begin{array}{lllll}\text { C } & 0.621163 & 5.067071 & -0.956483\end{array}$

$\mathrm{H} \quad 0.774729 \quad 3.597600 \quad-2.521258$

$\begin{array}{llll}\text { C } & -0.345036 & 4.331167 & 1.115215\end{array}$

$\mathrm{H} \quad \begin{array}{llll}\mathrm{H} & 0.899564 & 2.255151 & 1.209634\end{array}$

$\begin{array}{llll}\mathrm{Si} & -4.473259 & 0.443421 & 1.241197\end{array}$

$\begin{array}{lllll}\mathrm{Si} & & -4.492148 & -0.217552 & -1.726252\end{array}$

$\mathrm{N} \quad 4.515614 \quad-0.637056-1.027113$

$\begin{array}{llll}\text { C } & 3.486361 & 1.445842 & -0.216415\end{array}$

$\begin{array}{llll}\mathrm{H} & -1.380429 & 0.060475 & 2.985357\end{array}$

$\begin{array}{llll}\mathrm{H} & 0.062220 & -0.160866 & 3.979249\end{array}$

$\begin{array}{llll}\mathrm{H} & 0.170624 & 0.698420 & 2.436791\end{array}$

$\mathrm{H} \quad \begin{array}{llll}\mathrm{H} & -0.579465 & -4.156215 & 2.610608\end{array}$

$\begin{array}{lllll}\mathrm{H} & -0.468018 & -3.196406 & 4.093274\end{array}$

$\mathrm{H} \quad \begin{array}{llll}\mathrm{H} & -1.889694 & -3.055472 & 3.047765\end{array}$

$\begin{array}{llll}\mathrm{H} & 2.203489 & -3.096003 & 1.920331\end{array}$

$\mathrm{H} \quad 2.239620-1.991804 \quad 3.303861$

$\begin{array}{llll}\mathrm{H} & 2.503533 & -1.376005 & 1.657979\end{array}$

$\mathrm{H} \quad-\quad-0.482831 \quad-1.428545 \quad-2.696210$

$\begin{array}{llll}\mathrm{H} & -0.108673 & -3.079627 & -3.193982\end{array}$

$\begin{array}{llll}\mathrm{H} & 1.146221 & -2.066265 & -2.455701\end{array}$

$\mathrm{H} \quad 0.642514 \quad-4.850741 \quad 0.654360$ 


\begin{tabular}{|c|c|c|c|}
\hline $\mathrm{H}$ & 0.805979 & -5.137771 & -1.083664 \\
\hline $\mathrm{H}$ & 1.872516 & -3.979042 & -0.274153 \\
\hline $\mathrm{H}$ & -2.893561 & -3.088975 & -1.134170 \\
\hline $\mathrm{H}$ & -2.396635 & -4.221356 & 0.135315 \\
\hline $\mathrm{H}$ & -2.120734 & -4.602345 & -1.574067 \\
\hline $\mathrm{C}$ & 0.216213 & 5.356836 & 0.352430 \\
\hline $\mathrm{H}$ & 1.072591 & 5.846916 & -1.566012 \\
\hline $\mathrm{H}$ & -0.659441 & 4.520067 & 2.139326 \\
\hline $\mathrm{C}$ & -6.326722 & 0.822367 & 1.120906 \\
\hline $\mathrm{C}$ & -3.706773 & 1.921164 & 2.149970 \\
\hline $\mathrm{C}$ & -4.285222 & -1.079335 & 2.370791 \\
\hline $\mathrm{C}$ & -5.651235 & -1.717008 & -1.615506 \\
\hline $\mathrm{C}$ & -3.208448 & -0.600756 & -3.071545 \\
\hline $\mathrm{C}$ & -5.498476 & 1.258457 & -2.366025 \\
\hline $\mathrm{C}$ & 4.407407 & -2.014145 & -1.528790 \\
\hline $\mathrm{C}$ & 5.854031 & -0.291969 & -0.530741 \\
\hline $\mathrm{C}$ & 2.791781 & 1.814309 & 0.940983 \\
\hline $\mathrm{C}$ & 4.170339 & 2.419272 & -0.956937 \\
\hline $\mathrm{H}$ & 0.337041 & 6.354639 & 0.763043 \\
\hline $\mathrm{H}$ & -6.503348 & 1.771721 & 0.602485 \\
\hline $\mathrm{H}$ & -6.750262 & 0.914457 & 2.129049 \\
\hline $\mathrm{H}$ & -6.891228 & 0.045246 & 0.595294 \\
\hline $\mathrm{H}$ & -2.661988 & 1.749883 & 2.429982 \\
\hline $\mathrm{H}$ & -3.737371 & 2.828705 & 1.533050 \\
\hline $\mathrm{H}$ & -4.258599 & 2.133424 & 3.074461 \\
\hline $\mathrm{H}$ & -3.234845 & -1.333519 & 2.569562 \\
\hline $\mathrm{H}$ & -4.759450 & -1.959162 & 1.915279 \\
\hline $\mathrm{H}$ & -4.752542 & -0.920384 & 3.350635 \\
\hline $\mathrm{H}$ & -5.110858 & -2.614186 & -1.288777 \\
\hline $\mathrm{H}$ & -6.104427 & -1.940876 & -2.589518 \\
\hline $\mathrm{H}$ & -6.466968 & -1.549618 & -0.903027 \\
\hline $\mathrm{H}$ & -3.688519 & -0.666494 & -4.055733 \\
\hline $\mathrm{H}$ & -2.435321 & 0.177277 & -3.137325 \\
\hline $\mathrm{H}$ & -2.698030 & -1.552700 & -2.892762 \\
\hline $\mathrm{H}$ & -5.935721 & 1.044116 & -3.349411 \\
\hline $\mathrm{H}$ & -4.860957 & 2.146578 & -2.476072 \\
\hline $\mathrm{H}$ & -6.316446 & 1.523384 & -1.688385 \\
\hline $\mathrm{C}$ & 4.781178 & -3.021809 & -0.436838 \\
\hline $\mathrm{H}$ & 3.389999 & -2.166789 & -1.883422 \\
\hline $\mathrm{H}$ & 5.094707 & -2.105815 & -2.380387 \\
\hline $\mathrm{C}$ & 6.269141 & -1.258653 & 0.587016 \\
\hline $\mathrm{H}$ & 5.853093 & 0.736327 & -0.174985 \\
\hline
\end{tabular}




$\begin{array}{llll}\mathrm{H} & 6.556383 & -0.359052 & -1.372112 \\ \mathrm{C} & 2.803086 & 3.138391 & 1.369988 \\ \mathrm{H} & 2.246430 & 1.060808 & 1.500179 \\ \mathrm{C} & 4.159742 & 3.747363 & -0.536551 \\ \mathrm{H} & 4.686537 & 2.136569 & -1.870091 \\ \mathrm{C} & 6.176617 & -2.718707 & 0.125021 \\ \mathrm{H} & 4.737661 & -4.036516 & -0.849567 \\ \mathrm{H} & 4.034701 & -2.962511 & 0.363626 \\ \mathrm{H} & 7.287837 & -1.014755 & 0.911430 \\ \mathrm{H} & 5.605153 & -1.099317 & 1.446992 \\ \mathrm{C} & 3.481427 & 4.106656 & 0.629694 \\ \mathrm{H} & 2.252689 & 3.420938 & 2.259897 \\ \mathrm{H} & 4.670467 & 4.503720 & -1.125229 \\ \mathrm{H} & 6.411059 & -3.395282 & 0.954530 \\ \mathrm{H} & 6.930616 & -2.899695 & -0.654951 \\ \mathrm{H} & 3.460317 & 5.143237 & 0.949575\end{array}$

\section{S1t-1x}

$\mathrm{Li}$

N

N

$\mathrm{Si}$

$\mathrm{Si}$

$\mathrm{Li}$

$\mathrm{O}$

C

$\mathrm{Li}$

$\mathrm{H}$

C

C

$\mathrm{C}$

$\mathrm{C}$

$\begin{array}{llll}\text { C } & 0.225765 & 0.745237 & 2.796192\end{array}$

$\begin{array}{llll}\text { C } & 2.040105 & 3.256644 & 2.822255\end{array}$

$\begin{array}{lllll}\mathrm{N} & 3.601969 & -0.868279 & -0.069823\end{array}$

$\begin{array}{lllll}\text { C } & -3.089141 & 0.275539 & -0.055178\end{array}$

$\begin{array}{lllll}\text { C } & -1.591568 & -2.938366 & -1.758362\end{array}$

$\begin{array}{lllll}\text { C } & -1.099036 & -2.512609 & 0.565224\end{array}$

$\mathrm{H} \quad \begin{array}{llll}\mathrm{H} & 1.282693 & 5.211183 & 0.045549\end{array}$

$\mathrm{H} \quad 1.027098 \quad 5.335625 \quad-1.701448$

$\begin{array}{lllll}\mathrm{H} & -0.289752 & 4.790936 & -0.648743\end{array}$

$\mathrm{H} \quad 0.670858 \quad 1.230075 \quad-2.778001$ 


\begin{tabular}{|c|c|c|c|}
\hline $\mathrm{H}$ & -0.669552 & 2.318254 & -2.367088 \\
\hline $\mathrm{H}$ & 0.608772 & 2.856639 & -3.467580 \\
\hline $\mathrm{H}$ & 3.663743 & 2.087500 & -1.776766 \\
\hline $\mathrm{H}$ & 3.790737 & 3.511763 & -0.726719 \\
\hline $\mathrm{H}$ & 3.356396 & 3.684978 & -2.432452 \\
\hline $\mathrm{H}$ & -1.564653 & 3.144261 & 1.145649 \\
\hline $\mathrm{H}$ & -1.331677 & 3.414159 & 2.886779 \\
\hline $\mathrm{H}$ & -0.587253 & 4.508969 & 1.714755 \\
\hline $\mathrm{H}$ & 0.020635 & 0.941521 & 3.855814 \\
\hline $\mathrm{H}$ & -0.681232 & 0.300570 & 2.367855 \\
\hline $\mathrm{H}$ & 1.022856 & -0.006872 & 2.751906 \\
\hline $\mathrm{H}$ & 1.719880 & 3.499016 & 3.843748 \\
\hline $\mathrm{H}$ & 2.274574 & 4.203258 & 2.318061 \\
\hline $\mathrm{H}$ & 2.973029 & 2.685948 & 2.896350 \\
\hline $\mathrm{Si}$ & 4.078513 & -1.459062 & 1.478135 \\
\hline $\mathrm{Si}$ & 4.481491 & -1.025150 & -1.547747 \\
\hline $\mathrm{N}$ & -3.711914 & -0.078782 & -1.203582 \\
\hline $\mathrm{C}$ & -3.733810 & -0.022657 & 1.258183 \\
\hline $\mathrm{C}$ & -2.551006 & -3.873368 & -1.37650 \\
\hline $\mathrm{H}$ & -1.404601 & -2.753357 & -2.814758 \\
\hline $\mathrm{C}$ & -2.044919 & -3.462801 & 0.936700 \\
\hline $\mathrm{H}$ & -0.553160 & -1.963968 & 1.330309 \\
\hline $\mathrm{C}$ & 5.722049 & -2.394641 & 1.552422 \\
\hline $\mathrm{C}$ & 4.195320 & -0.023736 & 2.715112 \\
\hline $\mathrm{C}$ & 2.720979 & -2.635048 & 2.114874 \\
\hline $\mathrm{C}$ & 5.986642 & 0.125091 & -1.623655 \\
\hline $\mathrm{C}$ & 5.093978 & -2.787068 & -1.899363 \\
\hline $\mathrm{C}$ & 3.302076 & -0.608319 & -2.980591 \\
\hline $\mathrm{C}$ & -5.141895 & -0.355210 & -1.354279 \\
\hline $\mathrm{C}$ & -3.025654 & 0.056572 & -2.490582 \\
\hline $\mathrm{C}$ & -4.354057 & -1.252071 & 1.518586 \\
\hline $\mathrm{C}$ & -3.607539 & 0.921884 & 2.284964 \\
\hline $\mathrm{C}$ & -2.789931 & -4.151388 & -0.026578 \\
\hline $\mathrm{H}$ & -3.110055 & -4.401867 & -2.145717 \\
\hline $\mathrm{H}$ & -2.215821 & -3.651089 & 1.993614 \\
\hline $\mathrm{H}$ & 6.542559 & -1.805897 & 1.125084 \\
\hline $\mathrm{H}$ & 5.983979 & -2.620145 & 2.593670 \\
\hline $\mathrm{H}$ & 5.674413 & -3.344091 & 1.008763 \\
\hline $\mathrm{H}$ & 4.479576 & -0.376630 & 3.714225 \\
\hline $\mathrm{H}$ & 4.950745 & 0.708683 & 2.399233 \\
\hline $\mathrm{H}$ & 3.241740 & 0.504373 & 2.830077 \\
\hline $\mathrm{H}$ & 1.734632 & -2.151860 & 2.161332 \\
\hline
\end{tabular}




\begin{tabular}{|c|c|c|c|}
\hline $\mathrm{H}$ & 2.627149 & -3.525730 & 1.476725 \\
\hline $\mathrm{H}$ & 2.936606 & -2.991700 & 3.129567 \\
\hline $\mathrm{H}$ & 6.689016 & -0.117020 & -0.816065 \\
\hline $\mathrm{H}$ & 6.528049 & 0.031961 & -2.573380 \\
\hline $\mathrm{H}$ & 5.702799 & 1.177745 & -1.504953 \\
\hline $\mathrm{H}$ & 5.441536 & -2.875057 & -2.936552 \\
\hline $\mathrm{H}$ & 4.288706 & -3.521004 & -1.759742 \\
\hline $\mathrm{H}$ & 5.923625 & -3.078433 & -1.247408 \\
\hline $\mathrm{H}$ & 3.834508 & -0.594039 & -3.939563 \\
\hline $\mathrm{H}$ & 2.510588 & -1.366095 & -3.061950 \\
\hline $\mathrm{H}$ & 2.811205 & 0.363343 & -2.861857 \\
\hline $\mathrm{C}$ & -5.762865 & 0.709743 & -2.265385 \\
\hline $\mathrm{H}$ & -5.631118 & -0.339196 & -0.381834 \\
\hline $\mathrm{H}$ & -5.264172 & -1.351271 & -1.796870 \\
\hline $\mathrm{C}$ & -3.721974 & 1.115570 & -3.339003 \\
\hline $\mathrm{H}$ & -1.982275 & 0.304500 & -2.309185 \\
\hline $\mathrm{H}$ & -3.076087 & -0.915100 & -2.993252 \\
\hline $\mathrm{C}$ & -4.856758 & -1.522964 & 2.789810 \\
\hline $\mathrm{H}$ & -4.394188 & -2.011596 & 0.746312 \\
\hline $\mathrm{C}$ & -4.127386 & 0.654080 & 3.549266 \\
\hline $\mathrm{H}$ & -3.096548 & 1.855983 & 2.085424 \\
\hline $\mathrm{H}$ & -3.526522 & -4.893766 & 0.265840 \\
\hline $\mathrm{O}$ & -5.097320 & 0.796061 & -3.512547 \\
\hline $\mathrm{H}$ & -6.805449 & 0.459512 & -2.481753 \\
\hline $\mathrm{H}$ & -5.732376 & 1.683870 & -1.748433 \\
\hline $\mathrm{H}$ & -3.282932 & 1.159035 & -4.339208 \\
\hline $\mathrm{H}$ & -3.617740 & 2.104048 & -2.862329 \\
\hline $\mathrm{C}$ & -4.753203 & -0.568379 & 3.803793 \\
\hline $\mathrm{H}$ & -5.319261 & -2.484984 & 2.990410 \\
\hline $\mathrm{H}$ & -4.034438 & 1.394847 & 4.337937 \\
\hline $\mathrm{H}$ & -5.149217 & -0.780943 & 4.792776 \\
\hline
\end{tabular}

\section{TS-S2d-1aa}

$\begin{array}{llll}\mathrm{O} & -0.131517 & 1.105674 & 0.851459 \\ \mathrm{C} & -1.346366 & 0.958009 & 0.452686 \\ \mathrm{Li} & 1.268697 & -0.033928 & 1.256104 \\ \mathrm{~N} & -2.026147 & -0.156045 & 0.957082 \\ \mathrm{C} & -2.136404 & 2.227967 & 0.224303 \\ \mathrm{Li} & 0.783130 & 1.000603 & -0.907687 \\ \mathrm{~N} & 2.526340 & 0.361161 & -0.132623 \\ \mathrm{C} & -3.308645 & -0.678945 & 0.440307 \\ \mathrm{C} & -1.434951 & -1.123478 & 1.789858\end{array}$




\begin{tabular}{|c|c|c|c|}
\hline $\mathrm{C}$ & -1.588011 & 3.398261 & 0.768635 \\
\hline $\mathrm{C}$ & -3.342971 & 2.322885 & -0.484879 \\
\hline $\mathrm{Si}$ & 3.160852 & 1.947828 & 0.010365 \\
\hline $\mathrm{Si}$ & 3.298439 & -1.122482 & -0.516463 \\
\hline $\mathrm{C}$ & -3.239237 & -2.207348 & 0.676703 \\
\hline $\mathrm{H}$ & -3.404702 & -0.455614 & -0.61780 \\
\hline $\mathrm{H}$ & -4.140730 & -0.215313 & 0.984008 \\
\hline $\mathrm{C}$ & -0.401040 & -0.979002 & 2.727248 \\
\hline $\mathrm{C}$ & -2.100623 & -2.353684 & 1.656837 \\
\hline $\mathrm{C}$ & -2.231467 & 4.626824 & 0.621432 \\
\hline $\mathrm{H}$ & -0.650835 & 3.325996 & 1.307352 \\
\hline $\mathrm{C}$ & -3.984308 & 3.551598 & -0.633207 \\
\hline $\mathrm{H}$ & -3.780176 & 1.450007 & -0.951083 \\
\hline $\mathrm{C}$ & 4.926531 & 2.221622 & -0.614252 \\
\hline $\mathrm{C}$ & 3.069633 & 2.544971 & 1.812847 \\
\hline $\mathrm{C}$ & 2.054008 & 3.145535 & -0.995038 \\
\hline $\mathrm{C}$ & 3.000437 & -1.645061 & -2.311780 \\
\hline $\mathrm{C}$ & 5.171411 & -1.170576 & -0.215213 \\
\hline $\mathrm{C}$ & 2.596032 & -2.481484 & 0.633661 \\
\hline $\mathrm{H}$ & -4.182489 & -2.596318 & 1.072396 \\
\hline $\mathrm{H}$ & -3.021600 & -2.732183 & -0.258921 \\
\hline $\mathrm{C}$ & 0.021470 & -2.110432 & 3.442220 \\
\hline $\mathrm{H}$ & -0.006482 & 0.003168 & 2.968756 \\
\hline $\mathrm{C}$ & -1.671965 & -3.462691 & 2.368546 \\
\hline $\mathrm{C}$ & -3.433995 & 4.709034 & -0.079575 \\
\hline $\mathrm{H}$ & -1.788698 & 5.519552 & 1.054096 \\
\hline $\mathrm{H}$ & -4.914299 & 3.603832 & -1.192309 \\
\hline $\mathrm{H}$ & 5.044389 & 1.873142 & -1.647344 \\
\hline $\mathrm{H}$ & 5.177647 & 3.289691 & -0.592064 \\
\hline $\mathrm{H}$ & 5.662408 & 1.692825 & 0.000257 \\
\hline $\mathrm{H}$ & 3.691331 & 1.915100 & 2.463126 \\
\hline $\mathrm{H}$ & 2.035227 & 2.496333 & 2.180945 \\
\hline $\mathrm{H}$ & 3.409443 & 3.581390 & 1.932079 \\
\hline $\mathrm{H}$ & 1.018483 & 3.202621 & -0.630070 \\
\hline $\mathrm{H}$ & 2.032712 & 2.885403 & -2.063825 \\
\hline $\mathrm{H}$ & 2.450033 & 4.166396 & -0.929124 \\
\hline $\mathrm{H}$ & 3.450352 & -2.620119 & -2.538908 \\
\hline $\mathrm{H}$ & 3.442916 & -0.908161 & -2.994763 \\
\hline $\mathrm{H}$ & 1.930648 & -1.709352 & -2.540425 \\
\hline $\mathrm{H}$ & 5.424136 & -0.825052 & 0.795165 \\
\hline $\mathrm{H}$ & 5.547894 & -2.196383 & -0.318493 \\
\hline $\mathrm{H}$ & 5.719534 & -0.544211 & -0.926810 \\
\hline
\end{tabular}




$\begin{array}{llll}\mathrm{H} & 1.502607 & -2.576188 & 0.628333 \\ \mathrm{H} & 2.903714 & -2.305772 & 1.674968 \\ \mathrm{H} & 2.992841 & -3.464740 & 0.350587 \\ \mathrm{C} & -0.586322 & -3.349110 & 3.251045 \\ \mathrm{H} & 0.822537 & -2.005386 & 4.168744 \\ \mathrm{H} & -2.178221 & -4.416517 & 2.245342 \\ \mathrm{H} & -3.935563 & 5.664880 & -0.200230 \\ \mathrm{H} & -0.242929 & -4.216908 & 3.805054 \\ \mathrm{~N} & -1.006739 & 0.538455 & -1.567428 \\ \mathrm{C} & -1.269856 & -0.760624 & -1.967815 \\ \mathrm{H} & -1.592109 & 1.193905 & -2.082174 \\ \mathrm{C} & -0.539350 & -1.821059 & -1.387969 \\ \mathrm{C} & -2.261349 & -1.106728 & -2.916635 \\ \mathrm{C} & -0.776910 & -3.147463 & -1.733681 \\ \mathrm{H} & 0.233847 & -1.584042 & -0.668504 \\ \mathrm{C} & -2.494874 & -2.435269 & -3.261552 \\ \mathrm{H} & -2.841244 & -0.312857 & -3.384366 \\ \mathrm{C} & -1.759454 & -3.470984 & -2.673114 \\ \mathrm{H} & -0.186179 & -3.930387 & -1.265241 \\ \mathrm{H} & -3.258795 & -2.665988 & -4.000087 \\ \mathrm{H} & -1.943783 & -4.504641 & -2.949150\end{array}$

\section{TS-S2d-1ag}

$\begin{array}{llll}\mathrm{C} & -0.989705 & 0.061800 & -0.494964 \\ \mathrm{~N} & -1.817832 & 1.210055 & -0.008620 \\ \mathrm{C} & -1.663579 & -1.269961 & -0.237723 \\ \mathrm{C} & -3.151165 & 1.369579 & -0.383789 \\ \mathrm{C} & -1.150063 & 2.412066 & 0.396922 \\ \mathrm{O} & -0.389217 & 0.236610 & -1.617655 \\ \mathrm{C} & -1.474780 & -2.295352 & -1.164371 \\ \mathrm{C} & -2.412193 & -1.519221 & 0.917505 \\ \mathrm{O} & -3.549861 & 0.371027 & -1.183949 \\ \mathrm{O} & -3.832208 & 2.310546 & -0.013659 \\ \mathrm{C} & -0.273013 & 3.068984 & -0.473667 \\ \mathrm{C} & -1.334720 & 2.894687 & 1.697192 \\ \mathrm{Li} & 1.341989 & 0.006420 & -1.253238 \\ \mathrm{C} & -2.020892 & -3.560453 & -0.938931 \\ \mathrm{H} & -0.914517 & -2.079002 & -2.066639 \\ \mathrm{C} & -2.962320 & -2.778432 & 1.142675 \\ \mathrm{H} & -2.560428 & -0.723425 & 1.639510 \\ \mathrm{C} & -4.970381 & 0.149369 & -1.479469 \\ \mathrm{C} & 0.442412 & 4.182166 & -0.027087\end{array}$




\begin{tabular}{|c|c|c|c|}
\hline $\mathrm{H}$ & -0.157994 & 2.689357 & -1.481508 \\
\hline $\mathrm{C}$ & -0.628640 & 4.015646 & 2.132636 \\
\hline $\mathrm{H}$ & -2.034896 & 2.383659 & 2.349319 \\
\hline $\mathrm{N}$ & 3.221598 & -0.144989 & -0.712326 \\
\hline $\mathrm{Li}$ & 2.354545 & -0.179548 & 0.976625 \\
\hline $\mathrm{C}$ & -2.765626 & -3.806256 & 0.215449 \\
\hline $\mathrm{H}$ & -1.871332 & -4.350277 & -1.670038 \\
\hline $\mathrm{H}$ & -3.539258 & -2.961006 & 2.04498 \\
\hline $\mathrm{C}$ & -4.939248 & -1.155910 & -2.27451 \\
\hline $\mathrm{C}$ & -5.503702 & 1.303178 & -2.329669 \\
\hline $\mathrm{C}$ & -5.753400 & -0.028560 & -0.176820 \\
\hline $\mathrm{C}$ & 0.269872 & 4.655582 & 1.274551 \\
\hline $\mathrm{H}$ & 1.132391 & 4.680393 & -0.701657 \\
\hline $\mathrm{H}$ & -0.778582 & 4.388423 & 3.141826 \\
\hline $\mathrm{Si}$ & 4.019677 & 1.352030 & -0.474350 \\
\hline $\mathrm{Si}$ & 3.765329 & -1.630772 & -1.379977 \\
\hline $\mathrm{N}$ & 0.335630 & 0.062824 & 0.757324 \\
\hline $\mathrm{H}$ & -3.192236 & -4.789628 & 0.392581 \\
\hline $\mathrm{H}$ & -4.323889 & -1.039893 & -3.172087 \\
\hline $\mathrm{H}$ & -5.954696 & -1.431387 & -2.578374 \\
\hline $\mathrm{H}$ & -4.517395 & -1.964967 & -1.671394 \\
\hline $\mathrm{H}$ & -4.886027 & 1.425515 & -3.225607 \\
\hline $\mathrm{H}$ & -6.529402 & 1.083269 & -2.646676 \\
\hline $\mathrm{H}$ & -5.496062 & 2.236005 & -1.764509 \\
\hline $\mathrm{H}$ & -5.311503 & -0.834460 & 0.417913 \\
\hline $\mathrm{H}$ & -6.789081 & -0.299768 & -0.408 \\
\hline $\mathrm{H}$ & -5.755061 & 0.891781 & 0.409459 \\
\hline $\mathrm{H}$ & 0.826817 & 5.522621 & 1.617585 \\
\hline $\mathrm{C}$ & 3.545211 & 2.631792 & -1.785769 \\
\hline $\mathrm{C}$ & 5.905645 & 1.262093 & -0.352680 \\
\hline $\mathrm{C}$ & 3.394709 & 2.035329 & 1.210215 \\
\hline $\mathrm{C}$ & 5.153260 & -1.467092 & -2.657067 \\
\hline $\mathrm{C}$ & 4.342566 & -2.813328 & -0.011307 \\
\hline $\mathrm{C}$ & 2.289736 & -2.463665 & -2.247970 \\
\hline $\mathrm{C}$ & 0.336914 & -0.821734 & 1.859835 \\
\hline $\mathrm{H}$ & 0.255163 & 1.010706 & 1.121816 \\
\hline $\mathrm{H}$ & 3.891526 & 2.308002 & -2.775198 \\
\hline $\mathrm{H}$ & 3.974227 & 3.621723 & -1.586434 \\
\hline $\mathrm{H}$ & 2.455214 & 2.745448 & -1.839394 \\
\hline $\mathrm{H}$ & 6.328811 & 2.214710 & -0.010547 \\
\hline $\mathrm{H}$ & 6.216124 & 0.483568 & 0.355356 \\
\hline $\mathrm{H}$ & 6.359167 & 1.027173 & -1.321863 \\
\hline
\end{tabular}




$\begin{array}{llll}\mathrm{H} & 2.319234 & 2.259832 & 1.211307 \\ \mathrm{H} & 3.621314 & 1.373591 & 2.064714 \\ \mathrm{H} & 3.895856 & 2.982581 & 1.441842 \\ \mathrm{H} & 5.352457 & -2.426414 & -3.150741 \\ \mathrm{H} & 4.886299 & -0.741338 & -3.435458 \\ \mathrm{H} & 6.088775 & -1.131626 & -2.195876 \\ \mathrm{H} & 5.196353 & -2.388430 & 0.532398 \\ \mathrm{H} & 3.539484 & -2.987084 & 0.718398 \\ \mathrm{H} & 4.649652 & -3.792178 & -0.400417 \\ \mathrm{H} & 1.441990 & -2.633491 & -1.570833 \\ \mathrm{H} & 1.920301 & -1.865927 & -3.093233 \\ \mathrm{H} & 2.573996 & -3.444196 & -2.649555 \\ \mathrm{C} & 0.156833 & -0.354422 & 3.177623 \\ \mathrm{C} & 0.613923 & -2.191603 & 1.663478 \\ \mathrm{C} & 0.245733 & -1.229737 & 4.258557 \\ \mathrm{H} & -0.066351 & 0.697690 & 3.337058 \\ \mathrm{C} & 0.687071 & -3.060103 & 2.751021 \\ \mathrm{H} & 0.734564 & -2.565409 & 0.652153 \\ \mathrm{C} & 0.506359 & -2.587113 & 4.052721 \\ \mathrm{H} & 0.101764 & -0.849875 & 5.266309 \\ \mathrm{H} & 0.883446 & -4.113949 & 2.576104 \\ \mathrm{H} & 0.567710 & -3.267212 & 4.896625 \\ & & & \\ \mathrm{H} & & & \end{array}$

\section{TS-S2d-1r}

$\begin{array}{llll}\mathrm{Li} & -0.718486 & 0.069545 & 0.543471 \\ \mathrm{~N} & -2.620290 & -0.267681 & 0.117037 \\ \mathrm{O} & -0.231220 & 0.813846 & -1.248074 \\ \mathrm{Si} & -2.786308 & -1.878386 & -0.420653 \\ \mathrm{Si} & -3.623782 & 0.917410 & 0.824531 \\ \mathrm{Li} & -1.964430 & 0.608643 & -1.481455 \\ \mathrm{~N} & 0.944551 & 0.877514 & 1.103787 \\ \mathrm{C} & 0.992628 & 1.158233 & -0.999117 \\ \mathrm{C} & -4.357479 & -2.787197 & 0.108533 \\ \mathrm{C} & -1.285081 & -2.905429 & 0.127336 \\ \mathrm{C} & -2.735819 & -1.878354 & -2.336319 \\ \mathrm{C} & -5.362534 & 0.361115 & 1.320710 \\ \mathrm{C} & -2.779171 & 1.680333 & 2.341724 \\ \mathrm{C} & -3.825442 & 2.338252 & -0.446258 \\ \mathrm{C} & 2.025165 & 0.215228 & 1.664127 \\ \mathrm{H} & 1.017457 & 1.866825 & 1.335142 \\ \mathrm{~N} & 1.320616 & 2.534273 & -1.044602 \\ \mathrm{C} & 2.077358 & 0.188020 & -1.296378\end{array}$




\begin{tabular}{|c|c|c|c|}
\hline $\mathrm{H}$ & -4.437133 & -2.830811 & 1.201107 \\
\hline $\mathrm{H}$ & -4.361276 & -3.817392 & -0.268674 \\
\hline $\mathrm{H}$ & -5.258309 & -2.288986 & -0.266925 \\
\hline $\mathrm{H}$ & -1.197063 & -2.926738 & 1.221367 \\
\hline $\mathrm{H}$ & -0.348227 & -2.497130 & -0.275772 \\
\hline $\mathrm{H}$ & -1.352586 & -3.944322 & -0.21903 \\
\hline $\mathrm{H}$ & -3.582855 & -1.318549 & -2.759529 \\
\hline $\mathrm{H}$ & -1.802996 & -1.438138 & -2.72575 \\
\hline $\mathrm{H}$ & -2.783167 & -2.892676 & -2.751465 \\
\hline $\mathrm{H}$ & -5.322347 & -0.460989 & 2.044428 \\
\hline $\mathrm{H}$ & -5.921670 & 1.185614 & 1.780408 \\
\hline $\mathrm{H}$ & -5.936760 & 0.009709 & 0.455397 \\
\hline $\mathrm{H}$ & -2.658147 & 0.931841 & 3.135031 \\
\hline $\mathrm{H}$ & -1.774944 & 2.052253 & 2.096009 \\
\hline $\mathrm{H}$ & -3.349810 & 2.520761 & 2.756279 \\
\hline $\mathrm{H}$ & -4.327502 & 1.986906 & -1.360708 \\
\hline $\mathrm{H}$ & -2.856814 & 2.778512 & -0.731677 \\
\hline $\mathrm{H}$ & -4.429135 & 3.165197 & -0.052291 \\
\hline $\mathrm{C}$ & 3.113751 & 0.891130 & 2.259457 \\
\hline $\mathrm{C}$ & 2.095693 & -1.194586 & 1.593428 \\
\hline $\mathrm{C}$ & 0.206975 & 3.424418 & -0.743050 \\
\hline $\mathrm{C}$ & 2.069478 & 3.002142 & -2.214655 \\
\hline $\mathrm{C}$ & 1.742655 & -1.049173 & -1.860160 \\
\hline $\mathrm{C}$ & 3.410617 & 0.428807 & -0.927979 \\
\hline $\mathrm{C}$ & 4.217118 & 0.194073 & 2.745216 \\
\hline $\mathrm{H}$ & 3.082966 & 1.977325 & 2.323473 \\
\hline $\mathrm{C}$ & 3.207189 & -1.883178 & 2.063113 \\
\hline $\mathrm{H}$ & 1.272592 & -1.740434 & 1.141956 \\
\hline $\mathrm{H}$ & 0.600605 & 4.427166 & -0.548697 \\
\hline $\mathrm{H}$ & -0.518320 & 3.492127 & -1.570881 \\
\hline $\mathrm{H}$ & -0.327202 & 3.081224 & 0.144713 \\
\hline $\mathrm{H}$ & 2.462365 & 4.002764 & -2.006687 \\
\hline $\mathrm{H}$ & 1.429125 & 3.063052 & -3.111323 \\
\hline $\mathrm{H}$ & 2.904470 & 2.338988 & -2.431976 \\
\hline $\mathrm{C}$ & 2.719841 & -2.022730 & -2.063251 \\
\hline $\mathrm{H}$ & 0.710867 & -1.238239 & -2.134842 \\
\hline $\mathrm{C}$ & 4.384581 & -0.544347 & -1.128664 \\
\hline $\mathrm{H}$ & 3.669752 & 1.365382 & -0.449987 \\
\hline $\mathrm{C}$ & 4.280135 & -1.197360 & 2.641785 \\
\hline $\mathrm{H}$ & 5.038033 & 0.743211 & 3.200138 \\
\hline $\mathrm{H}$ & 3.237857 & -2.966308 & 1.977198 \\
\hline $\mathrm{C}$ & 4.043272 & -1.773580 & -1.696816 \\
\hline
\end{tabular}




\begin{tabular}{|c|c|c|c|}
\hline $\mathrm{H}$ & 2.445543 & -2.976953 & -2.504314 \\
\hline $\mathrm{H}$ & 5.406494 & -0.351663 & -0.816921 \\
\hline & 5.144959 & -1.739199 & 3.01309 \\
\hline & 4.803226 & -2.535633 & -1.84 \\
\hline
\end{tabular}

\section{TS-S2d-1s}

\begin{tabular}{|c|c|c|c|}
\hline $\mathrm{Si}$ & -3.189304 & 1.446795 & 0.916828 \\
\hline $\mathrm{N}$ & -2.540033 & 0.183378 & -0.062222 \\
\hline $\mathrm{C}$ & -4.989849 & 1.196352 & 1.455478 \\
\hline $\mathrm{Si}$ & -3.368666 & -1.000112 & -0.985090 \\
\hline $\mathrm{Li}$ & -1.188717 & 0.584933 & -1.370537 \\
\hline $\mathrm{Li}$ & -0.984083 & -0.468117 & 0.909196 \\
\hline $\mathrm{C}$ & -3.094112 & 3.113640 & 0.013196 \\
\hline $\mathrm{C}$ & -2.171773 & 1.573889 & 2.517841 \\
\hline $\mathrm{H}$ & -5.145256 & 0.198316 & 1.884143 \\
\hline $\mathrm{H}$ & -5.262008 & 1.931411 & 2.223381 \\
\hline $\mathrm{H}$ & -5.690191 & 1.314140 & 0.622042 \\
\hline $\mathrm{C}$ & -5.149858 & -0.599744 & -1.487037 \\
\hline $\mathrm{C}$ & -3.381312 & -2.692505 & -0.128668 \\
\hline $\mathrm{C}$ & -2.408492 & -1.189236 & -2.636158 \\
\hline $\mathrm{N}$ & 0.733400 & 0.330617 & -1.120013 \\
\hline $\mathrm{O}$ & 0.731756 & -0.003254 & 1.517854 \\
\hline $\mathrm{H}$ & -2.063509 & 3.371290 & -0.262584 \\
\hline $\mathrm{H}$ & -3.686516 & 3.077120 & -0.910808 \\
\hline $\mathrm{H}$ & -3.485322 & 3.937615 & 0.623200 \\
\hline $\mathrm{H}$ & -2.493657 & 2.438509 & 3.111826 \\
\hline $\mathrm{H}$ & -2.321928 & 0.682384 & 3.144308 \\
\hline $\mathrm{H}$ & -1.093266 & 1.675092 & 2.348994 \\
\hline $\mathrm{H}$ & -5.228908 & 0.406859 & -1.915576 \\
\hline $\mathrm{H}$ & -5.504391 & -1.312719 & -2.242230 \\
\hline $\mathrm{H}$ & -5.834579 & -0.652115 & -0.634485 \\
\hline $\mathrm{H}$ & -3.828993 & -3.478173 & -0.749822 \\
\hline $\mathrm{H}$ & -3.961378 & -2.631575 & 0.801216 \\
\hline $\mathrm{H}$ & -2.367806 & -3.015133 & 0.139673 \\
\hline $\mathrm{H}$ & -1.339647 & -1.406051 & -2.503870 \\
\hline $\mathrm{H}$ & -2.504712 & -0.290187 & -3.266178 \\
\hline $\mathrm{H}$ & -2.816212 & -2.017561 & -3.227609 \\
\hline $\mathrm{C}$ & 1.333971 & 1.596077 & -0.991833 \\
\hline $\mathrm{H}$ & 1.231073 & -0.166745 & -1.858595 \\
\hline $\mathrm{C}$ & 1.233919 & -0.682073 & 0.568560 \\
\hline $\mathrm{C}$ & 2.507042 & 1.959114 & -1.682843 \\
\hline $\mathrm{C}$ & 0.775745 & 2.533302 & -0.099235 \\
\hline
\end{tabular}




\begin{tabular}{|c|c|c|c|}
\hline $\mathrm{N}$ & 0.477640 & -1.924267 & 0.360884 \\
\hline $\mathrm{C}$ & 2.728749 & -0.603549 & 0.372585 \\
\hline $\mathrm{C}$ & 3.088187 & 3.210316 & -1.496694 \\
\hline $\mathrm{H}$ & 2.970881 & 1.237200 & -2.350404 \\
\hline $\mathrm{C}$ & 1.366746 & 3.781773 & 0.088315 \\
\hline $\mathrm{H}$ & -0.111373 & 2.264940 & 0.461431 \\
\hline $\mathrm{C}$ & 0.553316 & -2.751898 & -0.851125 \\
\hline $\mathrm{C}$ & 0.276760 & -2.691365 & 1.614974 \\
\hline $\mathrm{C}$ & 3.422737 & 0.263314 & 1.226210 \\
\hline $\mathrm{C}$ & 3.444833 & -1.257945 & -0.639542 \\
\hline $\mathrm{C}$ & 2.524189 & 4.131491 & -0.609478 \\
\hline $\mathrm{H}$ & 3.994090 & 3.463782 & -2.041235 \\
\hline $\mathrm{H}$ & 0.916078 & 4.483707 & 0.785079 \\
\hline $\mathrm{C}$ & 1.447314 & -4.000681 & -0.821465 \\
\hline $\mathrm{H}$ & 0.845555 & -2.105026 & -1.678309 \\
\hline $\mathrm{H}$ & -0.467370 & -3.083585 & -1.081660 \\
\hline $\mathrm{C}$ & 1.547021 & -3.080535 & 2.376297 \\
\hline $\mathrm{H}$ & -0.298843 & -3.582092 & 1.343193 \\
\hline $\mathrm{H}$ & -0.356774 & -2.112164 & 2.298151 \\
\hline $\mathrm{C}$ & 4.798485 & 0.446926 & 1.094484 \\
\hline $\mathrm{H}$ & 2.856736 & 0.793498 & 1.981935 \\
\hline $\mathrm{C}$ & 4.820254 & -1.076412 & -0.771472 \\
\hline $\mathrm{H}$ & 2.938307 & -1.893261 & -1.353562 \\
\hline $\mathrm{H}$ & 2.980950 & 5.106104 & -0.464887 \\
\hline $\mathrm{H}$ & 1.079827 & -4.738155 & -0.101703 \\
\hline $\mathrm{H}$ & 1.430981 & -4.474523 & -1.810044 \\
\hline $\mathrm{H}$ & 2.484409 & -3.770907 & -0.568240 \\
\hline $\mathrm{H}$ & 2.085660 & -2.186669 & 2.700860 \\
\hline $\mathrm{H}$ & 1.279564 & -3.660270 & 3.267557 \\
\hline $\mathrm{H}$ & 2.223220 & -3.682239 & 1.764329 \\
\hline $\mathrm{C}$ & 5.505419 & -0.226370 & 0.099208 \\
\hline $\mathrm{H}$ & 5.315778 & 1.125599 & 1.766873 \\
\hline $\mathrm{H}$ & 5.355628 & -1.594671 & -1.562439 \\
\hline 1 & 6.577027 & -0.083107 & -0.007 \\
\hline
\end{tabular}

\section{TS-S2d-1v}

\begin{tabular}{|c|c|c|c|}
\hline $\mathrm{Si}$ & -3.083639 & -1.464117 & -1.035678 \\
\hline $\mathrm{N}$ & -2.474872 & -0.161914 & -0.077460 \\
\hline $\mathrm{C}$ & -4.848053 & -1.222484 & -1.689624 \\
\hline $\mathrm{Si}$ & -3.362799 & 0.907636 & 0.927826 \\
\hline $\mathrm{Li}$ & -1.120540 & -0.569391 & 1.248201 \\
\hline $\mathrm{L}$ & -0.951042 & 0.567716 & -1.048028 \\
\hline
\end{tabular}




\begin{tabular}{|c|c|c|c|}
\hline $\mathrm{C}$ & -3.062910 & -3.094613 & -0.061638 \\
\hline $\mathrm{C}$ & -1.990879 & -1.670132 & -2.575816 \\
\hline $\mathrm{H}$ & -4.976146 & -0.227495 & -2.134150 \\
\hline $\mathrm{H}$ & -5.062554 & -1.962920 & -2.470673 \\
\hline $\mathrm{H}$ & -5.605481 & -1.338672 & -0.90792 \\
\hline $\mathrm{C}$ & -5.185168 & 0.500118 & 1.233359 \\
\hline $\mathrm{C}$ & -3.258094 & 2.691367 & 0.297251 \\
\hline $\mathrm{C}$ & -2.560935 & 0.862869 & 2.675227 \\
\hline $\mathrm{N}$ & 0.805701 & -0.302213 & 1.135030 \\
\hline $\mathrm{O}$ & 0.756493 & -0.011639 & -1.598889 \\
\hline $\mathrm{H}$ & -2.052156 & -3.359252 & 0.274099 \\
\hline $\mathrm{H}$ & -3.698046 & -3.012312 & 0.830561 \\
\hline $\mathrm{H}$ & -3.440145 & -3.935226 & -0.65761 \\
\hline $\mathrm{H}$ & -2.299951 & -2.556129 & -3.144634 \\
\hline $\mathrm{H}$ & -2.100436 & -0.806458 & -3.247988 \\
\hline $\mathrm{H}$ & -0.923462 & -1.772910 & -2.352741 \\
\hline $\mathrm{H}$ & -5.315728 & -0.540192 & 1.554903 \\
\hline $\mathrm{H}$ & -5.591001 & 1.146259 & 2.022044 \\
\hline $\mathrm{H}$ & -5.793017 & 0.648937 & 0.335220 \\
\hline $\mathrm{H}$ & -3.666262 & 3.421754 & 1.006725 \\
\hline $\mathrm{H}$ & -3.814060 & 2.791842 & -0.643683 \\
\hline $\mathrm{H}$ & -2.217232 & 2.966409 & 0.092069 \\
\hline $\mathrm{H}$ & -1.484248 & 1.088171 & 2.704220 \\
\hline $\mathrm{H}$ & -2.716374 & -0.115671 & 3.155911 \\
\hline $\mathrm{H}$ & -3.035194 & 1.604569 & 3.329097 \\
\hline $\mathrm{C}$ & 1.374867 & -1.573001 & 1.063553 \\
\hline $\mathrm{H}$ & 1.315795 & 0.240326 & 1.832133 \\
\hline $\mathrm{C}$ & 1.305264 & 0.689797 & -0.704591 \\
\hline $\mathrm{C}$ & 2.539947 & -1.944527 & 1.769334 \\
\hline $\mathrm{C}$ & 0.793775 & -2.536488 & 0.206818 \\
\hline $\mathrm{N}$ & 0.566814 & 1.919963 & -0.421522 \\
\hline $\mathrm{C}$ & 2.791122 & 0.614072 & -0.548459 \\
\hline $\mathrm{C}$ & 3.085506 & -3.217875 & 1.634349 \\
\hline $\mathrm{H}$ & 3.021542 & -1.209629 & 2.410625 \\
\hline $\mathrm{C}$ & 1.352997 & -3.804732 & 0.067337 \\
\hline $\mathrm{H}$ & -0.080688 & -2.264954 & -0.373902 \\
\hline $\mathrm{C}$ & 0.614944 & 2.593028 & 0.897396 \\
\hline $\mathrm{C}$ & 0.740123 & 2.940200 & -1.508819 \\
\hline $\mathrm{C}$ & 3.590047 & 1.660139 & -0.063248 \\
\hline $\mathrm{C}$ & 3.416550 & -0.577545 & -0.951009 \\
\hline $\mathrm{C}$ & 2.501127 & -4.158342 & 0.780717 \\
\hline $\mathrm{H}$ & 3.983744 & -3.473811 & 2.190646 \\
\hline
\end{tabular}




$\begin{array}{llll}\mathrm{H} & 0.886490 & -4.519950 & -0.605218 \\ \mathrm{C} & 0.441290 & 4.086374 & 0.584756 \\ \mathrm{H} & -0.188286 & 2.216561 & 1.533374 \\ \mathrm{H} & 1.558700 & 2.410639 & 1.420532 \\ \mathrm{C} & 1.097968 & 4.248113 & -0.792819 \\ \mathrm{H} & 1.509930 & 2.618913 & -2.215008 \\ \mathrm{H} & -0.197940 & 3.048701 & -2.069055 \\ \mathrm{C} & 4.972909 & 1.511453 & 0.036685 \\ \mathrm{H} & 3.149597 & 2.607197 & 0.217683 \\ \mathrm{C} & 4.796662 & -0.727069 & -0.842202 \\ \mathrm{H} & 2.803827 & -1.375715 & -1.349026 \\ \mathrm{H} & 2.932553 & -5.149191 & 0.674895 \\ \mathrm{H} & 0.888407 & 4.721423 & 1.355242 \\ \mathrm{H} & -0.623080 & 4.337923 & 0.525281 \\ \mathrm{H} & 0.743592 & 5.129094 & -1.336141 \\ \mathrm{H} & 2.185074 & 4.337067 & -0.691316 \\ \mathrm{C} & 5.580973 & 0.314158 & -0.344396 \\ \mathrm{H} & 5.575369 & 2.337071 & 0.405474 \\ \mathrm{H} & 5.258030 & -1.661974 & -1.146657 \\ \mathrm{H} & 6.658063 & 0.198335 & -0.261788\end{array}$

\section{TS-S2d-1w}

$\begin{array}{llll}\mathrm{Li} & -0.860305 & -0.528370 & 0.601223 \\ \mathrm{~N} & -2.641578 & 0.016456 & -0.051102 \\ \mathrm{O} & 0.701873 & -0.005712 & 1.612744 \\ \mathrm{Si} & -3.213799 & -1.161312 & -1.155696 \\ \mathrm{Si} & -3.455485 & 0.885574 & 1.191820 \\ \mathrm{Li} & -1.471106 & 1.066283 & -1.125121 \\ \mathrm{C} & 1.297541 & -0.428874 & 0.577589 \\ \mathrm{C} & -5.043792 & -1.043206 & -1.629732 \\ \mathrm{C} & -2.877655 & -2.929394 & -0.552653 \\ \mathrm{C} & -2.226362 & -0.953083 & -2.782689 \\ \mathrm{C} & -5.228201 & 0.319362 & 1.551021 \\ \mathrm{C} & -2.487573 & 0.731582 & 2.817508 \\ \mathrm{C} & -3.527269 & 2.729600 & 0.732404 \\ \mathrm{~N} & 0.804702 & -1.762743 & 0.185110 \\ \mathrm{C} & 2.750335 & -0.047112 & 0.395764 \\ \mathrm{H} & -5.310026 & -0.023088 & -1.933244 \\ \mathrm{H} & -5.269930 & -1.711742 & -2.470128 \\ \mathrm{H} & -5.699244 & -1.321759 & -0.798004 \\ \mathrm{H} & -3.405247 & -3.119422 & 0.390622 \\ \mathrm{H} & -1.806508 & -3.079847 & -0.365982\end{array}$




\begin{tabular}{|c|c|c|c|}
\hline $\mathrm{H}$ & -3.199147 & -3.689045 & -1.276105 \\
\hline $\mathrm{H}$ & -2.379118 & 0.037594 & -3.24168 \\
\hline $\mathrm{H}$ & -1.148872 & -1.097963 & -2.63288 \\
\hline $\mathrm{H}$ & -2.547938 & -1.686630 & -3.531932 \\
\hline $\mathrm{H}$ & -5.270055 & -0.757611 & 1.7561 \\
\hline $\mathrm{H}$ & -5.623585 & 0.839268 & 2.432739 \\
\hline $\mathrm{H}$ & -5.902768 & 0.524976 & 0.71293 \\
\hline $\mathrm{H}$ & -1.434978 & 1.021509 & 2.71292 \\
\hline $\mathrm{H}$ & -2.501458 & -0.307148 & 3.1759 \\
\hline $\mathrm{H}$ & -2.928212 & 1.355585 & 3.605383 \\
\hline $\mathrm{H}$ & -4.102002 & 2.872822 & -0.192904 \\
\hline $\mathrm{H}$ & -2.523800 & 3.146512 & 0.57011 \\
\hline $\mathrm{H}$ & -4.004848 & 3.334538 & 1.5133 \\
\hline $\mathrm{C}$ & 1.211584 & -2.424769 & -1.063931 \\
\hline $\mathrm{C}$ & 0.789990 & -2.730448 & 1.310917 \\
\hline $\mathrm{C}$ & 3.441157 & -0.045265 & -0.822627 \\
\hline $\mathrm{C}$ & 3.435064 & 0.340939 & 1.554050 \\
\hline $\mathrm{C}$ & 2.517107 & -3.232062 & -0.981649 \\
\hline $\mathrm{H}$ & 1.264158 & -1.685159 & -1.864202 \\
\hline $\mathrm{H}$ & 0.392087 & -3.106571 & -1.331574 \\
\hline $\mathrm{C}$ & 2.118316 & -3.473619 & 1.486596 \\
\hline $\mathrm{H}$ & 0.515854 & -2.185668 & 2.214420 \\
\hline $\mathrm{H}$ & -0.006078 & -3.458137 & 1.098417 \\
\hline $\mathrm{C}$ & 4.787399 & 0.309723 & -0.878117 \\
\hline $\mathrm{H}$ & 2.932184 & -0.306841 & -1.742873 \\
\hline $\mathrm{C}$ & 4.784472 & 0.689768 & 1.502168 \\
\hline $\mathrm{H}$ & 2.886255 & 0.369061 & 2.488041 \\
\hline $\mathrm{C}$ & 2.485651 & -4.213670 & 0.195735 \\
\hline $\mathrm{H}$ & 3.366321 & -2.552706 & -0.862427 \\
\hline $\mathrm{H}$ & 2.659789 & -3.766253 & -1.929764 \\
\hline $\mathrm{H}$ & 2.031149 & -4.174452 & 2.326295 \\
\hline $\mathrm{H}$ & 2.904894 & -2.752833 & 1.744211 \\
\hline $\mathrm{C}$ & 5.467060 & 0.672919 & 0.286091 \\
\hline $\mathrm{H}$ & 5.303085 & 0.310569 & -1.834338 \\
\hline $\mathrm{H}$ & 5.299478 & 0.983333 & 2.412682 \\
\hline $\mathrm{H}$ & 3.452728 & -4.720214 & 0.295418 \\
\hline $\mathrm{H}$ & 1.735563 & -4.995648 & 0.004789 \\
\hline $\mathrm{H}$ & 6.516274 & 0.950818 & 0.241627 \\
\hline $\mathrm{N}$ & 0.444729 & 0.592118 & -0.906590 \\
\hline $\mathrm{C}$ & 0.794230 & 1.952564 & -0.836659 \\
\hline $\mathrm{H}$ & 0.809574 & 0.214170 & -1.779553 \\
\hline $\mathrm{C}$ & 1.533634 & 2.602822 & -1.847055 \\
\hline
\end{tabular}




\begin{tabular}{|c|c|c|c|}
\hline $\mathrm{C}$ & 0.362866 & 2.718209 & 0.269844 \\
\hline $\mathrm{C}$ & 1.814942 & 3.963547 & -1.762832 \\
\hline $\mathrm{H}$ & 1.889870 & 2.024139 & -2.696498 \\
\hline $\mathrm{C}$ & 0.660719 & 4.078381 & 0.348350 \\
\hline $\mathrm{H}$ & -0.166992 & 2.223033 & 1.075843 \\
\hline $\mathrm{C}$ & 1.381076 & 4.712682 & -0.665193 \\
\hline $\mathrm{H}$ & 2.383756 & 4.440955 & -2.556468 \\
\hline $\mathrm{H}$ & 0.324622 & 4.644335 & 1.213022 \\
\hline $\mathrm{H}$ & 1.603687 & 5.773433 & -0.601713 \\
\hline
\end{tabular}

\section{TS-S2d-1x}

Li $\quad-1.035073 \quad 0.262013 \quad 0.627815$

$\begin{array}{llll}\mathrm{N} & -2.904859 & 0.067501 & 0.036318\end{array}$

$\mathrm{N} \quad 0.792939 \quad 0.450388 \quad 1.186156$

$\mathrm{Si} \quad \quad \quad-3.558702 \quad-1.491986 \quad-0.212675$

$\begin{array}{llll}\mathrm{Si} & -3.620044 & 1.515969 & 0.614451\end{array}$

$\begin{array}{lllll}\mathrm{Li} & & -1.918582 & 0.228777 & -1.618913\end{array}$

$\begin{array}{llll}\text { O } & -0.240997 & 0.573772 & -1.192418\end{array}$

C $\quad 1.775772-0.221133 \quad 1.889028$

$\mathrm{H} \quad 0.926703 \quad 1.454092 \quad 1.316664$

C $\quad-5.439216-1.641680-0.064858$

$\begin{array}{lllll}\text { C } & -2.757502 & -2.780665 & 0.923297\end{array}$

$\begin{array}{lllll}\text { C } & -3.111068 & -1.997756 & -2.010988\end{array}$

$\begin{array}{lllll}\text { C } & -4.486311 & 2.466318 & -0.784121\end{array}$

$\begin{array}{llll}\text { C } & -4.850852 & 1.288474 & 2.034794\end{array}$

$\begin{array}{llll}\text { C } & -2.213626 & 2.635215 & 1.249914\end{array}$

$\begin{array}{lllll}\text { C } & & 1.018943 & 0.467827 & -0.895451\end{array}$

$\begin{array}{llll}\text { C } & 1.734875 & -1.629533 & 1.985940\end{array}$

$\begin{array}{llll}\text { C } & 2.864842 & 0.439897 & 2.504604\end{array}$

$\mathrm{H} \quad \quad \quad-5.769946 \quad-1.495404 \quad 0.969469$

$\mathrm{H} \quad-\quad-5.785191 \quad-2.631653 \quad-0.387143$

$\mathrm{H} \quad \begin{array}{llll}-5.947497 & -0.892181 & -0.683901\end{array}$

$\begin{array}{llll}\mathrm{H} & -2.973270 & -2.550277 & 1.973991\end{array}$

$\begin{array}{lllll}\mathrm{H} & & -1.664784 & -2.785423 & 0.811712\end{array}$

$\mathrm{H} \quad \begin{array}{llll}-3.113140 & -3.798922 & 0.722449\end{array}$

$\begin{array}{lllll}\mathrm{H} & & -3.586051 & -1.331827 & -2.748978\end{array}$

$\mathrm{H} \quad-2.026428 \quad-2.011705 \quad-2.201349$

$\begin{array}{llll}\mathrm{H} & -3.462200 & -3.010925 & -2.240841\end{array}$

$\mathrm{H} \quad-3.775062 \quad 2.715998 \quad-1.585064$

$\mathrm{H} \quad \begin{array}{llll}-4.934071 & 3.408108 & -0.443144\end{array}$

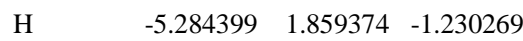

$\begin{array}{llll}\mathrm{H} & -5.754489 & 0.760818 & 1.709615\end{array}$ 


\begin{tabular}{|c|c|c|c|}
\hline $\mathrm{H}$ & -4.400501 & 0.706081 & 2.848324 \\
\hline $\mathrm{H}$ & -5.161849 & 2.255421 & 2.449645 \\
\hline $\mathrm{H}$ & -1.702244 & 2.203318 & 2.122325 \\
\hline $\mathrm{H}$ & -1.455517 & 2.822731 & 0.476847 \\
\hline $\mathrm{H}$ & -2.601013 & 3.612290 & 1.563573 \\
\hline $\mathrm{N}$ & 1.664646 & -0.745178 & -1.170296 \\
\hline $\mathrm{C}$ & 1.852552 & 1.708083 & -0.913181 \\
\hline $\mathrm{C}$ & 2.740717 & -2.338538 & 2.634571 \\
\hline $\mathrm{H}$ & 0.882482 & -2.153299 & 1.565098 \\
\hline $\mathrm{C}$ & 3.866415 & -0.275072 & 3.156226 \\
\hline $\mathrm{H}$ & 2.908688 & 1.525513 & 2.464260 \\
\hline $\mathrm{C}$ & 0.794177 & -1.913260 & -1.279433 \\
\hline $\mathrm{C}$ & 2.717043 & -0.798550 & -2.198755 \\
\hline $\mathrm{C}$ & 1.271179 & 2.900577 & -1.362684 \\
\hline $\mathrm{C}$ & 3.175347 & 1.732152 & -0.437019 \\
\hline $\mathrm{C}$ & 3.821405 & -1.670128 & 3.217996 \\
\hline $\mathrm{H}$ & 2.675868 & -3.422208 & 2.695518 \\
\hline $\mathrm{H}$ & 4.688670 & 0.262813 & 3.621837 \\
\hline $\mathrm{C}$ & 1.624145 & -3.192496 & -1.194337 \\
\hline $\mathrm{H}$ & 0.059600 & -1.897004 & -0.472471 \\
\hline $\mathrm{H}$ & 0.244321 & -1.900762 & -2.235404 \\
\hline $\mathrm{C}$ & 3.493076 & -2.102529 & -2.057349 \\
\hline $\mathrm{H}$ & 3.390643 & 0.050065 & -2.101326 \\
\hline $\mathrm{H}$ & 2.262814 & -0.758911 & -3.203534 \\
\hline $\mathrm{C}$ & 1.997498 & 4.091410 & -1.350782 \\
\hline $\mathrm{H}$ & 0.246052 & 2.879331 & -1.715069 \\
\hline $\mathrm{C}$ & 3.896572 & 2.922858 & -0.425414 \\
\hline $\mathrm{H}$ & 3.618511 & 0.826388 & -0.040643 \\
\hline $\mathrm{H}$ & 4.603950 & -2.225623 & 3.726093 \\
\hline $\mathrm{O}$ & 2.635515 & -3.225551 & -2.190699 \\
\hline $\mathrm{H}$ & 0.987566 & -4.065310 & -1.366340 \\
\hline $\mathrm{H}$ & 2.076800 & -3.265411 & -0.195409 \\
\hline $\mathrm{H}$ & 4.242674 & -2.186409 & -2.849550 \\
\hline $\mathrm{H}$ & 3.998808 & -2.121884 & -1.078600 \\
\hline $\mathrm{C}$ & 3.312947 & 4.106912 & -0.885441 \\
\hline $\mathrm{H}$ & 1.534426 & 5.007537 & -1.706538 \\
\hline $\mathrm{H}$ & 4.915045 & 2.927955 & -0.047443 \\
\hline $\mathrm{H}$ & 3.878629 & 5.034126 & 75620 \\
\hline
\end{tabular}

\section{TS-S2t-1aa}

$\begin{array}{llll}\mathrm{Si} & 2.224471 & 2.736079 & 1.072347\end{array}$

$\mathrm{N} \quad 1.666556 \quad 1.143275 \quad 1.490553$ 


\begin{tabular}{|c|c|c|c|}
\hline $\mathrm{C}$ & 2.658078 & 3.897232 & 2.499367 \\
\hline $\mathrm{Si}$ & 1.369288 & 0.641829 & 3.138136 \\
\hline L1 & 3.292307 & 0.327854 & 0.628765 \\
\hline $\mathrm{Li}$ & -0.265117 & 1.603640 & 1.106483 \\
\hline $\mathrm{C}$ & 0.923530 & 3.628644 & 0.018825 \\
\hline $\mathrm{C}$ & 3.815401 & 2.587143 & 0.027330 \\
\hline $\mathrm{H}$ & 1.836465 & 4.003715 & 3.216562 \\
\hline $\mathrm{H}$ & 2.878937 & 4.895541 & 2.101512 \\
\hline $\mathrm{H}$ & 3.540457 & 3.556388 & 3.050563 \\
\hline $\mathrm{C}$ & 1.036104 & -1.214396 & 3.216509 \\
\hline $\mathrm{C}$ & 2.794855 & 0.997963 & 4.336410 \\
\hline $\mathrm{C}$ & -0.189880 & 1.506180 & 3.800795 \\
\hline $\mathrm{N}$ & 2.800592 & -0.923331 & -0.743368 \\
\hline $\mathrm{Li}$ & 1.093594 & -0.282467 & 0.005692 \\
\hline $\mathrm{O}$ & -1.960996 & 1.337680 & 0.810119 \\
\hline $\mathrm{H}$ & 1.340191 & 4.561709 & -0.379685 \\
\hline $\mathrm{H}$ & 0.036920 & 3.918729 & 0.600742 \\
\hline $\mathrm{H}$ & 0.566020 & 3.049032 & -0.840073 \\
\hline $\mathrm{H}$ & 3.681515 & 2.044551 & -0.914859 \\
\hline $\mathrm{H}$ & 4.654329 & 2.129274 & 0.578004 \\
\hline $\mathrm{H}$ & 4.160432 & 3.592389 & -0.241145 \\
\hline $\mathrm{H}$ & 0.256762 & -1.531316 & 2.516302 \\
\hline $\mathrm{H}$ & 0.689049 & -1.478220 & 4.223592 \\
\hline $\mathrm{H}$ & 1.927925 & -1.813903 & 3.012745 \\
\hline $\mathrm{H}$ & 2.660976 & 0.415855 & 5.256582 \\
\hline $\mathrm{H}$ & 3.766717 & 0.715619 & 3.911706 \\
\hline $\mathrm{H}$ & 2.849436 & 2.053551 & 4.618446 \\
\hline $\mathrm{H}$ & -0.095941 & 2.600359 & 3.777216 \\
\hline $\mathrm{H}$ & -1.097134 & 1.232934 & 3.243995 \\
\hline $\mathrm{H}$ & -0.366947 & 1.221755 & 4.845438 \\
\hline $\mathrm{Si}$ & 2.977759 & -0.566794 & -2.417921 \\
\hline $\mathrm{Si}$ & 3.539290 & -2.271791 & 0.032665 \\
\hline $\mathrm{C}$ & -2.124548 & 0.862623 & -0.412836 \\
\hline $\mathrm{C}$ & 2.587654 & -2.015715 & -3.581293 \\
\hline $\mathrm{C}$ & 4.729835 & 0.019134 & -2.869179 \\
\hline $\mathrm{C}$ & 1.791698 & 0.842589 & -2.903950 \\
\hline $\mathrm{C}$ & 2.295062 & -3.571618 & 0.606421 \\
\hline $\mathrm{C}$ & 4.888048 & -3.186783 & -0.933449 \\
\hline $\mathrm{C}$ & 4.464596 & -1.564959 & 1.566391 \\
\hline $\mathrm{N}$ & -3.327879 & 0.059883 & -0.543413 \\
\hline $\mathrm{C}$ & -2.117235 & 1.967175 & -1.504013 \\
\hline $\mathrm{H}$ & 1.650269 & -2.510460 & -3.302970 \\
\hline
\end{tabular}




\begin{tabular}{|c|c|c|c|}
\hline $\mathrm{H}$ & 2.491393 & -1.670308 & -4.618697 \\
\hline $\mathrm{H}$ & 3.376234 & -2.775980 & -3.562560 \\
\hline $\mathrm{H}$ & 4.788982 & 0.324970 & -3.921673 \\
\hline $\mathrm{H}$ & 5.047811 & 0.874220 & -2.259768 \\
\hline $\mathrm{H}$ & 5.464398 & -0.780371 & -2.718539 \\
\hline $\mathrm{H}$ & 0.807181 & 0.464854 & -3.202227 \\
\hline $\mathrm{H}$ & 2.183806 & 1.395797 & -3.766108 \\
\hline $\mathrm{H}$ & 1.633090 & 1.570489 & -2.100745 \\
\hline $\mathrm{H}$ & 1.915501 & -4.119957 & -0.263338 \\
\hline $\mathrm{H}$ & 2.747827 & -4.299597 & 1.291567 \\
\hline $\mathrm{H}$ & 1.424132 & -3.135085 & 1.104307 \\
\hline $\mathrm{H}$ & 5.700983 & -2.519421 & -1.240367 \\
\hline $\mathrm{H}$ & 5.324562 & -3.974545 & -0.306242 \\
\hline $\mathrm{H}$ & 4.492244 & -3.668766 & -1.833698 \\
\hline $\mathrm{H}$ & 3.842106 & -0.994709 & 2.269613 \\
\hline $\mathrm{H}$ & 5.307766 & -0.928119 & 1.253318 \\
\hline $\mathrm{H}$ & 4.900056 & -2.376040 & 2.161591 \\
\hline $\mathrm{C}$ & -3.717142 & -0.580258 & -1.805886 \\
\hline $\mathrm{C}$ & -4.203192 & -0.329752 & 0.476697 \\
\hline $\mathrm{C}$ & -2.418736 & 3.266705 & -1.079796 \\
\hline $\mathrm{C}$ & -1.863989 & 1.758141 & -2.865402 \\
\hline $\mathrm{C}$ & -4.617400 & -1.761048 & -1.381755 \\
\hline $\mathrm{H}$ & -2.839005 & -0.924217 & -2.357907 \\
\hline $\mathrm{H}$ & -4.255677 & 0.132467 & -2.446759 \\
\hline $\mathrm{C}$ & -4.355546 & 0.163662 & 1.778180 \\
\hline $\mathrm{C}$ & -5.028797 & -1.377856 & 0.020941 \\
\hline $\mathrm{C}$ & -2.444088 & 4.329531 & -1.980674 \\
\hline $\mathrm{H}$ & -2.623531 & 3.419312 & -0.026220 \\
\hline $\mathrm{C}$ & -1.876749 & 2.821588 & -3.771214 \\
\hline $\mathrm{H}$ & -1.660953 & 0.762448 & -3.249008 \\
\hline $\mathrm{H}$ & -5.461354 & -1.898635 & -2.064979 \\
\hline $\mathrm{H}$ & -4.042348 & -2.696989 & -1.369836 \\
\hline $\mathrm{C}$ & -5.333187 & -0.413085 & 2.597428 \\
\hline $\mathrm{H}$ & -3.730348 & 0.971533 & 2.126905 \\
\hline $\mathrm{C}$ & -5.991950 & -1.936840 & 0.844564 \\
\hline $\mathrm{C}$ & -2.163200 & 4.113045 & -3.331566 \\
\hline $\mathrm{H}$ & -2.679904 & 5.329880 & -1.627197 \\
\hline $\mathrm{H}$ & -1.663260 & 2.634879 & -4.820097 \\
\hline $\mathrm{C}$ & -6.150959 & -1.451340 & 2.150151 \\
\hline $\mathrm{H}$ & -5.457200 & -0.029490 & 3.607347 \\
\hline $\mathrm{H}$ & -6.616119 & -2.749283 & 0.479003 \\
\hline $\mathrm{H}$ & -2.173369 & 4.941035 & -4.034815 \\
\hline
\end{tabular}




$\begin{array}{llll}\mathrm{H} & -6.905521 & -1.878341 & 2.804295 \\ \mathrm{~N} & -0.848736 & -0.082174 & -0.761682 \\ \mathrm{C} & -1.033305 & -1.471068 & -0.500009 \\ \mathrm{H} & -0.600208 & 0.037132 & -1.739885 \\ \mathrm{C} & -1.534225 & -1.892424 & 0.740128 \\ \mathrm{C} & -0.711356 & -2.431386 & -1.469894 \\ \mathrm{C} & -1.726903 & -3.250309 & 0.988930 \\ \mathrm{H} & -1.815190 & -1.148267 & 1.475282 \\ \mathrm{C} & -0.889294 & -3.788376 & -1.206670 \\ \mathrm{H} & -0.333597 & -2.107040 & -2.435835 \\ \mathrm{C} & -1.403032 & -4.205153 & 0.023163 \\ \mathrm{H} & -2.142818 & -3.557894 & 1.943720 \\ \mathrm{H} & -0.633701 & -4.518411 & -1.969611 \\ \mathrm{H} & -1.550672 & -5.261611 & 0.225031\end{array}$

\section{TS-S2t-1ag}

$\begin{array}{llll}\mathrm{Si} & 2.145174 & 2.980805 & -0.230546\end{array}$

$\begin{array}{lllll}\mathrm{N} & & 1.980990 & 1.570386 & -1.239687\end{array}$

C $\quad 0.968376 \quad 4.393097 \quad-0.683096$

$\begin{array}{llll}\mathrm{Si} & 2.228472 & 1.650318 & -2.957226\end{array}$

$\mathrm{Li} \quad 0.086744 \quad 0.967458 \quad-1.327964$

$\begin{array}{lllll}\mathrm{Li} & 3.698044 & 0.546725 & -0.982316\end{array}$

$\begin{array}{llll}\text { C } & 1.780594 & 2.517463 & 1.577344\end{array}$

$\begin{array}{llll}\text { C } & 3.911318 & 3.679492 & -0.322217\end{array}$

$\mathrm{H} \quad-0.037556 \quad 4.016104 \quad-0.899194$

$\begin{array}{llll}\mathrm{H} & 0.885291 & 5.106367 & 0.145947\end{array}$

$\begin{array}{lllll}\mathrm{H} & & 1.313903 & 4.945595 & -1.562327\end{array}$

C $\quad 2.335574 \quad 3.367769-3.739594$

$\begin{array}{lllll}\text { C } & & 0.826172 & 0.756797 & -3.868794\end{array}$

C $\quad 3.881610 \quad 0.776235 \quad-3.362894$

$\begin{array}{lllll}\mathrm{Li} & & 1.697339 & -0.070108 & 0.171263\end{array}$

$\begin{array}{lllll}\mathrm{O} & & -1.690579 & 1.017901 & -1.404507\end{array}$

N $\quad 3.459680 \quad-0.965554 \quad 0.133215$

$\begin{array}{llll}\mathrm{H} & 2.124131 & 1.519599 & 1.876090\end{array}$

$\begin{array}{llll}\mathrm{H} & 2.286901 & 3.225231 & 2.245591\end{array}$

$\begin{array}{llll}\mathrm{H} & 0.710538 & 2.586849 & 1.800064\end{array}$

$\mathrm{H} \quad 4.680373 \quad 2.928471 \quad-0.105200$

$\begin{array}{llll}\mathrm{H} & 4.053384 & 4.491682 & 0.401544\end{array}$

$\begin{array}{lllll}\mathrm{H} & & 4.119332 & 4.088745 & -1.317504\end{array}$

$\mathrm{H} \quad 1.388561 \quad 3.909636 \quad-3.641910$

$\begin{array}{llll}\mathrm{H} & 2.545156 & 3.265403 & -4.811867\end{array}$

$\begin{array}{llll}\mathrm{H} & 3.124904 & 3.992066 & -3.308509\end{array}$ 


\begin{tabular}{|c|c|c|c|}
\hline $\mathrm{H}$ & 1.038084 & 0.732749 & -4.944888 \\
\hline $\mathrm{H}$ & -0.133255 & 1.277882 & -3.746524 \\
\hline $\mathrm{H}$ & 0.687611 & -0.279829 & -3.545250 \\
\hline $\mathrm{H}$ & 3.927287 & -0.272633 & -3.040603 \\
\hline $\mathrm{H}$ & 4.744913 & 1.312050 & -2.934685 \\
\hline $\mathrm{H}$ & 4.048507 & 0.763153 & -4.446096 \\
\hline $\mathrm{C}$ & -2.026456 & 0.204982 & -0.468815 \\
\hline $\mathrm{Si}$ & 3.263073 & -2.461206 & -0.710616 \\
\hline $\mathrm{Si}$ & 4.423560 & -0.880129 & 1.568009 \\
\hline $\mathrm{N}$ & -3.022719 & 0.694184 & 0.513031 \\
\hline $\mathrm{C}$ & -2.216477 & -1.255725 & -0.795756 \\
\hline $\mathrm{C}$ & 2.926898 & -3.958533 & 0.401634 \\
\hline $\mathrm{C}$ & 4.765099 & -2.919059 & -1.781180 \\
\hline $\mathrm{C}$ & 1.801396 & -2.291031 & -1.919285 \\
\hline $\mathrm{C}$ & 3.386452 & -1.160471 & 3.125004 \\
\hline $\mathrm{C}$ & 5.898984 & -2.073345 & 1.605708 \\
\hline $\mathrm{C}$ & 5.208633 & 0.848604 & 1.705671 \\
\hline $\mathrm{C}$ & -4.393757 & 0.535880 & 0.275044 \\
\hline $\mathrm{C}$ & -2.690719 & 1.780430 & 1.388545 \\
\hline $\mathrm{C}$ & -1.837641 & -1.708369 & -2.061164 \\
\hline $\mathrm{C}$ & -2.727905 & -2.170718 & 0.132120 \\
\hline $\mathrm{H}$ & 3.824453 & -4.263822 & 0.950759 \\
\hline $\mathrm{H}$ & 2.597645 & -4.819844 & -0.193410 \\
\hline $\mathrm{H}$ & 2.150659 & -3.737040 & 1.138323 \\
\hline $\mathrm{H}$ & 4.565566 & -3.816306 & -2.381360 \\
\hline $\mathrm{H}$ & 5.042320 & -2.114989 & -2.475467 \\
\hline $\mathrm{H}$ & 5.640984 & -3.126770 & -1.156474 \\
\hline $\mathrm{H}$ & 1.461857 & -3.276372 & -2.260213 \\
\hline $\mathrm{H}$ & 0.918490 & -1.800182 & -1.491226 \\
\hline $\mathrm{H}$ & 2.093133 & -1.726144 & -2.810995 \\
\hline $\mathrm{H}$ & 2.570607 & -0.431791 & 3.203242 \\
\hline $\mathrm{H}$ & 3.988848 & -1.085342 & 4.039352 \\
\hline $\mathrm{H}$ & 2.918047 & -2.150536 & 3.107130 \\
\hline $\mathrm{H}$ & 6.572173 & -1.892332 & 0.758624 \\
\hline $\mathrm{H}$ & 6.479212 & -1.926212 & 2.525511 \\
\hline $\mathrm{H}$ & 5.600395 & -3.126094 & 1.572263 \\
\hline $\mathrm{H}$ & 5.845244 & 0.903418 & 2.597567 \\
\hline $\mathrm{H}$ & 5.857319 & 1.068179 & 0.844523 \\
\hline $\mathrm{H}$ & 4.470923 & 1.652675 & 1.789977 \\
\hline $\mathrm{O}$ & -4.609312 & -0.089899 & -0.889878 \\
\hline $\mathrm{O}$ & -5.250975 & 0.935565 & 1.042284 \\
\hline $\mathrm{C}$ & -2.953413 & 1.660026 & 2.756077 \\
\hline
\end{tabular}




\begin{tabular}{|c|c|c|c|}
\hline $\mathrm{C}$ & -2.122251 & 2.956626 & 0.888379 \\
\hline $\mathrm{C}$ & -1.954573 & -3.058016 & -2.395249 \\
\hline $\mathrm{H}$ & -1.480625 & -0.986664 & -2.787159 \\
\hline $\mathrm{C}$ & -2.856124 & -3.517075 & -0.203057 \\
\hline $\mathrm{H}$ & -3.028855 & -1.824059 & 1.113745 \\
\hline $\mathrm{C}$ & -5.934072 & -0.615646 & -1.245248 \\
\hline $\mathrm{C}$ & -2.623405 & 2.699996 & 3.622632 \\
\hline $\mathrm{H}$ & -3.406721 & 0.748181 & 3.125024 \\
\hline $\mathrm{C}$ & -1.772683 & 3.986546 & 1.765147 \\
\hline $\mathrm{H}$ & -1.966219 & 3.044217 & -0.179255 \\
\hline $\mathrm{C}$ & -2.463535 & -3.967714 & -1.466114 \\
\hline $\mathrm{H}$ & -1.658675 & -3.396024 & -3.384496 \\
\hline $\mathrm{H}$ & -3.252402 & -4.216680 & 0.527515 \\
\hline $\mathrm{C}$ & -5.642256 & -1.390446 & -2.530326 \\
\hline $\mathrm{C}$ & -6.887976 & 0.551663 & -1.500140 \\
\hline $\mathrm{C}$ & -6.443376 & -1.556812 & -0.151492 \\
\hline $\mathrm{C}$ & -2.019608 & 3.861328 & 3.132441 \\
\hline $\mathrm{H}$ & -2.830152 & 2.599736 & 4.684322 \\
\hline $\mathrm{H}$ & -1.322265 & 4.892410 & 1.371021 \\
\hline $\mathrm{H}$ & -2.559291 & -5.018430 & -1.725162 \\
\hline $\mathrm{H}$ & -5.221393 & -0.725143 & -3.290565 \\
\hline $\mathrm{H}$ & -6.567380 & -1.827796 & -2.920349 \\
\hline $\mathrm{H}$ & -4.923915 & -2.193091 & -2.339293 \\
\hline $\mathrm{H}$ & -6.466523 & 1.224509 & -2.254417 \\
\hline $\mathrm{H}$ & -7.845217 & 0.171508 & -1.874282 \\
\hline $\mathrm{H}$ & -7.061327 & 1.114290 & -0.581642 \\
\hline $\mathrm{H}$ & -5.711865 & -2.349939 & 0.032295 \\
\hline $\mathrm{H}$ & -7.378262 & -2.022181 & -0.482347 \\
\hline $\mathrm{H}$ & -6.629366 & -1.016498 & 0.777678 \\
\hline $\mathrm{H}$ & -1.754533 & 4.666638 & 3.811418 \\
\hline $\mathrm{N}$ & -0.424561 & 0.116332 & 0.546277 \\
\hline $\mathrm{C}$ & -0.321330 & -0.780722 & 1.640209 \\
\hline $\mathrm{H}$ & -0.496315 & 1.053082 & 0.946992 \\
\hline $\mathrm{C}$ & -0.098157 & -2.151471 & 1.413690 \\
\hline $\mathrm{C}$ & -0.398319 & -0.329740 & 2.972832 \\
\hline $\mathrm{C}$ & -0.008878 & -3.040885 & 2.484159 \\
\hline $\mathrm{H}$ & -0.009599 & -2.517460 & 0.398404 \\
\hline $\mathrm{C}$ & -0.288882 & -1.221878 & 4.035833 \\
\hline $\mathrm{H}$ & -0.549392 & 0.725916 & 3.167229 \\
\hline $\mathrm{C}$ & -0.106754 & -2.586517 & 3.799346 \\
\hline $\mathrm{H}$ & 0.141297 & -4.097607 & 2.282911 \\
\hline $\mathrm{H}$ & -0.348733 & -0.847039 & 5.053950 \\
\hline
\end{tabular}


$\begin{array}{llll}\mathrm{H} & -0.030669 & -3.283286 & 4.628622\end{array}$

\section{TS-S2t-1r}

$\begin{array}{llll}\mathrm{Li} & -0.743547 & -0.129702 & 0.070428\end{array}$

$\begin{array}{llll}\mathrm{N} & -2.448529 & -1.047632 & -0.182742\end{array}$

$\begin{array}{lllll}\mathrm{Li} & -2.862560 & 0.803485 & -0.304745\end{array}$

$\mathrm{Si} \quad \quad \quad-3.013021 \quad-1.635473-1.700008$

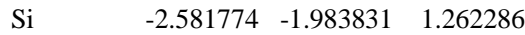

Li $\quad 0.560256 \quad 1.765435 \quad 0.965569$

$\mathrm{N} \quad-1.207499 \quad 1.927314 \quad-0.008514$

$\begin{array}{lllll}\text { C } & & -1.688791 & -2.537888 & -2.707301\end{array}$

C $\quad-4.530346-2.769654-1.604047$

$\begin{array}{lllll}\text { C } & -3.573104 & -0.123105 & -2.726232\end{array}$

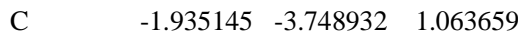

$\begin{array}{llll}\text { C } & -4.351699 & -2.071516 & 1.941886\end{array}$

$\begin{array}{lllll}\text { C } & & -1.552076 & -1.123466 & 2.613454\end{array}$

$\begin{array}{llll}\text { O } & 2.301977 & 1.991177 & 0.840831\end{array}$

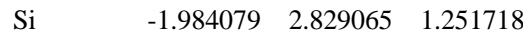

Si $\quad-0.710490 \quad 2.626058-1.531424$

$\mathrm{H} \quad \begin{array}{llll}-0.855341 & -1.875504 & -2.967751\end{array}$

$\mathrm{H} \quad-2.096820 \quad-2.942207 \quad-3.642278$

$\begin{array}{lllll}\mathrm{H} & & -1.270771 & -3.368672 & -2.127839\end{array}$

$\mathrm{H} \quad \begin{array}{llll}-5.377067 & -2.262975 & -1.125310\end{array}$

$\begin{array}{llll}\mathrm{H} & -4.847734 & -3.070635 & -2.610401\end{array}$

$\mathrm{H} \quad \begin{array}{llll}-4.330603 & -3.685421 & -1.036099\end{array}$

$\begin{array}{lllll}\mathrm{H} & -4.016182 & -0.450804 & -3.674510\end{array}$

$\begin{array}{llll}\mathrm{H} & -4.356170 & 0.465210 & -2.219095\end{array}$

$\mathrm{H} \quad-2.747929 \quad 0.550242 \quad-2.983100$

$\begin{array}{llll}\mathrm{H} & -2.553819 & -4.323346 & 0.363709\end{array}$

$\begin{array}{lllll}\mathrm{H} & & -1.931336 & -4.289137 & 2.018754\end{array}$

$\mathrm{H} \quad \begin{array}{llll}-0.910803 & -3.746326 & 0.677712\end{array}$

$\begin{array}{llll}\mathrm{H} & -4.378867 & -2.564757 & 2.922036\end{array}$

$\mathrm{H} \quad \begin{array}{llll}4.783644 & -1.069695 & 2.063902\end{array}$

$\begin{array}{llll}\mathrm{H} & -5.010215 & -2.632496 & 1.270011\end{array}$

$\begin{array}{lllll}\mathrm{H} & -0.514337 & -0.946994 & 2.307698\end{array}$

$\begin{array}{llll}\mathrm{H} & -1.504341 & -1.748961 & 3.513046\end{array}$

$\begin{array}{llll}\mathrm{H} & -1.991528 & -0.163867 & 2.908425\end{array}$

$\begin{array}{llll}\text { C } & 2.766207 & 0.767598 & 0.836529\end{array}$

$\begin{array}{llll}\text { C } & -2.286230 & 4.667079 & 0.938452\end{array}$

$\begin{array}{llll}\text { C } & -0.959586 & 2.711499 & 2.848257\end{array}$

$\begin{array}{llll}\text { C } & -3.704064 & 2.061029 & 1.580552\end{array}$

$\begin{array}{llll}\text { C } & 0.608105 & 3.967153 & -1.342563\end{array}$ 


\begin{tabular}{|c|c|c|c|}
\hline $\mathrm{C}$ & -2.167272 & 3.384531 & -2.485905 \\
\hline $\mathrm{C}$ & 0.063169 & 1.255674 & -2.601017 \\
\hline $\mathrm{N}$ & 3.156308 & 0.196665 & 2.121938 \\
\hline $\mathrm{C}$ & 3.751493 & 0.432570 & -0.274584 \\
\hline $\mathrm{H}$ & -1.353216 & 5.202386 & 0.732550 \\
\hline $\mathrm{H}$ & -2.740575 & 5.124872 & 1.825966 \\
\hline $\mathrm{H}$ & -2.963672 & 4.837897 & 0.095134 \\
\hline $\mathrm{H}$ & -1.485551 & 3.202199 & 3.676110 \\
\hline $\mathrm{H}$ & 0.007564 & 3.226727 & 2.751024 \\
\hline $\mathrm{H}$ & -0.764618 & 1.679788 & 3.164247 \\
\hline $\mathrm{H}$ & -3.678477 & 0.994425 & 1.836417 \\
\hline $\mathrm{H}$ & -4.390407 & 2.196734 & 0.727609 \\
\hline $\mathrm{H}$ & -4.184923 & 2.569351 & 2.424433 \\
\hline $\mathrm{H}$ & 1.441476 & 3.602285 & -0.729986 \\
\hline $\mathrm{H}$ & 1.009536 & 4.250438 & -2.324209 \\
\hline $\mathrm{H}$ & 0.210790 & 4.876109 & -0.876755 \\
\hline $\mathrm{H}$ & -3.046655 & 2.730996 & -2.524594 \\
\hline $\mathrm{H}$ & -1.878918 & 3.598907 & -3.522565 \\
\hline $\mathrm{H}$ & -2.477621 & 4.332280 & -2.032538 \\
\hline $\mathrm{H}$ & 1.133948 & 1.143694 & -2.390075 \\
\hline $\mathrm{H}$ & -0.018816 & 1.512127 & -3.664321 \\
\hline $\mathrm{H}$ & -0.417482 & 0.277934 & -2.477703 \\
\hline $\mathrm{C}$ & 2.205192 & 0.525854 & 3.176056 \\
\hline $\mathrm{C}$ & 4.498403 & 0.613905 & 2.539163 \\
\hline $\mathrm{C}$ & 3.931083 & 1.359187 & -1.306906 \\
\hline $\mathrm{C}$ & 4.407220 & -0.804856 & -0.346743 \\
\hline $\mathrm{H}$ & 2.463124 & -0.039997 & 4.078006 \\
\hline $\mathrm{H}$ & 2.193693 & 1.598652 & 3.426592 \\
\hline $\mathrm{H}$ & 1.195611 & 0.229259 & 2.879712 \\
\hline $\mathrm{H}$ & 4.748604 & 0.097729 & 3.472357 \\
\hline $\mathrm{H}$ & 4.563309 & 1.700791 & 2.714464 \\
\hline $\mathrm{H}$ & 5.238113 & 0.339834 & 1.787102 \\
\hline $\mathrm{C}$ & 4.745901 & 1.054710 & -2.398211 \\
\hline $\mathrm{H}$ & 3.428864 & 2.317033 & -1.231724 \\
\hline $\mathrm{C}$ & 5.225120 & -1.106121 & -1.435298 \\
\hline $\mathrm{H}$ & 4.269998 & -1.526828 & 0.449895 \\
\hline $\mathrm{C}$ & 5.394106 & -0.179926 & -2.466843 \\
\hline $\mathrm{H}$ & 4.880201 & 1.785117 & -3.191779 \\
\hline $\mathrm{H}$ & 5.726540 & -2.069126 & -1.479306 \\
\hline $\mathrm{H}$ & 6.029428 & -0.417814 & -3.315697 \\
\hline $\mathrm{N}$ & 1.380282 & -0.201550 & 0.329930 \\
\hline $\mathrm{C}$ & 1.529268 & -1.617895 & 0.286349 \\
\hline
\end{tabular}




\begin{tabular}{|c|c|c|c|}
\hline $\mathrm{H}$ & 1.399063 & 0.108161 & -0.644139 \\
\hline $\mathrm{C}$ & 1.655606 & -2.378615 & 1.460157 \\
\hline C & 1.541820 & -2.286556 & -0.949789 \\
\hline $\mathrm{C}$ & 1.795986 & -3.763305 & 1.388455 \\
\hline $\mathrm{H}$ & 1.665561 & -1.885105 & 2.420793 \\
\hline $\mathrm{C}$ & 1.661085 & -3.673580 & -1.014481 \\
\hline $\mathrm{H}$ & 1.467583 & -1.706942 & -1.866265 \\
\hline C & 1.795475 & -4.421354 & 0.156104 \\
\hline $\mathrm{H}$ & 1.896918 & -4.333417 & 2.307805 \\
\hline $\mathrm{H}$ & 1.661090 & -4.164755 & -1.983332 \\
\hline $\mathrm{H}$ & 1.894750 & -5.501641 & 0.110251 \\
\hline
\end{tabular}

\section{TS-S2t-1s}

\begin{tabular}{|c|c|c|c|}
\hline $\mathrm{Li}$ & 0.856779 & 0.131258 & 0.144095 \\
\hline $\mathrm{N}$ & 2.502691 & 1.201876 & 0.030829 \\
\hline $\mathrm{Li}$ & 3.065796 & -0.591231 & -0.233021 \\
\hline $\mathrm{Si}$ & 3.208157 & 1.875740 & -1.386771 \\
\hline $\mathrm{Si}$ & 2.440132 & 2.082412 & 1.513895 \\
\hline $\mathrm{Li}$ & -0.340590 & -2.089603 & 30.628401 \\
\hline $\mathrm{N}$ & 1.513142 & -1.880381 & -0.104750 \\
\hline $\mathrm{C}$ & 1.981265 & 2.753139 & -2.528473 \\
\hline $\mathrm{C}$ & 4.654276 & 3.062139 & -1.078051 \\
\hline $\mathrm{C}$ & 3.968286 & 0.421831 & -2.377556 \\
\hline $\mathrm{C}$ & 1.730487 & 3.824105 & 1.317900 \\
\hline $\mathrm{C}$ & 4.118709 & 2.235490 & 2.388646 \\
\hline $\mathrm{C}$ & 1.324927 & 1.114046 & 2.716123 \\
\hline $\mathrm{O}$ & -2.037117 & -2.038324 & 0.281814 \\
\hline $\mathrm{Si}$ & 2.281905 & -2.760485 & 1.176175 \\
\hline $\mathrm{Si}$ & 1.241581 & -2.521018 & -1.707763 \\
\hline $\mathrm{H}$ & 1.468646 & 3.556281 & -1.987197 \\
\hline $\mathrm{H}$ & 2.483520 & 3.192763 & -3.399433 \\
\hline $\mathrm{H}$ & 1.208269 & 2.069925 & -2.897795 \\
\hline $\mathrm{H}$ & 5.450236 & 2.574899 & -0.502337 \\
\hline $\mathrm{H}$ & 5.086661 & 3.397322 & -2.029166 \\
\hline $\mathrm{H}$ & 4.345903 & 3.955761 & -0.524237 \\
\hline $\mathrm{H}$ & 4.481712 & 0.807935 & -3.266266 \\
\hline $\mathrm{H}$ & 4.740451 & -0.124565 & -1.808942 \\
\hline $\mathrm{H}$ & 3.228909 & -0.300371 & -2.741816 \\
\hline $\mathrm{H}$ & 2.386653 & 4.454552 & 0.705437 \\
\hline $\mathrm{H}$ & 1.611780 & 4.321422 & 2.288960 \\
\hline $\mathrm{H}$ & 0.749323 & 3.796091 & 0.834109 \\
\hline $\mathrm{H}$ & 4.598196 & 1.257020 & 2.51963 \\
\hline
\end{tabular}




\begin{tabular}{|c|c|c|c|}
\hline $\mathrm{H}$ & 4.000916 & 2.682025 & 3.384459 \\
\hline $\mathrm{H}$ & 4.812071 & 2.867646 & 1.823485 \\
\hline $\mathrm{H}$ & 1.790789 & 0.172781 & 3.031671 \\
\hline $\mathrm{H}$ & 1.140546 & 1.702629 & 3.622958 \\
\hline $\mathrm{H}$ & 0.338190 & 0.886010 & 2.296652 \\
\hline $\mathrm{C}$ & -2.553836 & -0.820006 & 0.297330 \\
\hline $\mathrm{C}$ & 2.785106 & -4.544606 & 0.811309 \\
\hline $\mathrm{C}$ & 1.118891 & -2.810709 & 2.677425 \\
\hline $\mathrm{C}$ & 3.882493 & -1.848891 & 1.677485 \\
\hline $\mathrm{C}$ & 0.006538 & -3.954622 & -1.723840 \\
\hline $\mathrm{C}$ & 0.488871 & -1.153358 & -2.791435 \\
\hline $\mathrm{C}$ & 2.845011 & -3.149982 & -2.511628 \\
\hline $\mathrm{N}$ & -3.128007 & -0.362969 & 1.558556 \\
\hline $\mathrm{C}$ & -3.454913 & -0.507289 & -0.902787 \\
\hline $\mathrm{H}$ & 1.935053 & -5.149668 & 0.477428 \\
\hline $\mathrm{H}$ & 3.184403 & -5.011133 & 1.720557 \\
\hline $\mathrm{H}$ & 3.561200 & -4.605868 & 0.041070 \\
\hline $\mathrm{H}$ & 1.623809 & -3.253329 & 3.544480 \\
\hline $\mathrm{H}$ & 0.229353 & -3.432963 & 2.498121 \\
\hline $\mathrm{H}$ & 0.775442 & -1.815154 & 2.982101 \\
\hline $\mathrm{H}$ & 3.727559 & -0.803786 & 1.971811 \\
\hline $\mathrm{H}$ & 4.639958 & -1.873212 & 0.875978 \\
\hline $\mathrm{H}$ & 4.347394 & -2.350031 & 2.534466 \\
\hline $\mathrm{H}$ & -0.949161 & -3.656157 & -1.275412 \\
\hline $\mathrm{H}$ & -0.197575 & -4.266957 & -2.756211 \\
\hline $\mathrm{H}$ & 0.382778 & -4.832527 & -1.185420 \\
\hline $\mathrm{H}$ & 0.687352 & -1.346706 & -3.852665 \\
\hline $\mathrm{H}$ & -0.601874 & -1.126150 & -2.676840 \\
\hline $\mathrm{H}$ & 0.883148 & -0.154321 & -2.572010 \\
\hline $\mathrm{H}$ & 3.708264 & -2.503406 & -2.314329 \\
\hline $\mathrm{H}$ & 2.735591 & -3.220329 & -3.600787 \\
\hline $\mathrm{H}$ & 3.093424 & -4.151181 & -2.143153 \\
\hline $\mathrm{C}$ & -2.274597 & -0.640206 & 2.718467 \\
\hline $\mathrm{C}$ & -4.536105 & -0.703984 & 1.790079 \\
\hline $\mathrm{C}$ & -3.484093 & -1.417795 & -1.963472 \\
\hline $\mathrm{C}$ & -4.159973 & 0.699282 & -1.027671 \\
\hline $\mathrm{C}$ & -2.429881 & -2.007197 & 3.402140 \\
\hline $\mathrm{H}$ & -1.239709 & -0.517243 & 2.389569 \\
\hline $\mathrm{H}$ & -2.438818 & 0.147718 & 3.466687 \\
\hline $\mathrm{C}$ & -5.173176 & 0.211374 & 2.835931 \\
\hline $\mathrm{H}$ & -5.074554 & -0.591015 & 0.850321 \\
\hline $\mathrm{H}$ & -4.658882 & -1.757200 & 2.087228 \\
\hline
\end{tabular}




\begin{tabular}{llll}
$\mathrm{C}$ & -4.194620 & -1.130255 & -3.130267 \\
$\mathrm{H}$ & -2.945818 & -2.351638 & -1.849208 \\
$\mathrm{C}$ & -4.874153 & 0.984632 & -2.190731 \\
$\mathrm{H}$ & -4.147344 & 1.411981 & -0.211221 \\
$\mathrm{H}$ & -3.396334 & -2.103828 & 3.907327 \\
$\mathrm{H}$ & -1.650364 & -2.127876 & 4.163867 \\
$\mathrm{H}$ & -2.345774 & -2.814524 & 2.670969 \\
$\mathrm{H}$ & -4.722329 & 0.086654 & 3.826772 \\
$\mathrm{H}$ & -6.242603 & -0.008248 & 2.930733 \\
$\mathrm{H}$ & -5.056234 & 1.260309 & 2.541971 \\
$\mathrm{C}$ & -4.890774 & 0.073470 & -3.249072 \\
$\mathrm{H}$ & -4.208930 & -1.850706 & -3.944077 \\
$\mathrm{H}$ & -5.414625 & 1.923985 & -2.270879 \\
$\mathrm{H}$ & -5.445335 & 0.298878 & -4.156003 \\
$\mathrm{~N}$ & -1.245584 & 0.197905 & 0.004781 \\
$\mathrm{C}$ & -1.470309 & 1.607949 & -0.020693 \\
$\mathrm{H}$ & -1.079826 & -0.078634 & -0.965128 \\
$\mathrm{C}$ & -1.368672 & 2.315634 & -1.228468 \\
$\mathrm{C}$ & -1.781273 & 2.317423 & 1.150185 \\
$\mathrm{C}$ & -1.563896 & 3.695018 & -1.269730 \\
$\mathrm{H}$ & -1.148849 & 1.772522 & -2.143653 \\
$\mathrm{C}$ & -2.000545 & 3.693106 & 1.099224 \\
$\mathrm{H}$ & -1.867341 & 1.791606 & 2.089406 \\
$\mathrm{H}$ & -1.890904 & 4.391621 & -0.105585 \\
& -1.474344 & 4.219256 & -2.216895 \\
$\mathrm{H}$ & -2.246611 & 4.224798 & 2.014259 \\
\hline & -2.052654 & 5.465012 & -0.134474
\end{tabular}

\section{TS-S2t-1v}

$\begin{array}{llll}\mathrm{Li} & -0.828533 & -0.099878 & -0.256281 \\ \mathrm{~N} & -2.495166 & -1.128921 & -0.103048 \\ \mathrm{Li} & -3.035247 & 0.682608 & 0.133905 \\ \mathrm{Si} & -3.306430 & -1.465589 & -1.580106 \\ \mathrm{Si} & -2.389033 & -2.205529 & 1.236029 \\ \mathrm{Li} & 0.439152 & 1.641559 & 0.918154 \\ \mathrm{~N} & -1.451556 & 1.911725 & 0.208343 \\ \mathrm{C} & -2.102073 & -1.860982 & -2.984332 \\ \mathrm{C} & -4.603729 & -2.845264 & -1.524446 \\ \mathrm{C} & -4.296900 & 0.119218 & -2.022325 \\ \mathrm{C} & -1.122146 & -1.493163 & 2.461110 \\ \mathrm{C} & -1.855620 & -3.968313 & 0.781577 \\ \mathrm{C} & -4.024938 & -2.380721 & 2.187135\end{array}$




\begin{tabular}{|c|c|c|c|}
\hline $\mathrm{O}$ & 2.192331 & 1.761761 & 1.097257 \\
\hline $\mathrm{Si}$ & -2.008744 & 2.585459 & 1.706746 \\
\hline $\mathrm{Si}$ & -1.195760 & 2.876348 & -1.222184 \\
\hline $\mathrm{H}$ & -1.565871 & -2.789300 & -2.754601 \\
\hline $\mathrm{H}$ & -2.611332 & -1.991613 & -3.947545 \\
\hline $\mathrm{H}$ & -1.340133 & -1.084244 & -3.109971 \\
\hline $\mathrm{H}$ & -5.359892 & -2.652473 & -0.754403 \\
\hline $\mathrm{H}$ & -5.122003 & -2.924641 & -2.488392 \\
\hline $\mathrm{H}$ & -4.153776 & -3.821329 & -1.312486 \\
\hline $\mathrm{H}$ & -3.696486 & 1.039271 & -2.051103 \\
\hline $\mathrm{H}$ & -5.123197 & 0.270554 & -1.309574 \\
\hline $\mathrm{H}$ & -4.757468 & 0.037338 & -3.014263 \\
\hline $\mathrm{H}$ & -1.500601 & -0.576741 & 2.929347 \\
\hline $\mathrm{H}$ & -0.154813 & -1.252963 & 2.002945 \\
\hline $\mathrm{H}$ & -0.905637 & -2.201079 & 3.270075 \\
\hline $\mathrm{H}$ & -2.666862 & -4.509123 & 0.280647 \\
\hline $\mathrm{H}$ & -1.580528 & -4.542597 & 1.675108 \\
\hline $\mathrm{H}$ & -0.997873 & -3.965014 & 0.103053 \\
\hline $\mathrm{H}$ & -4.418661 & -1.410855 & 2.516055 \\
\hline $\mathrm{H}$ & -3.904001 & -3.007208 & 3.080328 \\
\hline $\mathrm{H}$ & -4.792281 & -2.847961 & 1.558848 \\
\hline $\mathrm{C}$ & 2.647921 & 0.651685 & 0.584984 \\
\hline $\mathrm{C}$ & -2.468525 & 4.418265 & 1.721405 \\
\hline $\mathrm{C}$ & -0.698456 & 2.341305 & 3.062016 \\
\hline $\mathrm{C}$ & -3.585481 & 1.649306 & 2.245540 \\
\hline $\mathrm{C}$ & 0.293406 & 4.029867 & -1.010662 \\
\hline $\mathrm{C}$ & -2.693377 & 3.936151 & -1.708075 \\
\hline $\mathrm{C}$ & -0.866382 & 1.769592 & -2.729112 \\
\hline $\mathrm{N}$ & 3.743103 & 0.749195 & -0.359509 \\
\hline $\mathrm{C}$ & 2.809908 & -0.526395 & 1.525380 \\
\hline $\mathrm{H}$ & -1.657084 & 5.053778 & 1.349984 \\
\hline $\mathrm{H}$ & -2.686703 & 4.731784 & 2.749925 \\
\hline $\mathrm{H}$ & -3.358111 & 4.622836 & 1.116641 \\
\hline $\mathrm{H}$ & -1.099209 & 2.668095 & 4.029318 \\
\hline $\mathrm{H}$ & 0.205969 & 2.939024 & 2.880256 \\
\hline $\mathrm{H}$ & -0.388818 & 1.296439 & 3.186986 \\
\hline $\mathrm{H}$ & -3.443078 & 0.568845 & 2.368245 \\
\hline $\mathrm{H}$ & -4.430806 & 1.814157 & 1.556530 \\
\hline $\mathrm{H}$ & -3.922509 & 2.025700 & 3.218252 \\
\hline $\mathrm{H}$ & 1.197151 & 3.500020 & -0.684925 \\
\hline $\mathrm{H}$ & 0.083784 & 4.797071 & -0.254803 \\
\hline $\mathrm{H}$ & 0.530402 & 4.548770 & -1.947818 \\
\hline
\end{tabular}




\begin{tabular}{|c|c|c|c|}
\hline H & -2.587458 & 4.290194 & -2.741149 \\
\hline $\mathrm{H}$ & -3.629874 & 3.365900 & -1.655184 \\
\hline $\mathrm{H}$ & -2.805436 & 4.817553 & -1.069229 \\
\hline $\mathrm{H}$ & -0.056434 & 1.044289 & -2.602892 \\
\hline $\mathrm{H}$ & -0.590198 & 2.407567 & -3.578267 \\
\hline $\mathrm{H}$ & -1.756792 & 1.208582 & -3.029716 \\
\hline $\mathrm{C}$ & 3.719761 & 1.936690 & -1.221557 \\
\hline $\mathrm{C}$ & 5.090798 & 0.793281 & 0.243921 \\
\hline $\mathrm{C}$ & 2.354929 & -0.404594 & 2.841241 \\
\hline $\mathrm{C}$ & 3.375913 & -1.739689 & 1.110885 \\
\hline $\mathrm{C}$ & 5.064179 & 1.859459 & -1.956992 \\
\hline $\mathrm{H}$ & 3.643800 & 2.857155 & -0.623577 \\
\hline $\mathrm{H}$ & 2.866288 & 1.920575 & -1.907715 \\
\hline $\mathrm{C}$ & 6.004604 & 1.154673 & -0.942169 \\
\hline $\mathrm{H}$ & 5.141958 & 1.572093 & 1.023078 \\
\hline $\mathrm{H}$ & 5.346959 & -0.160457 & 0.705782 \\
\hline $\mathrm{C}$ & 2.435135 & -1.482340 & 3.723853 \\
\hline $\mathrm{H}$ & 1.954929 & 0.550762 & 3.162936 \\
\hline $\mathrm{C}$ & 3.457457 & -2.817563 & 1.991856 \\
\hline $\mathrm{H}$ & 3.746333 & -1.831402 & 0.096776 \\
\hline $\mathrm{H}$ & 5.420296 & 2.850690 & -2.253138 \\
\hline $\mathrm{H}$ & 4.964431 & 1.256791 & -2.865181 \\
\hline $\mathrm{H}$ & 6.440147 & 0.252924 & -1.382758 \\
\hline $\mathrm{H}$ & 6.831032 & 1.795902 & -0.621516 \\
\hline $\mathrm{C}$ & 2.979808 & -2.695204 & 3.298840 \\
\hline $\mathrm{H}$ & 2.076431 & -1.372917 & 4.743720 \\
\hline $\mathrm{H}$ & 3.890927 & -3.755185 & 1.654824 \\
\hline $\mathrm{H}$ & 3.041481 & -3.536787 & 3.983410 \\
\hline $\mathrm{N}$ & 1.269656 & 0.129485 & -0.470802 \\
\hline $\mathrm{C}$ & 1.457896 & -1.039411 & -1.257250 \\
\hline $\mathrm{H}$ & 1.351853 & 0.918601 & -1.112574 \\
\hline $\mathrm{C}$ & 1.081588 & -2.290510 & -0.740704 \\
\hline $\mathrm{C}$ & 2.005687 & -0.985898 & -2.550664 \\
\hline $\mathrm{C}$ & 1.244953 & -3.450037 & -1.496673 \\
\hline $\mathrm{H}$ & 0.672608 & -2.344943 & 0.260981 \\
\hline $\mathrm{C}$ & 2.151298 & -2.145498 & -3.309740 \\
\hline $\mathrm{H}$ & 2.318494 & -0.028864 & -2.957186 \\
\hline $\mathrm{C}$ & 1.774977 & -3.384909 & -2.786489 \\
\hline $\mathrm{H}$ & 0.953376 & -4.407421 & -1.074306 \\
\hline $\mathrm{H}$ & 2.568501 & -2.080810 & -4.310956 \\
\hline $\mathrm{H}$ & 1.893754 & -4.288311 & -3.377207 \\
\hline
\end{tabular}




\section{TS-S2t-1w}

$\begin{array}{llll}\mathrm{Si} & 0.990342 & 2.308223 & 2.077896\end{array}$

$\mathrm{N} \quad 1.287936 \quad 1.961929 \quad 0.390297$

$\begin{array}{llll}\text { C } & -0.449679 & 3.507372 & 2.339118\end{array}$

Si $\quad 1.835203 \quad 3.141822-0.756579$

$\begin{array}{lllll}\mathrm{Li} & -0.604530 & 1.917660 & -0.256452\end{array}$

$\begin{array}{llll}\mathrm{Li} & 3.033816 & 0.952769 & 0.281712\end{array}$

$\begin{array}{llll}\text { C } & 2.512588 & 3.054751 & 2.936548\end{array}$

$\begin{array}{llll}\text { C } & 0.511363 & 0.691225 & 2.954221\end{array}$

$\mathrm{H} \quad-1.363445 \quad 3.131522 \quad 1.862506$

$\mathrm{H} \quad-0.658961 \quad 3.621998 \quad 3.410616$

$\mathrm{H} \quad \begin{array}{llll}\mathrm{H} & -0.235111 & 4.505374 & 1.939364\end{array}$

$\begin{array}{llll}\text { C } & 2.064085 & 4.909927 & -0.130475\end{array}$

$\begin{array}{lllll}\text { C } & 0.603041 & 3.233582 & -2.199993\end{array}$

$\begin{array}{llll}\text { C } & 3.536442 & 2.601105 & -1.435724\end{array}$

$\begin{array}{lllll}\mathrm{Li} & 0.955987 & -0.079332 & -0.128068\end{array}$

$\begin{array}{llll}\mathrm{O} & -2.255657 & 1.584636 & 0.162771\end{array}$

$\begin{array}{llll}\mathrm{N} & 2.757021 & -0.856450 & -0.222722\end{array}$

$\begin{array}{llll}\mathrm{H} & 3.452552 & 2.572420 & 2.643365\end{array}$

$\begin{array}{llll}\mathrm{H} & 2.428967 & 2.963281 & 4.026447\end{array}$

$\begin{array}{llll}\mathrm{H} & 2.603538 & 4.121522 & 2.704850\end{array}$

$\begin{array}{llll}\text { H } & 0.717729 & 0.757806 & 4.029396\end{array}$

$\begin{array}{llll}\mathrm{H} & -0.563801 & 0.498440 & 2.852817\end{array}$

$\begin{array}{llll}\mathrm{H} & 1.055545 & -0.184408 & 2.581454\end{array}$

$\begin{array}{llll}\mathrm{H} & 1.144296 & 5.310366 & 0.309343\end{array}$

$\begin{array}{llll}\mathrm{H} & 2.341776 & 5.564782 & -0.965883\end{array}$

$\begin{array}{llll}\mathrm{H} & 2.854780 & 4.984376 & 0.623519\end{array}$

$\begin{array}{llll}\mathrm{H} & 0.995705 & 3.866899 & -3.004671\end{array}$

$\begin{array}{lllll}\mathrm{H} & -0.350895 & 3.688784 & -1.895602\end{array}$

$\begin{array}{llll}\mathrm{H} & 0.381195 & 2.256403 & -2.645396\end{array}$

$\begin{array}{llll}\mathrm{H} & 3.538939 & 1.608101 & -1.900620\end{array}$

$\mathrm{H} \quad 4.318634 \quad 2.620924 \quad-0.658045$

$\mathrm{H} \quad 3.872180 \quad 3.307166-2.204176$

$\begin{array}{lllll}\text { C } & -2.581759 & 0.321080 & -0.051641\end{array}$

$\begin{array}{llll}\mathrm{Si} & 3.610849 & -1.621242 & 1.060999\end{array}$

$\begin{array}{lllll}\mathrm{Si} & 2.772344 & -1.486026 & -1.829548\end{array}$

$\begin{array}{lllll}\mathrm{N} & & -3.132760 & -0.012615 & -1.356088\end{array}$

$\begin{array}{lllll}\text { C } & & -3.361438 & -0.328405 & 1.095022\end{array}$

$\begin{array}{llll}\text { C } & 2.576877 & -2.848363 & 2.063613\end{array}$

$\begin{array}{lllll}\text { C } & 5.208064 & -2.506635 & 0.549366\end{array}$

$\begin{array}{llll}\text { C } & 4.172789 & -0.241324 & 2.264559\end{array}$

$\begin{array}{llll}\text { C } & 2.341146 & -3.324292 & -1.913848\end{array}$ 


\begin{tabular}{|c|c|c|c|}
\hline $\mathrm{C}$ & 4.422457 & -1.227275 & -2.731626 \\
\hline $\mathrm{C}$ & 1.472278 & -0.525596 & -2.836571 \\
\hline $\mathrm{C}$ & -2.439934 & 0.614858 & -2.482751 \\
\hline $\mathrm{C}$ & -4.588939 & 0.079183 & -1.526019 \\
\hline $\mathrm{C}$ & -3.500600 & 0.387695 & 2.287993 \\
\hline $\mathrm{C}$ & -3.847987 & -1.642982 & 1.033875 \\
\hline $\mathrm{H}$ & 1.722247 & -2.361510 & 2.546874 \\
\hline $\mathrm{H}$ & 3.173449 & -3.331230 & 2.847911 \\
\hline $\mathrm{H}$ & 2.174543 & -3.630041 & 1.409570 \\
\hline $\mathrm{H}$ & 5.906824 & -1.816918 & 0.060805 \\
\hline $\mathrm{H}$ & 5.712441 & -2.924947 & 1.429485 \\
\hline $\mathrm{H}$ & 5.020313 & -3.332768 & -0.145505 \\
\hline $\mathrm{H}$ & 4.790066 & -0.672938 & 3.061670 \\
\hline $\mathrm{H}$ & 4.811569 & 0.515941 & 1.778397 \\
\hline $\mathrm{H}$ & 3.342533 & 0.272288 & 2.762251 \\
\hline $\mathrm{H}$ & 3.094700 & -3.933854 & -1.400573 \\
\hline $\mathrm{H}$ & 2.282361 & -3.678177 & -2.950921 \\
\hline $\mathrm{H}$ & 1.375382 & -3.518874 & -1.437521 \\
\hline $\mathrm{H}$ & 4.347825 & -1.522200 & -3.786249 \\
\hline $\mathrm{H}$ & 4.737792 & -0.176521 & -2.702751 \\
\hline $\mathrm{H}$ & 5.222805 & -1.821711 & -2.277916 \\
\hline $\mathrm{H}$ & 1.380400 & -0.948055 & -3.844498 \\
\hline $\mathrm{H}$ & 0.470194 & -0.573026 & -2.394032 \\
\hline $\mathrm{H}$ & 1.748053 & 0.528962 & -2.952154 \\
\hline $\mathrm{C}$ & -2.890676 & 2.046354 & -2.823652 \\
\hline $\mathrm{H}$ & -1.364286 & 0.586516 & -2.285238 \\
\hline $\mathrm{H}$ & -2.606564 & -0.022498 & -3.365865 \\
\hline $\mathrm{C}$ & -5.103540 & 1.504484 & -1.773652 \\
\hline $\mathrm{H}$ & -5.080481 & -0.356341 & -0.657135 \\
\hline $\mathrm{H}$ & -4.850073 & -0.548769 & -2.392288 \\
\hline $\mathrm{C}$ & -4.108355 & -0.194624 & 3.401620 \\
\hline $\mathrm{H}$ & -3.127697 & 1.404988 & 2.318165 \\
\hline $\mathrm{C}$ & -4.459097 & -2.223392 & 2.144782 \\
\hline $\mathrm{H}$ & -3.747685 & -2.208353 & 0.114833 \\
\hline $\mathrm{C}$ & -4.411651 & 2.109554 & -2.999981 \\
\hline $\mathrm{H}$ & -2.602500 & 2.713342 & -2.006191 \\
\hline $\mathrm{H}$ & -2.376566 & 2.379283 & -3.735503 \\
\hline $\mathrm{H}$ & -4.899277 & 2.118706 & -0.888469 \\
\hline $\mathrm{H}$ & -6.191986 & 1.478259 & -1.915725 \\
\hline $\mathrm{C}$ & -4.588705 & -1.503283 & 3.334346 \\
\hline $\mathrm{H}$ & -4.211442 & 0.377051 & 4.320344 \\
\hline $\mathrm{H}$ & -4.830207 & -3.242713 & 2.080748 \\
\hline
\end{tabular}




$\begin{array}{llll}\mathrm{H} & -4.736392 & 3.144195 & -3.165264 \\ \mathrm{H} & -4.702407 & 1.539612 & -3.895985 \\ \mathrm{H} & -5.062890 & -1.958195 & 4.199836 \\ \mathrm{~N} & -1.102829 & -0.505822 & 0.027729 \\ \mathrm{C} & -1.101313 & -1.914902 & -0.198722 \\ \mathrm{H} & -0.939140 & -0.379393 & 1.028801 \\ \mathrm{C} & -1.357840 & -2.447078 & -1.472310 \\ \mathrm{C} & -0.831575 & -2.799678 & 0.857285 \\ \mathrm{C} & -1.360472 & -3.827227 & -1.668375 \\ \mathrm{H} & -1.570866 & -1.783862 & -2.297795 \\ \mathrm{C} & -0.811058 & -4.177825 & 0.650644 \\ \mathrm{H} & -0.651581 & -2.398830 & 1.851165 \\ \mathrm{C} & -1.085267 & -4.700958 & -0.613759 \\ \mathrm{H} & -1.567424 & -4.221457 & -2.659474 \\ \mathrm{H} & -0.594742 & -4.839884 & 1.484221 \\ \mathrm{H} & -1.078866 & -5.774369 & -0.777686\end{array}$

\section{TS-S2t-1x}

Si $\quad-1.614532 \quad 2.935707 \quad-0.919357$

$\begin{array}{lllll}\mathrm{N} & & -1.685407 & 1.832940 & 0.426957\end{array}$

C $\quad-3.275570 \quad 3.726219 \quad-1.377212$

$\begin{array}{llll}\mathrm{Si} & -2.267984 & 2.326933 & 1.990899\end{array}$

$\mathrm{Li} \quad-3.152171 \quad 0.439221 \quad 0.350910$

$\begin{array}{llll}\mathrm{Li} & 0.260012 & 1.615655 & 0.996673\end{array}$

$\begin{array}{lllll}\text { C } & -1.036278 & 1.967987 & -2.444645\end{array}$

$\begin{array}{llll}\text { C } & -0.386913 & 4.350756 & -0.617677\end{array}$

$\begin{array}{lllll}\mathrm{H} & & -4.093523 & 2.995611 & -1.392223\end{array}$

$\mathrm{H} \quad-3.220577 \quad 4.172562 \quad-2.378000$

$\begin{array}{llll}\mathrm{H} & -3.552157 & 4.521480 & -0.678081\end{array}$

$\begin{array}{llll}\text { C } & -2.982123 & 4.072004 & 2.127081\end{array}$

$\begin{array}{llll}\text { C } & -0.920744 & 2.170764 & 3.313110\end{array}$

$\begin{array}{llll}\text { C } & -3.695959 & 1.158544 & 2.500610\end{array}$

$\mathrm{N} \quad \begin{array}{llll}-2.467219 & -1.263298 & -0.161516\end{array}$

$\mathrm{Li} \quad \quad-0.966696 \quad-0.018823 \quad-0.391109$

$\begin{array}{lllll}\text { O } & 1.874538 & 1.362214 & 1.661518\end{array}$

$\begin{array}{llll}\mathrm{H} & -0.141512 & 1.360349 & -2.268472\end{array}$

$\begin{array}{llll}\mathrm{H} & -0.783485 & 2.663374 & -3.254449\end{array}$

$\begin{array}{llll}\mathrm{H} & -1.816739 & 1.302844 & -2.828868\end{array}$

$\mathrm{H} \quad 0.588181 \quad 3.996844 \quad-0.263117$

$\mathrm{H} \quad \quad-0.209053 \quad 4.921796 \quad-1.537569$

$\begin{array}{llll}\mathrm{H} & -0.769148 & 5.052584 & 0.132717\end{array}$

$\begin{array}{llll}\mathrm{H} & -3.895307 & 4.192062 & 1.534444\end{array}$ 


\begin{tabular}{|c|c|c|c|}
\hline $\mathrm{H}$ & -3.238413 & 4.276299 & 3.174081 \\
\hline $\mathrm{H}$ & -2.271849 & 4.842150 & 1.808583 \\
\hline $\mathrm{H}$ & -1.346056 & 2.405422 & 4.296922 \\
\hline $\mathrm{H}$ & -0.092602 & 2.872914 & 3.148028 \\
\hline $\mathrm{H}$ & -0.480233 & 1.169509 & 3.381476 \\
\hline $\mathrm{H}$ & -3.404723 & 0.104481 & 2.582808 \\
\hline $\mathrm{H}$ & -4.578996 & 1.227894 & 1.842218 \\
\hline $\mathrm{H}$ & -4.056176 & 1.451311 & 3.493381 \\
\hline $\mathrm{Si}$ & -3.225439 & -1.580609 & -1.677609 \\
\hline $\mathrm{Si}$ & -2.294800 & -2.454101 & 1.074749 \\
\hline $\mathrm{C}$ & 2.442712 & 0.504023 & 0.858783 \\
\hline $\mathrm{C}$ & -1.961353 & -1.743368 & -3.076808 \\
\hline $\mathrm{C}$ & -4.350148 & -3.102540 & -1.762196 \\
\hline $\mathrm{C}$ & -4.371682 & -0.091678 & -2.040167 \\
\hline $\mathrm{C}$ & -1.705410 & -4.154742 & 0.466616 \\
\hline $\mathrm{C}$ & -3.925716 & -2.774392 & 2.000902 \\
\hline $\mathrm{C}$ & -1.055841 & -1.855841 & 2.396247 \\
\hline $\mathrm{N}$ & 3.253380 & 1.032551 & -0.230448 \\
\hline $\mathrm{C}$ & 3.069311 & -0.718336 & 1.511453 \\
\hline $\mathrm{H}$ & -1.320908 & -2.614899 & -2.897083 \\
\hline $\mathrm{H}$ & -2.445205 & -1.873285 & -4.053323 \\
\hline $\mathrm{H}$ & -1.292403 & -0.879131 & -3.150865 \\
\hline $\mathrm{H}$ & -5.127888 & -3.080633 & -0.990081 \\
\hline $\mathrm{H}$ & -4.852743 & -3.140176 & -2.737056 \\
\hline $\mathrm{H}$ & -3.789190 & -4.035681 & -1.646207 \\
\hline $\mathrm{H}$ & -3.852832 & 0.875337 & -2.036709 \\
\hline $\mathrm{H}$ & -5.186245 & -0.045520 & -1.300313 \\
\hline $\mathrm{H}$ & -4.849101 & -0.178179 & -3.023789 \\
\hline $\mathrm{H}$ & -2.545285 & -4.763377 & 0.113589 \\
\hline $\mathrm{H}$ & -1.217125 & -4.711906 & 1.276534 \\
\hline $\mathrm{H}$ & -0.990588 & -4.063540 & -0.356956 \\
\hline $\mathrm{H}$ & -4.314781 & -1.875737 & 2.495896 \\
\hline $\mathrm{H}$ & -3.795902 & -3.543113 & 2.773687 \\
\hline $\mathrm{H}$ & -4.701065 & -3.129067 & 1.311736 \\
\hline $\mathrm{H}$ & -0.014350 & -2.110336 & 2.167459 \\
\hline $\mathrm{H}$ & -1.280694 & -2.329038 & 3.360319 \\
\hline $\mathrm{H}$ & -1.103977 & -0.772275 & 2.555915 \\
\hline $\mathrm{C}$ & 2.669412 & 2.228675 & -0.832641 \\
\hline $\mathrm{C}$ & 4.639363 & 1.346334 & 0.155354 \\
\hline $\mathrm{C}$ & 2.817589 & -0.947956 & 2.867927 \\
\hline $\mathrm{C}$ & 3.797656 & -1.668788 & 0.781456 \\
\hline $\mathrm{C}$ & 3.479971 & 2.634119 & -2.059858 \\
\hline
\end{tabular}




\begin{tabular}{|c|c|c|c|}
\hline $\mathrm{H}$ & 1.638237 & 2.018279 & -1.129184 \\
\hline $\mathrm{H}$ & 2.651027 & 3.067540 & -0.118865 \\
\hline $\mathrm{C}$ & 5.415377 & 1.761021 & -1.089910 \\
\hline $\mathrm{H}$ & 5.108629 & 0.468773 & 0.602172 \\
\hline $\mathrm{H}$ & 4.668310 & 2.163540 & 0.895835 \\
\hline $\mathrm{C}$ & 3.277580 & -2.110822 & 3.486178 \\
\hline $\mathrm{H}$ & 2.260821 & -0.197973 & 3.418886 \\
\hline $\mathrm{C}$ & 4.262469 & -2.828292 & 1.401950 \\
\hline $\mathrm{H}$ & 3.990613 & -1.497872 & -0.271404 \\
\hline $\mathrm{O}$ & 4.832339 & 2.893125 & -1.721076 \\
\hline $\mathrm{H}$ & 3.084344 & 3.558592 & -2.491690 \\
\hline $\mathrm{H}$ & 3.428148 & 1.836248 & -2.819344 \\
\hline $\mathrm{H}$ & 6.438656 & 2.046926 & -0.829324 \\
\hline $\mathrm{H}$ & 5.448929 & 0.912558 & -1.793425 \\
\hline $\mathrm{C}$ & 3.999607 & -3.055907 & 2.754305 \\
\hline $\mathrm{H}$ & 3.075981 & -2.277187 & 4.541041 \\
\hline $\mathrm{H}$ & 4.823592 & -3.558150 & 0.824789 \\
\hline $\mathrm{H}$ & 4.358068 & -3.962057 & 3.235030 \\
\hline $\mathrm{N}$ & 1.061258 & -0.263429 & 0.038881 \\
\hline $\mathrm{C}$ & 1.336372 & -1.173163 & -1.019822 \\
\hline $\mathrm{H}$ & 0.781806 & -0.836845 & 0.836883 \\
\hline $\mathrm{C}$ & 1.679651 & -0.708628 & -2.299482 \\
\hline $\mathrm{C}$ & 1.260260 & -2.561185 & -0.812857 \\
\hline $\mathrm{C}$ & 1.922322 & -1.605716 & -3.338236 \\
\hline $\mathrm{H}$ & 1.761588 & 0.353878 & -2.479338 \\
\hline $\mathrm{C}$ & 1.491715 & -3.454979 & -1.856207 \\
\hline $\mathrm{H}$ & 1.024981 & -2.934695 & 0.177997 \\
\hline $\mathrm{C}$ & 1.824671 & -2.982551 & -3.127189 \\
\hline $\mathrm{H}$ & 2.186286 & -1.222917 & -4.320218 \\
\hline $\mathrm{H}$ & 1.420175 & -4.522891 & -1.669324 \\
\hline $\mathrm{H}$ & 2.008687 & -3.677277 & -3 \\
\hline
\end{tabular}




\section{List of Known Compounds/General Methods}

All starting materials reported in the manuscript have been previously described in literature and prepared by the method reported previously. Amides and esters were prepared by standard methods. ${ }^{12-40}$ All products reported in the manuscript have been previously described in literature. ${ }^{41-56}$ All experiments were performed using standard Schlenk techniques under argon or nitrogen atmosphere unless stated otherwise. All solvents were purchased at the highest commercial grade and used as received or after purification by passing through activated alumina columns or distillation from sodium/benzophenone under nitrogen. All other chemicals were purchased at the highest commercial grade and used as received. Reaction glassware was ovendried at $140{ }^{\circ} \mathrm{C}$ for at least $24 \mathrm{~h}$ or flame-dried prior to use, allowed to cool under vacuum and purged with argon or nitrogen (three cycles). All products were identified using ${ }^{1} \mathrm{H}$ NMR analysis and comparison with authentic samples. GC and/or GC/MS analysis was used for volatile products. All yields refer to yields determined by ${ }^{1} \mathrm{H}$ NMR and/or GC or GC/MS using an internal standard (optimization) and isolated yields (preparative runs) unless stated otherwise. ${ }^{1} \mathrm{H}$ NMR and ${ }^{13} \mathrm{C}$ NMR spectra were recorded in $\mathrm{CDCl}_{3}$ on Bruker spectrometers at $500\left({ }^{1} \mathrm{H}\right.$ $\mathrm{NMR}$ ) and $125 \mathrm{MHz}\left({ }^{13} \mathrm{C} \mathrm{NMR}\right)$. All shifts are reported in parts per million (ppm) relative to residual $\mathrm{CHCl}_{3}$ peak (7.27 and $77.2 \mathrm{ppm},{ }^{1} \mathrm{H} \mathrm{NMR}$ and ${ }^{13} \mathrm{C} \mathrm{NMR}$, respectively). All coupling constants $(\mathrm{J})$ are reported in hertz $(\mathrm{Hz})$. Abbreviations are: s, singlet; d, doublet; t, triplet; q, quartet; brs, broad singlet. GC-MS chromatography was performed using Agilent HP6890 GC System and Agilent 5973A inert XL EI/CI MSD using helium as the carrier gas at a flow rate of $1 \mathrm{~mL} / \mathrm{min}$ and an initial oven temperature of $50{ }^{\circ} \mathrm{C}$. The injector temperature was $280{ }^{\circ} \mathrm{C}$. The detector temperature was $280{ }^{\circ} \mathrm{C}$. For runs with the initial oven temperature of $50{ }^{\circ} \mathrm{C}$, temperature was increased with a $10{ }^{\circ} \mathrm{C} / \mathrm{min}$ ramp after $50{ }^{\circ} \mathrm{C}$ hold for $3 \mathrm{~min}$ to a final temperature of $280^{\circ} \mathrm{C}$, then hold at $280^{\circ} \mathrm{C}$ for $10 \mathrm{~min}$ (splitless mode of injection, total run time of $33.00 \mathrm{~min}$ ). All flash chromatography was performed using silica gel, $60 \AA$ A, $300 \mathrm{mesh}$. TLC analysis was carried out on glass plates coated with silica gel 60 F254, $0.2 \mathrm{~mm}$ thickness. The plates were visualized using a $254 \mathrm{~nm}$ ultraviolet lamp or aqueous potassium permanganate solutions. ${ }^{1} H$ NMR and ${ }^{13} C N M R$ data are given for all compounds in the Supporting Experimental for characterization purposes. ${ }^{1} \mathrm{H} N M R,{ }^{13} \mathrm{C} N M R$ and HRMS data are reported for all new compounds. 


\section{Experimental Procedures and Characterization Data}

General Procedure for Transamidation Reactions. An oven-dried vial equipped with a stir bar was charged with an amide substrate (neat, 1.0 equiv), amine (typically, 2.0 equiv) placed under a positive pressure of argon, and subjected to three evacuation/backfilling cycles. Toluene (typically, 0.25 M) and LiHMDS (1.0 M in THF, typically, 3.0 equiv) were sequentially added with vigorous stirring at room temperature, and the reaction mixture was stirred at room temperature for an indicated time. After the indicated time, the reaction mixture was quenched with $\mathrm{NH}_{4} \mathrm{Cl}$ (aq., $\left.1.0 \mathrm{M}, 1 \mathrm{~mL}\right)$, diluted with EtOAc $(10 \mathrm{~mL})$, the organic layer was washed with water $(1 \times 10 \mathrm{~mL})$, brine $(1 \times 10 \mathrm{~mL})$, dried and concentrated. A sample was analyzed by ${ }^{1} \mathrm{H}$ $\mathrm{NMR}\left(\mathrm{CDCl}_{3}, 500 \mathrm{MHz}\right)$ and GC-MS to obtain conversion, yield and selectivity using internal standard and comparison with authentic samples. Purification by chromatography on silica gel (EtOAc/hexanes) afforded the title product.

General Procedure for Amidation Reactions. An oven-dried vial equipped with a stir bar was charged with an ester substrate (neat, 1.0 equiv), amine (typically, 1.2 equiv) placed under a positive pressure of argon, and subjected to three evacuation/backfilling cycles. Toluene (typically, $0.25 \mathrm{M}$ ) and LiHMDS (1.0 M in THF, typically, 2.0 equiv) were sequentially added with vigorous stirring at room temperature, and the reaction mixture was stirred at room temperature for an indicated time. After the indicated time, the reaction mixture was quenched with $\mathrm{NH}_{4} \mathrm{Cl}$ (aq., $\left.1.0 \mathrm{M}, 1 \mathrm{~mL}\right)$, diluted with EtOAc $(10 \mathrm{~mL})$, the organic layer was washed with water $(1 \times 10 \mathrm{~mL})$, brine $(1 \times 10 \mathrm{~mL})$, dried and concentrated. A sample was analyzed by ${ }^{1} \mathrm{H}$ $\mathrm{NMR}\left(\mathrm{CDCl}_{3}, 500 \mathrm{MHz}\right)$ and GC-MS to obtain conversion, yield and selectivity using internal standard and comparison with authentic samples. Purification by chromatography on silica gel (EtOAc/hexanes) afforded the title product.

Representative Procedure for Transamidation of Amides. Gram Scale. Ph/Me Amide. An oven-dried vial equipped with a stir bar was charged with $\mathrm{N}$-methyl-N-phenylbenzamide (1.00 g, $4.73 \mathrm{mmol}, 1.0$ equiv), $p$-toluidine (1.01 g, $9.46 \mathrm{mmol}, 2.0$ equiv), placed under a positive pressure of argon, and subjected to three evacuation/backfilling cycles. Toluene $(18.9 \mathrm{~mL})$ and LiHMDS (1.0 M in THF, $14.2 \mathrm{~mL}, 3.0$ equiv) were sequentially added with vigorous stirring at 
room temperature and the reaction mixture was stirred for $15 \mathrm{~h}$ at room temperature. After the indicated time, the reaction mixture was quenched with $\mathrm{NH}_{4} \mathrm{Cl}$ (aq., $1.0 \mathrm{M}, 40 \mathrm{~mL}$ ), extracted with EtOAc ( 3 x $50 \mathrm{~mL})$, the organic layers were combined, washed with $\mathrm{HCl}$ (aq., $2.0 \mathrm{M}, 2$ x 50 $\mathrm{mL})$, water $(1 \times 50 \mathrm{~mL})$, brine $(1 \times 50 \mathrm{~mL})$, dried over $\mathrm{Na}_{2} \mathrm{SO}_{4}$ and concentrated. Purification by chromatography on silica gel (EtOAc/hexanes) afforded the title product. Yield 97\% (0.970 g). White solid. Characterization data are included in the section below.

Representative Procedure for Amidation of Esters. Gram Scale. An oven-dried vial equipped with a stir bar was charged with methyl benzoate $(1.00 \mathrm{~g}, 7.35 \mathrm{mmol}, 1.0$ equiv), aniline $(0.821$ g, $8.81 \mathrm{mmol}, 1.2$ equiv), placed under a positive pressure of argon, and subjected to three evacuation/backfilling cycles. Toluene (29.4 mL) and LiHMDS (1.0 M in THF, $14.7 \mathrm{~mL}, 2.0$ equiv) were sequentially added with vigorous stirring at room temperature and the reaction mixture was stirred for $15 \mathrm{~h}$ at room temperature. After the indicated time, the reaction mixture was quenched with $\mathrm{NH}_{4} \mathrm{Cl}$ (aq., $1.0 \mathrm{M}, 40 \mathrm{~mL}$ ), extracted with EtOAc (3 x $50 \mathrm{~mL}$ ), the organic layers were combined, washed with $\mathrm{HCl}$ (aq., $2.0 \mathrm{M}, 2$ x $50 \mathrm{~mL})$, water $(1 \mathrm{x} 50 \mathrm{~mL})$, brine $(1 \mathrm{x}$ $50 \mathrm{~mL}$ ), dried over $\mathrm{Na}_{2} \mathrm{SO}_{4}$ and concentrated. Purification by chromatography on silica gel (EtOAc/hexanes) afforded the title product. Yield 93\% (1.35 g). White solid. Characterization data are included in the section below. 


\section{Mechanistic Studies Referred to from the Main Manuscript}

Additional studies were performed to gain insight into the reaction mechanism and investigate factors involved in controlling the transition-metal-free transamidation protocol.

(1) Intermolecular competition experiments revealed that the electronic nature of aniline significantly affects the reactivity of amides, however, the electronic nature of aniline does not affect the reactivity of esters (Scheme S5-A).

Intermolecular competition: anilines<smiles>COc1ccc(N)cc1</smiles><smiles>COc1ccc(NC(=O)c2ccccc2)cc1</smiles>

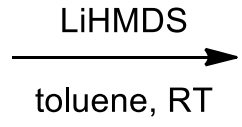

$3 b: 3 a d>20: 1$

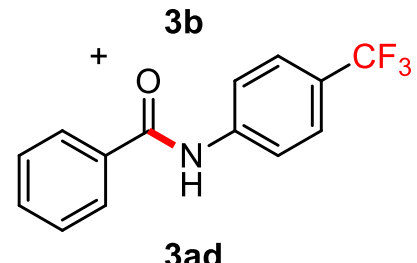<smiles>COC(=O)c1ccccc1</smiles><smiles>COc1ccc(NC(=O)c2ccccc2)cc1</smiles>

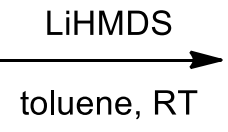

$3 b: 3 a d=1.1: 1.0$<smiles>O=C(Nc1ccc(C(F)(F)F)cc1)c1ccccc1</smiles>

Scheme S5-A. Intermolecular Competition Experiments: Anilines. Conditions: 1 or 4 (1.0 equiv), aniline (1.0 equiv each), LiHMDS (2.0 equiv), toluene (0.25 M), $23^{\circ} \mathrm{C}, 15 \mathrm{~h}$.

(2) Further intermolecular competition experiments revealed that electron-deficient amides and esters are inherently more reactive than their electron-rich counterparts (Scheme S5-B). 


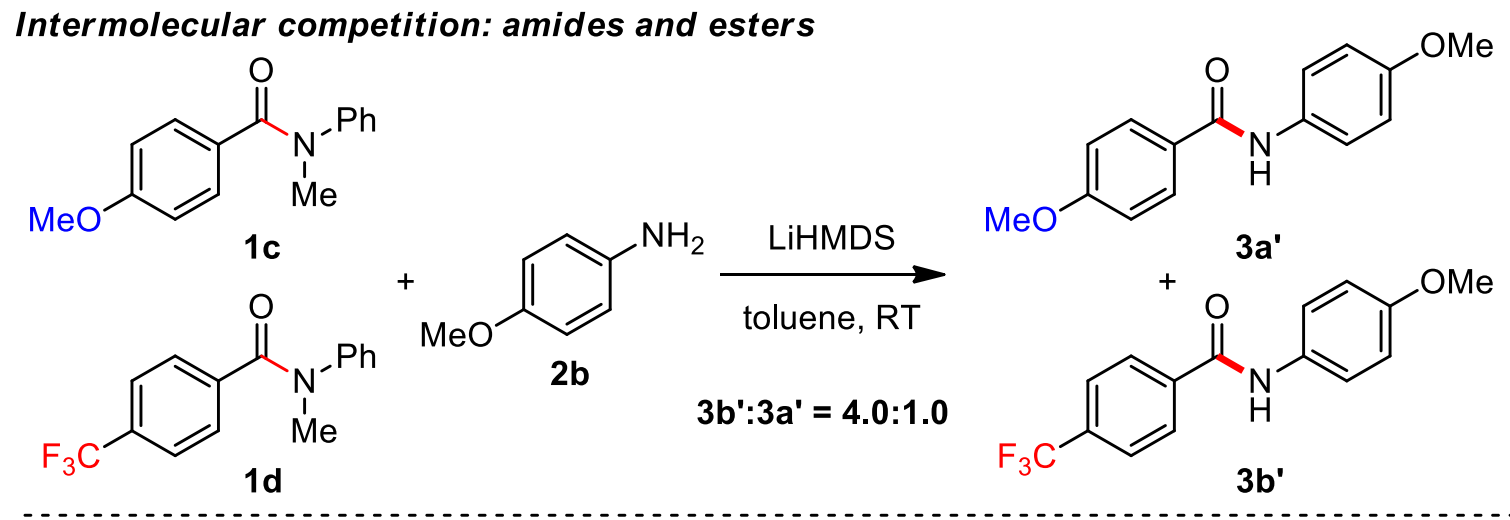<smiles>COC(=O)c1ccc(C(=O)OC)cc1</smiles><smiles>COc1ccc(C(=O)Nc2ccccc2)cc1</smiles>

Scheme S5-B. Intermolecular Competition Experiments: Amides and Esters. Conditions: 1 or 4 (1.0 equiv each), aniline (1.0 equiv), LiHMDS (2.0 equiv), toluene (0.25 M), $23{ }^{\circ} \mathrm{C}, 15 \mathrm{~h}$.

(3) Further competition experiments established a much higher order of reactivity of esters than amides (Scheme S5-C), as expected from the Nlp/Olp to $\pi^{*} \mathrm{C}=\mathrm{O}$ delocalization, and provided the basis for the extensive studies on the selectivity within different amide and ester electrophiles that is not feasible using existing methods (Charts 1-3).

Intermolecular competition: amides vs. esters<smiles>COC(=O)c1ccc(C)cc1</smiles><smiles>COc1ccc(N)cc1</smiles><smiles>COc1ccc(NC(=O)c2ccccc2)cc1</smiles><smiles>COc1ccc(NC(=O)c2ccc(C)cc2)cc1</smiles>

Scheme S5-C. Intermolecular Competition Experiments: Amides vs. Esters. Conditions: 1 and 4 (1.0 equiv each), aniline (1.0 equiv), LiHMDS (2.0 equiv), toluene (0.25 M), $23{ }^{\circ} \mathrm{C}, 15 \mathrm{~h}$. 
(4) Finally, intramolecular competition experiments revealed that anilines are inherently more reactive than aliphatic amines for both amide and ester substrates (Scheme S5-D).

Intermolecular competition: amines
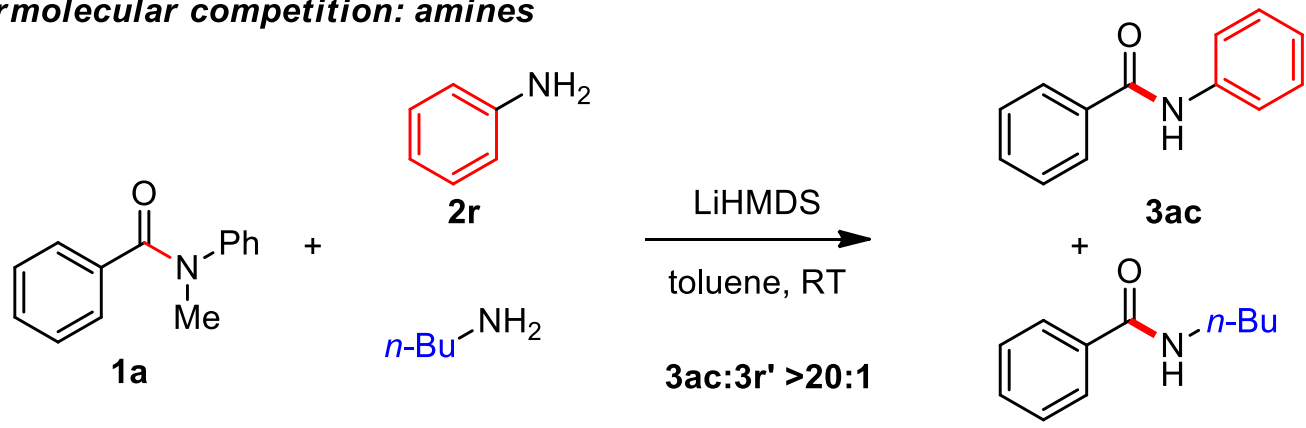

$2 y$ $3 r^{\prime}$

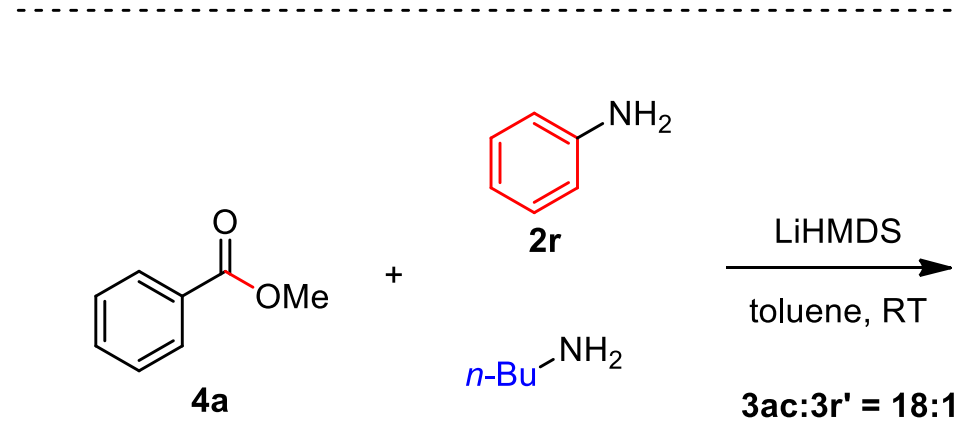

$2 y$<smiles>O=C(Nc1ccccc1)c1ccccc1</smiles><smiles>CCCCNC(=O)c1ccccc1</smiles>

$3 r^{\prime}$

Scheme S5-D. Intermolecular Competition Experiments: Amines. Conditions: 1 or 4 (1.0 equiv), amine (1.0 equiv each), LiHMDS (2.0 equiv), toluene (0.25 M), $23{ }^{\circ} \mathrm{C}, 15 \mathrm{~h}$.

Overall, the intermolecular competition studies support the key role of amine deprotonation (cf. direct nucleophilic addition) and suggest a common manifold for transition-metal-free functionalization of amides and esters by controlling Nlp/Olp to $\pi^{*} \mathrm{C}=\mathrm{O}$ delocalization. The experimental findings are further supported by extensive DFT studies on the mechanism of the direct transamidation and amidation reactions of amides and esters (vide supra). 


\section{Selected Optimization Studies Referred to from the Main Manuscript (Table S3)}

Table S3. Selected Optimization Studies in Transamidation of Amide 1a. ${ }^{a}$

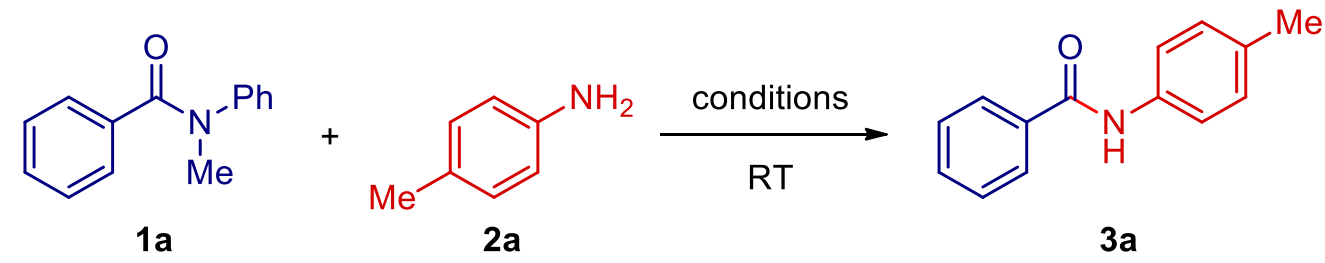

\begin{tabular}{|c|c|c|c|c|c|}
\hline entry & base & solvent & $\begin{array}{l}\text { temp } \\
\left({ }^{\circ} \mathrm{C}\right)\end{array}$ & $\begin{array}{c}\mathbf{2 a} \\
\text { (equiv) }\end{array}$ & $\begin{array}{c}\text { yield }^{b} \\
(\%)\end{array}$ \\
\hline $1^{c}$ & LiHMDS & toluene & 23 & 1.0 & 78 \\
\hline $2^{d}$ & LiHMDS & toluene & 23 & 1.5 & 85 \\
\hline 3 & LiHMDS & toluene & 23 & 2.0 & $>95$ \\
\hline $4^{c}$ & LiHMDS & toluene & 23 & 2.0 & 84 \\
\hline 5 & LiHMDS & - & 23 & 2.0 & 83 \\
\hline 6 & LiHMDS & $\mathrm{THF}$ & 23 & 2.0 & 87 \\
\hline 7 & LiHMDS & $\mathrm{CH}_{3} \mathrm{CN}$ & 23 & 2.0 & $<5$ \\
\hline 8 & NaHMDS & toluene & 23 & 2.0 & 86 \\
\hline 9 & KHMDS & toluene & 23 & 2.0 & 88 \\
\hline 10 & $n$-BuLi & toluene & 23 & 2.0 & $<5$ \\
\hline
\end{tabular}




\section{Charton and Taft Correlation Studies}

Table S4. Steric Influence on the Relative Rates in the Transition-Metal-Free Direct Amidation of Alkyl Esters - Charton and Taft Correlation Study. ${ }^{a}$
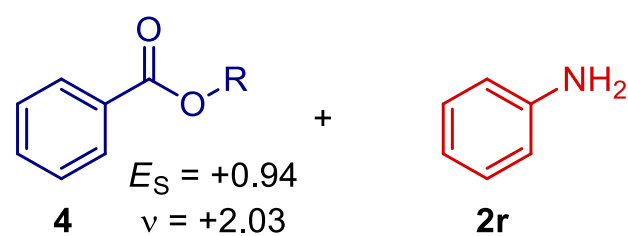

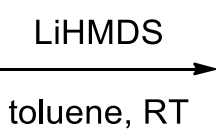

toluene, RT<smiles>O=C(Nc1ccccc1)c1ccccc1</smiles>

3ac

\begin{tabular}{ccccc}
\hline entry & $\mathrm{R}$ & $k_{\mathrm{X}} / k_{\mathrm{Me}}{ }^{a}$ & $\begin{array}{c}\text { Taft } \mathrm{E}_{S} \\
\text { parameter }\end{array}$ & $\begin{array}{c}\text { Charton } v \\
\text { parameter }\end{array}$ \\
\hline 1 & $\mathrm{Me}$ & 1.00 & 0 & -0.52 \\
2 & $\mathrm{Et}$ & 0.89 & -0.07 & -0.56 \\
3 & $i-\mathrm{Pr}$ & 0.29 & -0.47 & -0.76 \\
4 & $t$ - $\mathrm{Bu}$ & 0.04 & -1.54 & -1.24
\end{tabular}

${ }^{a}$ Relative reactivity values determined from product distribution by ${ }^{1} \mathrm{H}$ NMR and/or GC/MS of crude reaction mixtures. The correlation using Charton steric parameter $v\left(\mathrm{Y}=2.027 \mathrm{X}+1.05, \mathrm{R}^{2}=0.996\right)$ can be compared with the correlation obtained using Taft steric parameter $\mathrm{E}_{S}\left(\mathrm{Y}=0.941-0.021, \mathrm{R}^{2}=0.994\right)$, indicating involvement of steric effects in the transition state of the reaction.

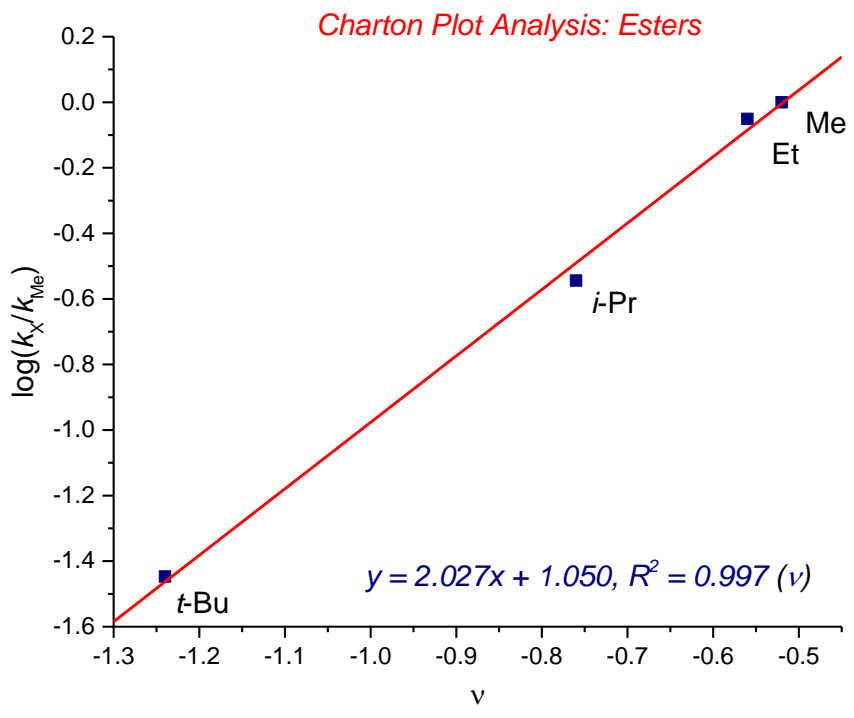

Figure S1. Steric Influence on the Relative Rates in the Transition-Metal-Free Direct Amidation of Alkyl Esters - Charton Correlation Study. 


\section{Kinetic Profile of Transamidation}

General Procedure. An oven-dried vial equipped with a stir bar was charged with an amide substrate (neat, 1.0 equiv), amine (2.0 equiv) placed under a positive pressure of argon, and subjected to three evacuation/backfilling cycles. Toluene (0.25 M) and LiHMDS (1.0 M in THF, 2.0 equiv) were sequentially added with vigorous stirring at room temperature, and the reaction mixture was stirred at room temperature for an indicated time. After the indicated time, the reaction mixture was quenched with $\mathrm{NH}_{4} \mathrm{Cl}$ (aq., $\left.1.0 \mathrm{M}, 1 \mathrm{~mL}\right)$, diluted with EtOAc $(10 \mathrm{~mL})$, the organic layer was washed with water $(1 \times 10 \mathrm{~mL})$, brine $(1 \times 10 \mathrm{~mL})$, dried and concentrated. A sample was analyzed by ${ }^{1} \mathrm{H} \mathrm{NMR}\left(\mathrm{CDCl}_{3}, 500 \mathrm{MHz}\right)$ and $\mathrm{GC}-\mathrm{MS}$ to obtain conversion, yield and selectivity using internal standard and comparison with authentic samples.<smiles>CN(C(=O)c1ccccc1)c1ccccc1</smiles>

$1 \mathrm{a}$<smiles>Cc1ccc(N)cc1</smiles>

$2 a$

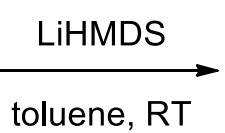
toluene, RT<smiles>Cc1ccc(NC(=O)c2ccccc2)cc1</smiles>

$3 a$

Scheme S6. Determination of Kinetic Profile in Transamidation of 1a. Conditions: 1 (1.0 equiv), amine (2.0 equiv), LiHMDS (2.0 equiv), toluene (0.25 M), $23{ }^{\circ} \mathrm{C}, 0-300 \mathrm{~s}$.

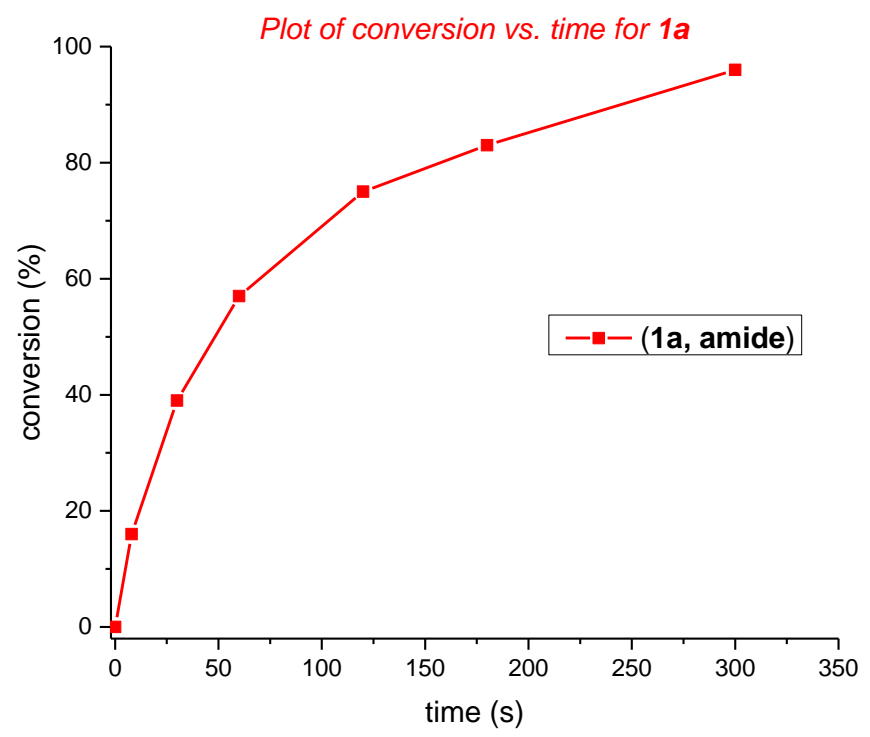

Figure S2. Kinetic Profile in Transamidation of 1a. 


\section{Full Chart 2 Referred to from the Main Manuscript}

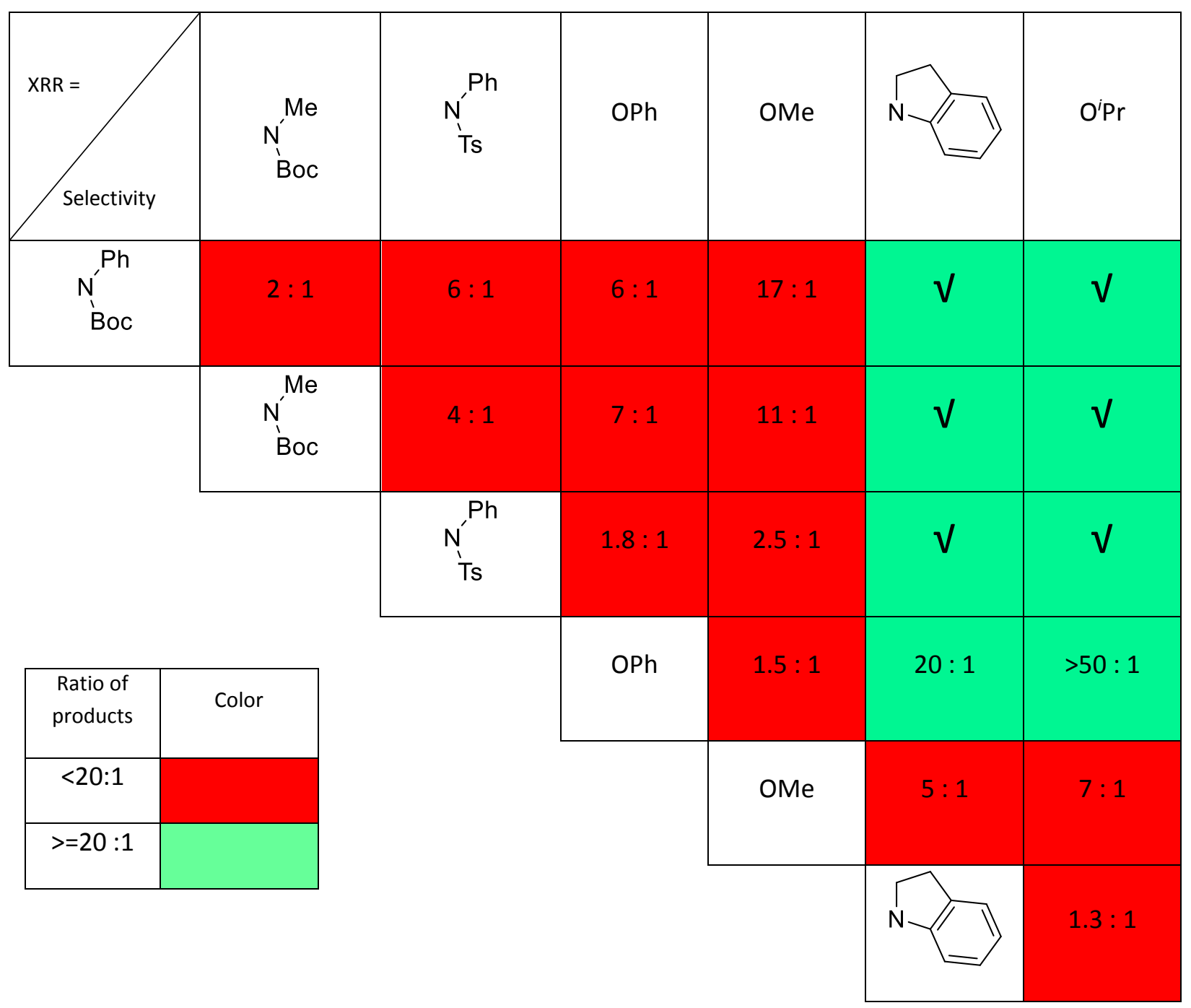

Figure S3. Selectivity in transition-metal-free transamidation of activated secondary amides vs. unactivated esters. Note: for all other entries denoted in green in Charts 1-3 in the main manuscript, $>50: 1$ selectivity favoring the more reactive electrophile has been observed. 


\section{Representative Competition Experiments used to Establish the Order of Reactivity of Amide and Ester Electrophiles}

General Procedure. An oven-dried vial equipped with a stir bar was charged with two substrates (neat, 1.0 equiv), amine (1.0 equiv) placed under a positive pressure of argon, and subjected to three evacuation/backfilling cycles. Toluene (0.25 M) and LiHMDS (1.0 M in THF, 2.0 equiv) were sequentially added with vigorous stirring at room temperature, and the reaction mixture was stirred at room temperature for an indicated time. After the indicated time, the reaction mixture was quenched with $\mathrm{NH}_{4} \mathrm{Cl}$ (aq., $\left.1.0 \mathrm{M}, 1 \mathrm{~mL}\right)$, diluted with EtOAc $(10 \mathrm{~mL})$, the organic layer was washed with water $(1 \times 10 \mathrm{~mL})$, brine $(1 \times 10 \mathrm{~mL})$, dried and concentrated. A sample was analyzed by ${ }^{1} \mathrm{H} \mathrm{NMR}\left(\mathrm{CDCl}_{3}, 500 \mathrm{MHz}\right)$ and GC-MS to obtain conversion, yield and selectivity using internal standard and comparison with authentic samples.<smiles>[R]c1ccc(C(=O)N([R])[X]([R])([R])C(=O)c2ccc([R])cc2)cc1</smiles><smiles>[R]c1ccc(N)cc1</smiles><smiles>[R]c1ccc(NC(=O)c2ccc(NC(=O)c3ccc([R])cc3)cc2)cc1</smiles>

Scheme S7. Intermolecular Competition Experiments. Conditions: 1-A and 1-B (1.0 equiv each), aniline (1.0 equiv), LiHMDS (2.0 equiv), toluene (0.25 M), $23{ }^{\circ} \mathrm{C}, 15 \mathrm{~h}$. 
Table S5. Representative Competition Experiments used to Establish the Relative Order of Reactivity of Amide and Ester Electrophiles. ${ }^{a}$

\begin{tabular}{|c|c|c|c|c|}
\hline entry & 1-A & 1-B & 2 & 3-A:3-B ${ }^{b}$ \\
\hline 1 & & & & $1.08: 1$ \\
\hline 2 & & & & $>50: 1$ \\
\hline 3 & & & & $>50: 1$ \\
\hline 4 & & & & $6.5: 1$ \\
\hline 5 & & & & $>50: 1$ \\
\hline 6 & & & & $2.0: 1$ \\
\hline 7 & & & & $>50: 1$ \\
\hline 8 & & & & $>50: 1$ \\
\hline
\end{tabular}


9<smiles>Cc1ccc(C(=O)N(C)C)cc1</smiles>

10<smiles>Cc1ccc(C(=O)N(C)C)cc1</smiles>

11<smiles>Cc1ccc(C(=O)N(C)C)cc1</smiles>

12<smiles>Cc1ccc(C(=O)N(C)C)cc1</smiles>

13<smiles>Cc1ccc(C(=O)N2CCCC2)cc1</smiles>

14<smiles>Cc1ccc(C(=O)N2CCCCC2)cc1</smiles>

15<smiles>CC(C)(C)OC(=O)N(C(=O)c1ccccc1)c1ccccc1</smiles>

16<smiles>CN(C(=O)c1ccccc1)C(=O)c1ccccc1</smiles>

17<smiles>CN(Cc1ccccc1)C(=O)c1ccccc1</smiles><smiles>O=C(c1ccccc1)N1CCCC1</smiles><smiles>O=C(c1ccccc1)N1CCCCC1</smiles><smiles>CCN(CC)C(=O)c1ccccc1</smiles><smiles>O=C(c1ccccc1)N1CCCCC1</smiles><smiles>CCN(CC)C(=O)c1ccccc1</smiles><smiles>COC(=O)c1ccc(C)cc1</smiles><smiles>COC(=O)c1ccc(C)cc1</smiles><smiles>COC(=O)c1ccc(C)cc1</smiles><smiles>Cc1ccc(N)cc1</smiles><smiles>COc1ccc(N)cc1</smiles>

2.0:1<smiles>COc1ccc(N)cc1</smiles><smiles>Cc1ccc(N)cc1</smiles><smiles>Cc1ccc(N)cc1</smiles>

$>50: 1^{c}$

17:1<smiles>COc1ccc(N)cc1</smiles>

11:1<smiles>COc1ccc(N)cc1</smiles>

2.64:1 
18<smiles>Cc1ccc(C(=O)c2ccccc2)cc1</smiles>

19<smiles>Cc1ccc(C(=O)c2ccccc2)cc1</smiles>

20<smiles>Cc1ccc(C(=O)c2ccccc2)cc1</smiles>

21<smiles>COC(=O)c1ccc(C)cc1</smiles>

22<smiles>COC(=O)c1ccc(C)cc1</smiles>

23<smiles>O=C(c1ccccc1)N1CCc2ccccc21</smiles>

24<smiles>Cc1ccc(C(=O)N(c2ccccc2)c2ccccc2)cc1</smiles>

25<smiles>COC(=O)c1ccccc1</smiles><smiles>Nc1ccccc1</smiles><smiles>[AlH2]</smiles><smiles>O=C(c1ccccc1)N1CCc2ccccc21</smiles><smiles>CCCOC(=O)c1ccccc1</smiles><smiles>Cc1ccc(N)cc1</smiles>

$>50: 1$

8:1<smiles>Cc1ccc(N)cc1</smiles><smiles>Cc1ccc(N)cc1</smiles><smiles>CCCOC(=O)c1ccc(C)cc1</smiles><smiles>Cc1ccc(N)cc1</smiles><smiles>COc1ccc(N)cc1</smiles>
$>50: 1$<smiles>Cc1ccc(C(=O)N(C)c2ccccc2)cc1</smiles>

10:1 
26<smiles>O=C(c1ccccc1)N([As])c1ccccc1</smiles>

27<smiles>Cc1ccc(C(=O)c2ccccc2)cc1</smiles>

28<smiles>COC(=O)c1ccc(C)cc1</smiles>

29<smiles>O=C(c1ccccc1)N1CCc2ccccc21</smiles>

30<smiles>O=C(c1ccccc1)N1CCc2ccccc21</smiles>

31<smiles>Cc1ccc(C(=O)OC(C)C)cc1</smiles>

32<smiles>Cc1ccc(C(=O)OC(C)C)cc1</smiles>

33<smiles>Cc1ccc(C(=O)N(C)c2ccccc2)cc1</smiles><smiles>CN(C(=O)c1ccccc1)c1ccccc1</smiles><smiles>CN(C(=O)c1ccccc1)c1ccccc1</smiles><smiles>Cc1ccc(C(=O)N(c2ccccc2)N(C)c2ccccc2)cc1</smiles><smiles>Cc1ccc(C(=O)N(C)c2ccccc2)cc1</smiles><smiles>CN(C(=O)c1ccccc1)c1ccccc1</smiles><smiles>Cc1ccc(C(=O)N2CCC2)cc1</smiles><smiles>O=C(c1ccccc1)N1CCOCC1</smiles><smiles>COc1ccc(N)cc1</smiles>

$>50: 1$

$>50: 1$<smiles>Cc1ccc(N)cc1</smiles><smiles>COc1ccc(N)cc1</smiles>

$>50: 1$

$>50: 1$

$>50: 1$<smiles>COc1ccc(N)cc1</smiles><smiles>COc1ccc(N)cc1</smiles>

$>50: 1$

$>50: 1$

${ }^{a}$ Conditions: 1-A (1.0 equiv), 1-B (1.0 equiv), 2 (1.0 equiv), LiHMDS (1.0 M in THF, 2.0 equiv), toluene (0.25 M), $23{ }^{\circ} \mathrm{C}, 15 \mathrm{~h}$. ${ }^{b}$ Relative reactivity values determined from product distribution by ${ }^{1} \mathrm{H}$ NMR and/or GC/MS of crude reaction mixtures. ${ }^{c} 60{ }^{\circ} \mathrm{C}$. 


\section{Characterization Data of Amide and Ester Starting Materials}

All amide and ester starting materials used in this study were prepared by procedures reported in the literature. 1a, ${ }^{12} \mathbf{1 b},{ }^{13} \mathbf{1 c},{ }^{13} \mathbf{1 d},{ }^{14} \mathbf{1 e},{ }^{15} \mathbf{1 f},{ }^{16} \mathbf{1 h},{ }^{13} \mathbf{1 i},{ }^{17} \mathbf{1 j},{ }^{18} \mathbf{1 k},{ }^{19} \mathbf{1 l},{ }^{20} \mathbf{1 m},{ }^{21} \mathbf{1 n},{ }^{13} \mathbf{1 o},{ }^{22} \mathbf{1 p},{ }^{23}$ $\mathbf{1 q},{ }^{12} \mathbf{1 r},{ }^{24} \mathbf{1 s},{ }^{12} \mathbf{1 t},{ }^{25} \mathbf{1 u},{ }^{25} \mathbf{1 v},{ }^{12} \mathbf{1 w},{ }^{12} \mathbf{1 x},{ }^{12} \mathbf{1 y},{ }^{12} \mathbf{1 z},{ }^{26} \mathbf{1 a a},{ }^{27} \mathbf{1 a b},{ }^{28} \mathbf{1 a c}^{29} \mathbf{1 a d},{ }^{30} \mathbf{1 a e},{ }^{30} \mathbf{1 a h},{ }^{31}$ $1 \mathbf{a i},{ }^{26} 4 \mathbf{a},{ }^{32} 4 \mathbf{b},{ }^{32} 4 \mathbf{c},{ }^{32} 4 \mathbf{d},{ }^{32} 4 \mathbf{e},{ }^{33} 4 \mathbf{f},{ }^{34} 4 \mathbf{g},{ }^{35} 4 \mathbf{h},{ }^{32} 4 \mathbf{j},{ }^{36} 4 \mathbf{k},{ }^{32} 4 \mathbf{l},{ }^{37} 4 \mathbf{m},{ }^{34} 4 \mathbf{n},{ }^{38} 4 \mathbf{o},{ }^{38} 4 \mathbf{p},{ }^{39} 4 \mathbf{q},{ }^{40} 4 \mathbf{s}^{32}$ have been previously reported. Spectroscopic data match those reported in the literature.

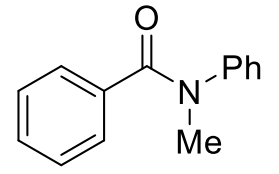

$N$-Methyl- $N$-phenylbenzamide (1a). White solid. ${ }^{1} \mathrm{H}$ NMR (500 MHz, $\left.\mathrm{CDCl}_{3}\right) \delta 7.31(\mathrm{~d}, J=7.8 \mathrm{~Hz}, 2 \mathrm{H}), 7.25-7.22(\mathrm{~m}, 3 \mathrm{H}), 7.19-7.15(\mathrm{~m}, 3 \mathrm{H})$, $7.06(\mathrm{~d}, J=7.8 \mathrm{~Hz}, 2 \mathrm{H}), 3.52(\mathrm{~s}, 3 \mathrm{H}) .{ }^{13} \mathrm{C} \mathrm{NMR}\left(125 \mathrm{MHz}, \mathrm{CDCl}_{3}\right) \delta 170.7$, $144.9,135.9,129.6,129.2,128.7,127.7,126.9,126.5,38.4$.<smiles>Cc1ccc(C(=O)N(C)c2ccccc2)cc1</smiles>

$\boldsymbol{N}, 4-D i m e t h y l-N$-phenylbenzamide (1b). White solid. ${ }^{1} \mathrm{H}$ NMR (500 $\left.\mathrm{MHz}, \mathrm{CDCl}_{3}\right) \delta$ 7.28-7.19 (m, $\left.4 \mathrm{H}\right), 7.16(\mathrm{t}, J=7.4 \mathrm{~Hz}, 1 \mathrm{H}), 7.06(\mathrm{~d}, J=$ $7.7 \mathrm{~Hz}, 2 \mathrm{H}), 6.98(\mathrm{~d}, J=7.9 \mathrm{~Hz}, 2 \mathrm{H}), 3.51$ (s, $3 \mathrm{H}), 2.27$ (s, $3 \mathrm{H}) .{ }^{13} \mathrm{C}$ NMR $\left(125 \mathrm{MHz}, \mathrm{CDCl}_{3}\right) \delta 170.7,145.2,139.8,133.0,129.1,128.9,128.3,126.9,126.3,38.5$, 21.3.<smiles>COc1ccc(C(=O)N(C)c2ccccc2)cc1</smiles>
4-Methoxy- $N$-methyl- $N$-phenylbenzamide (1c). White solid. ${ }^{1} \mathrm{H}$ NMR $\left(500 \mathrm{MHz}, \mathrm{CDCl}_{3}\right) \delta$ 7.31-7.23 (m, $\left.4 \mathrm{H}\right), 7.16(\mathrm{t}, J=7.4 \mathrm{~Hz}, 1 \mathrm{H}), 7.06$ $(\mathrm{d}, J=8.5 \mathrm{~Hz}, 2 \mathrm{H}), 6.68(\mathrm{~d}, J=8.9 \mathrm{~Hz}, 2 \mathrm{H}), 3.75(\mathrm{~s}, 3 \mathrm{H}), 3.50$ (s, $3 \mathrm{H})$. ${ }^{13} \mathrm{C} \mathrm{NMR}\left(125 \mathrm{MHz}, \mathrm{CDCl}_{3}\right) \delta 170.3,160.6,145.5,130.9,129.2,128.8,126.9,126.3,113.0$, $55.2,38.6$.<smiles>CN(C(=O)c1ccc(C(F)(F)F)cc1)c1ccccc1</smiles>

$N$-Methyl- $N$-phenyl-4-(trifluoromethyl)benzamide (1d). White solid. ${ }^{1} \mathrm{H}$ NMR (500 MHz, $\left.\mathrm{CDCl}_{3}\right) \delta 7.44(\mathrm{q}, J=8.4 \mathrm{~Hz}, 4 \mathrm{H}), 7.26(\mathrm{~d}, J=7.9$ $\mathrm{Hz}, 2 \mathrm{H}), 7.20$ (t, $J=7.4 \mathrm{~Hz}, 1 \mathrm{H}), 7.05$ (d, $J=7.6 \mathrm{~Hz}, 2 \mathrm{H}), 3.54$ (s, $3 \mathrm{H})$. ${ }^{13} \mathrm{C} \mathrm{NMR}\left(125 \mathrm{MHz}, \mathrm{CDCl}_{3}\right) \delta 169.2,144.3,139.5,131.3\left(\mathrm{q}, J^{F}=32.6 \mathrm{~Hz}\right), 129.4,129.0,127.1$, $126.9,124.8\left(\mathrm{q}, J^{F}=7.3,3.5 \mathrm{~Hz}\right), 122.6,38.4$. 


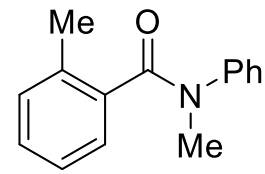

$\boldsymbol{N}$,2-Dimethyl- $N$-phenylbenzamide (1e). White solid. ${ }^{1} \mathrm{H}$ NMR (500 MHz, $\left.\mathrm{CDCl}_{3}\right) \delta 7.27-6.81(\mathrm{~m}, 9 \mathrm{H}), 3.50(\mathrm{~d}, J=15.2 \mathrm{~Hz}, 3 \mathrm{H}), 2.35(\mathrm{~s}, 3 \mathrm{H}) .{ }^{13} \mathrm{C}$ NMR (125 MHz, $\left.\mathrm{CDCl}_{3}\right) \delta 171.2,143.9,136.6,134.7,130.2,128.9,128.7$, 127.5, 126.6, 126.5, 125.1, 37.2, 19.5.<smiles>CN(C(=O)c1cccc(Br)c1)c1ccccc1</smiles>

3-Bromo- $\boldsymbol{N}$-methyl- $\boldsymbol{N}$-phenylbenzamide (1f). Oil. ${ }^{1} \mathrm{H}$ NMR $(500 \mathrm{MHz}$, $\left.\mathrm{CDCl}_{3}\right) \delta 7.52(\mathrm{~s}, 1 \mathrm{H}), 7.38(\mathrm{~d}, J=8.0 \mathrm{~Hz}, 1 \mathrm{H}), 7.28(\mathrm{dd}, J=9.0,4.6 \mathrm{~Hz}$, $2 \mathrm{H}), 7.18$ (dd, $J=19.8,7.6 \mathrm{~Hz}, 2 \mathrm{H}), 7.08-6.99(\mathrm{~m}, 3 \mathrm{H}), 3.51(\mathrm{~s}, 3 \mathrm{H}) .{ }^{13} \mathrm{C}$ NMR $\left(125 \mathrm{MHz}, \mathrm{CDCl}_{3}\right) \delta 168.9,144.4,137.9,132.6,131.9,129.4,129.2,127.2,126.9,121.9$, 38.4 .<smiles>CN(C(=O)c1ccc(F)c(F)c1)c1ccccc1</smiles>
3,4-Difluoro- $N$-methyl- $N$-phenylbenzamide (1g). New compound. Oil. ${ }^{1} \mathrm{H}$ NMR (500 MHz, $\left.\mathrm{CDCl}_{3}\right) \delta 7.28(\mathrm{t}, J=7.6 \mathrm{~Hz}, 2 \mathrm{H}), 7.24-7.15(\mathrm{~m}, 2 \mathrm{H})$, 7.08-7.01 (m, $3 \mathrm{H}), 6.95(\mathrm{dd}, J=17.8,8.5 \mathrm{~Hz}, 1 \mathrm{H}), 3.50(\mathrm{~s}, 3 \mathrm{H}) .{ }^{13} \mathrm{C} \mathrm{NMR}$ $\left(125 \mathrm{MHz} \mathrm{CDCl}_{3}\right) \delta 168.3,151.0\left(\mathrm{dd}, J^{F}=252.5,12.7 \mathrm{~Hz}\right), 149.6(\mathrm{dd}, J=249.5,12.9 \mathrm{~Hz})$, 144.5, $132.7\left(J^{F}=7.0,4.1 \mathrm{~Hz}\right), 129.5,127.0,126.8,125.5\left(J^{F}=6.8,3.8 \mathrm{~Hz}\right), 118.4\left(J^{F}=18.5 \mathrm{~Hz}\right)$, $116.7\left(J^{F}=17.6 \mathrm{~Hz}\right), 38.6 .{ }^{19} \mathrm{~F}$ NMR $\left(471 \mathrm{MHz}, \mathrm{CDCl}_{3}\right) \delta-134.75(\mathrm{~m}),-134.83(\mathrm{~s}),-137.40(\mathrm{~m})$. HRMS calcd for $\mathrm{C}_{14} \mathrm{H}_{11} \mathrm{NOF}_{2} \mathrm{Na}(\mathrm{M}+\mathrm{Na}) 270.0701$, found 270.0680.

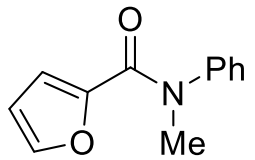

$N$-Methyl- $N$-phenylfuran-2-carboxamide (1h). White solid. ${ }^{1} \mathrm{H}$ NMR (500 $\left.\mathrm{MHz} \mathrm{CDCl}_{3}\right) \delta 7.42(\mathrm{t}, J=7.5 \mathrm{~Hz}, 2 \mathrm{H}), 7.37(\mathrm{t}, J=7.2 \mathrm{~Hz}, 1 \mathrm{H}), 7.32(\mathrm{~s}, 1 \mathrm{H})$, $7.22(\mathrm{~d}, J=7.8 \mathrm{~Hz}, 2 \mathrm{H}), 6.21(\mathrm{~d}, J=3.1 \mathrm{~Hz}, 1 \mathrm{H}), 5.85(\mathrm{~d}, J=3.1 \mathrm{~Hz}, 1 \mathrm{H})$, 3.45 (s, $3 \mathrm{H}) .{ }^{13} \mathrm{C}$ NMR $\left(125 \mathrm{MHz}, \mathrm{CDCl}_{3}\right) \delta 159.4,147.1,144.3,144.2,129.6,127.8,127.4$, $116.3,110.9,38.5$.

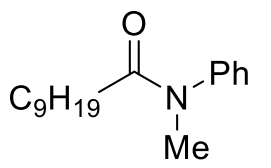

$N$-Methyl- $N$-phenyldecanamide (1i). Oil. ${ }^{1} \mathrm{H}$ NMR $\left(500 \mathrm{MHz}, \mathrm{CDCl}_{3}\right) \delta 7.42$ $(\mathrm{t}, J=7.6 \mathrm{~Hz}, 2 \mathrm{H}), 7.34(\mathrm{t}, J=7.3 \mathrm{~Hz}, 1 \mathrm{H}), 7.18(\mathrm{~d}, J=7.4 \mathrm{~Hz}, 2 \mathrm{H}), 3.27(\mathrm{~s}$, $3 \mathrm{H}), 2.07$ (t, $J=7.3 \mathrm{~Hz}, 2 \mathrm{H}), 1.56(\mathrm{~s}, 2 \mathrm{H}), 1.20$ (d, $J=12.7 \mathrm{~Hz}, 12 \mathrm{H}), 0.86$ $(\mathrm{d}, J=7.2 \mathrm{~Hz}, 3 \mathrm{H}) .{ }^{13} \mathrm{C} \mathrm{NMR}\left(125 \mathrm{MHz}, \mathrm{CDCl}_{3}\right) \delta 173.3,144.4,129.7,127.6,127.3,37.3$, $34.1,31.9,29.4,29.3,29.3,29.2,25.6,22.7,14.1$. 
<smiles>CC(C)C(=O)N(C)c1ccccc1</smiles>

$N$-Methyl- $N$-phenylisobutyramide (1j). White solid. ${ }^{1} \mathrm{H}$ NMR (500 MHz, $\left.\mathrm{CDCl}_{3}\right) \delta 7.43(\mathrm{t}, J=7.6 \mathrm{~Hz}, 2 \mathrm{H}), 7.35(\mathrm{t}, J=7.2 \mathrm{~Hz}, 1 \mathrm{H}), 7.20(\mathrm{~d}, J=7.3 \mathrm{~Hz}$, $2 \mathrm{H}), 3.26(\mathrm{~s}, 3 \mathrm{H}), 1.05(\mathrm{~s}, 4 \mathrm{H}), 1.03(\mathrm{~s}, 3 \mathrm{H}) .{ }^{13} \mathrm{C} \mathrm{NMR}\left(125 \mathrm{MHz}, \mathrm{CDCl}_{3}\right) \delta$ 177.4, 144.3, 129.7, 127.7, 127.3, 37.5, 31.0, 19.7.

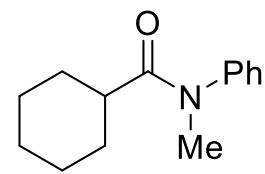

$N$-Methyl- $N$-phenylcyclohexanecarboxamide (1k). White solid. ${ }^{1} \mathrm{H}$ NMR $\left(500 \mathrm{MHz}, \mathrm{CDCl}_{3}\right) \delta 7.43(\mathrm{t}, J=7.2 \mathrm{~Hz}, 2 \mathrm{H}), 7.35(\mathrm{t}, J=7.1 \mathrm{~Hz}, 1 \mathrm{H}), 7.19(\mathrm{~d}$, $J=7.1 \mathrm{~Hz}, 2 \mathrm{H}), 3.25(\mathrm{~s}, 3 \mathrm{H}), 2.20(\mathrm{t}, J=10.4 \mathrm{~Hz}, 1 \mathrm{H}), 1.65$ (s, $4 \mathrm{H}), 1.58-$ $1.51(\mathrm{~m}, 3 \mathrm{H}), 1.19(\mathrm{~d}, J=15.2 \mathrm{~Hz}, 1 \mathrm{H}), 1.03-0.93(\mathrm{~m}, 2 \mathrm{H}) .{ }^{13} \mathrm{C} \mathrm{NMR}\left(125 \mathrm{MHz}, \mathrm{CDCl}_{3}\right) \delta$ 176.4, 144.3, 129.7, 127.6, 127.2, 41.4, 37.4, 29.5, 25.6, 25.5.

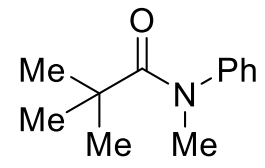

$N$-Methyl- $N$-phenylpivalamide (11). White solid. ${ }^{1} \mathrm{H}$ NMR $\left(500 \mathrm{MHz}, \mathrm{CDCl}_{3}\right)$ $\delta 7.41(\mathrm{t}, J=7.4 \mathrm{~Hz}, 2 \mathrm{H}), 7.35(\mathrm{t}, J=7.3 \mathrm{~Hz}, 1 \mathrm{H}), 7.24(\mathrm{~d}, J=7.2 \mathrm{~Hz}, 2 \mathrm{H})$, $3.24(\mathrm{~s}, 3 \mathrm{H}), 1.06(\mathrm{~s}, 9 \mathrm{H}) .{ }^{13} \mathrm{C} \mathrm{NMR}\left(125 \mathrm{MHz}, \mathrm{CDCl}_{3}\right) \delta 178.2,145.3,129.2$, $128.8,127.8,41.4,40.8,29.5$.<smiles>CCN(C(=O)c1ccccc1)c1ccccc1</smiles>

$\boldsymbol{N}$-Ethyl- $\boldsymbol{N}$-phenylbenzamide (1m). White solid. ${ }^{1} \mathrm{H}$ NMR (500 $\left.\mathrm{MHz}, \mathrm{CDCl}_{3}\right)$ $\delta 7.31(\mathrm{~d}, J=7.1 \mathrm{~Hz}, 2 \mathrm{H}), 7.27-7.21(\mathrm{~m}, 3 \mathrm{H}), 7.19-7.14(\mathrm{~m}, 3 \mathrm{H}), 7.05$ (d, $J=$ $7.5 \mathrm{~Hz}, 2 \mathrm{H}), 4.01(\mathrm{q}, J=7.1 \mathrm{~Hz}, 2 \mathrm{H}), 1.25(\mathrm{t}, J=7.1 \mathrm{~Hz}, 3 \mathrm{H}) .{ }^{13} \mathrm{C}$ NMR $(125$ $\left.\mathrm{MHz}_{\mathrm{CDCl}}\right) \delta 170.2,143.2,136.4,129.4,129.1,128.7,128.0,127.7,126.6,45.4,13.0$.<smiles>CCCN(C(=O)c1ccccc1)c1ccccc1</smiles>

$N$-Butyl- $N$-phenylbenzamide (1n). White solid. ${ }^{1} \mathrm{H}$ NMR $\left(500 \mathrm{MHz}, \mathrm{CDCl}_{3}\right)$ $\delta 7.30(\mathrm{~d}, J=7.3 \mathrm{~Hz}, 2 \mathrm{H}), 7.23(\mathrm{dt}, J=4.8,4.3 \mathrm{~Hz}, 3 \mathrm{H}), 7.16(\mathrm{dd}, J=11.2$, $7.3 \mathrm{~Hz}, 3 \mathrm{H}), 7.04$ (d, J= 7.5 Hz, $2 \mathrm{H}), 4.01-3.89$ (m, $2 \mathrm{H}), 1.67-1.61$ (m, $2 \mathrm{H})$, 1.43-1.36 (m, $2 \mathrm{H}), 0.94(\mathrm{t}, J=7.4 \mathrm{~Hz}, 3 \mathrm{H}) .{ }^{13} \mathrm{C} \mathrm{NMR}\left(125 \mathrm{MHz}, \mathrm{CDCl}_{3}\right) \delta 170.3,143.6,136.5$, 129.4, 129.1, 128.6, 127.9, 127.7, 126.5, 50.3, 29.8, 20.2, 13.9. 
<smiles>O=C(c1ccccc1)N(Cc1ccccc1)c1ccccc1</smiles>

$\boldsymbol{N}$-Benzyl- $\boldsymbol{N}$-phenylbenzamide (1o). White solid. ${ }^{1} \mathrm{H}$ NMR (500 $\left.\mathrm{MHz}, \mathrm{CDCl}_{3}\right)$ $\delta$ 7.40-7.30 (m, $6 \mathrm{H}), 7.30-7.23$ (m, $2 \mathrm{H}), 7.22-7.08$ (m, $5 \mathrm{H}), 6.94$ (d, J = 7.7 $\mathrm{Hz}, 2 \mathrm{H}), 5.16$ (s, $2 \mathrm{H}) .{ }^{13} \mathrm{C} \mathrm{NMR}\left(125 \mathrm{MHz}, \mathrm{CDCl}_{3}\right) \delta 170.6,143.6,137.6$, 136.0, 129.7, 129.0, 128.8, 128.5, 128.4, 127.8, 127.7, 127.4, 126.7, 53.9.<smiles>O=C(c1ccccc1)N(c1ccccc1)c1ccccc1</smiles>

$\boldsymbol{N}, \boldsymbol{N}$-Diphenylbenzamide (1p). White solid. ${ }^{1} \mathrm{H}$ NMR $\left(500 \mathrm{MHz}, \mathrm{CDCl}_{3}\right) \delta$ $7.48(\mathrm{~d}, J=7.5 \mathrm{~Hz}, 2 \mathrm{H}), 7.31(\mathrm{t}, J=7.6 \mathrm{~Hz}, 5 \mathrm{H}), 7.27-7.14(\mathrm{~m}, 8 \mathrm{H}) .{ }^{13} \mathrm{C}$ NMR $\left(125 \mathrm{MHz}, \mathrm{CDCl}_{3}\right) \delta 170.7,143.9,136.1,130.2,129.2,129.1,127.9$, $127.5,126.4$.<smiles>CN(Cc1ccccc1)C(=O)c1ccccc1</smiles>

$\boldsymbol{N}$-Benzyl- $\boldsymbol{N}$-methylbenzamide (1q). White solid. ${ }^{1} \mathrm{H} \mathrm{NMR}\left(500 \mathrm{MHz}, \mathrm{CDCl}_{3}\right)$ $\delta$ 7.50-7.48 (m, 2 H), 7.44-7.36 (m, 6 H), 7.32 (d, J = 5.7 Hz, 1 H), 7.20 (m, 1 $\mathrm{H}), 4.79(\mathrm{~s}, 1 \mathrm{H}), 4.53(\mathrm{~s}, 1 \mathrm{H}), 2.89(\mathrm{~s}, 3 \mathrm{H}) .{ }^{13} \mathrm{C} \mathrm{NMR}\left(125 \mathrm{MHz}, \mathrm{CDCl}_{3}\right) \delta$ $172.3,171.6,137.1,136.7,136.3,131.5,129.6,129.0,128.9,128.8,128.5,128.2$, 127.6, 127.0, $126.8,55.1,50.8,37.0,33.2$.<smiles>CN(C)C(=O)c1ccccc1</smiles>

$\boldsymbol{N}, \boldsymbol{N}$-Dimethylbenzamide (1r). White solid. ${ }^{1} \mathrm{H}$ NMR $\left(500 \mathrm{MHz}, \mathrm{CDCl}_{3}\right) \delta$ 7.39 (s, $5 \mathrm{H}), 3.10$ (s, $3 \mathrm{H}), 2.97$ (s, $3 \mathrm{H}) .{ }^{13} \mathrm{C} \mathrm{NMR}\left(125 \mathrm{MHz}, \mathrm{CDCl}_{3}\right) \delta 171.6$, $136.4,129.5,128.3,127.0,39.6,35.3$.

$\boldsymbol{N}, \boldsymbol{N}$-Diethylbenzamide (1s). White solid. ${ }^{1} \mathrm{H}$ NMR $\left(500 \mathrm{MHz}, \mathrm{CDCl}_{3}\right) \delta 7.40$<smiles>CCN(CC)C(=O)c1ccccc1</smiles>
(s, $5 \mathrm{H}), 3.57$ (s, $2 \mathrm{H}), 3.27$ (s, $2 \mathrm{H}), 1.26$ (s, $3 \mathrm{H}), 1.12$ (s, $3 \mathrm{H}) .{ }^{13} \mathrm{C}$ NMR (125 $\left.\mathrm{MHz}, \mathrm{CDCl}_{3}\right) \delta 171.3,137.3,129.1,128.4,126.3,43.2,39.2,14.2,12.9$.<smiles>CC1CN1C(=O)c1ccccc1</smiles>

(2-Methylaziridin-1-yl)(phenyl)methanone (1t). Oil. ${ }^{1} \mathrm{H}$ NMR (500 MHz, $\left.\mathrm{CDCl}_{3}\right) \delta 8.08-8.02(\mathrm{~m}, 2 \mathrm{H}), 7.57(\mathrm{td}, J=7.2,1.5 \mathrm{~Hz}, 1 \mathrm{H}), 7.48(\mathrm{t}, J=7.8$ $\mathrm{Hz}, 2 \mathrm{H}), 2.66-2.54(\mathrm{~m}, 2 \mathrm{H}), 2.17$ (d, J=3.6 Hz, $1 \mathrm{H}), 1.42$ (d, J = 5.4 Hz, 3 H). ${ }^{13} \mathrm{C} \mathrm{NMR}\left(125 \mathrm{MHz}, \mathrm{CDCl}_{3}\right) \delta 179.3,133.5,132.6,129.1,128.4,34.6,32.1,17.8$. 


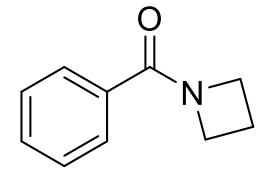

Azetidin-1-yl(phenyl)methanone (1u). Oil. ${ }^{1} \mathrm{H}$ NMR (500 $\left.\mathrm{MHz}, \mathrm{CDCl}_{3}\right) \delta$ 7.45-7.44 (d, $J=7.1 \mathrm{~Hz}, 2 \mathrm{H}), 7.26-7.23(\mathrm{t}, J=7.3 \mathrm{~Hz}, 1 \mathrm{H}), 7.21-7.18$ (t, $J=$ $7.7 \mathrm{~Hz}, 2 \mathrm{H}), 4.07-4.04$ (t, $J=7.5 \mathrm{~Hz}, 2 \mathrm{H}), 4.01-3.97$ (t, $J=7.7 \mathrm{~Hz}, 2 \mathrm{H}), 2.11$ 2.05 (m, $2 \mathrm{H}) .{ }^{13} \mathrm{C} \mathrm{NMR}\left(125 \mathrm{MHz}, \mathrm{CDCl}_{3}\right) \delta 169.9,133.1,130.7,128.2,127.6,53.2,48.7,15.9$.<smiles>O=C(c1ccccc1)N1CCCC1</smiles>

Phenyl(pyrrolidin-1-yl)methanone (1v). White solid. ${ }^{1} \mathrm{H}$ NMR $(500 \mathrm{MHz}$, $\left.\mathrm{CDCl}_{3}\right) \delta$ 7.69-7.35 (m, $\left.5 \mathrm{H}\right), 3.67-3.65$ (m, $\left.2 \mathrm{H}\right), 3.43-3.42(\mathrm{~m}, 2 \mathrm{H}), 1.97-1.96$ $(\mathrm{m}, 2 \mathrm{H}), 1.88-1.77(\mathrm{~m}, 2 \mathrm{H}) .{ }^{13} \mathrm{C} \mathrm{NMR}\left(125 \mathrm{MHz}, \mathrm{CDCl}_{3}\right) \delta$ 169.7, 137.3, 129.7, 128.2, 127.1, 49.6, 46.1, 26.4, 24.5.<smiles>O=C(c1ccccc1)N1CCCCC1</smiles>
Phenyl(piperidin-1-yl)methanone (1w). White solid. ${ }^{1} \mathrm{H}$ NMR (500 MHz, $\left.\mathrm{CDCl}_{3}\right) \delta$ 7.54-7.33 (m, $\left.5 \mathrm{H}\right), 3.52(\mathrm{~m}, 4 \mathrm{H}), 1.59(\mathrm{~m}, 6 \mathrm{H}) .{ }^{13} \mathrm{C}$ NMR $(125$ $\left.\mathrm{MHz}, \mathrm{CDCl}_{3}\right) \delta 170.3,136.5,129.3,128.4,126.8,48.8,43.1,26.6,25.6,24.6$.<smiles>O=C(c1ccccc1)N1CCOCC1</smiles>
Morpholino(phenyl)methanone (1x). White solid. ${ }^{1} \mathrm{H}$ NMR $(500 \mathrm{MHz}$, $\left.\mathrm{CDCl}_{3}\right) \delta 7.40(\mathrm{~s}, 5 \mathrm{H}), 3.84-3.36(\mathrm{~m}, 8 \mathrm{H}) .{ }^{13} \mathrm{C} \mathrm{NMR}\left(125 \mathrm{MHz}, \mathrm{CDCl}_{3}\right) \delta$ $170.3,135.1,129.7,128.4,126.9,66.7$.<smiles>CC(C)(C)OC(=O)N1CCN(C(=O)c2ccccc2)CC1</smiles>

tert-Butyl 4-benzoylpiperazine-1-carboxylate (1y). White solid. ${ }^{1} \mathrm{H}$ NMR (500 MHz, $\left.\mathrm{CDCl}_{3}\right) \delta$ 7.50-7.30 (m, $\left.5 \mathrm{H}\right), 3.57$ (m, $\left.8 \mathrm{H}\right), 1.48$ (s, 9 H). ${ }^{13} \mathrm{C} \mathrm{NMR}\left(125 \mathrm{MHz}, \mathrm{CDCl}_{3}\right) \delta 170.6,154.6,135.5,129.9,128.6$, 127.0, 80.3, 47.5, 44.0, 28.4 .<smiles>CC(C)(C)C(=O)N1CCN(C(=O)c2ccccc2)CC1</smiles>

1-(4-Benzoylpiperazin-1-yl)-2,2-dimethylpropan-1-one (1z). White solid. ${ }^{1} \mathrm{H}$ NMR (500 MHz, $\left.\mathrm{CDCl}_{3}\right) \delta$ 7.55-7.30 (m, $\left.5 \mathrm{H}\right), 3.68(\mathrm{~s}, 8 \mathrm{H})$, $1.26(\mathrm{~s}, 9 \mathrm{H}) .{ }^{13} \mathrm{C} \mathrm{NMR}\left(125 \mathrm{MHz}, \mathrm{CDCl}_{3}\right) \delta 176.7,170.6,135.3$, 130.0, 128.6, 127.1, 47.6, 45.8, 42.3, 38.7, 28.3. 


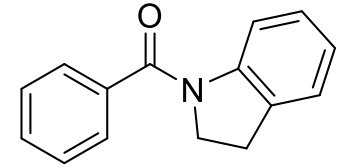

Indolin-1-yl(phenyl)methanone (1aa). White solid. ${ }^{1} \mathrm{H}$ NMR $(500 \mathrm{MHz}$, $\left.\mathrm{CDCl}_{3}\right) \delta 8.24(\mathrm{br}, 1 \mathrm{H}), 7.58(\mathrm{~d}, J=6.6 \mathrm{~Hz}, 2 \mathrm{H}), 7.53-7.44(\mathrm{~m}, 3 \mathrm{H}), 7.24$ (d, $J=7.6 \mathrm{~Hz}, 1 \mathrm{H}), 7.05(\mathrm{~s}, 1 \mathrm{H}), 4.10(\mathrm{~s}, 2 \mathrm{H}), 3.14(\mathrm{t}, J=8.3 \mathrm{~Hz}, 2 \mathrm{H})$.

${ }^{13} \mathrm{C}$ NMR $\left(125 \mathrm{MHz}, \mathrm{CDCl}_{3}\right) \delta 169.0,142.6,137.0,132.3,130.3,128.6,127,1,124.9,124.0$, 117.3, 50.6, 28.3.

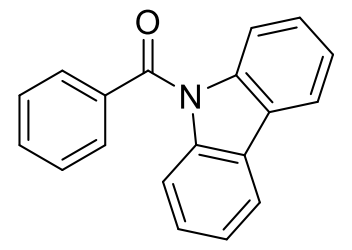

(9H-Carbazol-9-yl)(phenyl)methanone (1ab). White solid. ${ }^{1} \mathrm{H}$ NMR (500 $\left.\mathrm{MHz}, \mathrm{CDCl}_{3}\right) \delta 8.06-8.01(\mathrm{~m}, 2 \mathrm{H}), 7.81(\mathrm{~d}, J=7.1 \mathrm{~Hz}, 2 \mathrm{H}), 7.54(\mathrm{t}, J=$ 7.8 Hz, $1 \mathrm{H}), 7.77-7.58$ (m, $4 \mathrm{H}), 7.40-7.35$ (m, $4 \mathrm{H}) .{ }^{13} \mathrm{C}$ NMR (125 MHz, $\left.\mathrm{CDCl}_{3}\right) \delta 169.6,139.3,135.7,132.2,129.1,128.8,126.8,126.0,123.4$,

$119.8,115.7$.

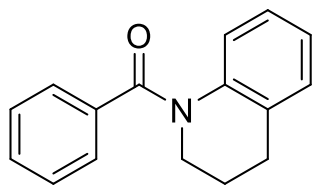

(3,4-Dihydroquinolin-1(2H)-yl)(phenyl)methanone (1ac). White solid. ${ }^{1} \mathrm{H}$ NMR (500 MHz, $\left.\mathrm{CDCl}_{3}\right) \delta$ 7.41-7.33 (m, $\left.3 \mathrm{H}\right), 7.29(\mathrm{dd}, J=9.1,5.7 \mathrm{~Hz}, 2$ H), $7.17(\mathrm{~d}, J=7.5 \mathrm{~Hz}, 1 \mathrm{H}), 7.01(\mathrm{t}, J=7.8 \mathrm{~Hz}, 1 \mathrm{H}), 6.89(\mathrm{t}, J=7.6 \mathrm{~Hz}, 1$ $\mathrm{H}), 6.75(\mathrm{~s}, 1 \mathrm{H}), 3.93(\mathrm{t}, J=6.5 \mathrm{~Hz}, 2 \mathrm{H}), 2.87(\mathrm{t}, J=6.6 \mathrm{~Hz}, 2 \mathrm{H}), 2.08(\mathrm{p}, J=6.6 \mathrm{~Hz}, 2 \mathrm{H}) .{ }^{13} \mathrm{C}$ NMR $\left(125 \mathrm{MHz}, \mathrm{CDCl}_{3}\right) \delta 170.3,139.4,136.4,131.6,130.1,128.6,128.4,128.1,125.7,125.5$, 124.6, 44.5, 27.0, 24.2.

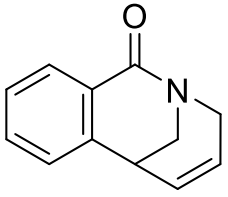

3,6-Dihydro-1H-2,6-methanobenzo[c]azocin-1-one (1ad). White solid. ${ }^{1} \mathrm{H}$ $\operatorname{NMR}\left(500 \mathrm{MHz}, \mathrm{CDCl}_{3}\right) \delta 7.82(\mathrm{t}, J=7.5 \mathrm{~Hz}, 1 \mathrm{H}), 7.38(\mathrm{dd}, J=12.5,6.9 \mathrm{~Hz}, 1$ H), $7.20(\mathrm{~d}, J=7.4 \mathrm{~Hz}, 1 \mathrm{H}), 7.06(\mathrm{q}, J=7.1 \mathrm{~Hz}, 1 \mathrm{H}), 5.91-5.81(\mathrm{~m}, 1 \mathrm{H}), 5.80$ $5.50(\mathrm{~m}, 1 \mathrm{H}), 4.42-4.08(\mathrm{~m}, 1 \mathrm{H}), 3.87$ (dd, $J=12.3,6.2 \mathrm{~Hz}, 1 \mathrm{H})$, 3.78-3.55 (m, $1 \mathrm{H}), 3.38-$ $3.23(\mathrm{~m}, 1 \mathrm{H}), 2.38-2.23(\mathrm{~m}, 1 \mathrm{H}), 2.23-2.04(\mathrm{~m}, 1 \mathrm{H}) .{ }^{13} \mathrm{C} \mathrm{NMR}\left(125 \mathrm{MHz}, \mathrm{CDCl}_{3}\right) \delta 177.0$, $147.1,132.4,130.6,130.1,129.8,128.3,127.7,125.5,52.4,52.1,36.6$.

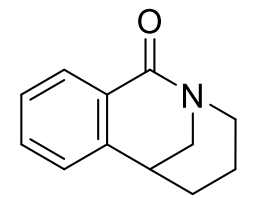

3,4,5,6-Tetrahydro-1H-2,6-methanobenzo[c]azocin-1-one (1ae). Colorless oil. ${ }^{1} \mathrm{H}$ NMR (500 MHz, $\left.\mathrm{CDCl}_{3}\right) \delta 7.99(\mathrm{~d}, J=7.6 \mathrm{~Hz}, 1 \mathrm{H}), 7.46(\mathrm{td}, J=7.4,1.1 \mathrm{~Hz}$, $1 \mathrm{H}), 7.36(\mathrm{dd}, J=10.9,4.1 \mathrm{~Hz}, 1 \mathrm{H}), 7.24(\mathrm{~d}, J=7.5 \mathrm{~Hz}, 1 \mathrm{H}), 3.99$ (dd, $J=$ 13.2, 4.6 Hz, $1 \mathrm{H}), 3.65$ (d, $J=13.1 \mathrm{~Hz}, 1 \mathrm{H}), 3.38(\mathrm{dd}, J=13.2,1.5 \mathrm{~Hz}, 1 \mathrm{H}), 3.17$ (td, $J=13.2$, 
$3.0 \mathrm{~Hz}, 1 \mathrm{H}), 2.87(\mathrm{~s}, 1 \mathrm{H}), 2.20-2.10(\mathrm{~m}, 1 \mathrm{H}), 1.90$ (d, J = 13.6 Hz, $1 \mathrm{H}), 1.85-1.68$ (m, $1 \mathrm{H})$, $1.37(\mathrm{~d}, J=14.1 \mathrm{~Hz}, 1 \mathrm{H}) .{ }^{13} \mathrm{C} \mathrm{NMR}\left(125 \mathrm{MHz}, \mathrm{CDCl}_{3}\right) \delta 178.0,145.7,132.9,132.8,128.7$, $127.5,127.2,54.2,53.8,35.2,29.3,21.6$.<smiles>O=C(Nc1ccccc1)c1ccccc1</smiles>

$N$-Benzylbenzamide (1ah). White solid. ${ }^{1} \mathrm{H}$ NMR $\left(500 \mathrm{MHz}, \mathrm{CDCl}_{3}\right) \delta 7.85$ $7.79(\mathrm{~m}, 2 \mathrm{H}), 7.52(\mathrm{t}, J=7.4 \mathrm{~Hz}, 1 \mathrm{H}), 7.45(\mathrm{t}, J=7.5 \mathrm{~Hz}, 2 \mathrm{H}), 7.37$ (t, $J=5.9$ $\mathrm{Hz}, 4 \mathrm{H}), 7.33(\mathrm{dd}, J=8.5,4.0 \mathrm{~Hz}, 1 \mathrm{H}), 6.54$ (s, $1 \mathrm{H}), 4.67$ (d, $J=5.6 \mathrm{~Hz}, 2$ H). ${ }^{13} \mathrm{C}$ NMR $\left(125 \mathrm{MHz}, \mathrm{CDCl}_{3}\right) \delta 167.4,138.2,134.4,131.6,128.8,128.6,127.9,127.6,127.0$, 44.2 .

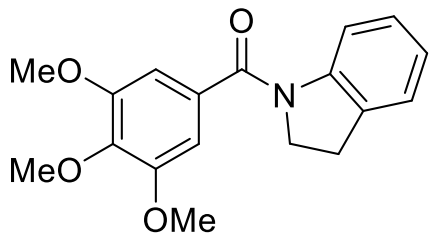

Indolin-1-yl(3,4,5-trimethoxyphenyl)methanone (1ai). White solid. ${ }^{1} \mathrm{H}$ NMR $\left(500 \mathrm{MHz}, \mathrm{CDCl}_{3}\right) \delta 7.80(\mathrm{~s}, 1 \mathrm{H}), 7.15(\mathrm{~d}, J=7.1$ $\mathrm{Hz}, 1 \mathrm{H}), 7.05$ (s, $1 \mathrm{H}), 6.95$ (s, $1 \mathrm{H}), 6.71$ (s, $2 \mathrm{H}), 4.05$ (s, $2 \mathrm{H})$, $3.83(\mathrm{~s}, 3 \mathrm{H}), 3.79(\mathrm{~s}, 6 \mathrm{H}), 3.06(\mathrm{t}, J=8.0 \mathrm{~Hz}, 2 \mathrm{H}) .{ }^{13} \mathrm{C} \mathrm{NMR}(125$ $\left.\mathrm{MHz}, \mathrm{CDCl}_{3}\right) \delta 168.6,153.4,143.5,139.8,132.8,132.2,127.3,125.0,123.9,104.5,61.0,56.3$, $50.6,22.7$.<smiles>COC(=O)c1ccccc1</smiles>
Methyl benzoate (4a). Colorless oil. ${ }^{1} \mathrm{H}$ NMR $\left(500 \mathrm{MHz}, \mathrm{CDCl}_{3}\right) \delta 8.06(\mathrm{~d}, J=$ 4.6, 3.7 Hz, $2 \mathrm{H}), 7.57$ (t, $J=7.4 \mathrm{~Hz}, 1 \mathrm{H}), 7.45$ (t, $J=7.6 \mathrm{~Hz}, 2 \mathrm{H}), 3.94$ (s, 3 H). ${ }^{13} \mathrm{C} \mathrm{NMR}\left(125 \mathrm{MHz}, \mathrm{CDCl}_{3}\right) \delta 167.1,132.9,130.2,129.6,128.4,52.1$.<smiles>COC(=O)c1ccc(C)cc1</smiles>

Methyl 4-methylbenzoate (4b). Colorless oil. ${ }^{1} \mathrm{H}$ NMR (500 MHz, $\left.\mathrm{CDCl}_{3}\right) \delta 7.95(\mathrm{~d}, J=8.2 \mathrm{~Hz}, 2 \mathrm{H}), 7.25(\mathrm{~d}, J=7.9 \mathrm{~Hz}, 2 \mathrm{H}), 3.92(\mathrm{~s}, 3 \mathrm{H})$, $2.42(\mathrm{~s}, 3 \mathrm{H}) .{ }^{13} \mathrm{C} \mathrm{NMR}\left(125 \mathrm{MHz}, \mathrm{CDCl}_{3}\right) \delta 167.3,143.6,129.6,129.1$, $127.5,51.9,21.6$.<smiles>COC(=O)c1ccc(OC)cc1</smiles>

Methyl 4-methoxybenzoate (4c). White solid. ${ }^{1} \mathrm{H}$ NMR (500 MHz, $\left.\mathrm{CDCl}_{3}\right) \delta 8.01(\mathrm{~d}, J=9.0 \mathrm{~Hz}, 2 \mathrm{H}), 6.93(\mathrm{~d}, J=8.9 \mathrm{~Hz}, 2 \mathrm{H}), 3.90(\mathrm{~s}, 3$ $\mathrm{H}), 3.87(\mathrm{~s}, 3 \mathrm{H}) .{ }^{13} \mathrm{C} \mathrm{NMR}\left(125 \mathrm{MHz}, \mathrm{CDCl}_{3}\right) \delta 166.9,163.3,131.6$, 122.6, 113.6, 55.4, 51.9. 
<smiles>COC(=O)c1ccc(C(F)(F)F)cc1</smiles>

Methyl 4-(trifluoromethyl)benzoate (4d). Colorless oil. ${ }^{1} \mathrm{H}$ NMR (500 $\left.\mathrm{MHz}, \mathrm{CDCl}_{3}\right) \delta 8.14(\mathrm{~d}, J=6.5 \mathrm{~Hz}, 2 \mathrm{H}), 7.70(\mathrm{~d}, J=6.5 \mathrm{~Hz}, 2 \mathrm{H}), 4.01-$ $3.91(\mathrm{~m}, 3 \mathrm{H}) .{ }^{13} \mathrm{C} \mathrm{NMR}\left(125 \mathrm{MHz}, \mathrm{CDCl}_{3}\right) \delta 165.8,134.4\left(J^{F}=32.5 \mathrm{~Hz}\right)$, 133.3, 130.0, $123.6\left(J^{F}=272.7 \mathrm{~Hz}\right), 125.4,52.5 .{ }^{19} \mathrm{~F} \mathrm{NMR}\left(471 \mathrm{MHz}, \mathrm{CDCl}_{3}\right) \delta-63.24(\mathrm{~s})$.<smiles>COC(=O)c1ccccc1C</smiles>

Methyl 2-methylbenzoate (4e). Colorless oil. ${ }^{1} \mathrm{H}$ NMR $\left(500 \mathrm{MHz}, \mathrm{CDCl}_{3}\right) \delta$ $7.94(\mathrm{dd}, J=8.1,1.3 \mathrm{~Hz}, 1 \mathrm{H}), 7.40$ (t, $J=7.5 \mathrm{~Hz}, 1 \mathrm{H}), 7.27-7.22$ (m, $2 \mathrm{H})$, $3.90(\mathrm{~s}, 3 \mathrm{H}), 2.63(\mathrm{~s}, 3 \mathrm{H}) .{ }^{13} \mathrm{C}$ NMR $\left(125 \mathrm{MHz}, \mathrm{CDCl}_{3}\right) \delta$ 168.0, 140.2, 132.0, 131.7, 130.6, 129.6, 125.7, 51.7, 21.7 .<smiles>COC(=O)c1cccc(Br)c1</smiles>

Methyl 3-bromobenzoate (4f). Colorless oil. ${ }^{1} \mathrm{H}$ NMR (500 MHz, $\left.\mathrm{CDCl}_{3}\right)$ $\delta 8.18(\mathrm{~s}, 1 \mathrm{H}), 7.97(\mathrm{~d}, J=7.8 \mathrm{~Hz}, 1 \mathrm{H}), 7.68(\mathrm{~d}, J=9.8 \mathrm{~Hz}, 1 \mathrm{H}), 7.31$ $(\mathrm{dd}, J=16.5,8.6 \mathrm{~Hz}, 1 \mathrm{H}), 3.93(\mathrm{~s}, 3 \mathrm{H}) .{ }^{13} \mathrm{C} \mathrm{NMR}\left(125 \mathrm{MHz}, \mathrm{CDCl}_{3}\right) \delta$ $165.7,135.9,132.6,132.1,129.9,128.3,122.5,52.4$.

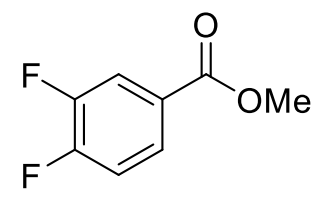

Methyl 3,4-difluorobenzoate (4g). Colorless oil. ${ }^{1} \mathrm{H}$ NMR (500 MHz, $\left.\mathrm{CDCl}_{3}\right) \delta$ 7.77-7.71 (m, $\left.2 \mathrm{H}\right)$, 7.15-7.09 (m, $\left.1 \mathrm{H}\right), 3.83(\mathrm{~d}, J=2.9 \mathrm{~Hz}, 3 \mathrm{H})$. ${ }^{13} \mathrm{C} \mathrm{NMR}\left(125 \mathrm{MHz}, \mathrm{CDCl}_{3}\right) \delta 163.2,152.4\left(\mathrm{dd}, J^{F}=260.5,12.8 \mathrm{~Hz}\right), 150.0$ $\left(\mathrm{dd}, J^{F}=248.5,12.8 \mathrm{~Hz}\right), 126.0\left(\mathrm{dd}, J^{F}=13.4,6.2 \mathrm{~Hz}\right), 125.2\left(\mathrm{dd}, J^{F}=7.0,3.2 \mathrm{~Hz}\right), 117.4\left(\mathrm{~d}, J^{F}\right.$ $=17.0 \mathrm{~Hz}), 116.3\left(\mathrm{~d}, J^{F}=17.0 \mathrm{~Hz}\right), 51.7 .{ }^{19} \mathrm{~F} \mathrm{NMR}\left(470 \mathrm{MHz}, \mathrm{CDCl}_{3}\right) \delta-130.38(\mathrm{~m}),-136.70$ (m).<smiles>COC(=O)c1cccs1</smiles>

Methyl thiophene-2-carboxylate (4h). Colorless oil. ${ }^{1} \mathrm{H}$ NMR (500 MHz, $\left.\mathrm{CDCl}_{3}\right) \delta$ 7.78-7.78 (m, $\left.1 \mathrm{H}\right)$, 7.54-7.52 (m, $\left.1 \mathrm{H}\right)$, 7.08-7.06 (m, $\left.1 \mathrm{H}\right), 3.86(\mathrm{~s}, 3$ H). ${ }^{13} \mathrm{C} \mathrm{NMR}\left(125 \mathrm{MHz}, \mathrm{CDCl}_{3}\right) \delta 162.6,133.6,133.4,132.4,127.7,52.1$.<smiles>CCOC(C)=O</smiles><smiles>COC(=O)c1ccccc1</smiles>
Methyl decanoate (4j). Colorless oil. ${ }^{1} \mathrm{H}$ NMR $\left(500 \mathrm{MHz}, \mathrm{CDCl}_{3}\right) \delta 3.65(\mathrm{~s}, 3$ H), 2.29 (t, $J=7.5 \mathrm{~Hz}, 2 \mathrm{H}), 2.03(\mathrm{~d}, J=2.0 \mathrm{~Hz}, 1 \mathrm{H}), 1.66-1.55$ (m, $2 \mathrm{H}), 1.32-$ 
$1.20(\mathrm{~s}, 12 \mathrm{H}), 0.87(\mathrm{t}, J=6.1 \mathrm{~Hz}, 3 \mathrm{H}) .{ }^{13} \mathrm{C} \mathrm{NMR}\left(125 \mathrm{MHz}, \mathrm{CDCl}_{3}\right) \delta 174.3,51.3,34.1,31.8$, 29.4, 29.2, 29.1, 24.9, 22.6, 14.0.<smiles>COC(=O)C1CCCCC1</smiles>

Methyl cyclohexanecarboxylate (4k). Colorless oil. ${ }^{1} \mathrm{H}$ NMR (500 MHz, $\left.\mathrm{CDCl}_{3}\right) \delta 3.64(\mathrm{~s}, 3 \mathrm{H}), 2.28(\mathrm{t}, J=10.6 \mathrm{~Hz}, 1 \mathrm{H}), 1.90-1.85(\mathrm{~m}, 2 \mathrm{H}), 1.76-1.70$ (m, $2 \mathrm{H}), 1.66-1.58(\mathrm{~m}, 1 \mathrm{H}), 1.42(\mathrm{~m}, 2 \mathrm{H}), 1.30-1.16$ (m, $3 \mathrm{H}) .{ }^{13} \mathrm{C}$ NMR $(125$ $\left.\mathrm{MHz}, \mathrm{CDCl}_{3}\right) \delta 176.5,51.4,43.1,29.0,25.7,25.4$.

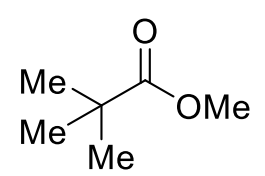

Methyl pivalate (4l). Colorless oil. ${ }^{1} \mathrm{H}$ NMR $\left(500 \mathrm{MHz}, \mathrm{CDCl}_{3}\right) \delta=3.65(\mathrm{~s}, 3$ $\mathrm{H}), 1.19$ (s, $9 \mathrm{H}) .{ }^{13} \mathrm{C} \mathrm{NMR}\left(125 \mathrm{MHz}, \mathrm{CDCl}_{3}\right) \delta 179.0,51.9,38.9,27.8$.<smiles>CCOC(=O)c1ccccc1</smiles>

Ethyl benzoate (4m). Colorless oil. ${ }^{1} \mathrm{H}$ NMR $\left(500 \mathrm{MHz}, \mathrm{CDCl}_{3}\right) \delta 8.06(\mathrm{~d}, J=$ 7.2 Hz, $2 \mathrm{H}), 7.57-7.49$ (m, $1 \mathrm{H}), 7.47-7.37$ (m, $2 \mathrm{H}), 4.38$ (dd, $J=13.4,6.3 \mathrm{~Hz}$, $2 \mathrm{H}), 1.38(\mathrm{t}, J=10.1 \mathrm{~Hz}, 3 \mathrm{H}) .{ }^{13} \mathrm{C} \mathrm{NMR}\left(\mathrm{CDCl}_{3}, 125 \mathrm{MHz}\right) \delta 166.1,132.2$, $129.9,129.0,127.9,60.2,14.0$.<smiles>CC(C)OC(=O)c1ccccc1</smiles>

Isopropyl benzoate (4n). Colorless oil. ${ }^{1} \mathrm{H} \mathrm{NMR}\left(500 \mathrm{MHz}, \mathrm{CDCl}_{3}\right) \delta 8.07$ $(\mathrm{d}, J=7.7 \mathrm{~Hz}, 2 \mathrm{H}), 7.56(\mathrm{t}, J=6.8 \mathrm{~Hz}, 1 \mathrm{H}), 7.45(\mathrm{t}, J=7.7 \mathrm{~Hz}, 2 \mathrm{H}), 5.28$ (hept, $J=6.3 \mathrm{~Hz}, 1 \mathrm{H}), 1.40$ (s, $3 \mathrm{H}), 1.39$ (s, $3 \mathrm{H}) .{ }^{13} \mathrm{C}$ NMR $(125 \mathrm{MHz}$, $\left.\mathrm{CDCl}_{3}\right) \delta 166.1,132.7,130.9,129.5,128.3,68.3,22.0$.<smiles>CC(C)(C)OC(=O)c1ccccc1</smiles>

81.0, 28.2.

tert-Butyl benzoate (4o). Colorless oil. ${ }^{1} \mathrm{H}$ NMR (500 MHz, $\left.\mathrm{CDCl}_{3}\right) \delta 7.92$ $(\mathrm{d}, J=8.1 \mathrm{~Hz}, 2 \mathrm{H}), 7.45(\mathrm{t}, J=6.8 \mathrm{~Hz}, 1 \mathrm{H}), 7.34(\mathrm{t}, J=7.7 \mathrm{~Hz}, 2 \mathrm{H}), 1.53$ (s, $9 \mathrm{H}) .{ }^{13} \mathrm{C} \mathrm{NMR}\left(125 \mathrm{MHz}, \mathrm{CDCl}_{3}\right) \delta 165.8,132.4,132.0,129.4,128.2$,<smiles>O=C(OCc1ccccc1)c1ccccc1</smiles>
Benzyl benzoate (4p). Colorless oil. ${ }^{1} \mathrm{H}$ NMR (500 MHz, $\left.\mathrm{CDCl}_{3}\right) \delta 8.17(\mathrm{~d}, J=$ $8.4 \mathrm{~Hz}, 2 \mathrm{H}), 7.60$ (t, $J=7.4 \mathrm{~Hz}, 1 \mathrm{H}), 7.45$ (ddd, $J=25.4,22.8,15.4 \mathrm{~Hz}, 7 \mathrm{H})$, 5.45 (s, $2 \mathrm{H}) .{ }^{13} \mathrm{C}$ NMR $\left(125 \mathrm{MHz}, \mathrm{CDCl}_{3}\right) \delta 166.5,136.3,133.1,130.2,129.8$, $128.7,128.7,128.5,128.3,128.3,66.8$. 


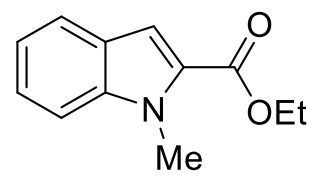

Ethyl 1-methyl-1H-indole-2-carboxylate (4q). White solid. ${ }^{1} \mathrm{H}$ NMR (500 $\left.\mathrm{MHz}_{\mathrm{CDCl}}\right) \delta 7.59(\mathrm{~d}, J=7.9 \mathrm{~Hz}, 1 \mathrm{H}), 7.33-7.24(\mathrm{~m}, 2 \mathrm{H}), 7.22$ (s, $\left.1 \mathrm{H}\right)$, $7.06(\mathrm{t}, J=7.3 \mathrm{~Hz}, 1 \mathrm{H}), 4.30(\mathrm{q}, J=7.1 \mathrm{~Hz}, 2 \mathrm{H}), 4.00$ (s, $3 \mathrm{H}), 1.33$ (t, $J=$

$7.1 \mathrm{~Hz}, 3 \mathrm{H}) .{ }^{13} \mathrm{C} \mathrm{NMR}\left(125 \mathrm{MHz}, \mathrm{CDCl}_{3}\right) \delta 162.3,139.7,128.1,125.9,124.9,122.6,120.5$, $110.2,110.1,60.5,31.6,14.4$.<smiles>CCOC(=O)c1cc2ccccc2[nH]1</smiles><smiles>COC(=O)c1ccc(Cl)cc1</smiles>

Methyl 4-chlorobenzoate (4s). White solid. ${ }^{1} \mathrm{H} \mathrm{NMR}\left(500 \mathrm{MHz}, \mathrm{CDCl}_{3}\right) \delta$ $7.98(\mathrm{~d}, J=8.8 \mathrm{~Hz}, 2 \mathrm{H}), 7.42(\mathrm{~d}, J=8.7 \mathrm{~Hz}, 2 \mathrm{H}), 3.93(\mathrm{~s}, 3 \mathrm{H}) .{ }^{13} \mathrm{C} \mathrm{NMR}$ $\left(125 \mathrm{MHz} \mathrm{CDCl}_{3}\right) \delta 166.3,139.4,131.0,128.7,128.6,52.3$.

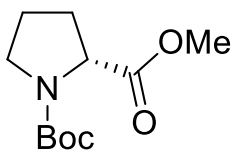
available. $\mathrm{CAS}=145681-01-2$. 


\section{Characterization Data of Transamidation Products}

$\mathbf{3 a},{ }^{41} \mathbf{3 b},{ }^{41} \mathbf{3 c},{ }^{42} \mathbf{3 d},{ }^{41} \mathbf{3 f},{ }^{43} \mathbf{3 g},{ }^{44} \mathbf{3 h},{ }^{41} \mathbf{3 i},{ }^{41} \mathbf{3 j},{ }^{41} \mathbf{3 k},{ }^{41} \mathbf{3 l},{ }^{45} \mathbf{3 m},{ }^{41} \mathbf{3 n},{ }^{41} \mathbf{3 o},{ }^{41} \mathbf{3 p},{ }^{46} \mathbf{3 q},{ }^{41} \mathbf{3 r},{ }^{47} \mathbf{3 s},{ }^{41}$ $\mathbf{3 t},{ }^{47} \mathbf{3 u},{ }^{48} \mathbf{3 v},{ }^{49} \mathbf{3 x},{ }^{50} \mathbf{3 y},{ }^{51} \mathbf{3 z},{ }^{47} \mathbf{3 a a},{ }^{52} \mathbf{3 a b},{ }^{53} \mathbf{3 a c},{ }^{41} \mathbf{3 a d},{ }^{41} \mathbf{3 a e},{ }^{41} \mathbf{3 a f},{ }^{54} \mathbf{3 a g},{ }^{41} \mathbf{3 a h},{ }^{41} \mathbf{3 a i},{ }^{41} \mathbf{3 a j},{ }^{41}$ 3ak, ${ }^{41}$ 3al,${ }^{41}$ 3am,${ }^{45}$ 3an, ${ }^{41}$ 3ao,${ }^{45}$ 3ap,${ }^{55}$ 3aq, ${ }^{41}$ 3ar, ${ }^{41}$ 3as, ${ }^{41}$ 3av,${ }^{56}$ 3ay, ${ }^{41} \mathbf{3 a z}^{41}$ have been previously reported. Spectroscopic data match those reported in the literature.

$N$-(p-Tolyl)benzamide (3a, Figure 2)

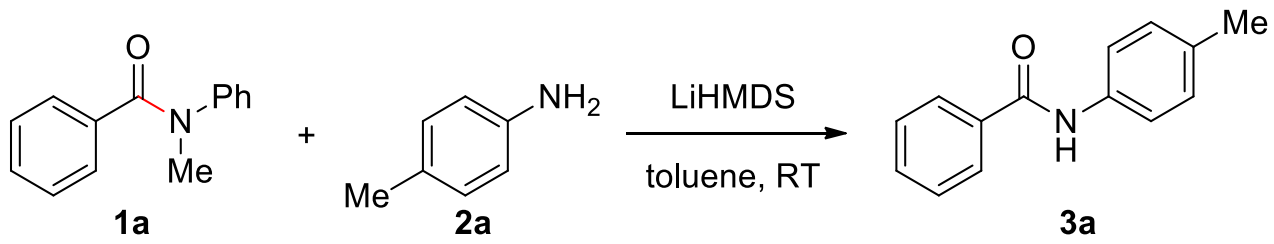

According to the general procedure, the reaction of $N$-methyl- $N$-phenylbenzamide $(4.73 \mathrm{mmol}$, 1.0 equiv), $p$-toluidine (2.0 equiv) and LiHMDS (1.0 M in THF, 3.0 equiv) in toluene $(0.25 \mathrm{M})$ for $15 \mathrm{~h}$ at room temperature, afforded after work-up and chromatography the title compound in 97\% yield (0.970 g). White solid. ${ }^{1} \mathrm{H}$ NMR (500 MHz, $\left.\mathrm{CDCl}_{3}\right) \delta 7.88(\mathrm{~d}, J=7.3 \mathrm{~Hz}, 2 \mathrm{H}), 7.83$ $(\mathrm{s}, 1 \mathrm{H}), 7.58-7.54(\mathrm{~m}, 3 \mathrm{H}), 7.50(\mathrm{t}, J=7.5 \mathrm{~Hz}, 2 \mathrm{H}), 7.19(\mathrm{~d}, J=8.2 \mathrm{~Hz}, 2 \mathrm{H}), 2.37(\mathrm{~s}, 3 \mathrm{H}) .{ }^{13} \mathrm{C}$ NMR (125 MHz, $\left.\mathrm{CDCl}_{3}\right) \delta 165.7,135.4,135.1,134.3,131.7,129.6,128.8,127.0,120.3,20.9$.

\section{$N$-(4-Methoxyphenyl)benzamide (3b, Figure 2)}<smiles>CN(C(=O)c1ccccc1)c1ccccc1</smiles>
$1 \mathrm{a}$<smiles>COc1ccc(N)cc1</smiles>

2b

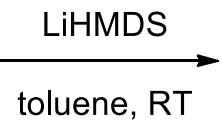

toluene, RT<smiles>COc1ccc(NC(=O)c2ccccc2)cc1</smiles>

According to the general procedure, the reaction of $N$-methyl- $N$-phenylbenzamide $(0.10$ mmol, 1.0 equiv), 4-methoxyaniline (2.0 equiv) and LiHMDS (1.0 M in THF, 3.0 equiv) in toluene $(0.25 \mathrm{M})$ for $15 \mathrm{~h}$ at room temperature, afforded after work-up and chromatography the title compound in $80 \%$ yield $(18.2 \mathrm{mg})$. White solid. ${ }^{1} \mathrm{H}$ NMR $\left(500 \mathrm{MHz}, \mathrm{CDCl}_{3}\right) \delta 8.00(\mathrm{~d}, J=7.6$ $\mathrm{Hz}, 2 \mathrm{H}), 7.94(\mathrm{~s}, 1 \mathrm{H}), 7.68(\mathrm{dd}, J=7.6,5.7 \mathrm{~Hz}, 3 \mathrm{H}), 7.61(\mathrm{t}, J=7.6 \mathrm{~Hz}, 2 \mathrm{H}), 7.04(\mathrm{~d}, J=8.9$ $\mathrm{Hz}, 2 \mathrm{H}), 3.95$ (s, $3 \mathrm{H}) .{ }^{13} \mathrm{C} \mathrm{NMR}\left(125 \mathrm{MHz}, \mathrm{CDCl}_{3}\right) \delta 164.6,155.7,134.1,130.7,130.0,127.8$, 126.0, 121.2, 113.3, 54.6. 


\section{$N$-(4-(Dimethylamino)phenyl)benzamide (3c, Figure 2)}

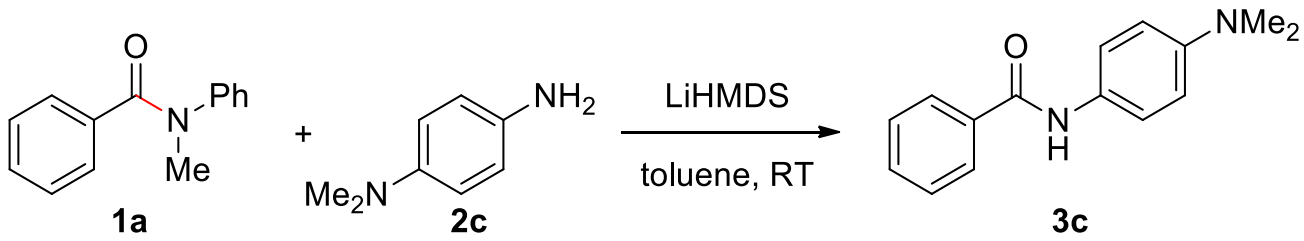

According to the general procedure, the reaction of $N$-methyl- $N$-phenylbenzamide $(0.10 \mathrm{mmol}$, 1.0 equiv), $N^{1}, N^{1}$-dimethylbenzene-1,4-diamine (2.0 equiv) and LiHMDS (1.0 $\mathrm{M}$ in THF, 3.0 equiv) in toluene $(0.25 \mathrm{M})$ for $15 \mathrm{~h}$ at room temperature, afforded after work-up chromatography the title compound in $82 \%$ yield $(19.7 \mathrm{mg})$. Yellow solid. ${ }^{1} \mathrm{H}$ NMR (500 MHz, DMSO- $\left.d_{6}\right) \delta 9.98$ $(\mathrm{s}, 1 \mathrm{H}), 7.95(\mathrm{~d}, J=7.3 \mathrm{~Hz}, 2 \mathrm{H}), 7.59-7.51(\mathrm{~m}, 3 \mathrm{H}), 7.51(\mathrm{t}, J=7.4 \mathrm{~Hz}, 2 \mathrm{H}), 6.73(\mathrm{~d}, J=9.0$ $\mathrm{Hz}, 2 \mathrm{H}), 2.88$ (s, $6 \mathrm{H}) .{ }^{13} \mathrm{C}$ NMR (125 MHz, DMSO-d $) \delta 165.2,147.8,135.7,131.6,129.3$, $128.8,127.9,122.3,112.9,41.0$.

\section{$N$-(4-Hydroxyphenyl)benzamide (3d, Figure 2)}

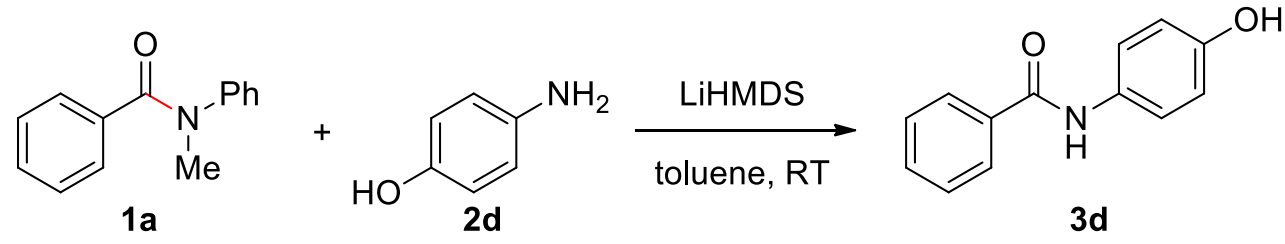

According to the general procedure, the reaction of $N$-methyl- $N$-phenylbenzamide $(0.10$ mmol, 1.0 equiv), 4-aminophenol (2.0 equiv) and LiHMDS (1.0 M in THF, 3.0 equiv) in toluene (0.25 M) for $15 \mathrm{~h}$ at room temperature, afforded after work-up and chromatography the title compound in $80 \%$ yield $(17.0 \mathrm{mg})$. White solid. ${ }^{1} \mathrm{H}$ NMR $\left(500 \mathrm{MHz}, \mathrm{DMSO}-d_{6}\right) \delta 10.04(\mathrm{~s}, 1 \mathrm{H}), 9.27(\mathrm{~s}, 1$ H), $7.96(\mathrm{~d}, J=7.4 \mathrm{~Hz}, 2 \mathrm{H}), 7.61-7.50(\mathrm{~m}, 5 \mathrm{H}), 6.77(\mathrm{~d}, J=8.8 \mathrm{~Hz}, 2 \mathrm{H}) .{ }^{13} \mathrm{C}$ NMR $(125 \mathrm{MHz}$, DMSO- $\left.d_{6}\right) \delta 165.9,154.7,136.2,132.2,131.7,129.3,128.5,123.2,115.9$.

\section{4-Benzamido- $N, N$-diethylbenzamide (3e, Figure 2)}<smiles>CN(C(=O)c1ccccc1)c1ccccc1</smiles>

$1 \mathrm{a}$

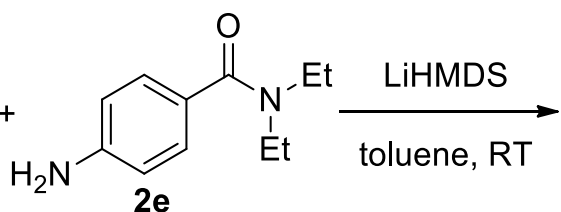


According to the general procedure, the reaction of $N$-methyl- $N$-phenylbenzamide $(0.10 \mathrm{mmol}$, 1.0 equiv), 4-amino- $N, N$-diethylbenzamide (2.0 equiv) and LiHMDS (1.0 M in THF, 3.0 equiv) in toluene $(0.25 \mathrm{M})$ for $15 \mathrm{~h}$ at room temperature, afforded after work-up and chromatography the title compound in $83 \%$ yield $(24.7 \mathrm{mg})$. New compound. White solid. $\mathrm{Mp}=143-144{ }^{\circ} \mathrm{C} .{ }^{1} \mathrm{H}$ NMR (500 MHz, $\left.\mathrm{CDCl}_{3}\right) \delta 8.29(\mathrm{~s}, 1 \mathrm{H}), 7.95(\mathrm{~d}, J=7.4 \mathrm{~Hz}, 2 \mathrm{H}), 7.65(\mathrm{~d}, J=7.5 \mathrm{~Hz}, 2 \mathrm{H})$, $7.58(\mathrm{t}, J=7.2 \mathrm{~Hz}, 1 \mathrm{H}), 7.51(\mathrm{t}, J=7.3 \mathrm{~Hz}, 2 \mathrm{H}), 7.36(\mathrm{~d}, J=7.5 \mathrm{~Hz}, 2 \mathrm{H}), 3.54-3.31(\mathrm{~m}, 4 \mathrm{H})$, $1.18(\mathrm{~s}, 6 \mathrm{H}) .{ }^{13} \mathrm{C} \mathrm{NMR}\left(125 \mathrm{MHz}, \mathrm{CDCl}_{3}\right) \delta 171.0,165.9,138.9,134.7,133.0,132.0,128.8$, $127.3(\mathrm{~d}, J=16.2 \mathrm{~Hz}), 120.2,43.4,39.5,14.2,13.0$. HRMS calcd for $\mathrm{C}_{18} \mathrm{H}_{20} \mathrm{~N}_{2} \mathrm{O}_{2} \mathrm{Na}(\mathrm{M}+\mathrm{Na})$ 319.1417 , found 319.1434 .

\section{$N$-(4-(N,N-Dimethylsulfamoyl)phenyl)benzamide (3f, Figure 2)}

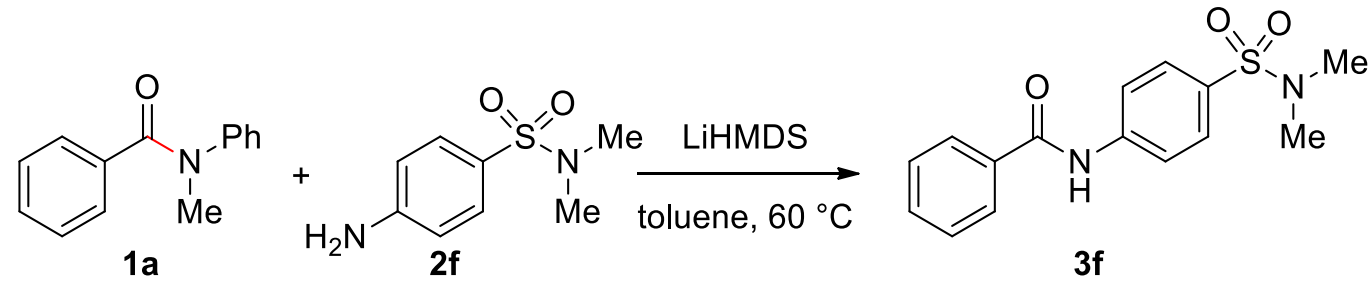

According to the general procedure, the reaction of $N$-methyl- $N$-phenylbenzamide $(0.10$ mmol, 1.0 equiv), 4-amino- $N, N$-dimethylbenzenesulfonamide (2.0 equiv) and LiHMDS (1.0 M in THF, 3.0 equiv) in toluene $(0.25 \mathrm{M})$ for $15 \mathrm{~h}$ at $60{ }^{\circ} \mathrm{C}$, afforded after work-up and chromatography the title compound in $80 \%$ yield $(24.3 \mathrm{mg})$. White solid. ${ }^{1} \mathrm{H}$ NMR $\left(500 \mathrm{MHz}, \mathrm{CDCl}_{3}\right) \delta 8.13(\mathrm{~s}, 1 \mathrm{H})$, $7.92(\mathrm{~d}, J=7.5 \mathrm{~Hz}, 2 \mathrm{H}), 7.87$ (d, $J=8.7 \mathrm{~Hz}, 2 \mathrm{H}), 7.79$ (d, $J=8.7 \mathrm{~Hz}, 2 \mathrm{H}), 7.62$ (t, $J=7.4 \mathrm{~Hz}$, $1 \mathrm{H}), 7.54(\mathrm{t}, J=7.6 \mathrm{~Hz}, 2 \mathrm{H}), 2.73(\mathrm{~s}, 6 \mathrm{H}) .{ }^{13} \mathrm{C} \mathrm{NMR}\left(125 \mathrm{MHz}, \mathrm{CDCl}_{3}\right) \delta 166.0,142.0,134.2$, $132.4,130.6,129.1,129.0,127.2,119.9,38.0$.

\section{$N$-(o-Tolyl)benzamide (3g, Figure 2)}

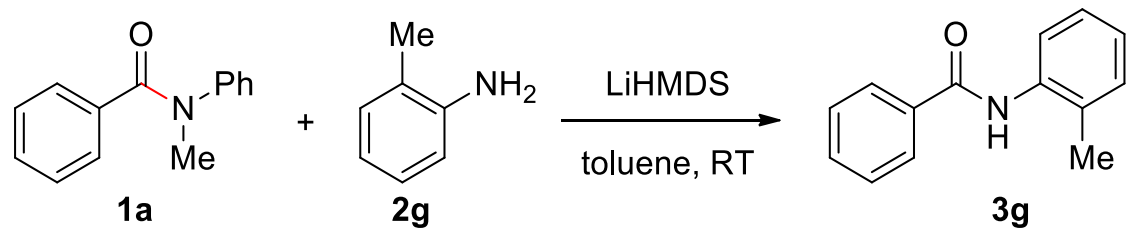

According to the general procedure, the reaction of $N$-methyl- $N$-phenylbenzamide $(0.10 \mathrm{mmol}$, 1.0 equiv), $o$-toluidine (2.0 equiv) and LiHMDS (1.0 M in THF, 3.0 equiv) in toluene ( $0.25 \mathrm{M})$ for $15 \mathrm{~h}$ at room temperature, afforded after work-up and chromatography the title compound in 
89\% yield (18.8 mg). White solid. ${ }^{1} \mathrm{H}$ NMR (500 MHz, $\left.\mathrm{CDCl}_{3}\right) \delta 7.97$ (d, J=7.8 Hz, $\left.1 \mathrm{H}\right), 7.92$ $(\mathrm{d}, J=7.4 \mathrm{~Hz}, 2 \mathrm{H}), 7.72(\mathrm{~s}, 1 \mathrm{H}), 7.59(\mathrm{t}, J=7.3 \mathrm{~Hz}, 1 \mathrm{H}), 7.53(\mathrm{t}, J=7.4 \mathrm{~Hz}, 2 \mathrm{H}), 7.32-7.24$ $(\mathrm{m}, 2 \mathrm{H}), 7.15(\mathrm{t}, J=7.4 \mathrm{~Hz}, 1 \mathrm{H}), 2.37(\mathrm{~s}, 3 \mathrm{H}) .{ }^{13} \mathrm{C} \mathrm{NMR}\left(125 \mathrm{MHz}, \mathrm{CDCl}_{3}\right) \delta 165.7,135.8$, $135.1,131.9,130.6,129.2,128.9,127.1,126.9,125.4,123.1,17.9$.

\section{$N$-([1,1'-Biphenyl]-2-yl)benzamide (3h, Figure 2)}

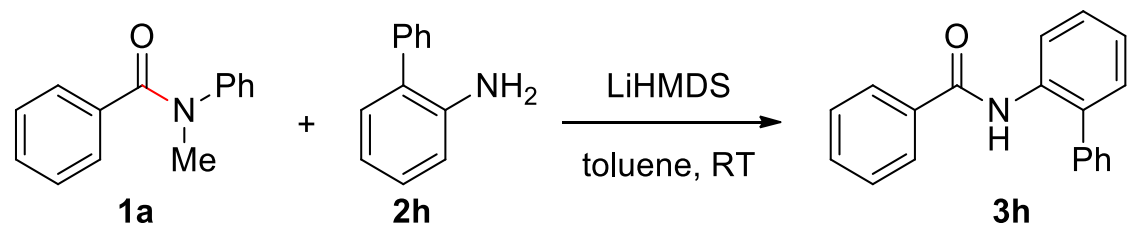

According to the general procedure, the reaction of $N$-methyl- $N$-phenylbenzamide $(0.10 \mathrm{mmol}$, 1.0 equiv), [1,1'-biphenyl]-2-amine (2.0 equiv) and LiHMDS (1.0 $\mathrm{M}$ in THF, 3.0 equiv) in toluene $(0.25 \mathrm{M})$ for $15 \mathrm{~h}$ at room temperature, afforded after work-up and chromatography the title compound in $85 \%$ yield $(23.2 \mathrm{mg})$. White solid. ${ }^{1} \mathrm{H}$ NMR $\left(500 \mathrm{MHz}, \mathrm{CDCl}_{3}\right) \delta 8.57(\mathrm{~d}, J=$ $8.2 \mathrm{~Hz}, 1 \mathrm{H}), 8.04(\mathrm{~s}, 1 \mathrm{H}), 7.63(\mathrm{~d}, J=7.8 \mathrm{~Hz}, 2 \mathrm{H}), 7.58-7.45(\mathrm{~m}, 7 \mathrm{H}), 7.41(\mathrm{t}, J=7.3 \mathrm{~Hz}, 2$ $\mathrm{H}), 7.33(\mathrm{~d}, J=7.5 \mathrm{~Hz}, 1 \mathrm{H}), 7.25(\mathrm{t}, J=7.4 \mathrm{~Hz}, 1 \mathrm{H}) .{ }^{13} \mathrm{C} \mathrm{NMR}\left(125 \mathrm{MHz}, \mathrm{CDCl}_{3}\right) \delta 165.0$, 138.1, 135.0, 134.8, 132.4, 131.8, 130.0, 129.4, 129.3, 128.8, 128.6, 128.2, 126.8, 124.4, 121.2.

\section{$N$-(2,6-Dimethylphenyl)benzamide (3i, Figure 2)}

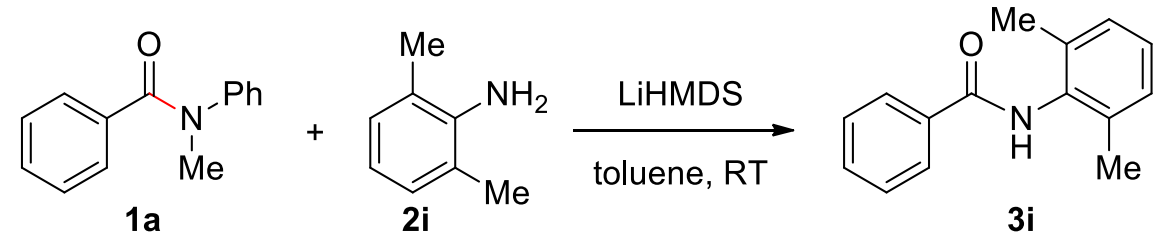

According to the general procedure, the reaction of $N$-methyl- $N$-phenylbenzamide $(0.10$ mmol, 1.0 equiv), 2,6-dimethylaniline (3.0 equiv) and LiHMDS (1.0 M in THF, 4.0 equiv) in toluene $(0.25 \mathrm{M})$ for $15 \mathrm{~h}$ at room temperature, afforded after work-up and chromatography the title compound in $77 \%$ yield $(17.3 \mathrm{mg})$. White solid. ${ }^{1} \mathrm{H}$ NMR $\left(500 \mathrm{MHz}, \mathrm{CDCl}_{3}\right) \delta 7.94(\mathrm{~d}, J=7.6$ $\mathrm{Hz}, 2 \mathrm{H}), 7.59$ (t, $J=7.3 \mathrm{~Hz}, 1 \mathrm{H}), 7.52$ (t, $J=7.6 \mathrm{~Hz}, 2 \mathrm{H}), 7.45$ (s, $1 \mathrm{H}), 7.20-7.12(\mathrm{~m}, 3 \mathrm{H})$, $2.30(\mathrm{~s}, 6 \mathrm{H}) .{ }^{13} \mathrm{C} \mathrm{NMR}\left(125 \mathrm{MHz}, \mathrm{CDCl}_{3}\right) \delta 165.7,135.4,134.4,133.7,131.7,128.7,128.2$, $127.3,127.1,18.4$. 


\section{$N$-(2,6-diisopropylphenyl)benzamide (3j, Figure 2)}

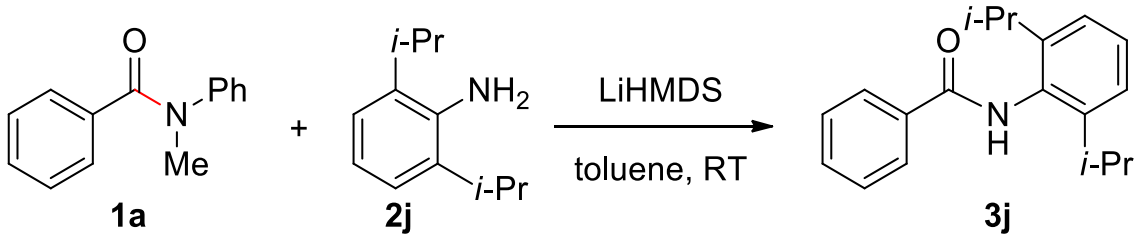

According to the general procedure, the reaction of $N$-methyl- $N$-phenylbenzamide $(0.10 \mathrm{mmol}$, 1.0 equiv), 2,6-diisopropylaniline (3.0 equiv) and LiHMDS (1.0 M in THF, 4.0 equiv) in toluene $(0.25 \mathrm{M})$ for $15 \mathrm{~h}$ at room temperature, afforded after work-up and chromatography the title compound in $75 \%$ yield $(21.0 \mathrm{mg})$. White solid. ${ }^{1} \mathrm{H}$ NMR $\left(500 \mathrm{MHz}, \mathrm{CDCl}_{3}\right) \delta 7.94(\mathrm{~d}, J=7.9$ $\mathrm{Hz}, 2 \mathrm{H}), 7.59$ (t, $J=7.4 \mathrm{~Hz}, 1 \mathrm{H}), 7.51(\mathrm{t}, J=7.6 \mathrm{~Hz}, 2 \mathrm{H}), 7.47(\mathrm{~s}, 1 \mathrm{H}), 7.37$ (t, $J=7.7 \mathrm{~Hz}, 1$ $\mathrm{H}), 7.26(\mathrm{~d}, J=7.7 \mathrm{~Hz}, 2 \mathrm{H}), 3.17(\mathrm{dt}, J=13.7,6.8 \mathrm{~Hz}, 2 \mathrm{H}), 1.25(\mathrm{~s}, 6 \mathrm{H}), 1.24(\mathrm{~s}, 6 \mathrm{H}) .{ }^{13} \mathrm{C}$ NMR $\left(125 \mathrm{MHz}, \mathrm{CDCl}_{3}\right) \delta 167.0,146.4,134.6,131.8,131.2,128.8,128.5,127.2,123.6,28.9$, 23.7 .

\section{$N$-(3-Chlorophenyl)benzamide (3k, Figure 2)}

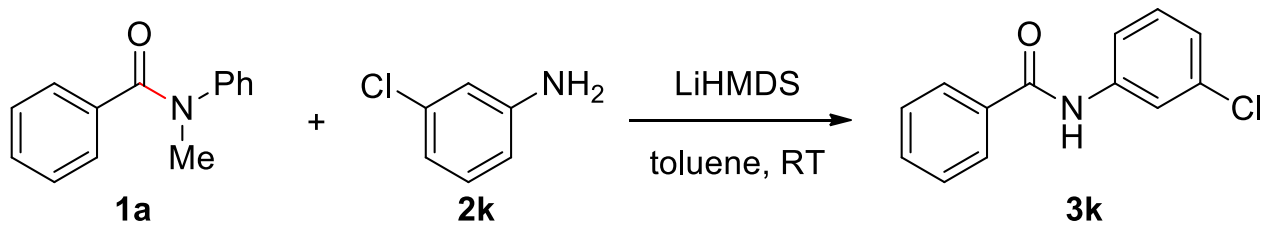

According to the general procedure, the reaction of $N$-methyl- $N$-phenylbenzamide $(0.10$ mmol, 1.0 equiv), 3-chloroaniline (2.0 equiv) and LiHMDS (1.0 M in THF, 3.0 equiv) in toluene (0.25 $\mathrm{M})$ for $15 \mathrm{~h}$ at room temperature, afforded after work-up and chromatography the title compound in $90 \%$ yield $(20.8 \mathrm{mg})$. White solid. ${ }^{1} \mathrm{H} \mathrm{NMR}\left(500 \mathrm{MHz}, \mathrm{CDCl}_{3}\right) \delta 7.97(\mathrm{~s}, 1 \mathrm{H}), 7.87(\mathrm{~d}, J=$ $7.4 \mathrm{~Hz}, 2 \mathrm{H}), 7.79$ (t, $J=1.7 \mathrm{~Hz}, 1 \mathrm{H}), 7.58$ (t, $J=7.4 \mathrm{~Hz}, 1 \mathrm{H}), 7.53-7.47$ (m, $3 \mathrm{H}), 7.30$ (t, $J=$ $6.7 \mathrm{~Hz}, 1 \mathrm{H}), 7.14(\mathrm{dd}, J=8.0,0.9 \mathrm{~Hz}, 1 \mathrm{H}) .{ }^{13} \mathrm{C} \mathrm{NMR}\left(125 \mathrm{MHz}, \mathrm{CDCl}_{3}\right) \delta 165.8,139.1,134.8$, 134.6, 132.1, 130.1, 128.9, 127.1, 124.6, 120.4, 118.2.

\section{$N$-(3-(Trifluoromethyl)phenyl)benzamide (3l, Figure 2)}

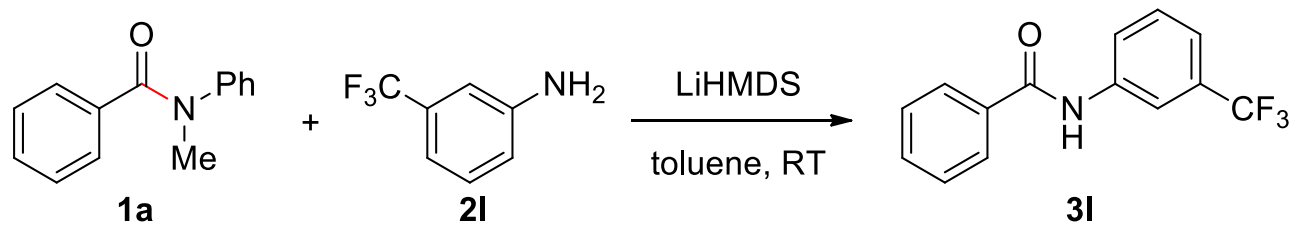


According to the general procedure, the reaction of $N$-methyl- $N$-phenylbenzamide $(0.10 \mathrm{mmol}$, 1.0 equiv), 3-(trifluoromethyl)aniline (2.0 equiv) and LiHMDS (1.0 M in THF, 3.0 equiv) in toluene $(0.25 \mathrm{M})$ for $15 \mathrm{~h}$ at room temperature, afforded after work-up and chromatography the title compound in $85 \%$ yield $(22.5 \mathrm{mg})$. White solid. ${ }^{1} \mathrm{H}$ NMR $\left(500 \mathrm{MHz}, \mathrm{CDCl}_{3}\right) \delta 8.07(\mathrm{~s}, 1 \mathrm{H})$, 7.96 (s, $1 \mathrm{H}), 7.89$ (d, $J=7.3 \mathrm{~Hz}, 3 \mathrm{H}), 7.59$ (t, $J=7.4 \mathrm{~Hz}, 1 \mathrm{H}), 7.50$ (dd, $J=12.8,7.6 \mathrm{~Hz}, 3 \mathrm{H})$, $7.42(\mathrm{~d}, J=7.7 \mathrm{~Hz}, 1 \mathrm{H}) .{ }^{13} \mathrm{C} \mathrm{NMR}\left(125 \mathrm{MHz} \mathrm{CDCl}_{3}\right) \delta 165.9,138.5,134.4,132.2,131.5(\mathrm{q}, J$ $=32.8 \mathrm{~Hz}), 129.7,128.9,127.0,123.9\left(\mathrm{q}, J^{F}=272.5 \mathrm{~Hz}\right), 123.3,121.1\left(\mathrm{q}, J^{F}=3.6 \mathrm{~Hz}\right), 117.0(\mathrm{q}$, $\left.J^{F}=3.8 \mathrm{~Hz}\right) .{ }^{19} \mathrm{~F}$ NMR $\left(471 \mathrm{MHz}, \mathrm{CDCl}_{3}\right) \delta-62.75(\mathrm{~s})$.

\section{$N$-(9-Ethyl-9H-carbazol-3-yl)benzamide (3m, Figure 2)}

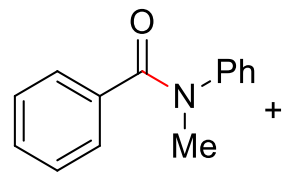

1a<smiles>CCn1c2ccccc2c2cc(N)ccc21</smiles>

$2 m \quad$ Et

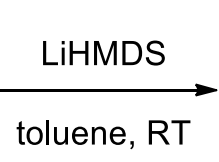

toluene, RT

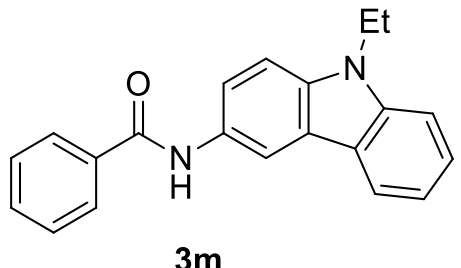

$3 m$

According to the general procedure, the reaction of $N$-methyl- $N$-phenylbenzamide $(0.10 \mathrm{mmol}$, 1.0 equiv), 9-ethyl-9H-carbazol-3-amine (2.0 equiv) and LiHMDS (1.0 $\mathrm{M}$ in THF, 3.0 equiv) in toluene $(0.25 \mathrm{M})$ for $15 \mathrm{~h}$ at room temperature, afforded after work-up and chromatography the title compound in $70 \%$ yield $(22.0 \mathrm{mg})$. White solid. ${ }^{1} \mathrm{H}$ NMR $\left(500 \mathrm{MHz}, \mathrm{CDCl}_{3}\right) \delta 8.42(\mathrm{~s}, 1 \mathrm{H})$, $8.05(\mathrm{~d}, J=7.7 \mathrm{~Hz}, 1 \mathrm{H}), 8.01(\mathrm{~s}, 1 \mathrm{H}), 7.92(\mathrm{~d}, J=7.5 \mathrm{~Hz}, 2 \mathrm{H}), 7.61(\mathrm{~d}, J=8.4 \mathrm{~Hz}, 1 \mathrm{H}), 7.53$ $(\mathrm{t}, J=7.2 \mathrm{~Hz}, 1 \mathrm{H}), 7.50-7.44(\mathrm{~m}, 3 \mathrm{H}), 7.39-7.34(\mathrm{~m}, 2 \mathrm{H}), 7.20(\mathrm{t}, J=7.4 \mathrm{~Hz}, 1 \mathrm{H}), 4.32(\mathrm{t}, J=$ $7.2 \mathrm{~Hz}, 2 \mathrm{H}), 1.40(\mathrm{t}, J=7.2 \mathrm{~Hz}, 3 \mathrm{H}) .{ }^{13} \mathrm{C} \mathrm{NMR}\left(125 \mathrm{MHz}, \mathrm{CDCl}_{3}\right) \delta 165.9,140.6,137.5$, $135.4,131.7,129.8,128.9,127.1,126.0,123.2,122.9$, 120.8, 119.8, 118.9, 113.3, 108.7, 37.7, 13.9.

\section{$N$-(Quinolin-8-yl)benzamide (3n, Figure 2)}<smiles>CN(C(=O)c1ccccc1)c1ccccc1</smiles>

$1 \mathrm{a}$<smiles>Nc1cccc2cccnc12</smiles>

2n

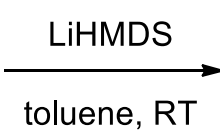

toluene, RT<smiles>O=C(Nc1cccc2cccnc12)c1ccccc1</smiles>

$3 n$

According to the general procedure, the reaction of $N$-methyl- $N$-phenylbenzamide $(0.10 \mathrm{mmol}$, 1.0 equiv), quinolin-8-amine (2.0 equiv) and LiHMDS (1.0 $\mathrm{M}$ in THF, 3.0 equiv) in toluene 
$(0.25 \mathrm{M})$ for $15 \mathrm{~h}$ at room temperature, afforded after work-up and chromatography the title compound in $75 \%$ yield $(18.6 \mathrm{mg})$. White solid. ${ }^{1} \mathrm{H}$ NMR $\left(500 \mathrm{MHz}, \mathrm{CDCl}_{3}\right) \delta 10.78(\mathrm{~s}, 1 \mathrm{H})$, $8.97(\mathrm{dd}, J=7.6,1.2 \mathrm{~Hz}, 1 \mathrm{H}), 8.88$ (dd, $J=4.2,1.6 \mathrm{~Hz}, 1 \mathrm{H}), 8.22(\mathrm{dd}, J=8.3,1.6 \mathrm{~Hz}, 1 \mathrm{H})$, 8.15-8.09 (m, $2 \mathrm{H}), 7.66-7.55(\mathrm{~m}, 5 \mathrm{H}), 7.51$ (dd, $J=8.2,4.2 \mathrm{~Hz}, 1 \mathrm{H}) .{ }^{13} \mathrm{C} \mathrm{NMR}(125 \mathrm{MHz}$, $\left.\mathrm{CDCl}_{3}\right) \delta 165.5,148.3,138.8,136.4,135.2,134.7,131.9,128.8,128.0,127.5,127.3,121.7$, 116.6.

\section{$N$-(Pyridin-2-yl)benzamide (3o, Figure 2)}

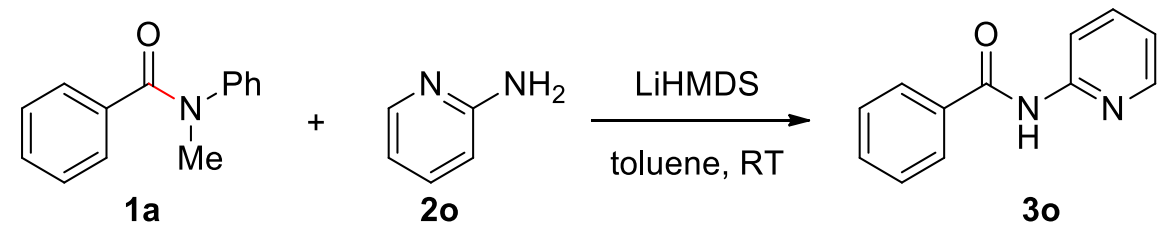

According to the general procedure, the reaction of $N$-methyl- $N$-phenylbenzamide $(0.10 \mathrm{mmol}$, 1.0 equiv), pyridin-2-amine (2.0 equiv) and LiHMDS (1.0 M in THF, 3.0 equiv) in toluene (0.25 M) for $15 \mathrm{~h}$ at room temperature, afforded after work-up and chromatography the title compound in 70\% yield $(13.9 \mathrm{mg})$. White solid. ${ }^{1} \mathrm{H} \mathrm{NMR}\left(500 \mathrm{MHz}, \mathrm{CDCl}_{3}\right) \delta 8.82(\mathrm{~s}, 1 \mathrm{H}), 8.37(\mathrm{~d}, J=$ $8.4 \mathrm{~Hz}, 1 \mathrm{H}), 8.21(\mathrm{~d}, J=4.6 \mathrm{~Hz}, 1 \mathrm{H}), 7.90(\mathrm{~d}, J=7.9 \mathrm{~Hz}, 2 \mathrm{H}), 7.73(\mathrm{t}, J=7.8 \mathrm{~Hz}, 1 \mathrm{H}), 7.54$ $(\mathrm{t}, J=7.3 \mathrm{~Hz}, 1 \mathrm{H}), 7.46(\mathrm{t}, J=7.5 \mathrm{~Hz}, 2 \mathrm{H}), 7.05-7.00(\mathrm{~m}, 1 \mathrm{H}) .{ }^{13} \mathrm{C} \mathrm{NMR}\left(125 \mathrm{MHz}, \mathrm{CDCl}_{3}\right) \delta$ $165.7,151.5,147.8,138.4,134.2,132.1,128.7,127.1,119.8,114.1$.

\section{$N$-(3,5-Dimethylisoxazol-4-yl)benzamide (3p, Figure 2)}<smiles>CN(C(=O)c1ccccc1)c1ccccc1</smiles>

$1 \mathrm{a}$<smiles>Cc1noc(C)c1N</smiles>
$2 p$

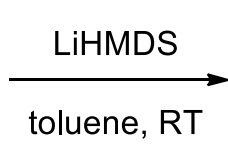

toluene, RT

According to the general procedure, the reaction of $N$-methyl- $N$-phenylbenzamide $(0.10 \mathrm{mmol}$, 1.0 equiv), 3,5-dimethylisoxazol-4-amine (2.0 equiv) and LiHMDS (1.0 M in THF, 3.0 equiv) in toluene $(0.25 \mathrm{M})$ for $15 \mathrm{~h}$ at room temperature, afforded after work-up and chromatography the title compound in $92 \%$ yield $(19.9 \mathrm{mg})$. White solid. ${ }^{1} \mathrm{H}$ NMR $\left(500 \mathrm{MHz}, \mathrm{CDCl}_{3}\right) \delta 7.94(\mathrm{~d}, J=$ $7.7 \mathrm{~Hz}, 2 \mathrm{H}), 7.64(\mathrm{t}, J=7.3 \mathrm{~Hz}, 1 \mathrm{H}), 7.58-7.51(\mathrm{~m}, 3 \mathrm{H}), 2.39$ (s, $3 \mathrm{H}), 2.24(\mathrm{~s}, 3 \mathrm{H}) .{ }^{13} \mathrm{C} \mathrm{NMR}$ $\left(125 \mathrm{MHz} \mathrm{CDCl}_{3}\right) \delta 166.5,164.0,157.9,133.2,132.3,128.8,127.3,113.4,11.2,9.7$. 


\section{Morpholino(phenyl)methanone (3q, Figure 2)}

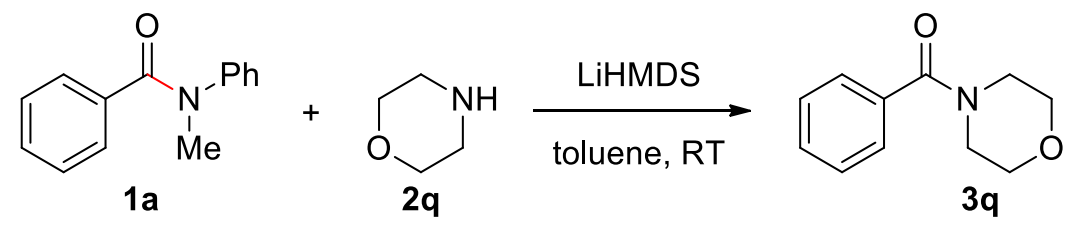

According to the general procedure, the reaction of $N$-methyl- $N$-phenylbenzamide $(0.10 \mathrm{mmol}$, 1.0 equiv), morpholine (2.0 equiv) and LiHMDS (1.0 M in THF, 3.0 equiv) in toluene (0.25 M) for $15 \mathrm{~h}$ at room temperature, afforded after work-up and chromatography the title compound in 95\% yield (18.1 mg). White solid. ${ }^{1} \mathrm{H}$ NMR (500 MHz, $\left.\mathrm{CDCl}_{3}\right) \delta 7.40(\mathrm{~s}, 5 \mathrm{H}), 3.84-3.36(\mathrm{~m}, 8$ H). ${ }^{13} \mathrm{C} \mathrm{NMR}\left(125 \mathrm{MHz}, \mathrm{CDCl}_{3}\right) \delta 170.3,135.1,129.7,128.4,126.9,66.7$.

\section{4-Methyl- $N$-(p-tolyl)benzamide (3r, Figure 2)}

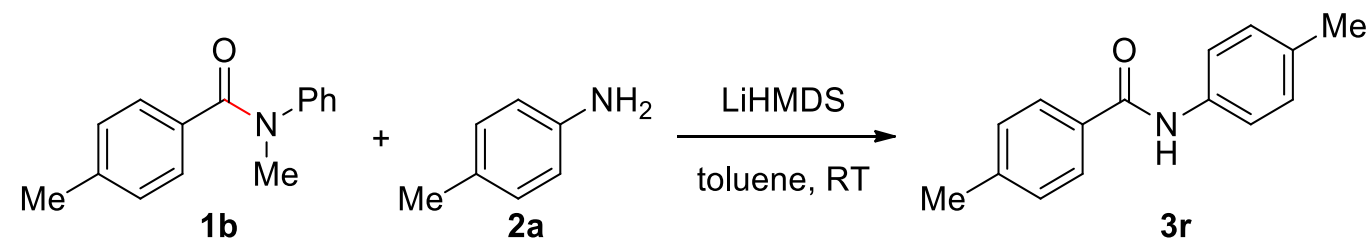

According to the general procedure, the reaction of $N, 4$-dimethyl- $N$-phenylbenzamide $(0.10$ mmol, 1.0 equiv), $p$-toluidine (2.0 equiv) and LiHMDS (1.0 M in THF, 3.0 equiv) in toluene $(0.25 \mathrm{M})$ for $15 \mathrm{~h}$ at room temperature, afforded after work-up and chromatography the title compound in $84 \%$ yield $(18.9 \mathrm{mg})$. White solid. ${ }^{1} \mathrm{H}$ NMR $\left(500 \mathrm{MHz}, \mathrm{CDCl}_{3}\right) \delta 7.83(\mathrm{~s}, 1 \mathrm{H})$, $7.78(\mathrm{~d}, J=7.8 \mathrm{~Hz}, 2 \mathrm{H}), 7.54(\mathrm{~d}, J=8.0 \mathrm{~Hz}, 2 \mathrm{H}), 7.29(\mathrm{~d}, J=7.4 \mathrm{~Hz}, 2 \mathrm{H}), 7.18(\mathrm{~d}, J=7.9 \mathrm{~Hz}$, $2 \mathrm{H}), 2.44(\mathrm{~s}, 3 \mathrm{H}), 2.36(\mathrm{~s}, 3 \mathrm{H}) .{ }^{13} \mathrm{C} \mathrm{NMR}\left(125 \mathrm{MHz}, \mathrm{CDCl}_{3}\right) \delta$ 165.6, 142.2, 135.5, 134.1, $132.2,129.6,129.6,129.4,127.0,120.3,21.5,20.9$.

\section{4-Methoxy- $N$-( $p$-tolyl)benzamide (3s, Figure 2$)$}<smiles>COc1ccc(C(=O)Nc2ccc(C(=O)N(C)c3ccc(C)cc3)cc2)cc1</smiles>

According to the general procedure, the reaction of 4-methoxy- $N$-methyl- $N$-phenylbenzamide (0.10 mmol, 1.0 equiv), $p$-toluidine (2.0 equiv) and LiHMDS (1.0 $\mathrm{M}$ in THF, 3.0 equiv) in toluene $(0.25 \mathrm{M})$ for $15 \mathrm{~h}$ at room temperature, afforded after work-up and chromatography the 
title compound in $80 \%$ yield $(19.3 \mathrm{mg})$. White solid. ${ }^{1} \mathrm{H}$ NMR $\left(500 \mathrm{MHz}, \mathrm{CDCl}_{3}\right) \delta 7.85(\mathrm{~d}, J=$ $8.3 \mathrm{~Hz}, 2 \mathrm{H}), 7.79$ (s, $1 \mathrm{H}), 7.53$ (d, $J=7.7 \mathrm{~Hz}, 2 \mathrm{H}), 7.18$ (d, $J=7.6 \mathrm{~Hz}, 2 \mathrm{H}), 6.97$ (d, $J=8.2$ $\mathrm{Hz}, 2 \mathrm{H}), 3.88$ (s, $3 \mathrm{H}), 2.35$ (s, $3 \mathrm{H}) .{ }^{13} \mathrm{C} \mathrm{NMR}\left(125 \mathrm{MHz}, \mathrm{CDCl}_{3}\right) \delta 165.2,162.4,135.6,134.0$, $129.6,128.9,127.3,120.3,113.9,55.5,20.9$.

\section{$N$-(p-Tolyl)-4-(trifluoromethyl)benzamide (3t, Figure 2)}

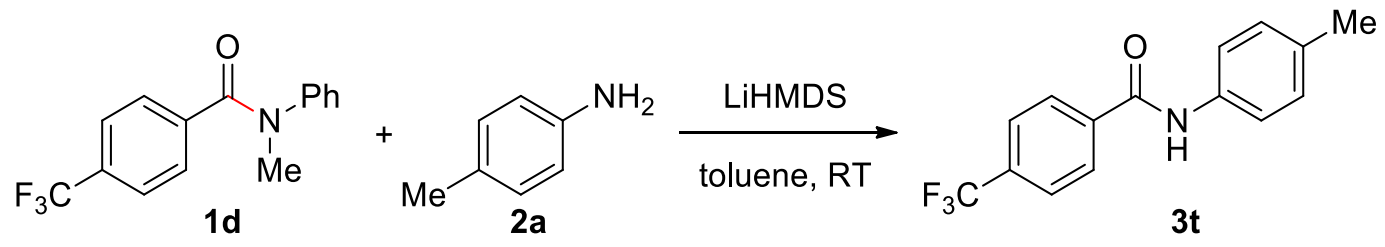

According to the general procedure, the reaction of $N$-methyl- $N$-phenyl-4-(trifluoromethyl) benzamide (0.10 mmol, 1.0 equiv), p-toluidine (2.0 equiv) and LiHMDS (1.0 M in THF, 3.0 equiv) in toluene $(0.25 \mathrm{M})$ for $15 \mathrm{~h}$ at room temperature, afforded after work-up and chromatography the title compound in $70 \%$ yield $(19.5 \mathrm{mg})$. White solid. ${ }^{1} \mathrm{H}$ NMR (500 MHz, $\left.\mathrm{CDCl}_{3}\right) \delta 8.00(\mathrm{~d}, J=7.8 \mathrm{~Hz}, 2 \mathrm{H}), 7.78(\mathrm{~d}, J=7.8 \mathrm{~Hz}, 2 \mathrm{H}), 7.75(\mathrm{~s}, 1 \mathrm{H}), 7.54$ (d, $J=7.4 \mathrm{~Hz}, 2$ $\mathrm{H}), 7.22(\mathrm{~d}, J=7.7 \mathrm{~Hz}, 2 \mathrm{H}), 2.38(\mathrm{~s}, 3 \mathrm{H}) .{ }^{13} \mathrm{C} \mathrm{NMR}\left(125 \mathrm{MHz}, \mathrm{CDCl}_{3}\right) \delta 164.3,138.4,134.9$, 134.8, $133.5\left(\mathrm{q}, J^{F}=32.7 \mathrm{~Hz}\right), 129.7,127.5,125.9\left(\mathrm{q}, J^{F}=3.6 \mathrm{~Hz}\right), 123.6\left(\mathrm{q}, J^{F}=272.5 \mathrm{~Hz}\right)$, 120.4, 20.9. ${ }^{19} \mathrm{~F}$ NMR (471 MHz, $\left.\mathrm{CDCl}_{3}\right) \delta-63.97$ (s).

\section{2-Methyl- $N$-(p-tolyl)benzamide (3u, Figure 2)}

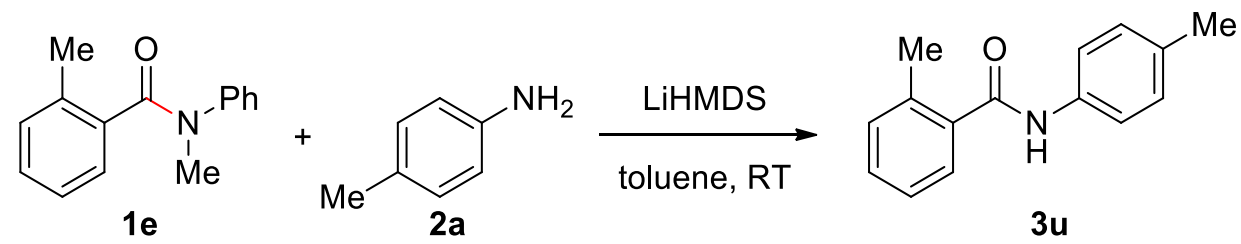

According to the general procedure, the reaction of $N, 2$-dimethyl- $N$-phenylbenzamide $(0.10$ mmol, 1.0 equiv), $p$-toluidine (2.0 equiv) and LiHMDS (1.0 M in THF, 3.0 equiv) in toluene $(0.25 \mathrm{M})$ for $15 \mathrm{~h}$ at room temperature, afforded after work-up and chromatography the title compound in $80 \%$ yield $(18.0 \mathrm{mg})$. White solid. ${ }^{1} \mathrm{H}$ NMR $\left(500 \mathrm{MHz}, \mathrm{CDCl}_{3}\right) \delta$ 7.53-7.48 (m, 4 H), $7.37(\mathrm{t}, J=7.3 \mathrm{~Hz}, 1 \mathrm{H}), 7.27(\mathrm{dd}, J=12.2,6.1 \mathrm{~Hz}, 2 \mathrm{H}), 7.19(\mathrm{~d}, J=7.6 \mathrm{~Hz}, 2 \mathrm{H}), 2.52(\mathrm{~s}, 3$ $\mathrm{H}), 2.37$ (s, $3 \mathrm{H}) .{ }^{13} \mathrm{C} \mathrm{NMR}\left(125 \mathrm{MHz}, \mathrm{CDCl}_{3}\right) \delta 168.0,136.6,136.4,135.5,134.2,131.2,130.2$, 129.6, 126.6, 125.9, 120.0, 20.9, 19.8. 


\section{3-Bromo- $N$-(p-tolyl)benzamide (3v, Figure 2$)$}<smiles>CN(C(=O)c1cccc(Br)c1)c1ccccc1</smiles>

$1 f$<smiles>Cc1ccc(N)cc1</smiles>

2a

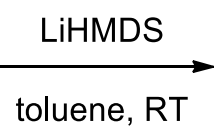

toluene, RT<smiles>Cc1ccc(NC(=O)c2cccc(Br)c2)cc1</smiles>

According to the general procedure, the reaction of 3-bromo- $N$-methyl- $N$-phenylbenzamide $(0.10$ mmol, 1.0 equiv), $p$-toluidine (2.0 equiv) and LiHMDS (1.0 M in THF, 3.0 equiv) in toluene $(0.25 \mathrm{M})$ for $15 \mathrm{~h}$ at room temperature, afforded after work-up and chromatography the title compound in 90\% yield (26.1 mg). White solid. ${ }^{1} \mathrm{H}$ NMR $\left(500 \mathrm{MHz}, \mathrm{CDCl}_{3}\right) \delta 8.00(\mathrm{~s}, 1 \mathrm{H})$, $7.90(\mathrm{~s}, 1 \mathrm{H}), 7.78(\mathrm{~d}, J=7.4 \mathrm{~Hz}, 1 \mathrm{H}), 7.67(\mathrm{~d}, J=7.8 \mathrm{~Hz}, 1 \mathrm{H}), 7.51(\mathrm{~d}, J=7.2 \mathrm{~Hz}, 2 \mathrm{H}), 7.34$ $(\mathrm{t}, J=7.5 \mathrm{~Hz}, 1 \mathrm{H}), 7.18(\mathrm{~d}, J=7.4 \mathrm{~Hz}, 2 \mathrm{H}), 2.36(\mathrm{~s}, 3 \mathrm{H}) .{ }^{13} \mathrm{C} \mathrm{NMR}\left(125 \mathrm{MHz}, \mathrm{CDCl}_{3}\right) \delta$ $164.3,137.1,135.0,134.7,134.6,130.3,129.6,125.6,122.9,120.5,20.9$.

\section{3,4-difluoro- $N$-( $p$-tolyl)benzamide (3w, Figure 2$)$}<smiles>CN(C(=O)c1ccc(F)c(F)c1)c1ccccc1</smiles>

$1 \mathrm{~g}$<smiles>Cc1ccc(N)cc1</smiles>

2a<smiles>Cc1ccc(NC(=O)c2ccc(F)c(F)c2)cc1</smiles>

According to the general procedure, the reaction of 3,4-difluoro- $N$-methyl- $N$-phenylbenzamide (0.10 mmol, 1.0 equiv), $p$-toluidine (2.0 equiv) and LiHMDS (1.0 $\mathrm{M}$ in THF, 3.0 equiv) in toluene $(0.25 \mathrm{M})$ for $15 \mathrm{~h}$ at room temperature, afforded after work-up and chromatography the title compound in $75 \%$ yield $(18.5 \mathrm{mg})$. New compound. White solid. $\mathrm{Mp}=166-167{ }^{\circ} \mathrm{C} .{ }^{1} \mathrm{H}$ NMR (500 MHz, $\left.\mathrm{CDCl}_{3}\right) \delta 7.74(\mathrm{~s}, 1 \mathrm{H}), 7.67-7.61(\mathrm{~m}, 1 \mathrm{H}), 7.55-7.49(\mathrm{~m}, 1 \mathrm{H}), 7.39(\mathrm{~d}, J=8.3$ $\mathrm{Hz}, 2 \mathrm{H}), 7.18-7.12(\mathrm{~m}, 1 \mathrm{H}), 7.08$ (d, $J=8.2 \mathrm{~Hz}, 2 \mathrm{H}), 2.26(\mathrm{~s}, 3 \mathrm{H}) .{ }^{13} \mathrm{C} \mathrm{NMR}(125 \mathrm{MHz}$, $\left.\mathrm{CDCl}_{3}\right) \delta 163.5,152.5\left(\mathrm{dd}, J^{F}=285.9,12.9 \mathrm{~Hz}\right), 150.5\left(\mathrm{dd}, J^{F}=282.0,12.9 \mathrm{~Hz}\right), 134.8\left(\mathrm{~d}, J^{F}=\right.$ $15.0 \mathrm{~Hz}), 132.1,129.7,123.4\left(\mathrm{q}, J^{F}=3.6 \mathrm{~Hz}\right), 120.5,117.6\left(\mathrm{~d}, J^{F}=17.9 \mathrm{~Hz}\right), 116.7\left(\mathrm{~d}, J^{F}=18.6\right.$ $\mathrm{Hz})$, 20.9. ${ }^{19} \mathrm{~F}$ NMR $\left(470 \mathrm{MHz}, \mathrm{CDCl}_{3}\right) \delta-132.02$, -135.61. HRMS calcd for $\mathrm{C}_{14} \mathrm{H}_{12} \mathrm{~F}_{2} \mathrm{NO}(\mathrm{M}+$ H) 248.0881, found 248.0898. 
$N$-(p-Tolyl)furan-2-carboxamide (3x, Figure 2)<smiles>CN(C(=O)c1ccco1)c1ccccc1</smiles>

$1 \mathrm{~h}$<smiles>Cc1ccc(N)cc1</smiles>

2a
LiHMDS toluene, RT<smiles>Cc1ccc(NC(=O)c2ccco2)cc1</smiles>

According to the general procedure, the reaction of $N$-methyl- $N$-phenylfuran-2-carboxamide (0.10 mmol, 1.0 equiv), $p$-toluidine (2.0 equiv) and LiHMDS (1.0 M in THF, 3.0 equiv) in toluene $(0.25 \mathrm{M})$ for $15 \mathrm{~h}$ at room temperature, afforded after work-up and chromatography the title compound in $91 \%$ yield $(18.3 \mathrm{mg})$. White solid. ${ }^{1} \mathrm{H} \mathrm{NMR}\left(500 \mathrm{MHz}, \mathrm{CDCl}_{3}\right) \delta 8.06(\mathrm{~s}, 1 \mathrm{H})$, 7.55 (d, $J=8.2 \mathrm{~Hz}, 2 \mathrm{H}), 7.52$ (s, $1 \mathrm{H}), 7.24$ (d, $J=3.2 \mathrm{~Hz}, 1 \mathrm{H}), 7.19$ (d, $J=8.1 \mathrm{~Hz}, 2 \mathrm{H}), 6.57$ $(\mathrm{d}, J=1.5 \mathrm{~Hz}, 1 \mathrm{H}), 2.36(\mathrm{~s}, 3 \mathrm{H}) .{ }^{13} \mathrm{C}$ NMR $\left(125 \mathrm{MHz}, \mathrm{CDCl}_{3}\right) \delta 156.0,148.0,144.1,134.8$, 134.2, 129.6, 120.0, 115.0, 112.6, 20.9.

\section{$N$-(p-Tolyl)decanamide (3y, Figure 2)}<smiles>CCC(C)(C)C(=O)N(C)c1ccccc1</smiles>
$1 \mathbf{i}$<smiles>Cc1ccc(N)cc1</smiles>

$2 a$

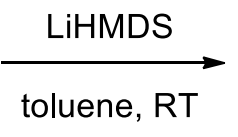

toluene, RT<smiles>CCC(C)(C)C(=O)Nc1ccc(C)cc1</smiles>

$3 y$

According to the general procedure, the reaction of $N$-methyl- $N$-phenyldecanamide $(0.10 \mathrm{mmol}$, 1.0 equiv), $p$-toluidine (2.0 equiv) and LiHMDS (1.0 M in THF, 3.0 equiv) in toluene ( $0.25 \mathrm{M})$ for $15 \mathrm{~h}$ at room temperature, afforded after work-up and chromatography the title compound in $78 \%$ yield (20.4 mg). White solid. ${ }^{1} \mathrm{H}$ NMR (500 MHz, $\left.\mathrm{CDCl}_{3}\right) \delta 7.41$ (d, $\left.J=7.9 \mathrm{~Hz}, 2 \mathrm{H}\right), 7.13$ (d, $J=7.8 \mathrm{~Hz}, 2 \mathrm{H}), 2.45-2.26$ (m, $5 \mathrm{H}), 1.73$ (dd, $J=14.8,7.5 \mathrm{~Hz}, 2 \mathrm{H}), 1.29$ (m, $12 \mathrm{H}), 0.90$ (t, $J=6.6 \mathrm{~Hz}, 3 \mathrm{H}) .{ }^{13} \mathrm{C} \mathrm{NMR}\left(125 \mathrm{MHz}, \mathrm{CDCl}_{3}\right) \delta 171.4,135.5,133.8,129.4,119.9,37.8,31.9$, 29.5, 29.4, 29.3, 29.3, 25.7, 22.7, 20.8, 14.1 .

\section{$N$-(p-Tolyl)isobutyramide (3z, Figure 2)}<smiles>CC(C)C(=O)N(C)c1ccccc1</smiles>

1j<smiles>Cc1ccc(N)cc1</smiles>

2a

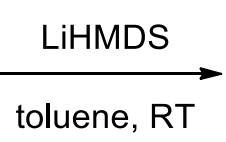

toluene, RT<smiles>Cc1ccc(NC(=O)C(C)C)cc1</smiles>

$3 z$ 
According to the general procedure, the reaction of $N$-methyl- $N$-phenylisobutyramide $(0.10$ mmol, 1.0 equiv), $p$-toluidine (2.0 equiv) and LiHMDS (1.0 M in THF, 3.0 equiv) in toluene $(0.25 \mathrm{M})$ for $15 \mathrm{~h}$ at room temperature, afforded after work-up and chromatography the title compound in $88 \%$ yield $(15.6 \mathrm{mg})$. White solid. ${ }^{1} \mathrm{H}$ NMR $\left(500 \mathrm{MHz}, \mathrm{CDCl}_{3}\right) \delta 7.43(\mathrm{~d}, J=7.9$ Hz, 2 H), $7.24(\mathrm{~s}, 1 \mathrm{H}), 7.13(\mathrm{~d}, J=7.9 \mathrm{~Hz}, 2 \mathrm{H}), 2.51(\mathrm{dt}, J=13.5,6.7 \mathrm{~Hz}, 1 \mathrm{H}), 2.33(\mathrm{~s}, 3 \mathrm{H})$, $1.27(\mathrm{~d}, J=6.7 \mathrm{~Hz}, 6 \mathrm{H}) .{ }^{13} \mathrm{C} \mathrm{NMR}\left(125 \mathrm{MHz}, \mathrm{CDCl}_{3}\right) \delta 175.1,135.5,133.7,129.4,119.9,36.6$, 20.8, 19.6.

\section{$N$-(p-Tolyl)cyclohexanecarboxamide (3aa, Figure 2)}<smiles>CN(C(=O)C1CCCCC1)c1ccccc1</smiles><smiles>Cc1ccc(N)cc1</smiles>

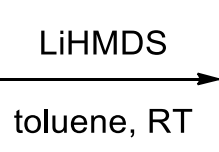

toluene, RT<smiles>Cc1ccc(NC(=O)C2CCCCC2)cc1</smiles>

According to the general procedure, the reaction of $N$-methyl- $N$-phenylcyclohexanecarboxamide (0.10 mmol, 1.0 equiv), p-toluidine (2.0 equiv) and LiHMDS (1.0 $\mathrm{M}$ in THF, 3.0 equiv) in toluene $(0.25 \mathrm{M})$ for $15 \mathrm{~h}$ at room temperature, afforded after work-up and chromatography the title compound in $87 \%$ yield $(18.9 \mathrm{mg})$. White solid. ${ }^{1} \mathrm{H}$ NMR $\left(500 \mathrm{MHz}, \mathrm{CDCl}_{3}\right) \delta 7.42(\mathrm{~d}, J=$ $7.7 \mathrm{~Hz}, 2 \mathrm{H}), 7.21(\mathrm{~s}, 1 \mathrm{H}), 7.13(\mathrm{~d}, J=7.7 \mathrm{~Hz}, 2 \mathrm{H}), 2.33(\mathrm{~s}, 3 \mathrm{H}), 2.23(\mathrm{t}, J=11.7 \mathrm{~Hz}, 1 \mathrm{H})$, $1.97(\mathrm{~d}, J=12.2 \mathrm{~Hz}, 2 \mathrm{H}), 1.85(\mathrm{~d}, J=10.1 \mathrm{~Hz}, 2 \mathrm{H}), 1.59-1.53(\mathrm{~m}, 2 \mathrm{H}), 1.38-1.26(\mathrm{~m}, 4 \mathrm{H}) .{ }^{13} \mathrm{C}$ NMR (125 MHz, $\left.\mathrm{CDCl}_{3}\right) \delta 174.2,135.6,133.7,129.4,119.9,46.5,29.7,25.7,20.8$.

\section{$N$-(p-Tolyl)pivalamide (3ab, Figure 2)}

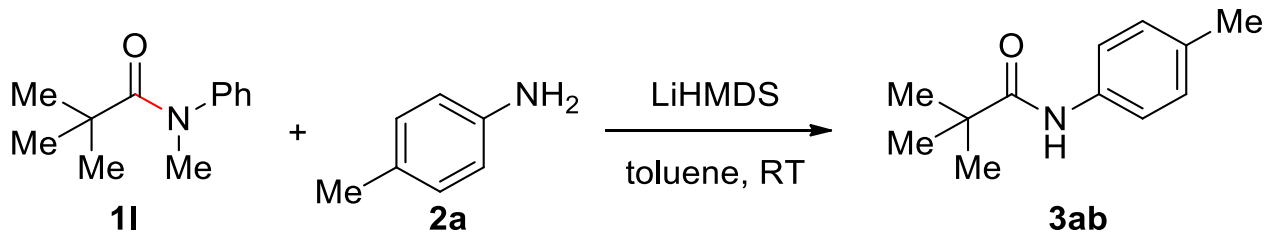

According to the general procedure, the reaction of $N$-methyl- $N$-phenylpivalamide $(0.10$ mmol, 1.0 equiv), $p$-toluidine (2.0 equiv) and LiHMDS (1.0 M in THF, 3.0 equiv) in toluene ( $0.25 \mathrm{M})$ for $15 \mathrm{~h}$ at room temperature, afforded after work-up and chromatography the title compound in $81 \%$ yield (15.5 mg). White solid. ${ }^{1} \mathrm{H}$ NMR (500 MHz, $\left.\mathrm{CDCl}_{3}\right) \delta 7.42$ (d, J=8.3 Hz, $\left.2 \mathrm{H}\right), 7.14$ 
$(\mathrm{d}, J=8.1 \mathrm{~Hz}, 2 \mathrm{H}), 2.33(\mathrm{~s}, 3 \mathrm{H}), 1.33(\mathrm{~s}, 9 \mathrm{H}) .{ }^{13} \mathrm{C} \mathrm{NMR}\left(125 \mathrm{MHz}, \mathrm{CDCl}_{3}\right) \delta$ 176.4, 135.5, $133.8,129.4,120.0,39.5,27.7,20.8$.

\section{$N$-(p-Tolyl)benzamide (3a, Figure 3)}

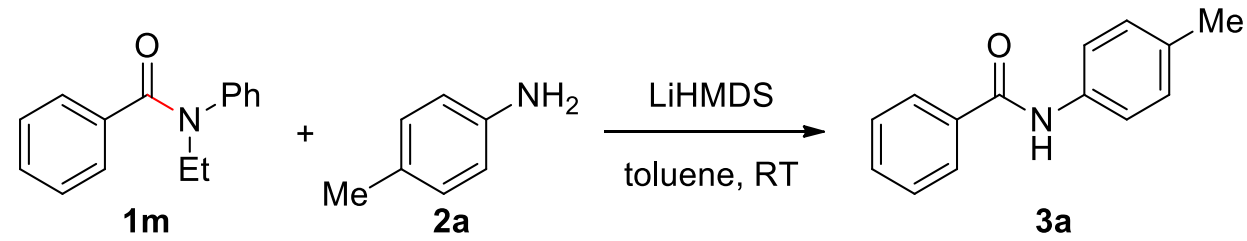

According to the general procedure, the reaction of $N$-ethyl- $N$-phenylbenzamide $(0.10 \mathrm{mmol}, 1.0$ equiv), $p$-toluidine (2.0 equiv) and LiHMDS (1.0 M in THF, 3.0 equiv) in toluene (0.25 M) for $15 \mathrm{~h}$ at room temperature, afforded after work-up and chromatography the title compound in $87 \%$ yield (18.4 mg). White solid. ${ }^{1} \mathrm{H}$ NMR (500 MHz, $\left.\mathrm{CDCl}_{3}\right) \delta 7.88(\mathrm{~d}, J=7.3 \mathrm{~Hz}, 2 \mathrm{H}), 7.83$ $(\mathrm{s}, 1 \mathrm{H}), 7.58-7.54(\mathrm{~m}, 3 \mathrm{H}), 7.50(\mathrm{t}, J=7.5 \mathrm{~Hz}, 2 \mathrm{H}), 7.19(\mathrm{~d}, J=8.2 \mathrm{~Hz}, 2 \mathrm{H}), 2.37(\mathrm{~s}, 3 \mathrm{H}) .{ }^{13} \mathrm{C}$ NMR (125 MHz, $\left.\mathrm{CDCl}_{3}\right) \delta 165.7,135.4,135.1,134.3,131.7,129.6,128.8,127.0,120.3,20.9$.

\section{$N$-(p-Tolyl)benzamide (3a, Figure 3)}

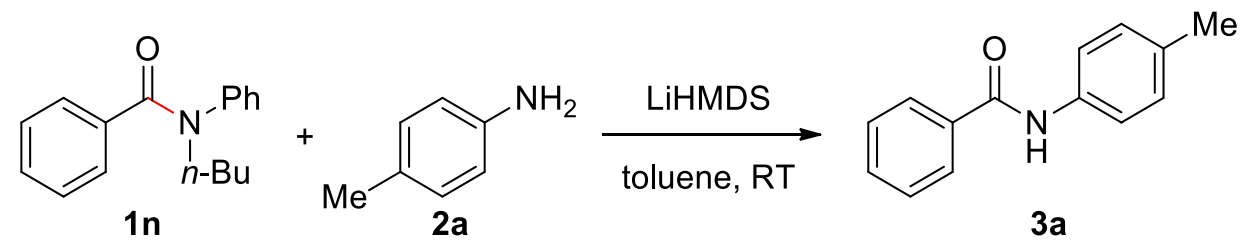

According to the general procedure, the reaction of $N$-butyl- $N$-phenylbenzamide $(0.10 \mathrm{mmol}, 1.0$ equiv), $p$-toluidine (2.0 equiv) and LiHMDS (1.0 M in THF, 3.0 equiv) in toluene (0.25 M) for $15 \mathrm{~h}$ at room temperature, afforded after work-up and chromatography the title compound in $85 \%$ yield (17.9 mg). White solid. ${ }^{1} \mathrm{H}$ NMR (500 MHz, $\left.\mathrm{CDCl}_{3}\right) \delta 7.88(\mathrm{~d}, J=7.3 \mathrm{~Hz}, 2 \mathrm{H}), 7.83$ (s, $1 \mathrm{H}), 7.58-7.54(\mathrm{~m}, 3 \mathrm{H}), 7.50(\mathrm{t}, J=7.5 \mathrm{~Hz}, 2 \mathrm{H}), 7.19(\mathrm{~d}, J=8.2 \mathrm{~Hz}, 2 \mathrm{H}), 2.37(\mathrm{~s}, 3 \mathrm{H}) .{ }^{13} \mathrm{C}$ NMR (125 MHz, $\left.\mathrm{CDCl}_{3}\right) \delta 165.7,135.4,135.1,134.3,131.7,129.6,128.8,127.0,120.3,20.9$. 


\section{$N$-(p-Tolyl)benzamide (3a, Figure 3)}

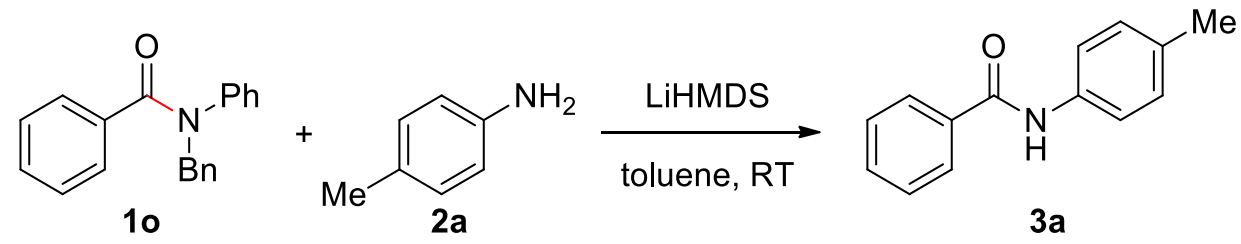

According to the general procedure, the reaction of $N$-benzyl- $N$-phenylbenzamide $(0.10 \mathrm{mmol}$, 1.0 equiv), $p$-toluidine (2.0 equiv) and LiHMDS (1.0 $\mathrm{M}$ in THF, 3.0 equiv) in toluene ( $0.25 \mathrm{M})$ for $15 \mathrm{~h}$ at room temperature, afforded after work-up and chromatography the title compound in $89 \%$ yield (18.8 mg). White solid. ${ }^{1} \mathrm{H}$ NMR $\left(500 \mathrm{MHz}, \mathrm{CDCl}_{3}\right) \delta 7.88(\mathrm{~d}, J=7.3 \mathrm{~Hz}, 2 \mathrm{H}), 7.83$ (s, $1 \mathrm{H}), 7.58-7.54(\mathrm{~m}, 3 \mathrm{H}), 7.50$ (t, $J=7.5 \mathrm{~Hz}, 2 \mathrm{H}), 7.19$ (d, $J=8.2 \mathrm{~Hz}, 2 \mathrm{H}), 2.37$ (s, $3 \mathrm{H}) .{ }^{13} \mathrm{C}$ NMR (125 MHz, $\left.\mathrm{CDCl}_{3}\right) \delta 165.7,135.4,135.1,134.3,131.7,129.6,128.8,127.0,120.3,20.9$.

\section{$N$-(p-Tolyl)benzamide (3a, Figure 3)}

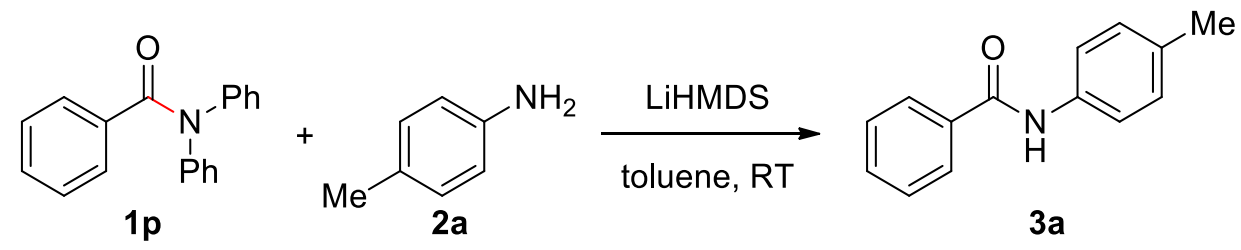

According to the general procedure, the reaction of $N, N$-diphenylbenzamide $(0.10 \mathrm{mmol}, 1.0$ equiv), $p$-toluidine (2.0 equiv) and LiHMDS (1.0 $\mathrm{M}$ in THF, 3.0 equiv) in toluene (0.25 M) for $15 \mathrm{~h}$ at room temperature, afforded after work-up and chromatography the title compound in 92\% yield (19.4 mg). White solid. ${ }^{1} \mathrm{H}$ NMR (500 MHz, $\left.\mathrm{CDCl}_{3}\right) \delta 7.88(\mathrm{~d}, J=7.3 \mathrm{~Hz}, 2 \mathrm{H}), 7.83$ (s, $1 \mathrm{H}), 7.58-7.54$ (m, $3 \mathrm{H}), 7.50$ (t, $J=7.5 \mathrm{~Hz}, 2 \mathrm{H}), 7.19$ (d, $J=8.2 \mathrm{~Hz}, 2 \mathrm{H}), 2.37$ (s, $3 \mathrm{H}) .{ }^{13} \mathrm{C}$ NMR (125 MHz, $\left.\mathrm{CDCl}_{3}\right) \delta 165.7,135.4,135.1,134.3,131.7,129.6,128.8,127.0,120.3,20.9$.

\section{$N$-Phenylbenzamide (3ac, Figure 3)}

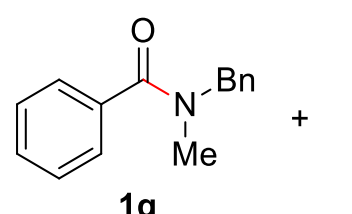

$1 q$<smiles>Nc1ccccc1</smiles><smiles>CO[AsH3]</smiles>
toluene, $60^{\circ} \mathrm{C}$<smiles>O=C(Nc1ccccc1)c1ccccc1</smiles>

According to the general procedure, the reaction of $N$-benzyl- $N$-methylbenzamide $(0.10 \mathrm{mmol}$, 1.0 equiv), aniline (2.0 equiv) and LiHMDS (1.0 M in THF, 3.0 equiv) in toluene $(0.25 \mathrm{M})$ for $15 \mathrm{~h}$ at $60{ }^{\circ} \mathrm{C}$, afforded after work-up and chromatography the title compound in $91 \%$ yield (17.9 
mg). White solid. ${ }^{1} \mathrm{H}$ NMR $\left(500 \mathrm{MHz}, \mathrm{CDCl}_{3}\right) \delta 7.78(\mathrm{~d}, J=7.7 \mathrm{~Hz}, 3 \mathrm{H}), 7.56(\mathrm{~d}, J=8.0 \mathrm{~Hz}, 2$ H), 7.46 (t, $J=7.3 \mathrm{~Hz}, 1 \mathrm{H}), 7.39$ (t, $J=7.4 \mathrm{~Hz}, 2 \mathrm{H}), 7.29$ (t, $J=7.7 \mathrm{~Hz}, 2 \mathrm{H}), 7.07$ (t, $J=7.4$ $\mathrm{Hz}, 1 \mathrm{H}) .{ }^{13} \mathrm{C} \mathrm{NMR}\left(125 \mathrm{MHz}, \mathrm{CDCl}_{3}\right) \delta 165.7,137.9,135.0,131.8,129.1,128.8,127.0,124.5$, 120.2 .

\section{N-Phenylbenzamide (3ac, Figure 3)}

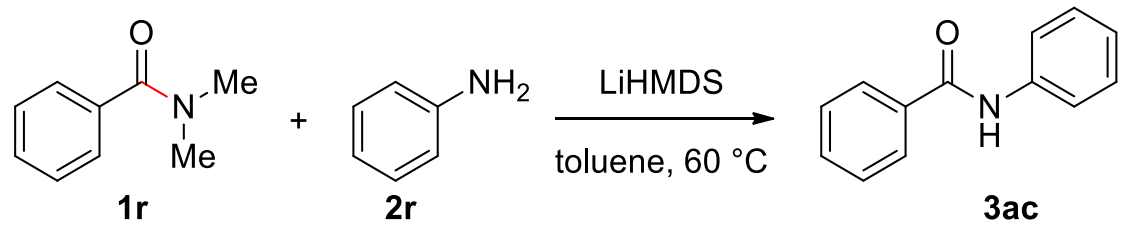

According to the general procedure, the reaction of $N, N$-dimethylbenzamide $(0.10 \mathrm{mmol}, 1.0$ equiv), aniline (2.0 equiv) and LiHMDS (1.0 M in THF, 3.0 equiv) in toluene (0.25 M) for $15 \mathrm{~h}$ at $60{ }^{\circ} \mathrm{C}$, afforded after work-up and chromatography the title compound in $86 \%$ yield (16.9 $\mathrm{mg}$ ). White solid. ${ }^{1} \mathrm{H}$ NMR $\left(500 \mathrm{MHz}, \mathrm{CDCl}_{3}\right) \delta 7.78(\mathrm{~d}, J=7.7 \mathrm{~Hz}, 3 \mathrm{H}), 7.56(\mathrm{~d}, J=8.0 \mathrm{~Hz}, 2 \mathrm{H})$, $7.46(\mathrm{t}, J=7.3 \mathrm{~Hz}, 1 \mathrm{H}), 7.39(\mathrm{t}, J=7.4 \mathrm{~Hz}, 2 \mathrm{H}), 7.29$ (t, $J=7.7 \mathrm{~Hz}, 2 \mathrm{H}), 7.07$ (t, $J=7.4 \mathrm{~Hz}, 1$ H). ${ }^{13} \mathrm{C} \mathrm{NMR}\left(125 \mathrm{MHz}, \mathrm{CDCl}_{3}\right) \delta 165.7,137.9,135.0,131.8,129.1,128.8,127.0,124.5,120.2$.

\section{N-Phenylbenzamide (3ac, Figure 3)}

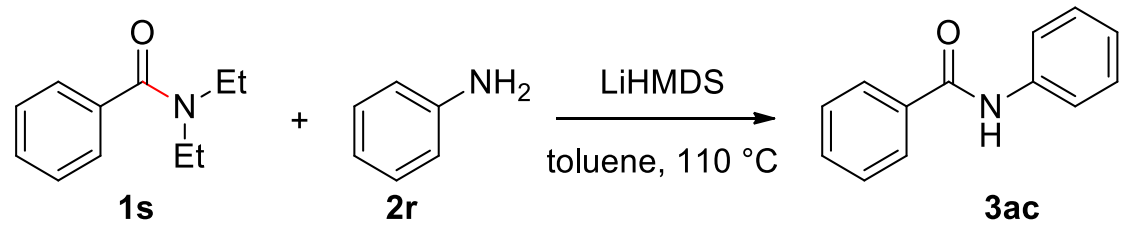

According to the general procedure, the reaction of $N, N$-diethylbenzamide $(0.10 \mathrm{mmol}, 1.0$ equiv), aniline (3.0 equiv) and LiHMDS (1.0 M in THF, 4.0 equiv) in toluene (0.25 M) for $15 \mathrm{~h}$ at $110{ }^{\circ} \mathrm{C}$, afforded after work-up and chromatography the title compound in $82 \%$ yield (16.1 mg). White solid. ${ }^{1} \mathrm{H}$ NMR $\left(500 \mathrm{MHz}, \mathrm{CDCl}_{3}\right) \delta 7.78(\mathrm{~d}, J=7.7 \mathrm{~Hz}, 3 \mathrm{H}), 7.56$ (d, J = 8.0 Hz, 2 H), $7.46(\mathrm{t}, J=7.3 \mathrm{~Hz}, 1 \mathrm{H}), 7.39$ (t, $J=7.4 \mathrm{~Hz}, 2 \mathrm{H}), 7.29$ (t, $J=7.7 \mathrm{~Hz}, 2 \mathrm{H}), 7.07$ (t, $J=7.4$ $\mathrm{Hz}, 1 \mathrm{H}) .{ }^{13} \mathrm{C} \mathrm{NMR}\left(125 \mathrm{MHz}, \mathrm{CDCl}_{3}\right) \delta 165.7,137.9,135.0,131.8,129.1,128.8,127.0,124.5$, 120.2 . 


\section{$N$-(p-Tolyl)benzamide (3a, Figure 3)}

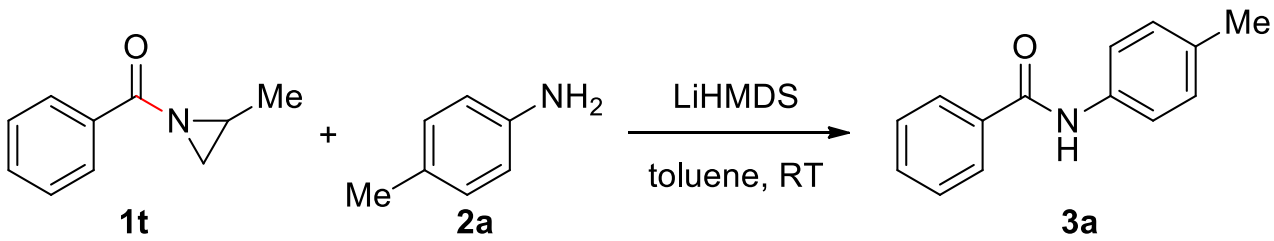

According to the general procedure, the reaction of (2-methylaziridin-1-yl)(phenyl)methanone (0.10 mmol, 1.0 equiv), $p$-toluidine (2.0 equiv) and LiHMDS (1.0 $\mathrm{M}$ in THF, 3.0 equiv) in toluene $(0.25 \mathrm{M})$ for $15 \mathrm{~h}$ at room temperature, afforded after work-up and chromatography the title compound in $91 \%$ yield $(19.2 \mathrm{mg})$. White solid. ${ }^{1} \mathrm{H}$ NMR $\left(500 \mathrm{MHz}, \mathrm{CDCl}_{3}\right) \delta 7.88(\mathrm{~d}, J=$ $7.3 \mathrm{~Hz}, 2 \mathrm{H}), 7.83$ (s, $1 \mathrm{H}), 7.58-7.54(\mathrm{~m}, 3 \mathrm{H}), 7.50$ (t, $J=7.5 \mathrm{~Hz}, 2 \mathrm{H}), 7.19$ (d, $J=8.2 \mathrm{~Hz}, 2$ H), 2.37 (s, $3 \mathrm{H}) .{ }^{13} \mathrm{C} \mathrm{NMR}\left(125 \mathrm{MHz}, \mathrm{CDCl}_{3}\right) \delta 165.7,135.4,135.1,134.3,131.7,129.6,128.8$, $127.0,120.3,20.9$.

\section{$N$-(p-Tolyl)benzamide (3a, Figure 3)}<smiles>O=C(c1ccccc1)N1CCC1</smiles><smiles>Cc1ccc(N)cc1</smiles>

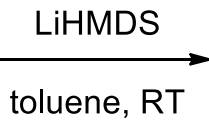
toluene, RT<smiles>Cc1ccc(NC(=O)c2ccccc2)cc1</smiles>

According to the general procedure, the reaction of azetidin-1-yl(phenyl)methanone $(0.10 \mathrm{mmol}$, 1.0 equiv), $p$-toluidine (2.0 equiv) and LiHMDS (1.0 M in THF, 3.0 equiv) in toluene ( $0.25 \mathrm{M}$ ) for $15 \mathrm{~h}$ at room temperature, afforded after work-up and chromatography the title compound in $82 \%$ yield (17.3 mg). White solid. ${ }^{1} \mathrm{H}$ NMR $\left(500 \mathrm{MHz}, \mathrm{CDCl}_{3}\right) \delta 7.88(\mathrm{~d}, J=7.3 \mathrm{~Hz}, 2 \mathrm{H}), 7.83$ (s, $1 \mathrm{H}), 7.58-7.54(\mathrm{~m}, 3 \mathrm{H}), 7.50$ (t, $J=7.5 \mathrm{~Hz}, 2 \mathrm{H}), 7.19$ (d, $J=8.2 \mathrm{~Hz}, 2 \mathrm{H}), 2.37$ (s, $3 \mathrm{H}) .{ }^{13} \mathrm{C}$ NMR (125 MHz, $\left.\mathrm{CDCl}_{3}\right) \delta 165.7,135.4,135.1,134.3,131.7,129.6,128.8,127.0,120.3,20.9$.

\section{$N$-(p-Tolyl)benzamide (3a, Figure 3)}<smiles>O=C(c1ccccc1)N1CCCC1</smiles><smiles>Cc1ccc(N)cc1</smiles>

$2 a$

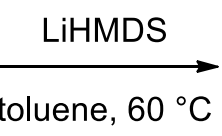

toluene, $60^{\circ} \mathrm{C}$<smiles>Cc1ccc(NC(=O)c2ccccc2)cc1</smiles>

According to the general procedure, the reaction of phenyl(pyrrolidin-1-yl)methanone $(0.10$ mmol, 1.0 equiv), $p$-toluidine (2.0 equiv) and LiHMDS (1.0 M in THF, 3.0 equiv) in toluene S211 
$(0.25 \mathrm{M})$ for $15 \mathrm{~h}$ at $60{ }^{\circ} \mathrm{C}$, afforded after work-up and chromatography the title compound in 92\% yield (19.4 mg). ${ }^{1} \mathrm{H} \mathrm{NMR}\left(500 \mathrm{MHz}, \mathrm{CDCl}_{3}\right) \delta 7.88(\mathrm{~d}, J=7.3 \mathrm{~Hz}, 2 \mathrm{H}), 7.83(\mathrm{~s}, 1 \mathrm{H})$, 7.58-7.54 (m, $3 \mathrm{H}), 7.50(\mathrm{t}, J=7.5 \mathrm{~Hz}, 2 \mathrm{H}), 7.19(\mathrm{~d}, J=8.2 \mathrm{~Hz}, 2 \mathrm{H}), 2.37(\mathrm{~s}, 3 \mathrm{H}) .{ }^{13} \mathrm{C} \mathrm{NMR}$ $\left(125 \mathrm{MHz}, \mathrm{CDCl}_{3}\right) \delta 165.7,135.4,135.1,134.3,131.7,129.6,128.8,127.0,120.3,20.9$.

\section{$N-(p$-Tolyl)benzamide (3a, Figure 3)}<smiles>O=C(c1ccccc1)N1CCCCC1</smiles><smiles>Cc1ccc(N)cc1</smiles>

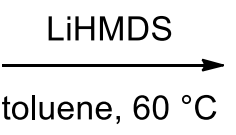

toluene, $60^{\circ} \mathrm{C}$<smiles>Cc1ccc(NC(=O)c2ccccc2)cc1</smiles>

According to the general procedure, the reaction of phenyl(piperidin-1-yl)methanone $(0.10$ mmol, 1.0 equiv), $p$-toluidine (2.0 equiv) and LiHMDS (1.0 M in THF, 3.0 equiv) in toluene $(0.25 \mathrm{M})$ for $15 \mathrm{~h}$ at $60{ }^{\circ} \mathrm{C}$, afforded after work-up and chromatography the title compound in 94\% yield (19.8 mg). ${ }^{1} \mathrm{H} \mathrm{NMR}\left(500 \mathrm{MHz}, \mathrm{CDCl}_{3}\right) \delta 7.88(\mathrm{~d}, J=7.3 \mathrm{~Hz}, 2 \mathrm{H}), 7.83(\mathrm{~s}, 1 \mathrm{H})$, 7.58-7.54 (m, $3 \mathrm{H}), 7.50(\mathrm{t}, J=7.5 \mathrm{~Hz}, 2 \mathrm{H}), 7.19(\mathrm{~d}, J=8.2 \mathrm{~Hz}, 2 \mathrm{H}), 2.37(\mathrm{~s}, 3 \mathrm{H}) .{ }^{13} \mathrm{C} \mathrm{NMR}$ $\left(125 \mathrm{MHz} \mathrm{CDCl}_{3}\right) \delta 165.7,135.4,135.1,134.3,131.7,129.6,128.8,127.0,120.3,20.9$.

\section{$N$-Phenylbenzamide (3ac, Figure 3)}<smiles>O=C(c1ccccc1)N1CCOCC1</smiles><smiles>Nc1ccccc1</smiles>

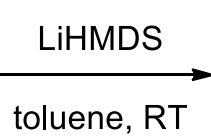

toluene, RT<smiles>O=C(Nc1ccccc1)c1ccccc1</smiles>

3ac

According to the general procedure, the reaction of morpholino(phenyl)methanone $(0.10 \mathrm{mmol}$, 1.0 equiv), aniline (2.0 equiv) and LiHMDS (1.0 M in THF, 3.0 equiv) in toluene ( $0.25 \mathrm{M})$ for $15 \mathrm{~h}$ at room temperature, afforded after work-up and chromatography the title compound in $82 \%$ yield (16.2 mg). White solid. ${ }^{1} \mathrm{H}$ NMR (500 MHz, $\left.\mathrm{CDCl}_{3}\right) \delta 7.78(\mathrm{~d}, J=7.7 \mathrm{~Hz}, 3 \mathrm{H}), 7.56$ $(\mathrm{d}, J=8.0 \mathrm{~Hz}, 2 \mathrm{H}), 7.46(\mathrm{t}, J=7.3 \mathrm{~Hz}, 1 \mathrm{H}), 7.39$ (t, $J=7.4 \mathrm{~Hz}, 2 \mathrm{H}), 7.29(\mathrm{t}, J=7.7 \mathrm{~Hz}, 2 \mathrm{H})$, $7.07(\mathrm{t}, J=7.4 \mathrm{~Hz}, 1 \mathrm{H}) .{ }^{13} \mathrm{C} \mathrm{NMR}\left(125 \mathrm{MHz}, \mathrm{CDCl}_{3}\right) \delta 165.7,137.9,135.0,131.8,129.1$, $128.8,127.0,124.5,120.2$. 


\section{$N$-Phenylbenzamide (3ac, Figure 3)}

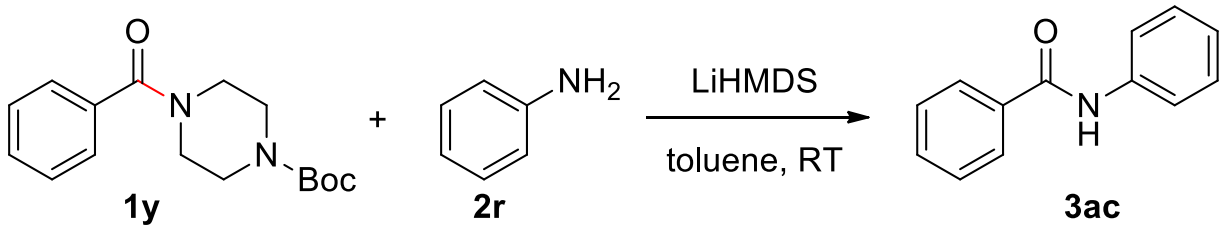

According to the general procedure, the reaction of tert-butyl 4-benzoylpiperazine-1-carboxylate (0.10 mmol, 1.0 equiv), aniline (2.0 equiv) and LiHMDS (1.0 M in THF, 3.0 equiv) in toluene $(0.25 \mathrm{M})$ for $15 \mathrm{~h}$ at room temperature, afforded after work-up and chromatography the title compound in $75 \%$ yield $(14.8 \mathrm{mg})$. White solid. ${ }^{1} \mathrm{H}$ NMR (500 MHz, $\left.\mathrm{CDCl}_{3}\right) \delta 7.78(\mathrm{~d}, J=7.7$ $\mathrm{Hz}, 3 \mathrm{H}), 7.56(\mathrm{~d}, J=8.0 \mathrm{~Hz}, 2 \mathrm{H}), 7.46(\mathrm{t}, J=7.3 \mathrm{~Hz}, 1 \mathrm{H}), 7.39$ (t, $J=7.4 \mathrm{~Hz}, 2 \mathrm{H}), 7.29$ (t, $J=$ $7.7 \mathrm{~Hz}, 2 \mathrm{H}), 7.07(\mathrm{t}, J=7.4 \mathrm{~Hz}, 1 \mathrm{H}) .{ }^{13} \mathrm{C}$ NMR $\left(125 \mathrm{MHz}, \mathrm{CDCl}_{3}\right) \delta$ 165.7, 137.9, 135.0, $131.8,129.1,128.8,127.0,124.5,120.2$.

\section{$N$-Phenylbenzamide (3ac, Figure 3)}

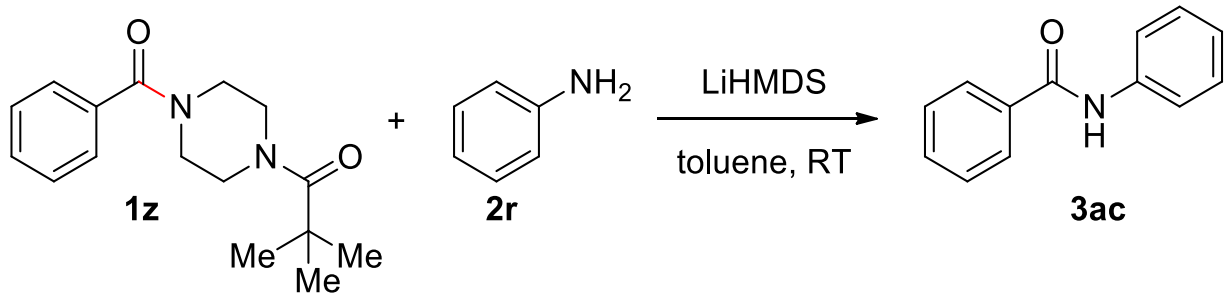

According to the general procedure, the reaction of 1-(4-benzoylpiperazin-1-yl)-2,2-dimethyl propan-1-one ( $0.10 \mathrm{mmol}, 1.0$ equiv), aniline (2.0 equiv) and LiHMDS (1.0 M in THF, 3.0 equiv) in toluene $(0.25 \mathrm{M})$ for $15 \mathrm{~h}$ at room temperature, afforded after work-up and chromatography the title compound in $77 \%$ yield $(15.2 \mathrm{mg})$. White solid. ${ }^{1} \mathrm{H}$ NMR (500 MHz, $\left.\mathrm{CDCl}_{3}\right) \delta 7.78(\mathrm{~d}, J=7.7 \mathrm{~Hz}, 3 \mathrm{H}), 7.56(\mathrm{~d}, J=8.0 \mathrm{~Hz}, 2 \mathrm{H}), 7.46(\mathrm{t}, J=7.3 \mathrm{~Hz}, 1 \mathrm{H}), 7.39(\mathrm{t}, J$ $=7.4 \mathrm{~Hz}, 2 \mathrm{H}), 7.29(\mathrm{t}, J=7.7 \mathrm{~Hz}, 2 \mathrm{H}), 7.07(\mathrm{t}, J=7.4 \mathrm{~Hz}, 1 \mathrm{H}) .{ }^{13} \mathrm{C} \mathrm{NMR}\left(125 \mathrm{MHz}, \mathrm{CDCl}_{3}\right) \delta$ 165.7, 137.9, 135.0, 131.8, 129.1, 128.8, 127.0, 124.5, 120.2.

\section{$N$-Phenylbenzamide (3ac, Figure 3)}<smiles>O=C(c1ccccc1)N1CCc2ccccc21</smiles><smiles>Nc1ccccc1</smiles>

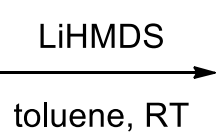
toluene, RT<smiles>O=C(Nc1ccccc1)c1ccccc1</smiles>

3ac 
According to the general procedure, the reaction of indolin-1-yl(phenyl)methanone $(0.10 \mathrm{mmol}$, 1.0 equiv), aniline (2.0 equiv) and LiHMDS (1.0 M in THF, 3.0 equiv) in toluene (0.25 M) for $15 \mathrm{~h}$ at room temperature, afforded after work-up and chromatography the title compound in 94\% yield (18.1 mg). White solid. ${ }^{1} \mathrm{H}$ NMR (500 MHz, $\left.\mathrm{CDCl}_{3}\right) \delta 7.78(\mathrm{~d}, J=7.7 \mathrm{~Hz}, 3 \mathrm{H}), 7.56$ $(\mathrm{d}, J=8.0 \mathrm{~Hz}, 2 \mathrm{H}), 7.46(\mathrm{t}, J=7.3 \mathrm{~Hz}, 1 \mathrm{H}), 7.39$ (t, $J=7.4 \mathrm{~Hz}, 2 \mathrm{H}), 7.29(\mathrm{t}, J=7.7 \mathrm{~Hz}, 2 \mathrm{H})$, $7.07(\mathrm{t}, J=7.4 \mathrm{~Hz}, 1 \mathrm{H}) .{ }^{13} \mathrm{C} \operatorname{NMR}\left(125 \mathrm{MHz}, \mathrm{CDCl}_{3}\right) \delta 165.7,137.9,135.0,131.8,129.1$, $128.8,127.0,124.5,120.2$.

\section{$N$-(p-Tolyl)benzamide (3a, Figure 3)}
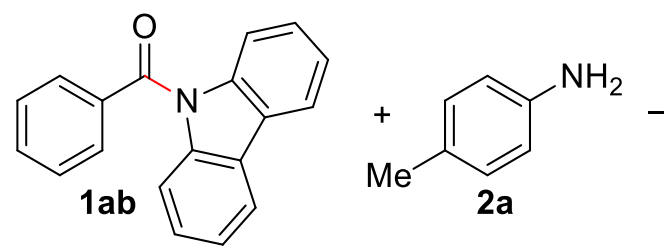

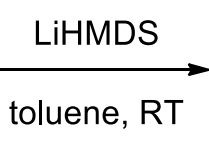

toluene, RT<smiles>Cc1ccc(NC(=O)c2ccccc2)cc1</smiles>

According to the general procedure, the reaction of $(9 H$-carbazol- $9-y l)($ phenyl)methanone $(0.10$ mmol, 1.0 equiv), aniline (2.0 equiv) and LiHMDS (1.0 M in THF, 3.0 equiv) in toluene (0.25 M) for $15 \mathrm{~h}$ at room temperature, afforded after work-up and chromatography the title compound in $90 \%$ yield $(19.0 \mathrm{mg})$. White solid. ${ }^{1} \mathrm{H} \mathrm{NMR}\left(500 \mathrm{MHz}, \mathrm{CDCl}_{3}\right) \delta 7.88(\mathrm{~d}, J=7.3 \mathrm{~Hz}, 2 \mathrm{H})$, $7.83(\mathrm{~s}, 1 \mathrm{H}), 7.58-7.54(\mathrm{~m}, 3 \mathrm{H}), 7.50$ (t, $J=7.5 \mathrm{~Hz}, 2 \mathrm{H}), 7.19$ (d, J = 8.2 Hz, $2 \mathrm{H}), 2.37(\mathrm{~s}, 3$ H). ${ }^{13} \mathrm{C}$ NMR $\left(125 \mathrm{MHz}, \mathrm{CDCl}_{3}\right) \delta 165.7,135.4,135.1,134.3,131.7,129.6,128.8,127.0,120.3$, 20.9 .

\section{$N$-Phenylbenzamide (3ac, Figure 3)}

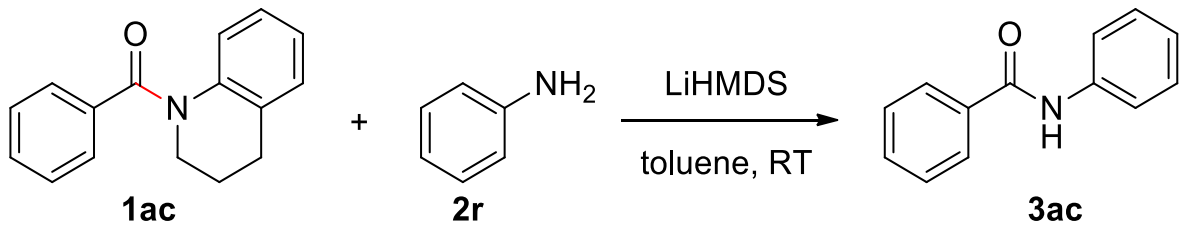

According to the general procedure, the reaction of $(3,4$-dihydroquinolin-1(2H)-yl)(phenyl) methanone (0.10 mmol, 1.0 equiv), aniline (2.0 equiv) and LiHMDS (1.0 M in THF, 3.0 equiv) in toluene $(0.25 \mathrm{M})$ for $15 \mathrm{~h}$ at room temperature, afforded after work-up and chromatography the title compound in $94 \%$ yield $(18.5 \mathrm{mg})$. White solid. ${ }^{1} \mathrm{H}$ NMR $\left(500 \mathrm{MHz}, \mathrm{CDCl}_{3}\right) \delta 7.78(\mathrm{~d}, J$ $=7.7 \mathrm{~Hz}, 3 \mathrm{H}), 7.56(\mathrm{~d}, J=8.0 \mathrm{~Hz}, 2 \mathrm{H}), 7.46(\mathrm{t}, J=7.3 \mathrm{~Hz}, 1 \mathrm{H}), 7.39$ (t, $J=7.4 \mathrm{~Hz}, 2 \mathrm{H}), 7.29$ 
(t, $J=7.7 \mathrm{~Hz}, 2 \mathrm{H}), 7.07(\mathrm{t}, J=7.4 \mathrm{~Hz}, 1 \mathrm{H}) .{ }^{13} \mathrm{C} \mathrm{NMR}\left(125 \mathrm{MHz}, \mathrm{CDCl}_{3}\right) \delta 165.7,137.9,135.0$, $131.8,129.1,128.8,127.0,124.5,120.2$.

\section{$N$-Phenylbenzamide (3ac, Figure 4)}

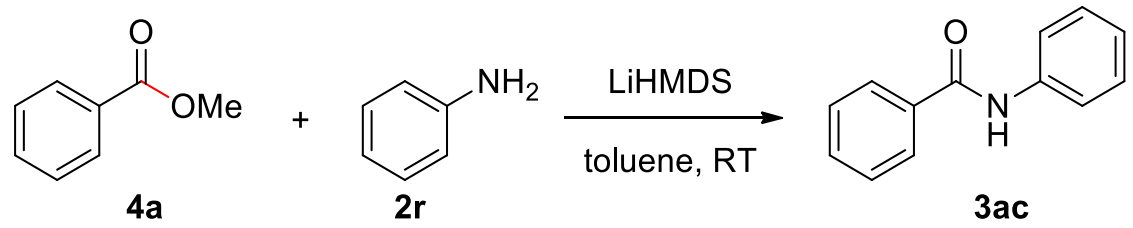

According to the general procedure, the reaction of methyl benzoate $(7.35 \mathrm{mmol})$, aniline $(1.2$ equiv) and LiHMDS (1.0 $\mathrm{M}$ in THF, 2.0 equiv) in toluene $(0.25 \mathrm{M})$ for $15 \mathrm{~h}$ at room temperature, afforded after work-up and chromatography the title compound in $93 \%$ yield (1.35 g). White solid. ${ }^{1} \mathrm{H}$ NMR $\left(500 \mathrm{MHz}, \mathrm{CDCl}_{3}\right) \delta 7.78(\mathrm{~d}, J=7.7 \mathrm{~Hz}, 3 \mathrm{H}), 7.56(\mathrm{~d}, J=8.0 \mathrm{~Hz}, 2$ H), $7.46(\mathrm{t}, J=7.3 \mathrm{~Hz}, 1 \mathrm{H}), 7.39$ (t, $J=7.4 \mathrm{~Hz}, 2 \mathrm{H}), 7.29(\mathrm{t}, J=7.7 \mathrm{~Hz}, 2 \mathrm{H}), 7.07$ (t, $J=7.4$ $\mathrm{Hz}, 1 \mathrm{H}) .{ }^{13} \mathrm{C} \mathrm{NMR}\left(125 \mathrm{MHz}, \mathrm{CDCl}_{3}\right) \delta 165.7,137.9,135.0,131.8,129.1,128.8,127.0,124.5$, 120.2 .

\section{$N$-(4-Methoxyphenyl)benzamide (3b, Figure 4)}

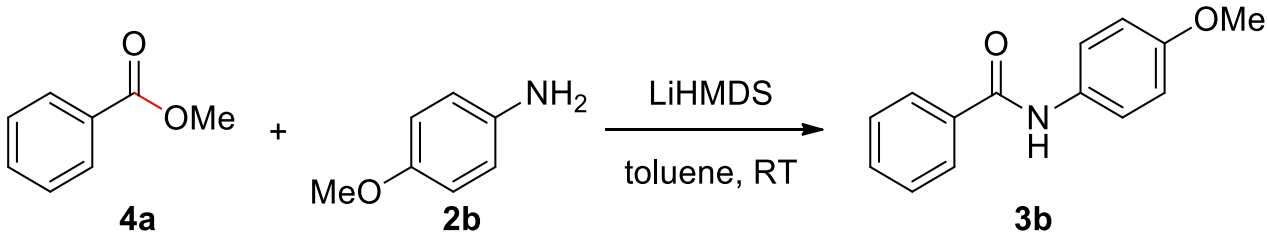

According to the general procedure, the reaction of methyl benzoate $(0.10 \mathrm{mmol}), p$-anisidine (1.2 equiv) and LiHMDS (1.0 $\mathrm{M}$ in THF, 2.0 equiv) in toluene $(0.25 \mathrm{M})$ for $15 \mathrm{~h}$ at room temperature, afforded after work-up and chromatography the title compound in $96 \%$ yield $(21.8$ mg). White solid. ${ }^{1} \mathrm{H}$ NMR (500 MHz, $\left.\mathrm{CDCl}_{3}\right) \delta 7.97$ (d, J= $\left.7.6 \mathrm{~Hz}, 2 \mathrm{H}\right), 7.92$ (s, $\left.1 \mathrm{H}\right)$, 7.67$7.64(\mathrm{~m}, 3 \mathrm{H}), 7.59$ (t, $J=7.6 \mathrm{~Hz}, 2 \mathrm{H}), 7.02(\mathrm{~d}, J=8.9 \mathrm{~Hz}, 2 \mathrm{H}), 3.93(\mathrm{~s}, 3 \mathrm{H}) .{ }^{13} \mathrm{C}$ NMR $(125$ $\left.\mathrm{MHz}, \mathrm{CDCl}_{3}\right) \delta 165.8,156.8,135.3,131.9,131.2,129.0,127.2,122.3,114.5,55.7$. 


\section{$N$-(4-(Trifluoromethyl)phenyl)benzamide (3ad, Figure 4)}

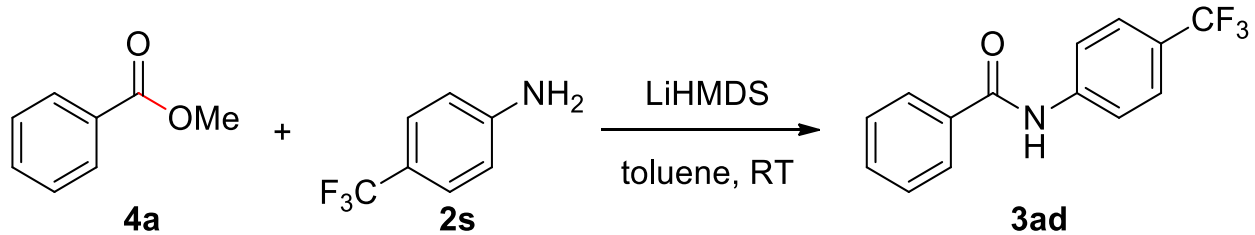

According to the general procedure, the reaction of methyl benzoate $(0.10 \mathrm{mmol})$, 4(trifluoromethyl)aniline (1.2 equiv) and LiHMDS (1.0 M in THF, 2.0 equiv) in toluene (0.25 M) for $15 \mathrm{~h}$ at room temperature, afforded after work-up and chromatography the title compound in $94 \%$ yield $(24.9 \mathrm{mg})$. White solid. ${ }^{1} \mathrm{H}$ NMR $\left(500 \mathrm{MHz}, \mathrm{DMSO}-d_{6}\right) \delta 10.60(\mathrm{~s}, 1 \mathrm{H}), 8.03(\mathrm{~d}, J=$ $8.4 \mathrm{~Hz}, 2 \mathrm{H}), 7.98$ (d, $J=7.4 \mathrm{~Hz}, 2 \mathrm{H}), 7.73$ (d, $J=8.5 \mathrm{~Hz}, 2 \mathrm{H}), 7.63$ (t, $J=7.3 \mathrm{~Hz}, 1 \mathrm{H}), 7.56$ $(\mathrm{t}, J=7.5 \mathrm{~Hz}, 2 \mathrm{H}) .{ }^{13} \mathrm{C}$ NMR $\left(125 \mathrm{MHz}, \mathrm{DMSO}-d_{6}\right) \delta 166.5,143.3,134.9,132.3,128.8,128.2$, $126.3\left(\mathrm{q}, J^{F}=3.8 \mathrm{~Hz}\right), 125.0\left(\mathrm{~d}, J^{F}=222.3 \mathrm{~Hz}\right), 123.8\left(\mathrm{~d}, J^{F}=14.9 \mathrm{~Hz}\right), 120.5 .{ }^{19} \mathrm{~F}$ NMR $(471$ MHz, DMSO- $\left.d_{6}\right) \delta-60.26$.

Ethyl 4-benzamidobenzoate (3ae, Figure 4)

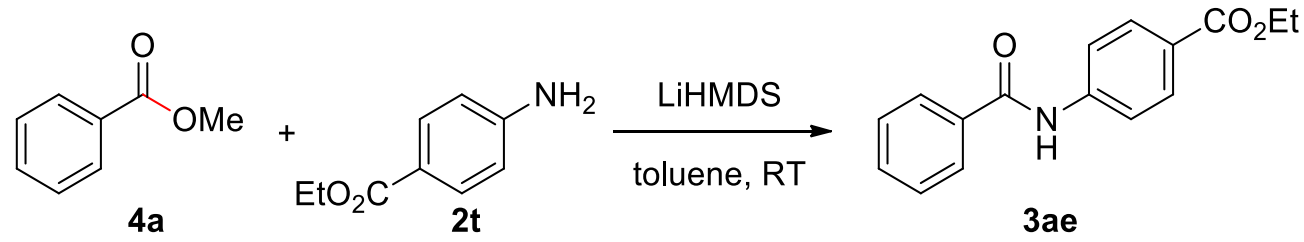

According to the general procedure, the reaction of methyl benzoate $(0.10 \mathrm{mmol})$, ethyl 4aminobenzoate (1.2 equiv) and LiHMDS (1.0 M in THF, 2.0 equiv) in toluene $(0.25 \mathrm{M})$ for $15 \mathrm{~h}$ at room temperature, afforded after work-up and chromatography the title compound in $84 \%$ yield (22.6 mg). White solid. ${ }^{1} \mathrm{H}$ NMR $\left(500 \mathrm{MHz}, \mathrm{CDCl}_{3}\right) \delta 8.05(\mathrm{~d}, J=8.4 \mathrm{~Hz}, 3 \mathrm{H}), 7.88(\mathrm{~d}, J$ $=7.6 \mathrm{~Hz}, 2 \mathrm{H}), 7.74(\mathrm{~d}, J=8.4 \mathrm{~Hz}, 2 \mathrm{H}), 7.57(\mathrm{t}, J=7.3 \mathrm{~Hz}, 1 \mathrm{H}), 7.49(\mathrm{t}, J=7.5 \mathrm{~Hz}, 2 \mathrm{H}), 4.37$ $(\mathrm{q}, J=7.1 \mathrm{~Hz}, 2 \mathrm{H}), 1.40(\mathrm{t}, J=7.1 \mathrm{~Hz}, 3 \mathrm{H}) .{ }^{13} \mathrm{C} \mathrm{NMR}\left(125 \mathrm{MHz}, \mathrm{CDCl}_{3}\right) \delta 166.3,165.9$, $142.2,134.7,132.4,131.0,129.0,127.2,126.4,119.3,61.1,14.5$. 


\section{$N$-([1,1'-Biphenyl]-2-yl)benzamide (3h, Figure 4)}<smiles>[X]OC(=O)c1ccccc1</smiles>

$4 a$

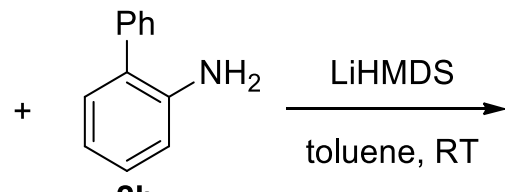

2h<smiles>O=C(Nc1ccccc1-c1ccccc1)c1ccccc1</smiles>

3h

According to the general procedure, the reaction of methyl benzoate $(0.10 \mathrm{mmol}),\left[1,1^{\prime}-\right.$ biphenyl]-2-amine (1.2 equiv) and LiHMDS (1.0 M in THF, 2.0 equiv) in toluene (0.25 M) for $15 \mathrm{~h}$ at room temperature, afforded after work-up and chromatography the title compound in 92\% yield (25.1 mg). White solid. ${ }^{1} \mathrm{H}$ NMR (500 MHz, $\left.\mathrm{CDCl}_{3}\right) \delta 8.57(\mathrm{~d}, J=8.2 \mathrm{~Hz}, 1 \mathrm{H}), 8.04$ $(\mathrm{s}, 1 \mathrm{H}), 7.63(\mathrm{~d}, J=7.8 \mathrm{~Hz}, 2 \mathrm{H}), 7.58-7.45(\mathrm{~m}, 7 \mathrm{H}), 7.41(\mathrm{t}, J=7.3 \mathrm{~Hz}, 2 \mathrm{H}), 7.33(\mathrm{~d}, J=7.5$ $\mathrm{Hz}, 1 \mathrm{H}), 7.25$ (t, $J=7.4 \mathrm{~Hz}, 1 \mathrm{H}) .{ }^{13} \mathrm{C} \mathrm{NMR}\left(125 \mathrm{MHz}, \mathrm{CDCl}_{3}\right) \delta 165.0,138.1,135.0,134.8$, $132.4,131.8,130.0,129.4,129.3,128.8,128.6,128.2,126.8,124.4,121.2$.

\section{$N$-(2-Cyano-3-methylphenyl)benzamide (3af, Figure 4)}<smiles>COC(=O)c1ccccc1</smiles>

$4 a$<smiles>Cc1cccc(N)c1C#N</smiles>
2u

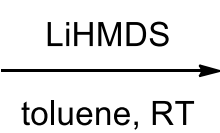

toluene, RT<smiles>Cc1cccc(NC(=O)c2ccccc2)c1C#N</smiles>

3af

According to the general procedure, the reaction of methyl benzoate $(0.10 \mathrm{mmol}), 2$-amino-6methylbenzonitrile (1.2 equiv) and LiHMDS (1.0 M in THF, 2.0 equiv) in toluene (0.25 M) for $15 \mathrm{~h}$ at room temperature, afforded after work-up and chromatography the title compound in 97\% yield (22.9 mg). White solid. ${ }^{1} \mathrm{H}$ NMR (500 MHz, $\left.\mathrm{CDCl}_{3}\right) \delta 8.43(\mathrm{~d}, J=8.5 \mathrm{~Hz}, 2 \mathrm{H}), 7.96$ $(\mathrm{d}, J=7.9 \mathrm{~Hz}, 2 \mathrm{H}), 7.62$ (t, $J=7.1 \mathrm{~Hz}, 1 \mathrm{H}), 7.56-7.52(\mathrm{~m}, 3 \mathrm{H}), 7.09$ (d, J = 7.6 Hz, $1 \mathrm{H}), 2.58$ (s, $3 \mathrm{H}) .{ }^{13} \mathrm{C} \mathrm{NMR}\left(125 \mathrm{MHz}, \mathrm{CDCl}_{3}\right) \delta 165.5,142.4,140.8,133.9,133.7,132.6,129.1,127.2$, $125.4,118.3,115.9,102.9,20.9$. 


\section{$N$-(2,6-Dimethylphenyl)benzamide (3i, Figure 4)}

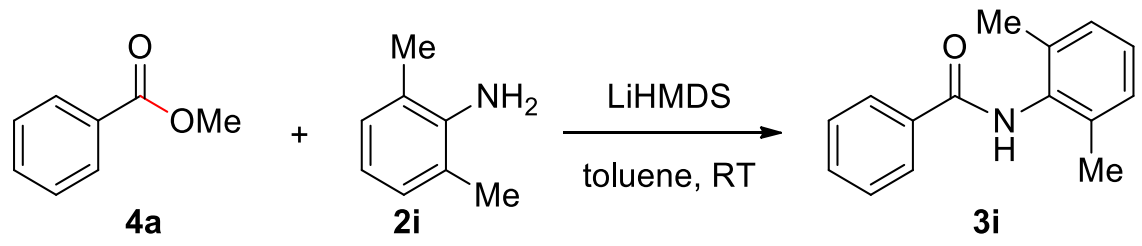

According to the general procedure, the reaction of methyl benzoate $(0.10 \mathrm{mmol}), 2,6$ dimethylaniline (1.2 equiv) and LiHMDS (1.0 M in THF, 2.0 equiv) in toluene (0.25 M) for $15 \mathrm{~h}$ at room temperature, afforded after work-up and chromatography the title compound in $89 \%$ yield $(20.0 \mathrm{mg})$. White solid. ${ }^{1} \mathrm{H} \mathrm{NMR}\left(500 \mathrm{MHz}, \mathrm{CDCl}_{3}\right) \delta 7.94(\mathrm{~d}, J=7.6 \mathrm{~Hz}, 2 \mathrm{H}), 7.59(\mathrm{t}, J=$ $7.3 \mathrm{~Hz}, 1 \mathrm{H}), 7.52$ (t, $J=7.6 \mathrm{~Hz}, 2 \mathrm{H}), 7.45(\mathrm{~s}, 1 \mathrm{H}), 7.20-7.12(\mathrm{~m}, 3 \mathrm{H}), 2.30(\mathrm{~s}, 6 \mathrm{H}) .{ }^{13} \mathrm{C} \mathrm{NMR}$ $\left(125 \mathrm{MHz}, \mathrm{CDCl}_{3}\right) \delta 165.7,135.4,134.4,133.7,131.7,128.7,128.2,127.3,127.1,18.4$.

\section{$N$-(2,6-Diisopropylphenyl)benzamide (3j, Figure 4)}<smiles>COC(=O)c1ccccc1</smiles>

$4 a$

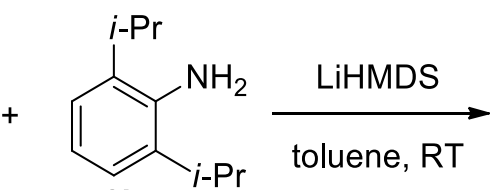

$2 \mathbf{j}$<smiles>CCCc1cccc(C(C)C)c1NC(=O)c1ccccc1</smiles>

3j

According to the general procedure, the reaction of methyl benzoate $(0.10 \mathrm{mmol}), 2,6-$ diisopropylaniline (1.2 equiv) and LiHMDS (1.0 M in THF, 2.0 equiv) in toluene $(0.25 \mathrm{M})$ for $15 \mathrm{~h}$ at room temperature, afforded after work-up and chromatography the title compound in 96\% yield (27.0 mg). White solid. ${ }^{1} \mathrm{H}$ NMR (500 MHz, $\left.\mathrm{CDCl}_{3}\right) \delta 7.94(\mathrm{~d}, J=7.9 \mathrm{~Hz}, 2 \mathrm{H}), 7.59$ $(\mathrm{t}, J=7.4 \mathrm{~Hz}, 1 \mathrm{H}), 7.51(\mathrm{t}, J=7.6 \mathrm{~Hz}, 2 \mathrm{H}), 7.47(\mathrm{~s}, 1 \mathrm{H}), 7.37(\mathrm{t}, J=7.7 \mathrm{~Hz}, 1 \mathrm{H}), 7.26(\mathrm{~d}, J=$ $7.7 \mathrm{~Hz}, 2 \mathrm{H}), 3.17$ (dt, $J=13.7,6.8 \mathrm{~Hz}, 2 \mathrm{H}), 1.25$ (s, $6 \mathrm{H}), 1.24$ (s, $6 \mathrm{H}) .{ }^{13} \mathrm{C} \mathrm{NMR}(125 \mathrm{MHz}$, $\left.\mathrm{CDCl}_{3}\right) \delta 167.0,146.4,134.6,131.8,131.2,128.8,128.5,127.2,123.6,28.9,23.7$.

\section{$N$-(3-Chlorophenyl)benzamide (3k, Figure 4)}<smiles>COC(=O)c1ccccc1</smiles>

4a<smiles>Nc1cccc(Cl)c1</smiles>

2k

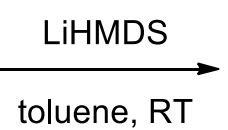

toluene, RT<smiles>Cc1cccc(NC(=O)c2ccccc2)c1</smiles>

3k

According to the general procedure, the reaction of methyl benzoate $(0.10 \mathrm{mmol})$, 3chloroaniline (1.2 equiv) and LiHMDS (1.0 M in THF, 2.0 equiv) in toluene $(0.25 \mathrm{M})$ for $15 \mathrm{~h}$ at S218 
room temperature, afforded after work-up and chromatography the title compound in $80 \%$ yield (18.6 mg). White solid. ${ }^{1} \mathrm{H}$ NMR (500 $\left.\mathrm{MHz} \mathrm{CDCl}_{3}\right) \delta 7.97$ (s, $\left.1 \mathrm{H}\right), 7.87$ (d, J = $\left.7.4 \mathrm{~Hz}, 2 \mathrm{H}\right)$, $7.79(\mathrm{t}, J=1.7 \mathrm{~Hz}, 1 \mathrm{H}), 7.58(\mathrm{t}, J=7.4 \mathrm{~Hz}, 1 \mathrm{H}), 7.53-7.47(\mathrm{~m}, 3 \mathrm{H}), 7.30(\mathrm{t}, J=6.7 \mathrm{~Hz}, 1 \mathrm{H})$, $7.14(\mathrm{dd}, J=8.0,0.9 \mathrm{~Hz}, 1 \mathrm{H}) .{ }^{13} \mathrm{C} \mathrm{NMR}\left(125 \mathrm{MHz}, \mathrm{CDCl}_{3}\right) \delta 165.8,139.1,134.8,134.6,132.1$, 130.1, 128.9, 127.1, 124.6, 120.4, 118.2.

\section{$N$-(Perfluorophenyl)benzamide (3ag, Figure 4)}<smiles>COC(=O)c1ccccc1</smiles>

$4 a$<smiles>Nc1c(F)c(F)c(F)c(F)c1F</smiles>

LiHMDS toluene, RT $2 v$<smiles>O=C(Nc1c(F)c(F)c(F)c(F)c1F)c1ccccc1</smiles>

$3 \mathrm{ag}$

According to the general procedure, the reaction of methyl benzoate $(0.10 \mathrm{mmol}), 2,3,4,5,6-$ pentafluoroaniline (1.2 equiv) and LiHMDS (1.0 M in THF, 2.0 equiv) in toluene (0.25 M) for $15 \mathrm{~h}$ at room temperature, afforded after work-up and chromatography the title compound in 94\% yield (27.0 mg). White solid. ${ }^{1} \mathrm{H}$ NMR (500 MHz, DMSO-d6) $\delta 10.56(\mathrm{~s}, 1 \mathrm{H}), 8.02(\mathrm{~d}, J=$ $7.8 \mathrm{~Hz}, 2 \mathrm{H}), 7.67$ (t, $J=7.3 \mathrm{~Hz}, 1 \mathrm{H}), 7.58$ (t, $J=7.5 \mathrm{~Hz}, 2 \mathrm{H}) .{ }^{13} \mathrm{C}$ NMR (125 MHz, DMSO- $\left.d_{6}\right)$ $\delta 170.7,149.2\left(\mathrm{dd}, J^{F}=9.9,3.6 \mathrm{~Hz}\right), 147.2\left(\mathrm{dd}, J^{F}=10.8,4.8 \mathrm{~Hz}\right), 146.2-144.7(\mathrm{~m}), 143.7-143.0$ (m), 142.3-140.4 (m), 137.8 (s), 137.6 (s), 133.9 (s), 133.3 (s), 118.4 (td, $J=15.0,3.1 \mathrm{~Hz}) .{ }^{19} \mathrm{~F}$ NMR (471 MHz, DMSO-d $d_{6} \delta-140.36--140.46(\mathrm{~m}),-152.53\left(\mathrm{t}, J^{F}=22.9 \mathrm{~Hz}\right),-158.47--$ $158.61(\mathrm{~m})$.

\section{$N$-(9-Ethyl-9H-carbazol-3-yl)benzamide (3m, Figure 4)}<smiles>COC(=O)c1ccccc1</smiles>

$4 a$<smiles>Cn1c2ccccc2c2cc(N)ccc21</smiles>

$2 m$ Et
LiHMDS toluene, RT

(t)<smiles>CCn1c2ccccc2c2cc(NC(=O)c3ccccc3)ccc21</smiles>

According to the general procedure, the reaction of methyl benzoate $(0.10 \mathrm{mmol})$, 9-ethyl-9Hcarbazol-3-amine (1.2 equiv) and LiHMDS (1.0 M in THF, 2.0 equiv) in toluene ( $0.25 \mathrm{M})$ for 15 $\mathrm{h}$ at room temperature, afforded after work-up and chromatography the title compound in $80 \%$ yield (25.1 mg). White solid. ${ }^{1} \mathrm{H}$ NMR $\left(500 \mathrm{MHz}, \mathrm{CDCl}_{3}\right) \delta 8.41$ (s, $\left.1 \mathrm{H}\right), 8.05$ (d, $J=7.7 \mathrm{~Hz}, 1$ 
H), $8.00(\mathrm{~s}, 1 \mathrm{H}), 7.91(\mathrm{~d}, J=7.5 \mathrm{~Hz}, 2 \mathrm{H}), 7.61(\mathrm{~d}, J=8.4 \mathrm{~Hz}, 1 \mathrm{H}), 7.53(\mathrm{t}, J=7.2 \mathrm{~Hz}, 1 \mathrm{H})$, 7.50-7.42 (m, $3 \mathrm{H}), 7.38-7.33$ (m, $2 \mathrm{H}), 7.19$ (t, $J=7.4 \mathrm{~Hz}, 1 \mathrm{H}), 4.32$ (q, J = 7.2 Hz, $2 \mathrm{H}), 1.40$ $(\mathrm{t}, J=7.2 \mathrm{~Hz}, 3 \mathrm{H}) .{ }^{13} \mathrm{C} \mathrm{NMR}\left(125 \mathrm{MHz}, \mathrm{CDCl}_{3}\right) \delta 165.8,140.5,137.4,135.3,131.7,129.7$, $128.8,127.0,125.9,123.1,122.8,120.7,119.8,118.8,113.2,108.6,37.7,13.8$.

\section{$N$-(Quinolin-8-yl)benzamide (3n, Figure 4)}

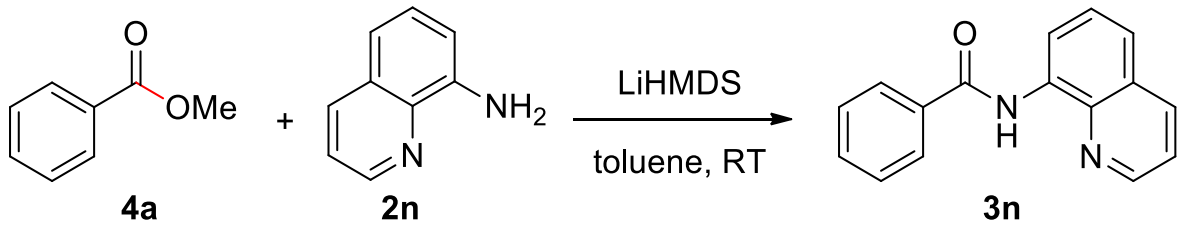

According to the general procedure, the reaction of methyl benzoate $(0.10 \mathrm{mmol})$, quinolin-8amine (1.2 equiv) and LiHMDS (1.0 M in THF, 2.0 equiv) in toluene (0.25 M) for $15 \mathrm{~h}$ at room temperature, afforded after work-up and chromatography the title compound in $80 \%$ yield (19.8 mg). White solid. ${ }^{1} \mathrm{H}$ NMR (500 MHz, $\left.\mathrm{CDCl}_{3}\right) \delta 10.78(\mathrm{~s}, 1 \mathrm{H}), 8.97$ (dd, J = 7.6, $\left.1.2 \mathrm{~Hz}, 1 \mathrm{H}\right)$, 8.88 (dd, $J=4.2,1.6 \mathrm{~Hz}, 1 \mathrm{H}), 8.22$ (dd, $J=8.3,1.6 \mathrm{~Hz}, 1 \mathrm{H}), 8.15-8.09$ (m, $2 \mathrm{H}), 7.66-7.55$ (m, $5 \mathrm{H}), 7.51(\mathrm{dd}, J=8.2,4.2 \mathrm{~Hz}, 1 \mathrm{H}) .{ }^{13} \mathrm{C} \mathrm{NMR}\left(125 \mathrm{MHz}, \mathrm{CDCl}_{3}\right) \delta 165.5,148.3,138.8,136.4$, $135.2,134.7,131.9,128.8,128.0,127.5,127.3,121.7,116.6$.

\section{$N$-(Pyridin-2-yl)benzamide (3o, Figure 4)}

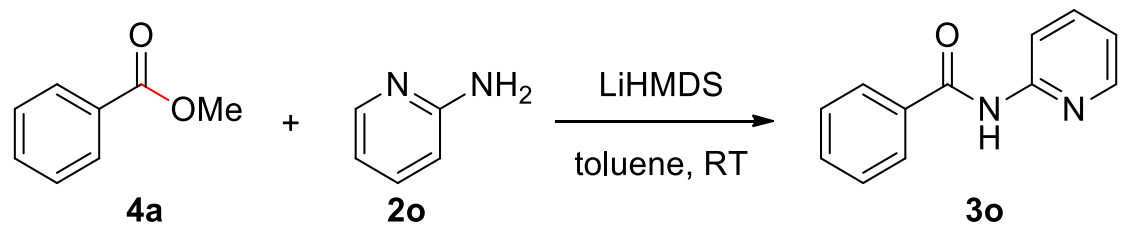

According to the general procedure, the reaction of methyl benzoate $(0.10 \mathrm{mmol})$, pyridin-2amine (1.2 equiv) and LiHMDS (1.0 M in THF, 2.0 equiv) in toluene $(0.25 \mathrm{M})$ for $15 \mathrm{~h}$ at room temperature, afforded after work-up and chromatography the title compound in $84 \%$ yield (16.6 mg). White solid. ${ }^{1} \mathrm{H}$ NMR $\left(500 \mathrm{MHz}, \mathrm{CDCl}_{3}\right) \delta 8.82(\mathrm{~s}, 1 \mathrm{H}), 8.37$ (d, J=8.4 Hz, $\left.1 \mathrm{H}\right), 8.21$ (d, $J=4.6 \mathrm{~Hz}, 1 \mathrm{H}), 7.90(\mathrm{~d}, J=7.9 \mathrm{~Hz}, 2 \mathrm{H}), 7.73(\mathrm{t}, J=7.8 \mathrm{~Hz}, 1 \mathrm{H}), 7.54(\mathrm{t}, J=7.3 \mathrm{~Hz}, 1 \mathrm{H})$, $7.46(\mathrm{t}, J=7.5 \mathrm{~Hz}, 2 \mathrm{H}), 7.05-7.00(\mathrm{~m}, 1 \mathrm{H}) .{ }^{13} \mathrm{C} \mathrm{NMR}\left(125 \mathrm{MHz}, \mathrm{CDCl}_{3}\right) \delta 165.7,151.5,147.8$, $138.4,134.2,132.1,128.7,127.1,119.8,114.1$. 


\section{$N$-(3,5-Dimethylisoxazol-4-yl)benzamide (3p, Figure 4)}<smiles>COC(=O)c1ccccc1</smiles>

$4 a$<smiles>Cc1noc(C)c1N</smiles>

$2 p$

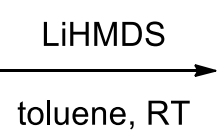

toluene, RT<smiles>Cc1noc(C)c1NC(=O)c1ccccc1</smiles>

$3 p$

According to the general procedure, the reaction of methyl benzoate $(0.10 \mathrm{mmol}), 3,5-$ dimethylisoxazol-4-amine (1.2 equiv) and LiHMDS (1.0 M in THF, 2.0 equiv) in toluene (0.25 M) for $15 \mathrm{~h}$ at room temperature, afforded after work-up and chromatography the title compound in $90 \%$ yield $(19.4 \mathrm{mg})$. White solid. ${ }^{1} \mathrm{H}$ NMR $\left(500 \mathrm{MHz}, \mathrm{CDCl}_{3}\right) \delta 7.94(\mathrm{~d}, J=7.7 \mathrm{~Hz}, 2 \mathrm{H})$, $7.64(\mathrm{t}, J=7.3 \mathrm{~Hz}, 1 \mathrm{H}), 7.58-7.51(\mathrm{~m}, 3 \mathrm{H}), 2.39(\mathrm{~s}, 3 \mathrm{H}), 2.24(\mathrm{~s}, 3 \mathrm{H}) .{ }^{13} \mathrm{C}$ NMR $(125 \mathrm{MHz}$, $\left.\mathrm{CDCl}_{3}\right) \delta 166.5,164.0,157.9,133.2,132.3,128.8,127.3,113.4,11.3,9.7$.

\section{$N$-Methyl- $N$-phenylbenzamide (3ah, Figure 4)}

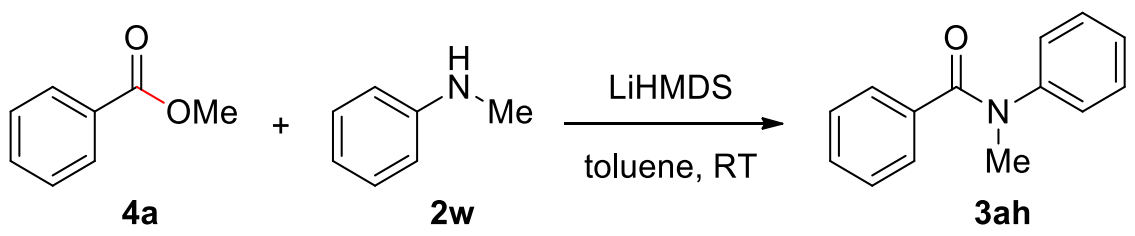

According to the general procedure, the reaction of methyl benzoate $(0.10 \mathrm{mmol}), \mathrm{N}$ methylaniline (1.2 equiv) and LiHMDS (1.0 M in THF, 2.0 equiv) in toluene $(0.25 \mathrm{M})$ for $15 \mathrm{~h}$ at room temperature, afforded after work-up and chromatography the title compound in 97\% yield (20.5 mg). White solid. ${ }^{1} \mathrm{H}$ NMR (500 MHz, $\left.\mathrm{CDCl}_{3}\right) \delta 7.31$ (d, $\left.J=7.8 \mathrm{~Hz}, 2 \mathrm{H}\right), 7.25-7.22$ (m, 3 $\mathrm{H}), 7.19-7.15(\mathrm{~m}, 3 \mathrm{H}), 7.06(\mathrm{~d}, J=7.8 \mathrm{~Hz}, 2 \mathrm{H}), 3.52(\mathrm{~s}, 3 \mathrm{H}) .{ }^{13} \mathrm{C}$ NMR $\left(125 \mathrm{MHz}, \mathrm{CDCl}_{3}\right) \delta$ 170.7, 144.9, 135.9, 129.6, 129.2, 128.7, 127.7, 126.9, 126.5, 38.4.

\section{Morpholino(phenyl)methanone (3q, Figure 4)}

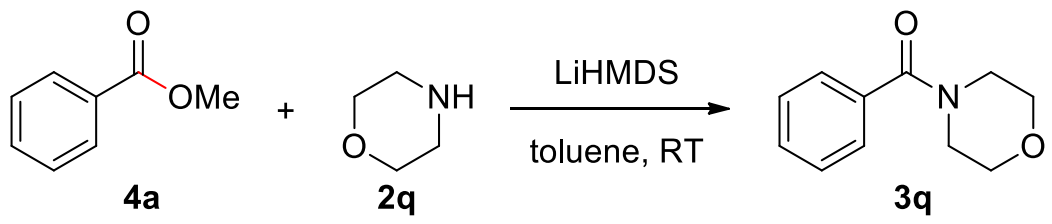

According to the general procedure, the reaction of methyl benzoate $(0.10 \mathrm{mmol})$, morpholine (1.2 equiv) and LiHMDS (1.0 $\mathrm{M}$ in THF, 2.0 equiv) in toluene $(0.25 \mathrm{M})$ for $15 \mathrm{~h}$ at room temperature, afforded after work-up and chromatography the title compound in 98\% yield (18.7 
mg). White solid. ${ }^{1} \mathrm{H}$ NMR (500 MHz, $\left.\mathrm{CDCl}_{3}\right) \delta 7.40(\mathrm{~s}, 5 \mathrm{H}), 3.84-3.36(\mathrm{~m}, 8 \mathrm{H}) .{ }^{13} \mathrm{C} \mathrm{NMR}$ $\left(125 \mathrm{MHz}, \mathrm{CDCl}_{3}\right) \delta 170.3,135.1,129.7,128.4,126.9,66.7$.

\section{4-Methyl-N-phenylbenzamide (3ai, Figure 4)}<smiles>COC(=O)c1ccc(C(=O)O[Na])cc1</smiles>

According to the general procedure, the reaction of methyl 4-methylbenzoate $(0.10 \mathrm{mmol})$, aniline (1.2 equiv) and LiHMDS (1.0 M in THF, 2.0 equiv) in toluene $(0.25 \mathrm{M})$ for $15 \mathrm{~h}$ at room temperature, afforded after work-up and chromatography the title compound in 93\% yield (19.6 mg). White solid. ${ }^{1} \mathrm{H}$ NMR $\left(500 \mathrm{MHz}, \mathrm{CDCl}_{3}\right) \delta 7.85(\mathrm{~s}, 1 \mathrm{H}), 7.79(\mathrm{~d}, J=8.0 \mathrm{~Hz}, 2 \mathrm{H}), 7.67$ (d, $J=7.9 \mathrm{~Hz}, 2 \mathrm{H}), 7.39(\mathrm{t}, J=7.8 \mathrm{~Hz}, 2 \mathrm{H}), 7.30(\mathrm{~d}, J=7.9 \mathrm{~Hz}, 2 \mathrm{H}), 7.17(\mathrm{t}, J=7.4 \mathrm{~Hz}, 1 \mathrm{H})$, 2.45 (s, $3 \mathrm{H}) .{ }^{13} \mathrm{C}$ NMR $\left(125 \mathrm{MHz}, \mathrm{CDCl}_{3}\right) \delta 165.7,142.4,138.0,132.1,129.5,129.1,127.0$, $124.5,120.2,21.5$.

\section{4-Methoxy- $N$-phenylbenzamide (3aj, Figure 4)}<smiles>COC(=O)c1ccc(OC)cc1</smiles>

$4 \mathrm{c}$

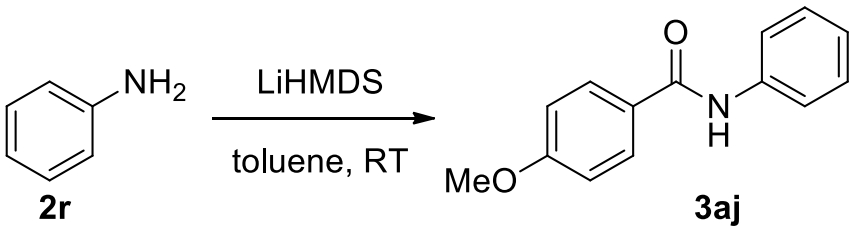

According to the general procedure, the reaction of methyl 4-methoxybenzoate $(0.10 \mathrm{mmol})$, aniline (3.0 equiv) and LiHMDS (1.0 M in THF, 3.0 equiv) in toluene (0.25 M) for $15 \mathrm{~h}$ at room temperature, afforded after work-up and chromatography the title compound in $70 \%$ yield (15.9 mg). White solid. ${ }^{1} \mathrm{H}$ NMR (500 MHz, DMSO- $\left.d_{6}\right) \delta 10.08(\mathrm{~s}, 1 \mathrm{H}), 7.97(\mathrm{~d}, J=8.6 \mathrm{~Hz}, 2 \mathrm{H})$, $7.77(\mathrm{~d}, J=8.0 \mathrm{~Hz}, 2 \mathrm{H}), 7.34$ (t, $J=7.1 \mathrm{~Hz}, 2 \mathrm{H}), 7.08$ (dd, $J=10.6,9.1 \mathrm{~Hz}, 3 \mathrm{H}), 3.85$ (s, $3 \mathrm{H})$. ${ }^{13} \mathrm{C}$ NMR $\left(125 \mathrm{MHz}, \mathrm{DMSO}-d_{6}\right) \delta 165.4,162.4$, 139.8, 130.0, 129.0, 127.5, 123.9, 120.8, 114.1, 55.9 . 


\section{$N$-Phenyl-4-(trifluoromethyl)benzamide (3ak, Figure 4)}<smiles>COC(=O)c1ccc(C(F)(F)F)cc1</smiles>

According to the general procedure, the reaction of methyl 4-(trifluoromethyl)benzoate $(0.10$ mmol), aniline(1.2 equiv) and LiHMDS (1.0 M in THF, 2.0 equiv) in toluene (0.25 M) for $15 \mathrm{~h}$ at room temperature, afforded after work-up and chromatography the title compound in $94 \%$ yield (22.1 mg). White solid. ${ }^{1} \mathrm{H}$ NMR (500 MHz, DMSO- $\left.d_{6}\right) \delta 10.47(\mathrm{~s}, 1 \mathrm{H}), 8.16(\mathrm{~d}, J=8.1$ $\mathrm{Hz}, 2 \mathrm{H}), 7.93$ (d, $J=8.2 \mathrm{~Hz}, 2 \mathrm{H}), 7.79(\mathrm{~d}, J=8.2 \mathrm{~Hz}, 2 \mathrm{H}), 7.39$ (t, $J=7.7 \mathrm{~Hz}, 2 \mathrm{H}), 7.14$ (t, $J$ $=7.4 \mathrm{~Hz}, 1 \mathrm{H}) \cdot{ }^{13} \mathrm{C}$ NMR $\left(125 \mathrm{MHz}\right.$, DMSO-$\left.d_{6}\right) \delta 164.8,139.3,139.2,131.8\left(\mathrm{q}, J^{F}=31.8 \mathrm{~Hz}\right)$, 129.1, 129.0, $125.8\left(\mathrm{q}, J^{F}=3.7 \mathrm{~Hz}\right), 124.4\left(\mathrm{q}, J^{F}=274.8 \mathrm{~Hz}\right), 124.4,120.9 .{ }^{19} \mathrm{~F}$ NMR $(471 \mathrm{MHz}$, $\left.\mathrm{CDCl}_{3}\right) \delta-61.31$.

\section{2-Methyl- $N$-phenylbenzamide (3al, Figure 4)}

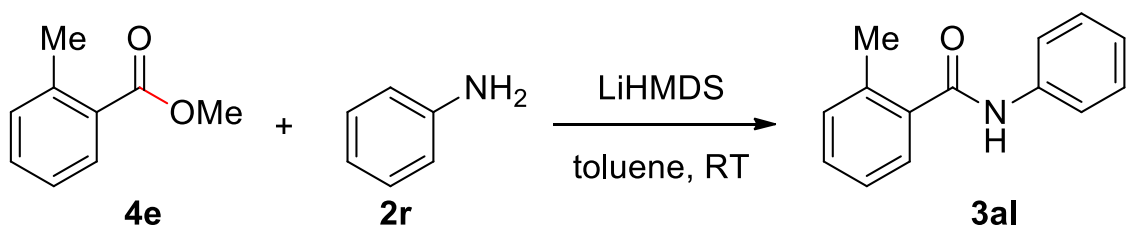

According to the general procedure, the reaction of methyl 2-methylbenzoate $(0.10 \mathrm{mmol}),(1.2$ equiv) and LiHMDS (1.0 $\mathrm{M}$ in THF, 2.0 equiv) in toluene $(0.25 \mathrm{M})$ for $15 \mathrm{~h}$ at room temperature, afforded after work-up and chromatography the title compound in 91\% yield $(19.2$ $\mathrm{mg}$ ). White solid. ${ }^{1} \mathrm{H} \mathrm{NMR}\left(500 \mathrm{MHz}, \mathrm{CDCl}_{3}\right) \delta 7.66(\mathrm{~d}, J=7.4 \mathrm{~Hz}, 2 \mathrm{H}), 7.52(\mathrm{~d}, J=6.6 \mathrm{~Hz}, 2$ H), 7.40 (t, $J=7.4 \mathrm{~Hz}, 3 \mathrm{H}), 7.31$ (d, $J=10.1 \mathrm{~Hz}, 2 \mathrm{H}), 7.19$ (t, $J=7.4 \mathrm{~Hz}, 1 \mathrm{H}), 2.55$ (s, $3 \mathrm{H})$. ${ }^{13} \mathrm{C}$ NMR $\left(125 \mathrm{MHz}, \mathrm{CDCl}_{3}\right) \delta 168.1,138.0,136.5,131.3,130.3,129.2,126.6,125.9,124.6$, $119.9,19.8$.

\section{3-Bromo- $N$-phenylbenzamide (3am, Figure 4)}<smiles>COC(=O)c1cccc(Br)c1</smiles> 
According to the general procedure, the reaction of methyl 3-bromobenzoate $(0.10 \mathrm{mmol})$, aniline (1.2 equiv) and LiHMDS (1.0 M in THF, 2.0 equiv) in toluene (0.25 M) for $15 \mathrm{~h}$ at room temperature, afforded after work-up and chromatography the title compound in $93 \%$ yield (25.7 mg). White solid. ${ }^{1} \mathrm{H}$ NMR $\left(500 \mathrm{MHz}, \mathrm{CDCl}_{3}\right) \delta 8.01(\mathrm{t}, J=1.8 \mathrm{~Hz}, 1 \mathrm{H}), 7.79(\mathrm{~d}, J=7.9 \mathrm{~Hz}, 2$ H), 7.68 (ddd, $J=8.0,1.8,0.9 \mathrm{~Hz}, 1 \mathrm{H}), 7.63$ (d, $J=7.8 \mathrm{~Hz}, 2 \mathrm{H}), 7.41-7.34(\mathrm{~m}, 3 \mathrm{H}), 7.18(\mathrm{t}, J$ $=7.4 \mathrm{~Hz}, 1 \mathrm{H}) .{ }^{13} \mathrm{C} \mathrm{NMR}\left(125 \mathrm{MHz}, \mathrm{CDCl}_{3}\right) \delta 164.3,137.6,137.0,134.8,130.4,130.3,129.2$, $125.6,124.9,123.0,120.3$.

\section{3,4-Difluoro- $N$-phenylbenzamide (3an, Figure 4)}<smiles>COC(=O)c1ccc(F)c(F)c1</smiles>

According to the general procedure, the reaction of methyl 3,4-difluorobenzoate $(0.10 \mathrm{mmol})$, aniline (1.2 equiv) and LiHMDS (1.0 M in THF, 2.0 equiv) in toluene $(0.25 \mathrm{M})$ for $15 \mathrm{~h}$ at room temperature, afforded after work-up and chromatography the title compound in $82 \%$ yield (19.1 mg). White solid. ${ }^{1} \mathrm{H}$ NMR (500 MHz, DMSO-d $\left.d_{6}\right) \delta 10.32(\mathrm{~s}, 1 \mathrm{H}), 8.11-8.02(\mathrm{~m}, 1 \mathrm{H}), 7.88$ (s, 1 H), $7.76(\mathrm{~d}, J=8.0 \mathrm{~Hz}, 2 \mathrm{H}), 7.63(\mathrm{dd}, J=18.5,8.6 \mathrm{~Hz}, 1 \mathrm{H}), 7.38$ (t, $J=7.7 \mathrm{~Hz}, 2 \mathrm{H}), 7.13$ (t, $J$ $=7.3 \mathrm{~Hz}, 1 \mathrm{H}) .{ }^{13} \mathrm{C} \mathrm{NMR}\left(125 \mathrm{MHz}, \mathrm{DMSO}-d_{6}\right) \delta 163.7,152.0\left(\mathrm{dd}, J^{F}=251.0,12.6 \mathrm{~Hz}\right), 149.6$ $\left(\mathrm{dd}, J^{F}=246.6,12.9 \mathrm{~Hz}\right), 139.3,132.8\left(\mathrm{dd}, J^{F}=4.9,3.6 \mathrm{~Hz}\right), 129.1,125.7\left(\mathrm{dd}, J^{F}=7.4,3.4 \mathrm{~Hz}\right)$, 124.4, 120.9, $118.1\left(\mathrm{~d}, J^{F}=17.6 \mathrm{~Hz}\right), 117.6\left(\mathrm{~d}, J^{F}=18.6 \mathrm{~Hz}\right) .{ }^{19} \mathrm{~F}$ NMR $\left(470 \mathrm{MHz}, \mathrm{DMSO}-d_{6}\right) \delta$ $-133.86--134.29(\mathrm{~m}),-137.74--137.97(\mathrm{~m})$.

\section{$N$-Phenylthiophene-2-carboxamide (3ao, Figure 4)}

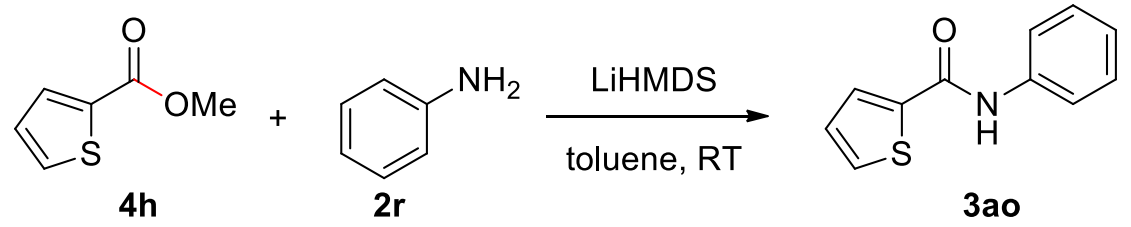

According to the general procedure, the reaction of methyl thiophene-2-carboxylate $(0.10 \mathrm{mmol})$, aniline (1.2 equiv) and LiHMDS (1.0 M in THF, 2.0 equiv) in toluene ( $0.25 \mathrm{M})$ for $15 \mathrm{~h}$ at room temperature, afforded after work-up and chromatography the title compound in $80 \%$ yield (16.2 mg). White solid. ${ }^{1} \mathrm{H}$ NMR (500 MHz, $\left.\mathrm{CDCl}_{3}\right) \delta 7.81(\mathrm{~s}, 1 \mathrm{H}), 7.65$ (dd, $\left.J=12.0,5.6 \mathrm{~Hz}, 3 \mathrm{H}\right)$, 
$7.56(\mathrm{~d}, J=4.8 \mathrm{~Hz}, 1 \mathrm{H}), 7.38(\mathrm{t}, J=7.7 \mathrm{~Hz}, 2 \mathrm{H}), 7.16(\mathrm{dd}, J=16.8,5.8 \mathrm{~Hz}, 2 \mathrm{H}) .{ }^{13} \mathrm{C}$ NMR $\left(125 \mathrm{MHz} \mathrm{CDCl}_{3}\right) \delta 160.0,139.3,137.6,130.8,129.1,128.5,127.8,124.6,120.3$.

\section{$N$-Phenylacetamide (3ap, Figure 4)}

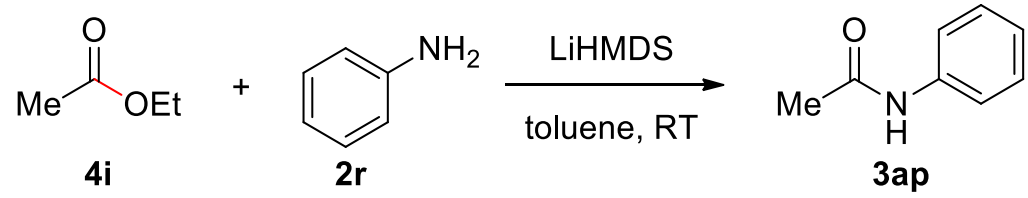

According to the general procedure, the reaction of ethyl acetate $(0.10 \mathrm{mmol})$, aniline (1.2 equiv) and LiHMDS (1.0 M in THF, 2.0 equiv) in toluene $(0.25 \mathrm{M})$ for $15 \mathrm{~h}$ at room temperature, afforded after work-up and chromatography the title compound in $86 \%$ yield $(11.6 \mathrm{mg})$. White solid. ${ }^{1} \mathrm{H}$ NMR $\left(500 \mathrm{MHz}, \mathrm{CDCl}_{3}\right) \delta 7.52(\mathrm{~d}, J=8.0 \mathrm{~Hz}, 2 \mathrm{H}), 7.39$ (s, $\left.1 \mathrm{H}\right), 7.33$ (t, $J=7.7 \mathrm{~Hz}$, $2 \mathrm{H}), 7.13(\mathrm{t}, J=7.3 \mathrm{~Hz}, 1 \mathrm{H}), 2.19(\mathrm{~s}, 3 \mathrm{H}) .{ }^{13} \mathrm{C} \mathrm{NMR}\left(125 \mathrm{MHz}, \mathrm{CDCl}_{3}\right) \delta 168.4,137.9,129.0$, $124.3,119.9,24.6$.

\section{$N$-Phenyldecanamide (3aq, Figure 4)}<smiles>CCC(C)(C)CC(=O)OC</smiles>

4j<smiles>Nc1ccccc1</smiles>

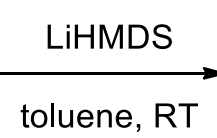
toluene, RT

$2 r$<smiles>CCC(C)(C)C(C)(C)C(N)=O</smiles>

$3 a q$

According to the general procedure, the reaction of methyl decanoate $(0.10 \mathrm{mmol})$, aniline $(1.2$ equiv) and LiHMDS (1.0 $\mathrm{M}$ in THF, 2.0 equiv) in toluene $(0.25 \mathrm{M})$ for $15 \mathrm{~h}$ at room temperature, afforded after work-up and chromatography the title compound in $85 \%$ yield $(21.0$ mg). White solid. ${ }^{1} \mathrm{H}$ NMR $\left(500 \mathrm{MHz}, \mathrm{CDCl}_{3}\right) \delta 7.54(\mathrm{~d}, J=7.9 \mathrm{~Hz}, 2 \mathrm{H}), 7.32$ (t, J = 7.6 Hz, 2 H), $7.11(\mathrm{t}, J=7.2 \mathrm{~Hz}, 1 \mathrm{H}), 2.37(\mathrm{t}, J=7.5 \mathrm{~Hz}, 2 \mathrm{H}), 1.78-1.71(\mathrm{~m}, 2 \mathrm{H}), 1.36-1.26(\mathrm{~m}, 12 \mathrm{H})$, $0.90(\mathrm{t}, J=6.9 \mathrm{~Hz}, 3 \mathrm{H}) .{ }^{13} \mathrm{C} \mathrm{NMR}\left(125 \mathrm{MHz}, \mathrm{CDCl}_{3}\right) \delta 171.7,138.1,129.0,124.2,119.9,37.8$, $31.9,29.5,29.4,29.3,29.3,25.7,22.7,14.1$.

\section{$N$-Phenylcyclohexanecarboxamide (3ar, Figure 4)}

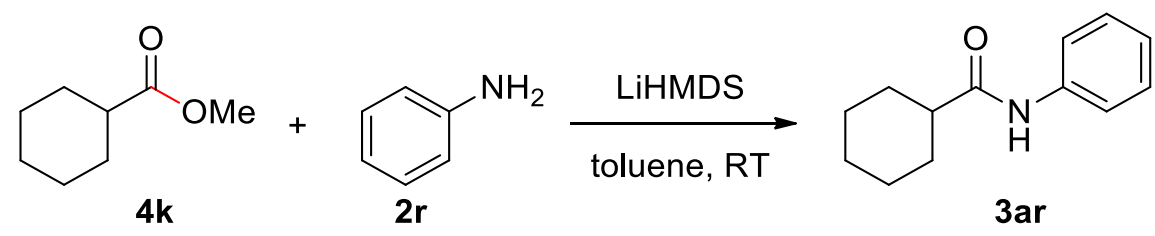


According to the general procedure, the reaction of methyl cyclohexanecarboxylate $(0.10 \mathrm{mmol})$, aniline (1.2 equiv) and LiHMDS (1.0 M in THF, 2.0 equiv) in toluene (0.25 M) for $15 \mathrm{~h}$ at room temperature, afforded after work-up and chromatography the title compound in $90 \%$ yield (18.3 mg). White solid. ${ }^{1} \mathrm{H}$ NMR $\left(500 \mathrm{MHz}, \mathrm{CDCl}_{3}\right) \delta 7.54(\mathrm{~d}, J=7.8 \mathrm{~Hz}, 2 \mathrm{H}), 7.41(\mathrm{~s}, 1 \mathrm{H}), 7.30$ (t, $J=7.2 \mathrm{~Hz}, 2 \mathrm{H}), 7.09$ (t, $J=7.3 \mathrm{~Hz}, 1 \mathrm{H}), 2.24(\mathrm{t}, J=11.7 \mathrm{~Hz}, 1 \mathrm{H}), 1.95(\mathrm{~d}, J=12.7 \mathrm{~Hz}, 2 \mathrm{H})$, 1.84-1.82 (m, $2 \mathrm{H}), 1.71-1.69$ (m, $1 \mathrm{H}), 1.58-1.49$ (m, $2 \mathrm{H}), 1.331 .21$ (m, $3 \mathrm{H}) .{ }^{13} \mathrm{C}$ NMR (125 $\left.\mathrm{MHz}_{\mathrm{CDCl}}\right) \delta 174.6,138.3,129.1,124.2,119.9,46.6,29.8,25.8$.

\section{$N$-Phenylpivalamide (3as, Figure 4)}

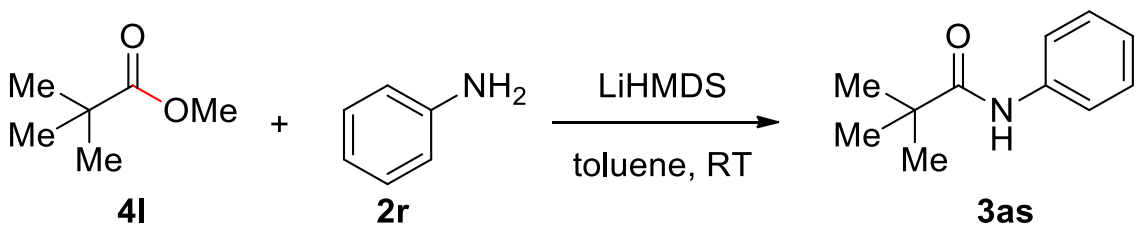

According to the general procedure, the reaction of methyl pivalate $(0.10 \mathrm{mmol})$, aniline $(1.2$ equiv) and LiHMDS (1.0 $\mathrm{M}$ in THF, 2.0 equiv) in toluene $(0.25 \mathrm{M})$ for $15 \mathrm{~h}$ at room temperature, afforded after work-up and chromatography the title compound in $88 \%$ yield (15.6 mg). White solid. ${ }^{1} \mathrm{H}$ NMR $\left(500 \mathrm{MHz}, \mathrm{CDCl}_{3}\right) \delta 7.55(\mathrm{~d}, J=8.1 \mathrm{~Hz}, 2 \mathrm{H}), 7.34(\mathrm{t}, J=7.5 \mathrm{~Hz}, 3$ $\mathrm{H}), 7.12(\mathrm{t}, J=7.4 \mathrm{~Hz}, 1 \mathrm{H}), 1.34(\mathrm{~s}, 9 \mathrm{H}) .{ }^{13} \mathrm{C} \mathrm{NMR}\left(125 \mathrm{MHz}, \mathrm{CDCl}_{3}\right) \delta$ 176.6, 138.0, 129.0, $124.2,120.0,39.6,27.7$.

\section{$N$-Phenylbenzamide (3ac, Figure 5)}

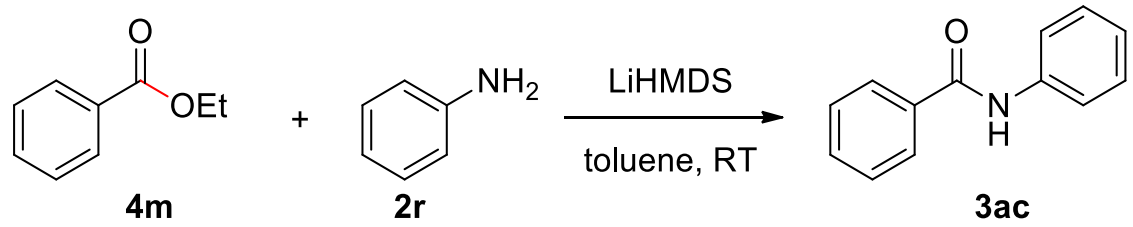

According to the general procedure, the reaction of ethyl benzoate $(0.10 \mathrm{mmol})$, aniline $(1.2$ equiv) and LiHMDS (1.0 $\mathrm{M}$ in THF, 2.0 equiv) in toluene $(0.25 \mathrm{M})$ for $15 \mathrm{~h}$ at room temperature, afforded after work-up and chromatography the title compound in 90\% yield (17.7 mg). White solid. ${ }^{1} \mathrm{H}$ NMR $\left(500 \mathrm{MHz}, \mathrm{CDCl}_{3}\right) \delta 7.78(\mathrm{~d}, J=7.7 \mathrm{~Hz}, 3 \mathrm{H}), 7.56(\mathrm{~d}, J=8.0 \mathrm{~Hz}, 2$ H), $7.46(\mathrm{t}, J=7.3 \mathrm{~Hz}, 1 \mathrm{H}), 7.39$ (t, $J=7.4 \mathrm{~Hz}, 2 \mathrm{H}), 7.29$ (t, $J=7.7 \mathrm{~Hz}, 2 \mathrm{H}), 7.07$ (t, $J=7.4$ $\mathrm{Hz}, 1 \mathrm{H}) .{ }^{13} \mathrm{C} \mathrm{NMR}\left(125 \mathrm{MHz}, \mathrm{CDCl}_{3}\right) \delta 165.7,137.9,135.0,131.8,129.1,128.8,127.0,124.5$, 120.2 . 


\section{$N$-Phenylbenzamide (3ac, Figure 5)}<smiles>CC(C)OC(=O)c1ccccc1</smiles>

According to the general procedure, the reaction of isopropyl benzoate $(0.10 \mathrm{mmol})$, aniline $(1.2$ equiv) and LiHMDS (1.0 $\mathrm{M}$ in THF, 2.0 equiv) in toluene $(0.25 \mathrm{M})$ for $15 \mathrm{~h}$ at room temperature, afforded after work-up and chromatography the title compound in 85\% yield (16.7 mg). White solid. ${ }^{1} \mathrm{H}$ NMR $\left(500 \mathrm{MHz}, \mathrm{CDCl}_{3}\right) \delta 7.78(\mathrm{~d}, J=7.7 \mathrm{~Hz}, 3 \mathrm{H}), 7.56(\mathrm{~d}, J=8.0 \mathrm{~Hz}, 2$ H), $7.46(\mathrm{t}, J=7.3 \mathrm{~Hz}, 1 \mathrm{H}), 7.39$ (t, $J=7.4 \mathrm{~Hz}, 2 \mathrm{H}), 7.29(\mathrm{t}, J=7.7 \mathrm{~Hz}, 2 \mathrm{H}), 7.07$ (t, $J=7.4$ $\mathrm{Hz}, 1 \mathrm{H}) .{ }^{13} \mathrm{C} \mathrm{NMR}\left(125 \mathrm{MHz}, \mathrm{CDCl}_{3}\right) \delta 165.7,137.9,135.0,131.8,129.1,128.8,127.0,124.5$, 120.2.

\section{$N$-Phenylbenzamide (3ac, Figure 5)}<smiles>CC(C)(C)OC(=O)c1ccccc1</smiles>

40<smiles>Nc1ccccc1</smiles>

$2 r$<smiles>O=C(Nc1ccccc1)c1ccccc1</smiles>

3ac

According to the general procedure, the reaction of tert-butyl benzoate $(0.10 \mathrm{mmol})$, aniline $(3.0$ equiv) and LiHMDS (1.0 $\mathrm{M}$ in THF, 3.0 equiv) in toluene $(0.25 \mathrm{M})$ for $15 \mathrm{~h}$ at room temperature, afforded after work-up and chromatography the title compound in $75 \%$ yield (14.8 mg). White solid. ${ }^{1} \mathrm{H}$ NMR $\left(500 \mathrm{MHz}, \mathrm{CDCl}_{3}\right) \delta 7.78(\mathrm{~d}, J=7.7 \mathrm{~Hz}, 3 \mathrm{H}), 7.56(\mathrm{~d}, J=8.0 \mathrm{~Hz}, 2$ H), $7.46(\mathrm{t}, J=7.3 \mathrm{~Hz}, 1 \mathrm{H}), 7.39$ (t, $J=7.4 \mathrm{~Hz}, 2 \mathrm{H}), 7.29$ (t, $J=7.7 \mathrm{~Hz}, 2 \mathrm{H}), 7.07$ (t, $J=7.4$ $\mathrm{Hz}, 1 \mathrm{H}) .{ }^{13} \mathrm{C} \mathrm{NMR}\left(125 \mathrm{MHz}, \mathrm{CDCl}_{3}\right) \delta 165.7,137.9,135.0,131.8,129.1,128.8,127.0,124.5$, 120.2 .

\section{$N$-Phenylbenzamide (3ac, Figure 5)}<smiles>O=C(OBr)c1ccccc1</smiles><smiles>Nc1ccccc1</smiles>

LiHMDS<smiles>O=C(Nc1ccccc1)c1ccccc1</smiles>
3ac 
According to the general procedure, the reaction of benzyl benzoate $(0.10 \mathrm{mmol})$, aniline $(1.2$ equiv) and LiHMDS (1.0 $\mathrm{M}$ in THF, 2.0 equiv) in toluene $(0.25 \mathrm{M})$ for $15 \mathrm{~h}$ at room temperature, afforded after work-up and chromatography the title compound in 94\% yield (18.5 mg). White solid. ${ }^{1} \mathrm{H}$ NMR (500 MHz, $\left.\mathrm{CDCl}_{3}\right) \delta 7.78(\mathrm{~d}, J=7.7 \mathrm{~Hz}, 3 \mathrm{H}), 7.56(\mathrm{~d}, J=8.0 \mathrm{~Hz}, 2$ H), 7.46 (t, $J=7.3 \mathrm{~Hz}, 1 \mathrm{H}), 7.39$ (t, $J=7.4 \mathrm{~Hz}, 2 \mathrm{H}), 7.29$ (t, $J=7.7 \mathrm{~Hz}, 2 \mathrm{H}), 7.07$ (t, $J=7.4$ $\mathrm{Hz}, 1 \mathrm{H}) .{ }^{13} \mathrm{C}$ NMR $\left(125 \mathrm{MHz}, \mathrm{CDCl}_{3}\right) \delta 165.7,137.9,135.0,131.8,129.1,128.8,127.0,124.5$, 120.2 . 


\section{Transamidation of Twisted Amides}

\section{2-(1,2,3,6-Tetrahydropyridin-3-yl)- $N$-(p-tolyl)benzamide (3at, Figure 6)}

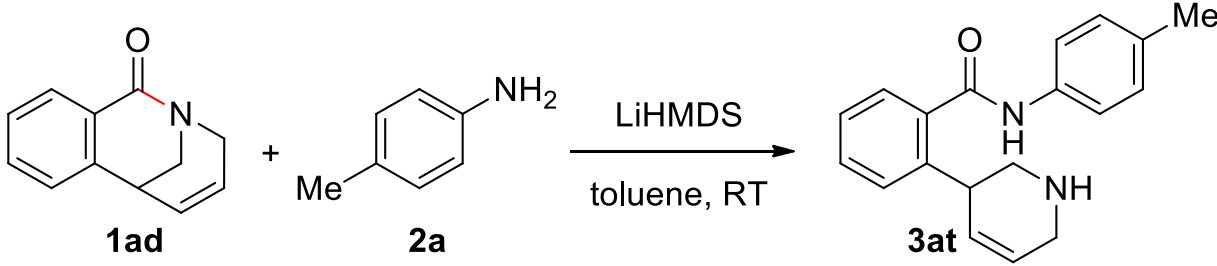

According to the general procedure, 3,6-dihydro-1H-2,6-methanobenzo[c]azocin-1-one $(0.05$ mmol) was reacted with $p$-toluidine (2.0 equiv) and LiHMDS (1.0 M in THF, 3.0 equiv) in toluene $(0.25 \mathrm{M})$ for $15 \mathrm{~h}$ at room temperature to afford the title compound in $80 \%$ yield. Yield determined by ${ }^{1} \mathrm{H}$ NMR spectroscopy vs. internal standard. Note: the title compound is unstable due to olefin isomerization. Purification by chromatography afforded an analytical sample for characterization purposes. New compound. Oil. ${ }^{1} \mathrm{H}$ NMR $\left(500 \mathrm{MHz}, \mathrm{CDCl}_{3}\right) \delta 7.60(\mathrm{~s}, 1 \mathrm{H})$, 7.55-7.42 (m, 5 H), 7.34-7.30 (m, $1 \mathrm{H}), 7.20$ (d, J=8.0 Hz, $2 \mathrm{H}), 6.00$ (d, J = 16.2 Hz, $1 \mathrm{H}), 5.85$ $(\mathrm{d}, J=10.3 \mathrm{~Hz}, 1 \mathrm{H}), 3.98(\mathrm{~s}, 1 \mathrm{H}), 3.49-3.35(\mathrm{~m}, 3 \mathrm{H}), 2.84(\mathrm{dd}, J=12.8,6.8 \mathrm{~Hz}, 1 \mathrm{H}), 2.37$ (s, $3 \mathrm{H}) .{ }^{13} \mathrm{C} \mathrm{NMR}\left(125 \mathrm{MHz}, \mathrm{CDCl}_{3}\right) \delta 167.9,142.1,136.8,135.4,134.4,130.3,129.6,129.1$, 128.7, 128.6, 126.6, 126.5, 120.0, 51.3, 44.7, 38.1, 20.9. HRMS calcd for $\mathrm{C}_{19} \mathrm{H}_{20} \mathrm{~N}_{2} \mathrm{ONa}(\mathrm{M}+$ $\mathrm{Na}$ 315.1468, found 315.1484. Note: products resulting from unselective $\sigma \mathrm{N}-\mathrm{C}$ bond cleavage were not detected. Selectivity of the N-C(O) opening $>20: 1 .^{30}$

\section{2-(Piperidin-3-yl)- $N$-(p-tolyl)benzamide (3au, Figure 6)}<smiles>O=C1c2ccccc2C2CCN1C2</smiles><smiles>Cc1ccc(N)cc1</smiles><smiles>Cc1ccc(NC(=O)c2ccccc2C2CCCCC2)cc1</smiles>

According to the general procedure, the reaction of 3,4,5,6-tetrahydro-1H-2,6methanobenzo[c]azocin-1-one $(0.05 \mathrm{mmol}), p$-toluidine (2.0 equiv) and LiHMDS (1.0 $\mathrm{M}$ in THF, 3.0 equiv) in toluene $(0.25 \mathrm{M})$ for $15 \mathrm{~h}$ at room temperature, afforded after work-up and chromatography the title compound in $90 \%$ yield $(26.5 \mathrm{mg})$. New compound. Oil. ${ }^{1} \mathrm{H}$ NMR (500 $\left.\mathrm{MHz}_{\mathrm{CDCl}}\right) \delta 7.55(\mathrm{~s}, 1 \mathrm{H}), 7.49(\mathrm{~d}, J=8.3 \mathrm{~Hz}, 2 \mathrm{H}), 7.42(\mathrm{dd}, J=12.0,7.5 \mathrm{~Hz}, 2 \mathrm{H}), 7.36(\mathrm{~d}$, 
$J=7.6 \mathrm{~Hz}, 1 \mathrm{H}), 7.28-7.24(\mathrm{~m}, 2 \mathrm{H}), 7.17(\mathrm{~d}, J=8.1 \mathrm{~Hz}, 2 \mathrm{H}), 3.22-3.04(\mathrm{~m}, 3 \mathrm{H}), 2.71-2.56(\mathrm{~m}$, $2 \mathrm{H}), 2.34$ (s, $3 \mathrm{H}), 2.06-2.01$ (m, $1 \mathrm{H}), 1.90$ (s, $1 \mathrm{H}), 1.76-1.64$ (m, $2 \mathrm{H}), 1.55$ (ddd, $J=12.5,8.0$, $3.9 \mathrm{~Hz}, 1 \mathrm{H}) .{ }^{13} \mathrm{C} \mathrm{NMR}\left(125 \mathrm{MHz}, \mathrm{CDCl}_{3}\right) \delta 168.1,143.0,137.0,135.4,134.3,130.2,129.6$, 127.1, 126.7, 126.2, 120.0, 53.6, 46.5, 40.8, 32.4, 27.3, 20.9. HRMS calcd for $\mathrm{C}_{19} \mathrm{H}_{23} \mathrm{~N}_{2} \mathrm{O}(\mathrm{M}+$ H) 295.1805, found 295.1820. Note: products resulting from unselective $\sigma \mathrm{N}-\mathrm{C}$ bond cleavage were not detected. Selectivity of the $\mathrm{N}-\mathrm{C}(\mathrm{O})$ opening $>20: 1 .^{30}$ 


\section{One-pot $N$-Activation/Transamidation}

$N$-(p-Tolyl)benzamide (3a, Figure 7)

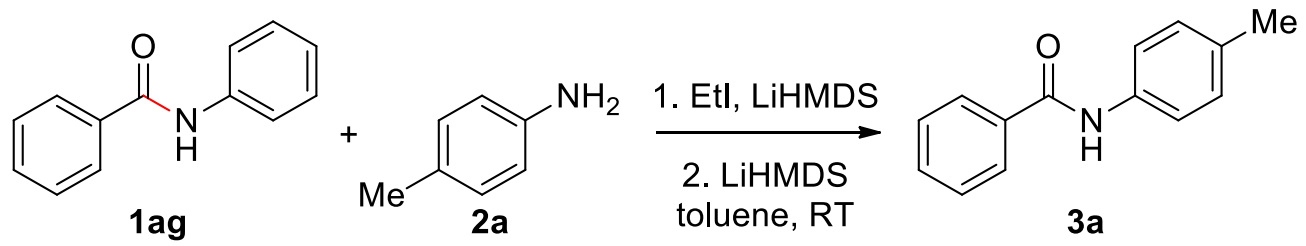

An oven-dried vial equipped with a stir bar was charged with $N$-phenylbenzamide $(0.10$ mmol, 1.0 equiv), placed under a positive pressure of argon and subjected to three evacuation/backfilling cycles. Toluene $(0.20 \mathrm{M})$ and LiHMDS (1.0 $\mathrm{M}$ in THF, 1.0 equiv) were sequentially added with vigorous stirring at room temperature, and the reaction mixture was stirred at room temperature for $10 \mathrm{~min}$. After the indicated time, iodoethane (2.0 equiv) was added and the resulting reaction mixture was stirred for $15 \mathrm{~h}$ at room temperature. After the indicated time, the reaction mixture was filtered and concentrated. The crude product was used in next step without further purification. According to the general procedure, an oven-dried vial equipped with a stir bar was charged with the above crude product, placed under a positive pressure of argon and subjected to three evacuation/backfilling cycles. $p$-Toluidine (2.0 equiv), toluene $(0.25 \mathrm{M})$ and LiHMDS (1.0 M in THF, 3.0 equiv) were sequentially added with vigorous stirring at room temperature, and the reaction mixture was stirred for $15 \mathrm{~h}$ at room temperature. Standard work-up and purification by chromatography on silica gel (EtOAc/hexanes) afforded the title product. Yield $83 \%(17.4 \mathrm{mg})$. White solid. ${ }^{1} \mathrm{H} \mathrm{NMR}\left(500 \mathrm{MHz}, \mathrm{CDCl}_{3}\right) \delta 7.88(\mathrm{~d}, J=$ $7.3 \mathrm{~Hz}, 2 \mathrm{H}), 7.83$ (s, $1 \mathrm{H}), 7.58-7.54(\mathrm{~m}, 3 \mathrm{H}), 7.50$ (t, $J=7.5 \mathrm{~Hz}, 2 \mathrm{H}), 7.19$ (d, J = 8.2 Hz, 2 $\mathrm{H}), 2.37$ (s, $3 \mathrm{H}) .{ }^{13} \mathrm{C}$ NMR $\left(125 \mathrm{MHz}, \mathrm{CDCl}_{3}\right) \delta 165.7,135.4,135.1,134.3,131.7,129.6,128.8$, $127.0,120.3,20.9$.

\section{$N$-Phenylbenzamide (3ac, Figure 7)}

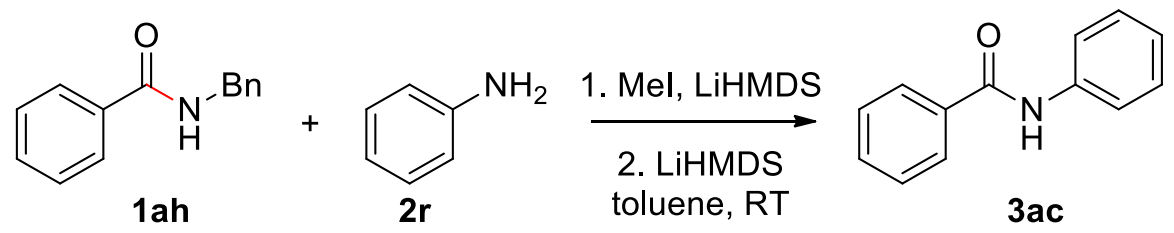

An oven-dried vial equipped with a stir was charged with $N$-benzylbenzamide $(0.10$ mmol, 1.0 equiv), placed under a positive pressure of argon and subjected to three evacuation/ S231 
backfilling cycles. Toluene (0.20 M) and LiHMDS (1.0 M in THF, 1.0 equiv) were sequentially added with vigorous stirring at room temperature, and the reaction mixture was stirred at room temperature for $10 \mathrm{~min}$. After the indicated time, iodomethane (2.0 equiv) was added and the resulting reaction mixture was stirred for $15 \mathrm{~h}$ at room temperature. After the indicated time, the reaction mixture was filtered and concentrated. The crude product was used in next step without further purification. According to the general procedure, an oven-dried vial equipped with a stir bar was charged with the above crude product, placed under a positive pressure of argon and subjected to three evacuation/backfilling cycles. Aniline (2.0 equiv), toluene $(0.25 \mathrm{M})$ and LiHMDS (1.0 M in THF, 3.0 equiv) were sequentially added with vigorous stirring at room temperature, and the reaction mixture was stirred for $15 \mathrm{~h}$ at room temperature. Standard workup and purification by chromatography on silica gel (EtOAc/hexanes) afforded the title product. Yield 81\% (16.0 mg). White solid. ${ }^{1} \mathrm{H}$ NMR (500 MHz, $\left.\mathrm{CDCl}_{3}\right) \delta 7.78(\mathrm{~d}, J=7.7 \mathrm{~Hz}, 3 \mathrm{H}), 7.56$ $(\mathrm{d}, J=8.0 \mathrm{~Hz}, 2 \mathrm{H}), 7.46(\mathrm{t}, J=7.3 \mathrm{~Hz}, 1 \mathrm{H}), 7.39(\mathrm{t}, J=7.4 \mathrm{~Hz}, 2 \mathrm{H}), 7.29(\mathrm{t}, J=7.7 \mathrm{~Hz}, 2 \mathrm{H})$, $7.07(\mathrm{t}, J=7.4 \mathrm{~Hz}, 1 \mathrm{H}) .{ }^{13} \mathrm{C} \mathrm{NMR}\left(125 \mathrm{MHz}, \mathrm{CDCl}_{3}\right) \delta 165.7,137.9,135.0,131.8,129.1$, $128.8,127.0,124.5,120.2$. 


\section{Application in Medicinal Chemistry}

Synthesis of $N$-(2,6-dimethylphenyl)-3,4,5-trimethoxybenzamide (3av, Figure 9)<smiles>COc1cc(C(=O)N2CCc3ccccc32)cc(OC)c1OC</smiles>

1 ai<smiles>Cc1cccc(C)c1N</smiles>

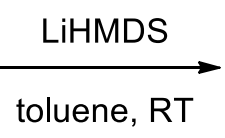

$2 i$<smiles>COc1cc(C(=O)Nc2c(C)cccc2C)cc(OC)c1OC</smiles>

3av

According to the general procedure, an oven-dried vial equipped with a stir bar was charged with indolin-1-yl(3,4,5-trimethoxyphenyl)methanone (0.10 $\mathrm{mmol}), 2,6$-dimethylaniline (2.0 equiv) placed under a positive pressure of argon, and subjected to three evacuation/backfilling cycles. Toluene $(0.25 \mathrm{M})$ and LiHMDS (1.0 M in THF, 2.0 equiv) were added with vigorous stirring at room temperature, and the reaction mixture was stirred for $15 \mathrm{~h}$ at room temperature. After the indicated time, the reaction mixture was quenched with $\mathrm{NH}_{4} \mathrm{Cl}$ (aq., $1.0 \mathrm{M}, 5 \mathrm{~mL}$ ), extracted with EtOAc $(3 \times 10 \mathrm{~mL})$, the organic layers were combined, washed with water $(1 \mathrm{x} 5 \mathrm{~mL})$, brine $(1 \mathrm{x}$ $5 \mathrm{~mL}$ ), dried over $\mathrm{Na}_{2} \mathrm{SO}_{4}$ and concentrated. Purification by chromatography on silica gel (EtOAc/hexanes) afforded the title product. Yield 83\% $(26.1 \mathrm{mg})$. White solid. ${ }^{1} \mathrm{H}$ NMR (500 $\mathrm{MHz}_{\mathrm{CDCl}}$ ) $\delta 7.35(\mathrm{~s}, 1 \mathrm{H}), 7.20-7.13(\mathrm{~m}, 5 \mathrm{H}), 3.96(\mathrm{~s}, 6 \mathrm{H}), 3.94(\mathrm{~s}, 3 \mathrm{H}), 2.31(\mathrm{~s}, 6 \mathrm{H}) .{ }^{13} \mathrm{C}$ NMR $\left(125 \mathrm{MHz} \mathrm{CDCl}_{3}\right) \delta 165.5,153.4,141.4,135.4,133.8,129.9,128.3,127.5,104.8,61.0$, $56.4,18.5$. Note: Transamidation of 1ai under previous conditions proceeded in $42 \%$ yield at 120 ${ }^{\circ} \mathrm{C},{ }^{26}$ clearly demonstrating the practicality and potential of the present method in the production of biologically-active building blocks by diversification. 


\section{Application in Heterocyclic Chemistry}

\section{$N$-(2,6-dimethylphenyl)-1-methyl-1H-indole-2-carboxamide (3aw, Figure 10)}

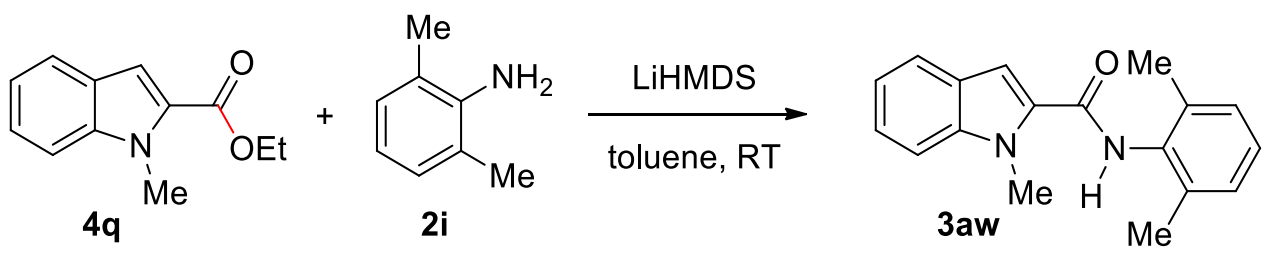

According to the general procedure, an oven-dried vial equipped with a stir bar was charged with ethyl 1-methyl-1H-indole-2-carboxylate $(0.10 \mathrm{mmol}), 2,6$-dimethylaniline $(1.2$ equiv) placed under a positive pressure of argon, and subjected to three evacuation/backfilling cycles. Toluene $(0.25 \mathrm{M})$ and LiHMDS (1.0 $\mathrm{M}$ in THF, 2.2 equiv) were added with vigorous stirring at room temperature, and the reaction mixture was stirred for $15 \mathrm{~h}$ at room temperature. After the indicated time, the reaction mixture was quenched with $\mathrm{NH}_{4} \mathrm{Cl}$ (aq., $1.0 \mathrm{M}, 5 \mathrm{~mL}$ ), extracted with EtOAc $(3 \times 10 \mathrm{~mL})$, the organic layers were combined, washed with water $(1 \mathrm{x} 5 \mathrm{~mL})$, brine $(1 \mathrm{x}$ $5 \mathrm{~mL}$ ), dried over $\mathrm{Na}_{2} \mathrm{SO}_{4}$ and concentrated. Purification by chromatography on silica gel (EtOAc/hexanes) afforded the title product. Yield 92\% (25.6 mg). New compound. White solid. $\mathrm{Mp}=177-178{ }^{\circ} \mathrm{C} .{ }^{1} \mathrm{H}$ NMR $\left(500 \mathrm{MHz}, \mathrm{CDCl}_{3}\right) \delta 7.69(\mathrm{~d}, J=7.9 \mathrm{~Hz}, 1 \mathrm{H}), 7.47(\mathrm{~s}, 1 \mathrm{H}), 7.43(\mathrm{~d}$, $J=8.4 \mathrm{~Hz}, 1 \mathrm{H}), 7.37(\mathrm{t}, J=7.6 \mathrm{~Hz}, 1 \mathrm{H}), 7.22-7.11(\mathrm{~m}, 4 \mathrm{H}), 7.08$ (s, $1 \mathrm{H}), 4.09$ (s, $3 \mathrm{H}), 2.32$ (s, $6 \mathrm{H}) .{ }^{13} \mathrm{C} \mathrm{NMR}\left(125 \mathrm{MHz}, \mathrm{CDCl}_{3}\right) \delta 160.9,139.3,135.6,133.4,131.7,128.4,127.5,126.0$, 124.4, 121.9, 120.7, 110.3, 104.2, 31.6, 18.6. HRMS calcd for $\mathrm{C}_{18} \mathrm{H}_{18} \mathrm{~N}_{2} \mathrm{ONa}(\mathrm{M}+\mathrm{Na})$ 301.1311 , found 301.1327.

\section{$N$-(2,6-diisopropylphenyl)-1H-indole-2-carboxamide (3ax, Figure 10)}

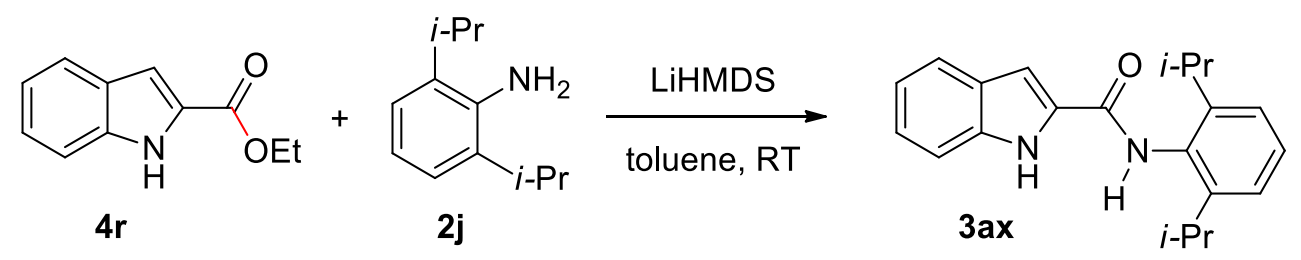

According to the general procedure, an oven-dried vial equipped with a stir bar was charged with ethyl $1 H$-indole-2-carboxylate $(0.10 \mathrm{mmol}), 2,6$-diisopropylaniline (2.0 equiv) placed under a positive pressure of argon, and subjected to three evacuation/backfilling cycles. Toluene (0.25 $\mathrm{M}$ ) and LiHMDS (1.0 $\mathrm{M}$ in THF, 4.0 equiv) were added with vigorous stirring at room 
temperature, and the reaction mixture was stirred for $15 \mathrm{~h}$ at room temperature. After the indicated time, the reaction mixture was quenched with $\mathrm{NH}_{4} \mathrm{Cl}$ (aq., $1.0 \mathrm{M}, 5 \mathrm{~mL}$ ), extracted with EtOAc $(3 \times 10 \mathrm{~mL})$, the organic layers were combined, washed with water $(1 \mathrm{x} 5 \mathrm{~mL})$, brine $(1 \mathrm{x}$ $5 \mathrm{~mL}$ ), dried over $\mathrm{Na}_{2} \mathrm{SO}_{4}$ and concentrated. Purification by chromatography on silica gel (EtOAc/hexanes) afforded the title product. Yield 75\% (24.0 mg). New compound. White solid. $\mathrm{Mp}=349-350{ }^{\circ} \mathrm{C} .{ }^{1} \mathrm{H}$ NMR $\left(500 \mathrm{MHz}, \mathrm{DMSO}-d_{6}\right) \delta 11.73(\mathrm{~s}, 1 \mathrm{H}), 9.77$ (s, $\left.1 \mathrm{H}\right), 7.67$ (d, $J=$ $7.9 \mathrm{~Hz}, 1 \mathrm{H}), 7.45$ (d, $J=8.2 \mathrm{~Hz}, 1 \mathrm{H}), 7.39$ (s, $1 \mathrm{H}), 7.33$ (t, $J=7.7 \mathrm{~Hz}, 1 \mathrm{H})$, 7.24-7.20 (m, 3 $\mathrm{H}), 7.08(\mathrm{t}, J=7.5 \mathrm{~Hz}, 1 \mathrm{H}), 3.16(\mathrm{dt}, J=13.6,6.8 \mathrm{~Hz}, 2 \mathrm{H}), 1.16(\mathrm{~m}, 12 \mathrm{H}) .{ }^{13} \mathrm{C}$ NMR $(125$ MHz, DMSO- $\left.d_{6}\right) \delta 161.4,146.9,137.1,132.7,131.8,128.2,127.6,124.0,123.5,122.0,120.3$, 112.8, 103.6, 28.7, 23.9. HRMS calcd for $\mathrm{C}_{21} \mathrm{H}_{24} \mathrm{~N}_{2} \mathrm{ONa}(\mathrm{M}+\mathrm{Na}) 343.1781$, found 343.1797. Note: Since the synthesis of five-membered benzofused heterocycles traditionally relies on the presence of $\alpha$-keto-ester moiety for their synthesis, ${ }^{57}$ the present approach permits rapid preparation of amide analogues and is compatible with both acidic NH and extremely challenging sterically-bulky anilines. 


\section{Synthesis of Moclobemide 3ay (Figure 11)}<smiles>CN(C(=O)c1ccc(Cl)cc1)N(C)c1ccccc1</smiles>

According to the general procedure, an oven-dried vial equipped with a stir bar was charged with 4-chloro- $N$-methyl- $N$-phenylbenzamide ( $0.10 \mathrm{mmol}, 1.0$ equiv), 2-morpholinoethan-1-amine (3.5 equiv) placed under a positive pressure of argon, and subjected to three evacuation/backfilling cycles. Toluene $(0.25 \mathrm{M})$ and LiHMDS (1.0 M in THF, 4.5 equiv) were added with vigorous stirring at room temperature, and the reaction mixture was stirred for $15 \mathrm{~h}$ at room temperature. After the indicated time, the reaction mixture was quenched with $\mathrm{NH}_{4} \mathrm{Cl}$ (aq., $1.0 \mathrm{M}, 5 \mathrm{~mL}$ ), extracted with EtOAc $(3 \times 10 \mathrm{~mL})$, the organic layers were combined, washed with water $(1 \times 5$ $\mathrm{mL}$ ), brine ( $1 \times 5 \mathrm{~mL})$, dried over $\mathrm{Na}_{2} \mathrm{SO}_{4}$ and concentrated. Purification by chromatography on silica gel (EtOAc/hexanes) afforded the title product. Yield $81 \%(21.7 \mathrm{mg})$. White solid. ${ }^{1} \mathrm{H}$ NMR (500 MHz, CDCl $) \delta 7.77(\mathrm{~d}, J=8.1 \mathrm{~Hz}, 2 \mathrm{H}), 7.42(\mathrm{~d}, J=8.2 \mathrm{~Hz}, 2 \mathrm{H}), 7.09(\mathrm{~s}, 1 \mathrm{H})$, 3.84-3.75 (m, $4 \mathrm{H}), 3.61(\mathrm{dd}, J=10.8,5.3 \mathrm{~Hz}, 2 \mathrm{H}), 2.71(\mathrm{t}, J=5.4 \mathrm{~Hz}, 2 \mathrm{H}), 2.63(\mathrm{~s}, 4 \mathrm{H}) .{ }^{13} \mathrm{C}$ NMR $\left(125 \mathrm{MHz}, \mathrm{CDCl}_{3}\right) \delta 166.3,137.7,132.7,128.8,128.4,66.4,57.1,53.2,35.7$. Note: analogous reaction using methyl 4-chlorobenzoate instead of 4-chloro- $N$-methyl- $N$ phenylbenzamide, afforded moclobemide in 90\% yield (2-morpholinoethan-1-amine, 2.0 equiv; LiHMDS, 1.0 M in THF, 3.0 equiv), demonstrating that both unactivated amides and alkyl esters are competent electrophiles in the reaction. 


\section{Direct Amidation of Enantiopure Amino Acid Derivatives}

tert-Butyl (R)-2-(phenylcarbamoyl)pyrrolidine-1-carboxylate (3az, Figure 12)<smiles>COC(=O)[C@@H]1CCCN1C(=O)OCc1ccccc1</smiles>

$4 \mathbf{t}$<smiles>Nc1ccccc1</smiles>

$2 \mathbf{r}$

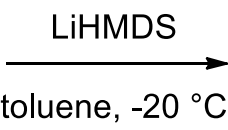

toluene, $-20{ }^{\circ} \mathrm{C}$<smiles>O=C(Nc1ccccc1)[C@@H]1CCCN1C(=O)O</smiles>

$3 a z$

According to the general procedure, an oven-dried vial equipped with a stir bar was charged with 1-(tert-butyl) 2-phenyl $(R)$-pyrrolidine-1,2-dicarboxylate $(0.10 \mathrm{mmol})$ and aniline (1.0 equiv), placed under a positive pressure of argon, and subjected to three evacuation/backfilling cycles. Toluene $(0.25 \mathrm{M})$ and LiHMDS (1.0 M in THF, 2.0 equiv) were added with vigorous stirring at $20{ }^{\circ} \mathrm{C}$, and the reaction mixture was stirred for $4 \mathrm{~h}$ at $-20{ }^{\circ} \mathrm{C}$. After the indicated time, the reaction mixture was carefully quenched with $\mathrm{NH}_{4} \mathrm{Cl}$ (aq., $1.0 \mathrm{M}, 5 \mathrm{~mL}$ ), extracted with EtOAc (3 x $10 \mathrm{~mL})$, the organic layers were combined, washed with water (1 x $5 \mathrm{~mL})$, brine $(1 \times 5 \mathrm{~mL})$, dried over $\mathrm{Na}_{2} \mathrm{SO}_{4}$ and concentrated. Purification by chromatography on silica gel (EtOAc/hexanes) afforded the title product. Yield 75\% (21.8 mg). 96.5\% ee. White solid. ${ }^{1} \mathrm{H}$ NMR (500 MHz, CDCl $) \delta 9.49(\mathrm{~s}, 1 \mathrm{H}), 7.54(\mathrm{~d}, J=7.8 \mathrm{~Hz}, 2 \mathrm{H}), 7.36-7.30(\mathrm{~m}, 2 \mathrm{H}), 7.10(\mathrm{~s}, 1$ $\mathrm{H}), 4.50(\mathrm{~s}, 1 \mathrm{H}), 3.70-3.34(\mathrm{~m}, 2 \mathrm{H}), 2.53(\mathrm{~s}, 1 \mathrm{H}), 2.04-1.88(\mathrm{~m}, 3 \mathrm{H}), 1.52(\mathrm{~s}, 9 \mathrm{H}) .{ }^{13} \mathrm{C}$ NMR $\left(125 \mathrm{MHz}, \mathrm{CDCl}_{3}\right) \delta 169.9,156.8,138.4,129.0,123.9,119.7,81.0,57.2,47.2,28.4,25.7,23.1$. HPLC analysis (chiralcel OD-H, $n$-hexane/ $i$-PrOH 90/10, $1.0 \mathrm{~mL} / \mathrm{min}, 254 \mathrm{~nm}$ ) indicated 96.5\% ee: $\mathrm{t}_{\mathrm{R}}($ major $)=6.28$ minutes, $\mathrm{t}_{\mathrm{R}}($ minor $)=7.43$ minutes. Note: analogous reaction at $-78{ }^{\circ} \mathrm{C}$ afforded the title product in $60 \%$ yield and $>99.5 \%$ ee, indicating minimal racemization under these conditions. 


\section{References}

1. Frisch, M. J.; Trucks, G. W.; Schlegel, H. B.; Scuseria, G. E.; Robb, M. A.; Cheeseman, J. R.; Scalmani, G.; Barone, V.; Mennucci, B.; Petersson, G. A.; Nakatsuji, H.; Caricato, M.; Li, X.; Hratchian, H. P.; Izmaylov, A. F.; Bloino, J.; Zheng, G.; Sonnenberg, J. L.; Hada, M.; Ehara, M.; Toyota, K.; Fukuda, R.; Hasegawa, J.; Ishida, M.; Nakajima, T.; Honda, Y.; Kitao, O.; Nakai, H.; Vreven, T.; Montgomery, J. A., Jr.; Peralta, J. E.; Ogliaro, F.; Bearpark, M.; Heyd, J. J.; Brothers, E.; Kudin, K. N.; Staroverov, V. N.; Kobayashi, R.; Normand, J.; Raghavachari, K.; Rendell, A.; Burant, J. C.; Iyengar, S. S.; Tomasi, J.; Cossi, M.; Rega, N.; Millam, M. J.; Klene, M.; Knox, J. E.; Cross, J. B.; Bakken, V.; Adamo, C.; Jaramillo, J.;

Gomperts, R.; Stratmann, R. E.; Yazyev, O.; Austin, A. J.; Cammi, R.; Pomelli, C.; Ochterski, J. W.; Martin, R. L.; Morokuma, K.; Zakrzewski, V. G.; Voth, G. A.; Salvador, P.; Dannenberg, J. J.; Dapprich, S.; Daniels, A. D.; Farkas, Ö.; Foresman, J. B.; Ortiz, J. V.; Cioslowski, J.; Fox, D. J. Gaussian, 09, revision C.01; Gaussian.: Inc., Wallingford CT, 2009.

2. Becke, D. J. Chem. Phys. 1993, 98, 5648-5652.

3. Lee, C.; Yang, W.; Parr, R. G. Phys. Rev. B: Condens. Matter Mater. Phys. 1988, 37, 785789.

4. Grimme, S.; Antony, J.; Ehrlich, S.; Krieg, H. J. Chem. Phys. 2010, 132, 154104-154119.

5. Zhao, Y.; Truhlar, D. G. Theor. Chem. Acc. 2008, 120, 215-241.

6. Marenich, A. V.; Cramer, C. J.; Truhlar, D. G. J. Phys. Chem. B 2009, 113, 6378-6396.

7. CYLview, 1.0b; Legault, C. Y.; Université de Sherbrooke, 2009. (http://www.cylview.org)

8. Lucht, B. L.; Collum, D. B. J. Am. Chem. Soc. 1996, 118, 2217-2226.

9. Greenberg, A.; Venanzi, C. A. J. Am. Chem. Soc. 1993, 115, 6951-6957.

10. Greenberg, A.; Moore, D. T.; DuBois, T. D. J. Am. Chem. Soc. 1996, 118, 8658-8668.

11. Szostak, R.; Meng, G.; Szostak, M. J. Org. Chem. 2017, 82, 6373-6378.

12. Gu, J.; Fang, Z.; Liu, C.; Yang, Z.; Li, X.; Wei, P.; Guo, K. RSC Adv. 2015, 5, 95014-95019.

13. Wang, S.; Wang, Ji; Guo, R.; Wang, G.; Chen, S. Y.; Yu, X. Q. Tetrahedron Lett. 2013, 54, 6233-6236.

14. Panini, P.; Chopra, D. New J. Chem. 2015, 39, 8720-8738.

15. Yin, Z.; Wang, Z.; Wu, X. F. Eur. J. Org. Chem. 2017, 3992-3995. 
16. Suzuki, T.; Khan, M. N. A.; Sawada, H.; Imai, E.; Itoh, Y.; Yamatsuta, K.; Tokuda, N.; Takeuchi, J.; Seko, T.; Nakagawa, H.; Miyata, N. J. Med. Chem. 2012, 55, 5760-5773.

17. Bedenbaugh, A. O.; Payton, A. L.; Bedenbaugh, J. H. J. Chem. Eng. Data 1976, 21, 247-249.

18. Ojha, D. P.; Gadde, K.; Prabhu, K. R. Org. Lett. 2016, 18, 5062-5065.

19. Chow, S. Y.; Stevens, M. Y.; Åkerbladh, L.; Bergman, S.; Odell, L. R. Chem. Eur. J. 2016, 22, 9155-9161.

20. Yi, Y. Q.; Yang, W. C.; Zhai, D. D.; Zhang, X. Y.; Li, S. Q.; Guan, B. T. Chem. Commun. 2016, 52, 10894-10897.

21. Manea, R. S.; Bhanage, B. M. Adv. Synth. Catal. 2017, 359, 2621-2629.

22. Racine, E.; Monnier, F.; Vors, J. P.; Taillefer, M. Org. Lett. 2011, 13, 2818-2821.

23. Zhou, S.; Junge, K.; Addis, D.; Das, S.; Beller, M. Angew. Chem. Int. Ed. 2009, 48, 95079510.

24. Gao, L.; Tang, H.; Wang, Z. Chem. Commun. 2014, 50, 4085-4088.

25. Meng, G.; Szostak, M. Angew. Chem. Int. Ed. 2015, 54, 14518-1452.

26. Cheung, C.; Ma, J. A.; Hu, X. J. Am. Chem. Soc. 2018, 140, 6789-6792.

27. Anima, B.; Prasenjit, M. Chem. Commun. 2017, 53, 11368-11371.

28. Quesnel, J. S.; Arndtsen, B. A. J. Am. Chem. Soc. 2013, 135, 16841-16844.

29. Li, J. Q.; Subramaniam, K.; Smith, D.; Qiao, Je, X.; Li, J. J.; Qian-Cutrone, J.; Kadow, J. F.; Vite, G. D.; Chen, B. C. Org. Lett. 2012, 14, 214-217.

30. Hu, F.; Lalancette, R.; Szostak, M. Angew. Chem. Int. Ed. 2016, 55, 5062-5066.

31. Liu, Y.; Shi, S.; Achtenhagen, M.; Liu, R.; Szostak, M. Org. Lett. 2017, 19, 1614-1617.

32. Powell, A. B.; Stahl, S. S. Org. Lett. 2013, 15, 5072-5075.

33. Wang, J.; Zhao, J.; Gong, H. Chem. Commun. 2017, 53, 10180-10183.

34. McTiernan, C. D.; Pitre, S. P.; Ismaili, H.; Scaiano, J. C. Adv. Synth. Catal. 2014, 356, 28192824.

35. Ferazzano, L.; Viola, A.; Lonati, E.; Bulbarelli, A.; Musumeci, R.; Cocuzza, C.; Lombardo, M.; Tolomelli, A. Eur. J. Med. Chem. 2016, 124, 906-919.

36. Guo, L.; Ma, X.; Fang, H.; Jia, X.; Huang, Z. Angew. Chem. Int. Ed. 2015, 54, 4023-4027.

37. Heller, S. T.; Sarpong, R. Org. Lett. 2010, 12, 4572-4575.

38. Pramanik, S.; Reddy, R. R.; Ghorai, P. Org. Lett. 2015, 17, 1393-1396.

39. Liu, H.; Shi, G.; Pan, S.; Jiang, Y.; Zhang, Y. Org. Lett. 2013, 15, 4098-4101. 
40. Mietke, T.; Cruchter, T.; Larionov, V. A.; Faber, T.; Harms, K.; Meggers, E. Adv. Synth. Catal. 2018, 360, 2093-2100.

41. Li, G.; Szostak, M. Nat. Commun. 2018, 9, 4165.

42. Yu, S.; Shin, T.; Zhang, M.; Xia, Y.; Kim, H.; Lee, S. Org. Lett. 2018, 20, 7563-7566.

43. Mangini, A. Chem. Zentr. 1940, 1, 146-147.

44. De, S.; Yin, J.; Ma, D. Org. Lett. 2017, 19, 4864-4867.

45. Kumar, S.; Vanjari, R.; Guntreddi, T.; Singh, K. N. RSC Adv. 2015, 5, 9920-9924.

46. Li, J.; Wang, P.; Zhou, B.; Shi, J.; Liu, J.; Li, X.; Fan, L.; Zheng, Y.; Ouyang, L. Eur. J. Med. Chem. 2016, 121, 294-299.

47. Xu, W. T.; Huang, B.; Dai, J. J.; Xu, H. J. Org. Lett. 2016, 18, 3114-3117.

48. Braddock, D. C.; Lickiss, P. D.; Rowley, B. C.; Pugh, D.; Purnomo, T.; Santhakumar, G.; Fussell, S. J. Org. Lett. 2018, 20, 950-953.

49. Waisser, K.; Houngbedji, N.; Machacek, M.; Sekera, M.; Urban, J.; Odlerova, Z. Collect. Czech. Chem. Commun. 1990, 55, 307-316.

50. Ding, Y.; Zhang, X.; Zhang, D.; Chen, Y.; Wu, Z.; Wang, P.; Xue, W.; Song, B.; Yang, S. Tetrahedron Lett. 2015, 56, 831-833.

51. Sugiyama, S.; Sato, S.; Yamaguchi, K. J. Pharm. Soc. Jpn. 1973, 93, 1415-1417.

52. Zhang, H.; Dai, C.; Liu, L.; Ma, H.; Bu, H.; Li, Y. Tetrahedron 2018, 74, 3712-3718.

53. Kathiravan, S.; Nicholls, I. A. Tetrahedron Lett. 2017, 58, 1-4.

54. Wu, X. F.; Oschatz, S.; Sharif, M.; Beller, M.; Langer, P. Tetrahedron 2014, 70, 23-29.

55. Kashyap, B.; Phukan, P. RSC Adv. 2013, 3, 15327-15336.

56. Brunhofer, G.; Studenik, C.; Ecker, G. F.; Erker, T. Eur. J. Pharm. Sci. 2011, 42, 37-44.

57. Joule, J. A.; Mills, K. Heterocyclic Chemistry; Wiley, 2010. 


\section{HPLC Traces}

Compound 3az: $96.5 \%$ ee was determined by chiral HPLC (chiralcel OD-H, $n$-hexane $/ i-\operatorname{PrOH}=$ $90 / 10,1 \mathrm{~mL} / \mathrm{min}, 254 \mathrm{~nm}): \mathrm{t}_{\mathrm{R}}($ major $)=6.28 \mathrm{~min}, \mathrm{t}_{\mathrm{R}}($ minor $)=7.43 \mathrm{~min}$.

(R)-3az:

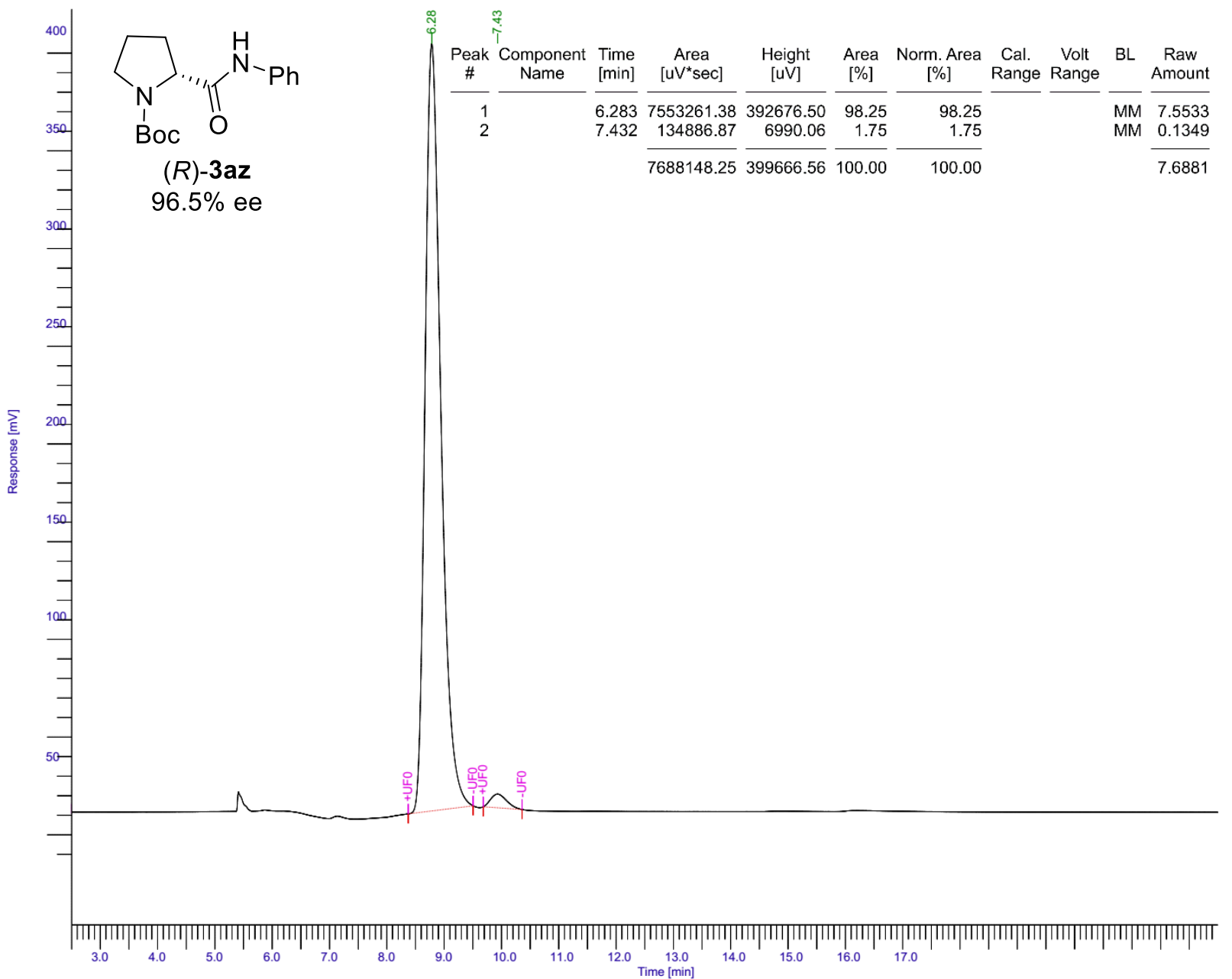




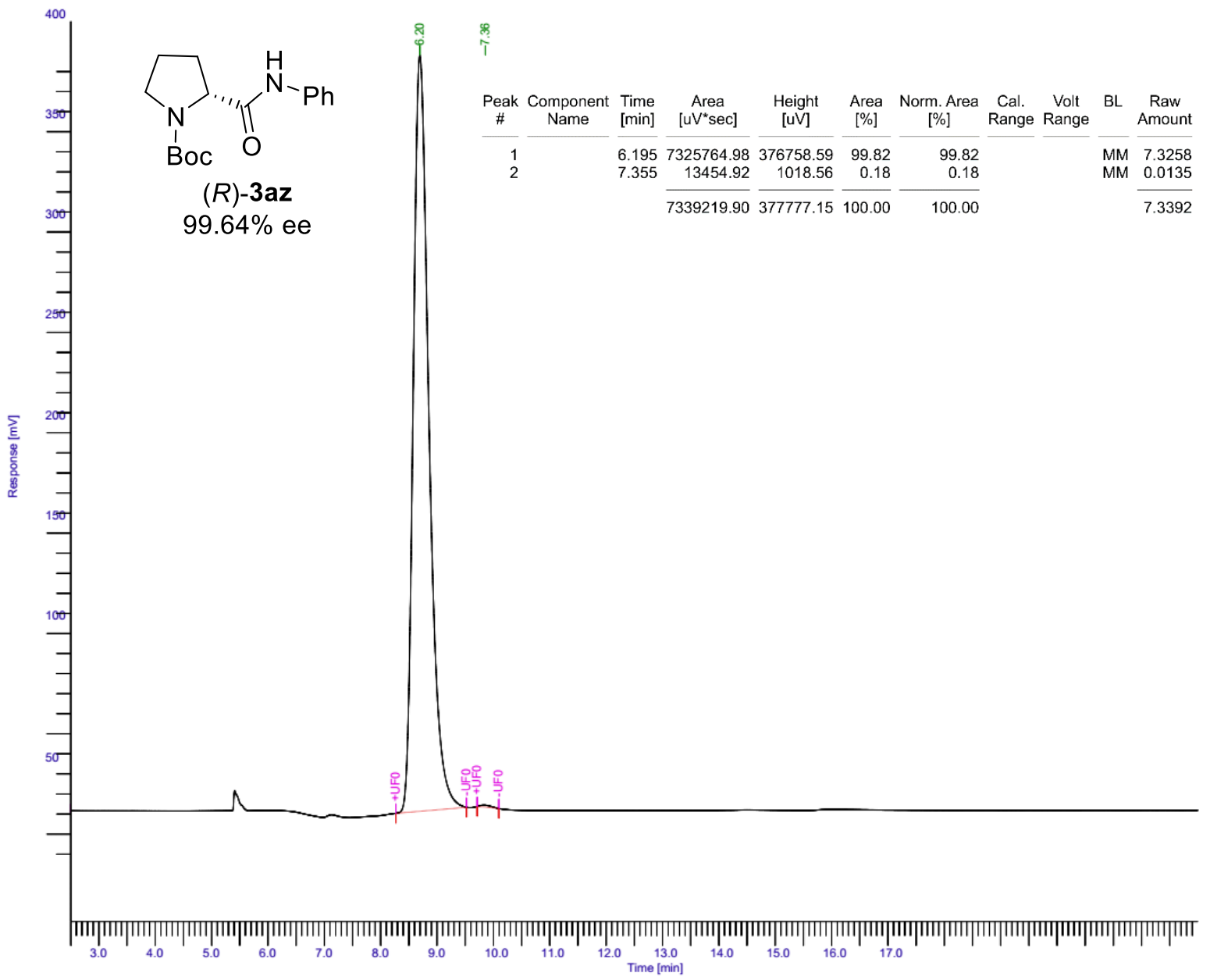


(rac)-3az:

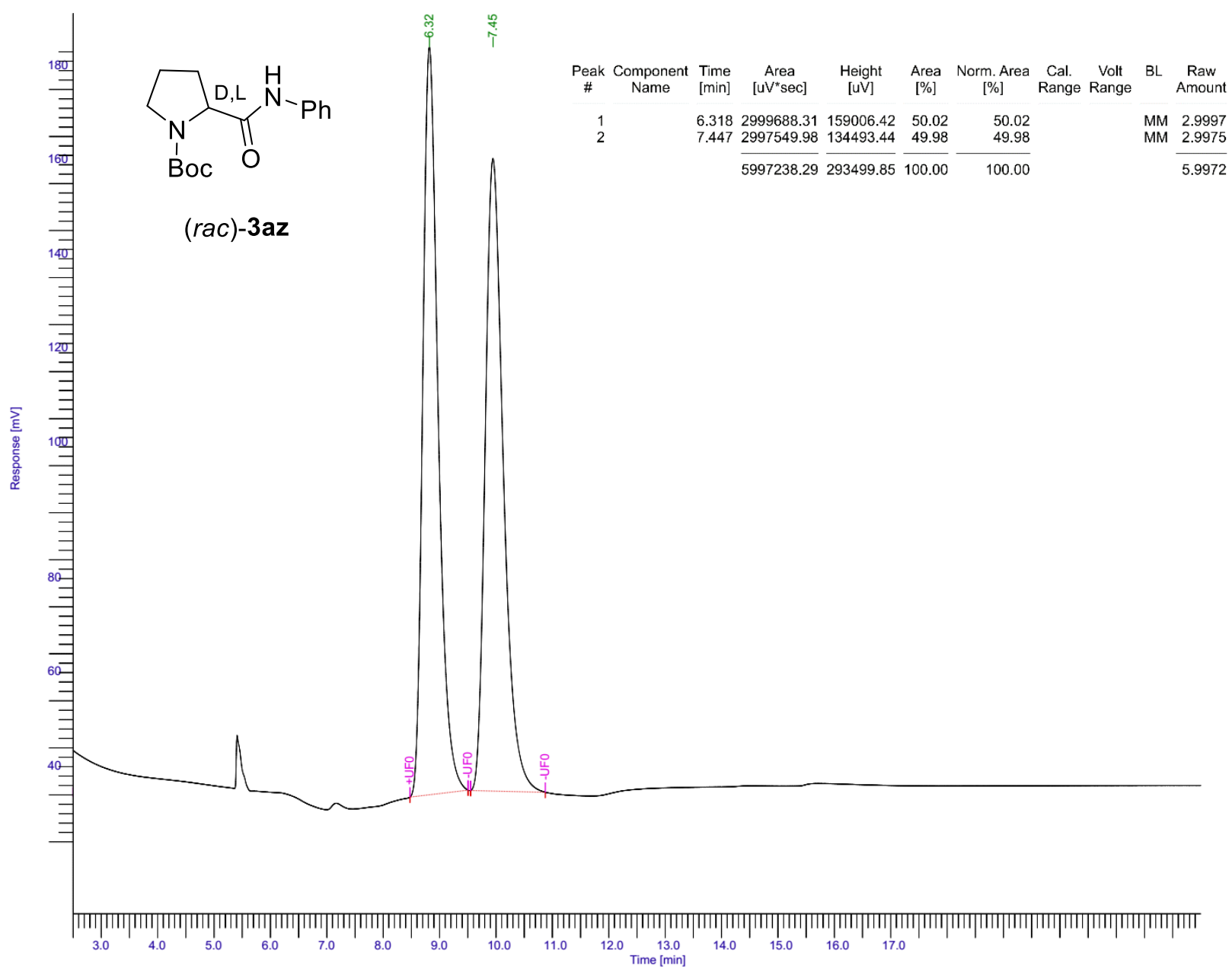


${ }^{1} \mathrm{H} \mathrm{NMR}\left(500 \mathrm{MHz}, \mathrm{CDCl}_{3}\right)$
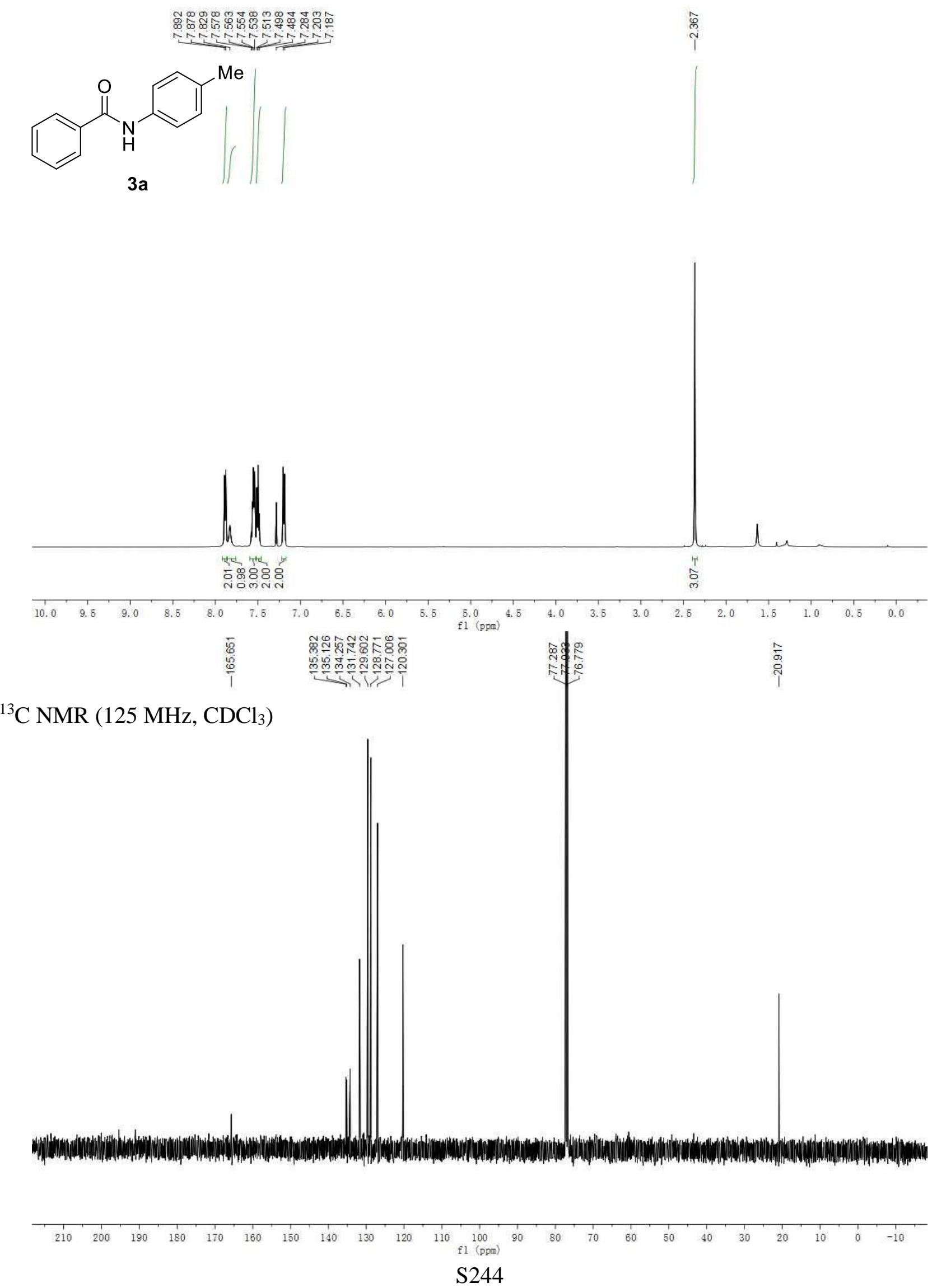
${ }^{1} \mathrm{H}$ NMR (500 $\left.\mathrm{MHz}, \mathrm{CDCl}_{3}\right)$

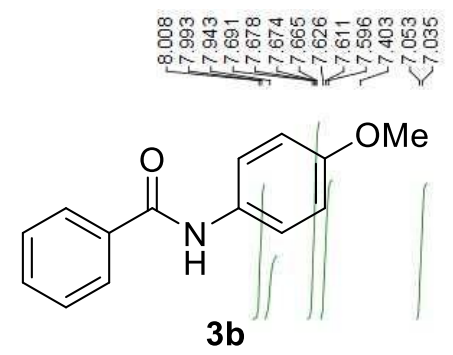

$$
\text { ॠ }
$$

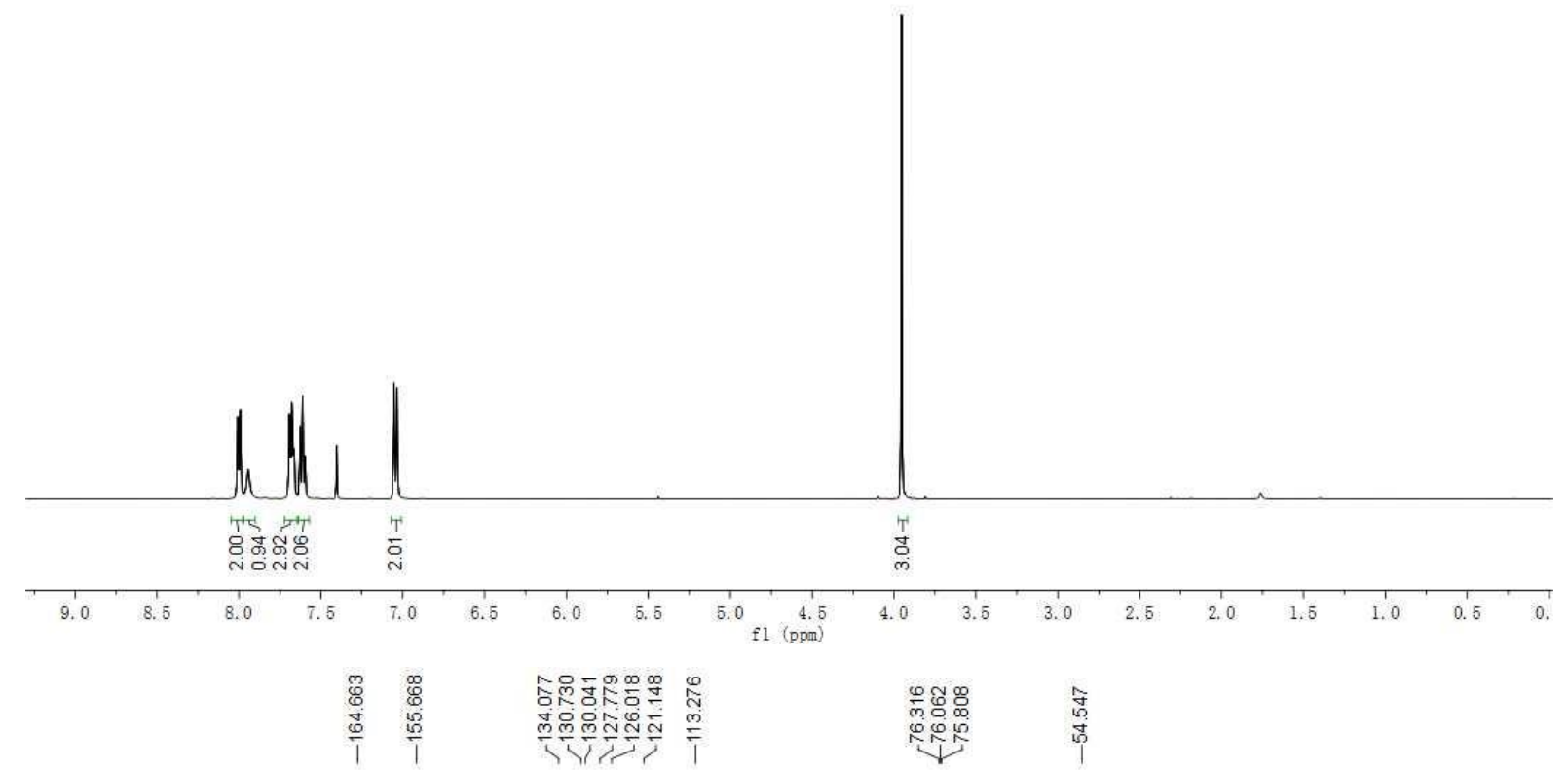

${ }^{13} \mathrm{C} \mathrm{NMR}\left(125 \mathrm{MHz}, \mathrm{CDCl}_{3}\right)$
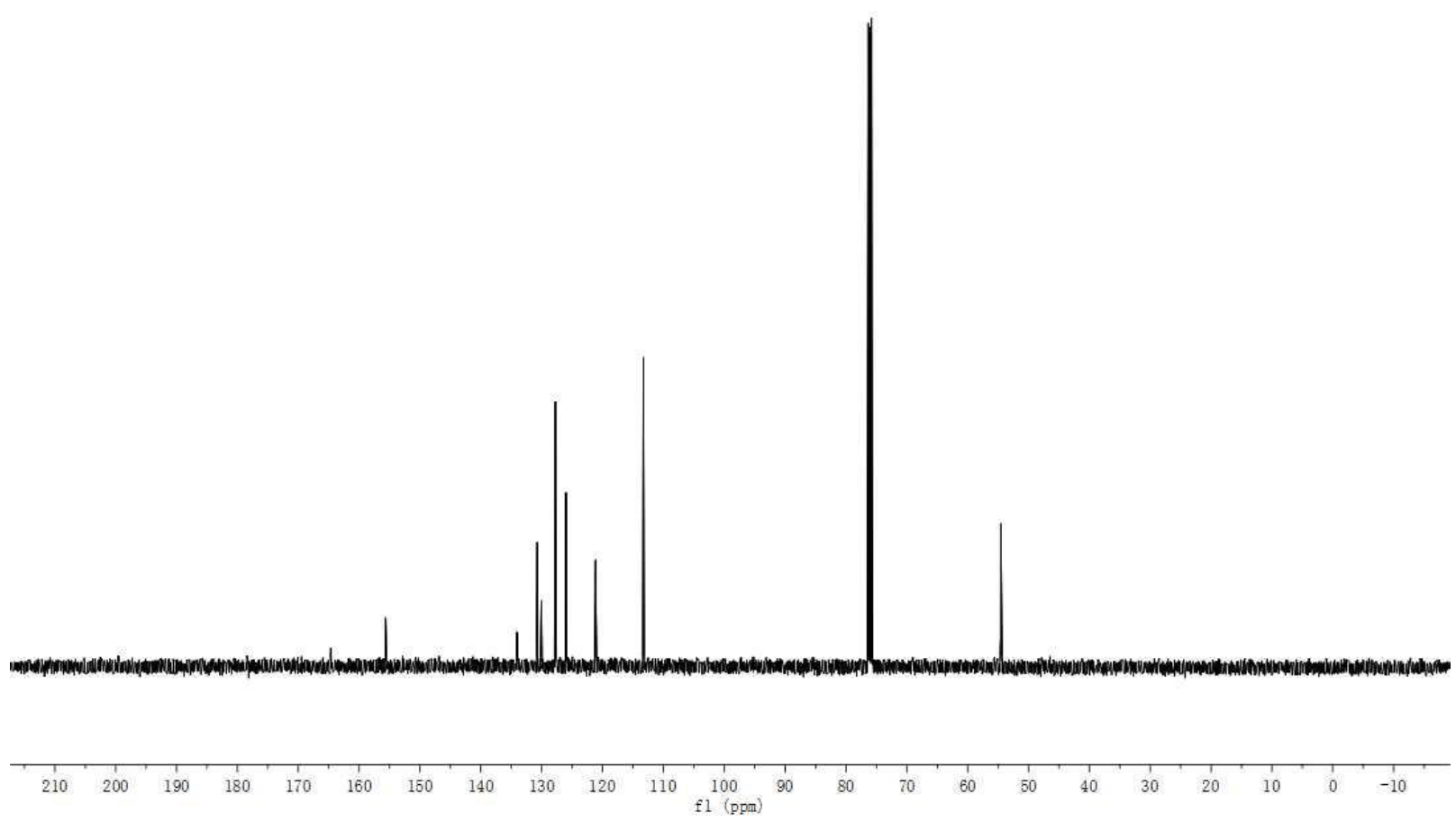
S245 
${ }^{1} \mathrm{H}$ NMR (500 MHz, DMSO- $\left.d_{6}\right)$
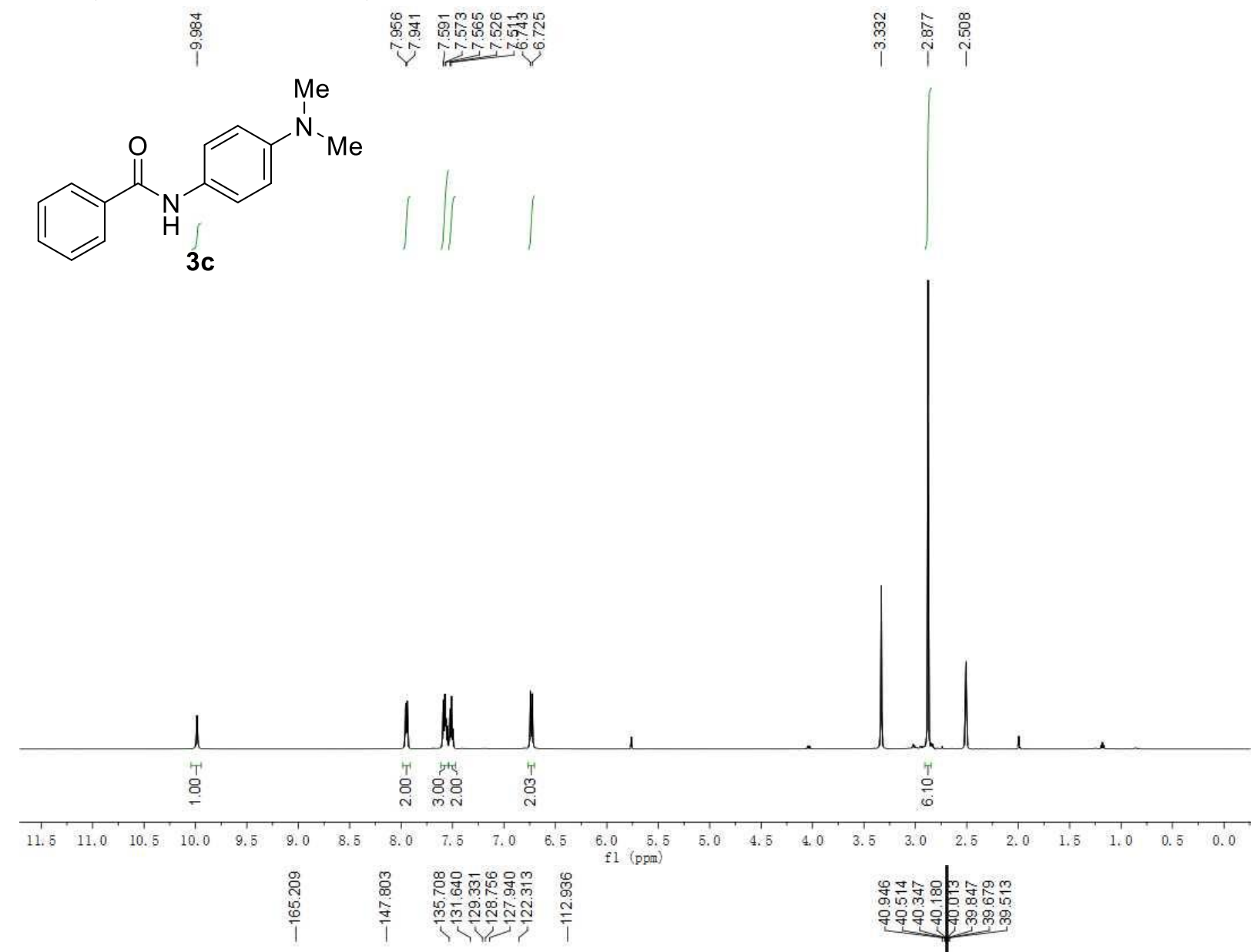

${ }^{13} \mathrm{C}$ NMR (125 MHz, DMSO- $\left.d_{6}\right)$
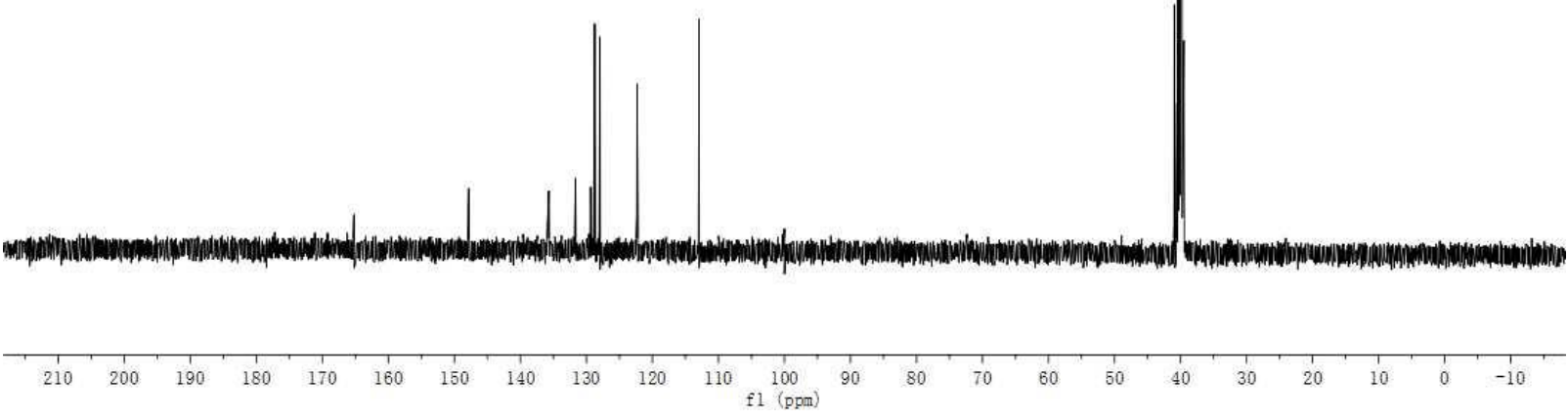
S246 
${ }^{1} \mathrm{H}$ NMR (500 MHz, DMSO-d $)$
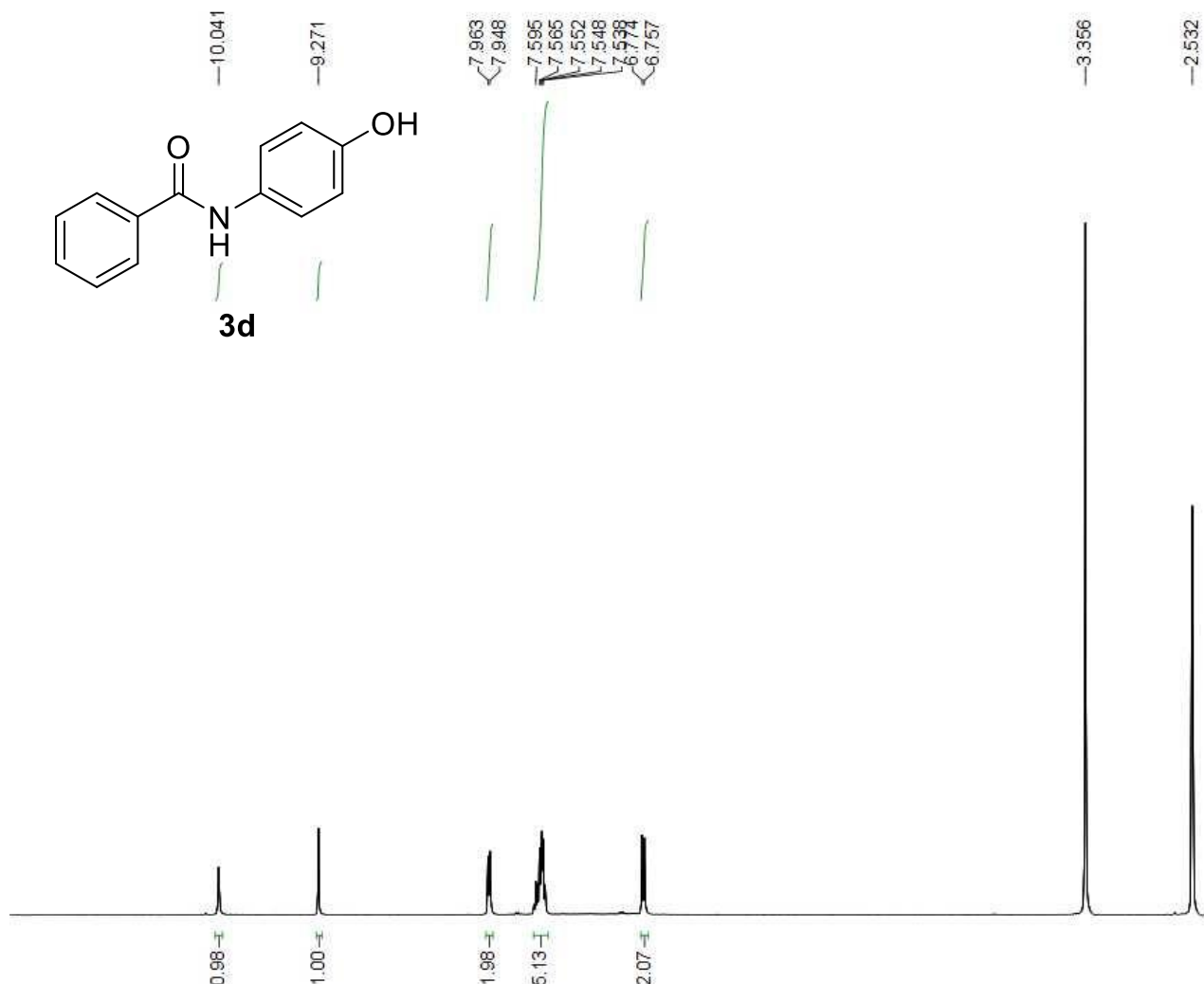

11.

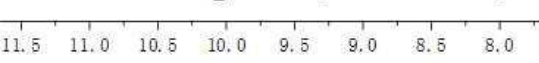

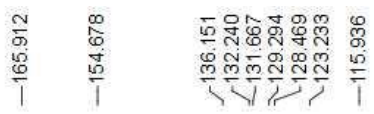

${ }^{13} \mathrm{C}$ NMR (125 MHz, DMSO-d6)
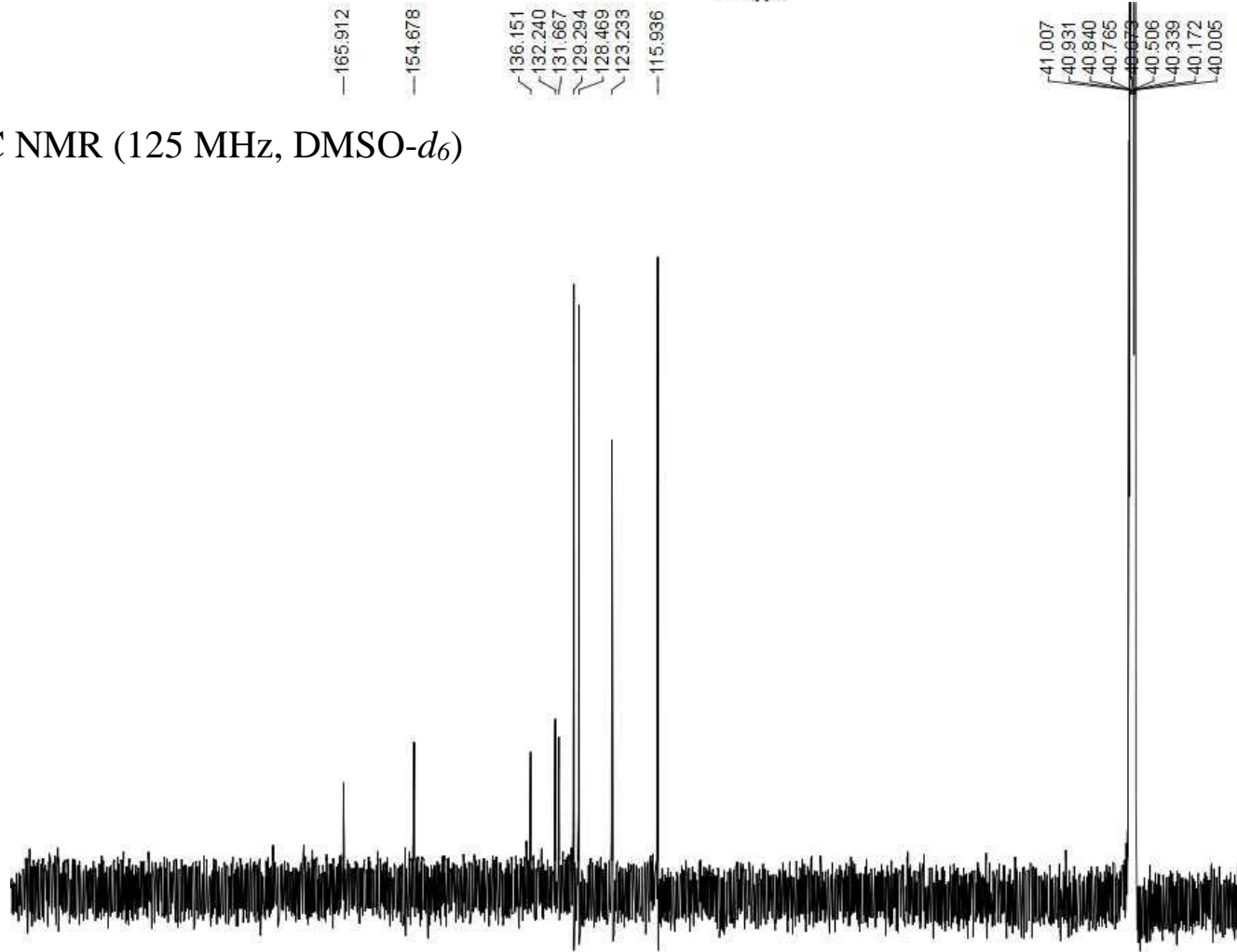

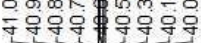

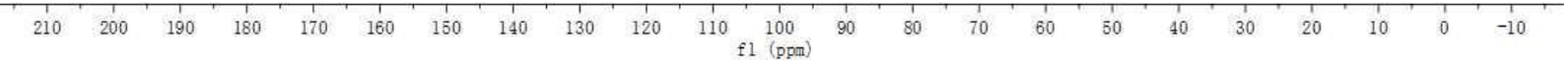


${ }^{1} \mathrm{H}$ NMR $\left(500 \mathrm{MHz}, \mathrm{CDCl}_{3}\right)$
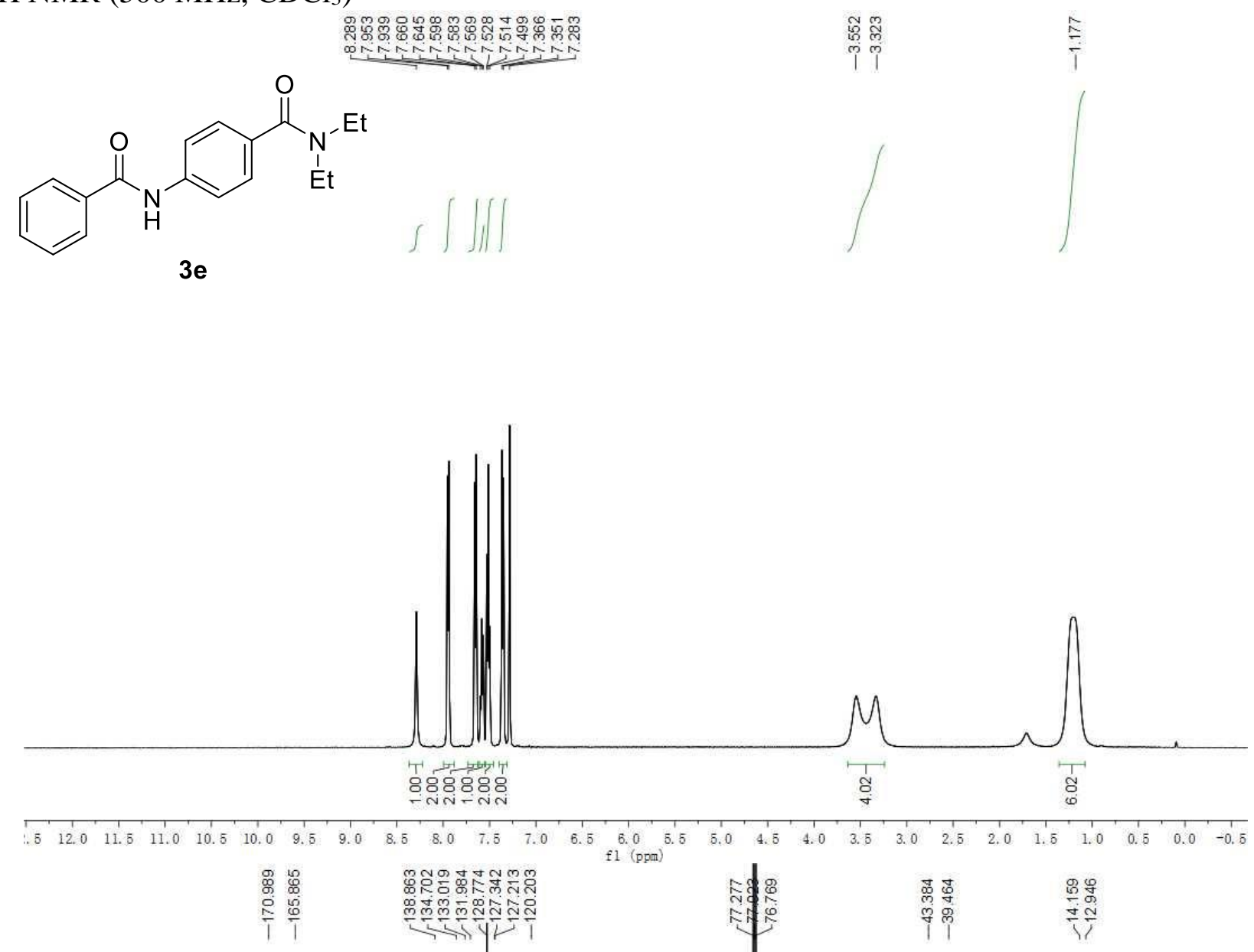

${ }^{13} \mathrm{C} \mathrm{NMR}\left(125 \mathrm{MHz}, \mathrm{CDCl}_{3}\right)$

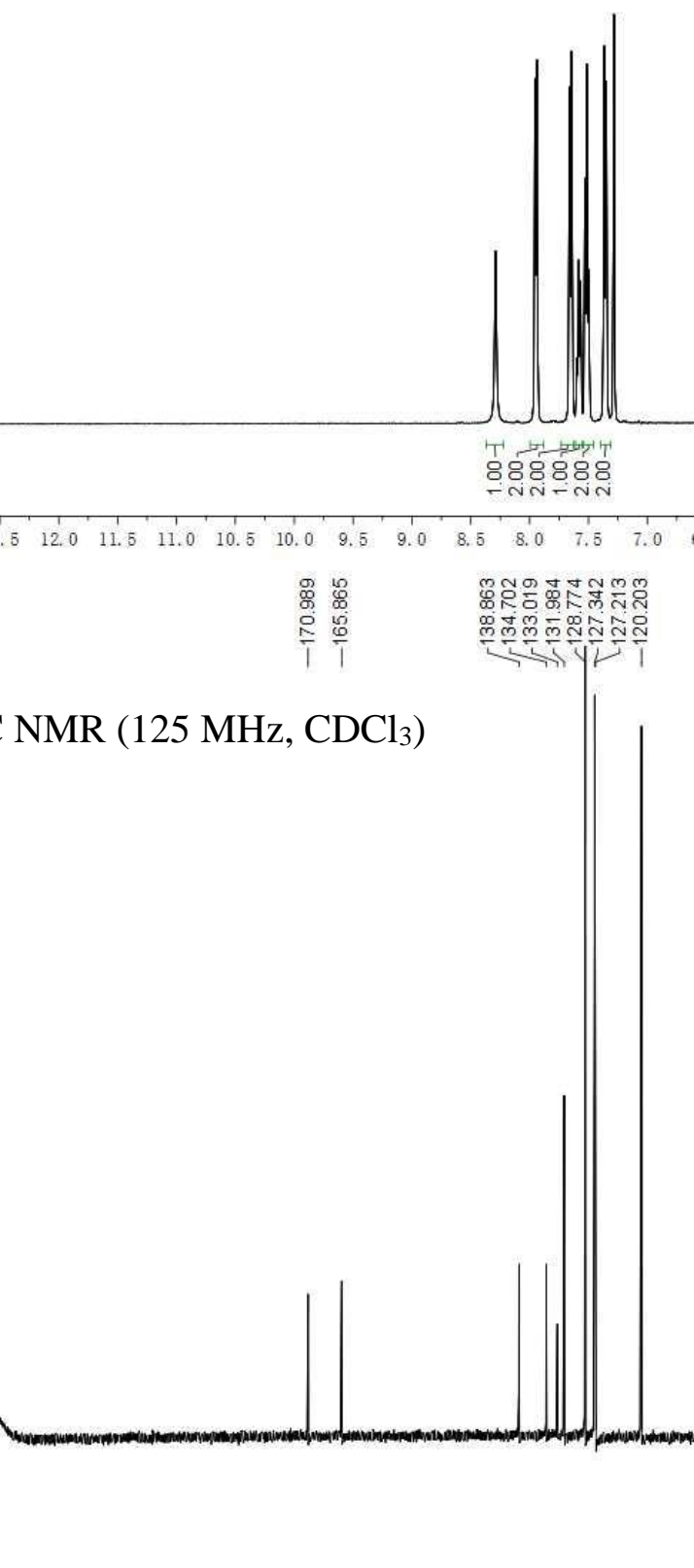

$10 \mathrm{fl}(\mathrm{ppm})$ 
${ }^{1} \mathrm{H} \mathrm{NMR}\left(500 \mathrm{MHz}, \mathrm{CDCl}_{3}\right)$
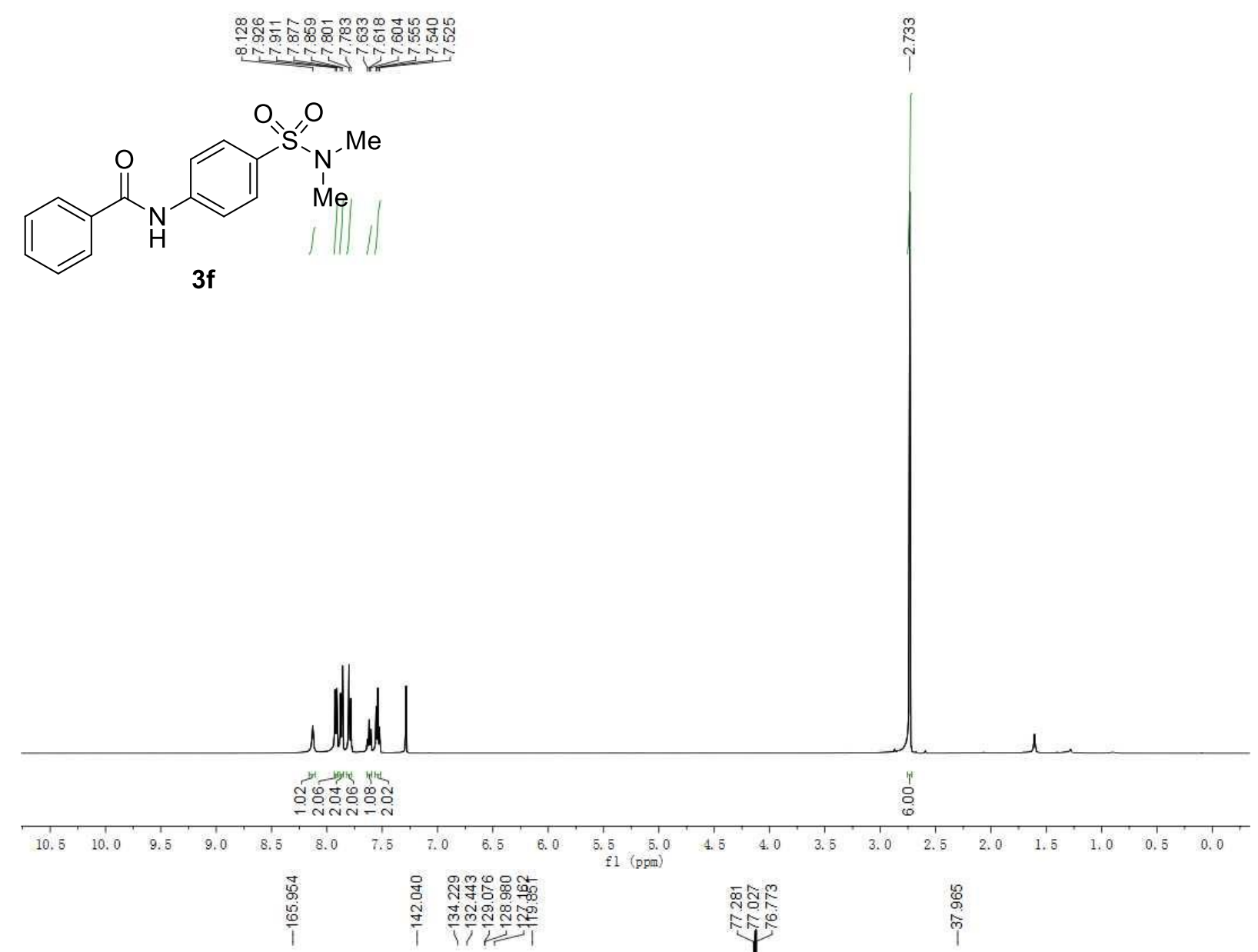

${ }^{13} \mathrm{C} \mathrm{NMR}\left(125 \mathrm{MHz}, \mathrm{CDCl}_{3}\right)$

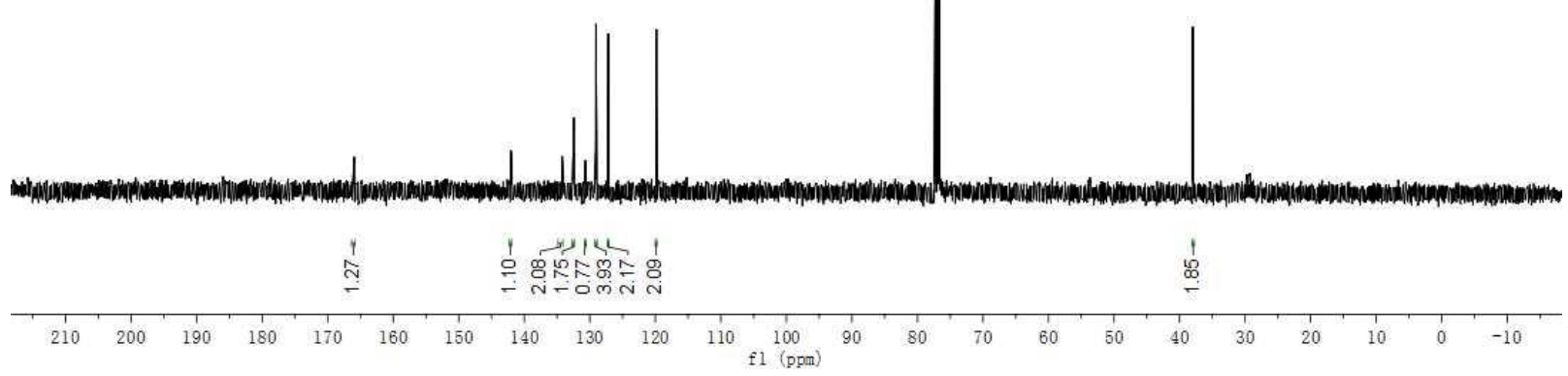
S249 
${ }^{1} \mathrm{H}$ NMR (500 MHz, $\mathrm{CDCl}_{3}$ )<smiles>Cc1ccccc1NC(=O)c1ccccc1</smiles>

$3 g$

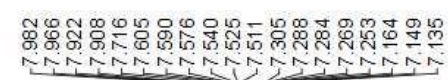
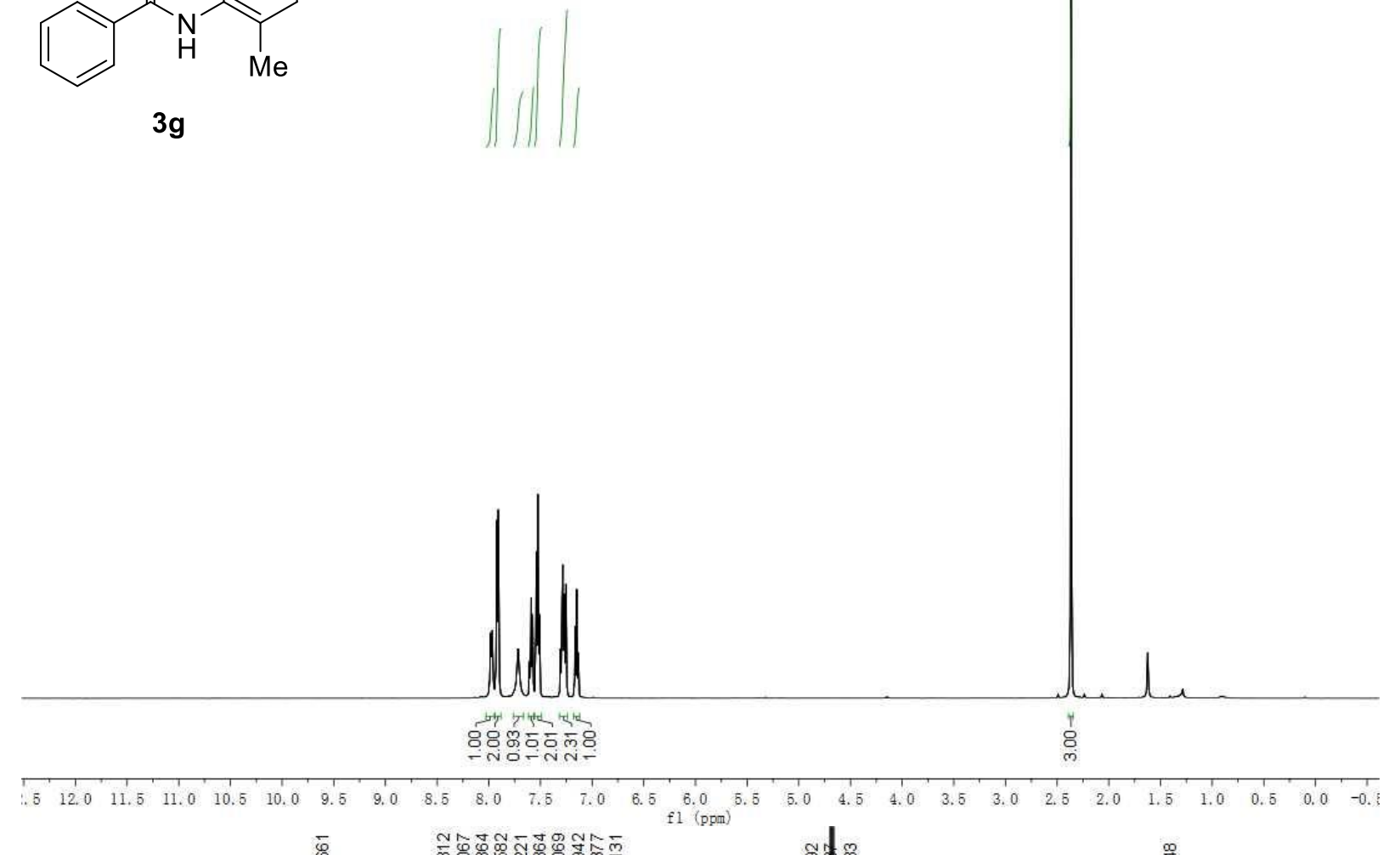

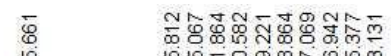

每

${ }^{13} \mathrm{C}$ NMR $\left(125 \mathrm{MHz}, \mathrm{CDCl}_{3}\right)$

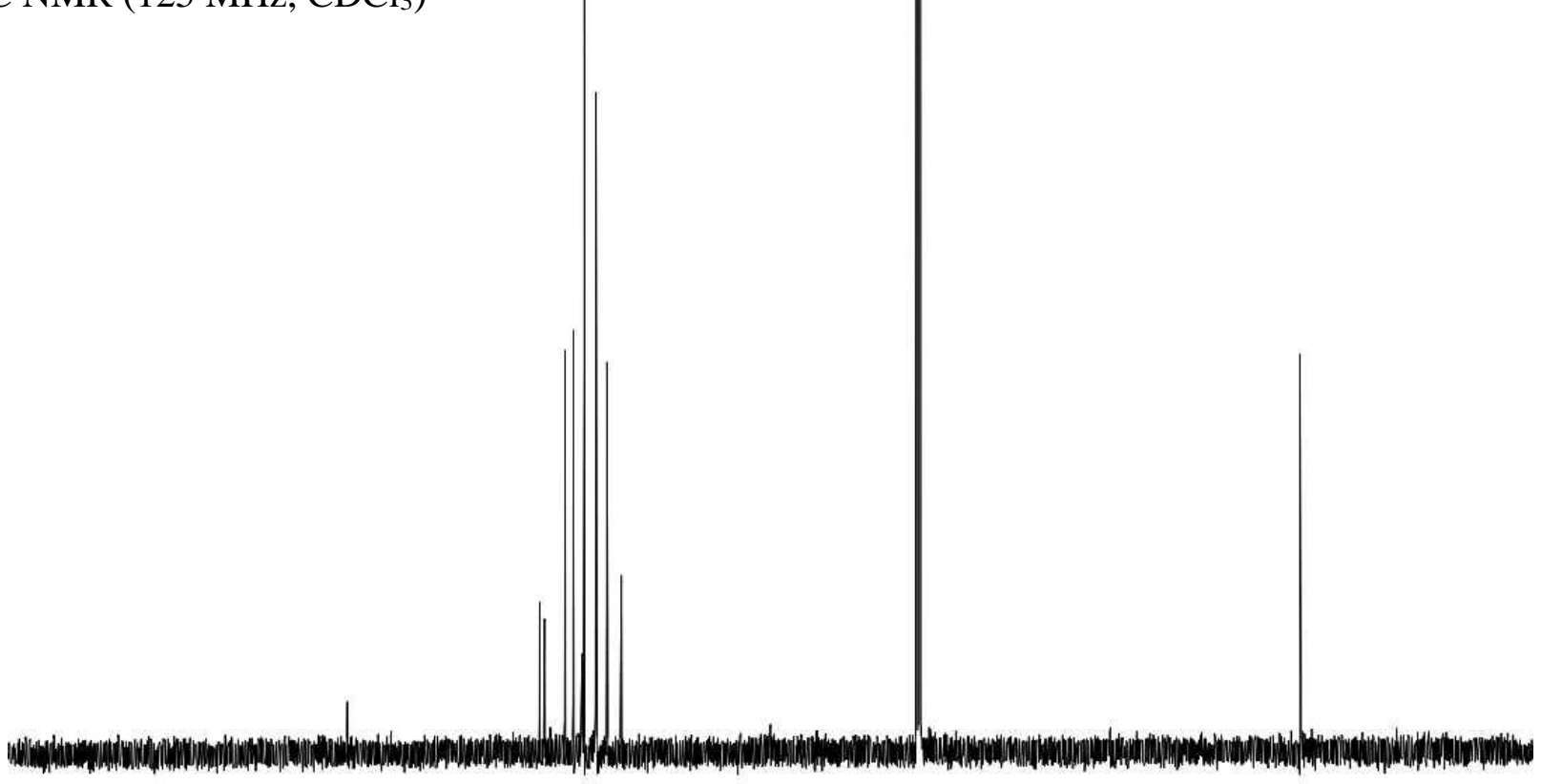

$10 \underset{f 1}{100}(\mathrm{ppm})$

$\mathrm{S} 250$ 
${ }^{1} \mathrm{H} \mathrm{NMR}\left(500 \mathrm{MHz}, \mathrm{CDCl}_{3}\right)$
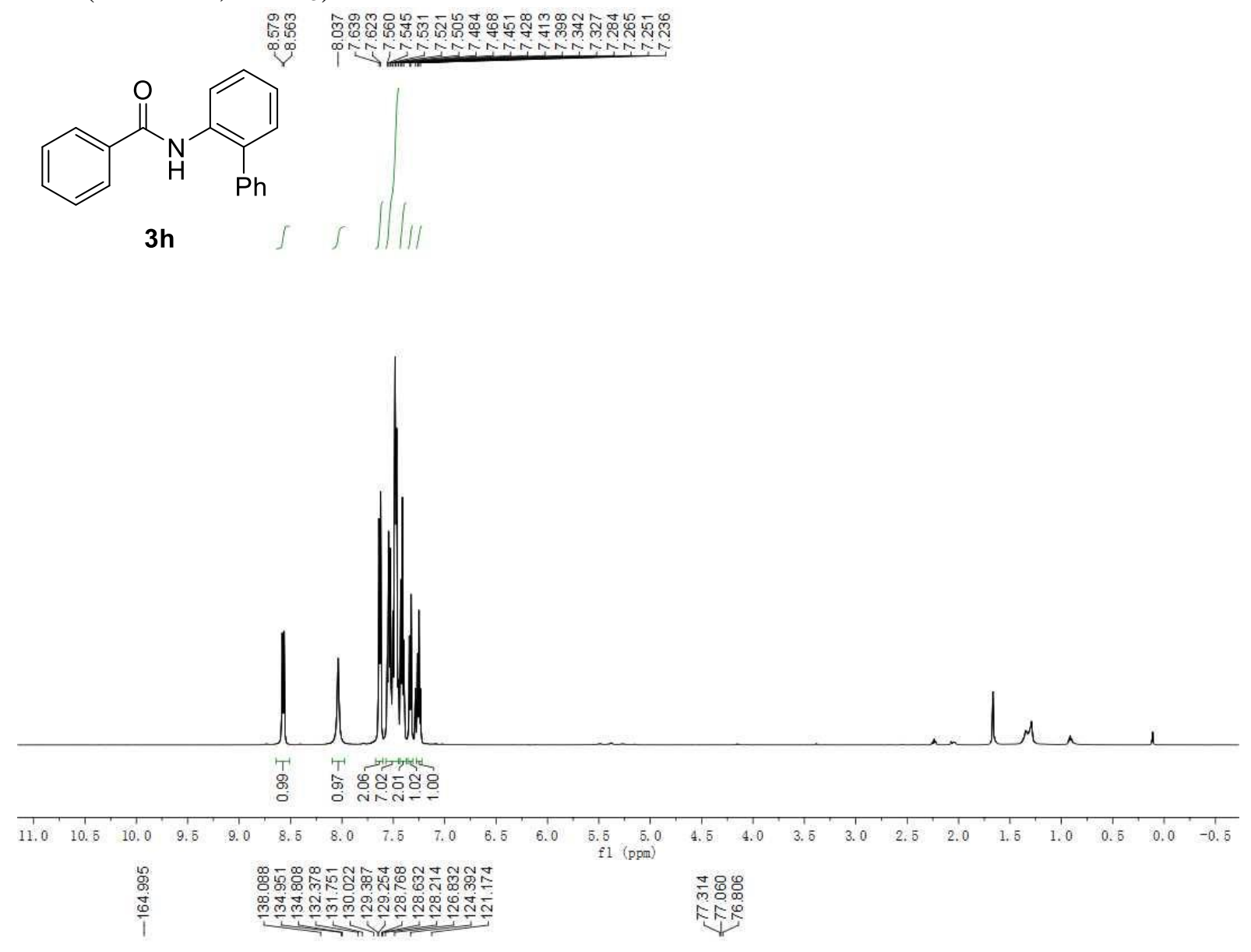

${ }^{13} \mathrm{C} \mathrm{NMR}\left(125 \mathrm{MHz}, \mathrm{CDCl}_{3}\right)$

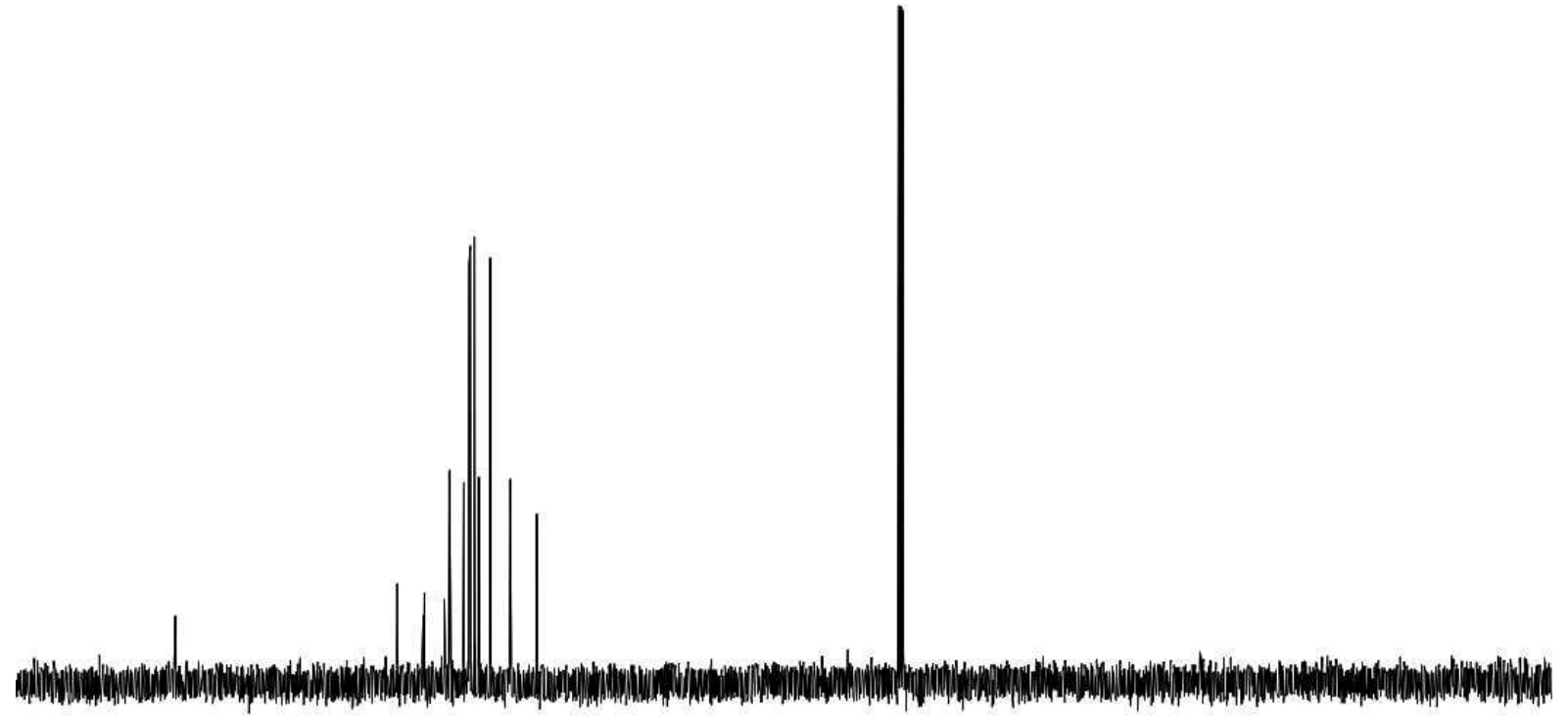

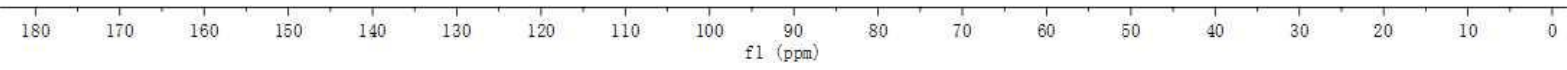


${ }^{1} \mathrm{H} \mathrm{NMR}\left(500 \mathrm{MHz}, \mathrm{CDCl}_{3}\right)$<smiles>Cc1cccc(C)c1NC(=O)c1ccccc1</smiles>
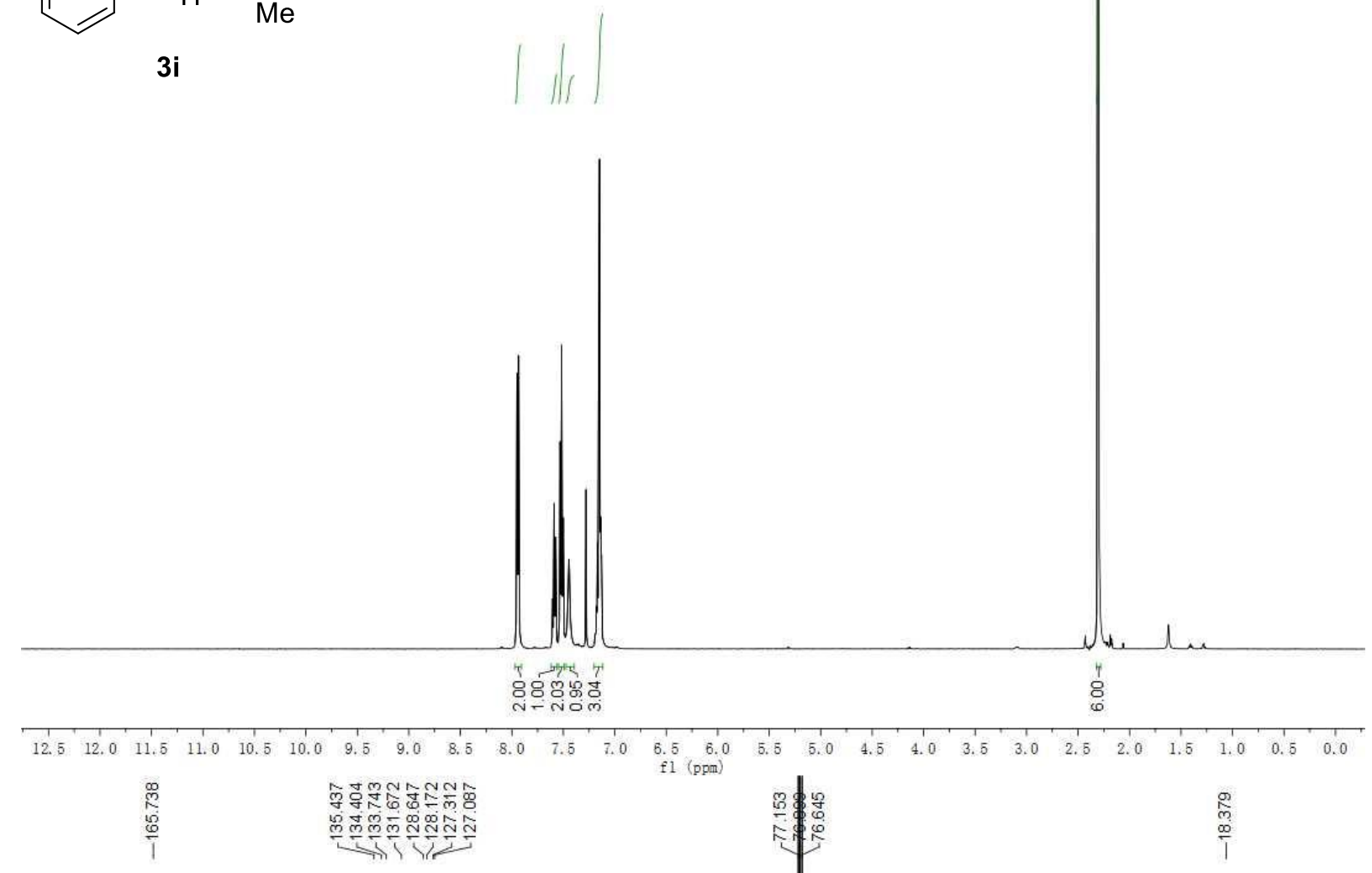

${ }^{13} \mathrm{C} \mathrm{NMR}\left(125 \mathrm{MHz}, \mathrm{CDCl}_{3}\right)$

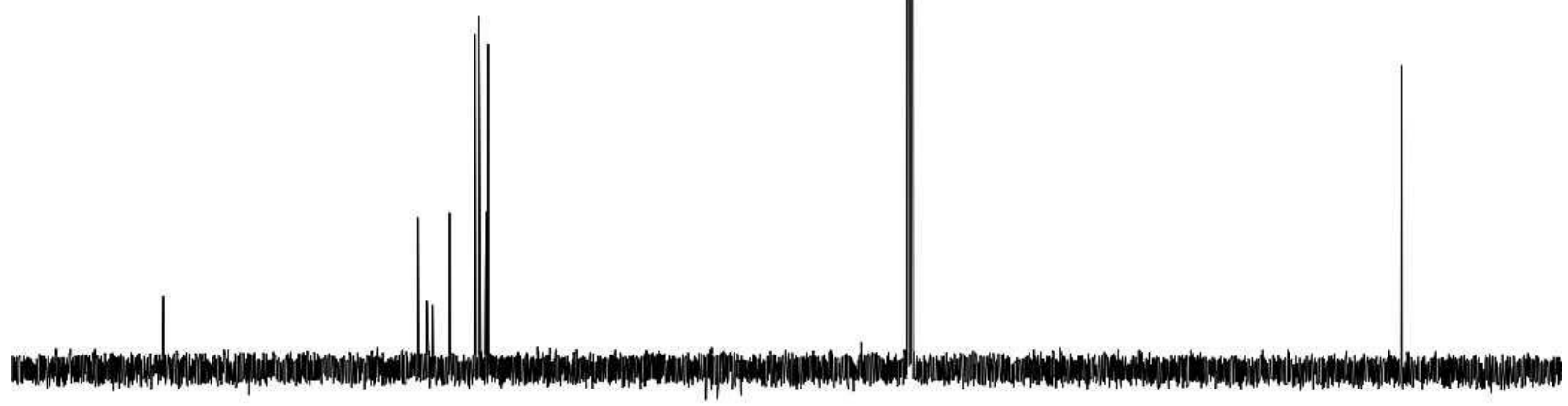

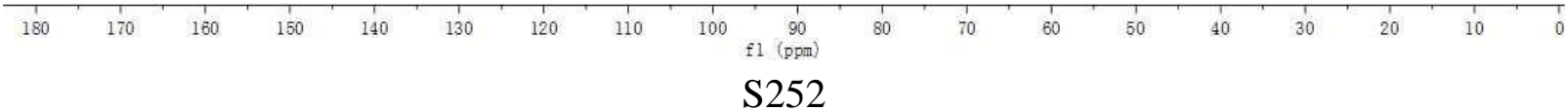


${ }^{1} \mathrm{H} \mathrm{NMR}\left(500 \mathrm{MHz}, \mathrm{CDCl}_{3}\right)$
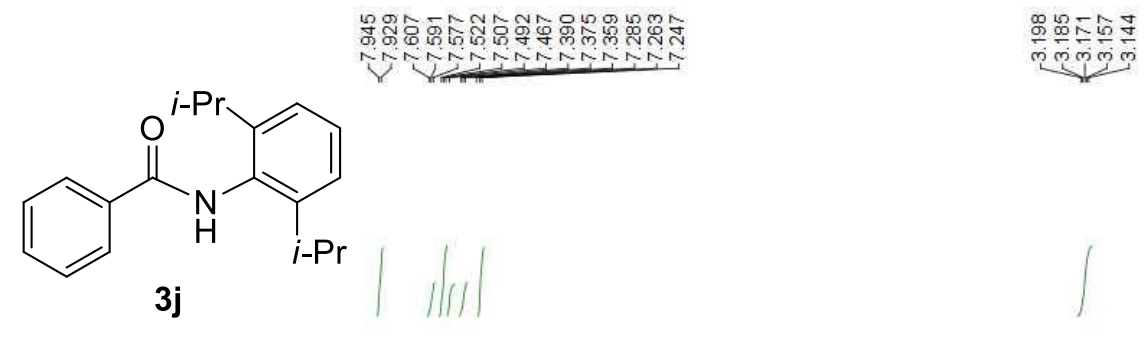

3j
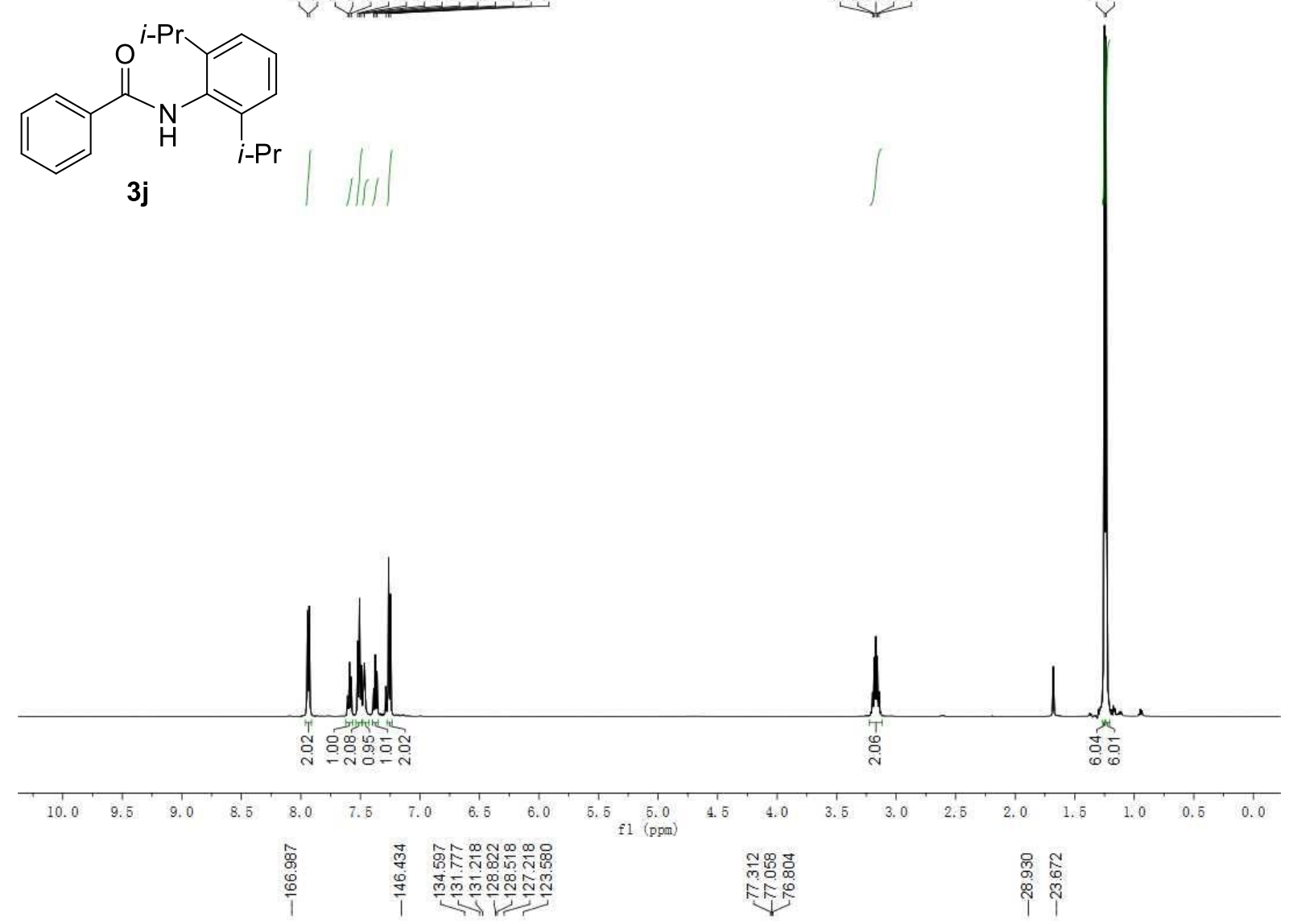

${ }^{13} \mathrm{C} \mathrm{NMR}\left(125 \mathrm{MHz}, \mathrm{CDCl}_{3}\right)$
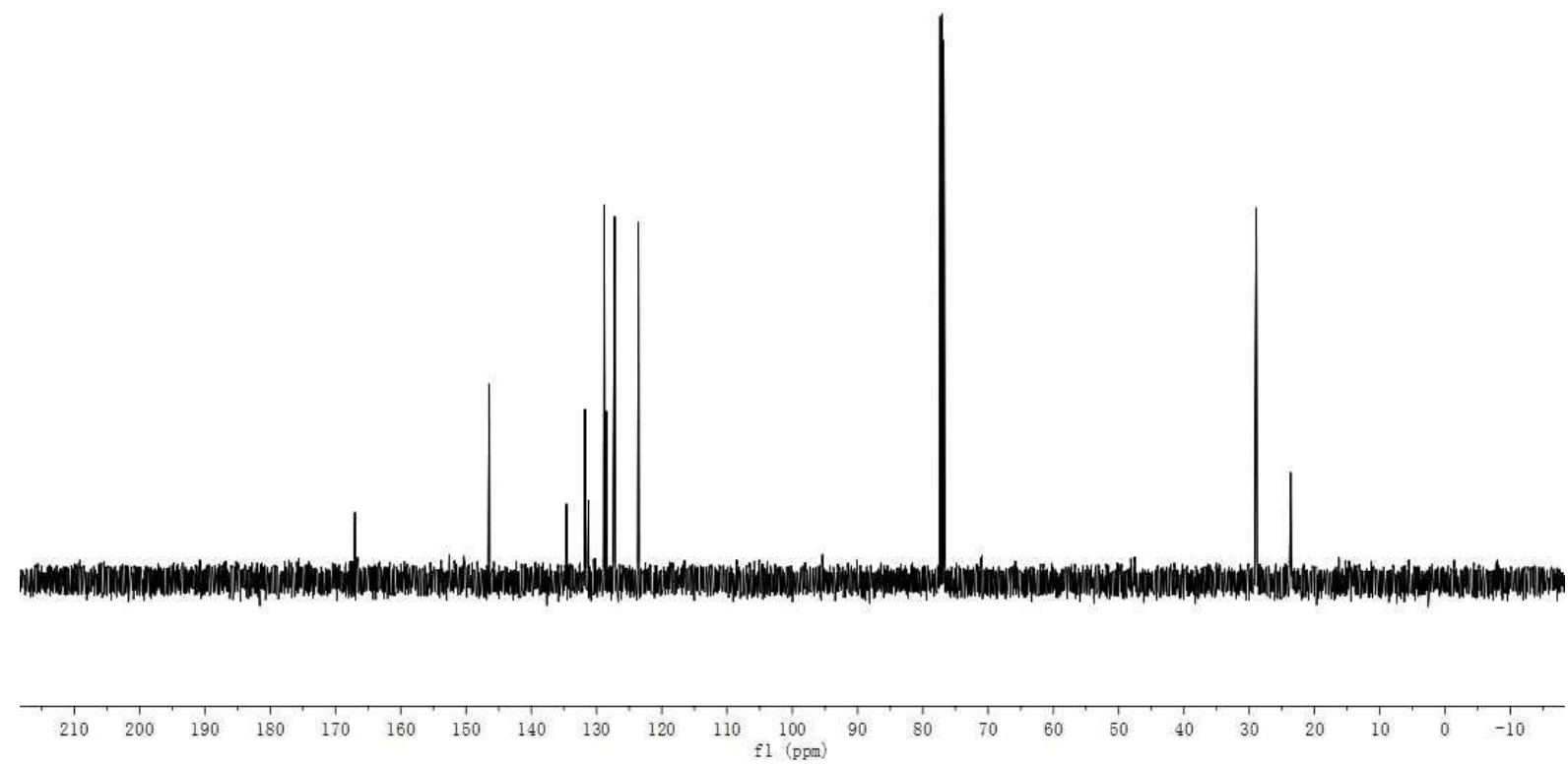
${ }^{1} \mathrm{H} \mathrm{NMR}\left(500 \mathrm{MHz}, \mathrm{CDCl}_{3}\right)$

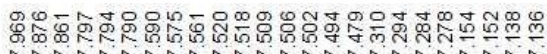

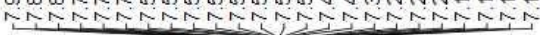<smiles>C#CC=C</smiles>

3k

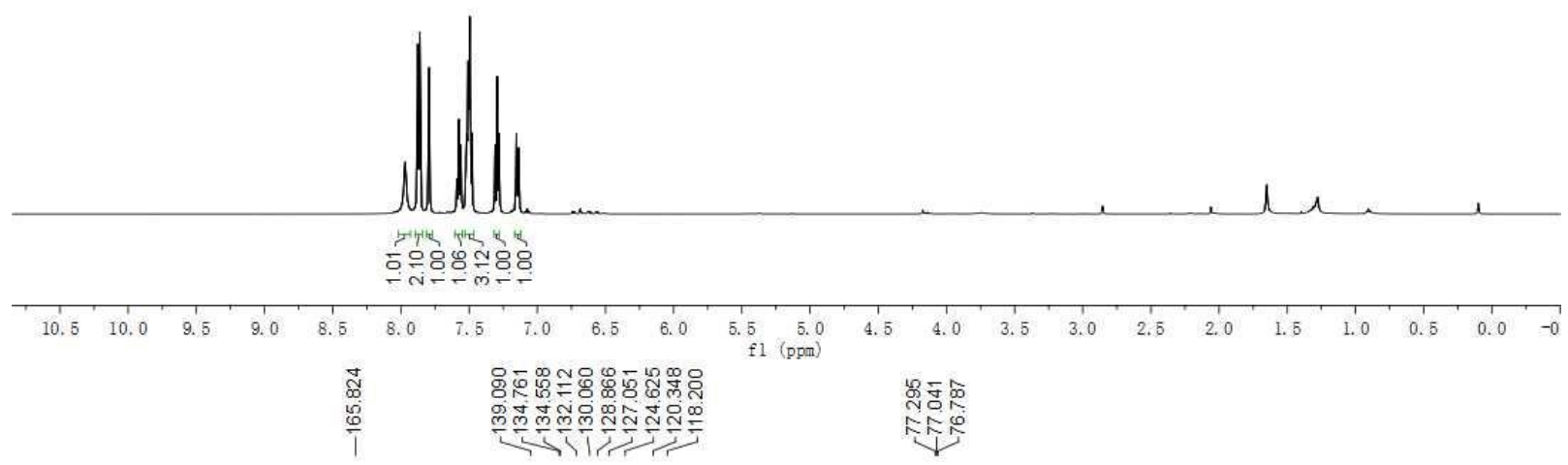

${ }^{13} \mathrm{C} \mathrm{NMR}\left(125 \mathrm{MHz}, \mathrm{CDCl}_{3}\right)$

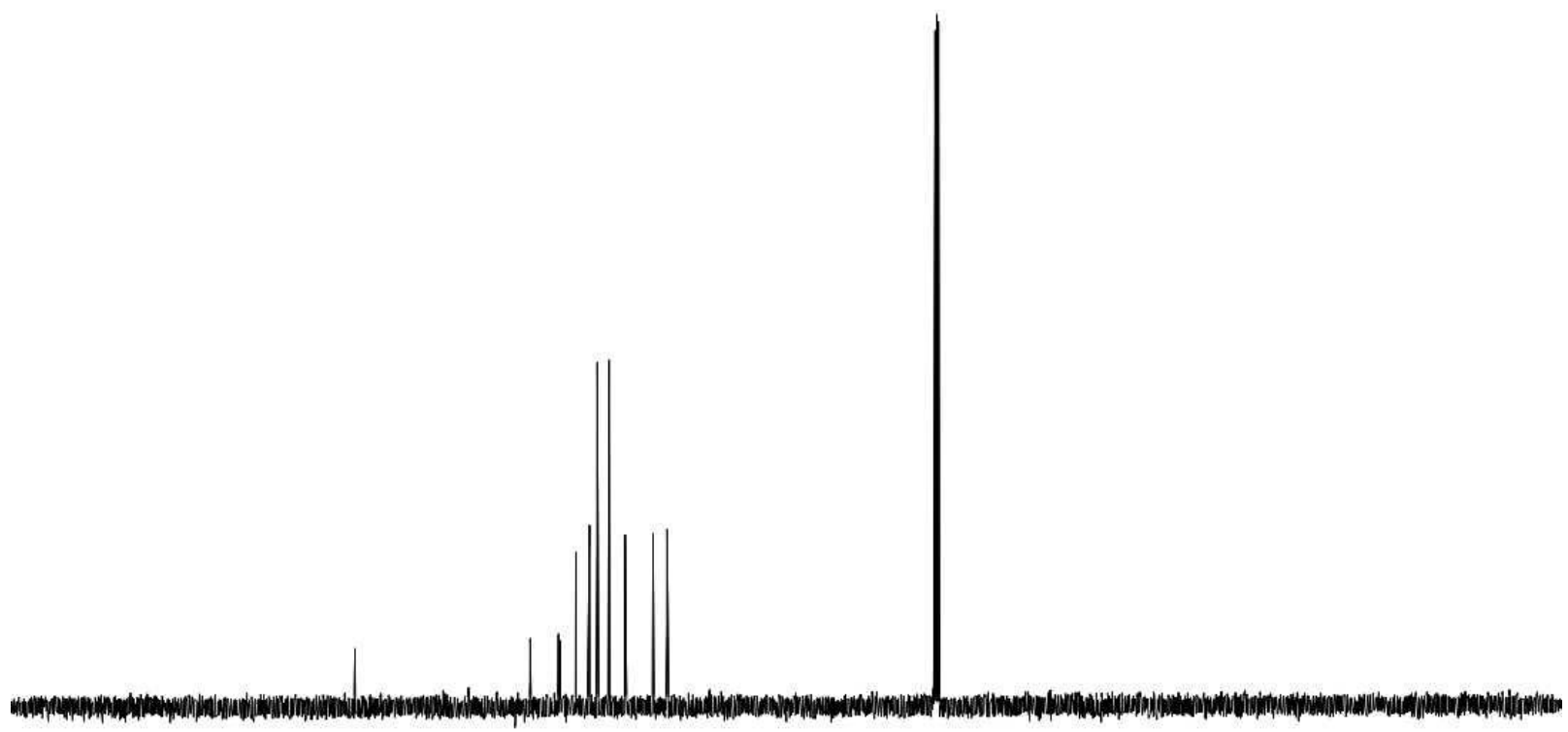

$\begin{array}{lllllllllllllllllllllllllllllllll}210 & 200 & 190 & 180 & 170 & 160 & 150 & 140 & 130 & 120 & 110 & 100 & 90 & 80 & 70 & 60 & 50 & 40 & 30 & 20 & 10 & 0 & -10 & \end{array}$ S254 
${ }^{1} \mathrm{H}$ NMR $\left(500 \mathrm{MHz}, \mathrm{CDCl}_{3}\right)$<smiles>O=C(Nc1cccc(C(F)(F)F)c1)c1ccccc1</smiles>

3)

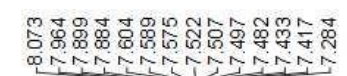

oin

dex
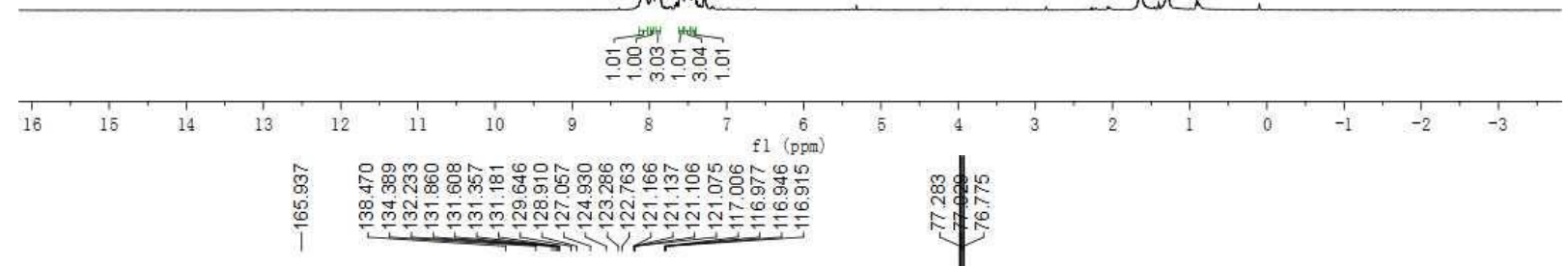

${ }^{13} \mathrm{C} \mathrm{NMR}\left(125 \mathrm{MHz}, \mathrm{CDCl}_{3}\right)$
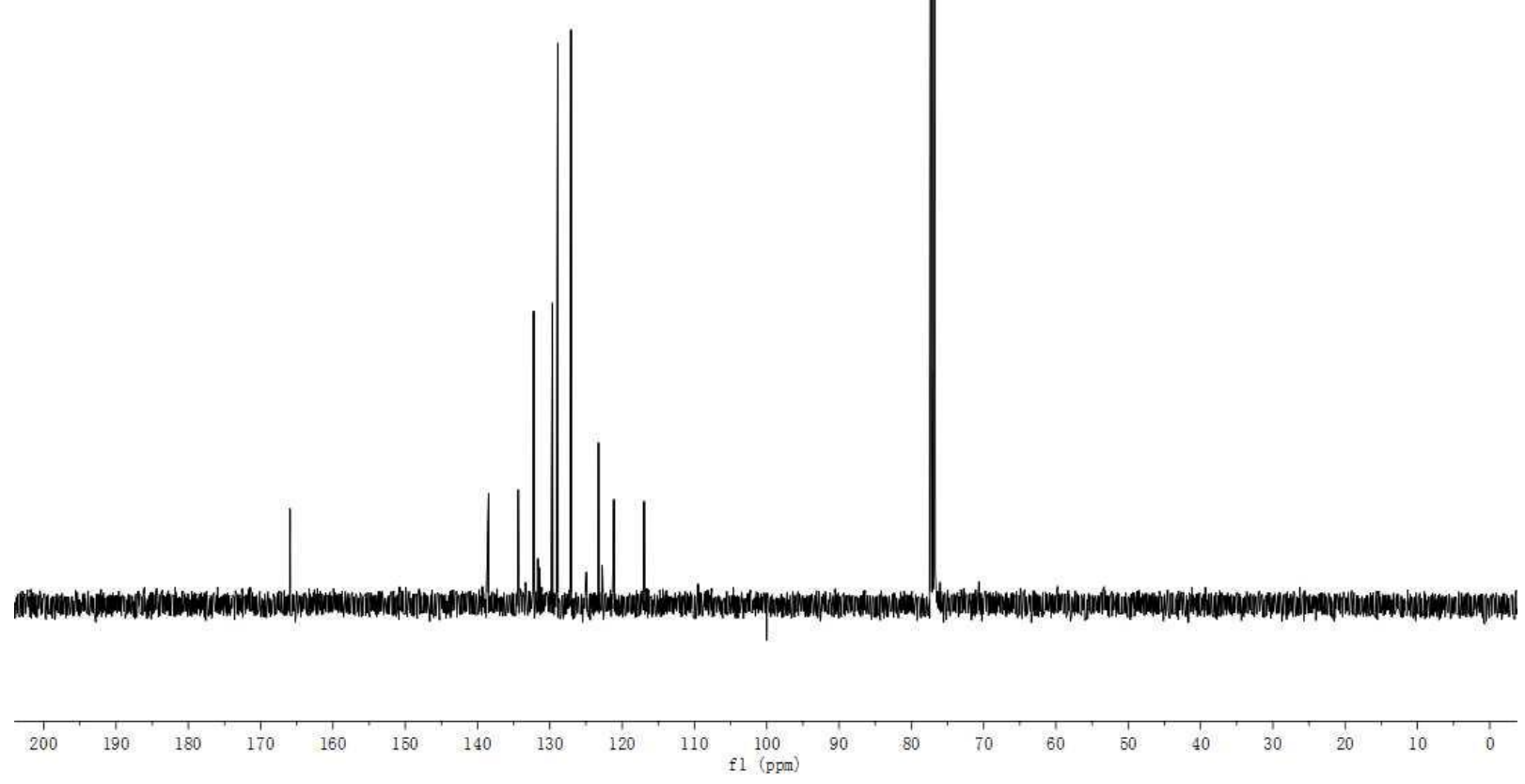
S255 
${ }^{19} \mathrm{~F}$ NMR (471 MHz, $\left.\mathrm{CDCl}_{3}\right)$<smiles>O=C(Nc1cccc(C(F)(F)F)c1)c1ccccc1</smiles>

31

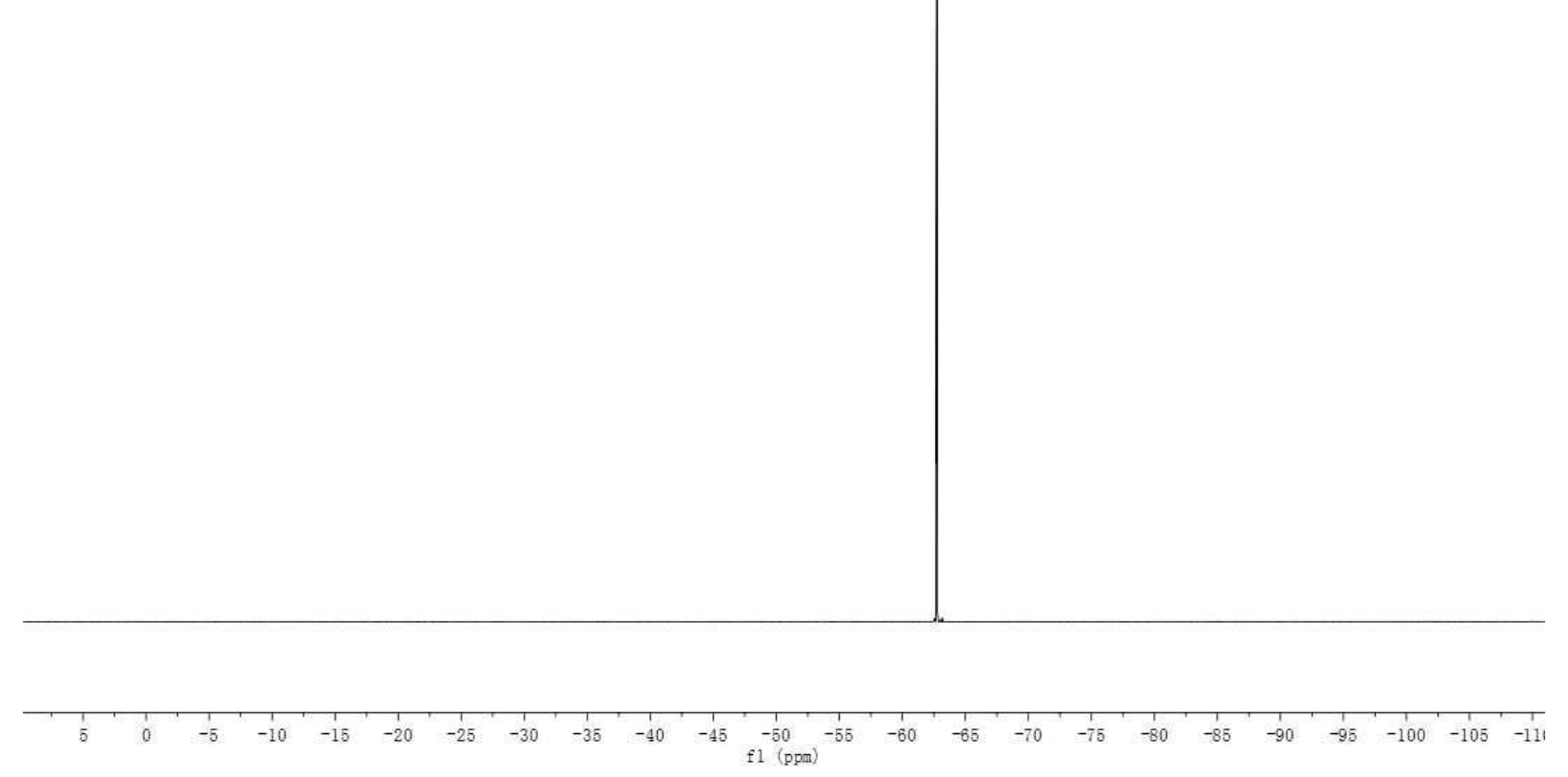


${ }^{1} \mathrm{H}$ NMR (500 MHz, $\mathrm{CDCl}_{3}$ )
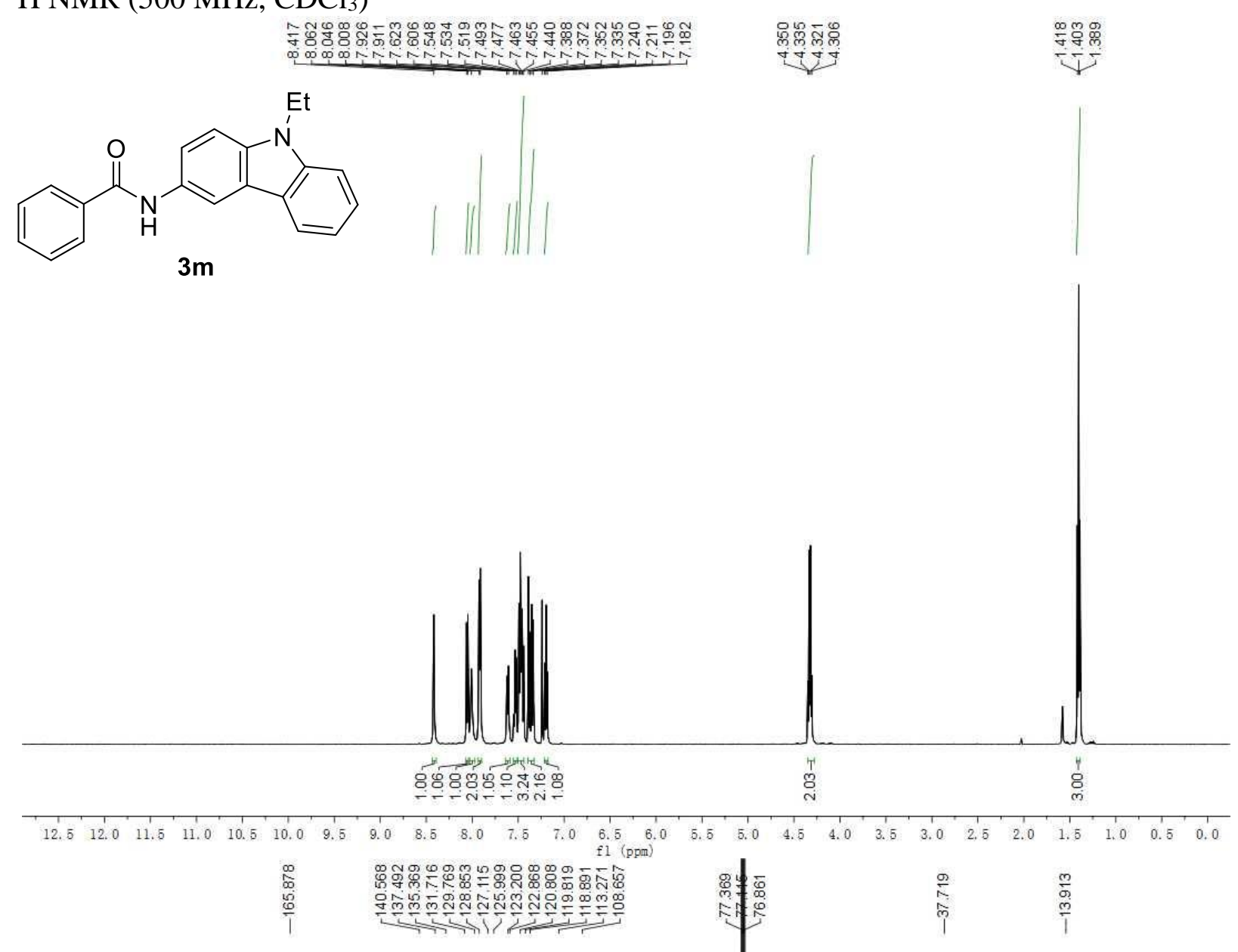

${ }^{13} \mathrm{C} \mathrm{NMR}\left(125 \mathrm{MHz}, \mathrm{CDCl}_{3}\right)$
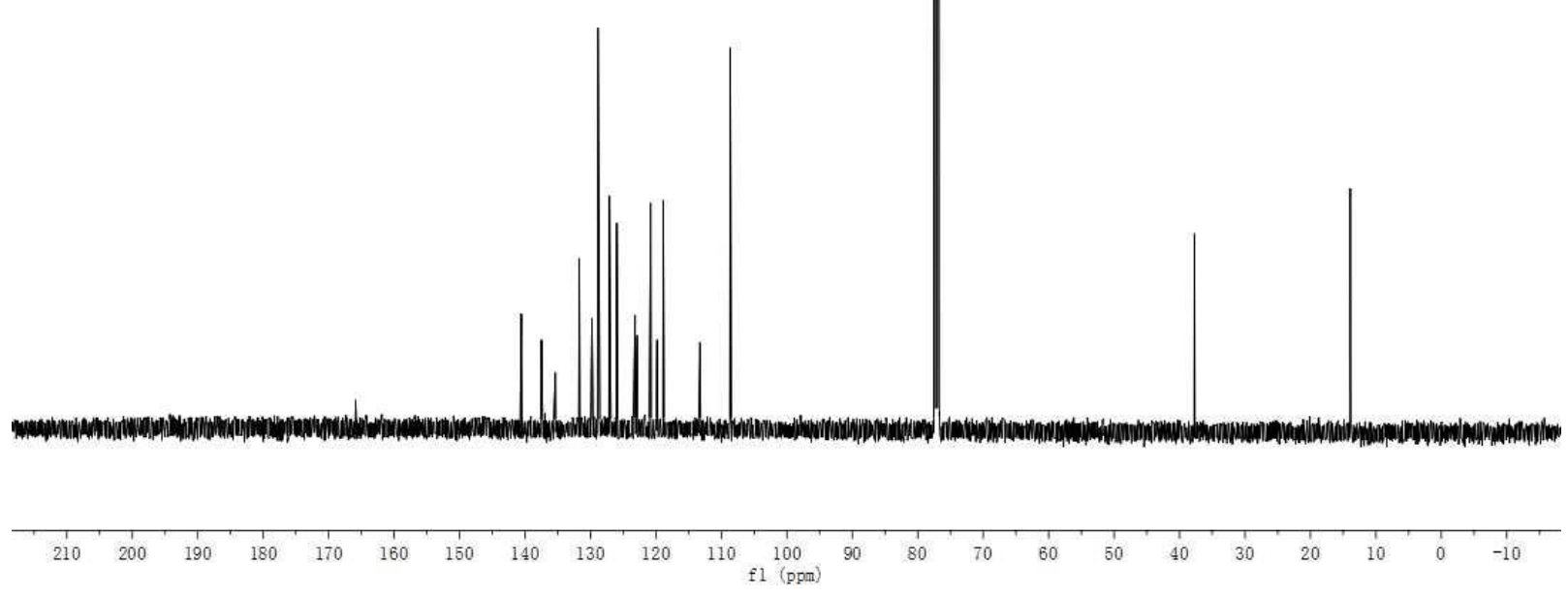
S 257 
${ }^{1} \mathrm{H}$ NMR $\left(500 \mathrm{MHz}, \mathrm{CDCl}_{3}\right)$

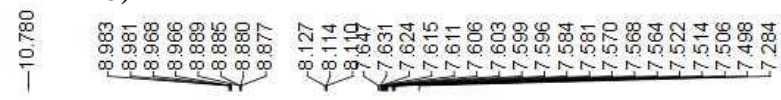<smiles>O=C(Nc1cccc2cccnc12)c1ccccc1</smiles>

$3 n$

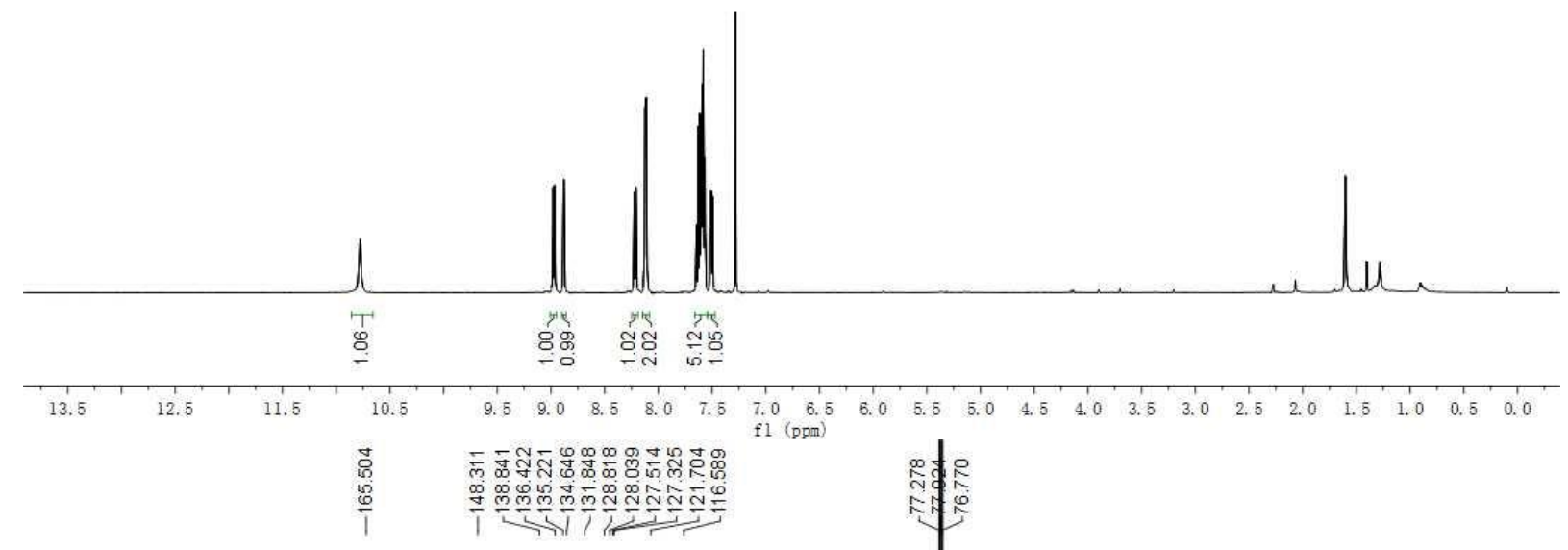

${ }^{13} \mathrm{C}$ NMR $\left(125 \mathrm{MHz}, \mathrm{CDCl}_{3}\right)$
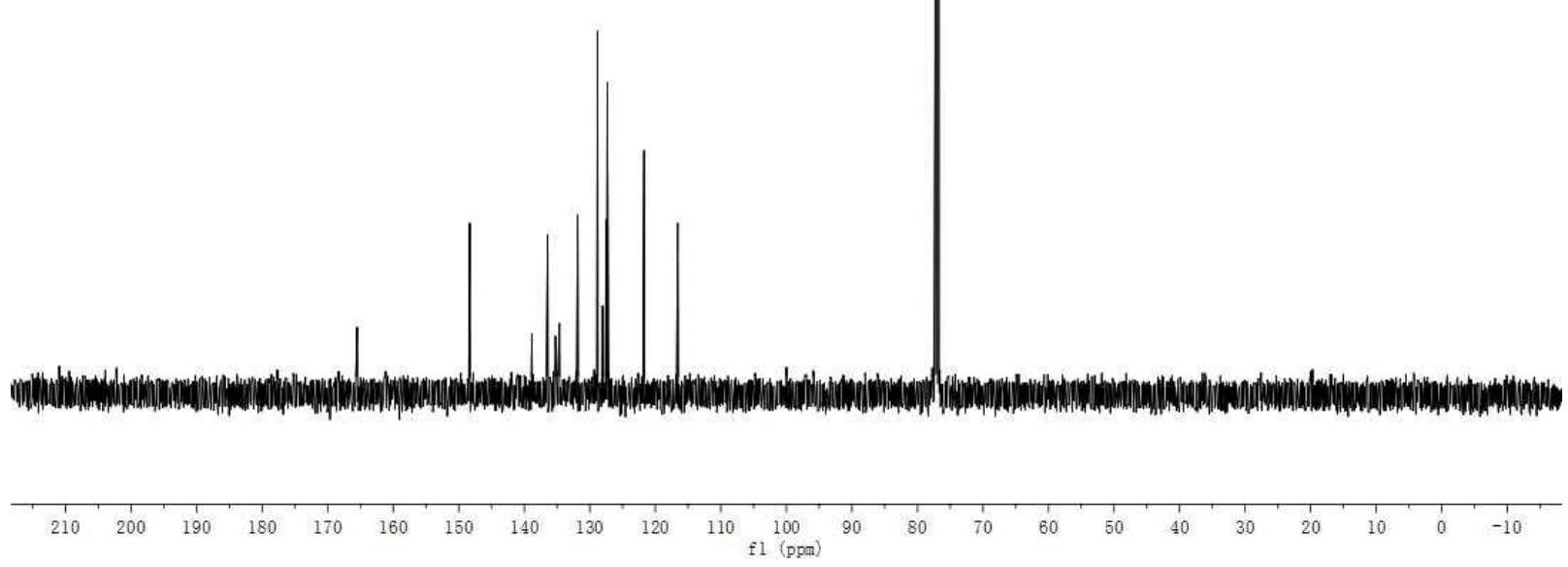
S258 
${ }^{1} \mathrm{H} \mathrm{NMR}\left(500 \mathrm{MHz}, \mathrm{CDCl}_{3}\right)$
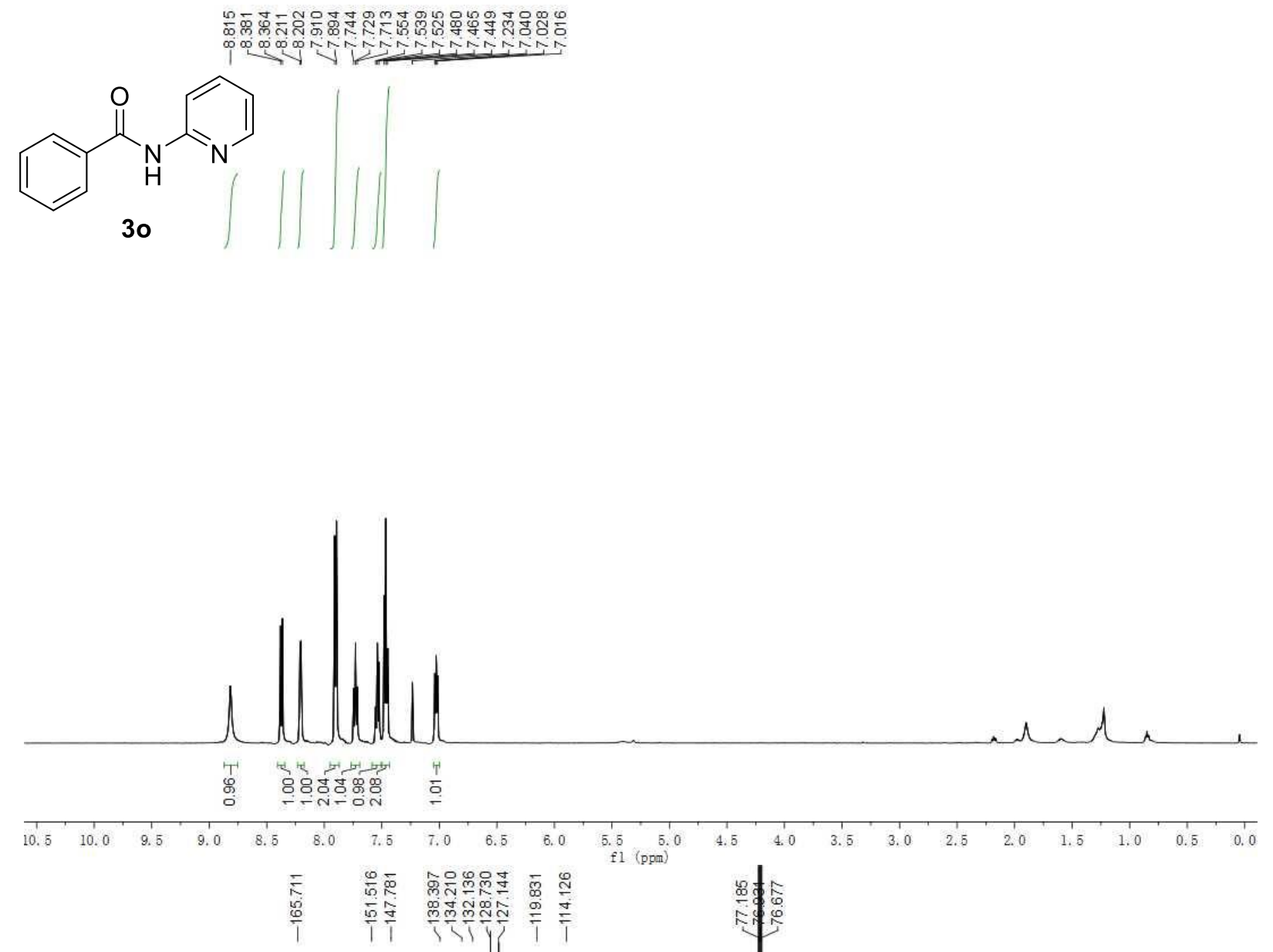

${ }^{13} \mathrm{C} \mathrm{NMR}\left(125 \mathrm{MHz}, \mathrm{CDCl}_{3}\right)$
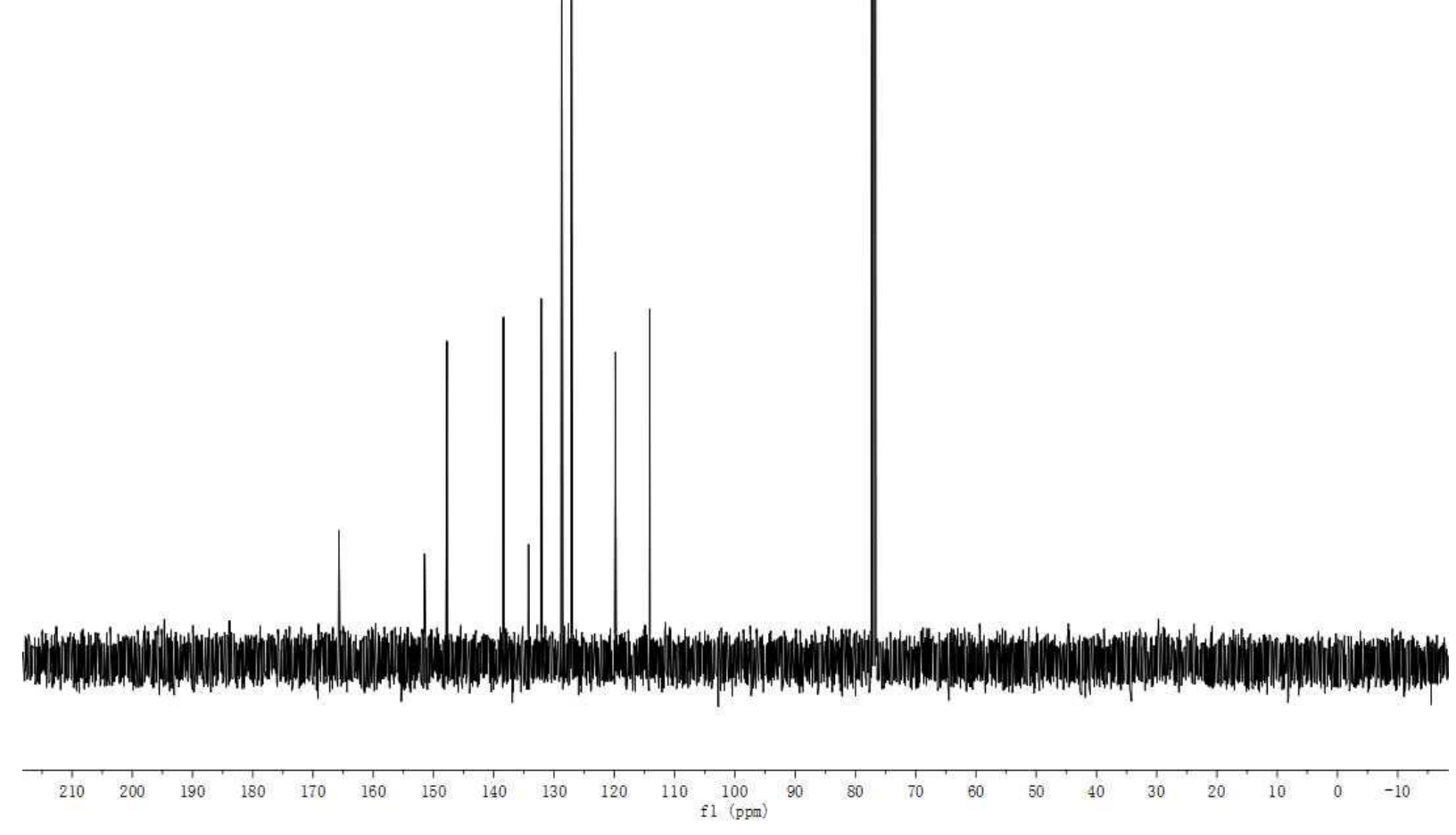
S259 
${ }^{1} \mathrm{H}$ NMR $\left(500 \mathrm{MHz}, \mathrm{CDCl}_{3}\right)$

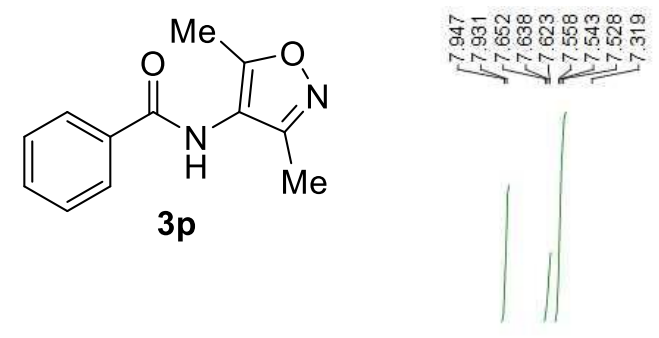

๙ึiñ
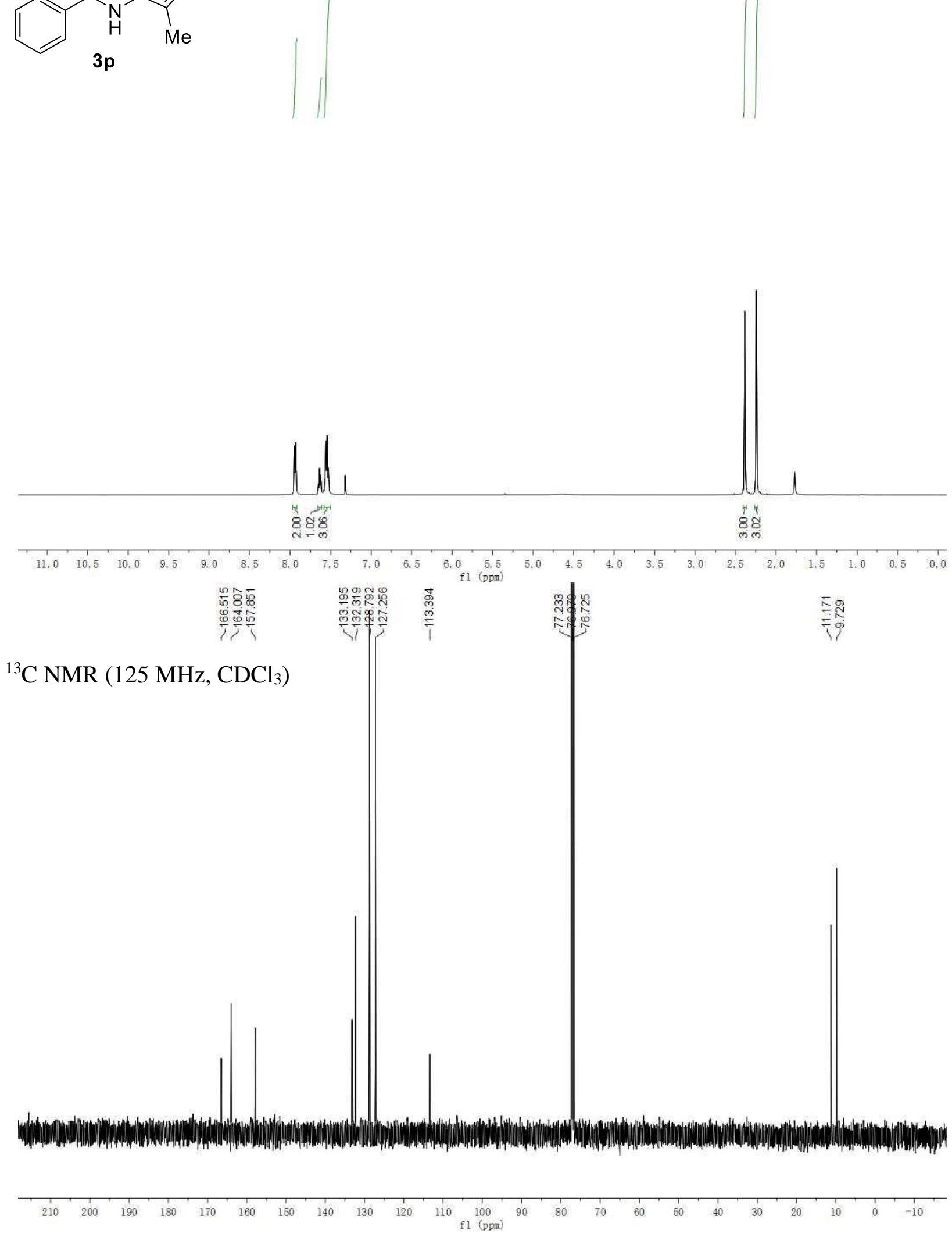
S260 
${ }^{1} \mathrm{H}$ NMR (500 MHz, $\left.\mathrm{CDCl}_{3}\right)$
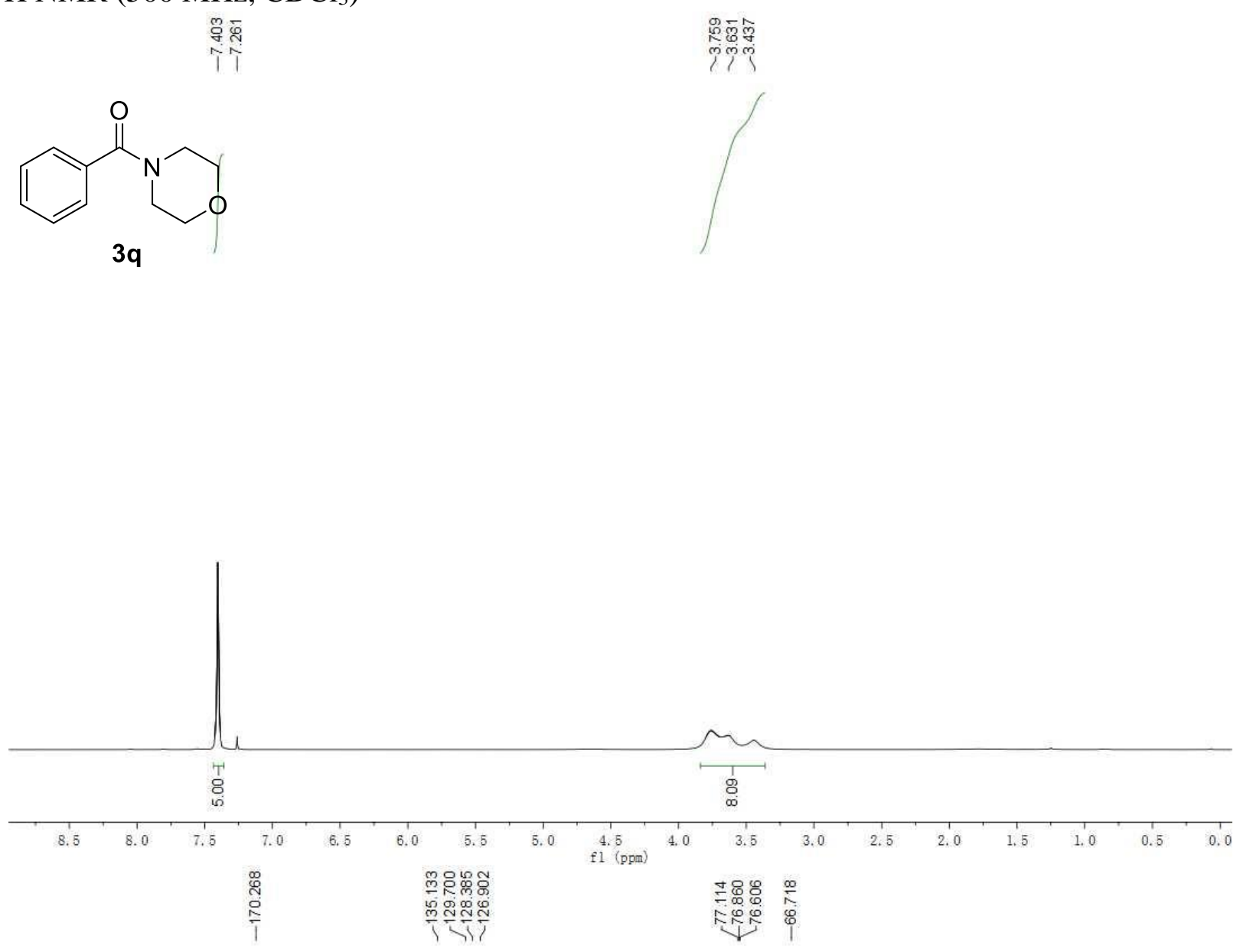

${ }^{13} \mathrm{C}$ NMR $\left(125 \mathrm{MHz}, \mathrm{CDCl}_{3}\right)$
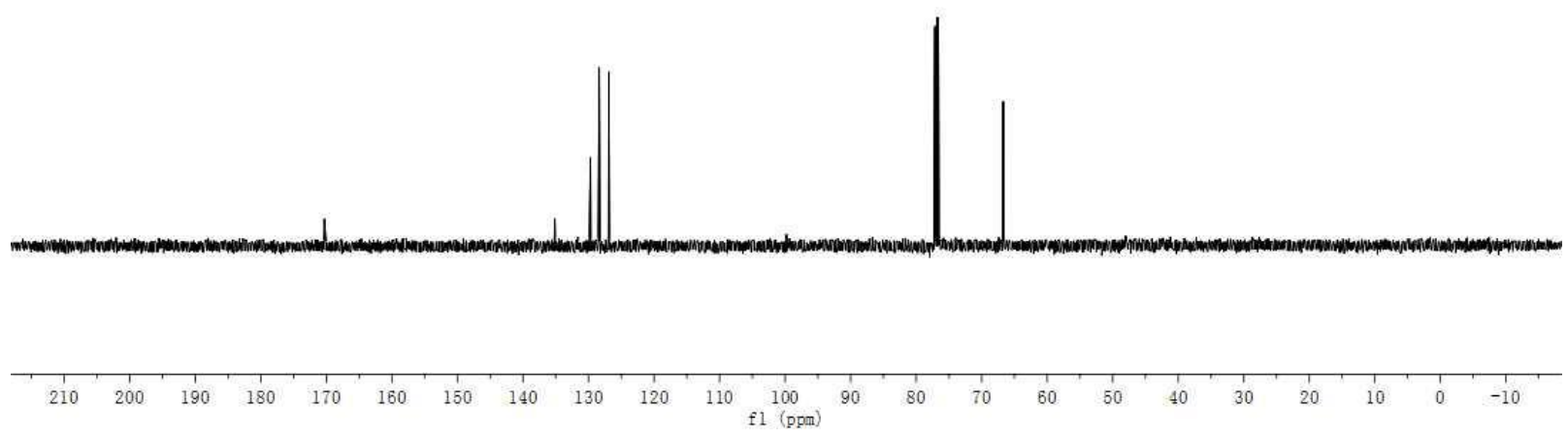
${ }^{1} \mathrm{H} \mathrm{NMR}\left(500 \mathrm{MHz}, \mathrm{CDCl}_{3}\right)$
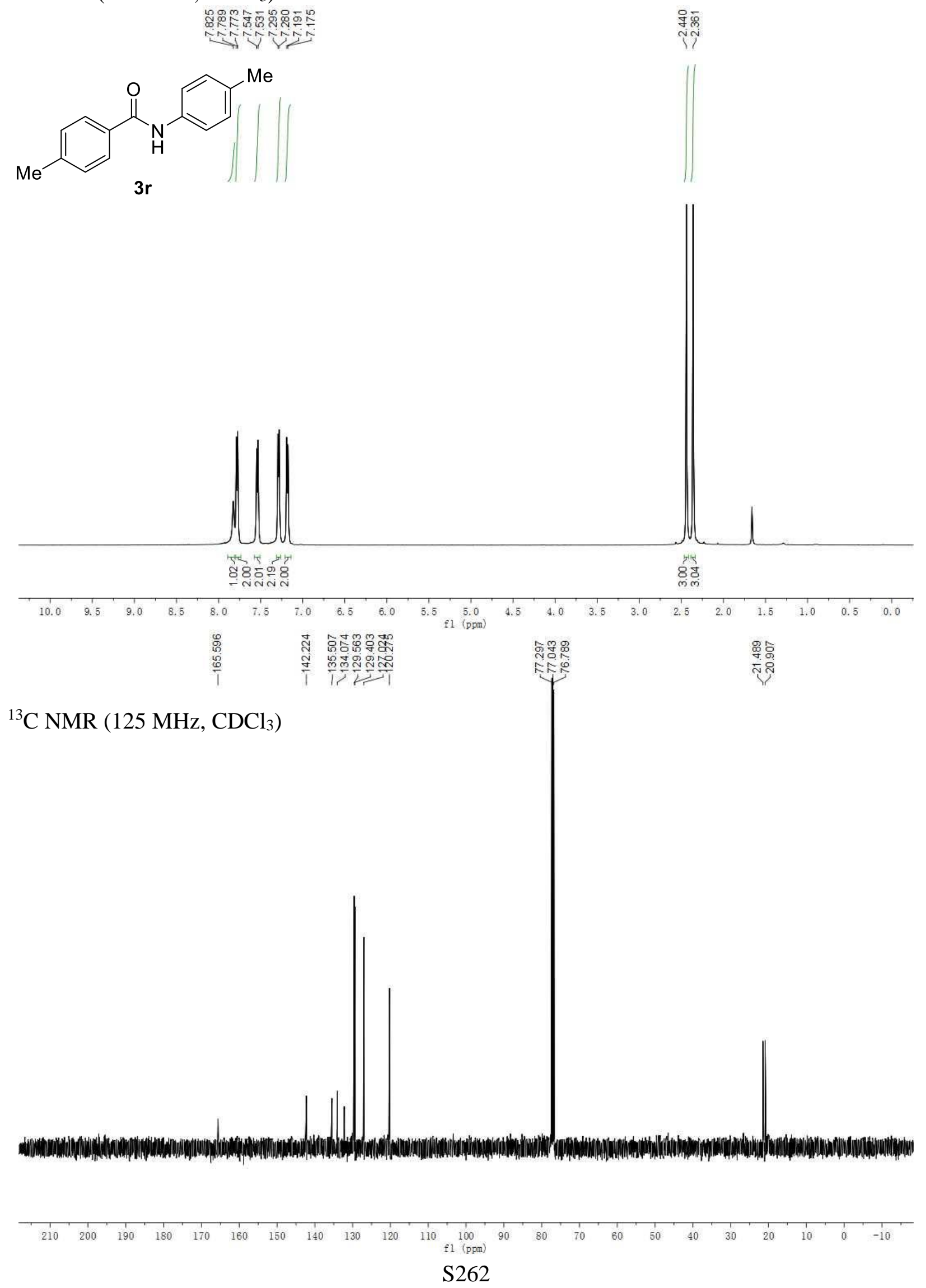
${ }^{1} \mathrm{H} \mathrm{NMR}\left(500 \mathrm{MHz}, \mathrm{CDCl}_{3}\right)$

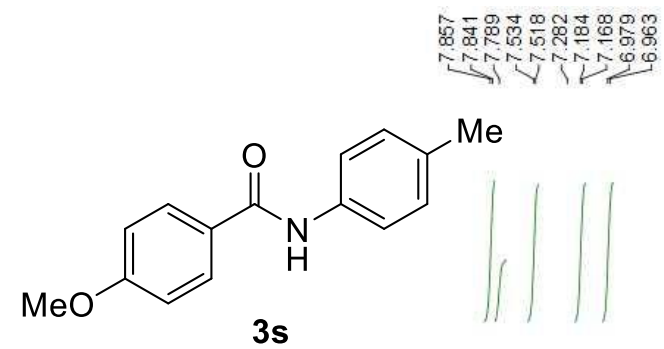

3s
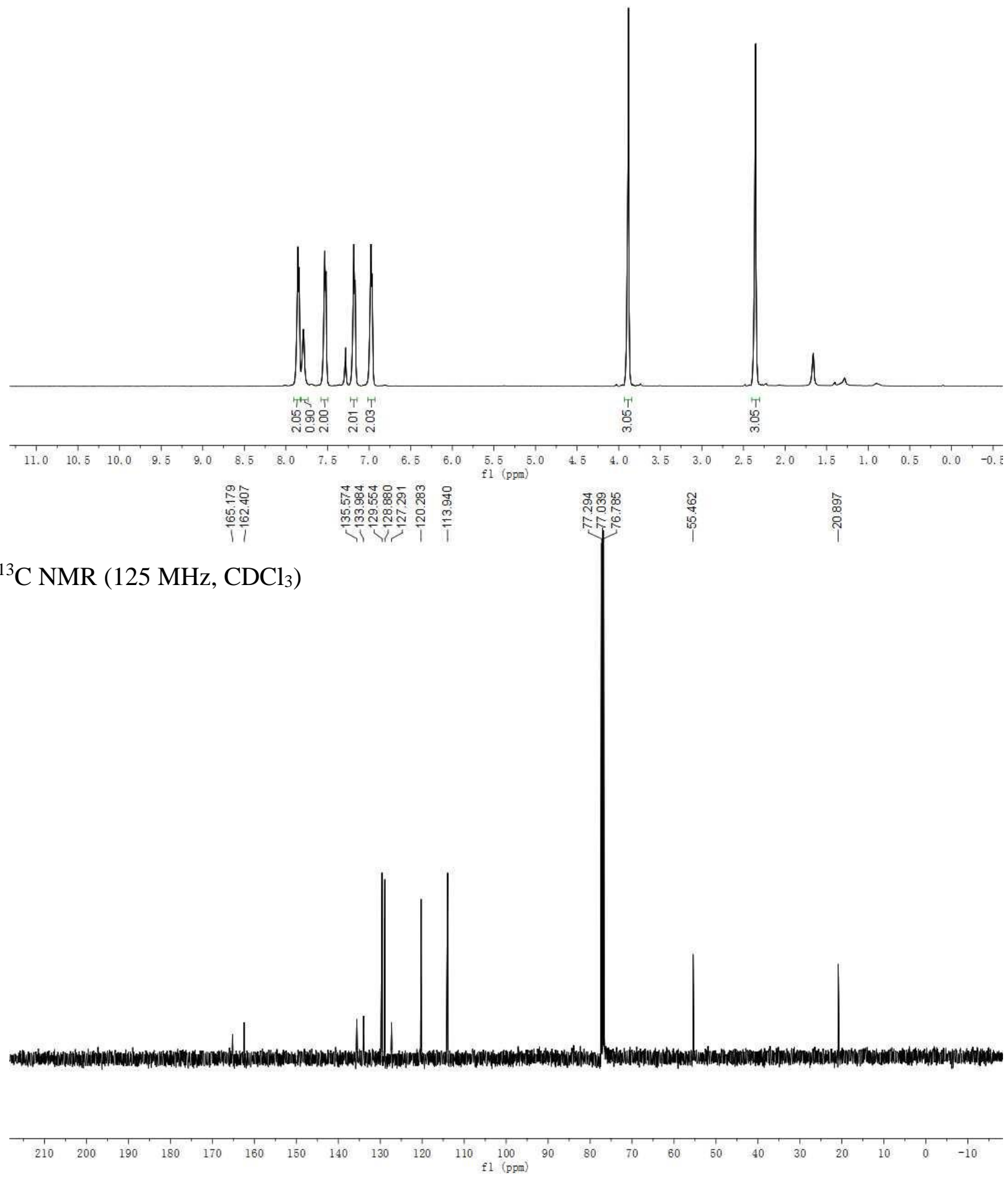
S263 
${ }^{1} \mathrm{H} \mathrm{NMR}\left(500 \mathrm{MHz}, \mathrm{CDCl}_{3}\right)$
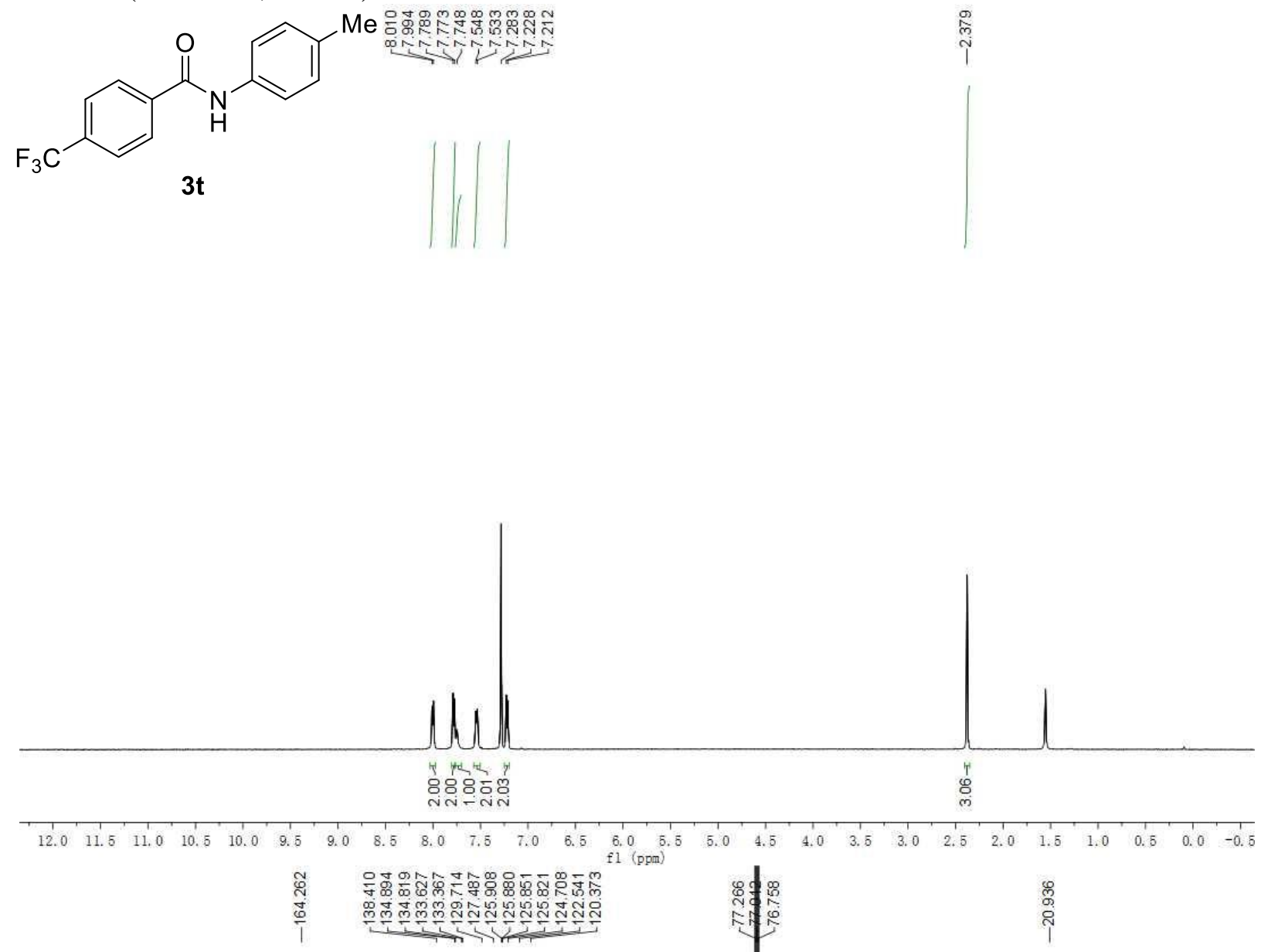

${ }^{13} \mathrm{C} \mathrm{NMR}\left(125 \mathrm{MHz}, \mathrm{CDCl}_{3}\right)$
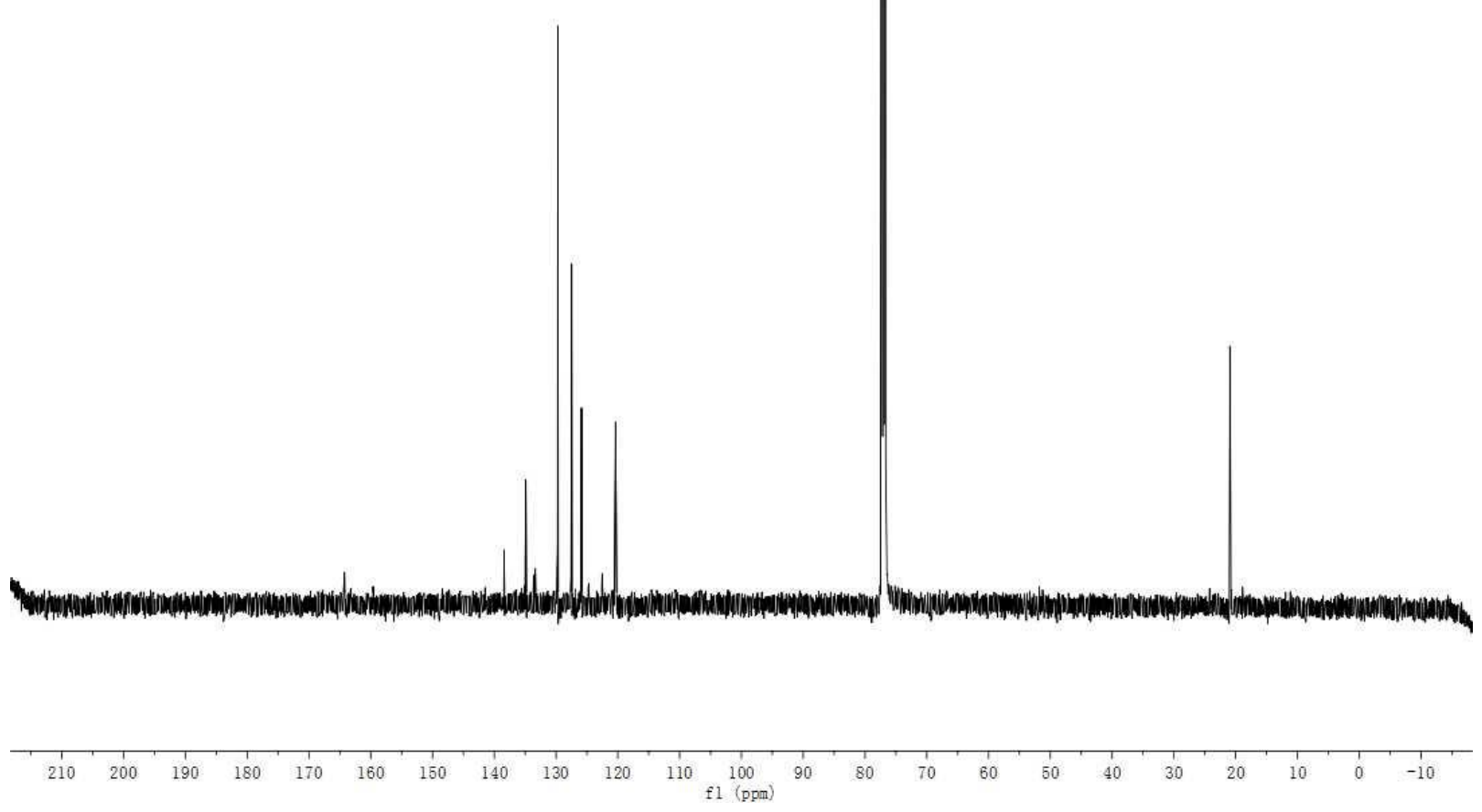
S264 
${ }^{19} \mathrm{~F}$ NMR (471 MHz, $\mathrm{CDCl}_{3}$ )<smiles>Cc1ccc(NC(=O)c2ccc(C(F)(F)F)cc2)cc1</smiles>

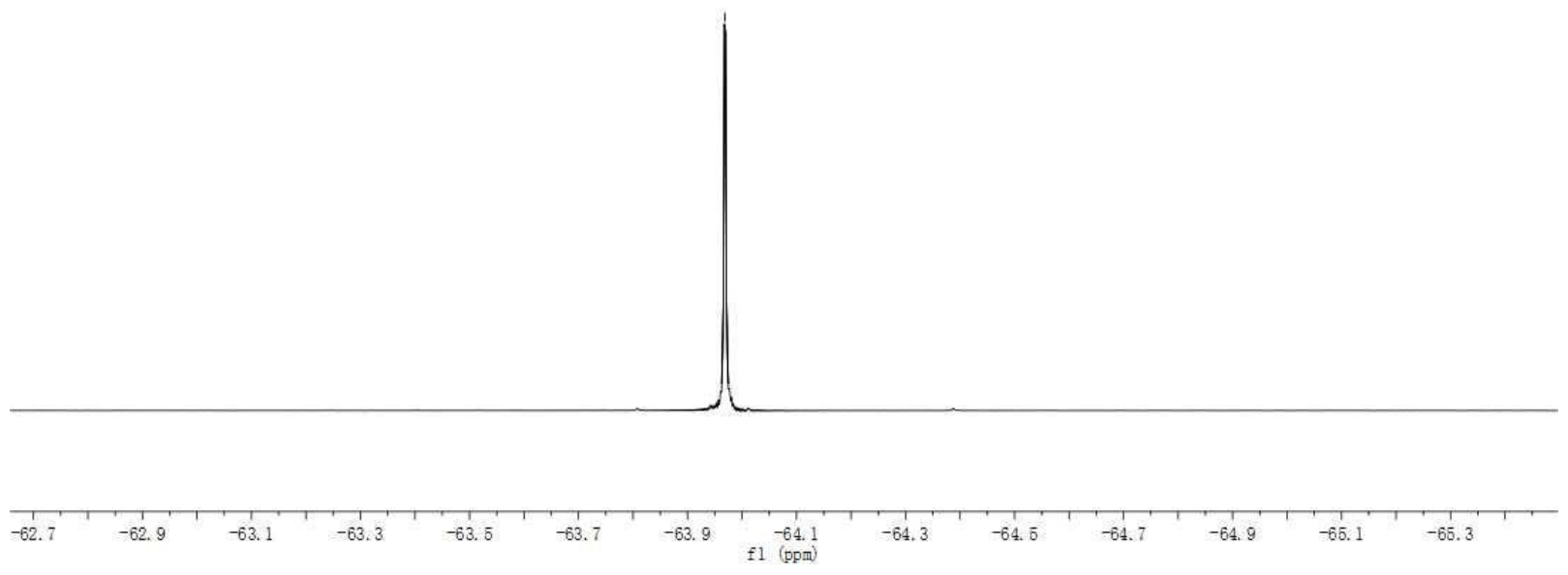


${ }^{1} \mathrm{H}$ NMR (500 MHz, $\left.\mathrm{CDCl}_{3}\right)$
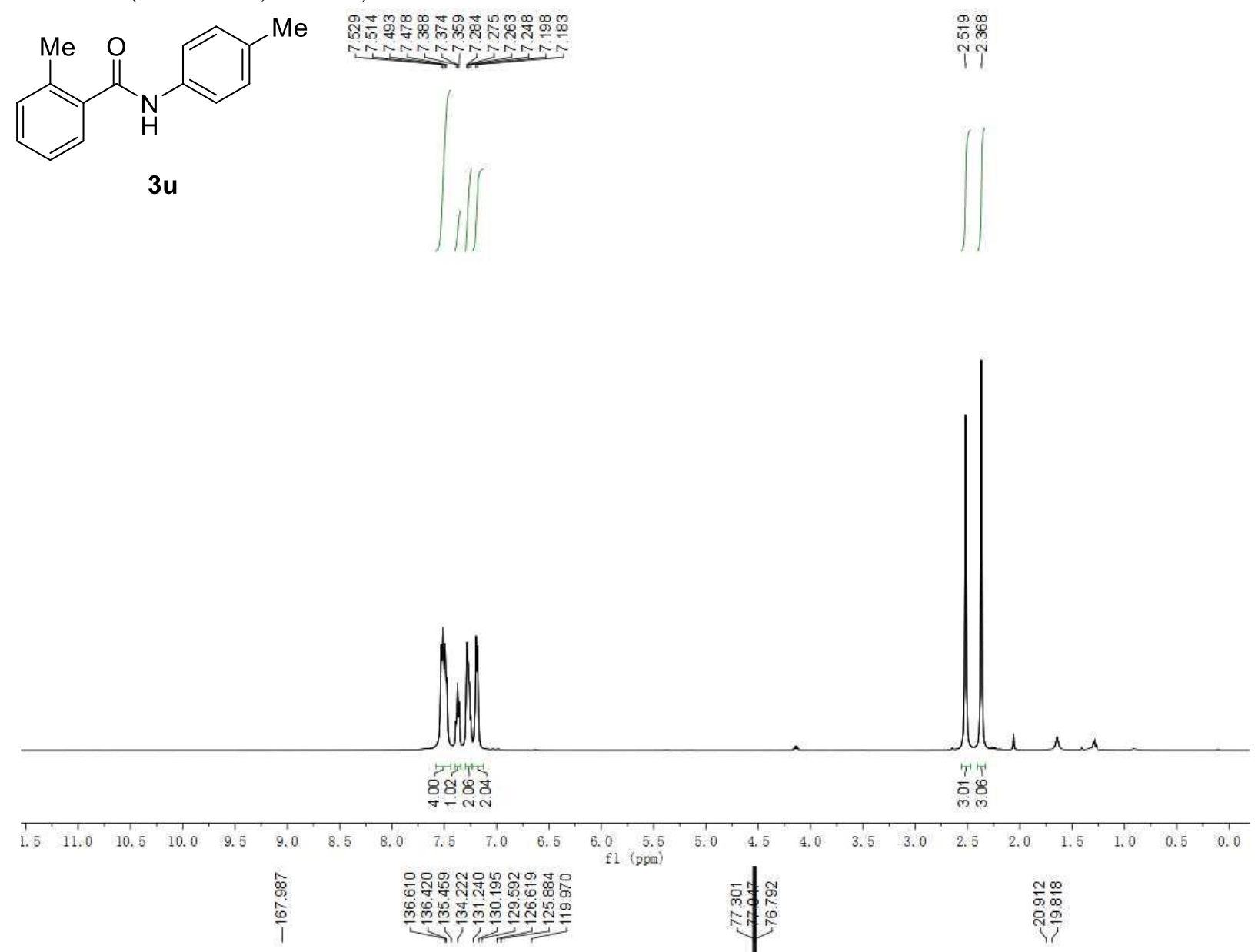

${ }^{13} \mathrm{C} \mathrm{NMR}\left(125 \mathrm{MHz}, \mathrm{CDCl}_{3}\right)$
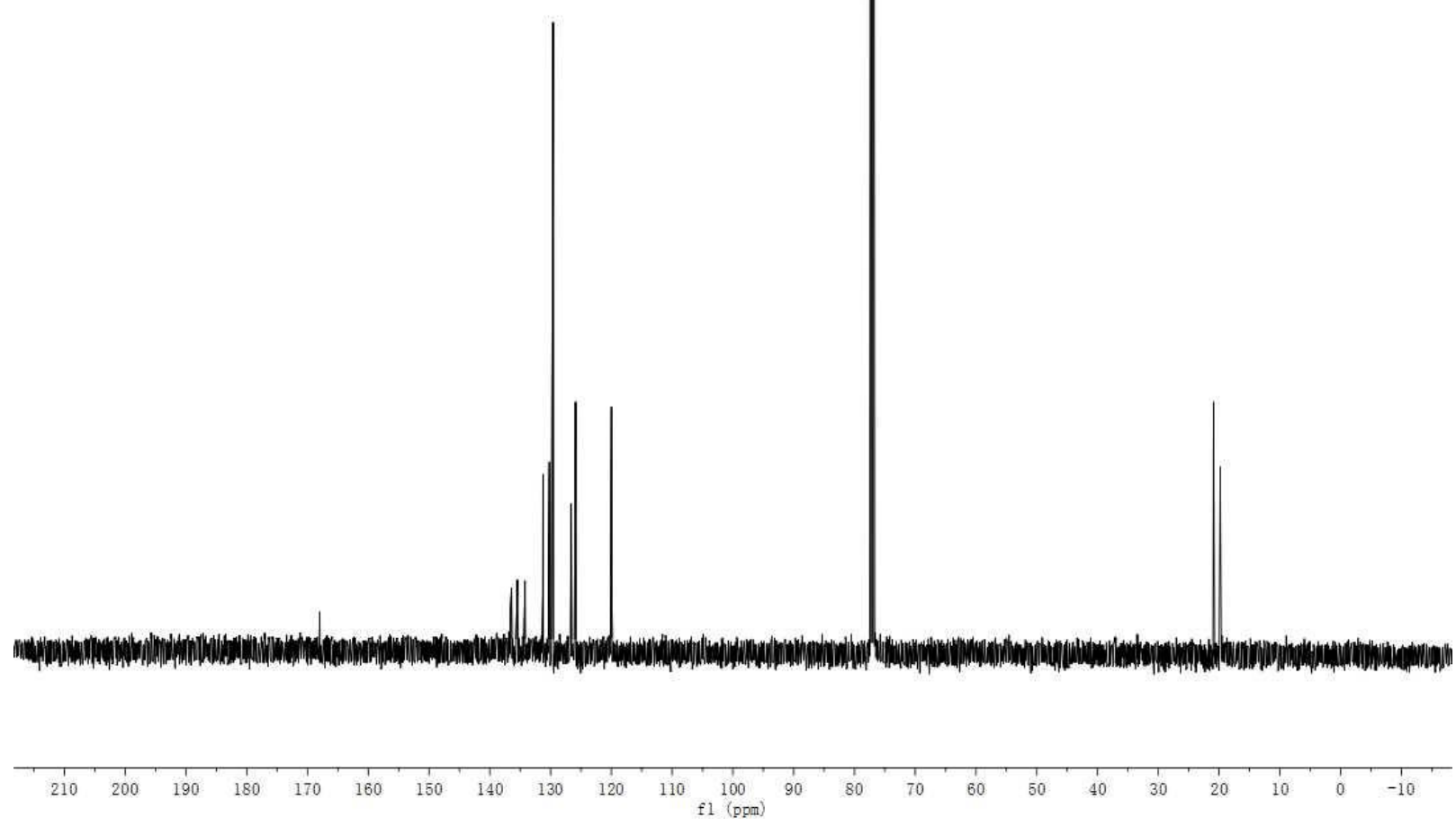
S266 
${ }^{1} \mathrm{H} \mathrm{NMR}\left(500 \mathrm{MHz}, \mathrm{CDCl}_{3}\right)$
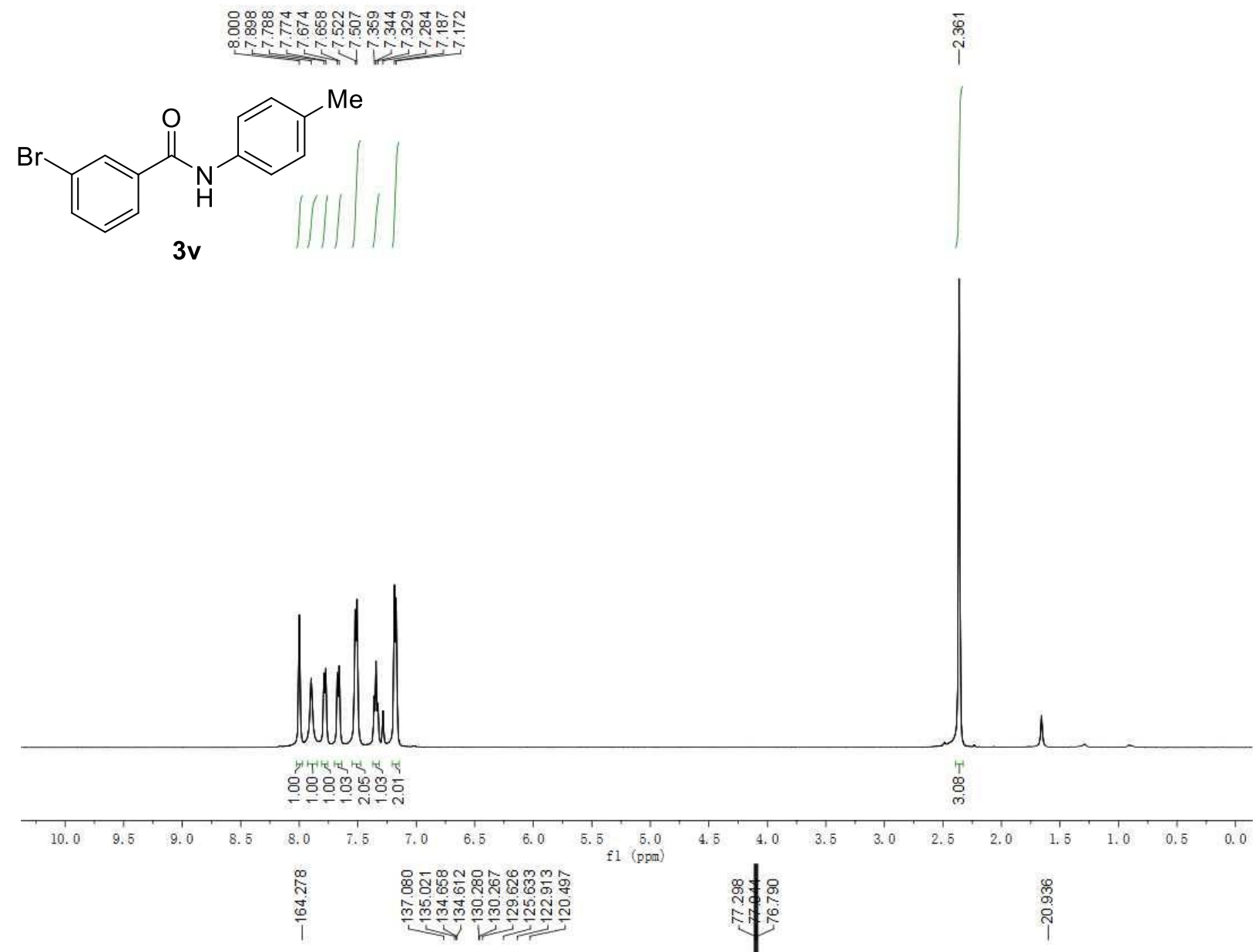

${ }^{13} \mathrm{C} \mathrm{NMR}\left(125 \mathrm{MHz}, \mathrm{CDCl}_{3}\right)$
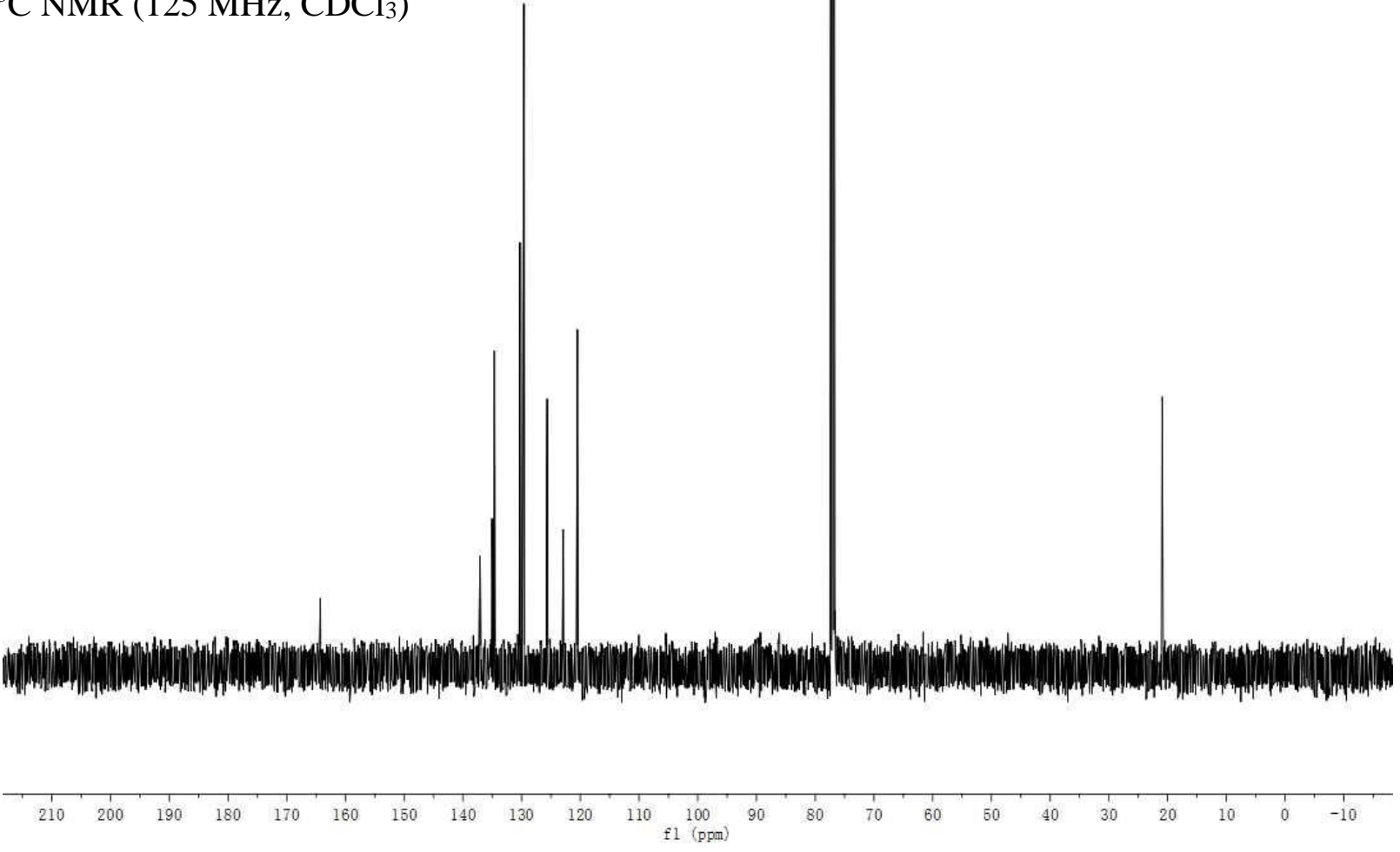
${ }^{1} \mathrm{H}$ NMR (500 MHz, $\left.\mathrm{CDCl}_{3}\right)$

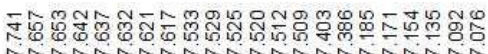

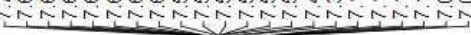<smiles>C#CCCCCC</smiles>

$3 w$

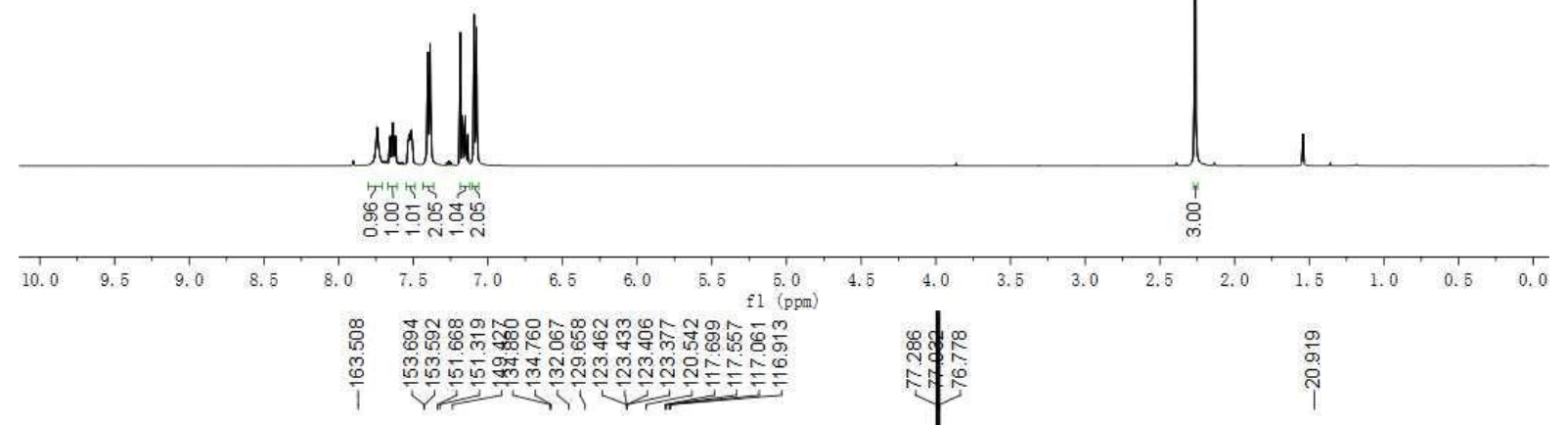

${ }^{13} \mathrm{C} \mathrm{NMR}\left(125 \mathrm{MHz}, \mathrm{CDCl}_{3}\right)$
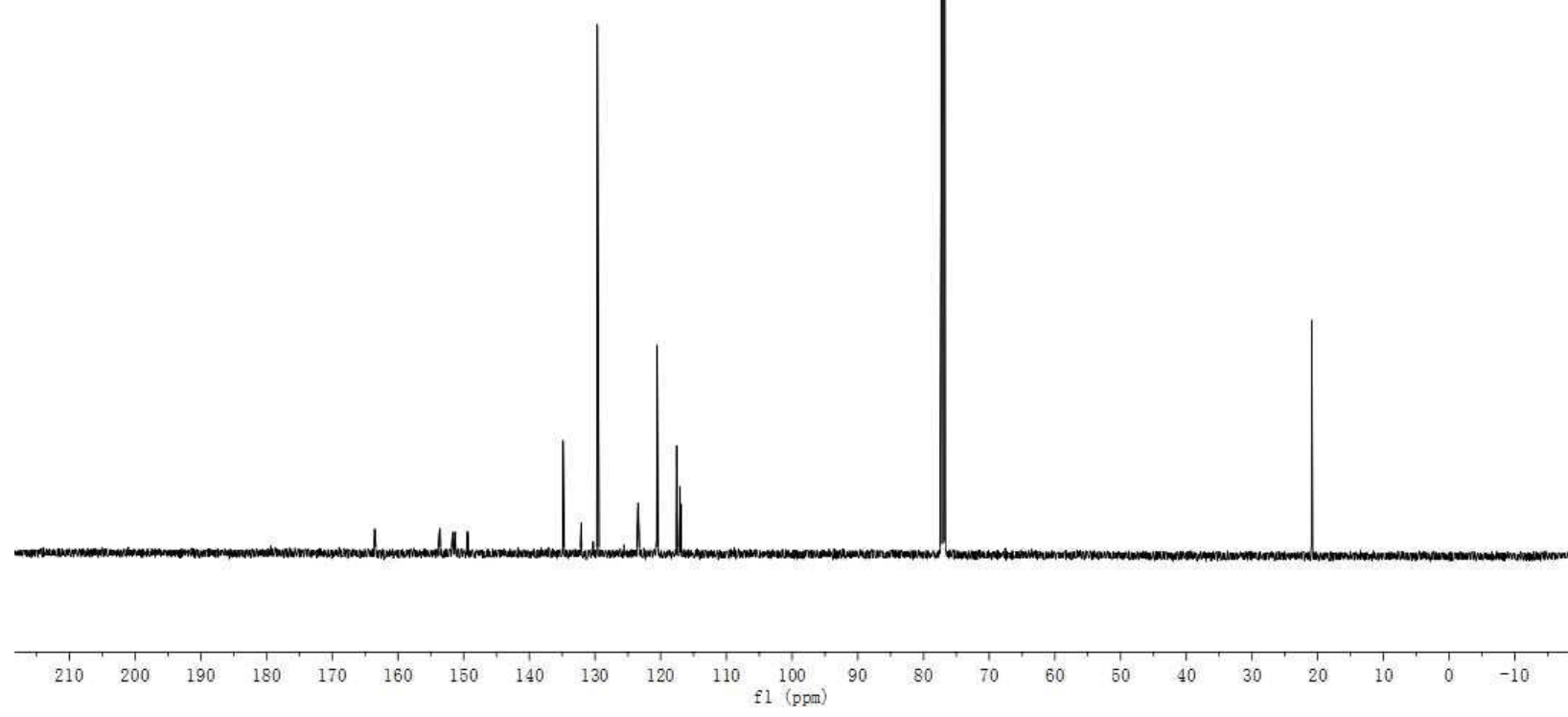
S268 
${ }^{19}$ F NMR (471 MHz, $\left.\mathrm{CDCl}_{3}\right)$<smiles>Cc1ccc(NC(=O)c2ccc(F)c(F)c2)cc1</smiles>

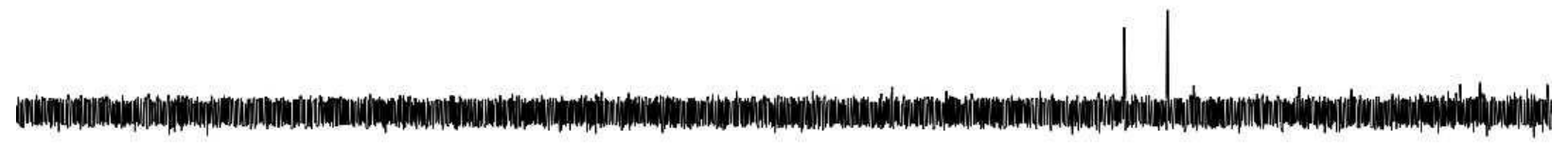

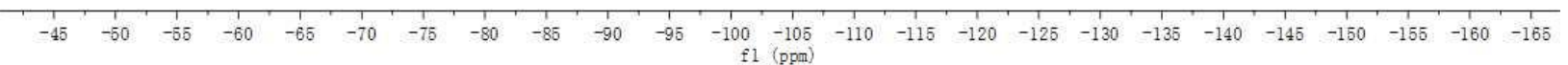


${ }^{1} \mathrm{H}$ NMR (500 MHz, $\left.\mathrm{CDCl}_{3}\right)$
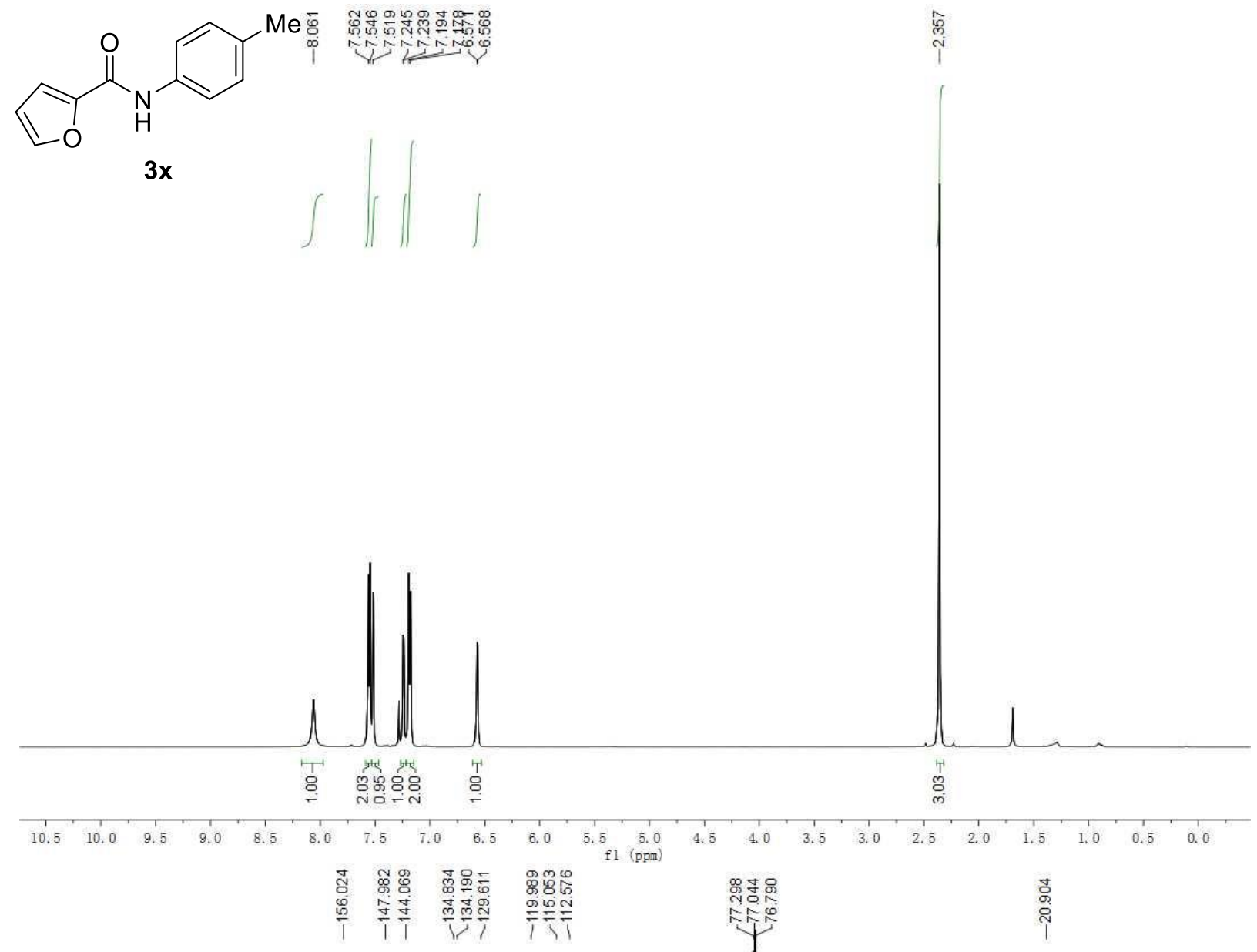

${ }^{13} \mathrm{C} \mathrm{NMR}\left(125 \mathrm{MHz}, \mathrm{CDCl}_{3}\right)$
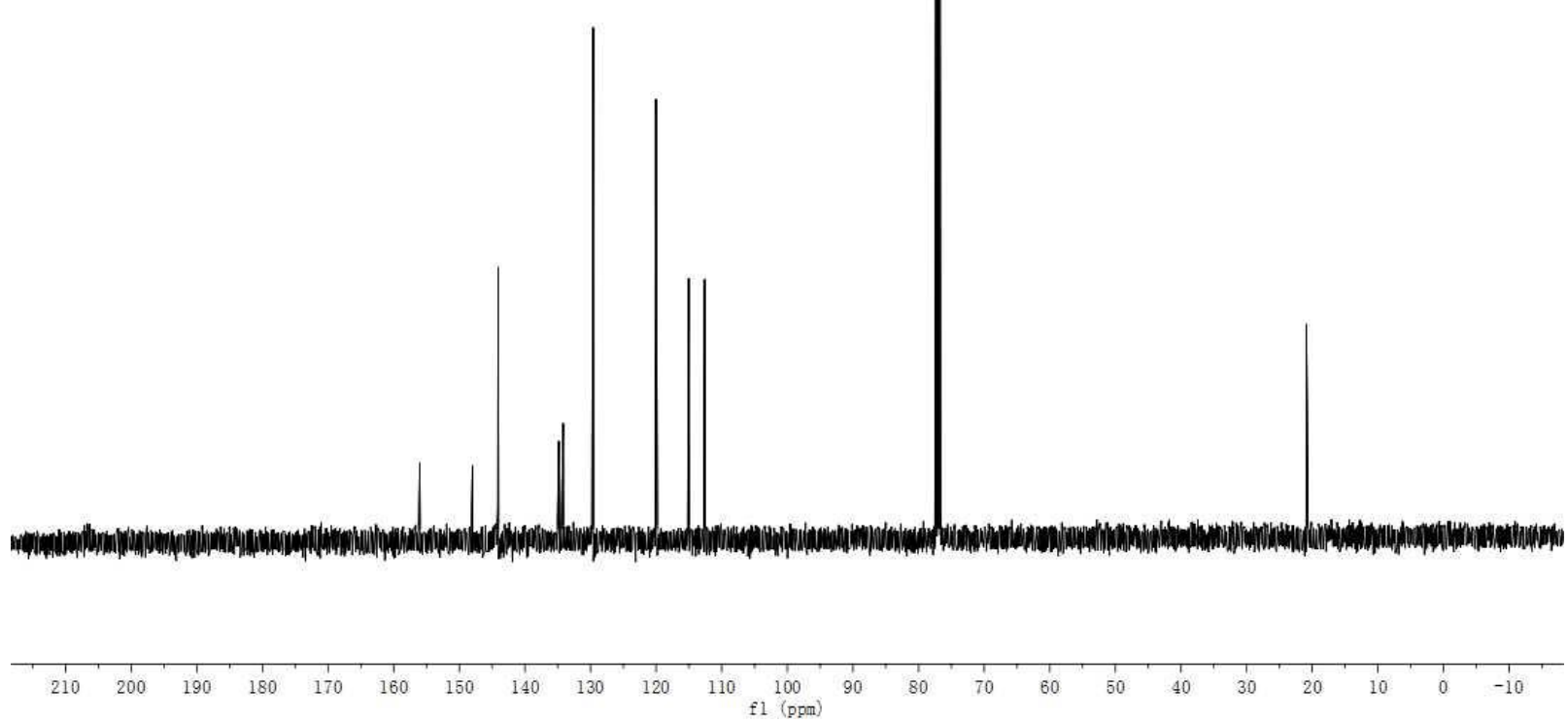
S270 
${ }^{1} \mathrm{H} \mathrm{NMR}\left(500 \mathrm{MHz}, \mathrm{CDCl}_{3}\right)$

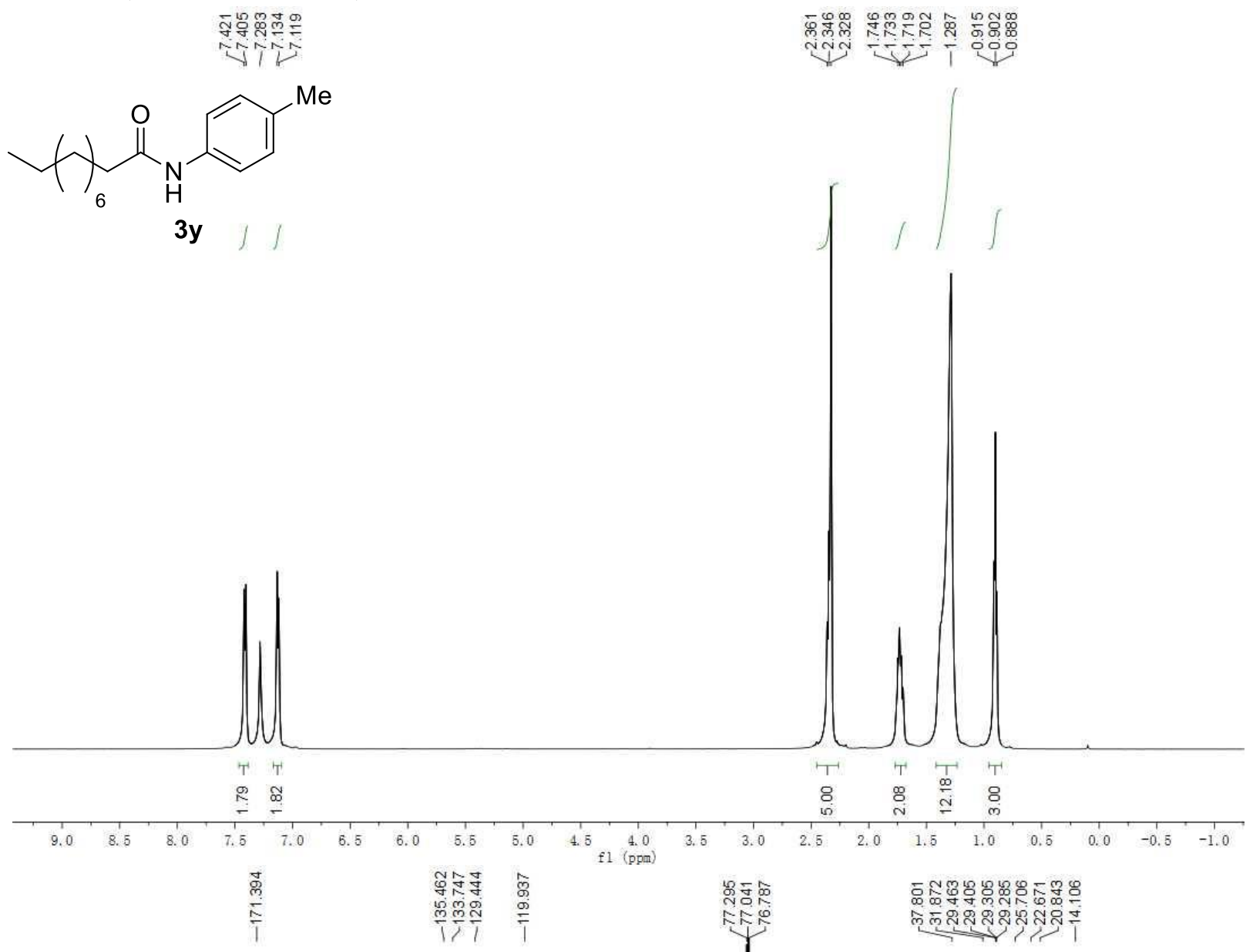

${ }^{13} \mathrm{C} \mathrm{NMR}\left(125 \mathrm{MHz}, \mathrm{CDCl}_{3}\right)$
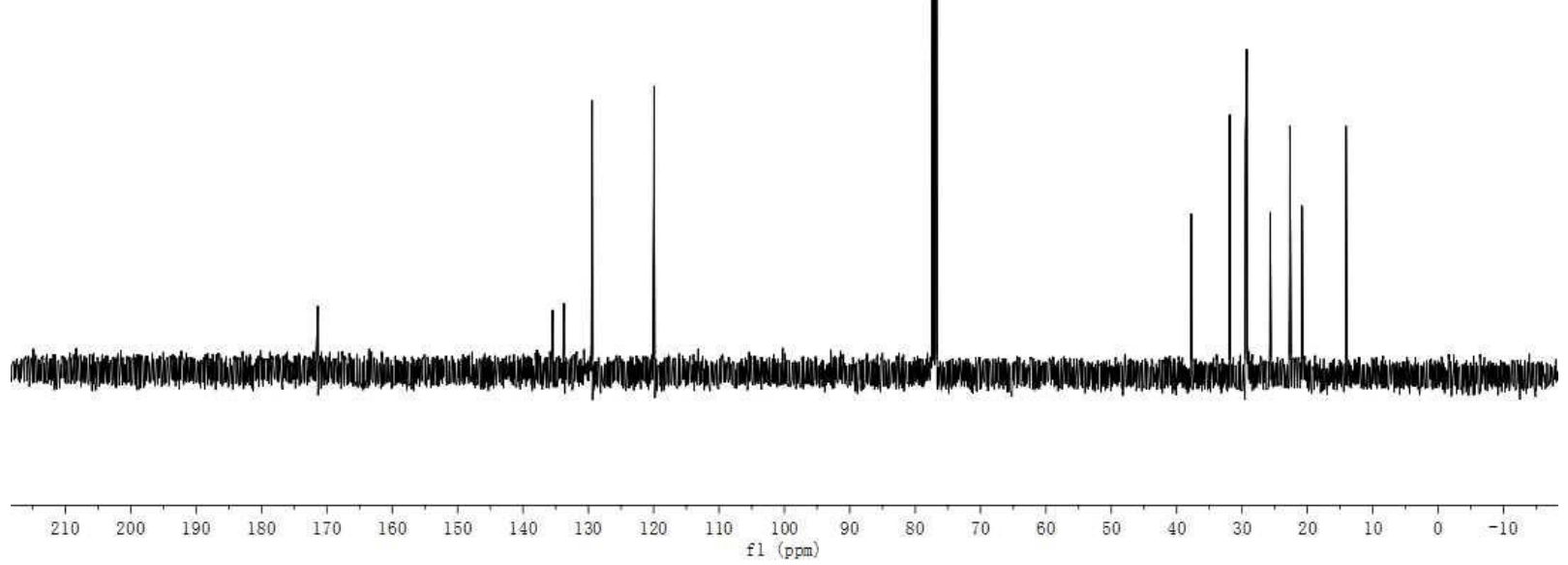
S271 
${ }^{1} \mathrm{H} \mathrm{NMR}\left(500 \mathrm{MHz}, \mathrm{CDCl}_{3}\right)$<smiles>Cc1ccc(NC(=O)C(C)C)cc1</smiles>
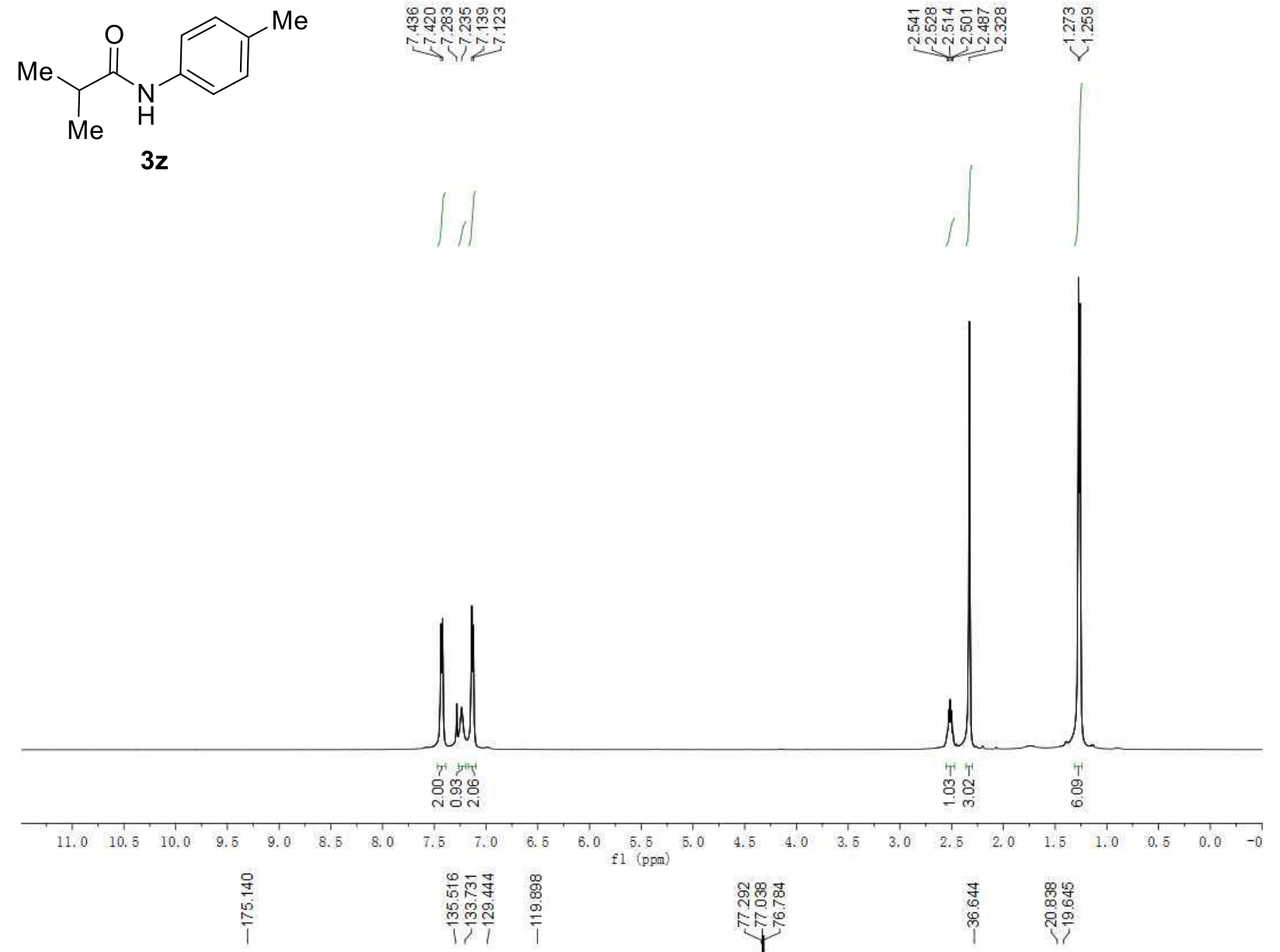

${ }^{13} \mathrm{C} \mathrm{NMR}\left(125 \mathrm{MHz}, \mathrm{CDCl}_{3}\right)$
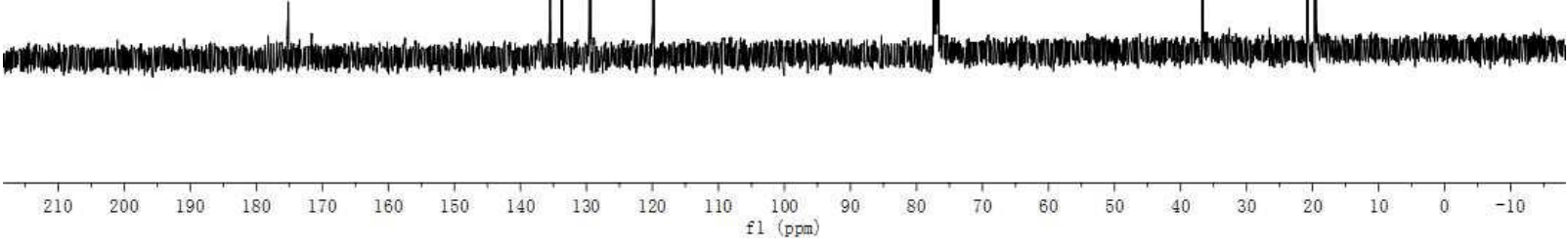
S272 
${ }^{1} \mathrm{H} \mathrm{NMR}\left(500 \mathrm{MHz}, \mathrm{CDCl}_{3}\right)$
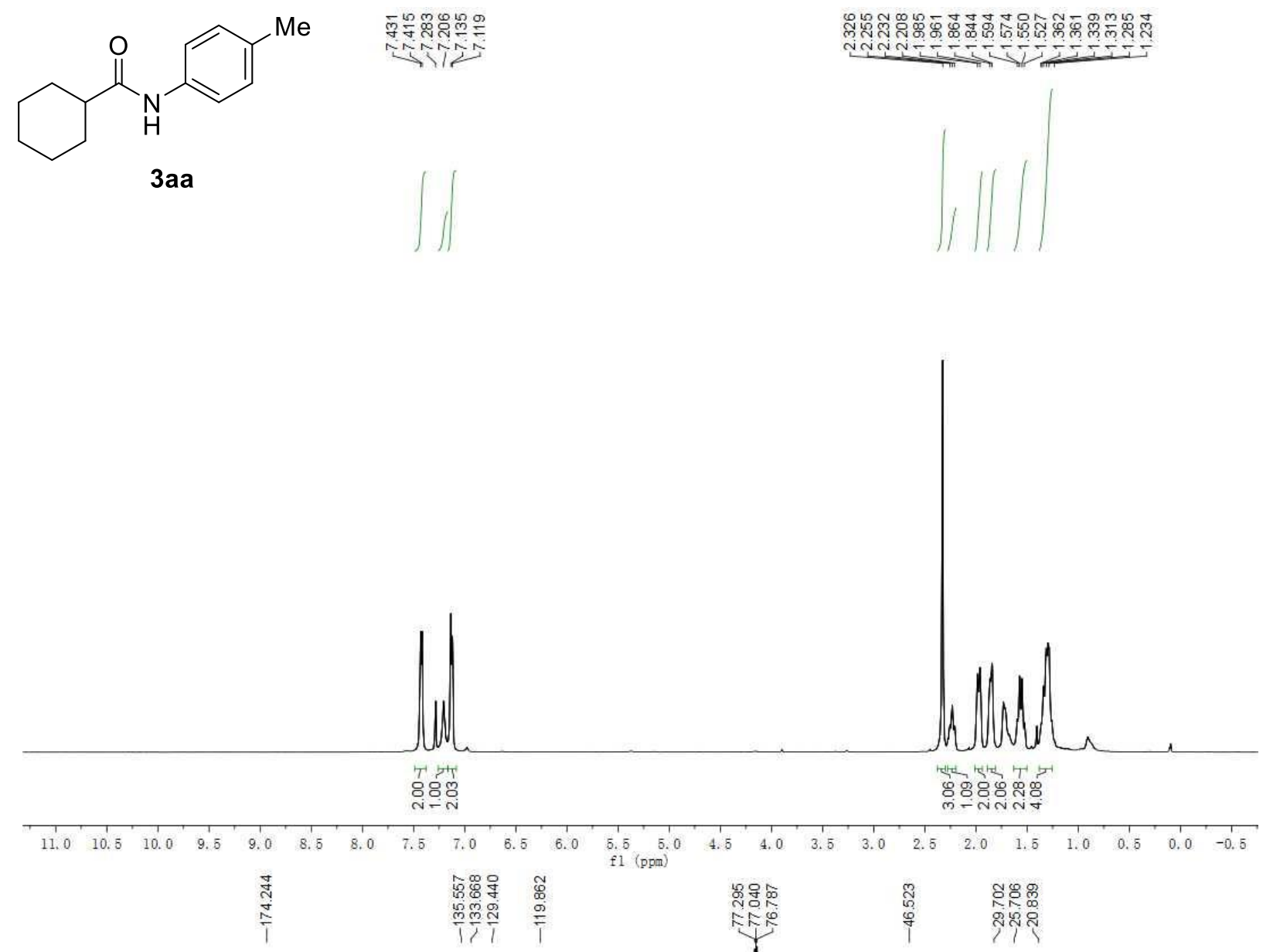

${ }^{13} \mathrm{C} \mathrm{NMR}\left(125 \mathrm{MHz}, \mathrm{CDCl}_{3}\right)$
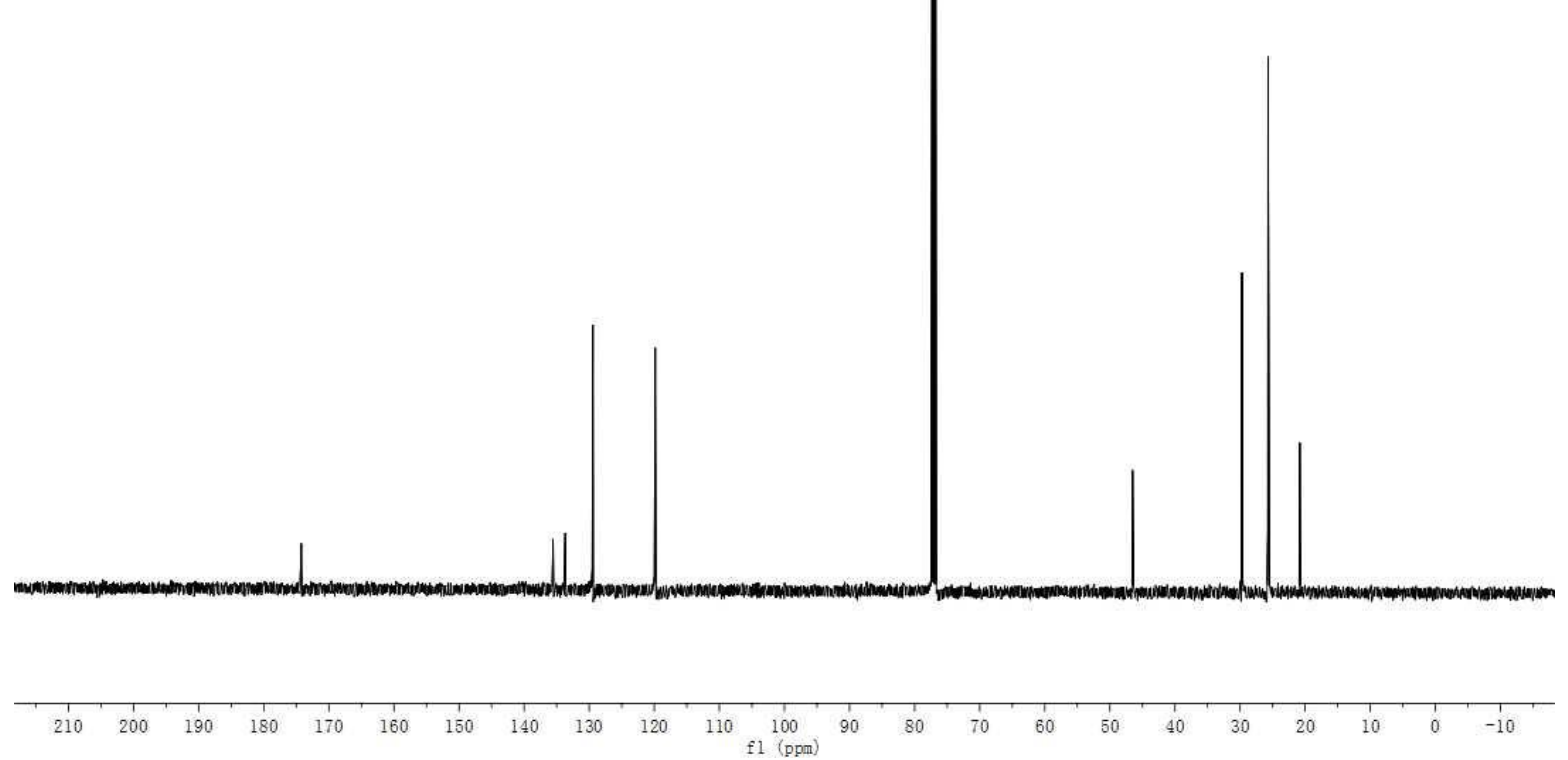
${ }^{1} \mathrm{H}$ NMR (500 MHz, $\left.\mathrm{CDCl}_{3}\right)$
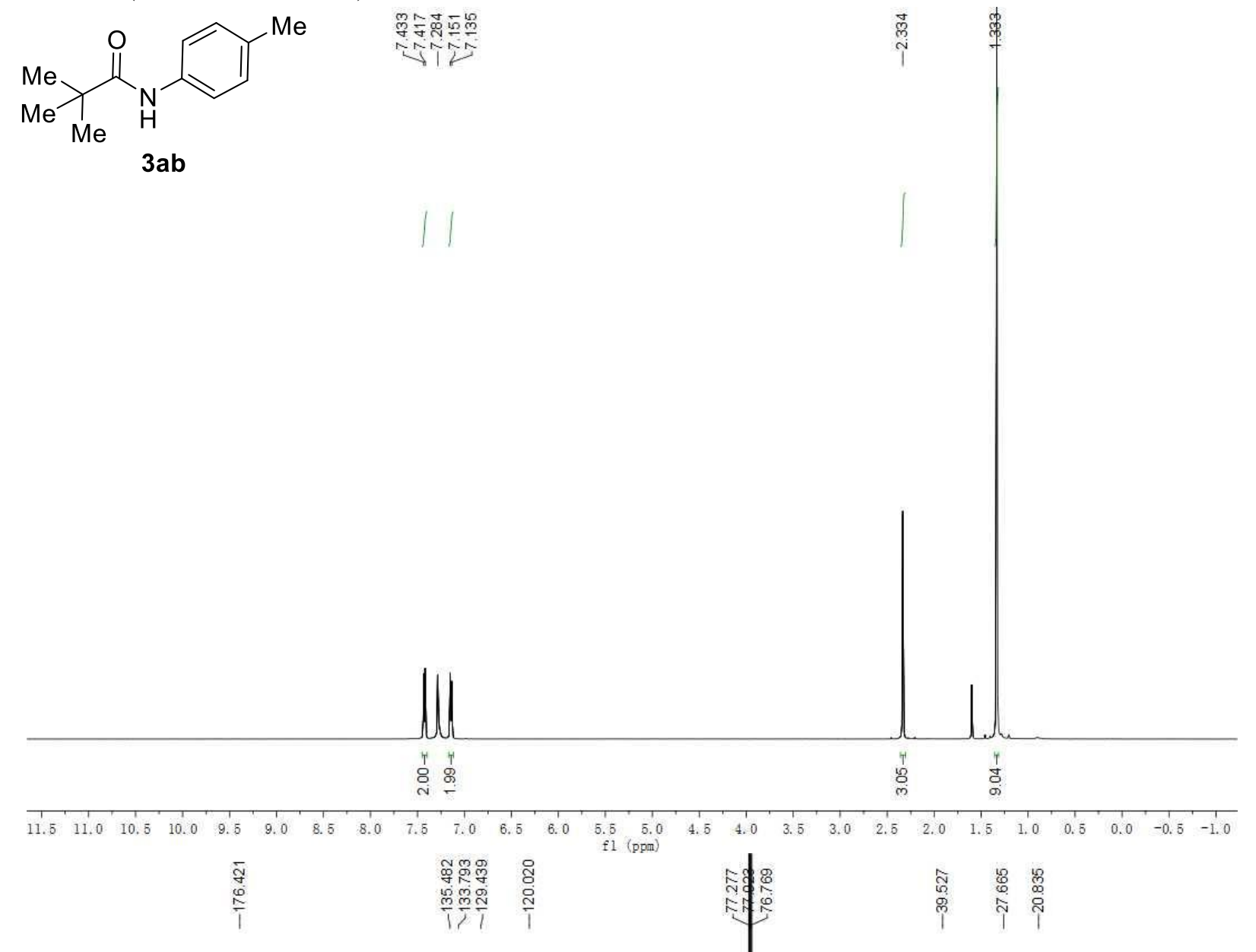

${ }^{13} \mathrm{C}$ NMR $\left(125 \mathrm{MHz}, \mathrm{CDCl}_{3}\right)$
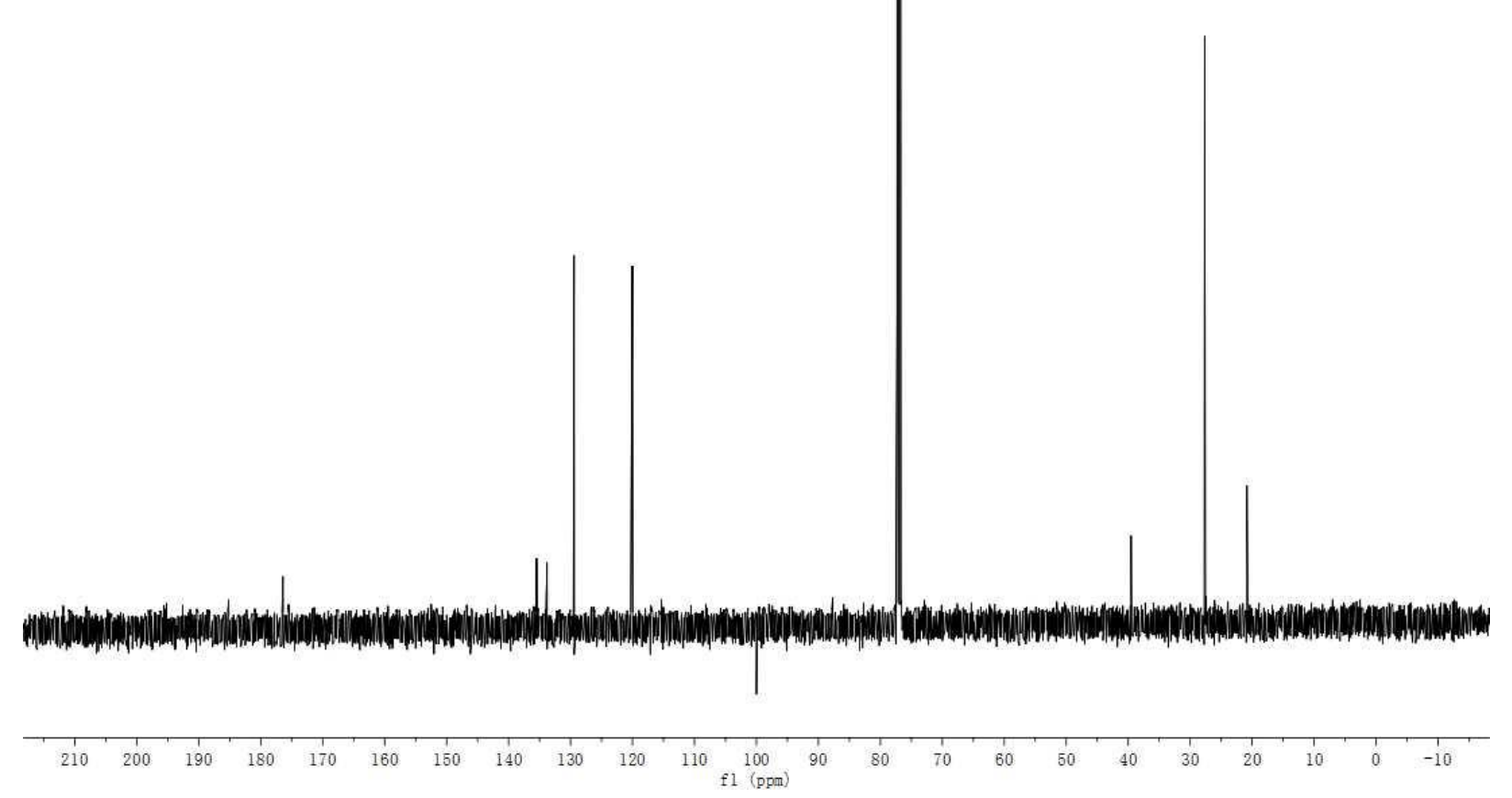
S274 
${ }^{1} \mathrm{H}$ NMR (500 MHz, $\mathrm{CDCl}_{3}$ )<smiles>O=C(Nc1ccccc1)c1ccccc1</smiles>

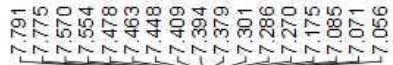
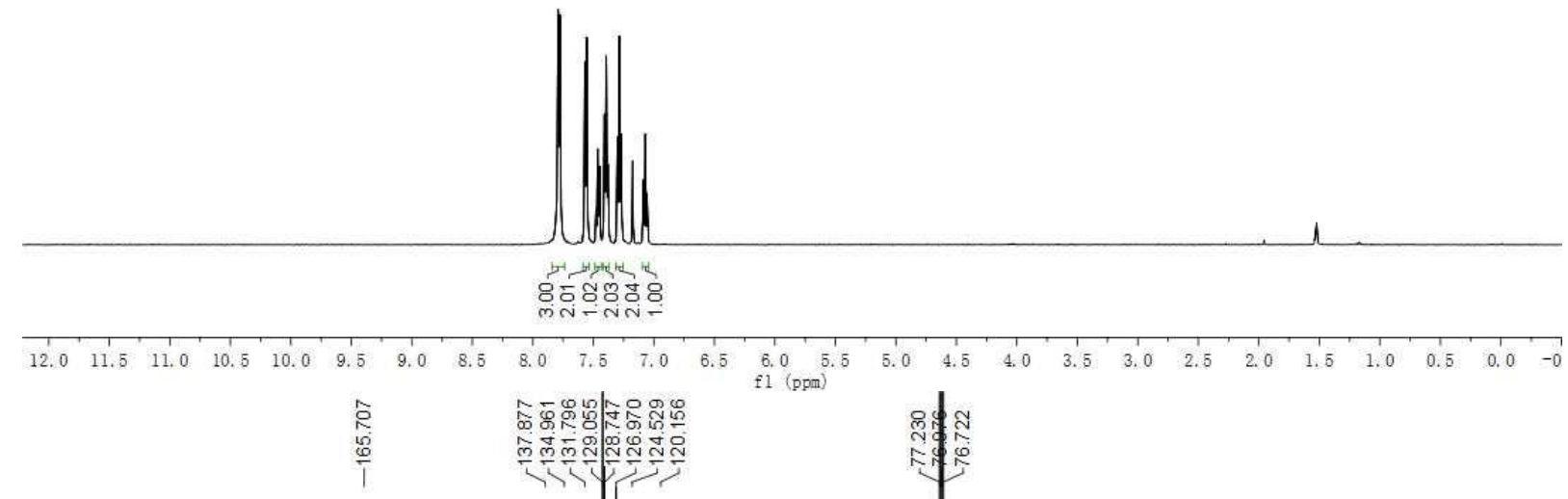

${ }^{13} \mathrm{C}$ NMR $\left(125 \mathrm{MHz}, \mathrm{CDCl}_{3}\right)$

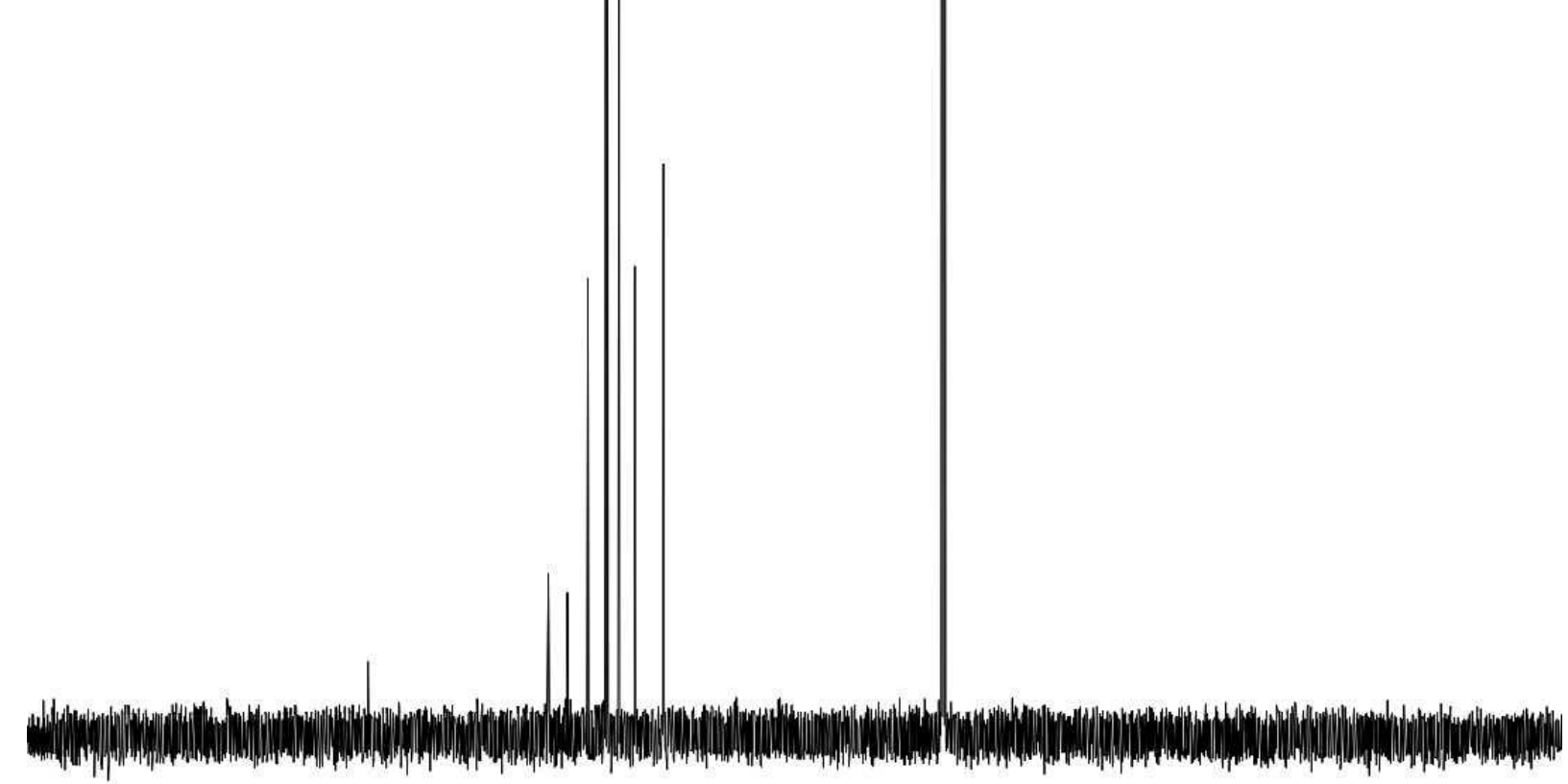

$\begin{array}{llllllll}210 & 200 & 190 & 180 & 170 & 160 & 150 & 140\end{array}$

$10 \stackrel{100}{\mathrm{fl}}(\mathrm{ppm})$ S275 
${ }^{1} \mathrm{H}$ NMR (500 MHz, DMSO-d6)

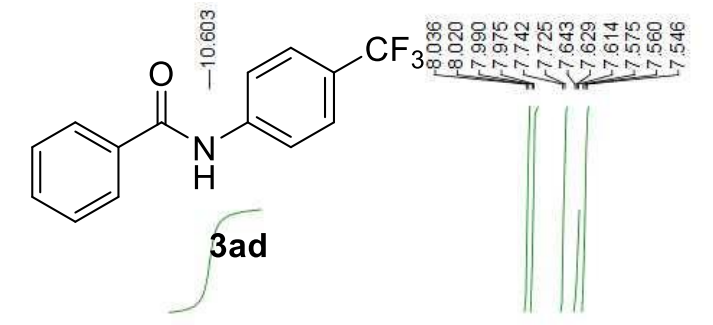

$\stackrel{\substack{j \\ \prod}}{\substack{0 \\ i}}$

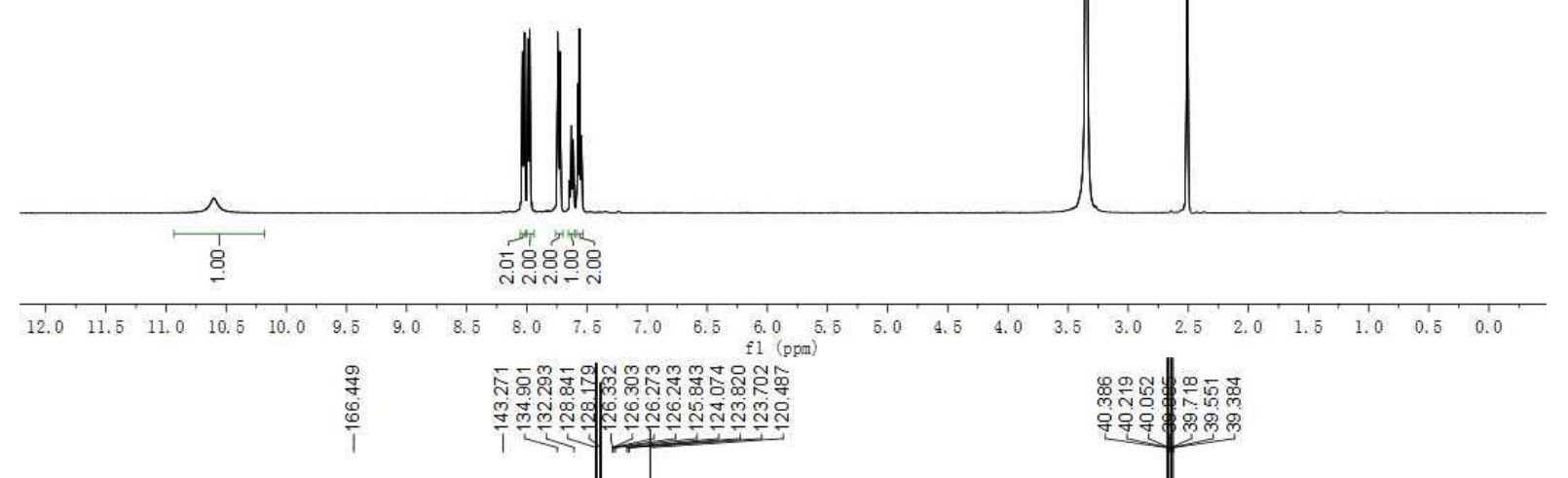

${ }^{13} \mathrm{C}$ NMR (125 MHz, DMSO-d6)

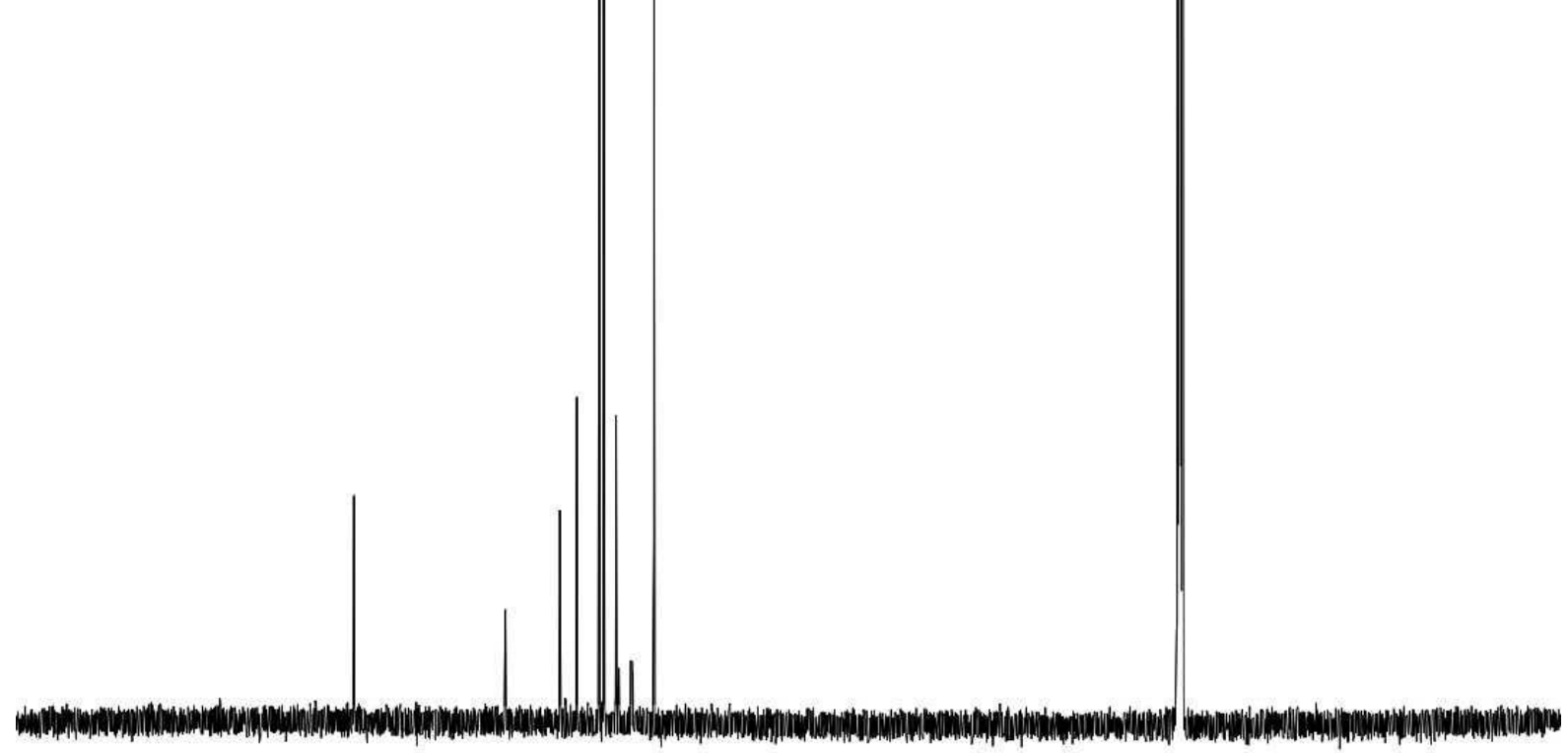

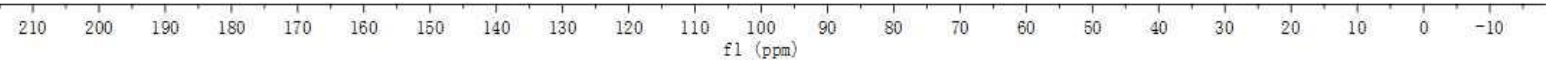


${ }^{19} \mathrm{~F}$ NMR (471 MHz, DMSO-d6)<smiles>CCCCCC(C)(F)F</smiles>

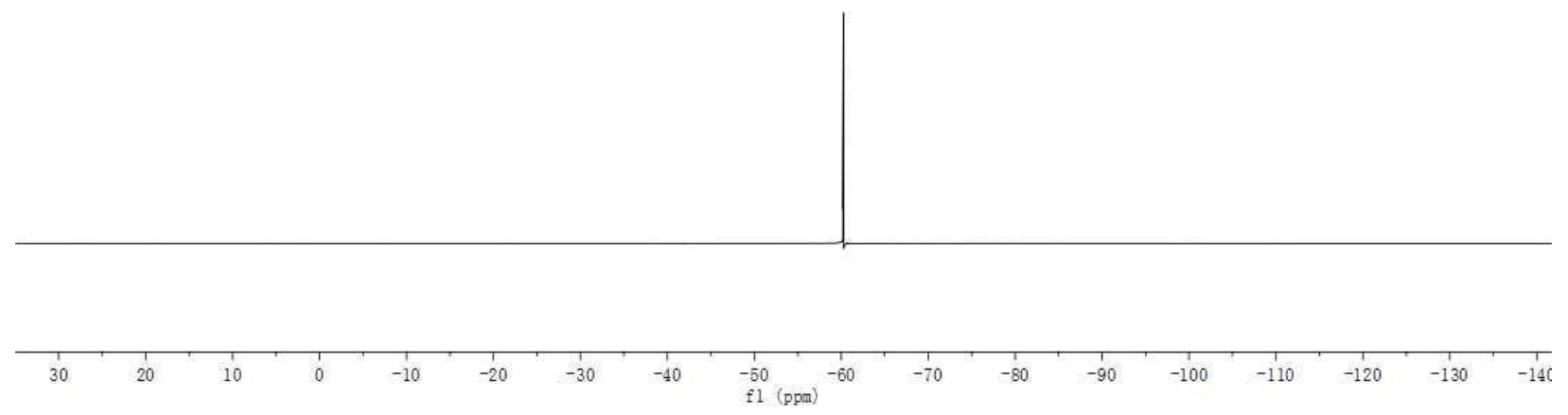


${ }^{1} \mathrm{H} \mathrm{NMR}\left(500 \mathrm{MHz}, \mathrm{CDCl}_{3}\right)$
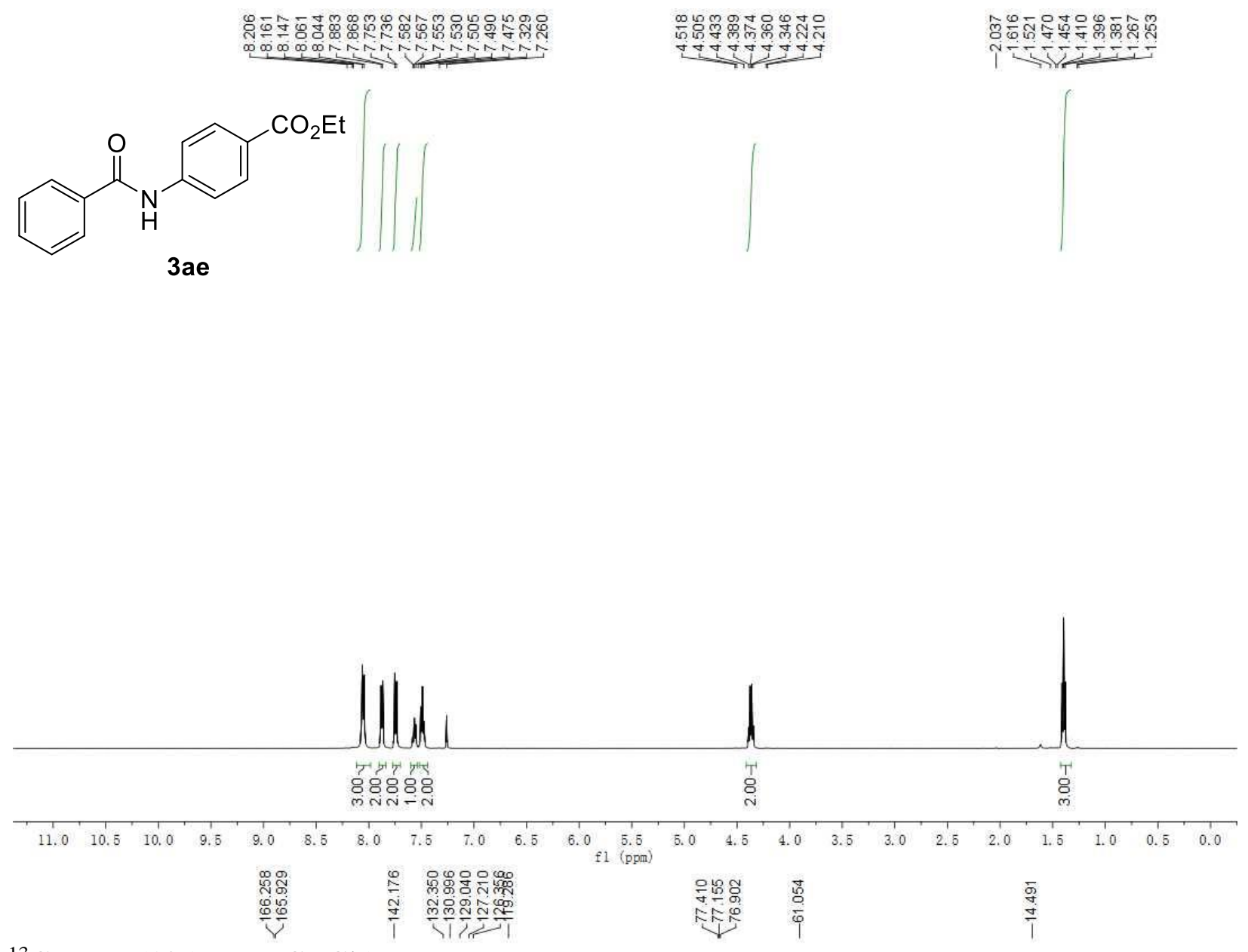

${ }^{13} \mathrm{C} \mathrm{NMR}\left(125 \mathrm{MHz}, \mathrm{CDCl}_{3}\right)$
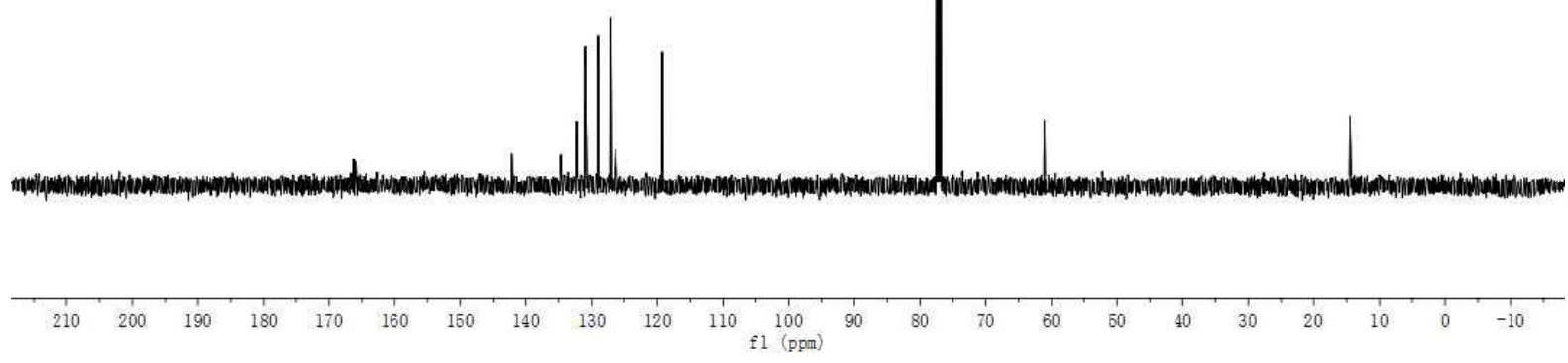
${ }^{1} \mathrm{H} \mathrm{NMR}\left(500 \mathrm{MHz}, \mathrm{CDCl}_{3}\right)$

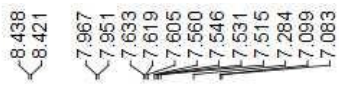<smiles>Cc1cccc(NC(=O)c2ccccc2)c1C#N</smiles>

$3 a f$
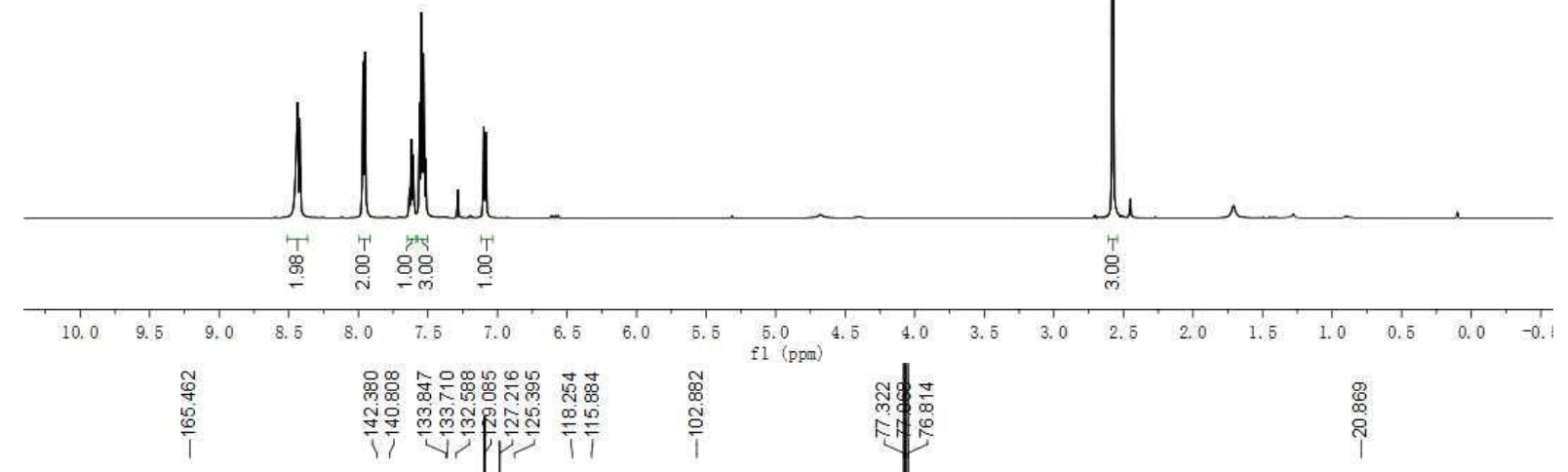

${ }^{13} \mathrm{C} \mathrm{NMR}\left(125 \mathrm{MHz}, \mathrm{CDCl}_{3}\right)$

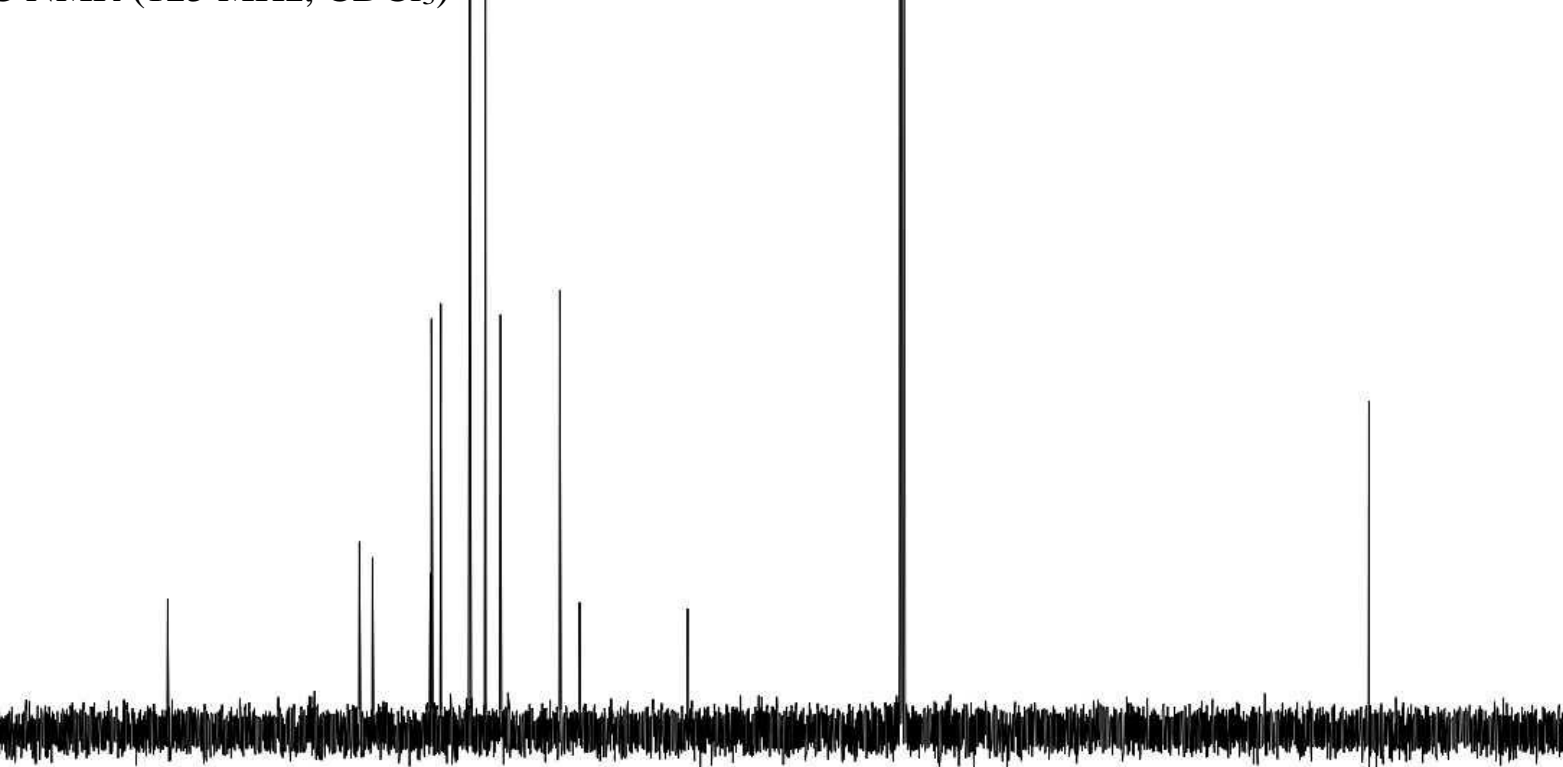

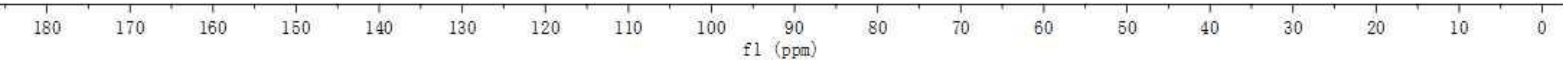


${ }^{1} \mathrm{H}$ NMR (500 MHz, DMSO-d 6 )<smiles>O=C(Nc1c(F)c(F)c(F)c(F)c1F)c1ccccc1</smiles>

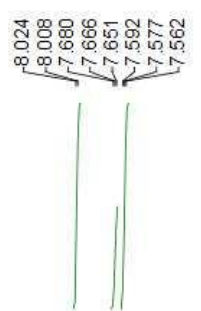

$\stackrel{\substack{0 \\ i}}{\substack{6 \\ i}}$
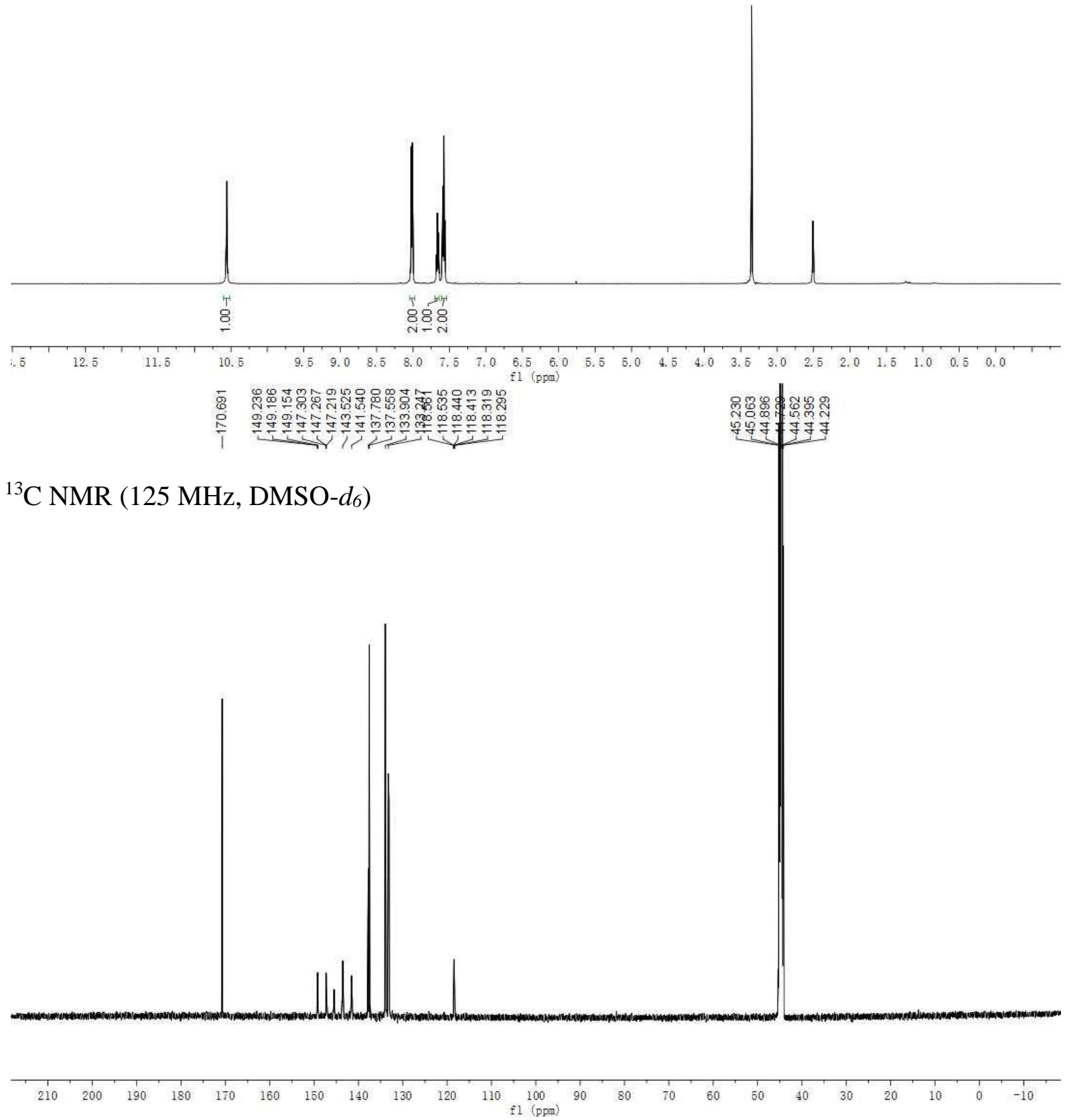
${ }^{19}$ F NMR (500 MHz, DMSO-d $)$<smiles>O=C(Nc1c(F)c(F)c(F)c(F)c1F)c1ccccc1</smiles>

3ag

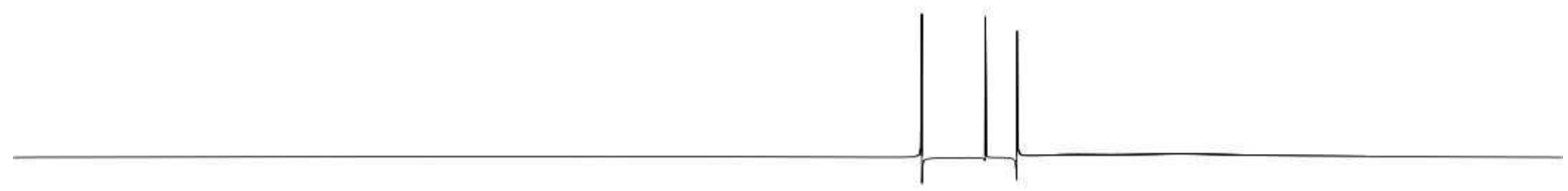

\begin{tabular}{|c|c|c|c|c|c|c|c|c|c|c|c|c|c|c|c|c|c|c|c|c|}
\hline 30 & 20 & 10 & 0 & -10 & -20 & -30 & -40 & $\frac{1}{-50}$ & -60 & -70 & -80 & -90 & $\begin{array}{l}-110 \\
\text { fl }(p p\end{array}$ & -130 & -150 & -170 & -190 & -210 & -230 & -250 \\
\hline
\end{tabular}


${ }^{1} \mathrm{H} \mathrm{NMR}\left(500 \mathrm{MHz}, \mathrm{CDCl}_{3}\right)$
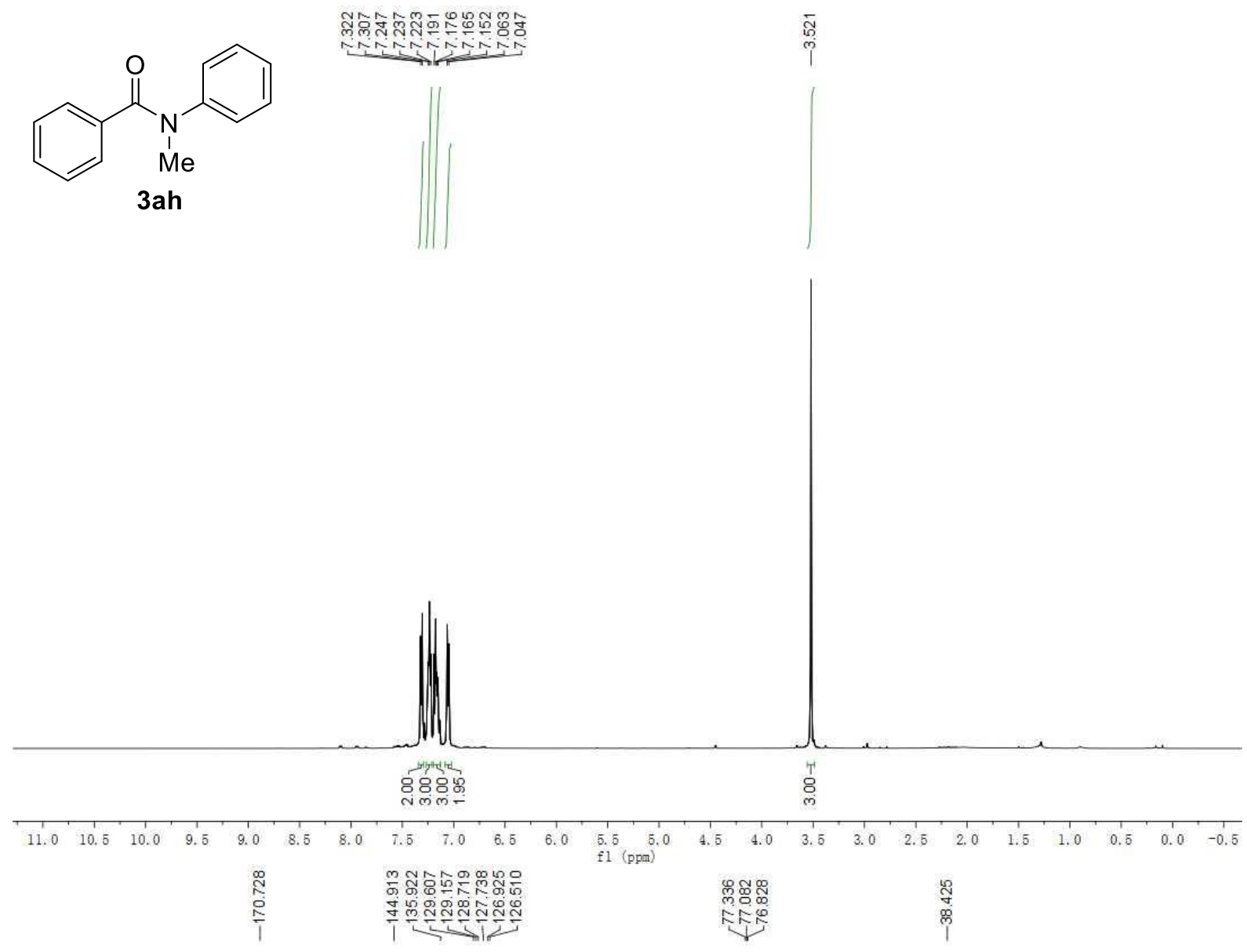

${ }^{13} \mathrm{C} \mathrm{NMR}\left(125 \mathrm{MHz}, \mathrm{CDCl}_{3}\right)$
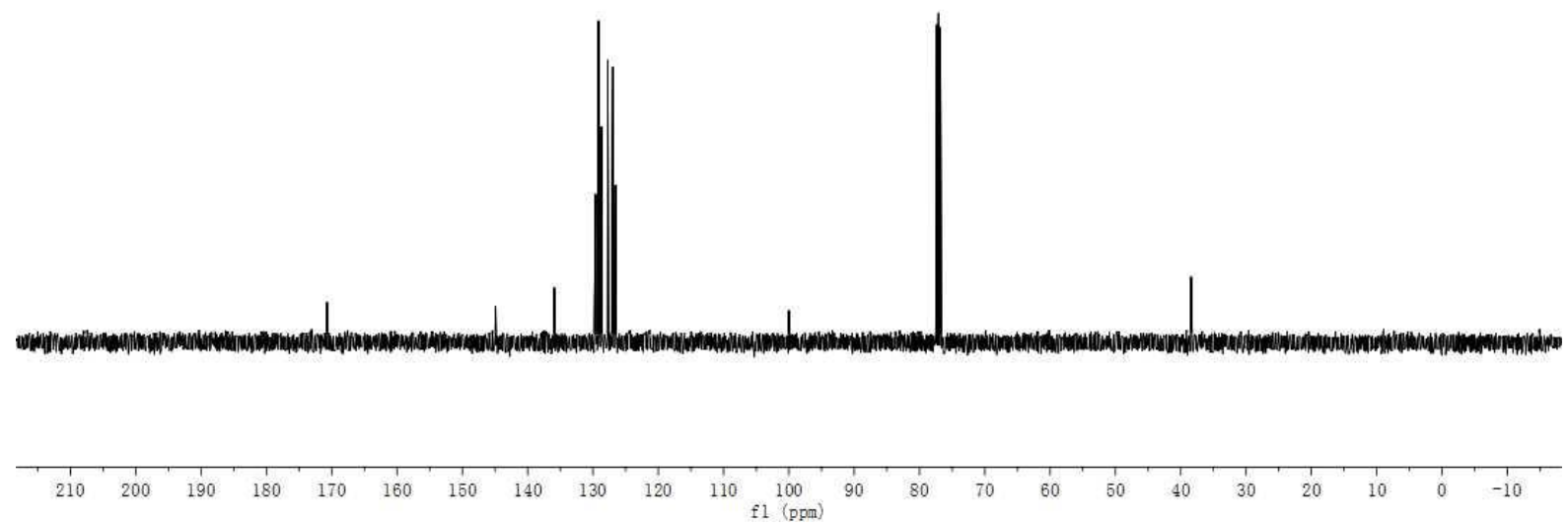
${ }^{1} \mathrm{H} \mathrm{NMR}\left(500 \mathrm{MHz}, \mathrm{CDCl}_{3}\right)$

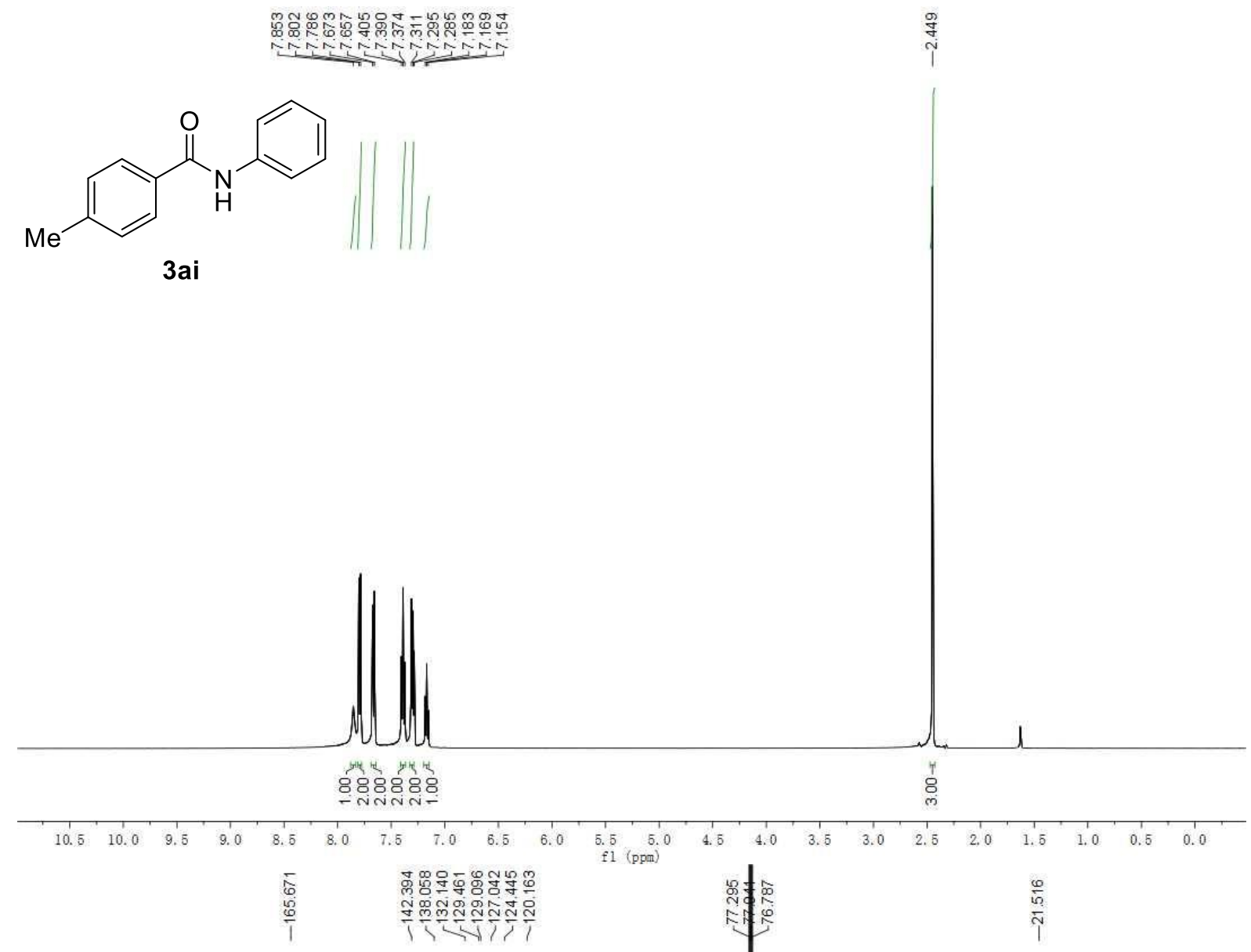

${ }^{13} \mathrm{C} \mathrm{NMR}\left(125 \mathrm{MHz}, \mathrm{CDCl}_{3}\right)$

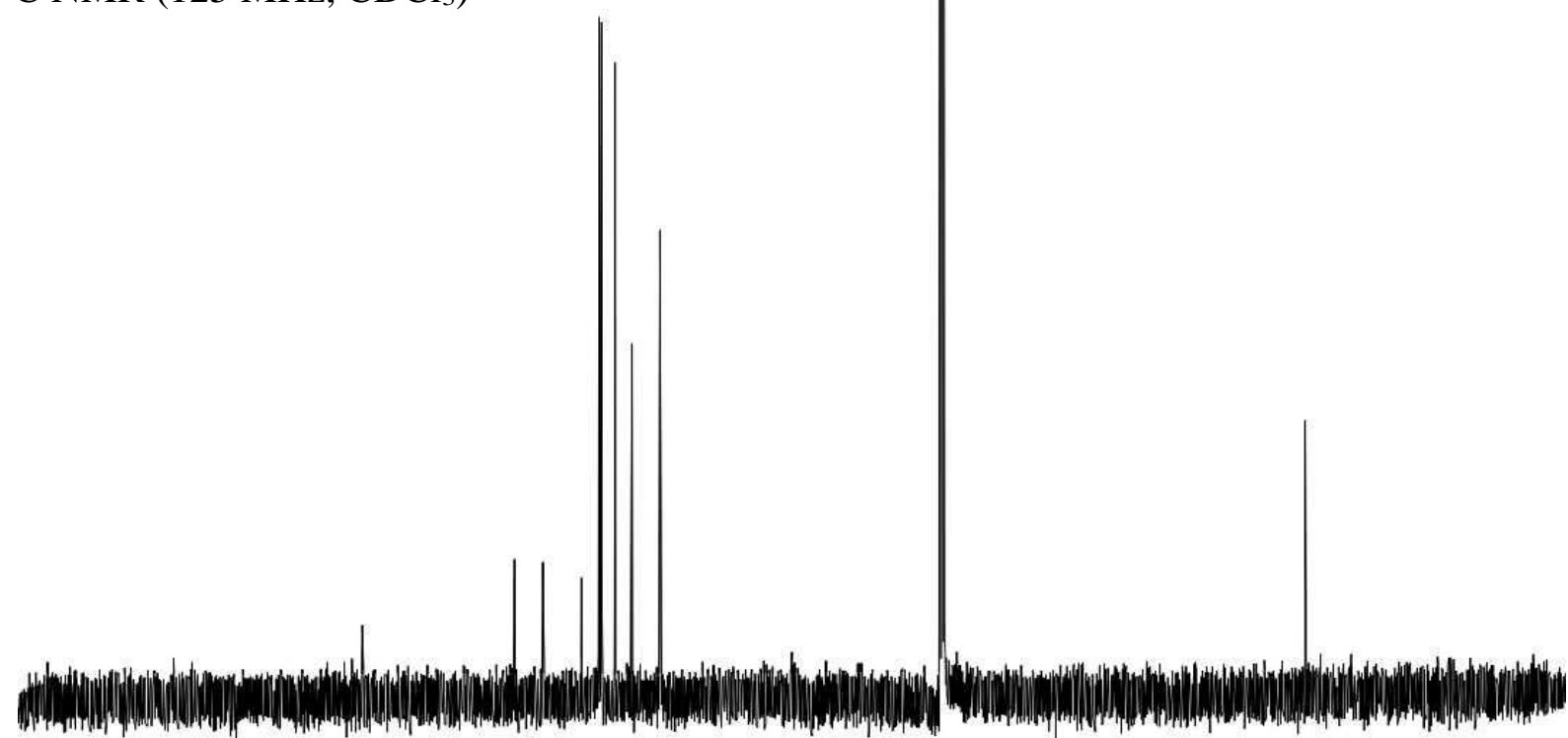

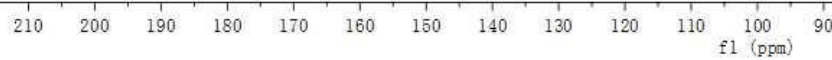


${ }^{1} \mathrm{H}$ NMR (500 MHz, DMSO-d 6 )

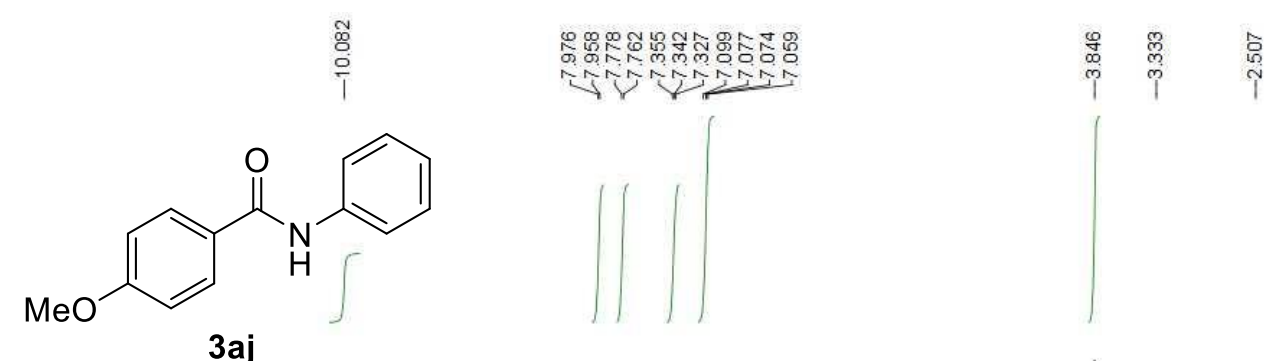

3aj
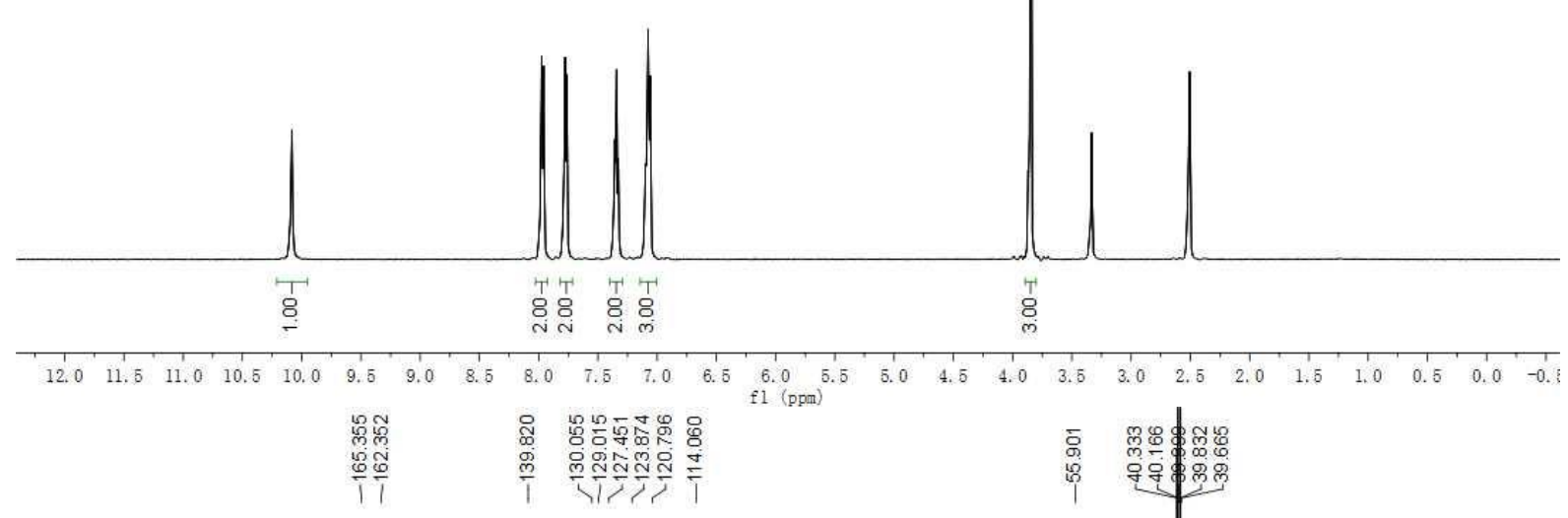

${ }^{13} \mathrm{C}$ NMR $\left(125 \mathrm{MHz}, \mathrm{DMSO}-d_{6}\right)$
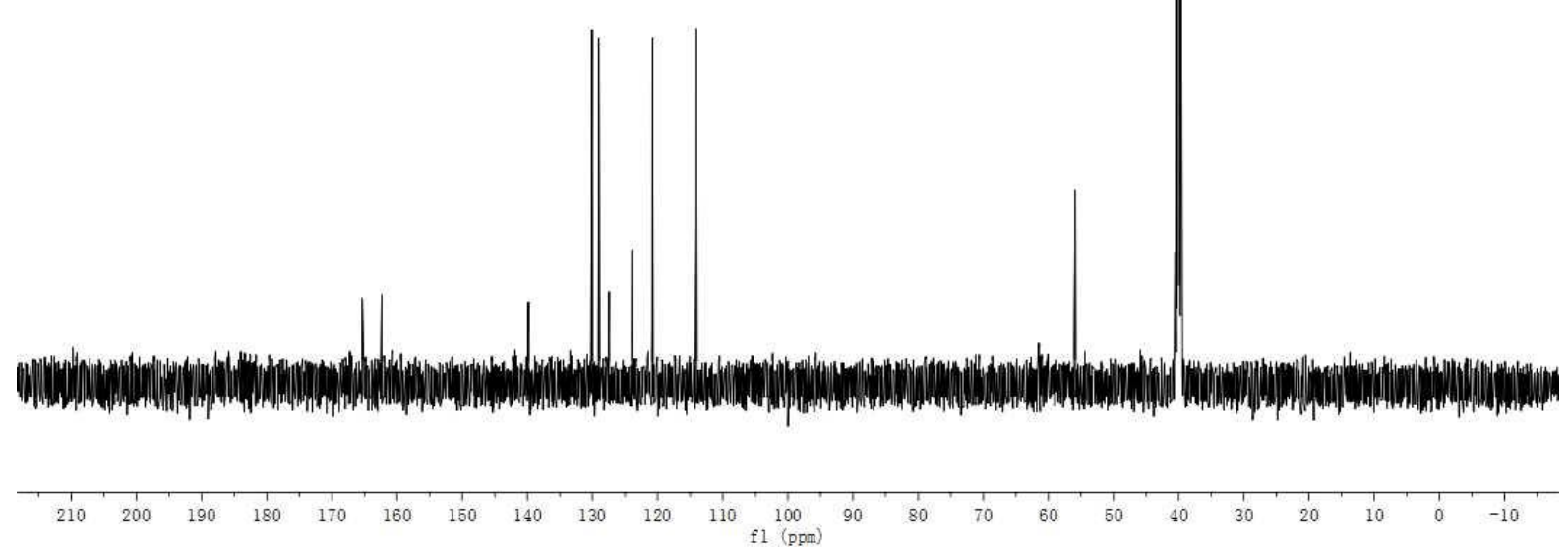
${ }^{1} \mathrm{H}$ NMR (500 MHz, DMSO-d $)$<smiles>Cc1ccc(NC(=O)c2ccc(C(F)(F)F)cc2)cc1</smiles>
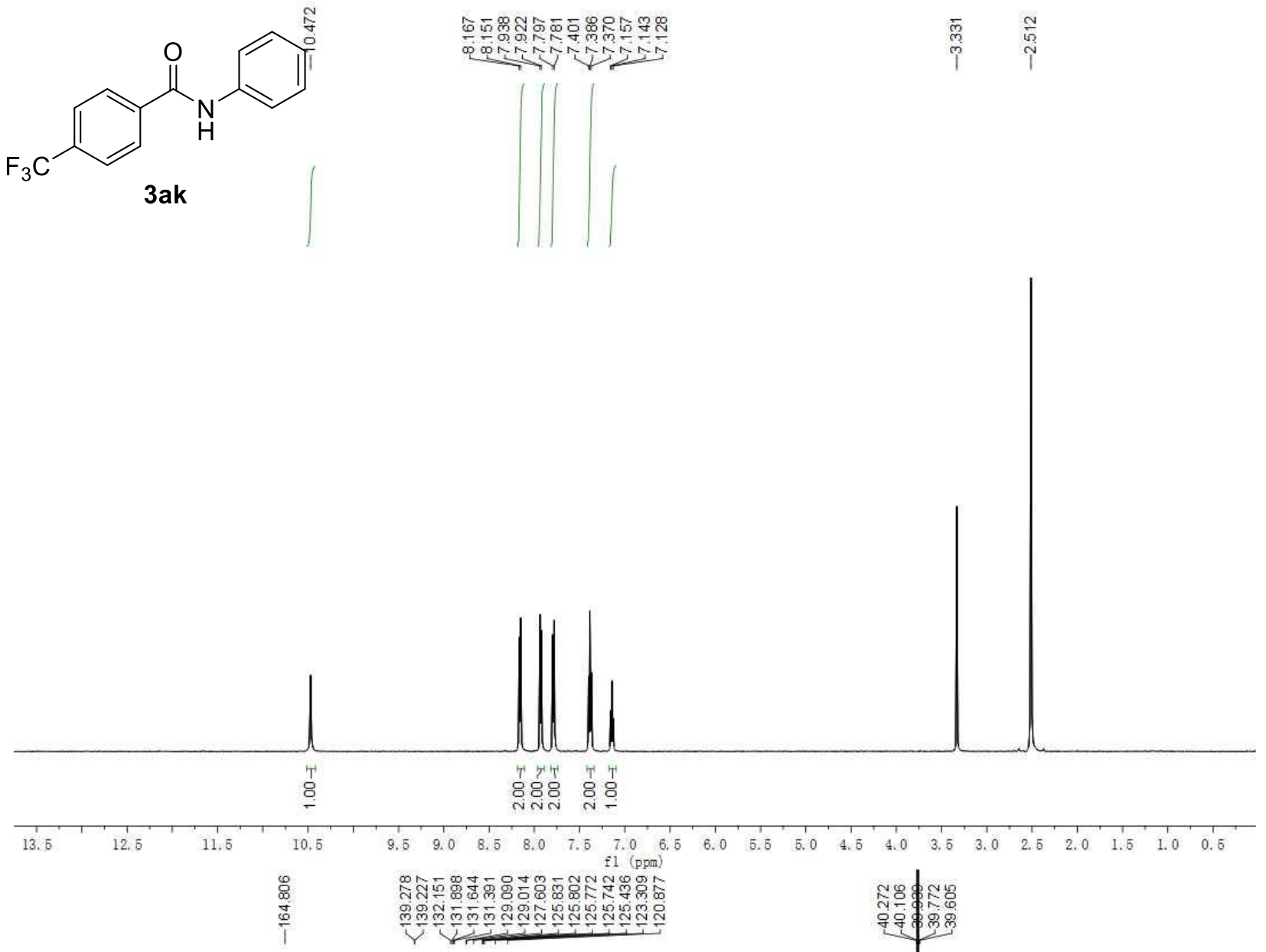

${ }^{13} \mathrm{C}$ NMR (125 MHz, DMSO-d6)
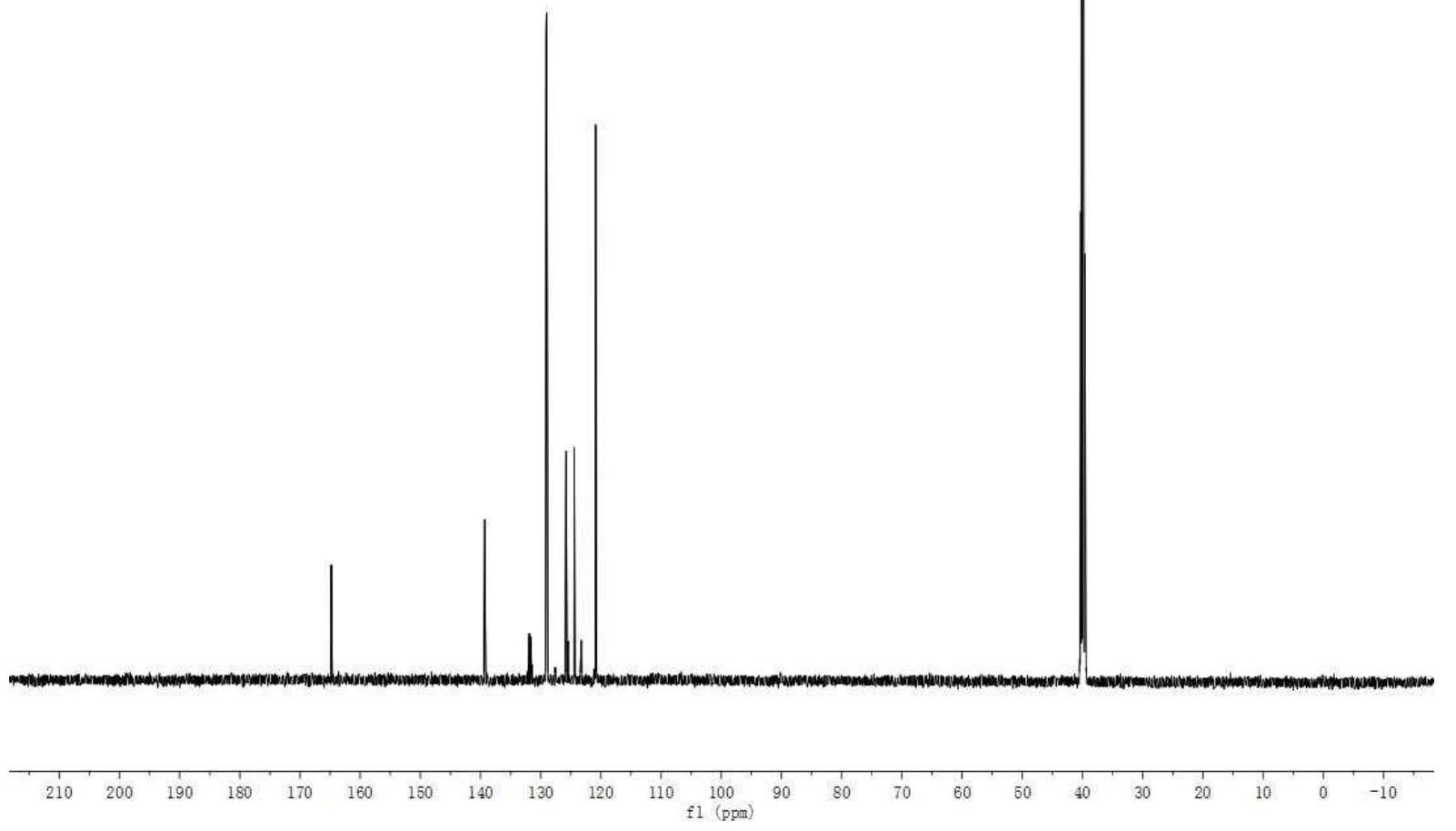
${ }^{19}$ F NMR (471 MHz, DMSO- $\left.d_{6}\right)$<smiles>O=C(Nc1ccccc1)c1ccc(C(F)(F)F)cc1</smiles>

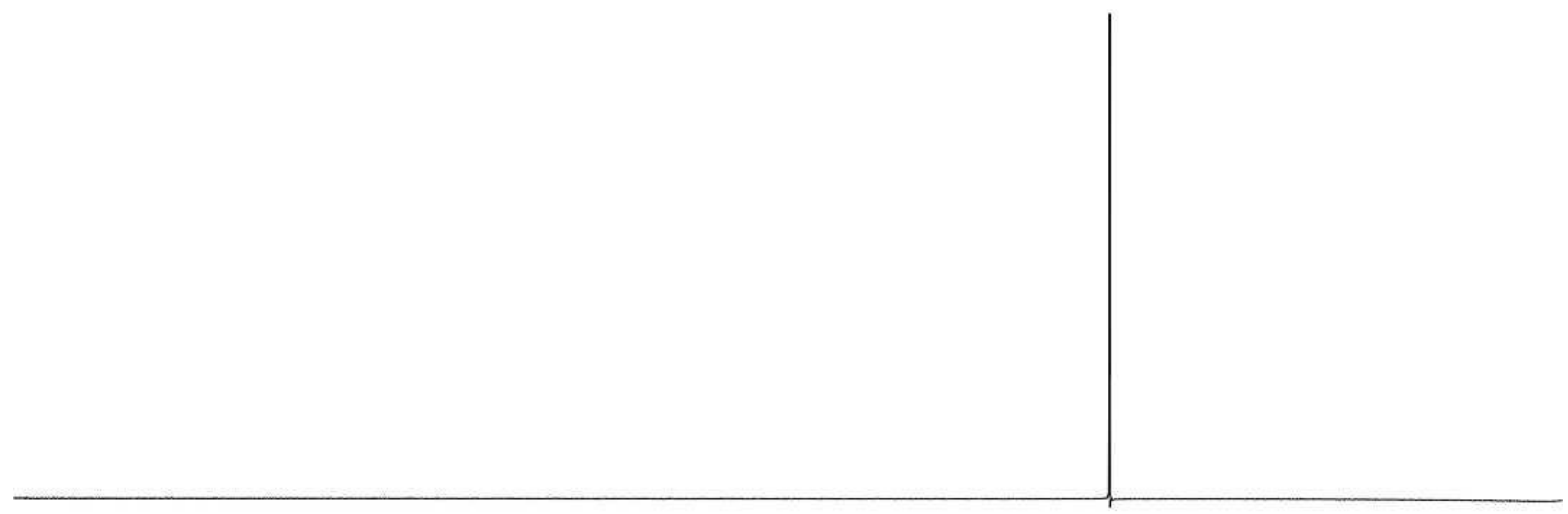

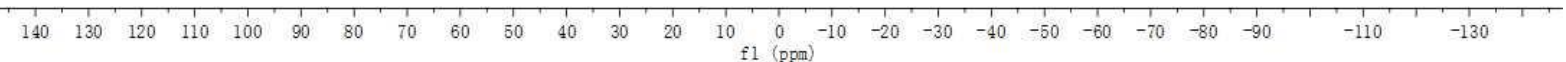


${ }^{1} \mathrm{H} \mathrm{NMR}\left(500 \mathrm{MHz}, \mathrm{CDCl}_{3}\right)$
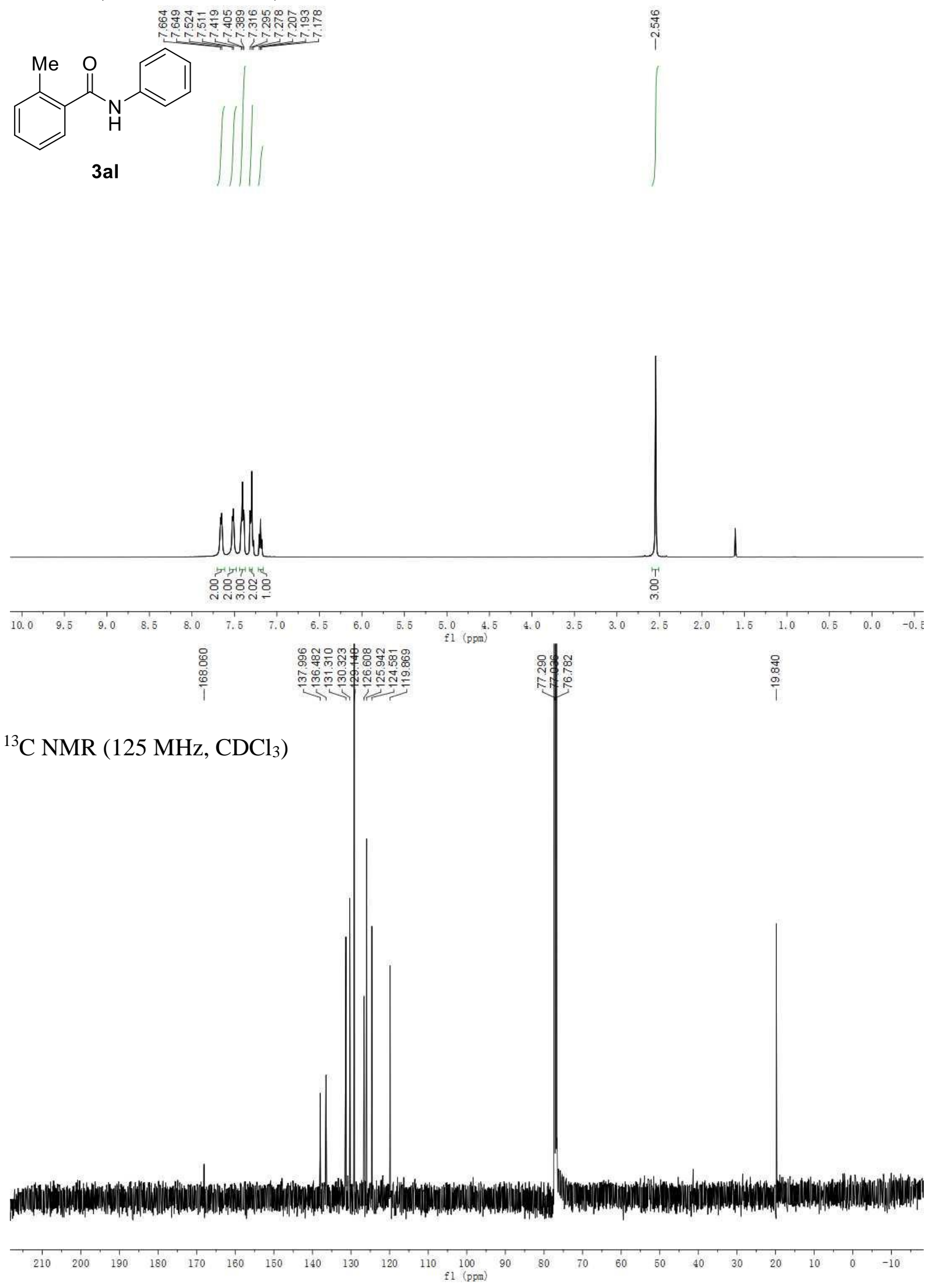
${ }^{1} \mathrm{H} \mathrm{NMR}\left(500 \mathrm{MHz}, \mathrm{CDCl}_{3}\right)$

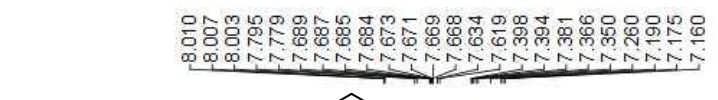<smiles>O=C(Nc1ccccc1)c1cccc(Br)c1</smiles>
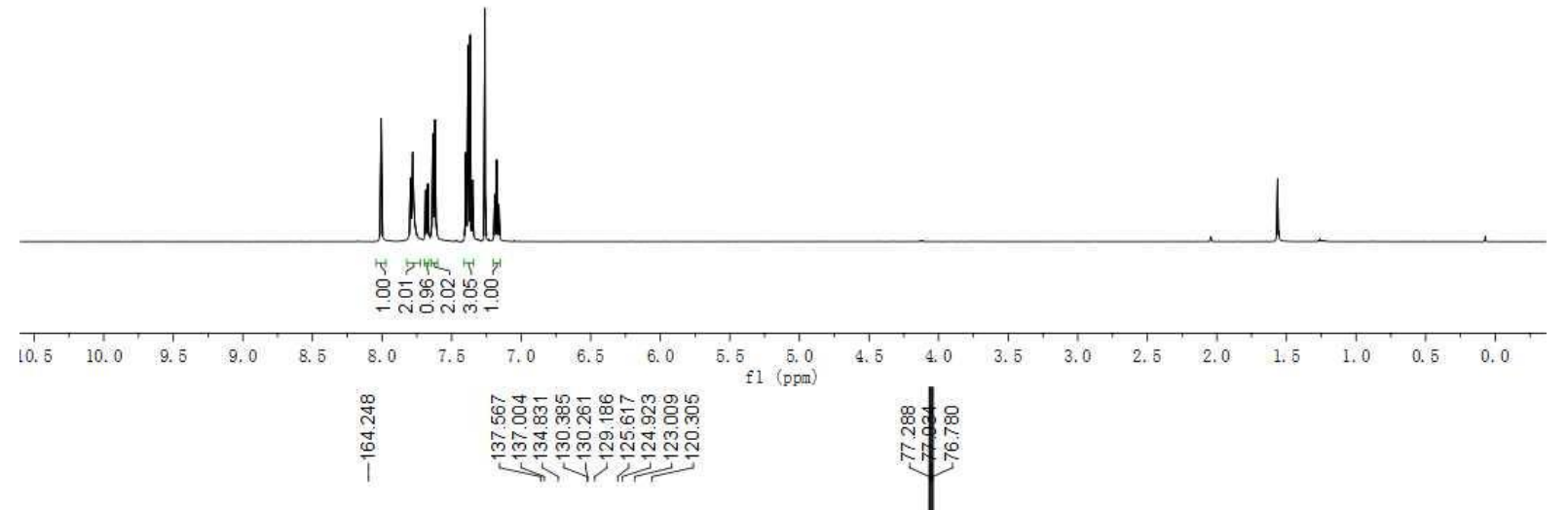

${ }^{13} \mathrm{C} \mathrm{NMR}\left(125 \mathrm{MHz}, \mathrm{CDCl}_{3}\right)$
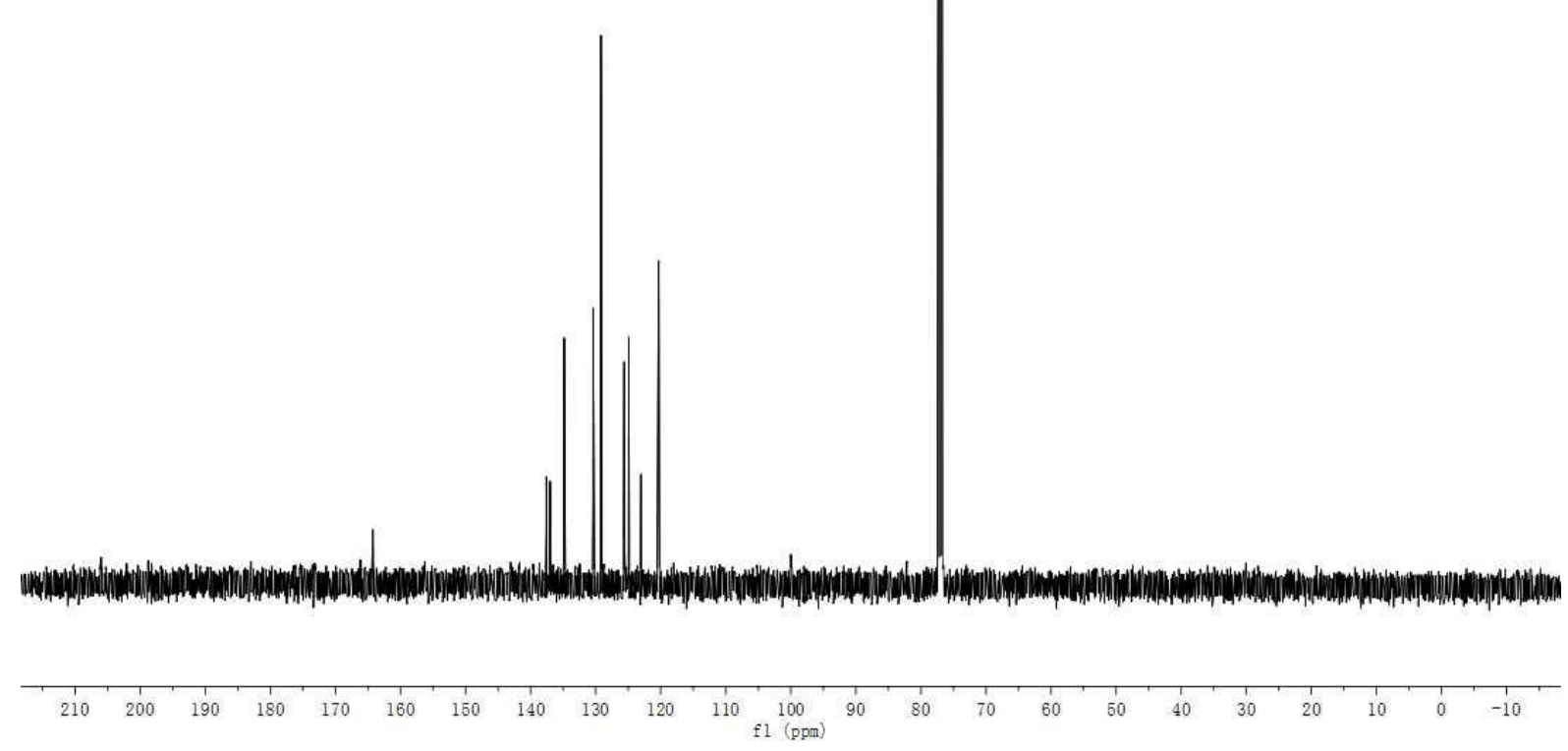
${ }^{1} \mathrm{H}$ NMR (500 MHz, DMSO-d6)
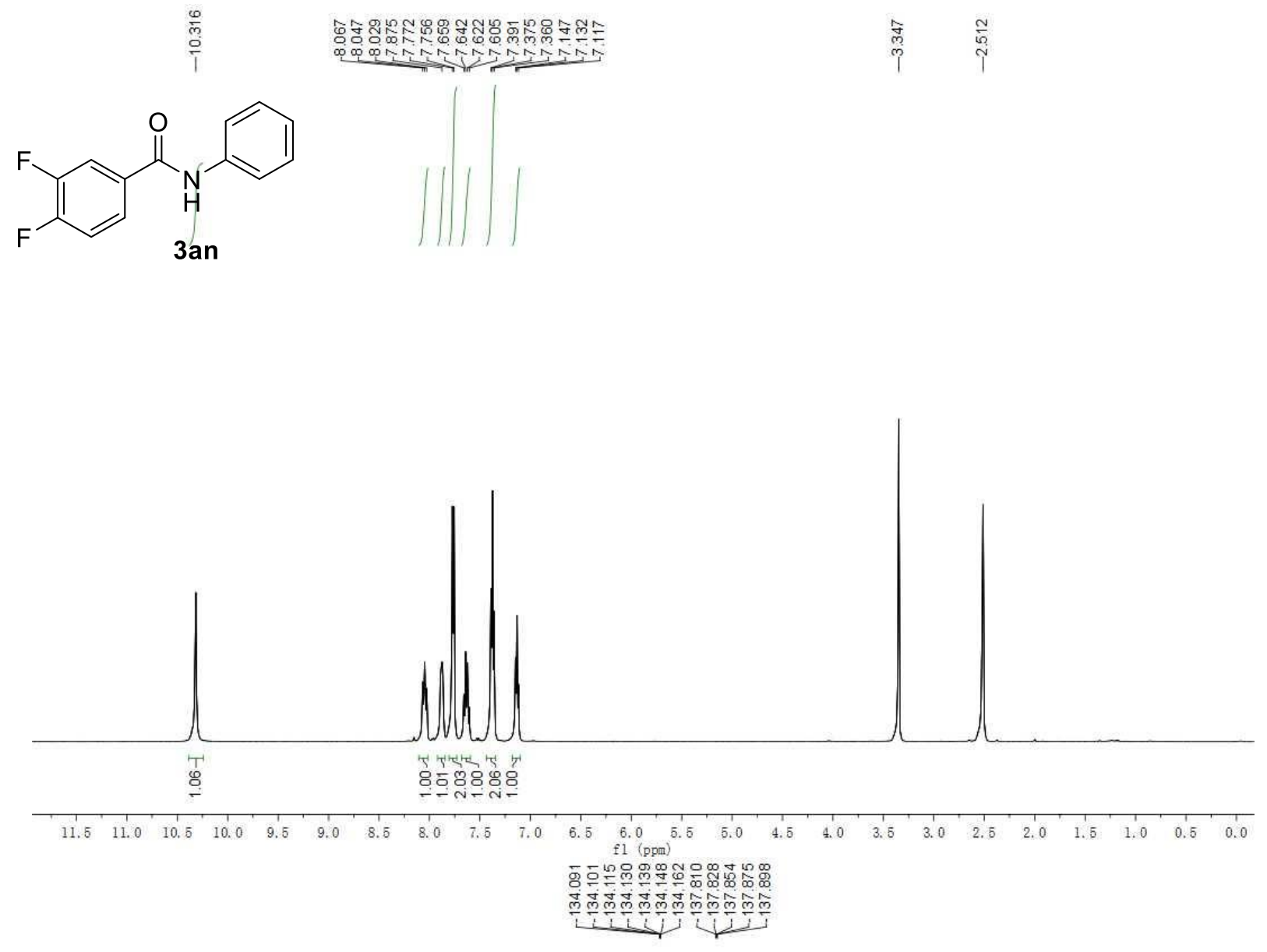

${ }^{19}$ F NMR (471 MHz, DMSO-d6)

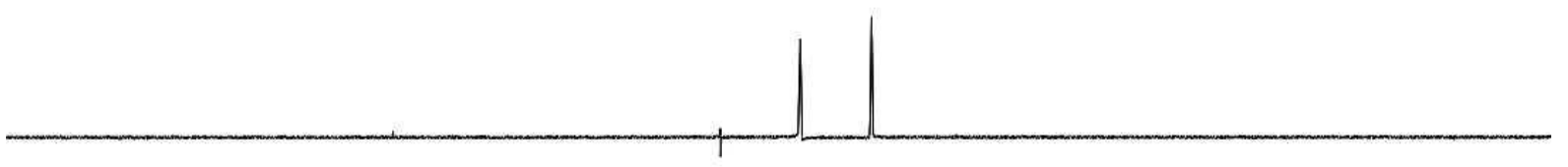

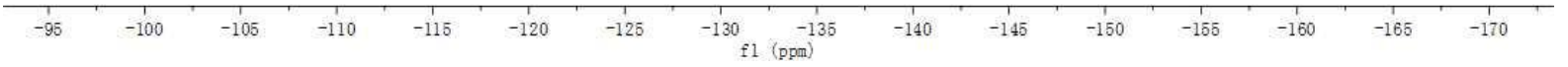


${ }^{1} \mathrm{H}$ NMR (500 MHz, $\left.\mathrm{CDCl}_{3}\right)$
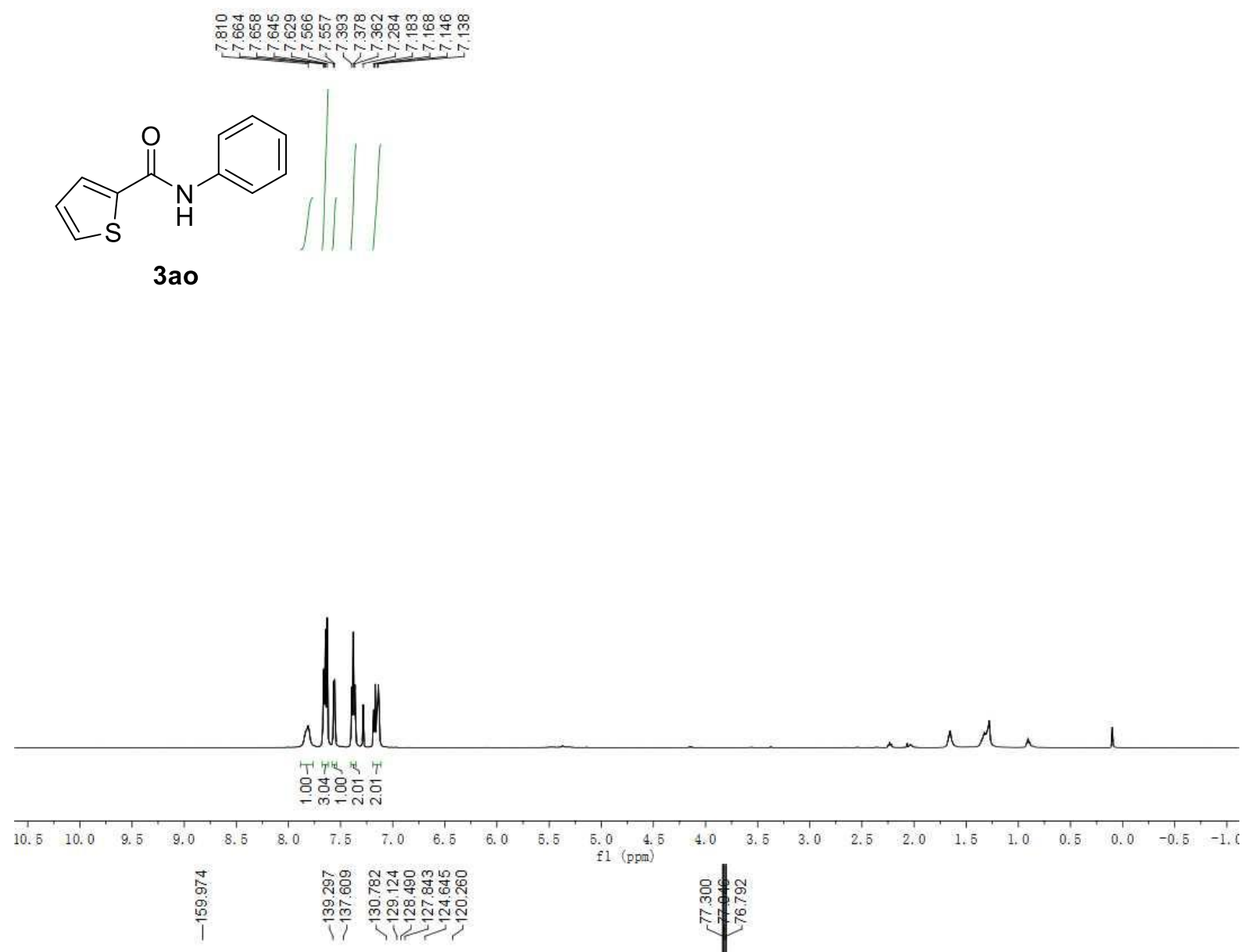

${ }^{13} \mathrm{C} \mathrm{NMR}\left(125 \mathrm{MHz}, \mathrm{CDCl}_{3}\right)$
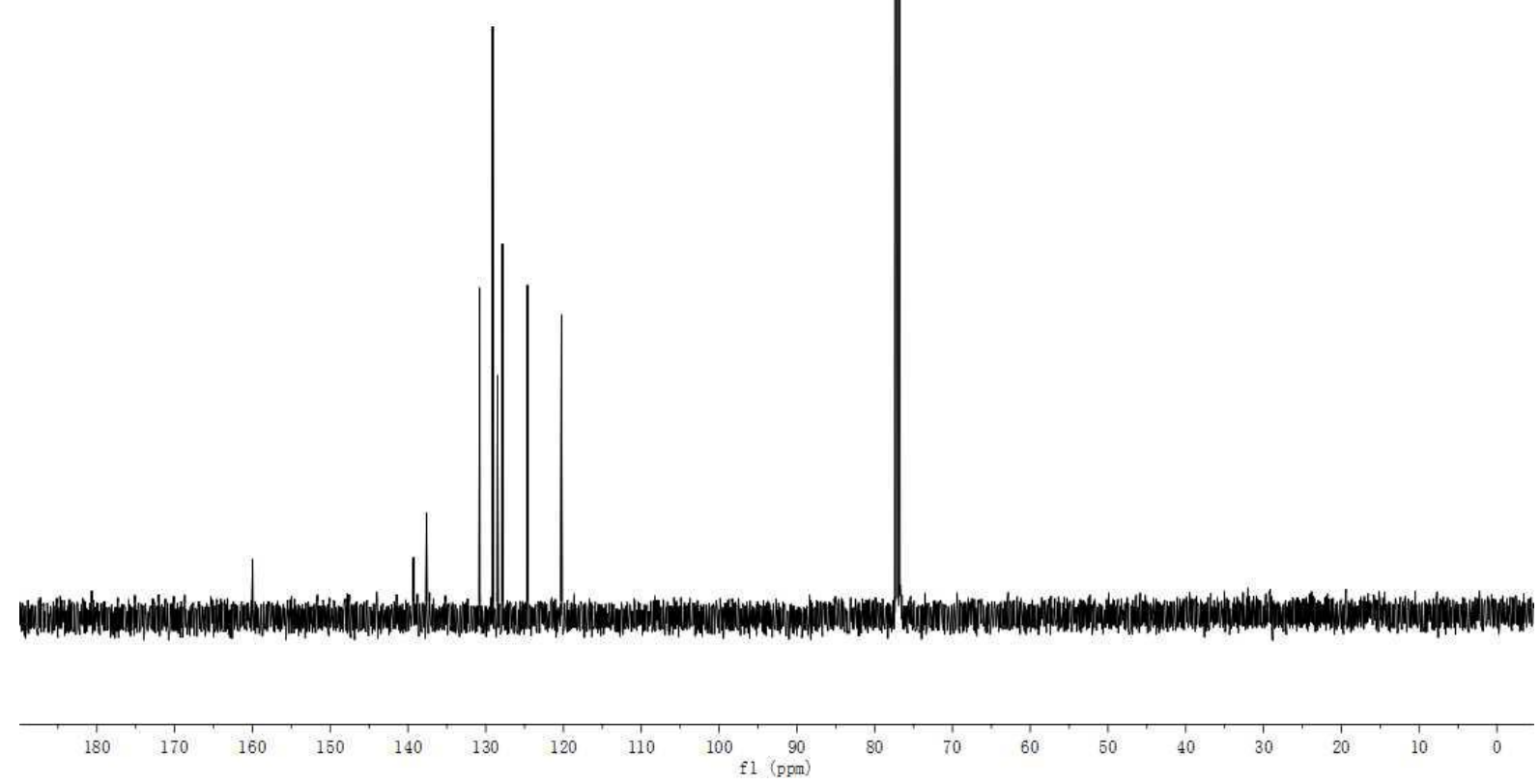
${ }^{1} \mathrm{H} \mathrm{NMR}\left(500 \mathrm{MHz}, \mathrm{CDCl}_{3}\right)$<smiles>CC(=O)Nc1ccccc1</smiles>

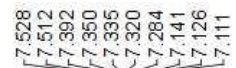

$3 a p$
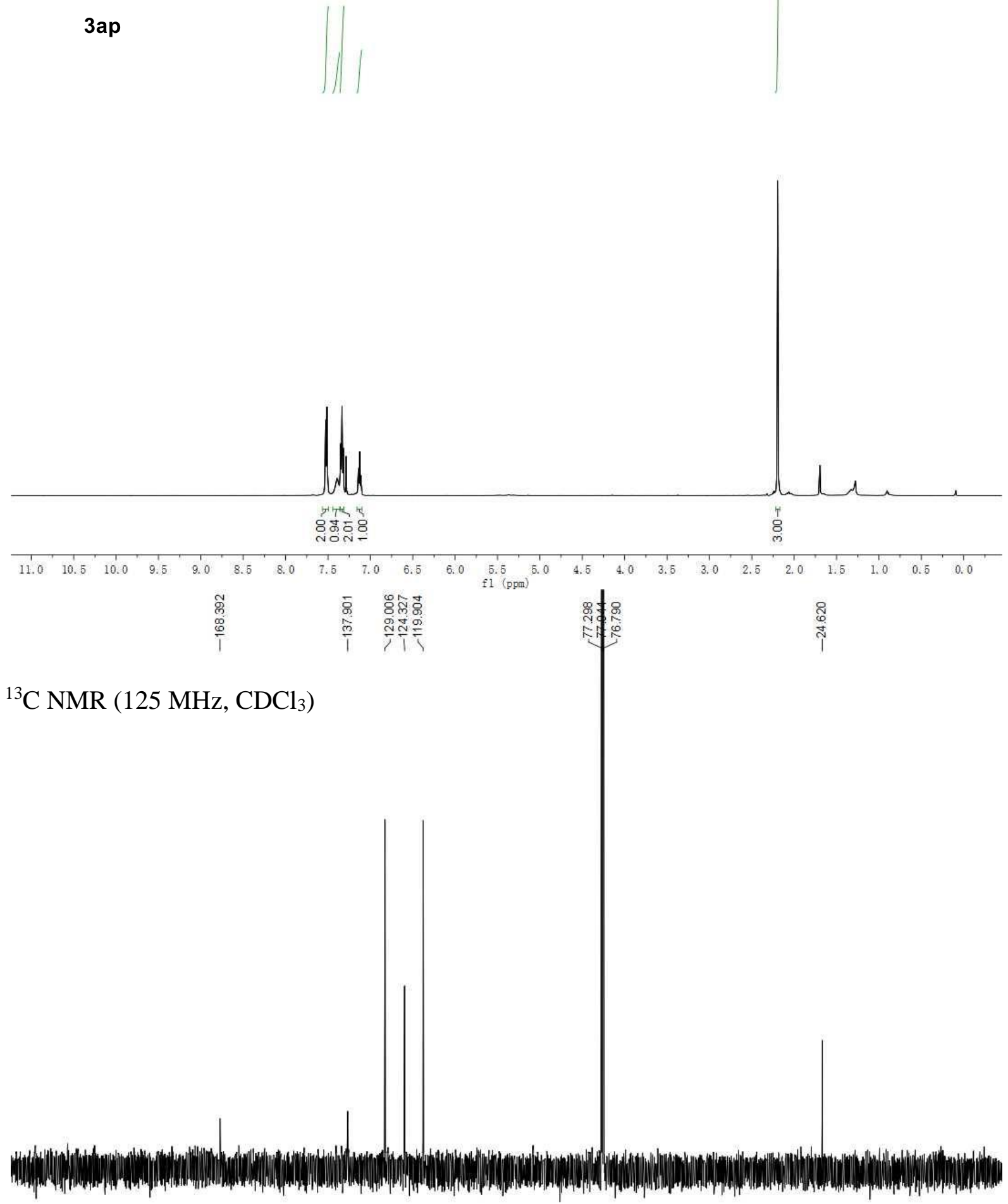

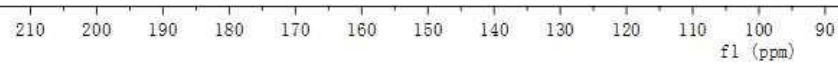


${ }^{1} \mathrm{H}$ NMR (500 MHz, $\mathrm{CDCl}_{3}$ )
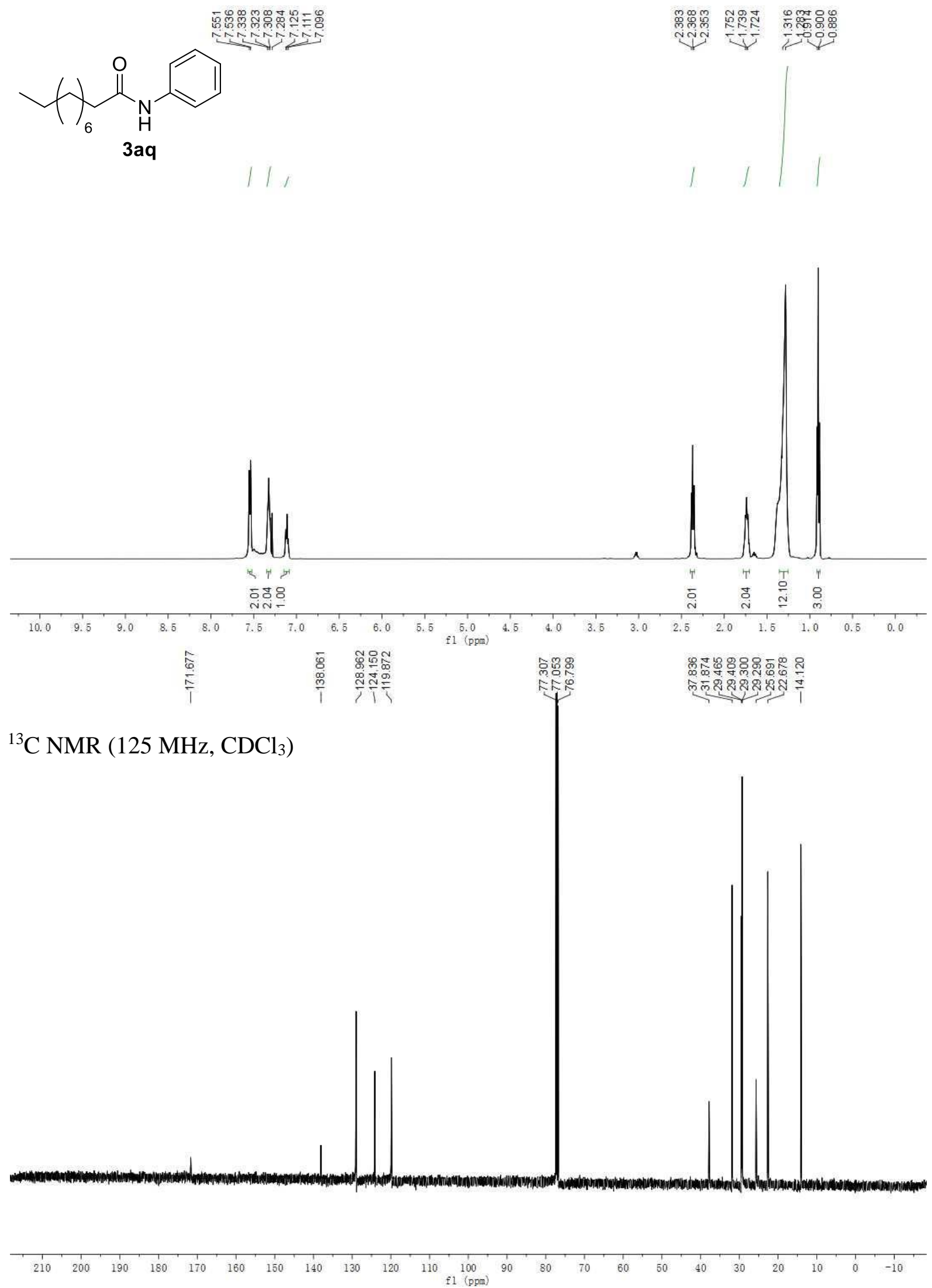
${ }^{1} \mathrm{H} \mathrm{NMR}\left(500 \mathrm{MHz}, \mathrm{CDCl}_{3}\right)$
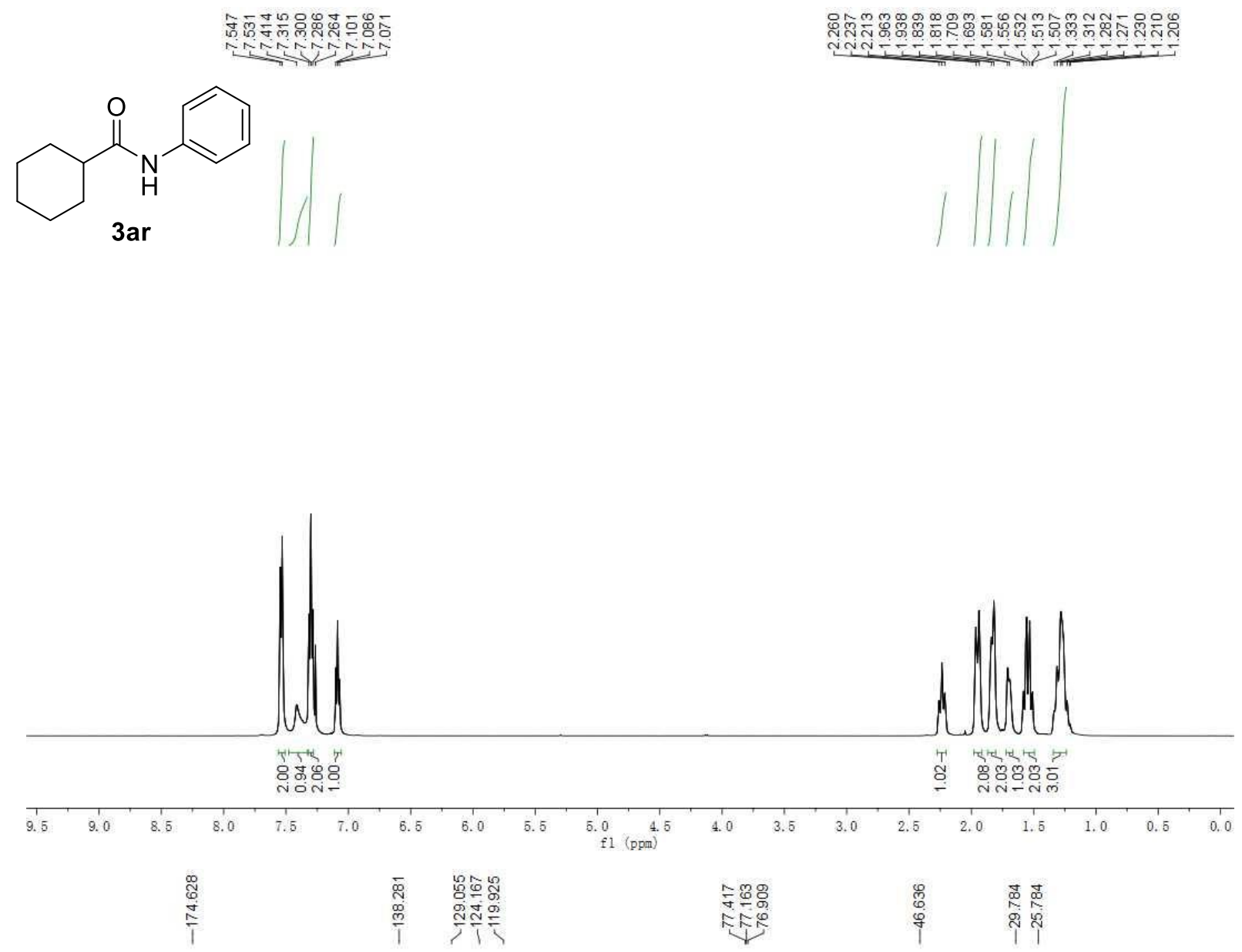

${ }^{13} \mathrm{C}$ NMR (125 MHz, $\left.\mathrm{CDCl}_{3}\right)$
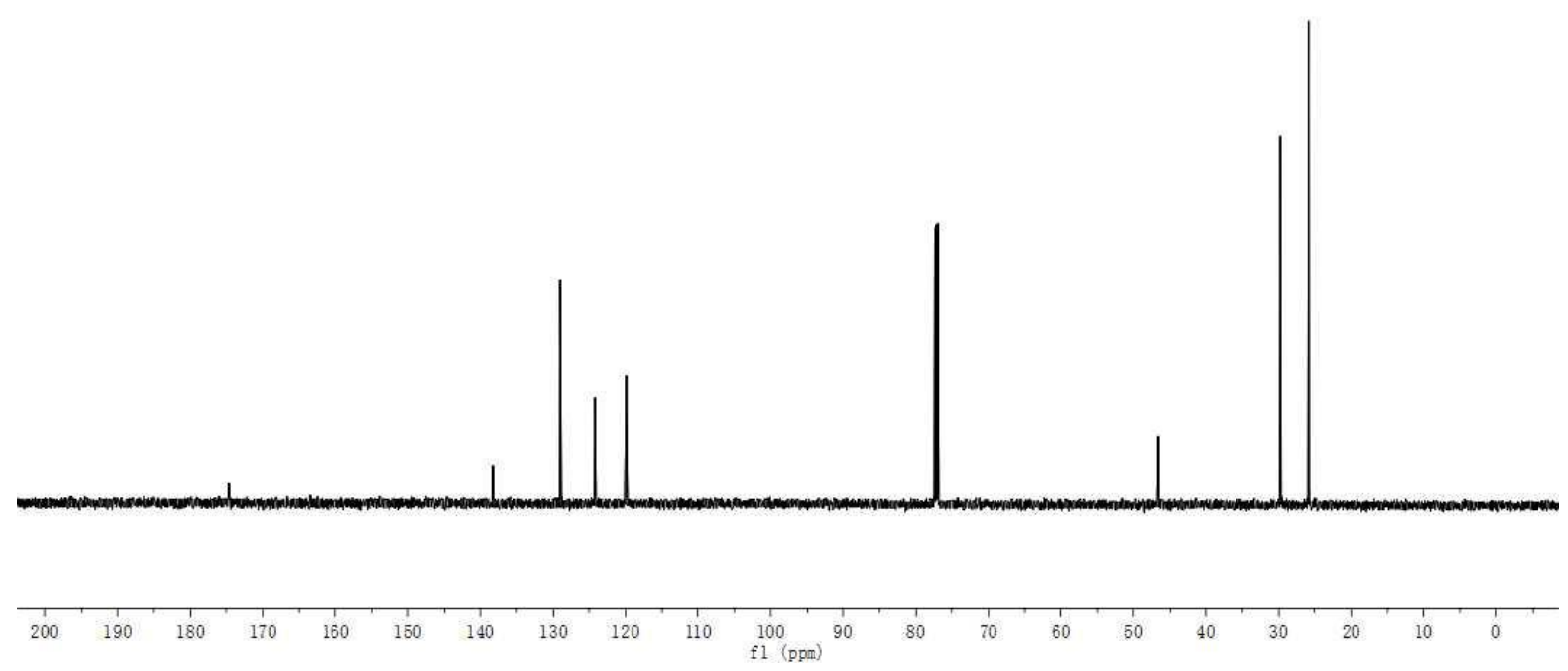
${ }^{1} \mathrm{H} \mathrm{NMR}\left(500 \mathrm{MHz}, \mathrm{CDCl}_{3}\right)$
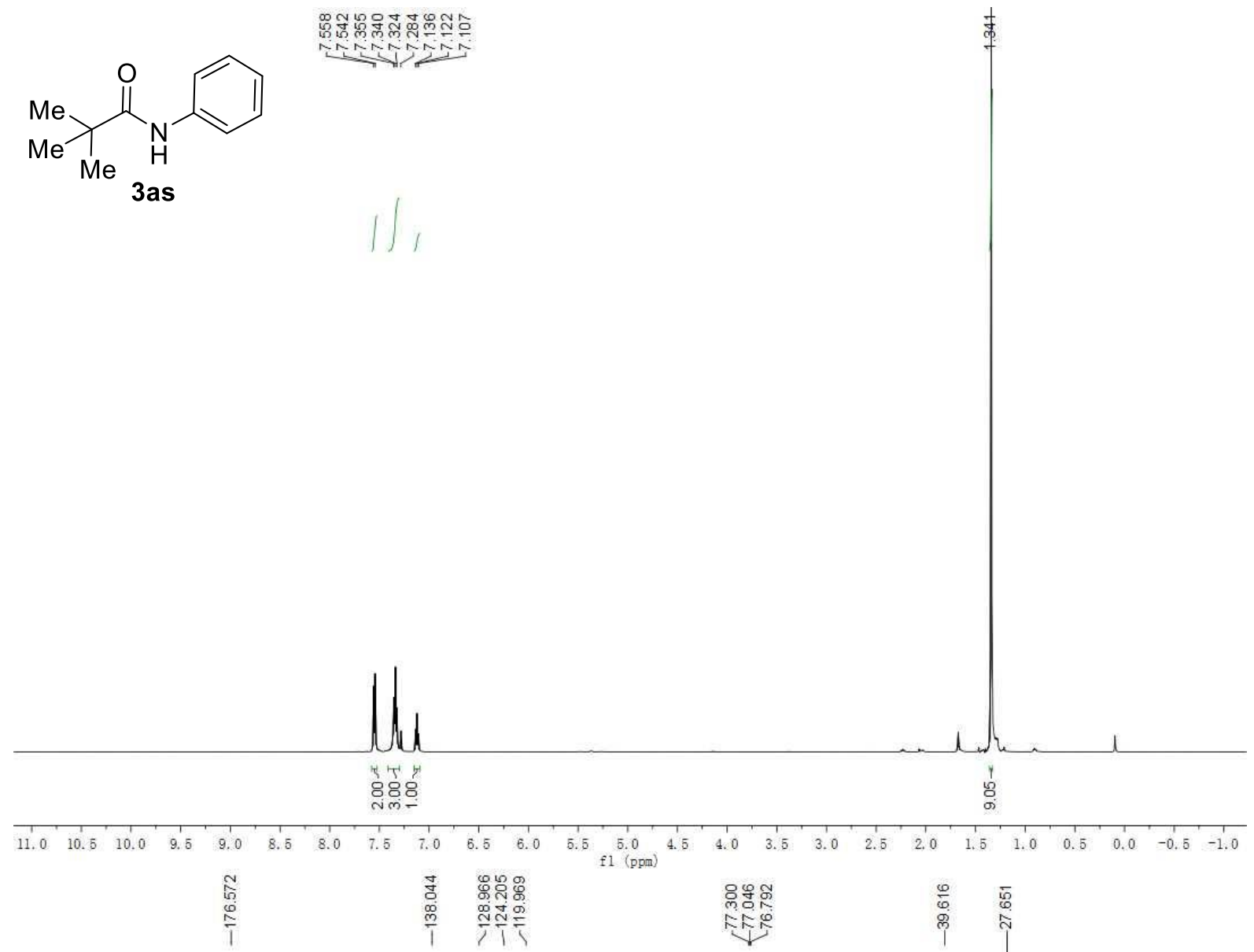

${ }^{13} \mathrm{C} \mathrm{NMR}\left(125 \mathrm{MHz}, \mathrm{CDCl}_{3}\right)$
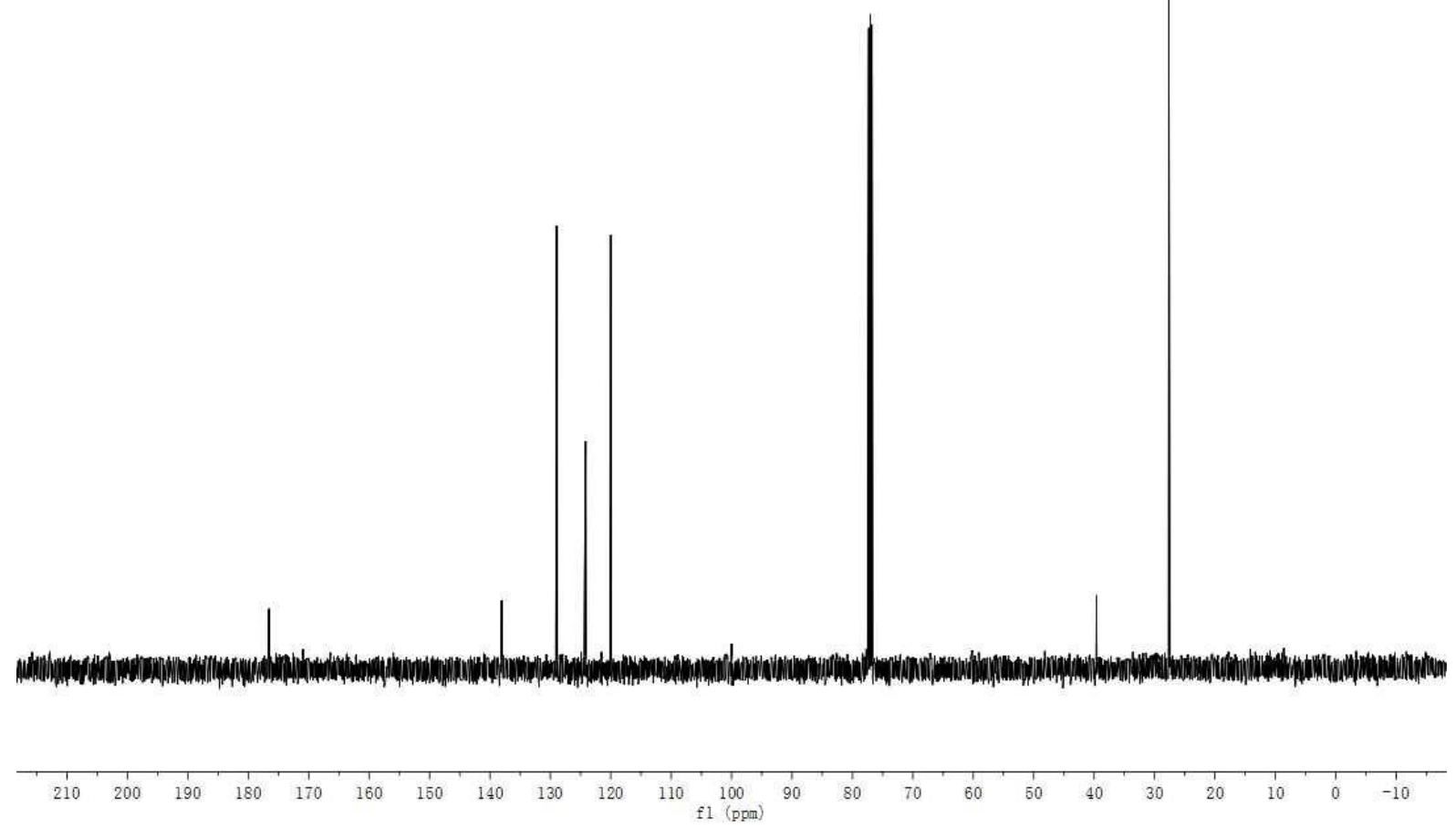
${ }^{1} \mathrm{H} \mathrm{NMR}\left(500 \mathrm{MHz}, \mathrm{CDCl}_{3}\right)$

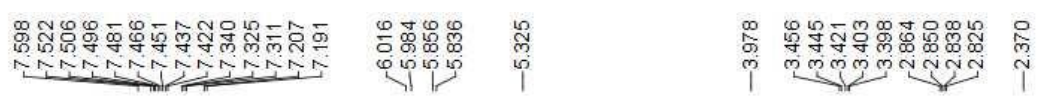<smiles>Cc1ccc(NC(=O)c2ccccc2C2C=CCNC2)cc1</smiles>

3at

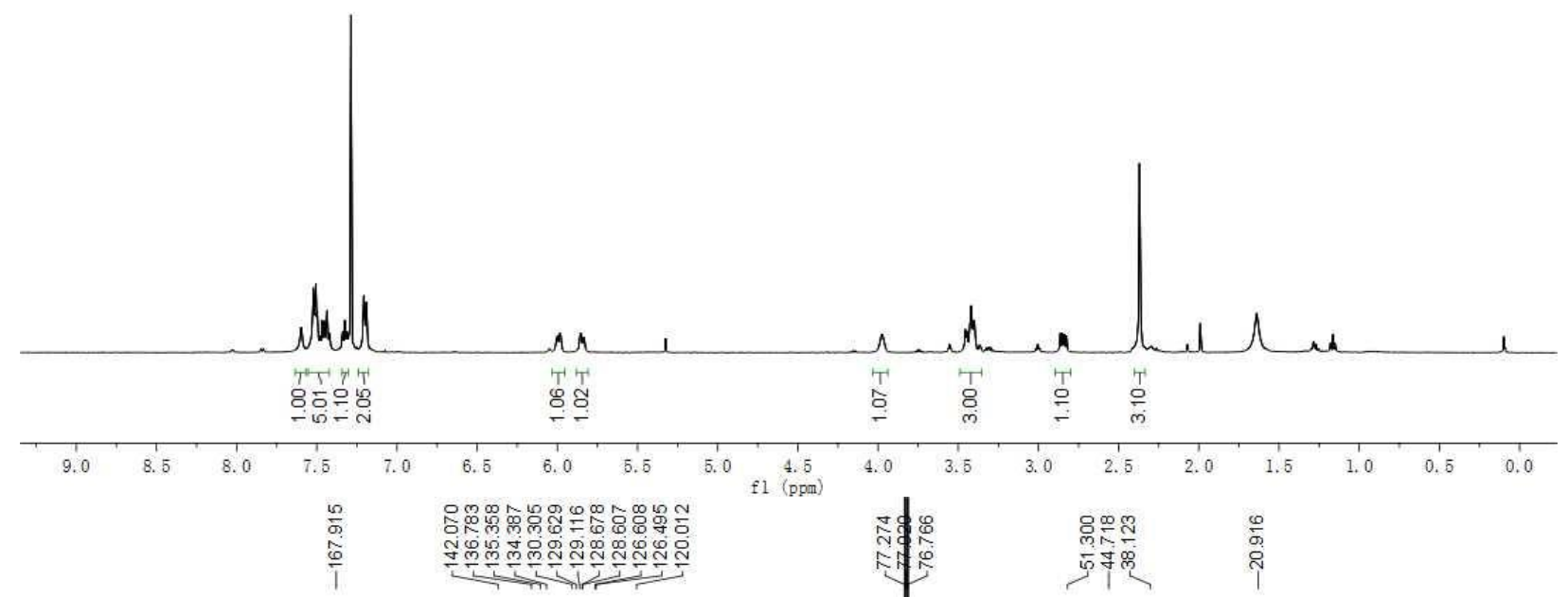

${ }^{13} \mathrm{C}$ NMR $\left(125 \mathrm{MHz}, \mathrm{CDCl}_{3}\right)$
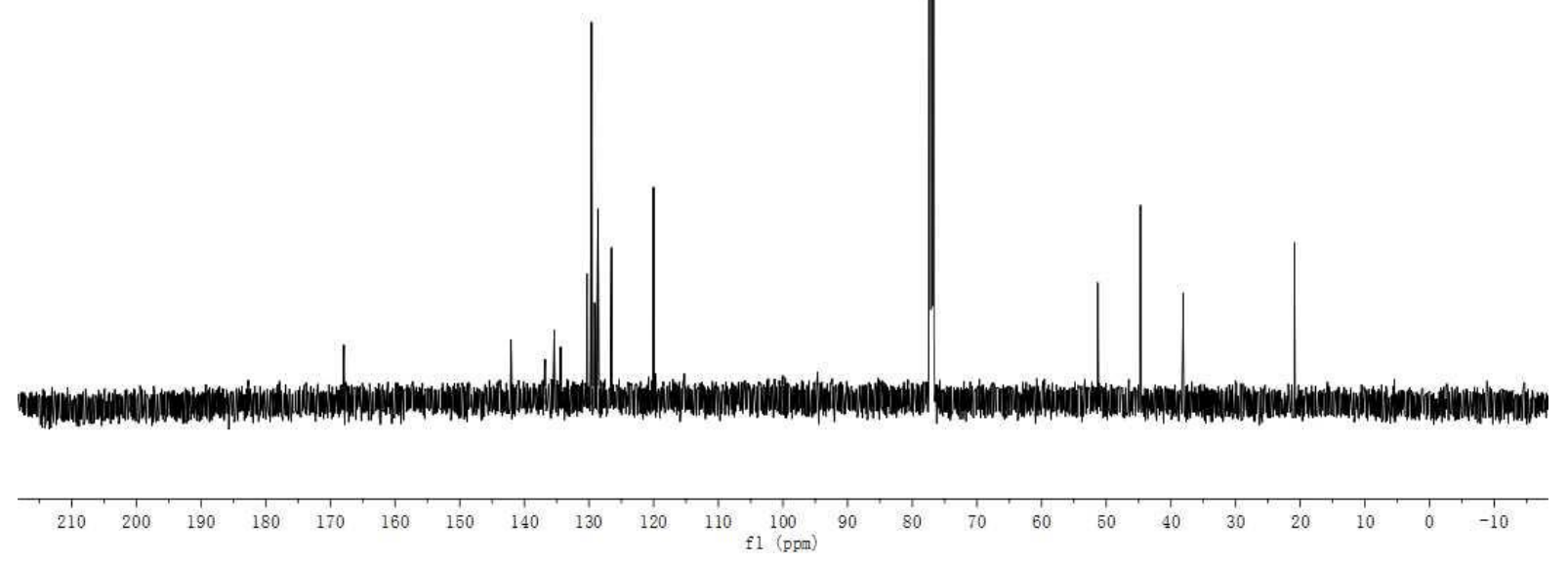
${ }^{1} \mathrm{H} \mathrm{NMR}\left(500 \mathrm{MHz}, \mathrm{CDCl}_{3}\right)$

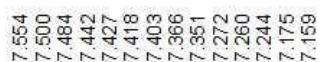

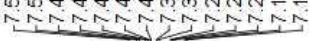<smiles>Cc1ccc(NC(=O)c2ccccc2C2CCCNC2)cc1</smiles>

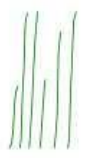

3au

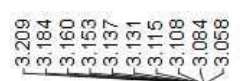

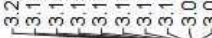

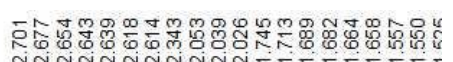

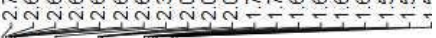

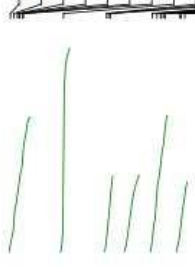

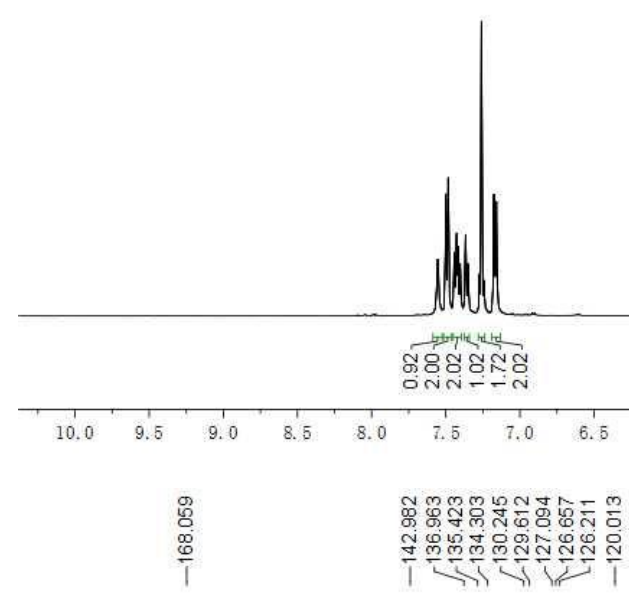

${ }^{13} \mathrm{C} \mathrm{NMR}\left(125 \mathrm{MHz}, \mathrm{CDCl}_{3}\right)$
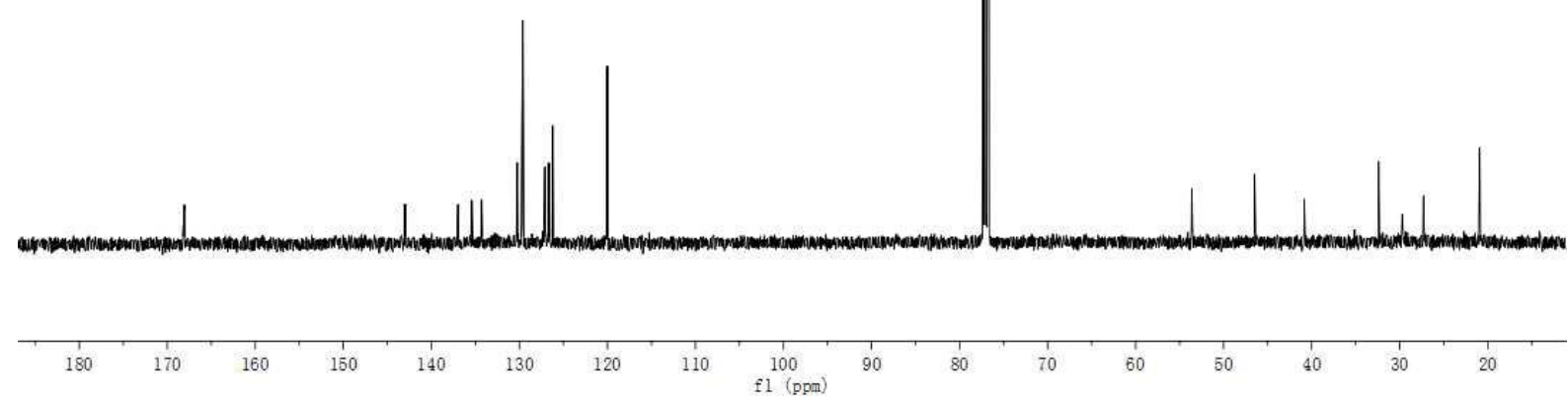
${ }^{1} \mathrm{H}$ NMR (500 MHz, $\left.\mathrm{CDCl}_{3}\right)$

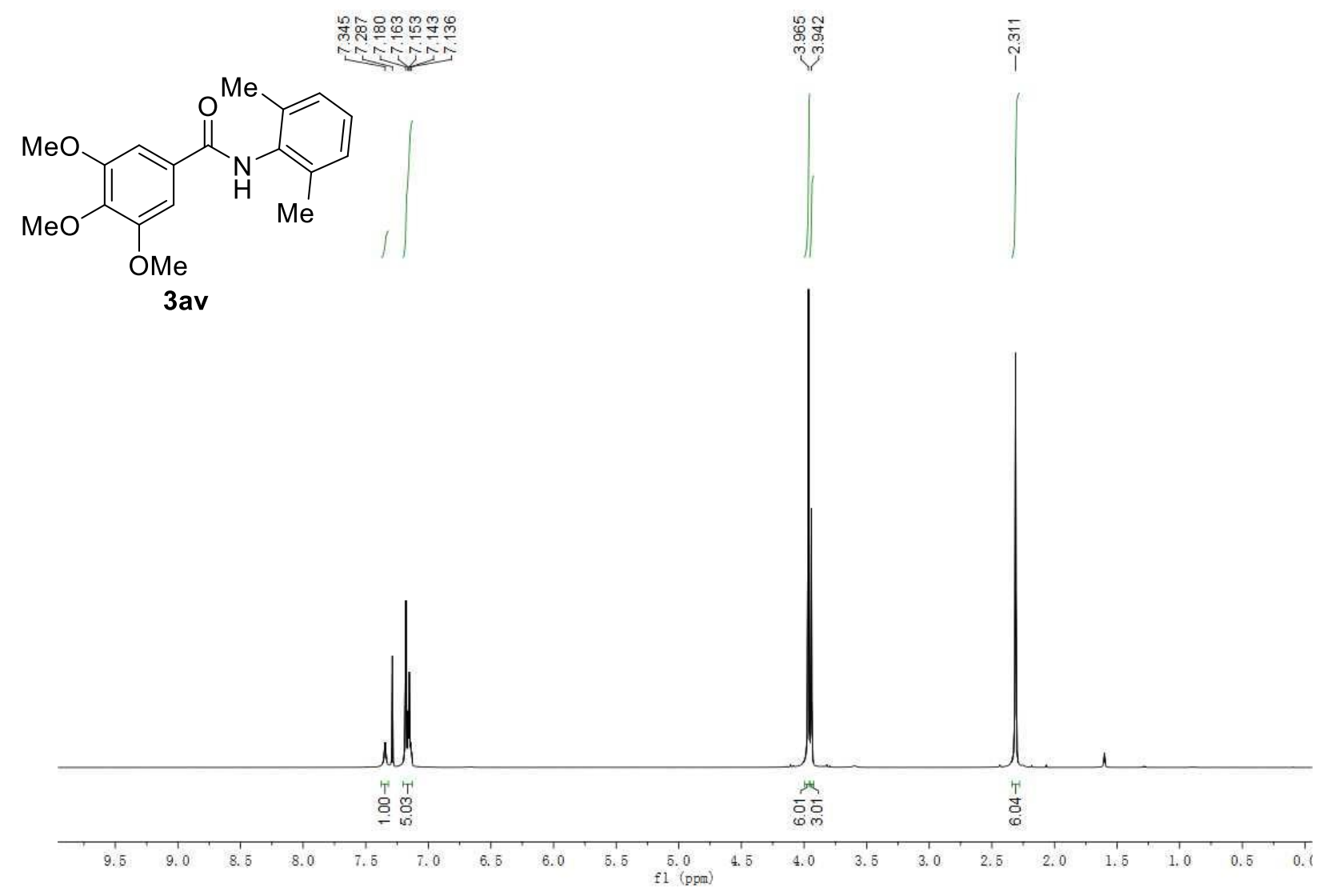

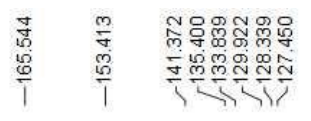

总

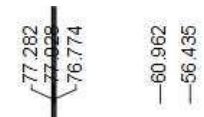

สู
ก
$\infty$

${ }^{13} \mathrm{C} \mathrm{NMR}\left(125 \mathrm{MHz}, \mathrm{CDCl}_{3}\right)$

(ppm)

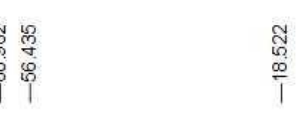

C
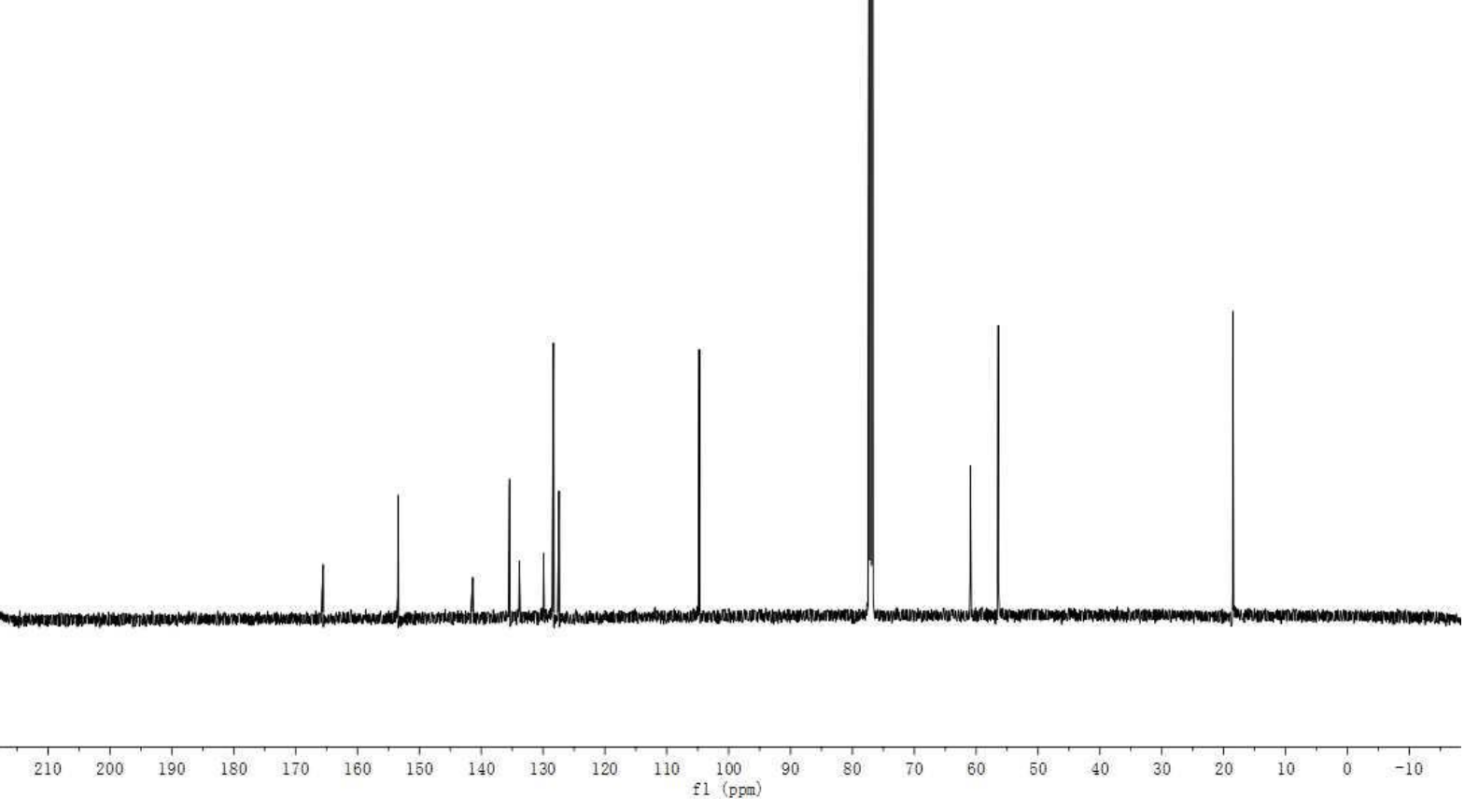
${ }^{1} \mathrm{H}$ NMR $\left(500 \mathrm{MHz}, \mathrm{CDCl}_{3}\right)$

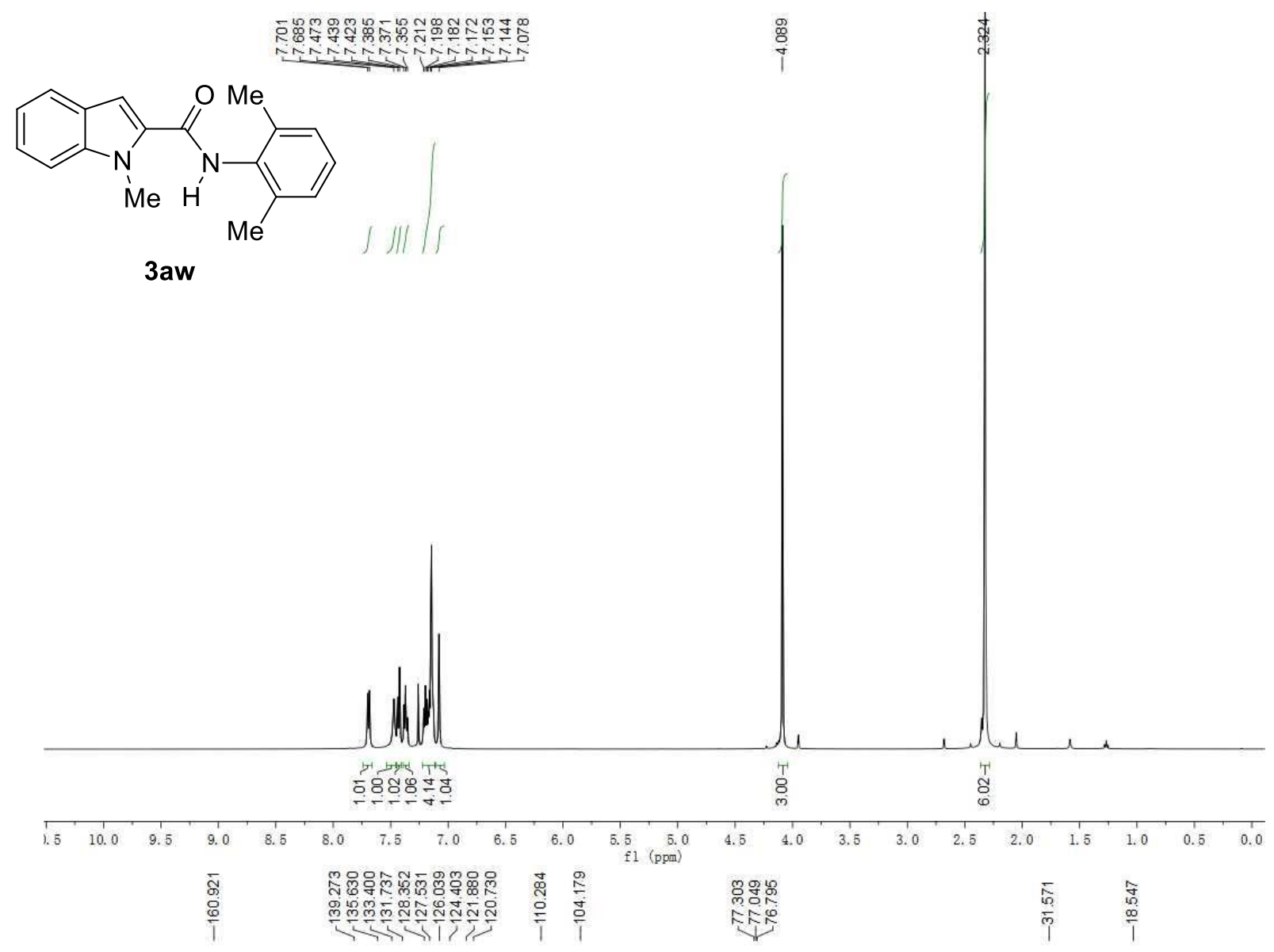

${ }^{13} \mathrm{C} \mathrm{NMR}\left(125 \mathrm{MHz}, \mathrm{CDCl}_{3}\right)$

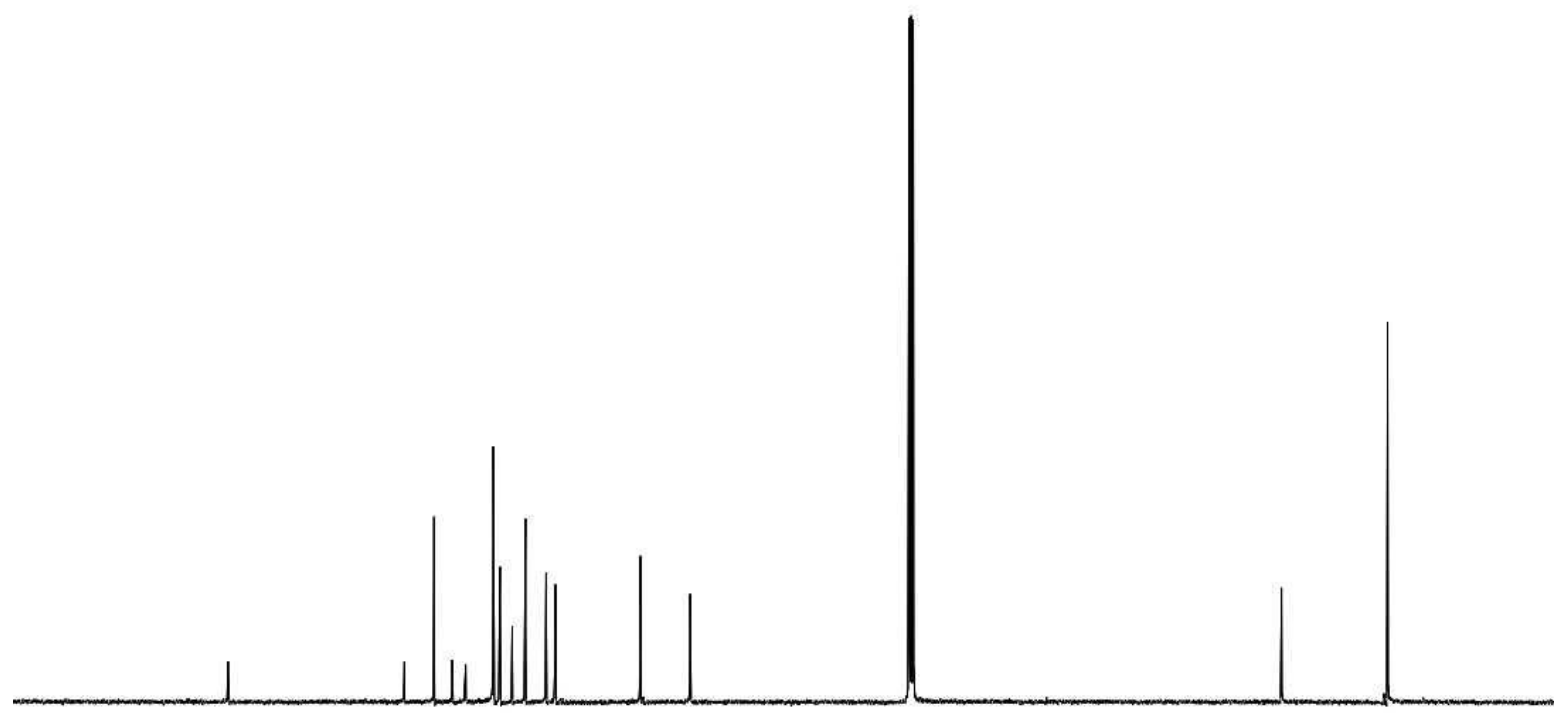

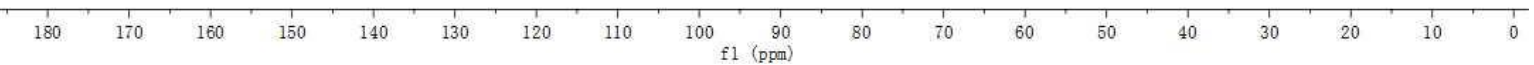


${ }^{1} \mathrm{H}$ NMR (500 MHz, DMSO-d6)

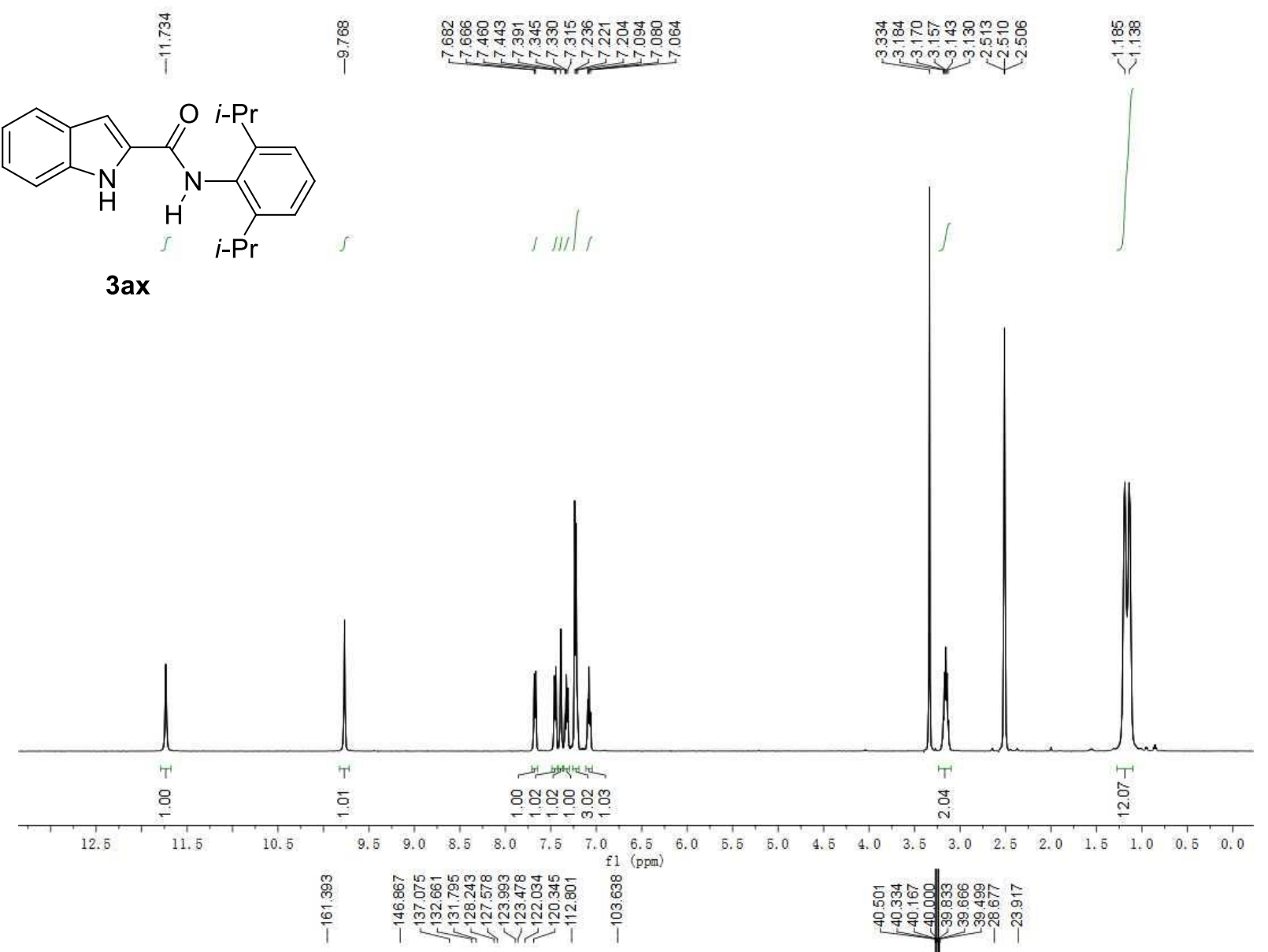

${ }^{13} \mathrm{C}$ NMR (125 MHz, DMSO-d6)

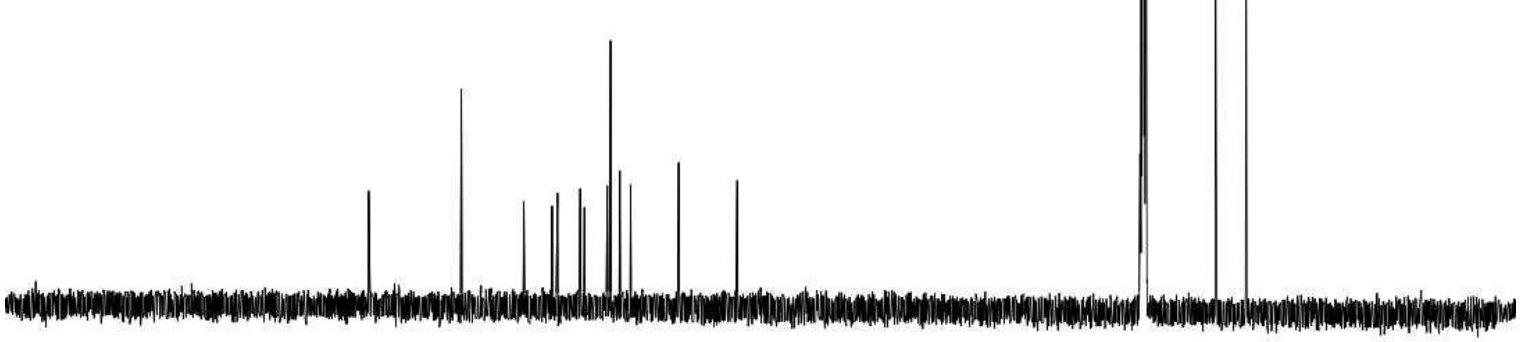

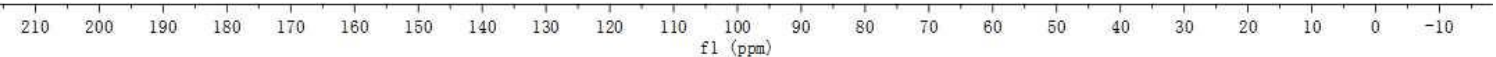


${ }^{1} \mathrm{H} \mathrm{NMR}\left(500 \mathrm{MHz}, \mathrm{CDCl}_{3}\right)$

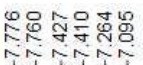

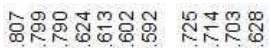

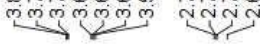<smiles>CCCN1CCOCC1CCNC(=O)c1ccc(Cl)cc1</smiles>

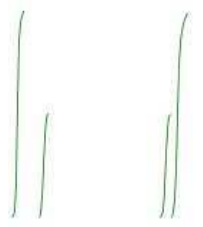

3ay

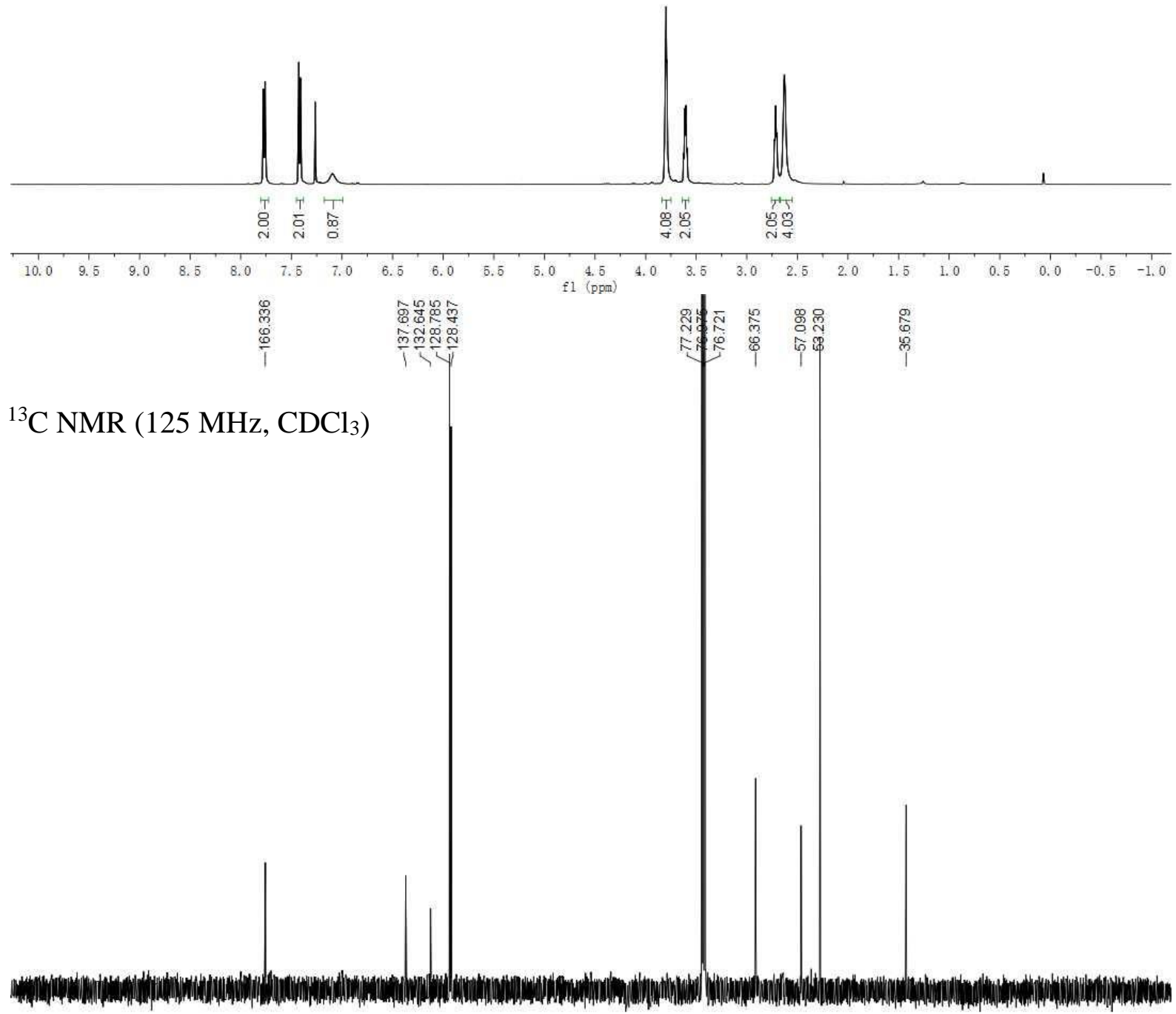

$\begin{array}{lllllllllllllllllllllllll}1 & 210 & 200 & 190 & 180 & 170 & 160 & 150 & 140 & 130 & 120 & 110 & 100 & 90 & 80 & 70 & 60 & 50 & 40 & 30 & 20 & 10 & 0 & -10 & 1\end{array}$ 
${ }^{1} \mathrm{H}$ NMR (500 MHz, $\left.\mathrm{CDCl}_{3}\right)$
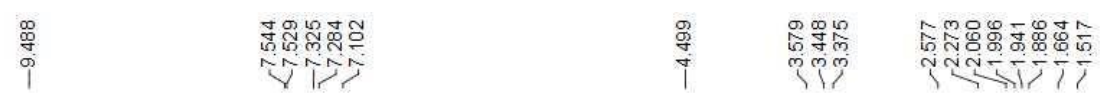<smiles>O=C(Nc1ccccc1)[C@@H]1CCCN1C(=O)O</smiles>

$3 \mathrm{az}$
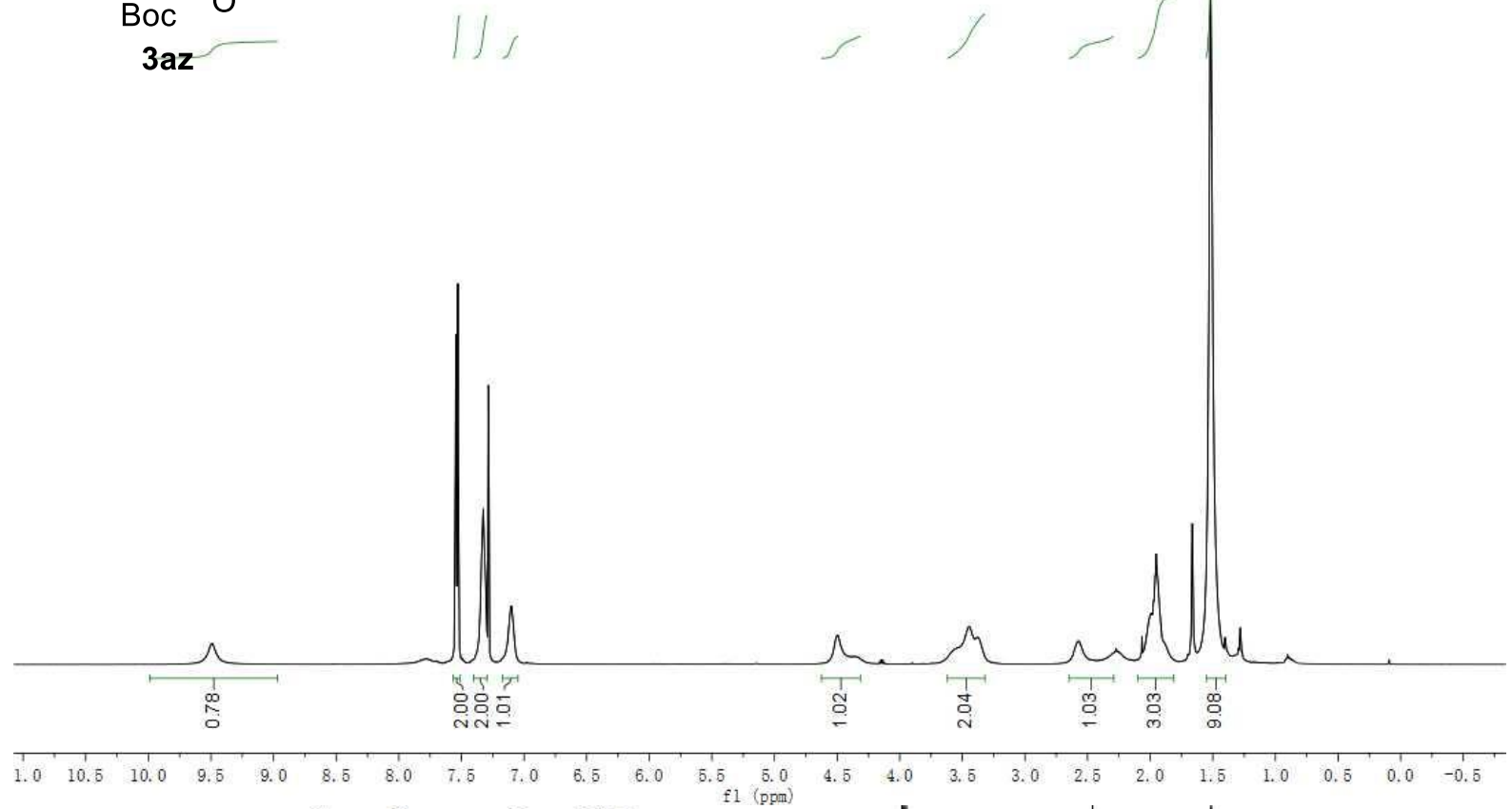

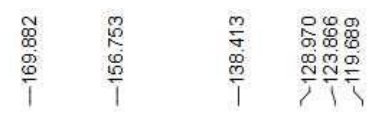

${ }^{13} \mathrm{C} \mathrm{NMR}\left(125 \mathrm{MHz}, \mathrm{CDCl}_{3}\right)$

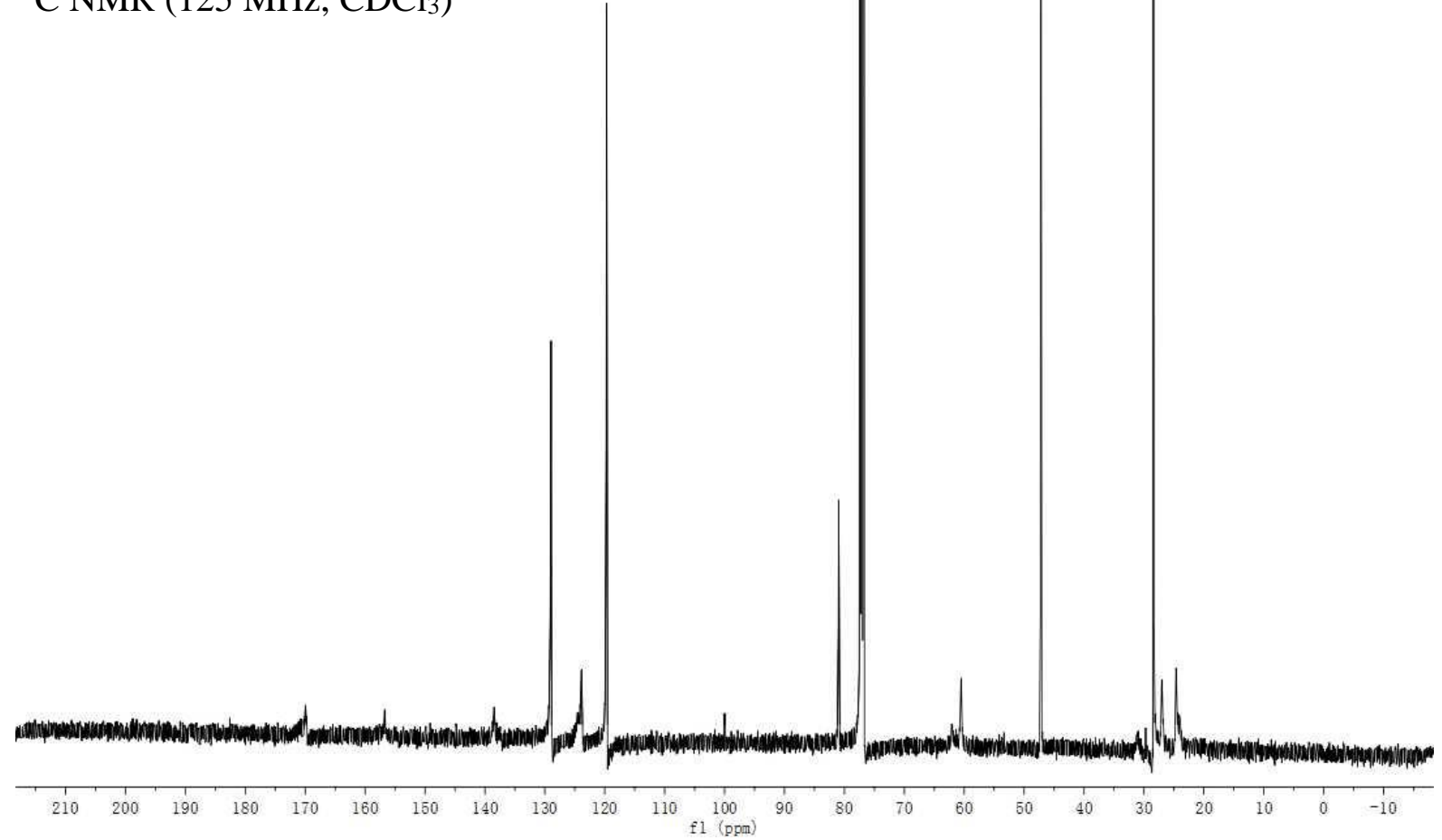


${ }^{1} \mathrm{H}$ NMR $\left(500 \mathrm{MHz}, \mathrm{CDCl}_{3}\right)$

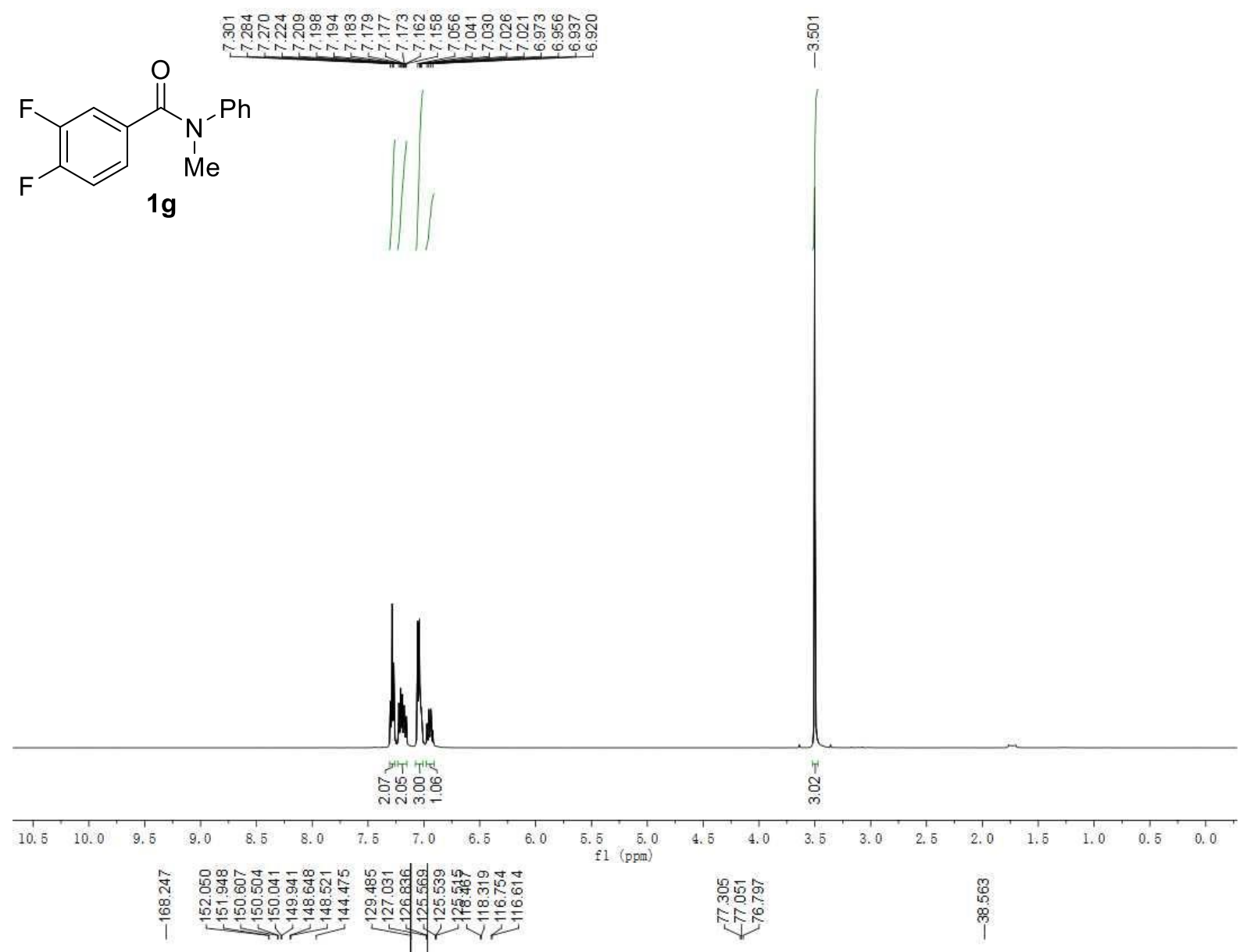

${ }^{13} \mathrm{C} \mathrm{NMR}\left(125 \mathrm{MHz}, \mathrm{CDCl}_{3}\right)$
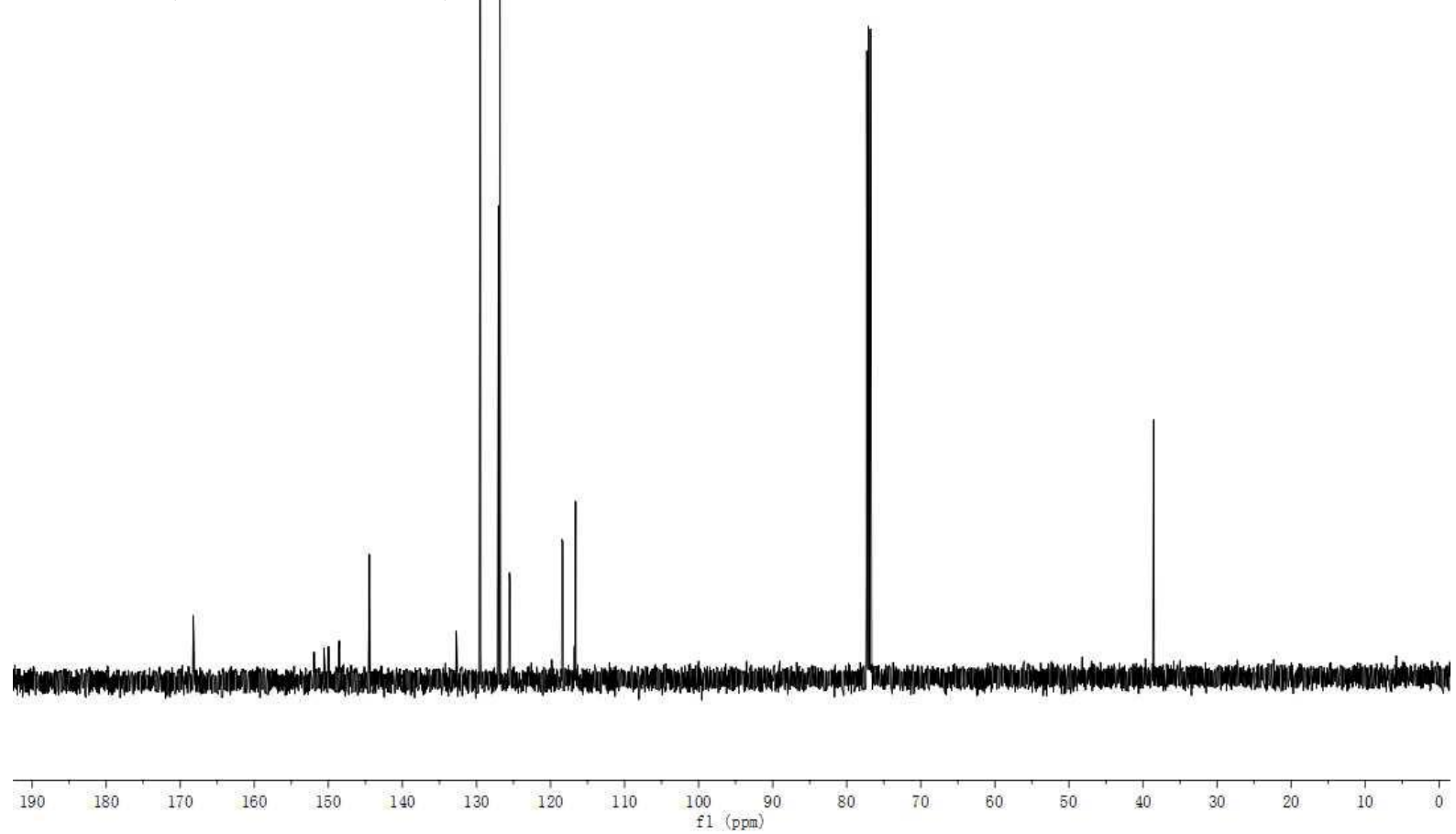
${ }^{19} \mathrm{~F}$ NMR $\left(500 \mathrm{MHz}, \mathrm{CDCl}_{3}\right)$

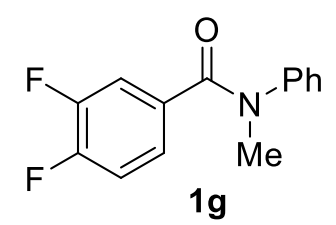

$1 \mathrm{~g}$

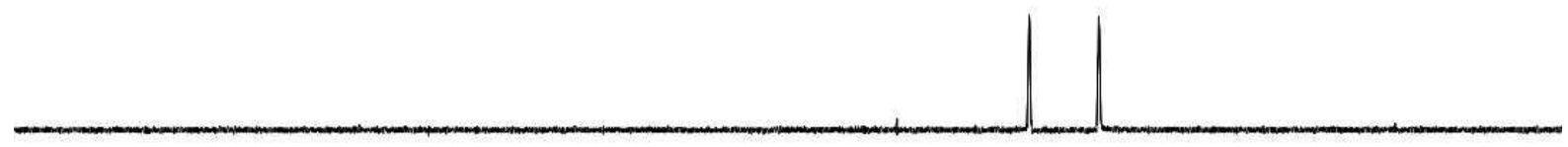

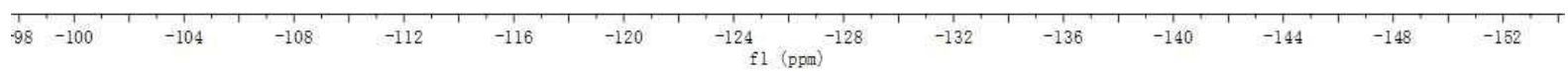

